\title{
Chemical Composition of
}

Sedimentary Rocks in

\section{California and Hawaii}

GEOLOGICALSURVEY PROFESSIONALAAPER 1097

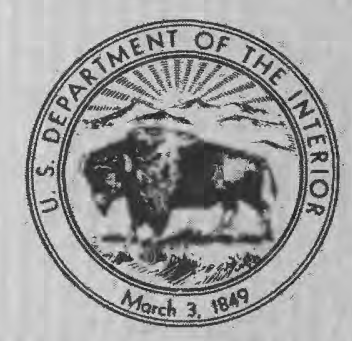




\section{Chemical Composition of Sedimentary Rocks in California and Hawaii}

Compiled by Thelma P. HiL

GE O L O G I A L S URVEY PROFES S I N A L PA PER 1097

A compilation of 2,312 analyses

published before 1974

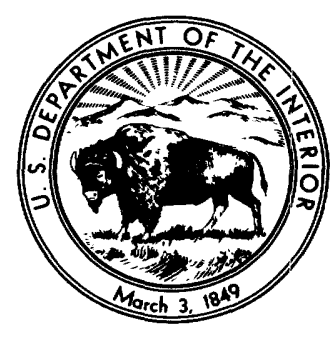

UNITED STATES GOVERNMENT PRINTING OFFICE, WASHINGTON : 1981 


\section{UNITED STATES DEPARTMENT OF THE INTERIOR JAMES G. WATT, Secretary}

\section{GEOLOGICAL SURVEY}

Dallas L. Peck, Director

Library of Congress catalog-card No. 81-600092 


\section{CONTENTS}

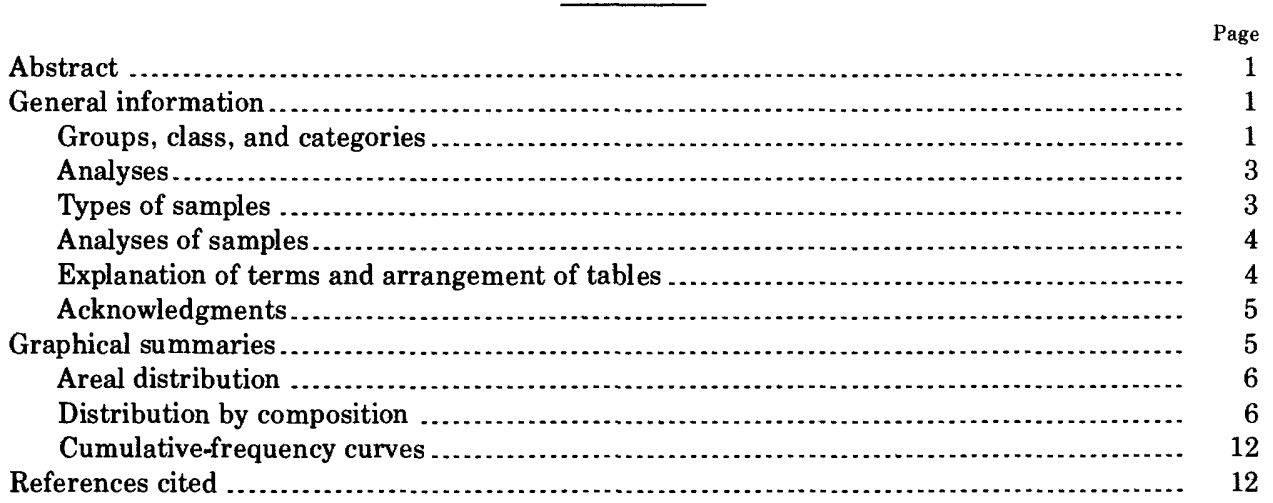

\section{ILLUSTRATIONS}

FigurE 1. Triangular diagram of composition of sedimentary rocks, showing the nine fields into which the chemical analyses are grouped

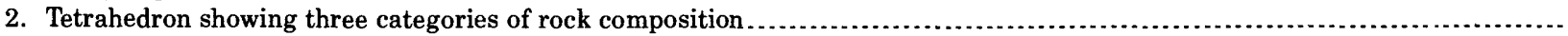

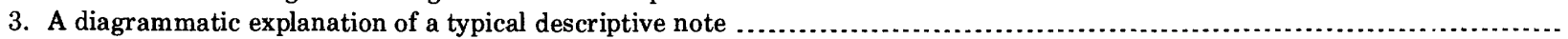

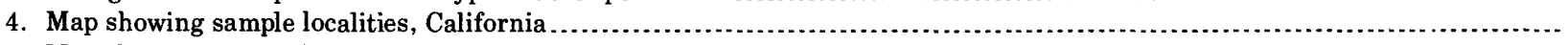

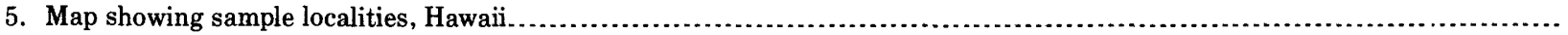

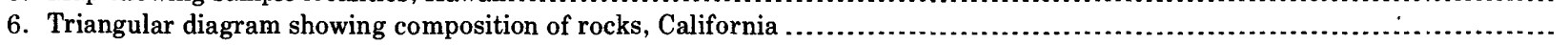

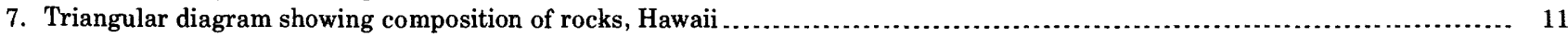

8. Cumulative-frequency curves showing percentages of various constituents:

$A$, Group A, California.

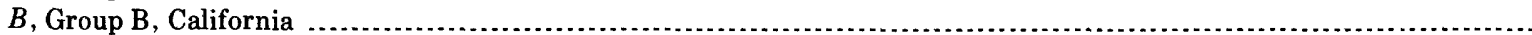

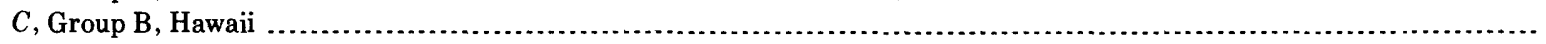

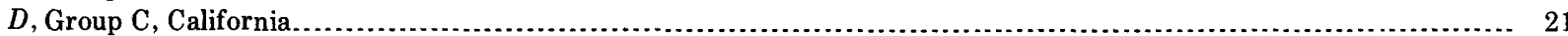

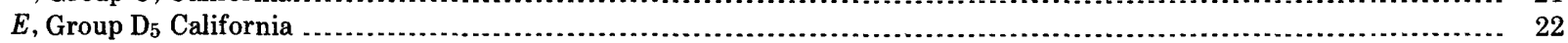

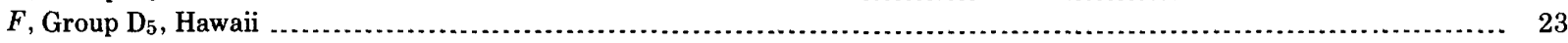

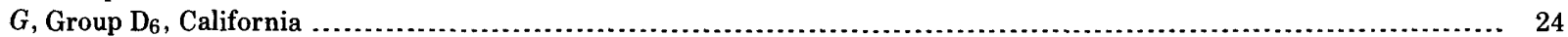

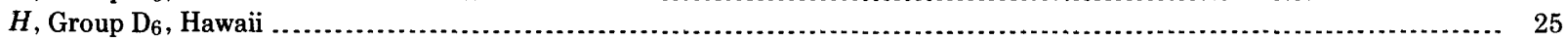

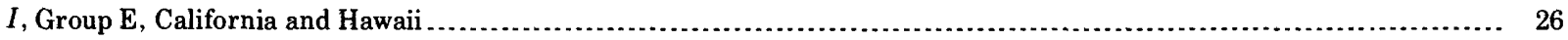

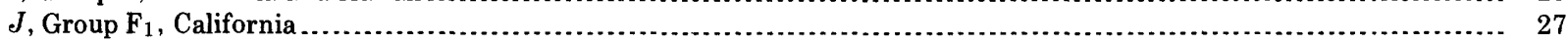

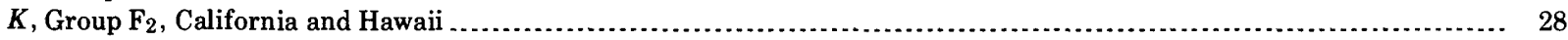

\section{TABLES}

TABLE 1. Analyses of samples from California containing 90-100 percent uncombined silica (Group $A_{3}$ ), common- and mixed-rock

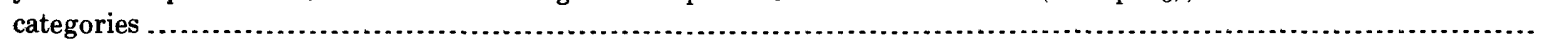

2. Analyses of samples from California containing 75-90 percent uncombined silica (Group A4), common- and mixed-rock categories

3. Analyses of samples from California and Hawaii containing uncombined silica and clay each less then 75 percent; uncombined silica and clay each greater than carbonate (Group B), common- and mixed-rock categories ..........................

4. Analyses of samples from California containing uncombined silica and carbonate each less than 75 percent; each greater than clay (group C), common- and mixed-rock categories.

5. Analyses of samples from California and Hawaii containing 75-90 percent clay (Group $\mathrm{D}_{5}$ ), common- and mixed-rock categories 
TABLE 6. Analyses of samples from California and Hawaii containing 90-100 percent clay (Group $\mathrm{D}_{6}$ ), from all rock categories .

7. Analyses of samples from California and Hawaii containing clay and carbonate each less than 75 percent: clay and carbonate each greater than uncombined silica (Group E), common- and mixed-rock categories .

8. Analyses of samples from California containing 75-90 percent carbonate (Group $F_{1}$ ), common- and mixed-rock categories...

9. Analyses of samples from California and Hawaii containing more than 90 percent carbonate (Group $\mathrm{F}_{2}$ ), common-rock category

10. Analyses of samples from California containing 50 percent or more sodium chloride (Group Na), special-rock category .

11. Analyses of samples from California containing 50 percent or more miscellaneous sulfate- and carbonate-bearing material (Group S), special-rock category

12. Analyses of samples from California containing 50 percent or more gypsum, anhydrite, or gypsite (Group G), special-rock category

13. Analyses of samples celestite and strontianite (Group Sr), from California, special-rock category

14. Analyses of samples of boron-bearing rocks (Group $B_{1}$ ) from California, special-rock category .

15. Analyses of samples of various types of rocks (Group M) from California and Hawaii, special-rock category

16. Analyses of samples of phosphate rock (Group P) from California, special-rock category

17. Analyses of samples of manganiferous opal (Group $\mathrm{Mn}$ ) from California, special-rock category

18. Number of samples of various rock types in the classification groups $A \cdot F_{2}$, common- and mixed-rock categories, California ...

19. Numbers of samples of various rock types in the classification groups of the special-rock category,California

20. Number of samples of various rock types in the classification groups, Hawaii

21. Distribution of samples by State, age, stratigraphic unit, county, and classification group

22. Distribution of 2,312 samples by State, category, and group

23. Code numbers for State and County

24. Code numbers for use of rock.

25. Code numbers of authors. 


\title{
CHEMICAL COMPOSITION OF SEDIMENTARY ROCKS IN CALIFORNIA AND HAWAII
}

\author{
Compiled by Thelma P. Hill
}

\begin{abstract}
A compilation of published chemical analyses of sedimentary rocks of the United States was undertaken by the U.S. Geological Survey in 1952 to make available scattered data that are needed for a wide range of economic and scientific uses. About 20,000-25,000 chemical analyses of sedimentary rocks in the United States have been published. This report brings together 2,312 of these analyses from California and Hawaii.
\end{abstract}

The samples are arranged by general lithologic characteristics and locality. Indexes of stratigraphy, rock name, commercial uses, and minor elements are provided.

The sedimentary rocks are classified into groups and into categories according to the chemical analyses. The groups (A through $\mathbf{F}_{2}$ ) are defined by a system similar to that proposed by Brian Mason in 1952, in which the main parameters are the three major components of sedimentary rocks: (1) uncombined silica, (2) clay $\left(\mathrm{R}_{2} \mathrm{O}_{3} \cdot 3 \mathrm{SiO}_{2} \cdot \mathrm{nH}_{2} \mathrm{O}\right)$, and (3) calcium-magnesium carbonate. The categories are based on the degree of admixture of these three major components with other components, such as sulfate, phosphate, and iron oxide. Common-rock, mixed-rock, and special-rock categories apply to rocks consisting of 85 percent or more, 50-84 percent, and less than 49 percent, respectively, of the three major components combined.

Maps show distribution of sample localities by States; triangular diagrams show the lithologic characteristics and classification groups. Cumulative-frequency curves of each constituent in each classification group of the common-rock and mixed-rock categories are also included.

The numerous analyses may not adequately represent the geochemical nature of the rock types and formations of the region because of sampling bias. Maps showing distribution of sample localities indicate that many of the localities are in areas where, for economic or other reasons, special problems attracted interest.

Most of the analyzed rocks tended to be fairly simple in composition - mainly mixtures of just two of the three major components or a mixture of these and a fourth component such as phosphate, gypsum, or iron oxide.

\section{GENERAL INFORMATION}

In 1952 , the U.S. Geological Survey began a project to compile published analyses of sedimentary rocks of the United States. The first report, covering the States of Colorado, Kansas, Montana, Nebraska, North Dakota, South Dakota, and Wyoming, was published as U.S. Geological Survey Professional Paper 561 (Hill and others, 1967); the second report, covering the States of Alaska, Idaho, Oregon, and Washington, was published as Professional Paper 771 (Hill and Werner, 1972). The present report covers the States of Califor- nia and Hawaii, and in most respects it is similar to the first and second reports.

The classification of the analyses into groups and categories was based on work done by computer. The tables of analyses and descriptive notes were arranged and printed out by computer. The cumulative-frequency curves were also based on statistical work done by computer. The triangular diagrams were drawn by a computer-controlled plotter.

The chemical analyses of sedimentary rocks were taken from many publications, and as a result the constituents determined, as well as the methods used for determination, vary widely. Uniformity seemed desirable in presenting the analyses in this report, so several general rules were formulated. Although information concerning some of the analyses is probably inadequate for certain purposes, a more critical selection can be made by the reader. The chemical analyses are shown in tables 1-17.

\section{GROUPS, CLASS, AND CATEGORIES}

Groups.-The analyses are classified into groups $\mathrm{A}_{3}$ through $\mathrm{F}_{2}$, following a system modified from the one proposed by Mason (1952, p. 130, 131), in which the relative proportions of the three major components of sedimentary rocks are utilized. These components are (1) uncombined silica, (2) an arbitrary clay molecule $\left(\mathrm{R}_{2} \mathrm{O}_{3} \cdot 3 \mathrm{SiO}_{2} \cdot n \mathrm{H}_{2} \mathrm{O}\right)$, and (3) calcium and magnesium carbonate. When the proportions are plotted on a triangular diagram (fig. 1), the following symmetrical groups can be identified:

\begin{tabular}{|c|c|c|}
\hline Group & Identification & Explanation \\
\hline$A_{3} \ldots$ & Silica & $\begin{array}{l}\text { Uncombined silica, } 90- \\
100 \text { percent. }\end{array}$ \\
\hline$A_{4} \ldots$ & Silica _.. & $\begin{array}{l}\text { Uncombined silica, } 75-90 \\
\text { percent. }\end{array}$ \\
\hline B ... & Mixed silica and clay $\ldots \ldots$ & $\begin{array}{l}\text { Uncombined silica and } \\
\text { clay, each less than } \\
75 \text { percent; uncom- } \\
\text { bined silica and clay, } \\
\text { each equal to or more } \\
\text { than carbonate. }\end{array}$ \\
\hline
\end{tabular}


C .... Mixed silica and carbonate _

$\mathrm{D}_{5} \ldots$ Clay
$\mathrm{D}_{6} \ldots$ Clay
$\mathrm{E} \ldots \mathrm{n}$... Mixed clay and carbonate

$F_{1} \ldots$ Carbonate $\ldots \ldots \ldots \ldots \ldots$

$\mathrm{F}_{2}$ _.. Carbonate

For the purpose of assigning analyses into compositional groups, the percentage of each major component was based on the total of all three major components. However, the percentages shown in the tables of analyses are based on total sample weight; therefore, those percentages are somewhat different than the compositional percentages.

Class.-The class figures are the calculated total amounts of the three major components in weight percent. The figures for silica, clay, and carbonate are given in that order at the bottom of each analysis.

Samples that contained 33 percent or more calcium-magnesium carbonate were given a notation indicating the ratio of calcium to magnesium unless it exceeded 100 , and then the notation is simply $\mathrm{Ca} / \mathrm{Mg}>$ 100. The ratio appears at the end of each descriptive note.

Categories.-The categories are based on the degree to which the three major components used to define the groups are admixed with other materials. The categories are defined by the following amounts of the

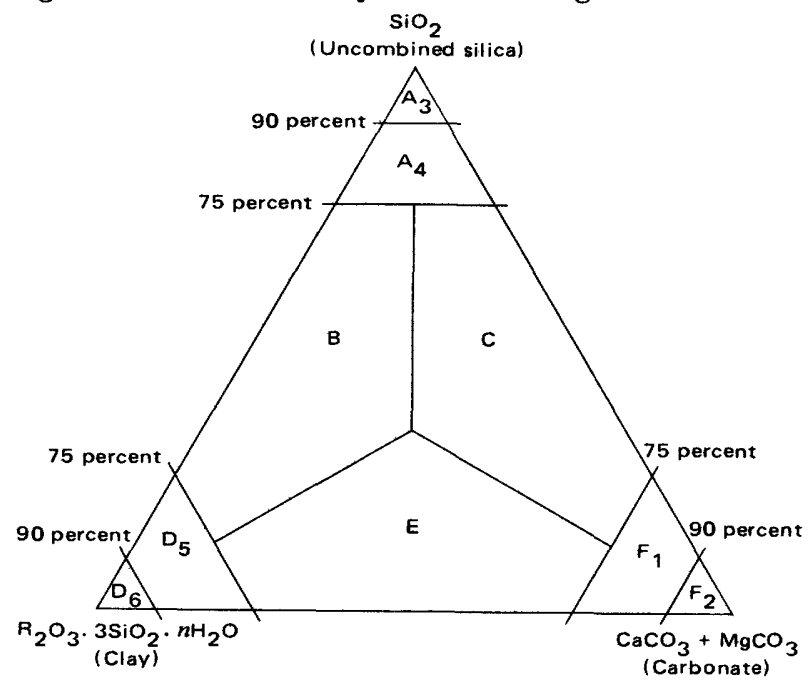

FIGURE 1.-Compósition of sedimentary rocks, showing the nine fields into which the chemical analyses are grouped. three main components: silica, clay, and carbonate.

Percent
85 or more

The common- and mixed-rock categories are assembled by group, State, county, and position within the county. Samples of common- and mixed-rock categories are intermixed in the tables of analyses, but the two may be distinguished by adding up the three class figures (last line of each analysis). A total of 85-100 percent indicates the common-rock category; 50-84 percent, the mixed-rock category. Table 6 also includes some Hawaiian clay samples from the special-rock category in which the class total of the three main constituents is less than 50 percent. The rest of the analyses of the special-rock category are in tables 10-17.

The special-rock category is divided according to the kind of material admixed with the major component of silica, clay, and carbonate. The numerical limits of the special-rock category were not strictly observed for all samples; some samples were included in this category because of unusual composition or other factors.

The relation of the three general categories to one another is illustrated by the tetrahedron in figure 2 , which represents a four-component classification system. The base of the tetrahedron may be taken as the triangular diagram of figure 1 , its three corners being,

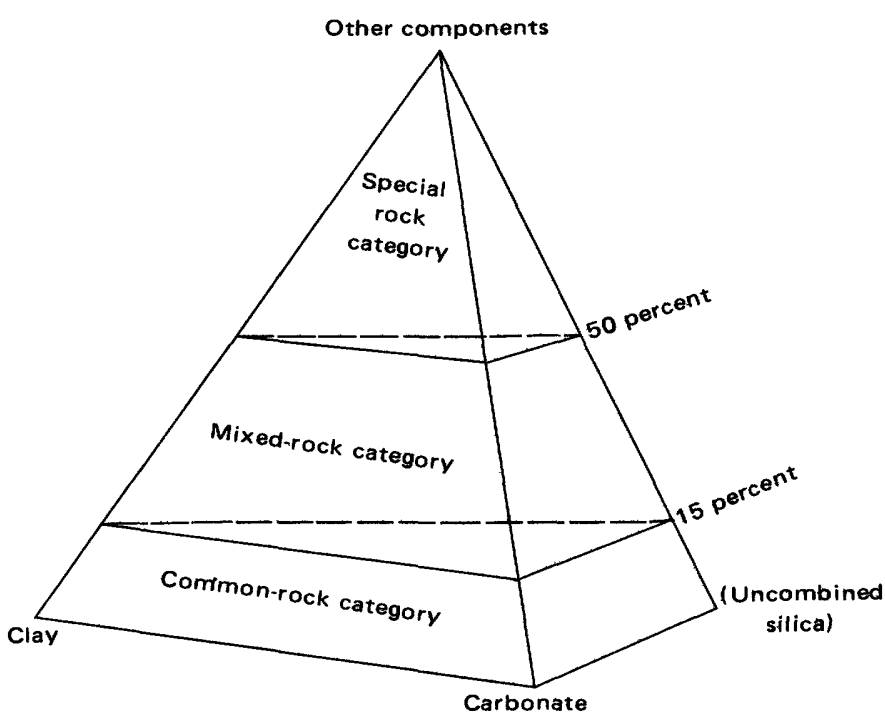

FIGURE 2.-Tetrahedron showing three categories of rock composition; common-rock category consisting of rocks with 85 percent or more uncombined silica, clay, and carbonate; mixed-rock category that includes rocks with one or more additional components; and special-rock category, in which other components, such as gypsum or phosphate rock, make up more than 50 percent of the rock sample. 
respectively, 100 percent uncombined silica, 100 percent clay, and 100 percent carbonate, The apex of the tetrahedron is then 100 percent of some component, or components, other than uncombined silica, clay, or carbonate. The interior of the tetrahedron, the volume above the basal triangle, represents those rocks composed partly of the three major components (uncombined silica, clay, and carbonate) and partly of one or more additional components. If, for example, an analysis shows 19 percent uncombined silica, 19 percent clay, 57 percent carbonate, and 5 percent calcium sulfate, its location within the tetrahedron is determined by first recalculating the three major component figures into percentages based on the total of those three components only: that is, 20 percent uncombined silica, 20 percent percent clay, and 60 percent carbonate. The point thus defined is located in the basal triangle, and is projected up towards the apex for a distance representing the amount of the fourth component. In this case, the point would be projected 5 percent of the distance from the base to the apex, and it would lie within the volume designated as commonrock category in figure 2 .

For an analysis that shows 15 percent uncombined silica, 30 percent clay, 15 percent carbonate, and 40 percent calcium phosphate, the recalculation explained above would yield these figures: 25 percent uncombined silica, 50 percent clay, and 25 percent carbonate. The defined point is located on the basal plane and then projected toward the apex for a distance equivalent to 40 percent (the amount of calcium phosphate in the analysis). The point representing the analysis will then fall in the upper part of the volume designated as mixed-rock category in figure 2 . If the analysis had shown 60 percent calcium phosphate, the point representing it would have fallen in the lower part of the volume designated as special-rock category.

To keep the nomenclature of the classification scheme simple and reasonably consistent, the subdivisions of the special-rock category are designated as "groups" by analogy with the use of that term for the different mixtures of the three main components represented on the basal plane of the tetrahedron. The nomenclature of the special-rock category is as follows:
Table
10 Group Description
11 $\mathrm{Na} . . .$. Sodium chloride
11 -.......-. S........ Miscellaneous sodium-sulfate and sodium-carbonate rocks
12 ........ G ......- Gypsum, gypsite, and anhydrite
13 .......... Sr ......- Celestite, strontianite
14 -.------ B ------ Boron-bearing rocks
15 -.....-. M......- Miscellaneous rocks
16 .......... P.-.-.-.- Phosphorite
17 .......- Mn ...... Manganiferous opal

ANALYSES

Analysis section. - Analyses were taken only from reports published prior to 1974 and from open-file reports of the U.S. Geological Survey. The analyses are generally recorded in this report as they are given in the original publication; the few recalculations made by the compiler are noted.

Arrangement of analyses. - The 2,312 analyses compiled are presented in tables 1-17 of this report. The tables are arranged in sequence according to classification groups of the common- and mixed-rock categories. For A through $\mathrm{F}_{2}$, each table lists the analyses by States and contains all the analyses in the group.

The user should note that the published analyses of California rocks, despite their large number, are probably not truly representative of the composition of all sedimentary rocks of the region. Most of the analyzed rocks were collected because they are (or were thought to be) of special economic interest and hence are probably of rather unusual chemical composition. For example, limestone that contains 95 percent or more $\mathrm{CaCO}_{3}$ is grossly overrepresented in the published analyses; natural limestone analyses show a wide range in $\mathrm{CaCO}_{3}$. This geochemically unrepresentative or biased nature of the published analyses does not necessarily detract from the potential usefulness of these analyses. Carbonate rocks of unusual purity were specially selected for analysis; the analysis of these pure rocks affords the potential manufacturer of lime, industrial fluxes, and other mineral commodities valuable information on localities where the best source materials for his particular purpose can most readily be obtained.

\section{TYPES OF SAMPLES}

Sedimentary and metamorphic rocks. - 'The dividing line between sedimentary and metamorphic rocks is not a sharp one in nature, and some authors do not always clarify where a given analyzed sample lies in relation to this line. In this report, the usage of the individual author was followed wherever possible. Slightly altered rocks are generally included in this compilation; considerably altered rocks are not. An "altered" rock is included if the "alteration" is interpreted to be due primarily to sedimentary or diagenetic processes rather than to metamorphic or hydrothermal processes. In general, Precambrian sedimentary rocks have been greatly modified by pressure, heat, and circulating fluids; only those that seem from the description to have been slightly modified are included here. "Marble" is sometimes applied as a trade name to any carbonate rock that will take a high polish, and for this reason, analyses of some rocks called marble are also included here. Analyses of some rocks of doubtful ori- 


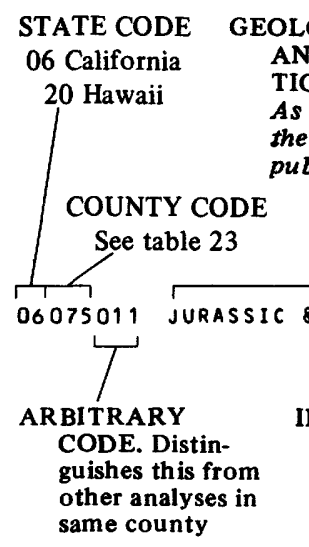

AGE SAMPLE LOCALITY BY TOWN. AND FORMATION NAME As reported in the original publication
SHIP AND RANGE SYSTEM California samples only. Quotation marks indicate information was added by compilers

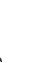
NEAREST TOWN OR
GEOGRAPHIC POINT

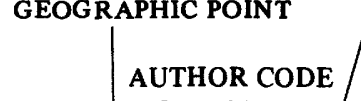

PAGE IN ORIGINAL PUBLICATION WHICH CONTAINS ANALYSIS

PAGES, PLATES, AND FIGURES CONTAINING ADDITIONAL INFORMATION ABOUT ANALYSIS
LITHOLOGY original publication

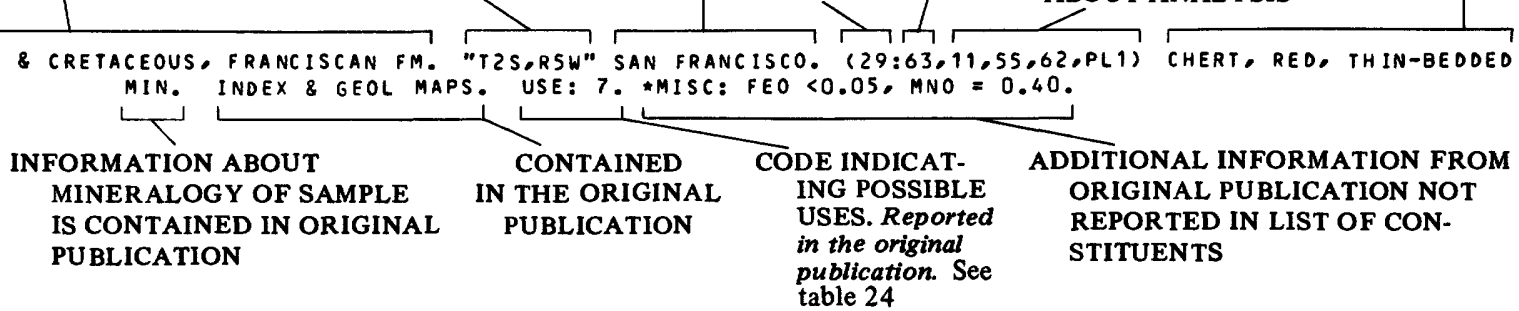

FIGURE 3.-Diagrammatic explanation of a typical descriptive note.

gin, or ones that have undergone an unknown amount of metamorphism, are included if the rock is of special economic interest.

Washed and purified samples.-Analyses of washed and purified samples are included if no more than 10 percent of the material was removed during the processing.

\section{ANALYSES OF SAMPLES}

Completeness.-No selection based on the known or inferred quality of the analyses was made, except that analyses totaling less than 95 percent or more than 102.5 percent were excluded. Selection and grading of analyses based on quality and completeness are left to the reader. A few analyses that do not precisely meet the standards outlined above are nevertheless included if the sample is part of a related suite, is the only one from a given formation, is of particular economic or scientific interest, or contains constituents not commonly determined.

Analyses were not graded into categories of superior, good, and fair. The original publication dates of the analyses afford the user a general means by which the reliability of such analyses might be appraised. This inclusive compilation can facilitate a later, more critical selection of truly superior analyses.

Insoluble residues.-Analyses that record more than 25 percent of the rock as insoluble in acid and that do not report $\mathrm{SiO}_{2}$ are not included.

Totals.-In general, analyses are reported if their totals are within the range of 95 to 102.5 percent. For those analyses whose totals were outside the range because the percentage of a readily calculated constituent had not previously been determined, the omitted constituent was calculated so the total was brought within these limits. For example, in some limestone and dolomite analyses, $\mathrm{CO}_{2}$ was not determined, and the analysis total is accordingly too low to meet the 95percent criterion. However, analyses of limestone and dolomite are included if the $\mathrm{CO}_{2}$ calculated from the determined $\mathrm{CaO}$ and $\mathrm{MgO}$ brings the total above the 95 percent limit. If the total is not in the source reference, the amount has been added by the compiler.

\section{EXPLANATION OF TERMS AND ARRANGEMENT OF TABLES}

Sample location.-Only rock samples that have reasonably adequate information concerning their localities are included. Localities are shown in the descriptive notes by State and county codes (see table 23 ), by the nearest town or geographic point, and for California only by township and range numbers (see fig. 3).

References.-Analyses utilized in this study were obtained mostly from readily available publications. If more than one reference is given for an analysis, the first given is generally the reference from which the analysis was taken. References other than the first supply additional information for the descriptive notes or for the analysis itself.

To simplify the computer printout of the descriptive notes, authors were given code numbers. Figure 3 shows how these codes are used in the descriptive notes, and a complete list of author codes is given in table 25. Authors are also listed alphabetically in the section on "References Cited" at the back of this report.

Lithology.-The rock name given to a sample is that used in the original reference. If the reference gives no name, or if the compiler thought there was some doubt as to the accuracy of the name, a name was supplied on the basis of either the position of the analysis in the classification system or the compiler's interpretation of the original publication.

Treatment of stratigraphic nomenclature.-Because the stratigraphic nomenclature used in this report is from many published sources, the names and ages do not necessarily reflect the latest usage of the U.S. Geological Survey. The age and formation of each sample are given in the descriptive notes as reported in the 
published source of the analysis, unless the reported assignment is so out of date as to be misleading and better information could be conveniently obtained. For some of the rock samples, age and the stratigraphic unit are not known because of the complex structure of the rocks or because the area has not been fully investigated.

Chemical analyses.-The lists of constituents in the tables were simplified so that the analyses could be printed by computer. Also, for the sake of simplicity, the footnotes do not vary from page to page, although not all footnotes are required on every page. For information that did not fit the standard footnote form, an asterisk is put on the total of the sample and the additional information is printed out in the descriptive notes. (See fig. 3.) The list of constituents for the common- and mixed-rock categories was not suitable for the special-rock category, so each set of analyses in that category has its own constituent list derived from the original publication. The footnotes for that category pertain only to the page where they appear.

Additional information.-If the original publication gave a spectrographic analysis or described the mineralogy of the sample, this is indicated in the descriptive notes, although these data are not reproduced here (see fig. 3). Quotation marks in the descriptive notes indicate information supplied by the compilers.

Many analyses are published and republished over the years. Most of these analyses are quoted exactly, but some are not consistent in constituents and amounts when republished. These inconsistencies may reflect the interpretation of a later author, or they may simply be the result of typographic errors.

Pages as a unit.-Each set of facing pages with chemical analyses and accompanying descriptive notes is considered a unit, and the footnotes apply to the unit as a whole.

English units, metric units.-English units of measure were not converted to metric units. For a few analyses the original publication used metric units, and these are recorded as given. Most of the units used are feet, inches, and miles, and the conversion factors are:

$$
\begin{aligned}
& 1 \text { mile }=1.609 \text { kilometers } \\
& 1 \text { foot }=0.3048 \text { meters } \\
& 1 \text { inch }=2.54 \text { centimeters }
\end{aligned}
$$

Reported use of the rock.-Generally, the information of the actual or potential economic use is given only if it is stated in the original publication. Uses have been grouped, and each type has been given a code number (1-18). This code number is used in the descriptive notes. (See table 24 for explanation of the code number.)

Abbreviations.-For the sake of saving space and computer printout time, many abbreviations are used in the descriptive notes. Most of these abbreviations are standard and probably are familiar to most readers. However, the notes also contain a few unusual or little-known abbreviations, and these are listed here.

B.D., Bulk density

COMPOS., Composite

DH, Drill hole

DIF., Difference

GR., Group or Grain

I.L., Ignition loss

MIN., Mineralogy (information SMPL., Sample about the sample's mineralogy is contained in the original publication)

SOL., Soluble

TEX., Texture

Acknowledgments:-My thanks to the following: Marian A. Werner for some of the research; Harry A. Tourtelot for help during different stages of assembling the material; Margaret Roberts for writing the computer programs; and Lillian Kennedy and Pamela Ketterer for their contribution to the work of preparing and typing the manuscript.

\section{GRAPHICAL SUMMARIES}

The analyses assembled in this report may be considered primarily as individual pieces of information about the composition of particular sedimentary rocks in particular places. Also, they may be thought of collectively as groups of data that tend to cluster around various modal points of composition corresponding to rocks of different lithologic types, geologic ages, economic uses, and so forth. Collectively, then, the data of the analyses need to be summarized if any clustering of points or systematic relations that may exist are to be recognized.

Many of the samples had been collected for analysis because the rock was, or was thought to be, of economic value. For this reason, the resulting analyses do not represent random sampling of all sedimentary rocks of the report region. Yet, despite systematic bias in the choice of samples, the compiler hopes that the wide range in composition of the samples may tend to offset the purely local peculiarities in the data and provide information of more regional interest and utility. 


\section{AREAL DISTRIBUTION}

Figures 4 and 5 show the approximate locations of analyzed samples from each of the rock-classification groups. These maps are crowded in some areas, and, accordingly, some symbols may indicate more than one sample; in addition, where symbols are closely spaced on the map, some of them are plotted in the county adjacent to their true location. More detailed locations are given in the descriptive notes accompanying the tables of analyses.

\section{DISTRIBUTION BY COMPOSITION}

Figures 6 and 7 are computer plots of the analyses within the fields of the triangular diagram that is the basis for the classification system. In some of the more crowded areas of the diagrams, much overlap occurs.

These computer plots show that much more data is available for some kinds of rocks, notably mixtures of siliceous and clayey materials (group B) and carbonate rocks (group $F_{1}$, and $F_{2}$ ), than for others. Whether these larger amounts of data are a reflection of the economic value of such rocks or of the relative abundance of the rocks is not certain.

The summaries also emphasize the difficulties of designing a classification system that consistently groups sedimentary rocks according to the names commonly applied to them. Rocks called clay, for example, fall into several of the classification groups when classified according to their chemical composition. The inverse is also true and is perhaps more important. Common names for sedimentary rocks are not always closely related to the actual rock composition, nor are they always consistently applied.

Tables 18,19 , and 20 categorize the numbers of analyses of various rock types by classification group and States as an aid in understanding the distribution of data.

Table 21 lists, by State, the number of analyses in each classification group, stratigraphic unit, and county. The stratigraphic units are those of the original author and do not necessarily conform to the usage of the U.S. Geological Survey. This table is useful for finding the available data on rocks of a particular composition within a reasonably restricted geographic area.

Table 22 shows the general distribution of the sam. ples by State, category, and group.

The geologist or geochemist interested in estimating the average composition of the Earth's materials probably cannot find the gross averages of thousands of published analyses of unusually pure limestones particularly useful for his purpose. Careful observation, however, of the areal distribution and thickness of different rock types and of different geologic formations represented by the published analyses should enable the geologist or geochemist to make closer estimates of average compositions than most of those now available, or at least enable him to discover which rock types and formations are most in need of new analytical data.

Several noteworthy questions and facts about sedimentary rocks resulted from the compilation. One possibly significant relation became apparent when the analyses of this report were plotted on triangular diagrams (figs. 5 and 6). A very large proportion of all the analyses fall not within the central region of the diagram, as might reasonably be expected, but within about 10 percent of the exterior boundaries of the triangle. This means that many samples of uncombined silica and clay mixtures contain very little carbonate, and that many samples of carbonate contain very little uncombined silica or clay. Such relations probably are not artifacts resulting from systematic bias in the choice of analyzed samples or in the classification system used; rather, they are largely results of natural sedimentation processes. Thus, environments favorable for deposition of sand and clay are unfavorable for deposition of carbonates, and vice versa. It follows that the physicochemical factors underlying deposition of sand and clay are different from those that result in carbonate deposition. 
$42^{\circ}$

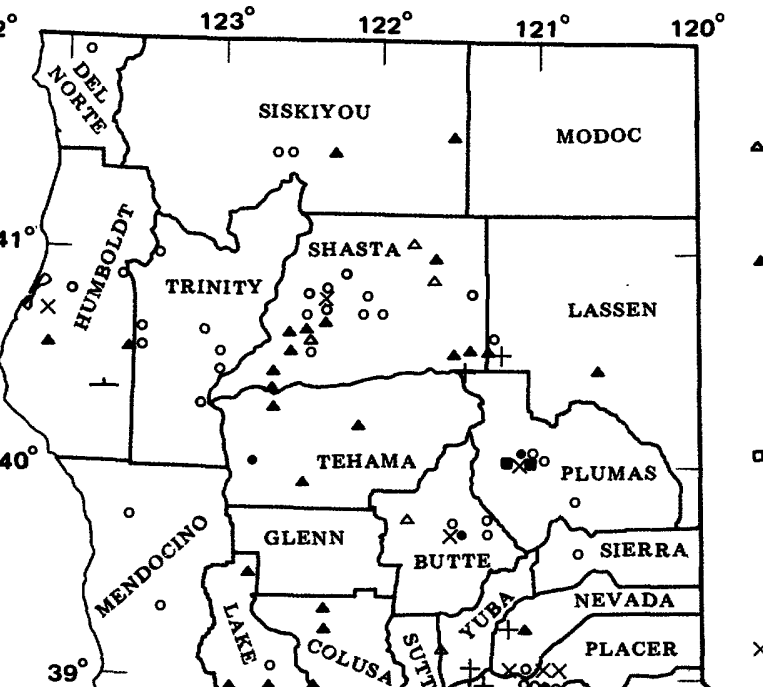

\section{EXPLANATION}

Symbol may indicate more than one sample

- Group A, common- and mixedrock categories-Rocks containing uncombined silica more than 75 percent

- Group B, common- and mixedrock categories-Rocks containing uncombined silica and clay, each less than 75 percent; uncombined silica and clay, each more than carbonate

Group C, common- and mixedrock categories-Rocks containing uncombined silica and carbonate, each less than 75 percent; uncombined silica and carbonate, each more than clay

$\times$ Group D, common-, mixed-, and special-rock cat egories-Rocks containing clay more than $\mathbf{7 5}$ percent

- Group E, common- and mixedrock categories-Rocks containing clay and carbonate, each less than 75 percent; clay and carbonate, each more than uncombined silica

- Group $F_{1}$, common- and mixedrock categories-Rocks containing carbonate from 75 to 90 percent

- Group $F_{2}$, common-rock category Rocks containing carbonate more than 90 percent

+ Groups Na, G, S, P, special-rock category-Saline materials and phosphorite

-Groups B, Sr, Mn, special-rock category-Boron-bearing, strontianite-bearing, manga nese-bearing rocks
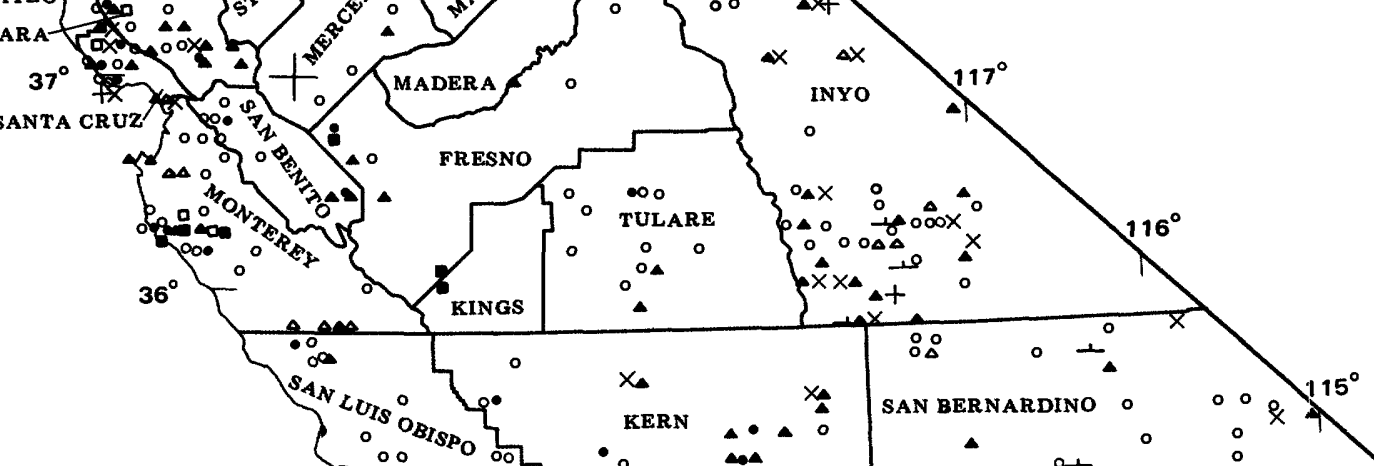
$35^{\circ}$ oे

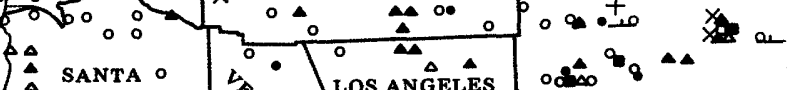

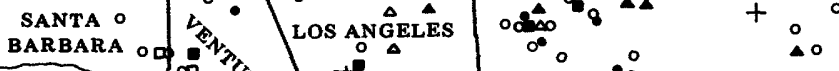
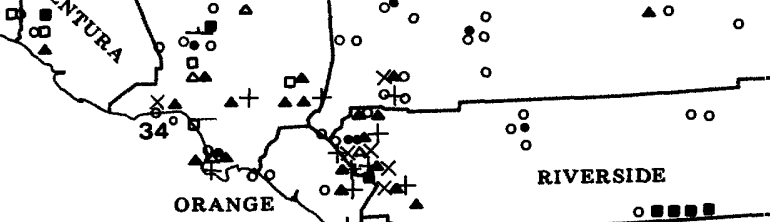

RIVERSIDE

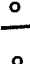



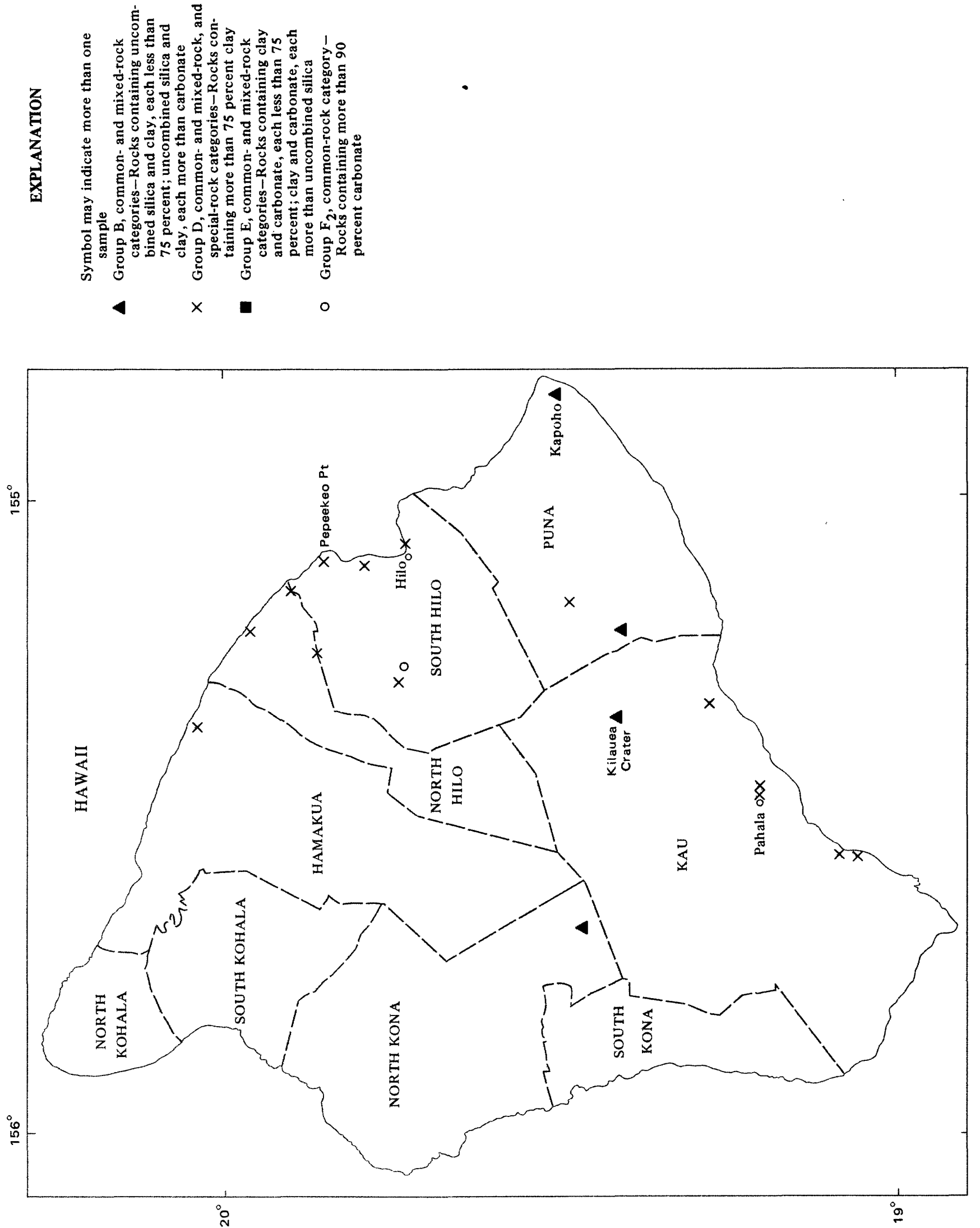

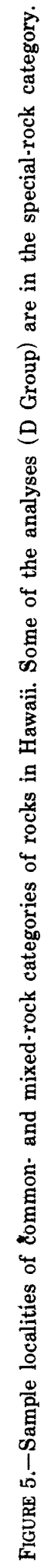


GRAPHICAL SUMMARIES
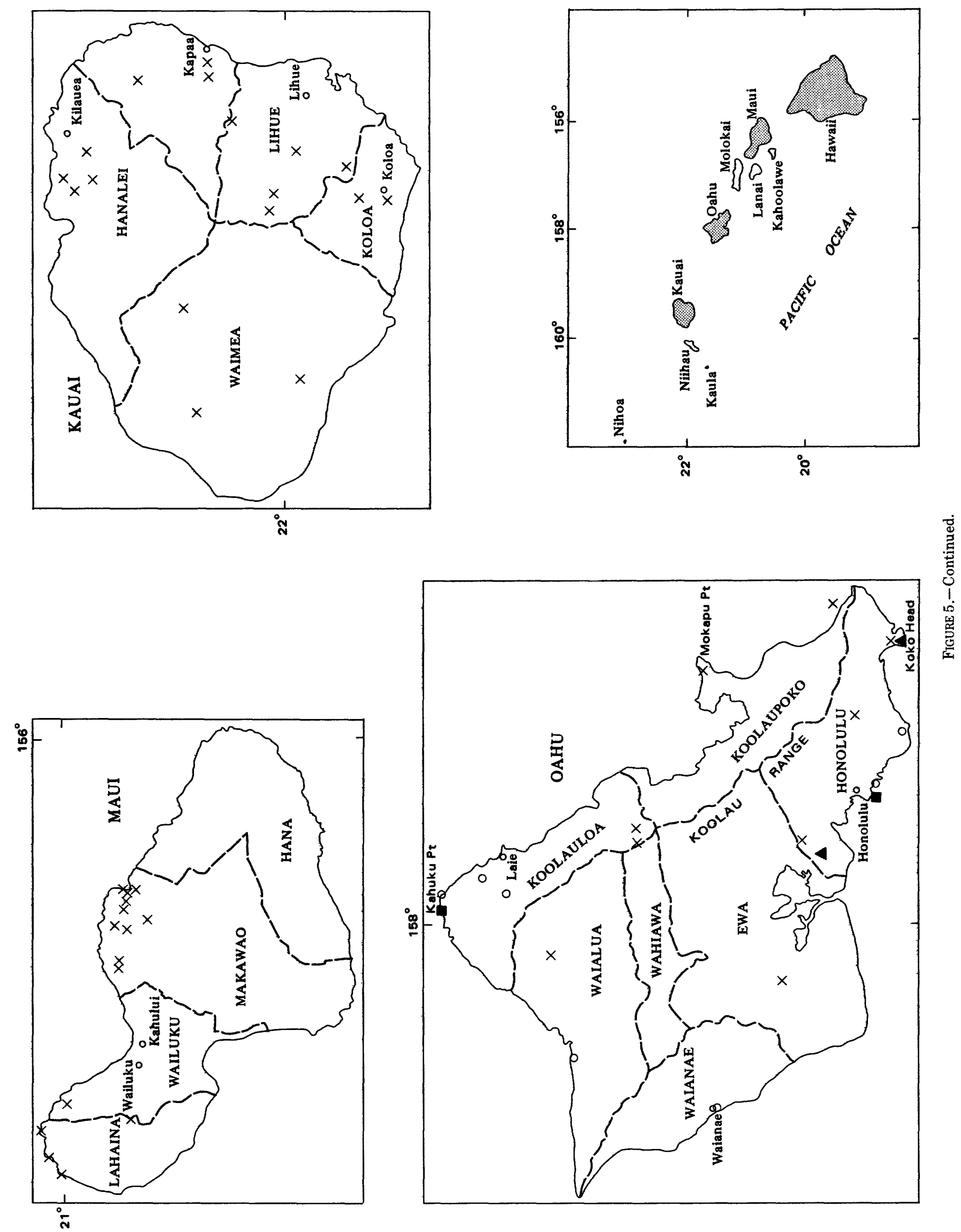


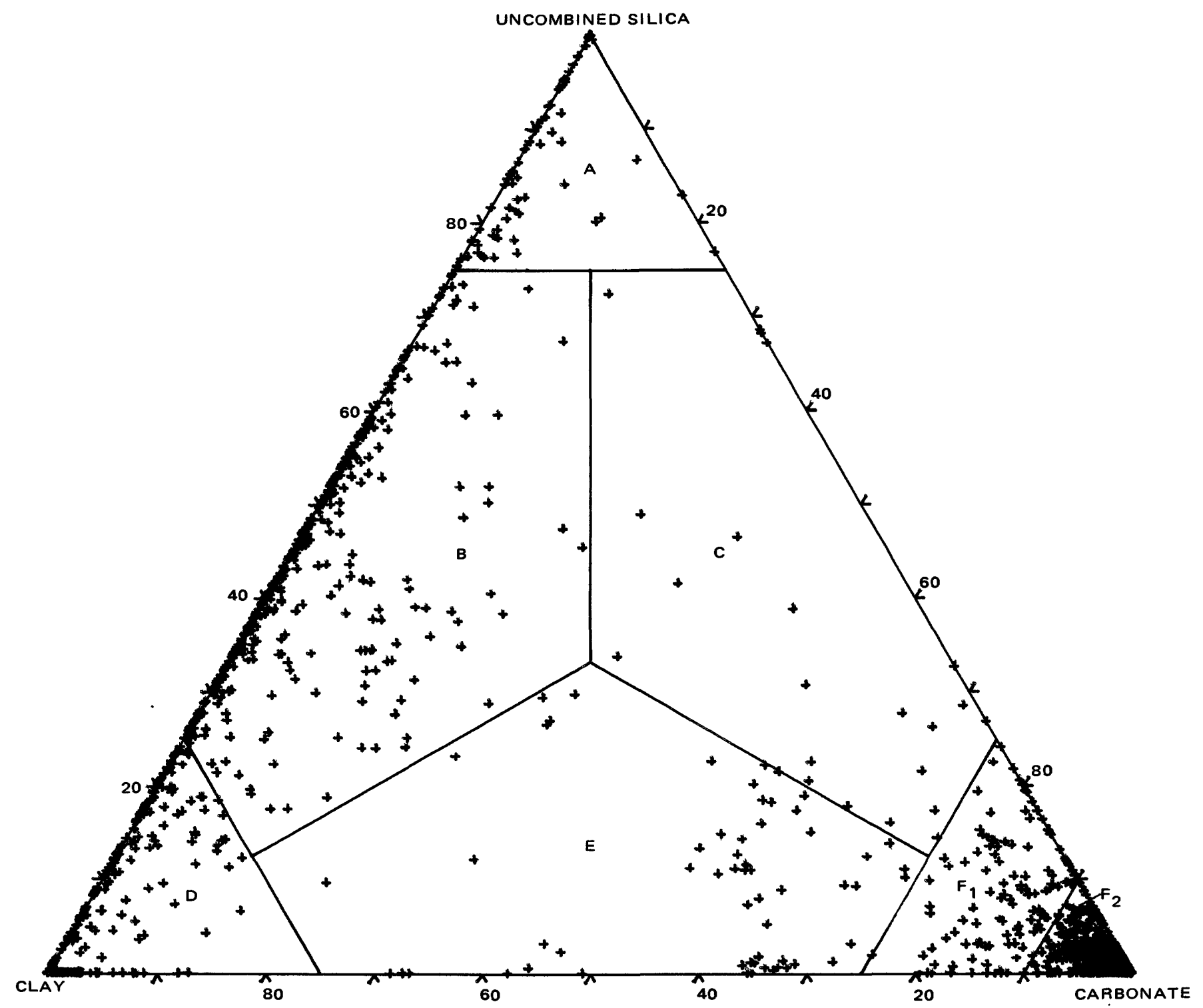

FIGURE 6.-Distribution among compositional groups of analyses of samples from California; common- and mixed-rock categories. 
GRAPHICAL SUMMARIES

11

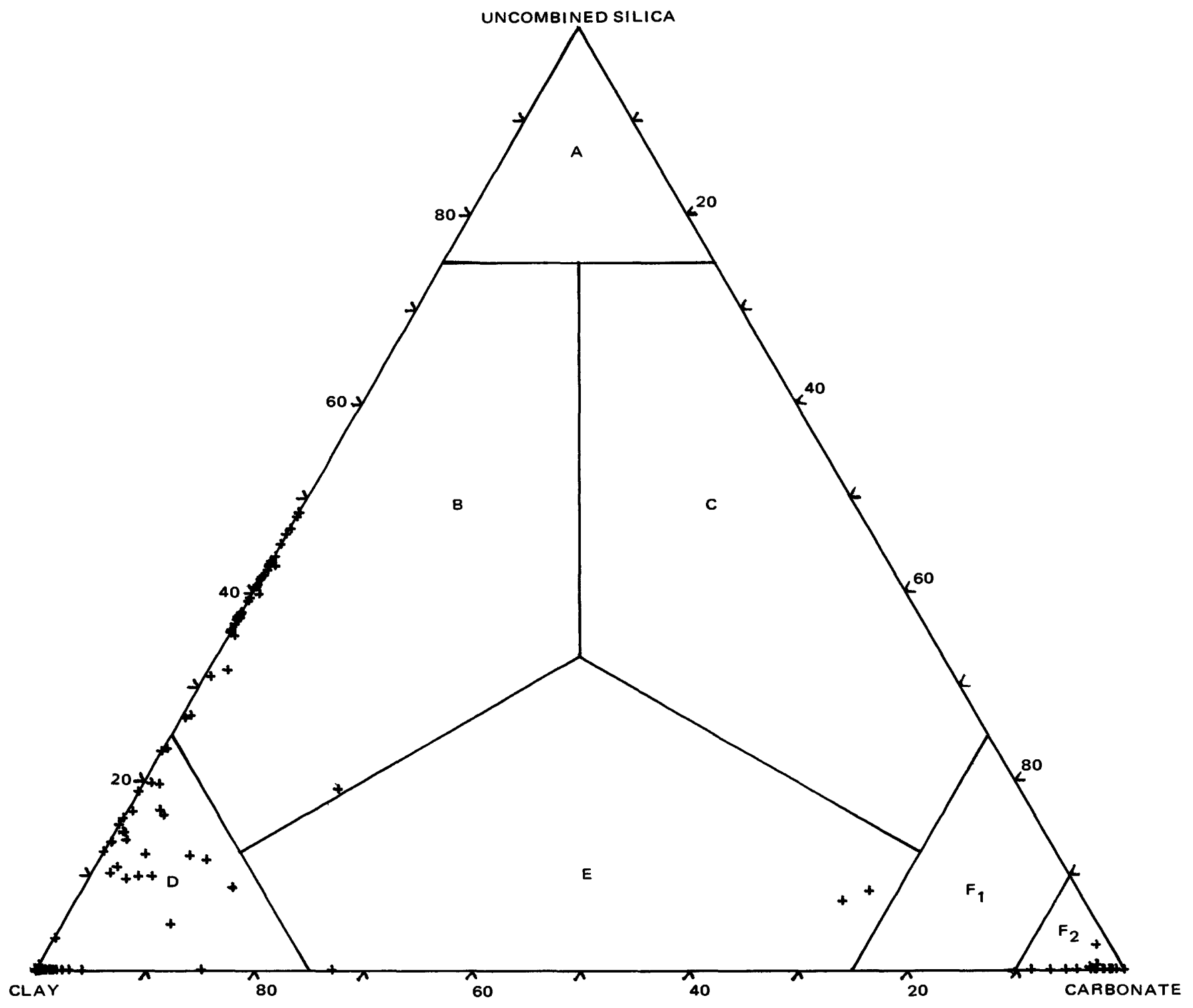

FIGURE 7.-Distribution among compositional groups of analyses of samples from Hawaii; common- and mixed-rock categories. Some of the analyses, Group D, are in the special-rock category. 
TABLE 18. - Number of samples of various rock types in the classification groups $A \cdot F_{2}$, common-and mixed-rock categories, California

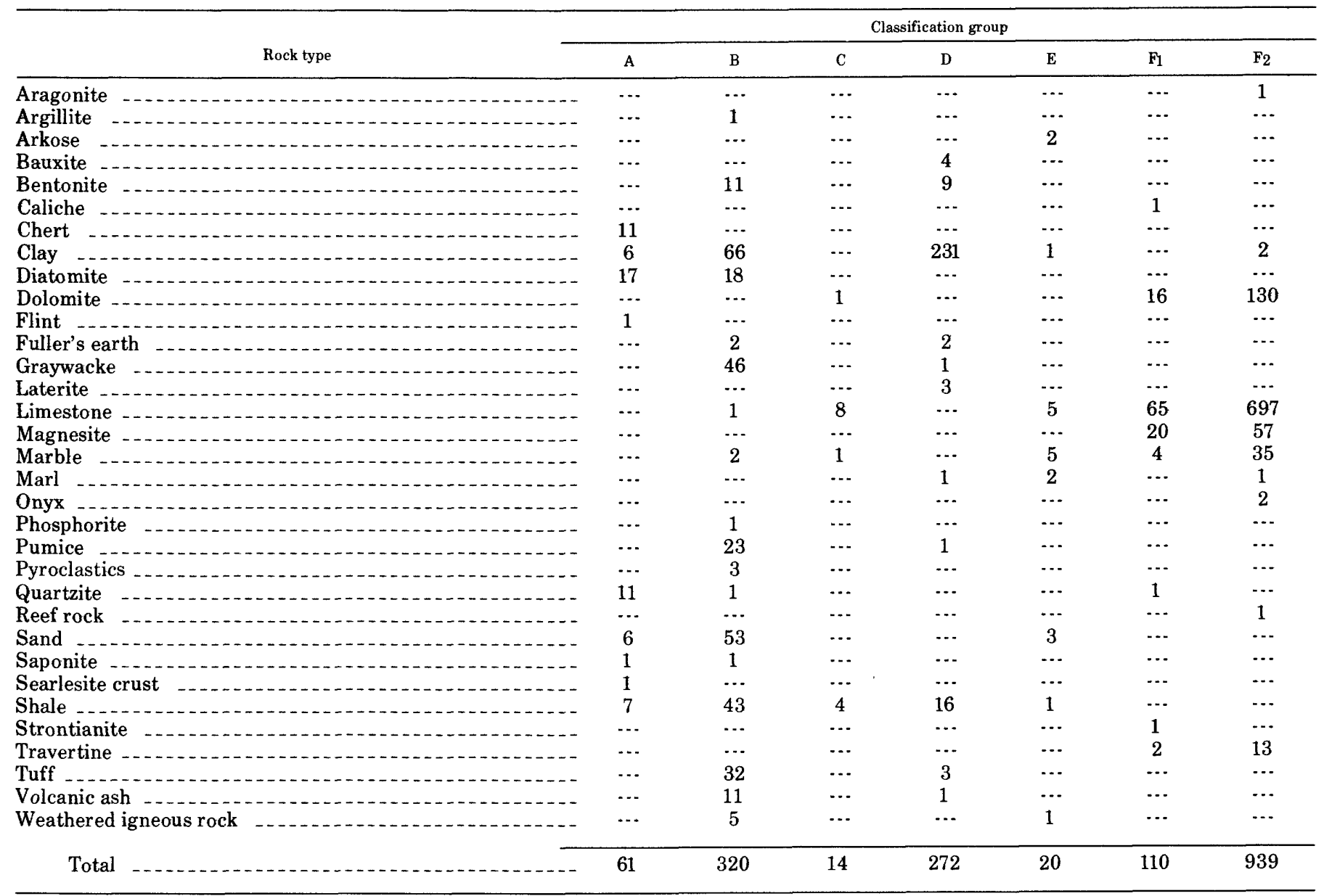

\section{CUMULATIVE-FREQUENCY CURVES}

Figures $8 A-K$ are cumulative-frequency curves of the constituents determined in all samples of each classification group. These curves show on one diagram the wide range in abundance of some of the constituents. Note that the minor constituents generally vary much more widely than do the major constituents.

Many of the constituents, particularly those determined in only a few samples, are distributed very irregularly, and several of them do not fit very well into either normal or log-normal distributions. Nevertheless, most of the more abundant constituents approximate a normal distribution, and many of the constituents of intermediate and lesser abundance show a tendency toward a log-normal distribution.

\section{REFERENCES CITED}

Allen, J. E., 1946, Geology of the San Juan Bautista quadrangle, California: California Div. Mines Bull. 133, p. 9-75.

Allen, R. D., and Kramer, Henry, 1953, Occurrence of bassanite in two desert basins in southeastern California: Am. Mineralogist, v. 38, no. 12, p. 1266-1268.

Allen, V. T., 1929, The Ione Formation of California: California Univ. Pubs. Geol. Sci., v. 18, no. 14, p. 347-448.

1941, Eocene anauxite clays and sands in the Coast Range of California: Geol. Soc. America Bull., v. 52, no. 2, p. 271-293.

Ames, L. L., Jr., Sand, L. B., and Goldich, S. S., 1958, A contribution on the Hector, California bentonite deposit: Econ. Geology, v. 53 , no. 1, p. 22-37.

Anderson, C. A., 1933a, Volcanic history of Glass Mountain, northern California: Am. Jour. Sci., 5th ser., v. 26, no. 155, p. 485-506.

1933b, The Tuscan Formation of northern California, with a discussion concerning the origin of volcanic breccias: California Univ. Pubs. Geol. Sci., v. 23, no. 7, p. 215-276. 
TABLE19. - Number of samples of various rock types in the classification groups of the special-rock category, California

\begin{tabular}{|c|c|c|c|c|c|c|c|c|}
\hline \multirow[b]{2}{*}{ Rock type } & \multicolumn{8}{|c|}{ Classification group } \\
\hline & $\mathrm{Na}$ & $\mathrm{s}$ & G & $\mathrm{Sr}$ & $\mathbf{B}_{1}$ & M & Mn & $P$ \\
\hline Barite nodule & $\cdots$ & $\cdots$ & $\cdots$ & $\cdots$ & $\cdots$ & 1 & $\cdots$ & $\cdots$ \\
\hline Bassanite & $\cdots$ & ... & 1 & -.. & $\cdots$ & $\cdots$ & $\cdots$ & $\cdots$ \\
\hline Borax crust & ... & ... & ... & ... & 1 & $\cdots$ & ... & ... \\
\hline Burkeite & $\cdots$ & 2 & $\cdots$ & $\cdots$ & $\cdots$ & $\cdots$ & $\cdots$ & $\cdots$ \\
\hline Celestite & $\cdots$ & $\cdots$ & $\cdots$ & 9 & $\cdots$ & $\cdots$ & $\cdots$ & $\cdots$ \\
\hline Colemanite & $\cdots$ & $\cdots$ & $\cdots$ & $\cdots$ & 5 & $\cdots$ & $\cdots$ & $\cdots$ \\
\hline Copiate & $\cdots$ & ... & $\cdots$ & $\cdots$ & $\cdots$ & 1 & $\cdots$ & $\cdots$ \\
\hline Gaylussite & $\cdots$ & 1 & $\cdots$ & $\cdots$ & $\cdots$ & $\cdots$ & $\cdots$ & $\cdots$ \\
\hline Gypsum & $\cdots$ & $\cdots$ & 23 & $\cdots$ & $\cdots$ & $\cdots$ & $\cdots$ & $\cdots$ \\
\hline Halite & 27 & $\cdots$ & $\cdots$ & $\cdots$ & $\cdots$ & $\cdots$ & $\cdots$ & $\cdots$ \\
\hline Hanksite & 1 & 6 & ... & ... & ... & $\cdots$ & ... & ... \\
\hline Howlite & $\cdots$ & $\ldots$ & ... & $\ldots$ & 4 & $\cdots$ & $\cdots$ & $\cdots$ \\
\hline Hydroboracite & $\cdots$ & $\cdots$ & $\cdots$ & $\cdots$ & 1 & $\cdots$ & $\cdots$ & $\cdots$ \\
\hline Laterite & $\cdots$ & $\cdots$ & $\cdots$ & $\cdots$ & $\cdots$ & 32 & $\cdots$ & $\cdots$ \\
\hline Magnesium sulfate pentahydrate & ... & 1 & ... & ... & ... & ... & ... & $\cdots$ \\
\hline Mirabilite crust & ... & 2 & $\cdots$ & ... & $\cdots$ & $\cdots$ & $\cdots$ & ... \\
\hline Natroalumite & $\cdots$ & $\cdots$ & $\cdots$ & $\cdots$ & $\cdots$ & 2 & $\cdots$ & $\cdots$ \\
\hline Neocolemanite & $\cdots$ & ... & ... & ... & 1 & $\cdots$ & $\cdots$ & -.. \\
\hline Niter & 4 & 1 & ... & ... & ... & 1 & ... & ... \\
\hline Opal & $\cdots$ & ... & $\cdots$ & ... & ... & $\cdots$ & 3 & $\cdots$ \\
\hline Phosphorite & $\cdots$ & ... & $\cdots$ & $\cdots$ & $\cdots$ & $\cdots$ & $\cdots$ & 2 \\
\hline Pickeringite & $\cdots$ & ... & ... & ... & ... & 2 & $\cdots$ & $\cdots$ \\
\hline Pirsonite & 1 & ... & ... & $\ldots$ & ... & ... & ... & ... \\
\hline Salines & 36 & ... & $\cdots$ & $\cdots$ & $\cdots$ & $\cdots$ & $\cdots$ & $\cdots$ \\
\hline Sand & $\cdots$ & $\cdots$ & $\cdots$ & $\cdots$ & $\cdots$ & 2 & $\cdots$ & $\cdots$ \\
\hline Sodium sulfate, sodium carbonate & ... & 28 & $\cdots$ & ... & $\cdots$ & $\cdots$ & $\cdots$ & $\cdots$ \\
\hline Strontianite & ... & ... & ... & 10 & ... & $\cdots$ & ... & ... \\
\hline Thenardite & ... & 1 & $\cdots$ & $\cdots$ & 1 & $\cdots$ & $\cdots$ & $\cdots$ \\
\hline Tincalconite & $\cdots$ & 1 & $\cdots$ & ... & ... & $\cdots$ & $\cdots$ & -.. \\
\hline Trona & 3 & 19 & $\cdots$ & $\cdots$ & $\cdots$ & $\cdots$ & $\cdots$ & $\cdots$ \\
\hline Ulexite & $\cdots$ & $\cdots$ & $\cdots$ & $\cdots$ & 2 & $\cdots$ & $\cdots$ & $\cdots$ \\
\hline Urao & $\cdots$ & 2 & ... & ... & ... & $\cdots$ & $\cdots$ & ... \\
\hline Veatchite & $\cdots$ & $\cdots$ & $\cdots$ & $\cdots$ & 1 & $\cdots$ & $\cdots$ & $\cdots$ \\
\hline Total & 72 & 64 & 24 & 19 & 16 & 41 & 3 & 2 \\
\hline
\end{tabular}

1936, Volcanic history of the Clear Lake area, California: Geol. Soc. America Bull., v. 47, no. 5, p. 629-664.

Anderson, C. A., and Russell, R. D., 1939, Tertiary formations of northern Sacramento Valley, California: California Jour. Mines and Geology, v. 35, no. 3, p. 219-253 [1940].

Anderson, F. M., 1899, The geology of Point Reyes Peninsula: California Univ. Pubs. Geol. Sci., v. 2, no. 5, p. 119-153.

1911, The Neocene deposits of Kern River, California, and the Temblor Basin: California Acad. Sci. Proc., 4th ser., v. 3, p. 73-146.

Anderson, Robert, and Pack, R. W., 1915, Geology and oil resources of the west border of the San Joaquin Valley north of Coalinga, California: U.S. Geol. Survey Bull. 603, 220 p.

Angel, Myron, 1890, San Luis Obispo County: California State Mining Bur. 10th Ann. Rept., p. 567-585.

Arnold, Ralph, and Anderson, Robert, 1907, Diatomaceous deposits
TABLE 20. - Number of samples of various rock types in the classification groups, Hawaii

\begin{tabular}{|c|c|c|c|c|c|}
\hline & B & D & $\mathrm{E}$ & $\mathrm{F}_{2}$ & M \\
\hline Bauxite & $\cdots$ & 113 & $\cdots$ & ... & $\cdots$ \\
\hline Chalk & ... & 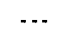 & ... & 2 & ... \\
\hline Clay & $\cdots$ & 32 & 2 & $\cdots$ & $\cdots$ \\
\hline Limestone $\ldots \ldots \ldots \ldots$ & ... & 5 & ... & 18 & ... \\
\hline Pumice & 36 & $\cdots$ & $\cdots$ & ... & ... \\
\hline Pyroclastic & 2 & 4 & 1 & ... & ... \\
\hline Saprolite & $\cdots$ & 85 & $\cdots$ & $\cdots$ & ... \\
\hline Soda-alenite & ... & $\cdots$ & $\cdots$ & ... & 1 \\
\hline Tuff & 4 & 23 & $\cdots$ & $\cdots$ & ... \\
\hline Volcanic ash & 2 & 5 & $\cdots$ & $\cdots$ & $\cdots$ \\
\hline -.................. & 44 & 267 & 3 & 20 & 1 \\
\hline
\end{tabular}


TABLE 21. - Distribution of samples by State, age, stratigraphic unit, county, and classification group

[The stratigraphic nomenclature used in this table does not necessarily reflect the latest usage of the U.S. Geological Survey; names and ages were taken from many published sources. Stratigraphic units are in alphabetical order in each age grouping]

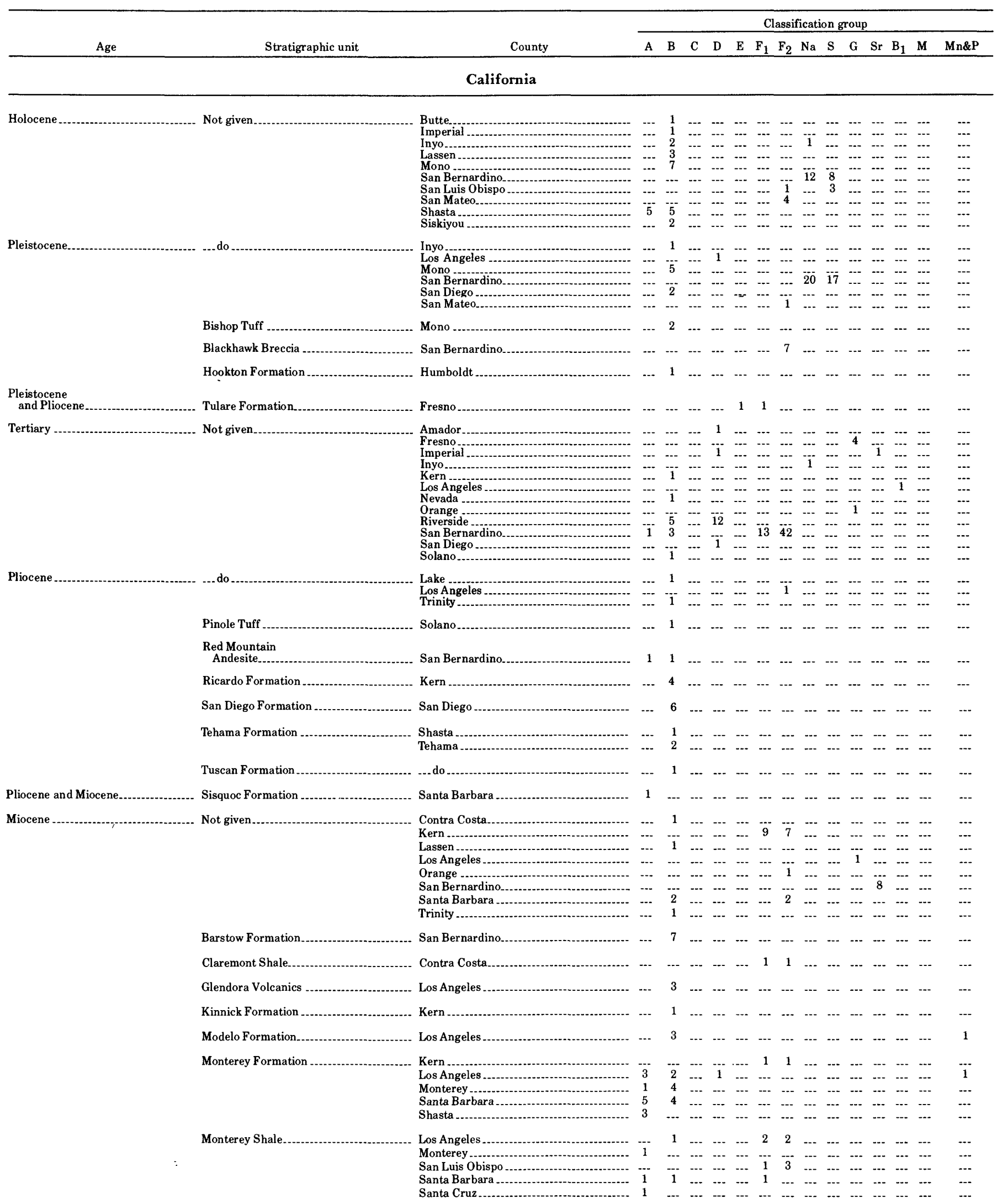


TABLE 21. - Distribution of samples by State, age, stratigraphic unit, county, and classification group - Continued

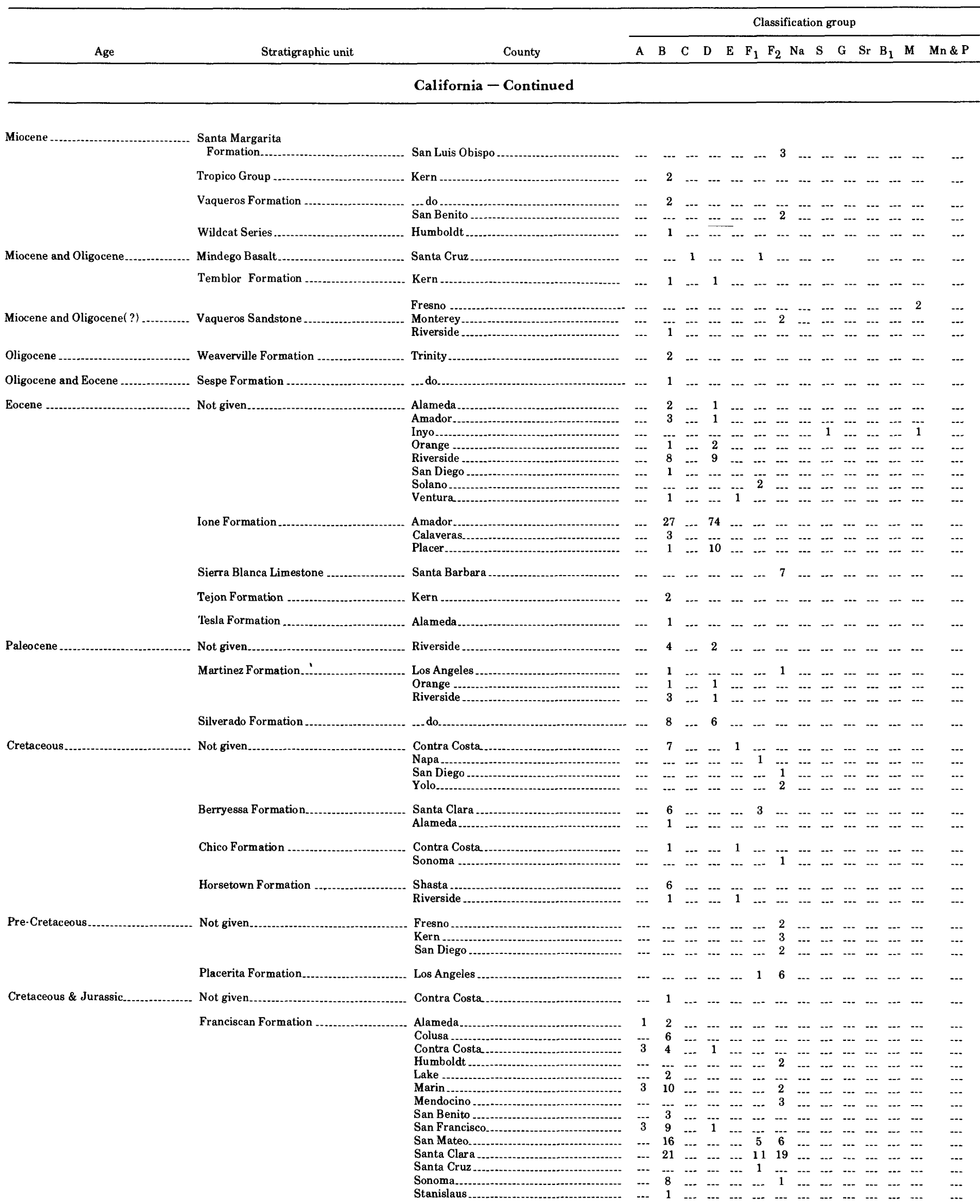


TABLE 21. - Distribution of samples by State, age, stratigraphic unit, county, and classification group - Continued

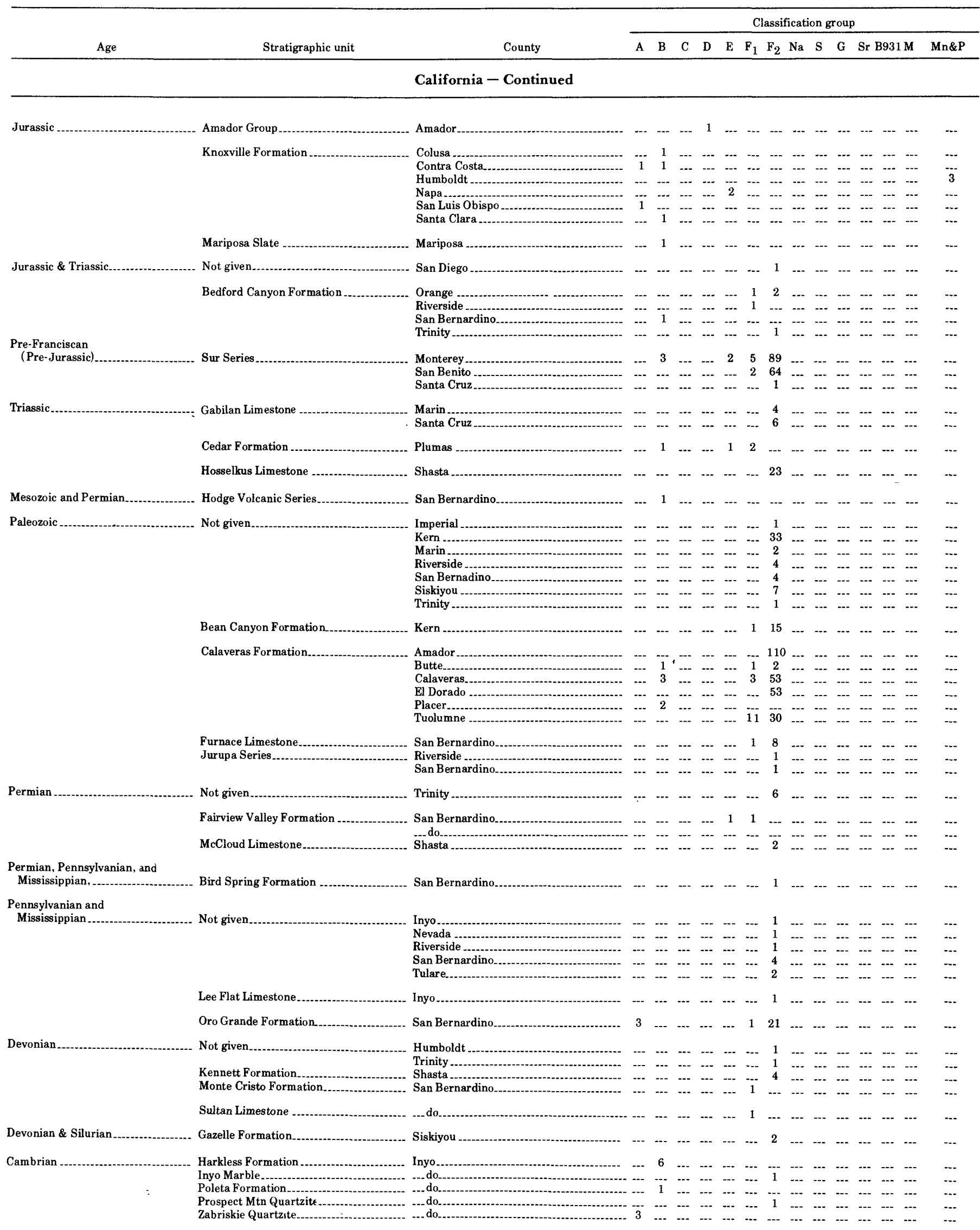


TABLE 21. - Distribution of samples by State, age, stratigraphic unit, county, and classification group - Continued

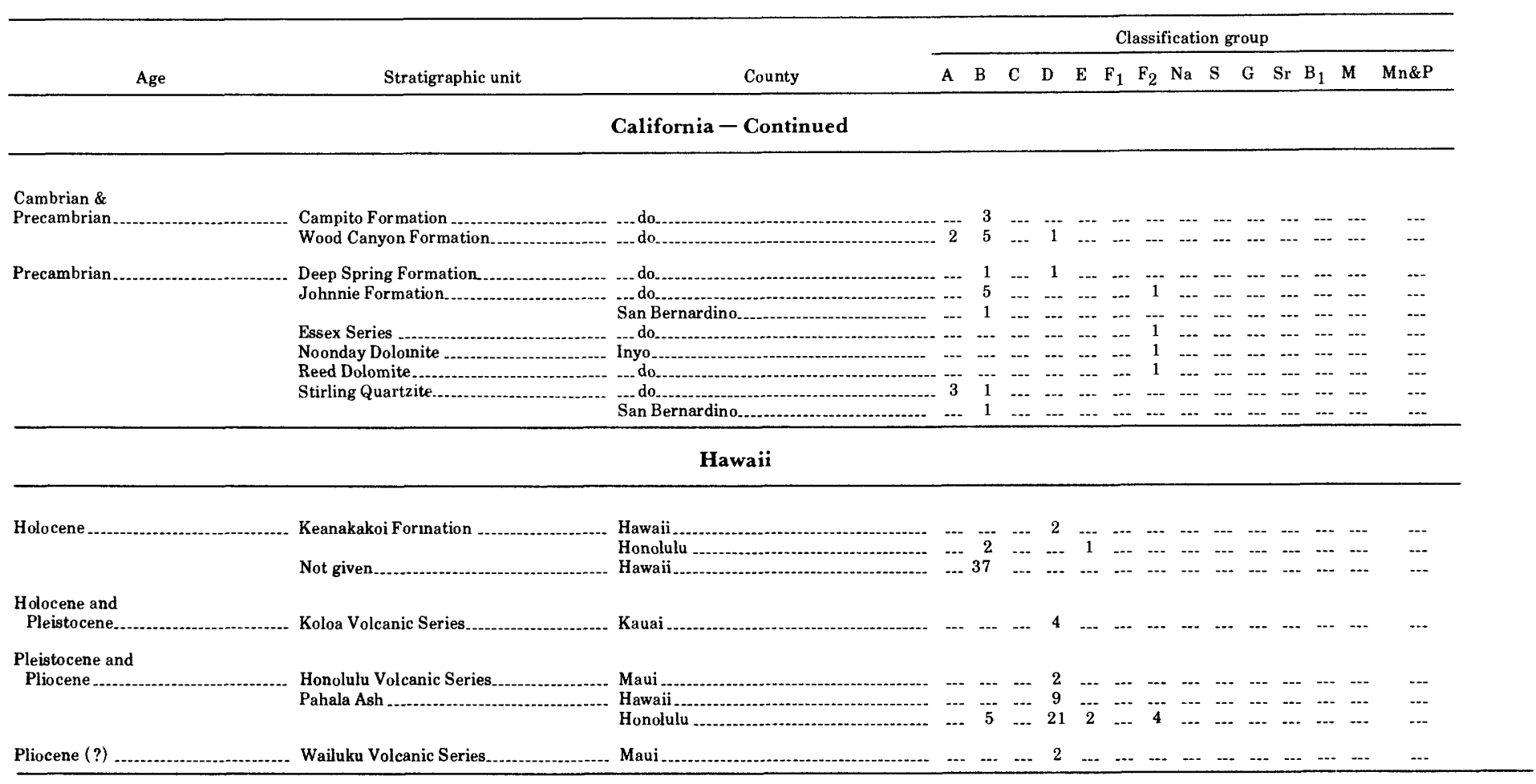

of northern Santa Barbara County, California: U.S. Geol. Survey Bull. 315-0, p. 0438-0447.

Arnold, Ralph, and Johnson, H. R., 1909, Sodium sulfate in Soda Lake, Carriso Plain, San Luis Obispo County, California: U.S. Geol. Survey Bull. 380-L, p. L369-L371.

Aubury, L. E., 1906, The structural and industrial materials of California: California State Mining Bur. Bull. 38, p. 13-378.

Aune, Q. A., 1965, Limestone, in O'Brien, J. C., Mines and mineral resources of Trinity County, California: California Div. Mines and Geology County Rept. 4, p. 39-42.

Averill, C. V., 1929, Redding Field Division: California Div. Mines and Mining Rept. 24, no. 4, p. 261-316.

1937, Mineral resources of Plumas County: California Jour. Mines and Geology, v. 33, no. 2, p. 79-143.

TABLE 22. - Distribution of 2,312 samples by State, category, and group

\begin{tabular}{|c|c|c|}
\hline \multicolumn{3}{|c|}{ State } \\
\hline \multicolumn{3}{|c|}{$\begin{array}{l}\text { California } \\
\text { Hawaii }\end{array}$} \\
\hline \multicolumn{3}{|c|}{ Category } \\
\hline \multicolumn{3}{|c|}{$\begin{array}{l}\text { Common and mixed rock } \\
\text { Special rock }\end{array}$} \\
\hline \multicolumn{3}{|c|}{ Group } \\
\hline 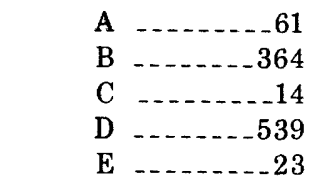 & $\begin{array}{l}\mathrm{F}_{1} \ldots \ldots 10 \\
\mathrm{~F}_{2} \ldots \ldots \\
\mathrm{Na} \ldots \ldots \\
\mathrm{S} \ldots \ldots \\
\mathrm{G} \ldots \ldots\end{array}$ & $\begin{array}{l}\mathrm{Sr} \ldots \ldots \ldots \\
\mathrm{B}_{1} \ldots \ldots \ldots \\
\mathrm{M} \ldots \ldots \\
\mathrm{P} \ldots \ldots \\
\mathrm{Mn} \ldots \ldots\end{array}$ \\
\hline
\end{tabular}

1939, Mineral resources of Shasta County: California Jour. Mines and Geology, v. 35, no. 2, p. 108-191.

1941, Mineral resources of Humboldt County: California Jour. Mines and Geology, v. 37, no. 4, p. 499-528.

1947, Mines and mineral resources of Lake County, California: California Jour. Mines and Geology, v. 43, no. 1, p. 15-40.

Bailey, E. H., and Everhart, D. L., 1964, Geology and quicksilver deposits of the New Almaden district, Santa Clara County, California: U.S. Geol. Survey Prof. Paper 360, 206 p.

Bailey, E. H., Irwin, W. P., and Jones, D. L., 1964, Franciscan and related rocks, and their significance in the geology of western California: California Div. Mines and Geology Bull. 183, 177 p.

Bailey, G. E., 1902, The saline deposits of California: California State Mining Bur. Bull. 24, 216 p.

Bateman, P. C., 1965, Geology and tungsten mineralization of the Bishop district, California: U.S. Geol. Survey Prof. Paper 470, $208 \mathrm{p}$.

Bates, P. H., Young, R. N., and Rapp, Paul, 1923, Tests of caustic magnesia made from magnesite from several sources: U.S. Natl. Bur. Standards Tech. Paper 239, v. 17, p. 529-558.

Bates, T. F., 1945, Origin of the Edwin clay, Ione, California: Geol. Soc. America Bull., v. 56 , no. 1, p. 1-38.

Becker, G. F., 1888, Geology of the quicksilver deposits of the Pacific slope, with an atlas: U.S. Geol. Survey Mon. 13, 486 p.

Benson, W. T., 1963, Pine Flat and Diamond Flat nickel-bearing laterite deposits, Del Norte County, California: U.S. Bur. Mines Rept. Inv. 6206, 19 p.

Bloxam, T. W., 1956, Jadeite-bearing metagraywackes in California: Am. Mineralogist, v. 41, no. 5-6, p. 488-496.

1959, Glaucophane-schists and associated rocks near Valley Ford, California: Am. Jour. Sci., v. 257, no. 2. p. 95-112.

1960, Jadeite-rocks and glaucophane-schists from Angel Island, San Francisco Bay, California: Am. Jour. Sci.. v. 258, no. 8 , p. 555-573. 

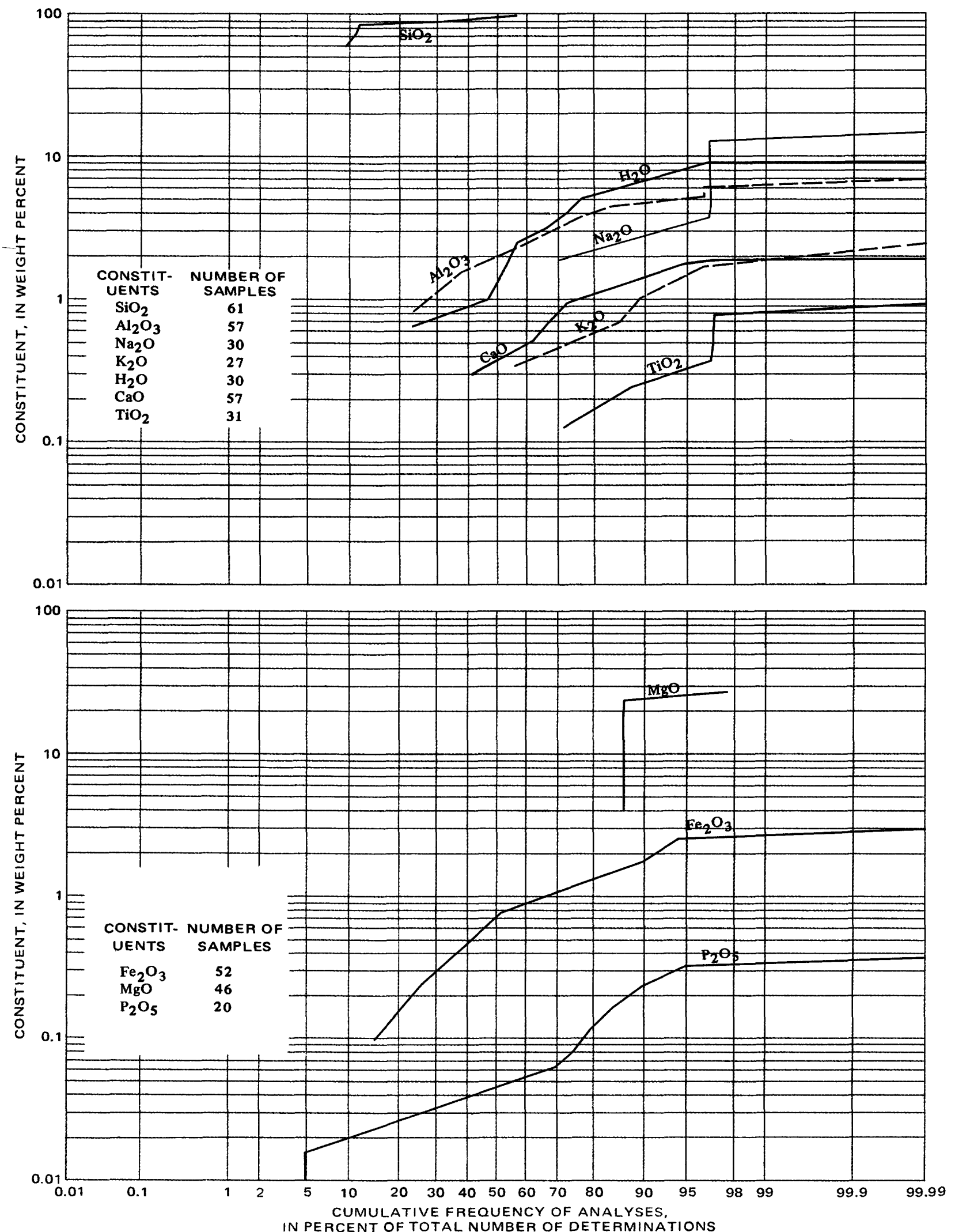

FIGURE 8.-Cumulative-frequency curves $A-K$ showing the proportion of all analyses that contain as much as, but not more than, the indicated percentages of various constituents. Constituents determined in fewer than nine samples and constituents with consistently low values were not plotted. $A$, Group $A$, California. 

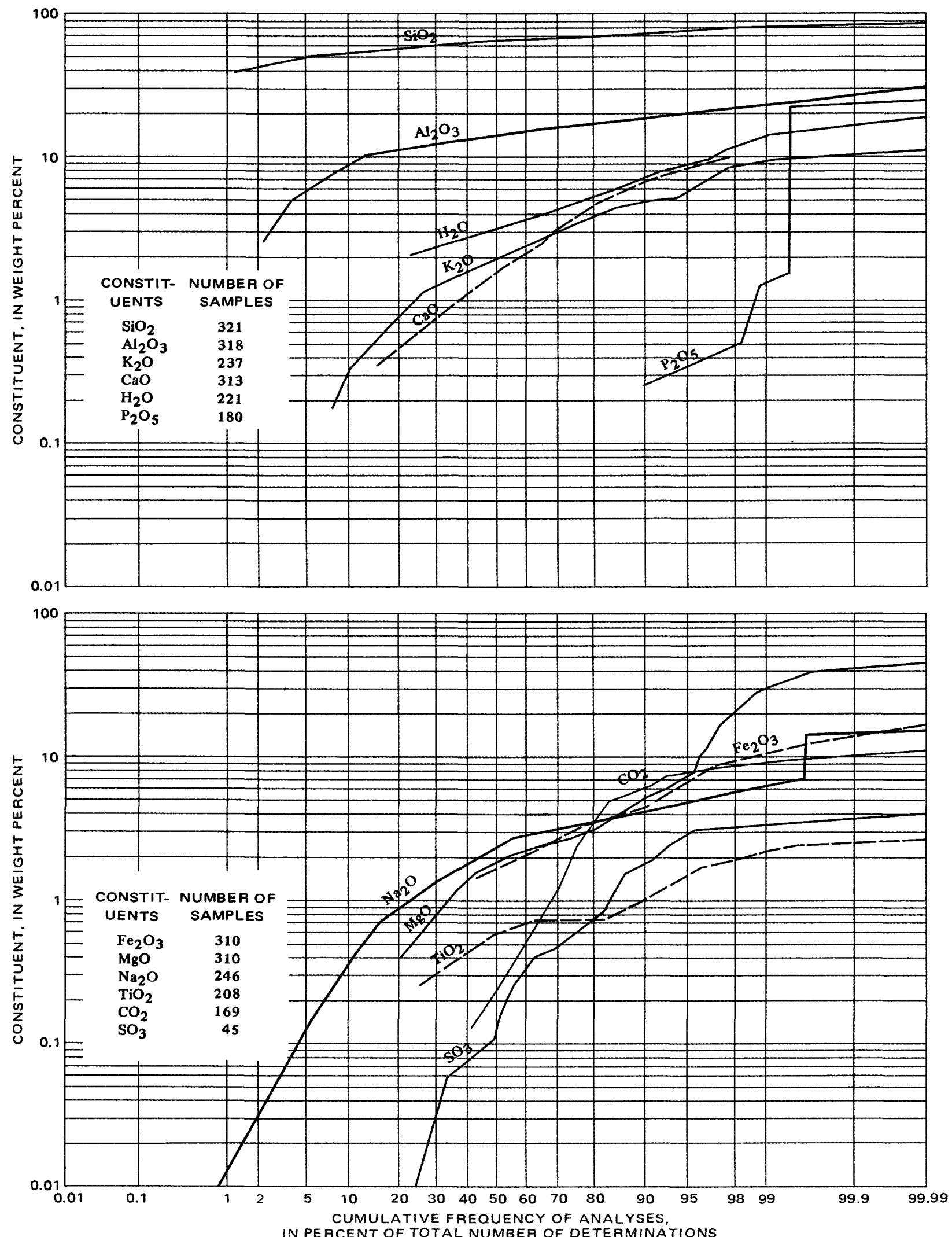

Figure 8.-Cumulative-frequency curve showing percentages of various constituents: $B$, Group $B$, California. 

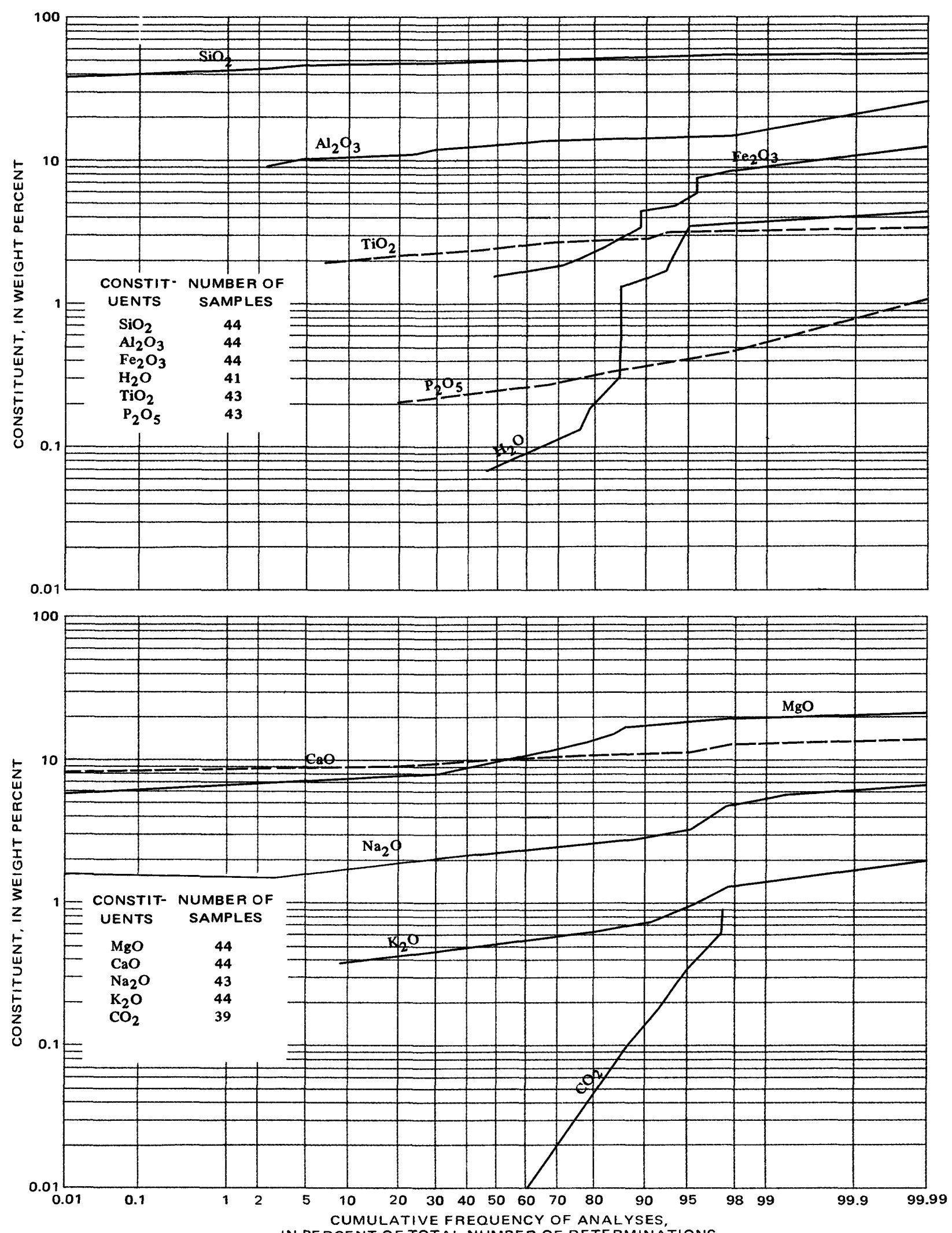

IN PERCENT OF TOTAL NUMBER OF DETERMINÁTIONS

FIGURE 8.-Cumulative-frequency curve showing percentages of various constituents: $C$, Group $B$, Hawaii. 


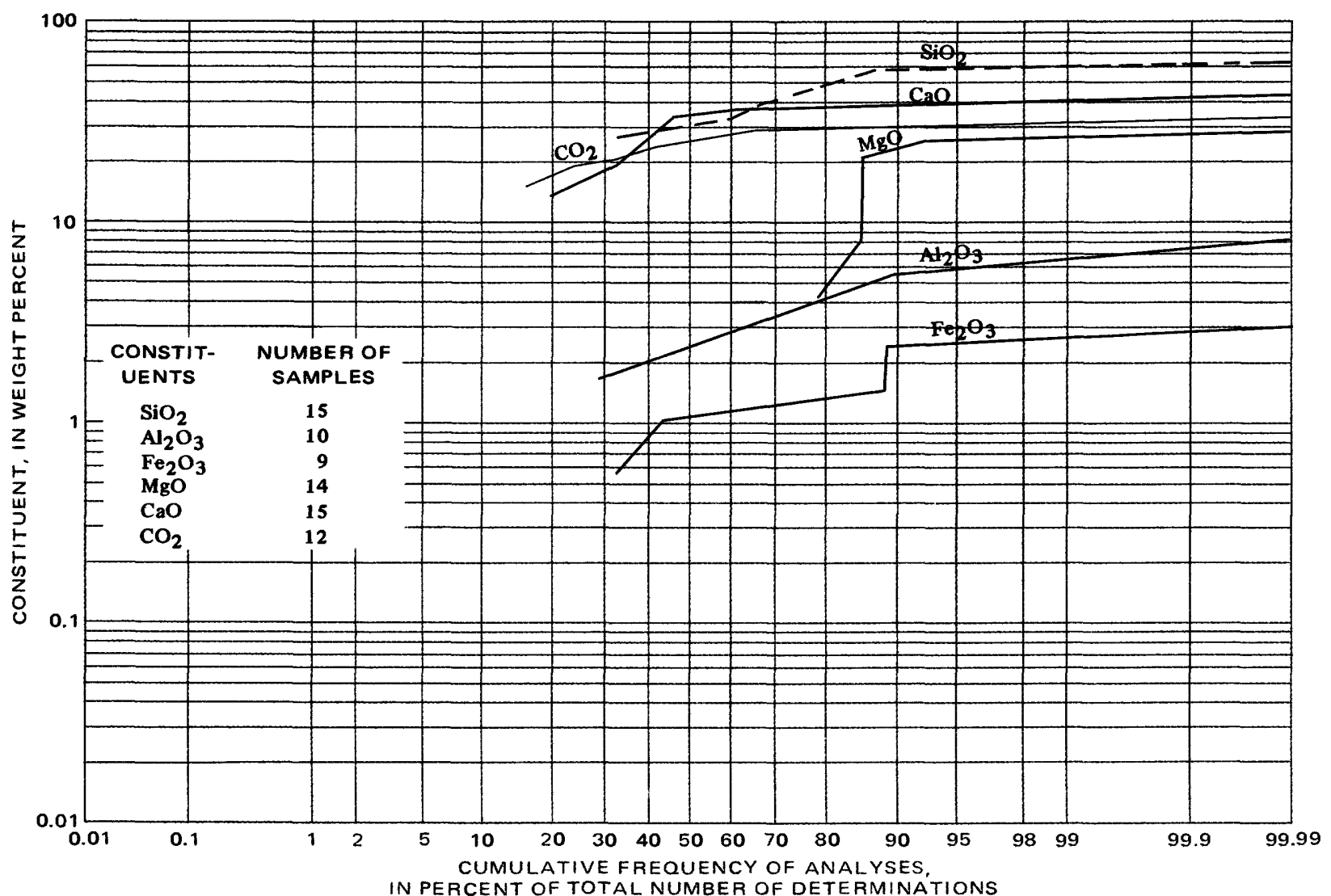

FIGURE 8.-Cumulative-frequency curve showing percentages of various constituents: $D$, Group $C$, California.

Boalich, E. S., Castello, W. O., Huguenin, Emile, Logan, C. A., and Tucker, W. B., 1920, The clay industry in California: California State Mining Bur. Prelim. Rept. 7, 102 p.

Booth, Edward, 1880, Report of the chemist: California State Mining Bur. Ann. Rept., June 1, 1880 to Dec. 1, 1880, p. 15-17.

Bowen, N. L., and Aurousseau, M., 1923, Fusion of sedimentary rocks in drill holes: Geol. Soc. America Bull., v. 34, no. 3, p. 431-448.

Bowen, O. E., Jr., 1954, Geology and mineral deposits of Barstow quadrangle, San Bernardino County, California: California Div. Mines Bull. 165, p. 7-185.

Bowen, O. E., Jr., and Gray, C. H., Jr., 1957, Mines and mineral deposits of Mariposa County, California: California Jour. Mines and Geology, v. 53, no. 1-2, p. 34-343.

1959 , Geology and economic possibilities of the limestone and dolomite deposits of the northern Gabilan Range, California: California Div. Mines and Geology Spec. Rept. 56, 40 p.

1962. The portland cement industry in California, 1962-Part 2: California Div. Mines and Geology Mineral Inf. Service, v. 15 , no. 8, p. 1-11.

Bowen, O. E., Jr., and Ver Planck, W. E., 1965, Stratigraphy, structure, and mineral deposits in the Oro Grande Series near Victorville, California: California Div. Mines and Geology Spec. Rept. 84,41 p.

Bradley, W. W., 1916, Mines and mineral resources of the counties of Colusa, Glenn, Lake, Marin, Napa, Solano, Sonoma, Yolo, California: California State Mining Bur. Rept. 14, pt. 2, p. 173-370.
1925, Magnesite in California: California State Mining Bur. Bull. 79, 147 p.

Bradley, W. W., and Logan, C. A., 1919, San Benito County: California State Mining Bur. Rept. 15, pt. 5, p. 616-673.

Bramlette, M. N., 1946, The Monterey Formation of California and the origin of its siliceous rocks: U.S. Geol. Survey Prof. Paper $212,57 \mathrm{p}$.

Burchard, E. F., 1911, Glass sand, other sand, and gravel: U.S. Geol. Survey Mineral Resources U.S., 1909, pt. 2, p. 519-542. 1912, Lime: U.S. Geol. Survey Mineral Resources U.S., 1911, pt. 2, p. $645-718$.

Burchfiel, B. M., 1923, Refractory clays of the Alberhill, California deposits: Am. Ceramic Soc. Jour., v. 6, no. 11, p. 1167-1175.

Burgess, P. S., 1917, Nitrification as a measure of the availability of different forms of calcium carbonate when employed as correctors of soil acidity: Soil Sci., v. 4, p. 327-336.

Burnett, J. L., 1965, Expansible shale resources of the San JoseGilroy area, California: California Div. Mines and Geology Spec. Rept. 87, 32 p.

Burnham, C. W., 1959, Contact metamorphism of magnesite limestones at Crestmore, California: Geol. Soc. America Bull., v. 70, p. 879-919.

Calhoun, W. A., and Hill, T. E., Jr., 1962, Metallurgical testing of Hawaiian ferruginous bauxites: U.S. Bur. Mines Rept. Inv. $6003,43 \mathrm{p}$.

California Division of Mines, 1948, Unique portland cement operation at Victorville: California Div. Mines Mineral Inf. Service, v. 1, no. 8 , p. 9 . 

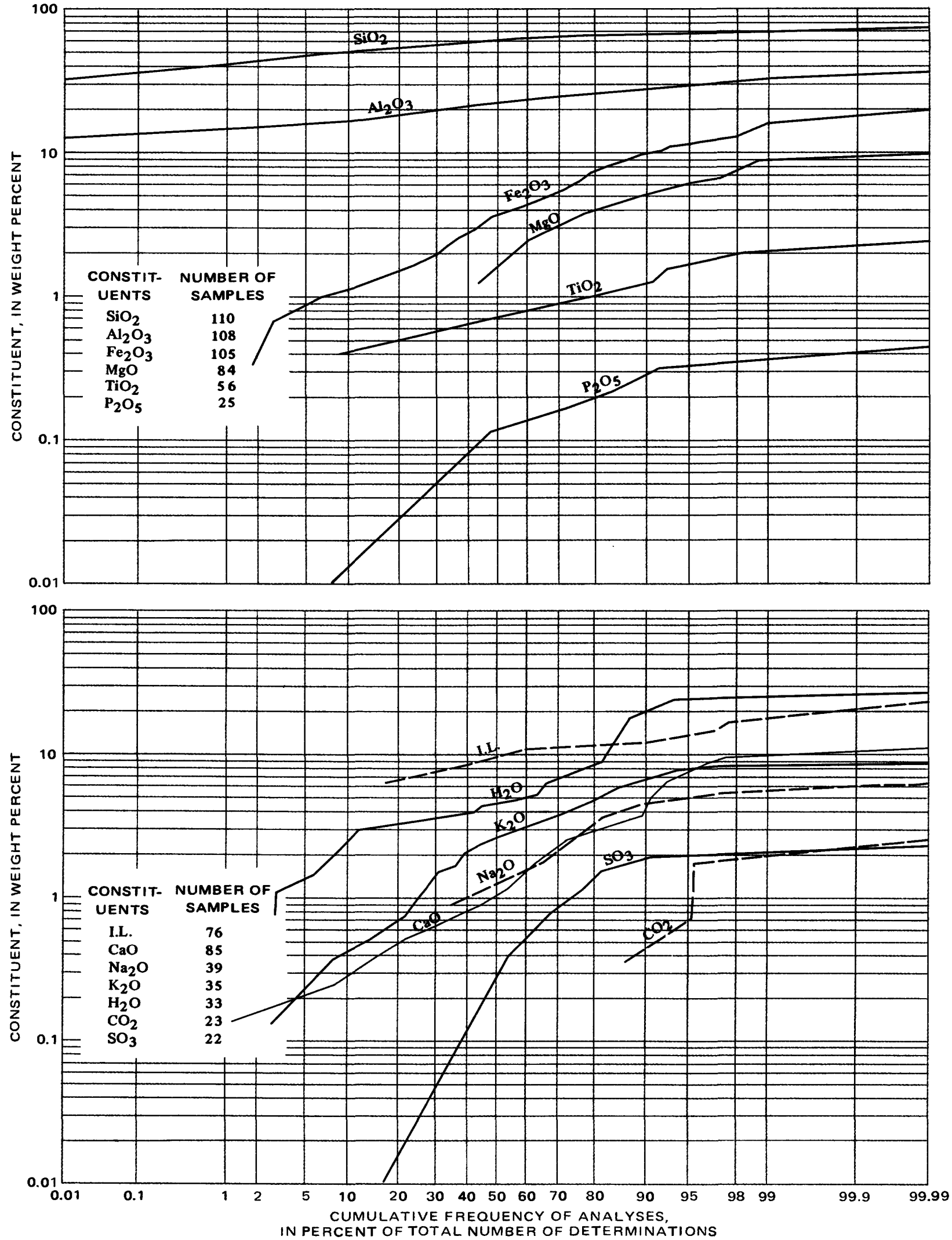

FTGURE 8.-Cumulative-frequency curve showing percentages of various constituents: $E$, Group $D_{5}$, California. (I.L., ignition loss.) 

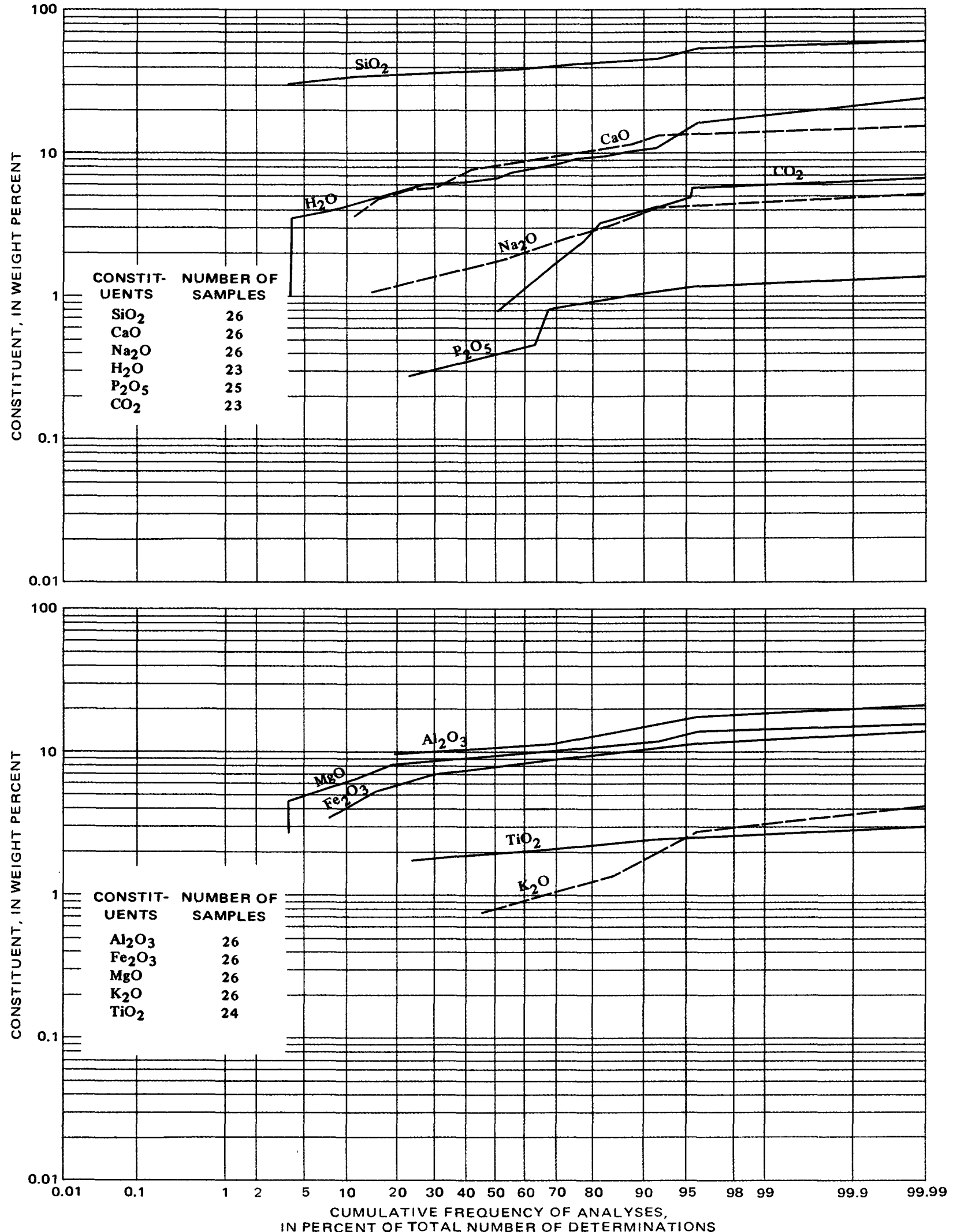

FIGURE 8.-Cumulative-frequency curve showing percentages of various constituents: $F$, Group $D_{5}$, Hawaii. 

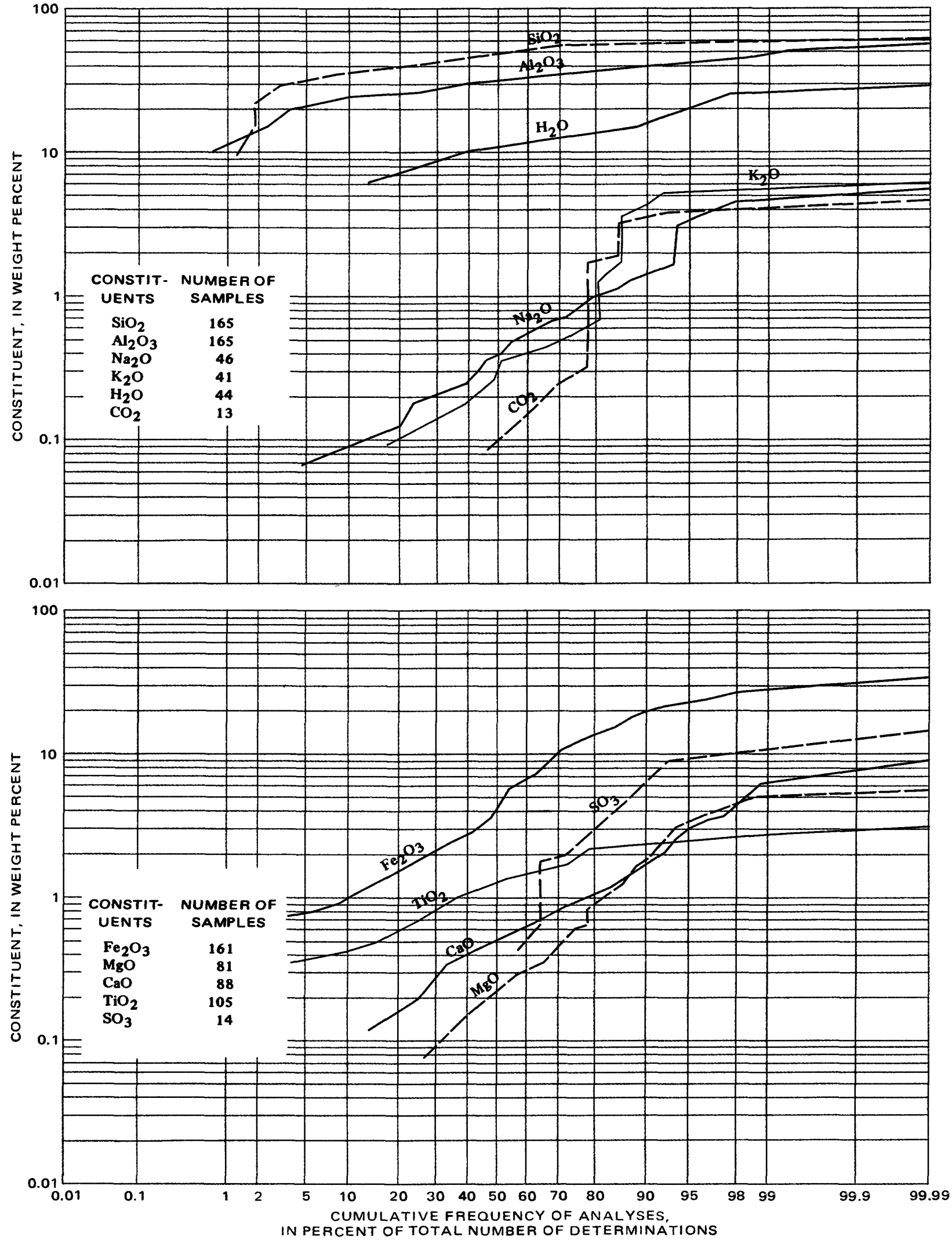

FIGURE 8.-Cumulative-frequency curve showing percentages of various constituents: $G$, Group $D_{6}$, California. 

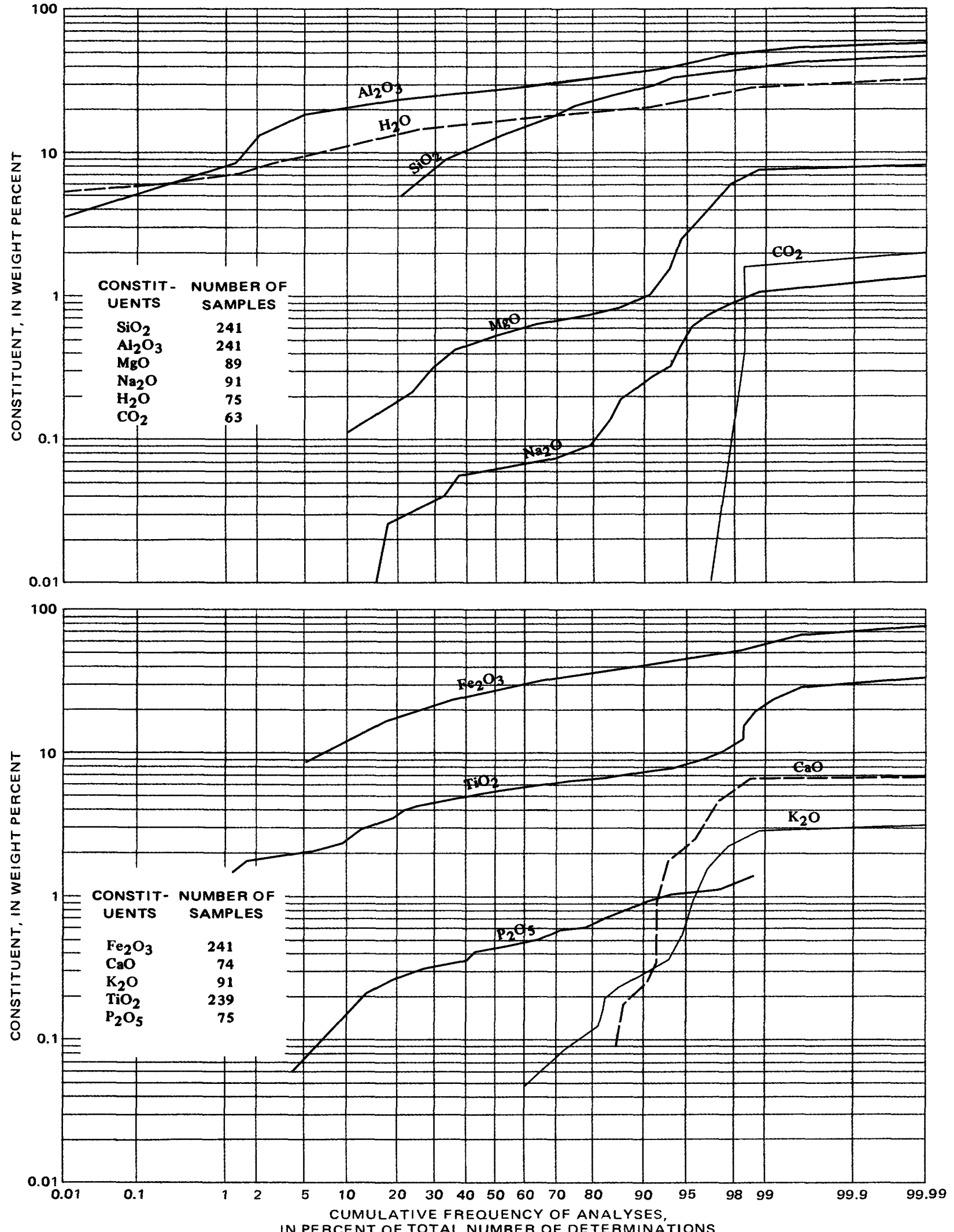

FIGURE 8.-Cumulative-frequency curve showing percentages of various constituents: $H$, Group $D_{6}$, Hawaii. 

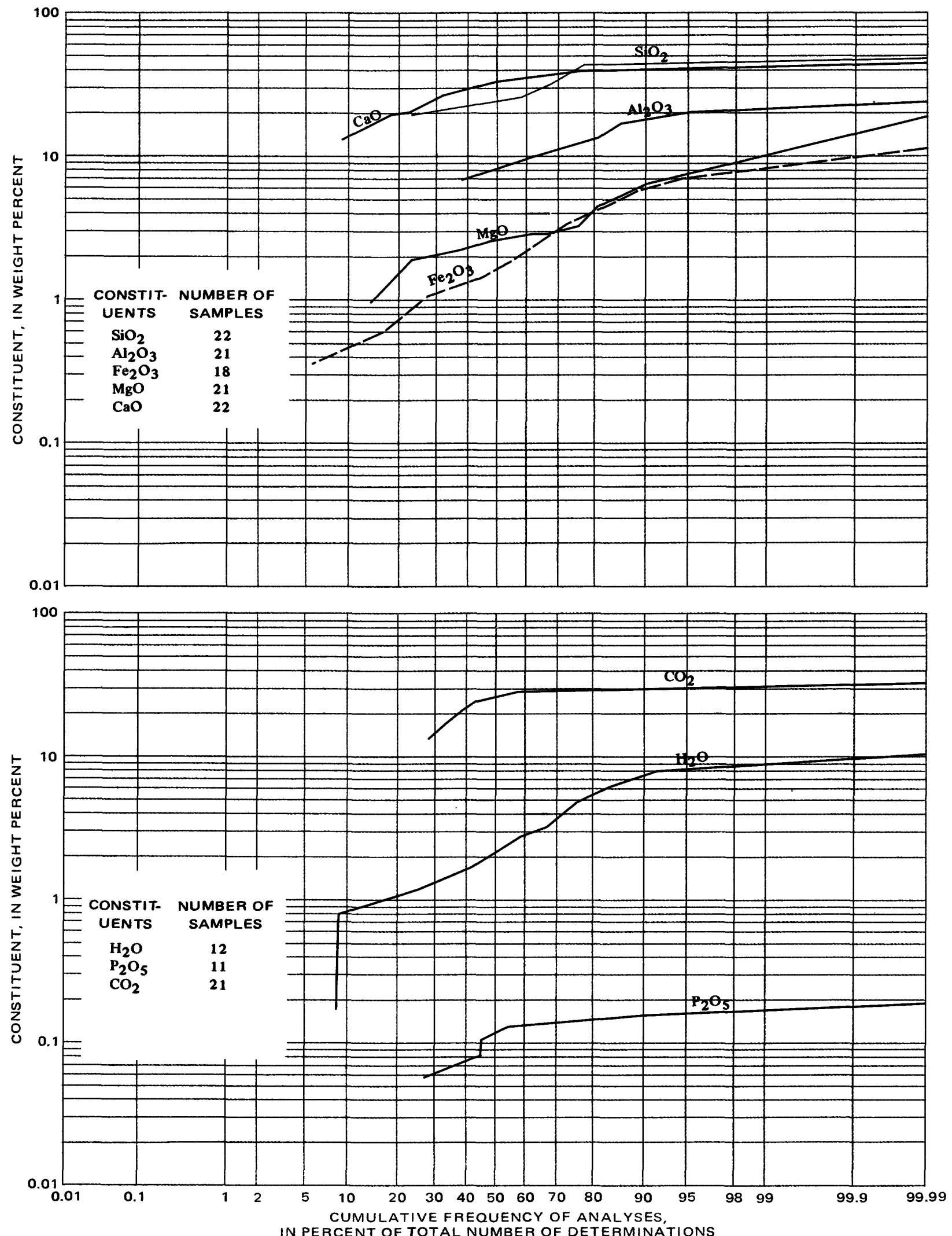

FIGURE 8.-Cumulative-frequency curve showing percentages of various constituents: $I$, Group $E$, California and Hawaii. 

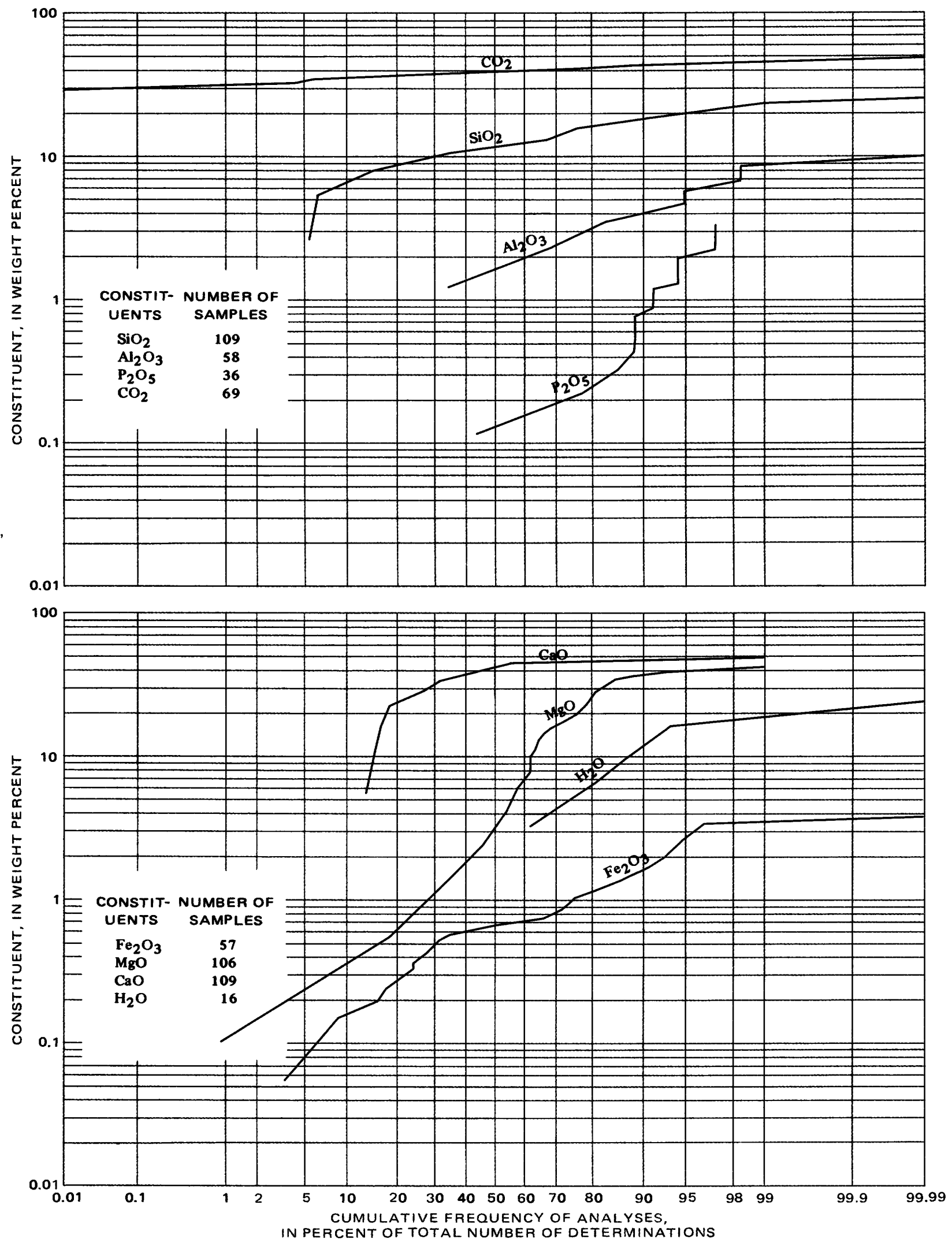

FIGURE 8.-Cumulative-frequency curve showing percentages of various constituents: $J$, Group $F_{1}$, California. 

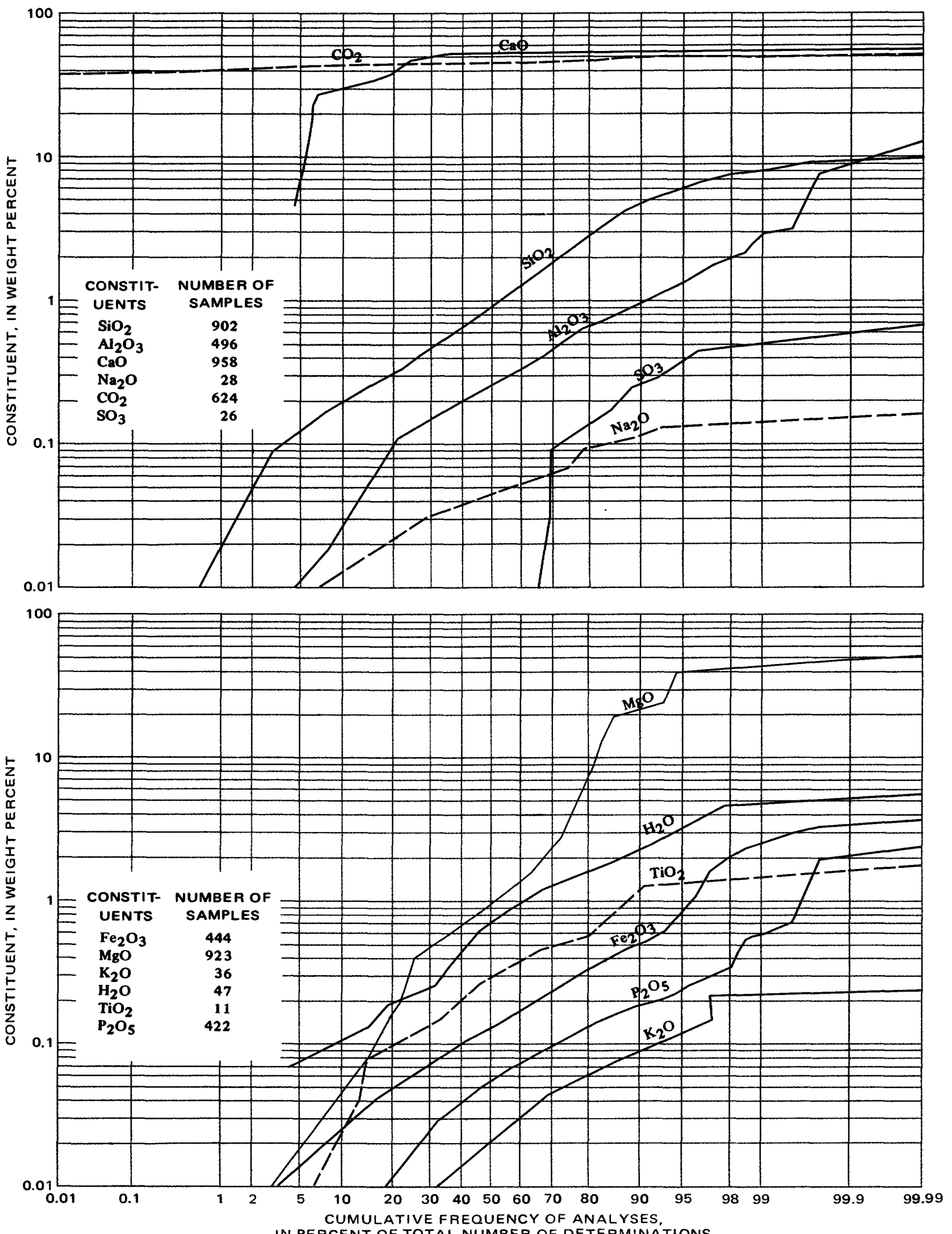

IN PERCENT OF TOTAL NUMBER OF DETERMINATIONS

FIGURE 8.-Cumulative-frequency curve showing percentages of various constituents: $K$, Group $F_{2}$, California and Hawaii. 
1957, Aluminum: California Div. Mines Mineral Inf. Service, v. 10 , no. 7 , p. 1-6.

Campbell, M. R., 1902, Reconnaissance of borax deposits of Death Valley and Mohave Desert: U.S. Geol. Survey Bull. 200, 23 p.

Carlson, D. W., and Clark, W. B., 1954, Mines and mineral resources of Amador County, California: California Jour. Mines and Geology, v. 50, no. 1, p. 149-285.

Cathcart, J. B., 1958, Bauxite deposits of Hawaii, Maui, and Kauai, Territory of Hawaii-A preliminary report: U.S. Geol. Survey open-file rept., $72 \mathrm{p}$.

Chandra, D. K., 1961, Geology and mineral deposits of the Colfax and Foresthill quadrangles, California: California Div. Mines and Geology Spec. Rept. 67, 50 p.

Chapman, R. W., 1946, Lithification of Pleistocene clay at Kahuku Point, Oahu [Hawaii]: Geol. Soc. America Bull., v. 57, no. 11, p. $985-995$.

Chatard, T. M., 1890, Natural soda-its occurrence and utilization: U.S. Geol. Survey Bull. 60, p. 27-101.

Chesterman, C. W., 1943, Contact metamorphic rocks of the Twin Lakes region, Fresno County, California: California Jour. Mines and Geology, v. 38, no. 3-4, p. 243-281.

1956, Pumice, pumicite, and volcanic cinders in California: California Div. Mines Bull. 174, 119 p.

Clark, L. D., 1954, Geology and mineral deposits of the Calaveritas quadrangle, Calaveras County, California: California Div. Mines Spec. Rept. 40, 23 p.

Clark, W. B., 1954, The Cool-Cave Valley limestone deposits, El Dorado and Placer Counties, California: California Jour. Mines and Geology, v. 50, nos. 3 and 4, p. 438-465.

Clark, W. B., and Carlson, D. W., 1956, Mines and mineral resources of El Dorado County, California: California Jour. Mines and Geology, v. 52 , no. 4, p. 369-591.

Clark, W. B., and Lydon, P. A., 1962, Mines and mineral resources of Calaveras County, California: California Div. Mines and Geology County Rept. 2, 217 p.

Clarke, F. W., 1889, Report of work done in the division of chemistry and physics, mainly during the fiscal year 1886-87: U.S. Geol. Survey Bull. 55, 96 p.

1890 , Report of work done in the division of chemistry and physics, mainly during the fiscal year 1887-88: U.S. Geol. Survey Bull. 60, $174 \mathrm{p}$.

1891, Report of work done in the division of chemistry and physics, mainly during the fiscal year 1889-90: U.S. Geol. Survey Bull. 78, $131 \mathrm{p}$.

1903. Mineral analyses from the laboratories of the United States Geological Survey, 1880-1903: U.S. Geol. Survey Bull. 220,119 p.

1908. The data of geochemistry: U.S. Geol. Survey Bull. 330. $716 \mathrm{p}$.

1915, Analyses of rocks and minerals from the laboratory of the United States Geological Survey, 1880 to 1914: U.S. Geol. Survey Bull. 591, $376 \mathrm{p}$,

Clarke, F. W., and Hillebrand, W. F., 1897, Analyses of rocks, with a chapter on analytical methods, laboratory of the United States Geological Survey 1880 to 1896: U.S. Geol. Survey Bull. 148, $306 \mathrm{p}$.

Cleveland, G. B., 1957, Clay, in Mineral commodities of California: California Div. Mines Bull. 176, p. 131-152.

1958, Poverty Hills diatomaceous earth deposit, Inyo County, California: California Jour. Mines and Geology, v. 54, no. 3, p. 305-316.

1960, Geology of the Otay bentonite deposits, San Diego County, California: California Div. Mines Spec. Rept. 64, 16 p.
1961, Economic geology of the Long Valley diatomaceous earth deposits, Mono County, California: California Div. Mines and Geology Map, sheet 1 .

1962, Clay, in Troxel, B. W., and Morton, P. K., Mines and mineral resources of Kern County, California: California Div. Mines and Geology County Rept. 1, p. 69-82.

Cloudman, H. C., Huguenin, Emile, and Merrill, F. J. H., 1919, San Bernardino County: California State Mining Bur. Rept. 15, pt. 6, p. 775-899.

Crawford, J. J., 1894, Report of the State mineralogist, two years ending September 15, 1894: California State Mining Bur. Rept. 12,412 p.

1896, Report of the State mineralogist for the two years ending September 15, 1896: California State Mining Bureau Rept. $13,726 \mathrm{p}$.

Creely, R. S., 1965, Geology of the Oroville quadrangle, California: California Div. Mines and Geology Bull. 184, 86 p.

Cressman, E. R., 1962, Nondetrital siliceous sediments, in Data of geochemistry: U.S. Geol. Survey Prof. Paper 440-T, 23 p.

Cross, Whitman, 1915, Lavas of Hawaii and their relations: U.S. Geol. Survey Prof. Paper 88, $97 \mathrm{p}$.

Dana, J. D., 1852, On coral reefs and islands: Am. Jour. Sci., 2nd ser., v. 14 , no. 40 , p. $76-84$.

Davis, C. W., and Vacher, H. C., 1928, Bentonite, its properties, mining, preparation, and utilization: U.S. Bur. Mines Tech. Paper 439, $51 \mathrm{p}$.

Davis, E. F., 1918a, The Franciscan sandstone: California Univ. Pubs. Geol. Sci., v. 11, no. 1, p. 1-44.

1918b, The radiolarian cherts of the Franciscan Group: California Univ. Pubs. Geol. Sci., v. 11, no. 3, p. 235-432.

Davis, F. F., 1955, Mines and mineral resources of San Mateo County, California: California Jour. Mines and Geology, v. 51, no. 4, p. 401-458.

Day, A. L., and Allen, E. T., 1925, The volcanic activity and hot springs of Lassen Peak [California]: Carnegie Inst. Washington Pub. 360,190 p.

Day, W. C., 1892, Stone: U.S. Geol. Survey Mineral Resources U.S., 1889-90, p. $373-440$.

1898, Stone: U.S. Geol. Survey 19th Ann. Rept. for 1897-98, pt. 6 , p. $205-309$.

1899, Stone: U.S. Geol. Survey 20th Ann. Rept. for 1898-99, pt. 6 , p. $269-464$

Dickinson, W. R., 1962, Metasomatic quartz keratophyre in central Oregon: Am. Jour. Sci., v. 260, no. 4, p. 249-266.

Dietrich. W. F., 1928. The clay resources and the ceramic industry of California: California State Mining Bur. Bull. 99, 383 p.

Dietrich, W. F., and Meyer, W. W., 1930, An investigation of the possible uses of certain California clays in vitreous china sanitary-ware bodies: Am. Ceramic Soc. Jour., v. 13, no. 3, p. 202-217.

Diller, J. S., 1890, Sandstone dikes: Geol. Soc. America Bull., v. 1, p. 411-442.

1891, A late volcanic eruption in northern California and its peculiar lava: U.S. Geol. Survey Bull. 79, 33 p.

1903, Klamath Mountain section, California: Am. Jour. Sci., 4 th ser., v. 15 , p. 342-362.

1904, Mining and mineral resources in the Redding quadrangle, California, in 1903: U.S. Geol. Survey Bull. 225, p. 169-179.

1906, Description of the Redding quadrangle, California: U.S. Geol. Survey Geol. Atlas, Folio 138, 14 p.

1911, The auriferous gravels of the Trinity River basin, California: U.S. Geol. Survey Bull. 470, p. 11-29.

Dolbear, C. E., 1913, The Searles Lake potash deposit: Eng. and Min- 
ing Jour., v. 95, no. 5, p. 259-261.

Dupont, F. M., 1910, The borax industry: Jour. Indus. and Eng. Chem., v. 2, no. 12 , p. 500-503.

Durrell, Cordell, 1953, Geological investigations of strontium deposits in southern California: California Div. Mines Spec. Rept. 32,48 p.

Eakle, A. S., 1911, Neocolemanite, a variety of colemanite, and howlite from Lang, Los Angeles County, California: California Univ. Pubs. Geol. Sci., v. 6, no. 9, p. 179-189.

1914, Minerals of California: California State Mining Bur. Bull. $67,226 \mathrm{p}$.

1917, Minerals associated with the crystalline limestone at Crestmore, Riverside County, California: California Univ. Pubs. Geol. Sci., v. 10, no. 19, p. 327-360.

1923, Minerals of California: California State Mining Bur. Bull. 91, 328 p.

Eardley-Wilmot, V. L., 1928, Diatomite, its occurrence, preparation and uses: Canada Dept. Mines, Mines Branch Pub. 691, 182 p.

Eckel, E. B., and Meyers, W. B., 1946, Quicksilver deposits of the New Idria district, San Benito and Fresno Counties, California: California Jour. Mines and Geology, v. 42, no. 2, p. 81-124.

Eckel, E. C., 1905, Cement materials and industry of the United States: U. S. Geol. Survey Bull. 243, 395 p.

1912 , Building stones and clays-their origin, characters and examination, 1st edition: New York, John Wiley and Sons, 264 p.

1913, Portland cement materials and industry in the United States, with contributions by E. F. Burchard, A. F. Crider, G. B. Richardson, E. A. Smith, J. S. Taff, E. O. Ulrich, and W. H. Weed: U. S. Geol. Survey Bull. 522, 401 p.

1928, Cements, limes, and plasters-their materials, manufacture, and properties, 3d edition: New York, John Wiley and Sons, $699 \mathrm{p}$.

1934, Limestone deposits of the San Francisco region:

- California Jour. Mines and Geology, v. 29, no. 3-4, p. 348361.

Engle, René, Gay, T. E., Jr., and Rogers, B. L., 1959, Geology and mineral deposits of the Lake Elsinore quadrangle, California: California Div. Mines Bull. 146, 154 p.

Ernst, W. G., Seki, Yotaro, Onuki, H., Gilbert, M. C., 1970, Comparative study of low-grade metamorphism in the California Coast Ranges and the Outer Metamorphic Belt of Japan: Geol. Soc. America Mem. 124, 276 p.

Everhart, J. O., 1957, Use of auxiliary fluxes to improve structural clay bodies: Am. Ceramic Soc. Bull. 36, no. 7, p. 268-271.

Fairbanks, H. W., 1896, The geology of Point Sal: California Univ. Pubs. Geol. Sci. Bull. 2, 91 p. 1904, Gypsum deposits in California: U.S. Geol. Survey Bull. 223, p. 119-123.

Fiske, R. S., and Koyanagi, R. Y., 1968, The December 1965 eruption or Kilauea Volcano, Hawaii: U.S. Geol. Survey Prof. Paper $607,21 \mathrm{p}$.

Foshag, W. F., 1918, Ulexite from Lang, California: Am. Mineralogist, v. 3 , no. 4 , p. 35 .

1935, Burkeite, a new mineral species from Searles Lake, California: Am. Mineralogist, v. 20, no. 1, p. 50-56.

1938, Sodium bicarbonate from Searles Lake, California [abs.]: Am. Mineralogist, v. 23, no. 3, p. 169.

Foshag, W. F., and Woodford, A. O., 1936, Bentonitic magnesian clay mineral from California: Am. Mineralogist, v. 21, no. 4, p. 238-244.

Franke, H. A., 1930, San Francisco Field Division: California Div. Mines Rept. 26, no. 1, p. 2-39. 1931, San Francisco Field Division: California Div. Mines
Rept. 26, no. 4, p. 413-471.

1936, Mines and mineral resources of San Luis Obispo County: California Jour. Mines and Geology, v. 31, no. 4, p. 402-461.

Fraser, H. J., Wilson, H. D. B., and Hendry, N. W., 1943, Hot springs deposits of the Coso Mountains: California Jour. Mines and Geology, v. 38, nos. 3 and 4, p. 223-242.

Gale, H. S., 1912, Magnesite: U.S. Geol. Survey Mineral Resources U.S., 1911, pt. 2, p. 1113-1127.

1914a, Prospecting for potash in Death Valley, California: U.S. Geol. Survey Bull. 540, p. 407-415.

$1914 \mathrm{~b}$, Salt, borax, and potash in Saline Valley, Inyo County, California: U.S. Geol. Survey Bull. 540, p. 416-421.

$1914 \mathrm{c}$, Late developments of magnesite deposits in California and Nevada: U.S. Geol. Survey Bull. 540, p. 483-520.

1915, Salines in the Owens, Searles, and Panamint basirs, southeastern California: U.S. Geol. Survey Bull. 580, p. 251-323.

1946, Geology of the Kramer borate district, Kern County, California: California Jour. Mines and Geology, v. 42, no. 4, p. 325-378.

Glaser, J. D., 1971, Geology and mineral resources of southern Maryland: Maryland Geol. Survey Rept. Inv. 15, 85 p.

Goldman, H. B., 1959, Franciscan chert in California concrete aggregates: California Div. Mines and Geology Spec. Rept. 55, 28 p.

Goldsmith, J. R., Graf, D. L., Witters, Juanita, and Northrop, D. A., 1962, Studies in the system $\mathrm{CaCO}_{3}-\mathrm{MgCO}_{3}-\mathrm{FeCO}_{3}-$ Part 1 , Phase relations; Part 2, A method for major-element spectrochemical analysis; Part 3, Composition of some ferroan dolomites: Jour. Geology, v. 70, no. 6, p. 659-688.

Goodyear, W. A., 1890, Marble quarries, in Degroot, Henry, Inyo County: California State Mining Bur. 10th Ann. Rept., p. 215-218.

Gower, H. D., and Madsen, B. M., 1964, The occurrence of phosphate rock in California: U.S. Geol. Survey Prof. Paper 501-D, p. D79-D85.

Gray, C. H., Jr., 1961, Mines and mineral deposits of the Corona South quadrangle, Riverside and Orange Counties, California: California Div. Mines and Geology Bull. 178, p. 59-120.

1962a, Limestone resources of southern California, Part 1: California Div. Mines and Geology Mineral Inf. Service, v. 15, no. 5, p. 1-5.

1962b, Limestone resources of southern California, Part 2: California Div. Mines and Geology Mineral Inf. Service, v. 15, no. 6, p. 4-7.

$1962 \mathrm{c}$, Limestone, dolomite, and cement, in Troxel, B. W., and Morton, P. K., Mines and mineral resources of Kern County, California: California Div. Mines and Geology County Rept. 1, p. 212-236.

Hagner, A. F., 1939, Adsorptive clays of the Texas Gulf Coast: Am. Mineralogist, v. 24, no. 2, p. 67-108.

Hall, C. W., and Sardeson, F. W., 1895, The magnesian series of the Northwestern States: Geol. Soc. America Bull., v. 6, p. 167-198.

Hall, W. E., and Stephens, H. G., 1963, Economic geology of the Panamint Butte quadrangle and Modoc district, Inyo County, California: California Div. Mines and Geology, Spec. Rept. 73, $39 \mathrm{p}$.

Hanks, H. G., 1882, Mud volcanoes and Colorado Desert: California State Mining Bur. 2d Ann. Rept., 1880-82, p. 227-240.

1883, Report on the borax deposits of California and Nevada: California State Mining Bur. 3rd Ann. Rept., 1882-83, pt. 2, $111 \mathrm{p}$.

1884, Catalogue and description of the minerals of California as far as known, with special reference to those having an 
economic value: California State Mining Bur. 4th Ann. Rept., 1883-84, p. 61-397.

1885. Fifth annual report of the State mineralogist, for the year ending May 15, 1885: California State Mining Bur. 5th Ann. Rept., 235 p.

Harder, E. C., 1910, The gypsum deposits of the Palen Mountains, Riverside County, California: U.S. Geol. Survey Bull. 430, p. 407-416.

1912. Iron-ore deposits of the Eagle Mountains, California: U.S. Geol. Survey Bull. 503, 81 p.

Hart, E. W., 1959, Geology of limestone and dolomite deposits in the southern half of Standard quadrangle, Tuolumne County, California: California Div. Mines Spec. Rept. 58, 25 p. 1966, Mines and mineral resources of Monterey County: California Div. Mines and Geology County Rept. 5, 142 p.

Hay, R. L., and Iijima, A., 1968, Nature and origin of palagonite tuffs of the Honolulu Group on Oahu, Hawaii, in Studies in volcanology- A memoir in honor of Howel Williams: Geol. Soc. America Mem. 116, p. 331-376.

Hay, R. L., and Jones, B. F., 1972, Weathering of basaltic tephra on the Island of Hawaii: Geol. Soc. America Bull., v. 83, no. 2, p. 317-332.

Hazzard, J. C., 1938, Paleozoic section in the Nopah and Resting Springs Mountains, lnyo County, California: California Jour. Mines and Geology, v. 33, no. 4, p. 237-339.

Heiken, Grant, 1972, Morphology and petrography of volcanic ashes: Geol. Soc. America Bull., v. 83, no. 7, p. 1961-1988.

Hess, F. L., 1910, A reconnaissance of the gypsum deposits of California: U.S. Geol. Survey Bull. 413, 36 p.

Hewett, D. F., 1956, Geology and mineral resources of the Ivanpah quadrangle, California and Nevada: U.S. Geol. Survey Prof. Paper 275, $172 \mathrm{p}$.

Heyl, G. R., and Walker, G. W., 1949, Geology of the limestone near Gazelle, Siskiyou County, California: California Jour. Mines and Geology, v. 45, no. 4, p. 514-520.

Heyl, G. R., and Wiese, J. H., 1949, Geology of the limestone near Sonora, Tuolumne County, California: California Jour. Mines and Geology, v. 45, no. 4, p. 509-513.

Hidden, W. E., 1885, On hanksite, in Hanks, H. G., Fifth Annual Report of the State Mineralogist: California State Mining Bur. 5 th Ann. Rept., p. 62-64.

Hietanen, Anna, 1951, Metamorphic and igneous rocks of the Merrimac area, Plumas National Forest, California: Geol. Soc. America Bull., v. 62 , no. 6 , p. 565-607.

Hilgard, E. W., 1892, A report on the relations of soil to climate: U.S. Dept. Agriculture Weather Bur. Bull. 3, 59 p.

Hill, J. H., 1923, Clay deposits of the Alberhill Coal and Clay Company: California State Mining Bur. Rept. 19, no. 4, p. 185-210.

Hill, T. P., Werner, M. A., and Horton, M. J., compilers, 1967, Chemical composition of sedimentary rocks in Colorado, Kansas, Montana, Nebraska, North Dakota, South Dakota, and Wyoming, with an introduction by W. W. Rubey: U.S. Geol. Survey Prof. Paper 561, 241 p.

Hill, T. P., and Werner, M. A., compilers, 1972, Chemical composition of sedimentary rocks in Alaska, Idaho, Oregon, and Washington: U.S. Geol. Survey Prof. Paper 771, 319 p. [1973].

Hinds, N. E. A., 1929, The weathering of the Hawaiian lavas, pt. 1, The compositions of lavas and soils from Kauai: Am. Jour. Sci., 5 th ser., v. 17 , no. 100 , p. 297-320.

Hodge, E. T., 1935, Fluxes and refractories, App. L-1 of Report on available raw materials for a Pacific Coast iron industry: Port land, Oregon, U.S. Army Corps Engineers, North Pacific Div., v. 4, App. L-1, 84 p.

1938a, Silica localities other than the Pacific Northwest, in
Market for Columbia River hydroelectric power using northwest minerals: Portland, Oregon, U.S. War Dept., Corps Engineers, North Pacific Div., sec. 2, v. 2, pt. 2, p. 177-189.

1938b, Other limestone occurrences available to or competitive with the Lower Columbia River area, in Market for Columbia River hydroelectric power using northwest minerals: Portland, Oregon, U.S. War Dept., Corps Engineers, North Pacific Div., sec. 3, v. 2, pt. 2, p. 373-382.

$1938 \mathrm{c}$, Uses, technology, and market of limestone and lime, in Market for Columbia River hydroelectric power using northwest minerals: Portland, Oregon, U.S. War Dept., Corps Engineers, North Pacific Div., sec 3, v. 2, pt. 3, p. 383-621.

1944, Limestone of the Pacific Northwest-Available limestones suitable for calcium carbide and for flint glass industries: Portland, Oregon, U.S. Bonneville Power Adm., 104 p.

Hoots, H. W., 1930, Geology and oil resources along the southern border of San Joaquin Valley, California: U.S. Geol. Survey Bull 812-D, p. D243-D332.

1931, Geology of the eastern part of the Santa Monica Mountains, Los Angeles County, California: U.S. Geol. Survey Prof. Paper 165, p. 83-134.

Huguenin, Emile, 1919, Ventura County: California State Mining Bur. Rept. 15, p. 751-769.

Huguenin, Emile, and Castello, W. O., 1921a, San Mateo County: California State Mining Bur. Rept. 17, p. 167-179.

1921b, Santa Clara County: California State Mining Bur. Rept. 17, p. 180-227.

Irelan, William, Jr., 1888, Report of the State mineralogist: California State Mining Bur. 8th Ann. Rept., 948 p.

1890, California cement: California State Mining Bur. 9th Ann. Rept., p. 309-311.

Johnson, F. T., and Ricker, Spangler, 1949, Ione-Carbondale clays, Amador County, California; California Jour. Mines and Geology, v. 45 , no. 3 , p. 491-498.

Johnston, W. D., 1890, Clays: California State Mining Bur. 9th Ann. Rept., p. 287-308.

Jones, C. C., 1916, The Pacific Coast iron situation-The iron ores of California and possibilities of smelting: Am. lnst. Mining Engineers Trans., v. 53, p. 306-323.

Jones, W. F., 1911, The geology of the Sargent oil field: California Univ. Pubs. Geol. Sci., v. 6, no. 3, p. 55-78.

Keenan, M. F., 1932, The Eocene Sierra Blanca Limestone at the type locality in Santa Barbara County, California: San Diego Soc. Nat. History Trans., v. 7, no. 8, p. 53-84.

Kelley, F. R., Cleveland, G. B., and Arkey, R. J., 1959, Field trip to the Ione clay area [California] held in conjunction with the 6th National Clay Conference, August 21, 1957, in Swineford, Ada, ed., Clays and clay minerals: Internat. Ser. Mons. Earth Sci., v. 2, p. 1-17.

Kerr, P. F., and Cameron, E. N., 1936, Fuller's earth of bentonitic origin from Tehachapi, California: Am. Mineralogist, v. 21, no. 4, p. 230-237.

Kerr, P. F., Hamilton, P. K., and Pill, R. J., 1950, X-ray diffraction measurements, in Analytical data on reference clay minerals: Am. Petroleum lnst. Research Proj. 49, Clay Mineral Standards, Prelim. Rept. 7, secs. 1A and 1B, p. 1-58.

Kerrick, D. M., and Cotton, W. R., 1971, Stability relations of jadeite pyroxene in Franciscan metagraywackes near San Jose, California: Am. Jour. Sci., v. 271, no. 4, p. 350-369.

Kessler, D. W., 1919, Physical and chemical tests of the commercial marbles of the United States: U.S. Natl. Bur. Standards Tech. Paper 123, 54 p.

King, C. R., 1948, Pumice and perlite as industrial materials in California: California Jour. Mines and Geology, v. 44, no. 3, p. 293-319. 
Kinkel, A. R., Jr., Hall, W. E., and Albers, J. P., 1956, Geology and base-metal deposits of west Shasta copper-zinc district, Shasta County, California: U.S. Geol. Survey Prof. Paper 285 . $156 \mathrm{p}$.

Kivari, A. M., 1942, Milling at the Permanente Cement Plant: Am. Inst. Mining Metall. Engineers Trans., v. 148, p. 374-397.

Knopf, Adolph, 1918, Strontianite deposits near Barstow, California: U.S. Geol. Survey Bull. 660, p. 257-270.

Kümmel, H. B., 1901, Report on the portland cement industry: New Jersey Geol. Survey Ann. Rept., 1900, p. 9-101.

Ladoo, R. B., 1920, The mining and preparation of tripoli: U.S. Bur. Mines Rept. Inv. 2190, 9 p.

1921, Bentonite: U.S. Bur. Mines Rept. Inv. 2289, 5 p.

1948, Industrial silica for Pacific Northwest industries: Portland, Oregon, Raw Materials Survey, Inc. Resource Rept. 1, 40 p.

Ladoo, R. B., and Myers. W. M., 1951, Nonmetallic minerals, 2nd ed: New York, McGraw-Hill, 605 p.

Laizure, C. M., 1925a, San Francisco Field Division: California State Mining Bur. Rept. 21. no. 1, p. 23-57.

1925b, San Francisco Field Division: California State Mining Bur. Rept. 21, no. 3, p. 281-324.

1925c, San Francisco Field Division: California State Mining Bur. Rept. 21, no. 4, p. 499-538.

1926, San Francisco Field Division: California State Mining Bur. Rept. 22, no. 1, p. 68-93.

1929, San Francisco Field Division: California Div. Mines and Mining Rept. 25, no. 3, p. 301-365.

Lamar, R. S., 1953, Absorbent clays in California: California Jour. Mines and Geology, v. 49, no. 3, p. 297-337.

Lamey, C. A., 1946, Shasta and California iron-ore deposits, Shasta County, California: California Div. Mines Bull. 129, pt. K, p. 137-164.

Laudermilk, J. D., 1935, Soda-alunite from Molokai, Hawaiian Islands: Am. Mineralogist, v. 20, no. 1, p. 57-58.

Laudermilk, J. D., and Woodford, A. O., 1932, Concerning rillensteine: Am. Jour. Sci., ser. 5, v. 23, no. 134, p. 135-154. 1934, Secondary montmorillonite in a California pegmatite: Am. Mineralogist, v. 19 , no. 6, p. 260-267.

Lawson, A. C., 1893, The geology of Carmelo Bay: California Univ. Pubs. Geol. Sci., v. 1, p. 1-59.

1914, Description of the San Francisco district, CaliforniaTamalpais, San Francisco, Concord, San Mateo, and Hayward quadrangles: U.S. Geol. Survey Geol. Atlas, Folio 193, 24 p.

Lindgren, Waldemar, 1896, The gold-quartz veins of Nevada City and Grass Valley districts, California: U.S. Geol. Survey 17th Ann. Rept., pt. 2, p. 1-262.

1898, Rhyolitic pumice, in Diller, J. S., The educational series of rock specimens collected and distributed by the United States Geological Survey: U.S. Geol. Survey Bull. 150, p. 148-149.

Linton, Robert, 1928, Tertiary clays of southern California: Am. Ceramic Soc. Jour., v. 11 , no. 10, p. 771-781.

Lockwood, J. P., 1971, Sedimentary and gravity-slide emplacement of serpentine: Geol. Soc. America Bull., v. 82, no. 4, p. 919-936.

Loew, Oscar, 1876, Report on the alkaline lakes, thermal springs, mineral springs, and brackish waters of southern California and adjacent country: U.S. Geog. Surveys West of the One Hundreth Meridian (Wheeler), App. JJ, p. 188-199.

Logan, C. A., 1926, Sacramento Field Division: California State Mining Bur. Rept. 22, no. 2, p. 121-216.

1947, Limestone in California: California Jour. Mines and Geology, v. 43, no. 3, p. 175-357.

Loney, R. A., 1968, Flow structure and composition of the Southern
Coulee, Mono Craters, California-A pumiceous rhyolite flow, in Studies in volcanology $-\mathrm{A}$ memoir in honor of Howel Williams: Geol. Soc. America Mem. 116, p. 415-440.

Lowell, F. L., 1916, The counties of Del Norte, Humboldt, Mendocino: California State Mining Bur. Rept. 14, pt. 3, p. $371-425$.

Lydon. P. A., 1964, Unusual phosphatic rock-New deposit near Hyampom opens economic possibilities: California Div. Mines and Geology Mineral Inf. Service, v. 17, no. 5, p. 65-74.

Lyons, A. B., 1896, Chemical composition of Hawaiian soils and of the rocks from which they have been derived: Am. Jour. Sci., ser. 4, v. 2, p. 421-429.

MacBoyle, Errol, 1920, Mines and mineral resources of Plumas County: California State Mining Bur. Rept. 16, 188 p.

Macdonald, G. A., and Katsura, Takashi, 1965, Eruption of Lassen Peak, Cascade Range, California, in 1915-Example of mixed magmas: Geol. Soc. America Bull., v. 76, no. 5, p. 475-482.

Mason, Brian, 1952, Principles of geochemistry [1st ed.]: New York, John Wiley \& Sons, Inc., 276 p.

Merrill, F. J. H., 1916, The counties of San Diego, Imperial: California State Mining Bur. Rept. 14, pt. 4, p. 635-743.

1919, Los Angeles County, Orange County, Riverside County: California State Mining Bur. Rept. 15, pt. 4, p. 465-589.

Milton, Charles, and Johnston, W. D., Jr., 1938, Sulphate minerals of the Comstock Lode, Nevada: Econ. Geology, v. 33, no. 7, p. 749-771.

Moore, B. N., 1935, Some strontium deposits of southeastern California and western Arizona: Am. Inst. Mining Metall. Engineers Trans., v. 115 , p. 356-377.

1936. Celestite and strontianite, in Hewett, D. F., and others, Mineral resources of the region around Boulder Dam: U.S. Geol. Survey Bull. 871, p. 151-162.

Muffler, L. J. P., and Doe, B. R., 1968, Composition and mean age of detritus of the Colorado River delta in the Salton Trough, southeastern California: Jour. Sed. Petrology, v. 38, no. 2, p. 384-399.

Muffler, L. J. P., and White, D. E., 1969, Active metamorphism of upper Cenozoic sediments in the Salton Sea geothermal field and the Salton Trough, southeastern California: Geol. Soc. America Bull., v. 80, no. 2, p. 157-182.

Murata, K. J., Friedman, Irving, and Cremer, Marcelyn, 1972, Geochemistry of diagenetic dolomites in Miocene marine formations of California and Oregon: U.S. Geol. Survey Prof. Paper 724-C, $12 \mathrm{p}$.

Murata, K. J., and Richter, D. H., 1966, Chemistry of the lavas of the 1959-60 eruption of Kilauea Volcano, Hawaii: U.S. Geol. Survey Prof. Paper 537-A, $26 \mathrm{p}$.

Murdoch, Joseph, and Webb, R. W., 1948, Minerals of California: California Div. Mines Bull. 136, 402 p.

1956, Minerals of California: California Div. Mines Bull. 173, $452 \mathrm{p}$.

Norman, James, and Ralston, O. C., 1942, Purification of diatomite by froth flotation: Am. Inst. Mining Metall. Engineers Trans., v. 148 , p. $356-366$.

Norman, L. A., Jr., and Stewart, R. M., 1951, Mines and mineral resources of Inyo County, California: California Jour. Mines and Geology, v. 47, no. 1, p. 17-223.

Norton, F. H., 1939, Critical study of the differential thermal method for the identification of the clay minerals: Am. Ceramic Soc. Jour., v. 22, no. 2, p. 54-63.

1952, Elements of ceramics: Cambridge, Mass., AddisonWesley, $246 \mathrm{p}$.

Nutting, P. G., 1943, Absorbent clays, their distribution, properties, production, and uses: U.S. Geol. Survey Bull. 928-C, p. C127-C221. 
Oakeshott, G. B., 1937, Geology and mineral deposits of the western San Gabriel Mountains, Los Angeles County: California Jour. Mines and Geology, v. 33, no. 3, p. 215-249.

O'Brien, J. C., 1953, Mines and mineral resources of Mendocino County, California: California Jour. Mines and Geology, v. 49. no. 4 , p. 347-398

Oesterling, W. A., and Spurck, W. H., 1964a, Klamath Mountains and Cascade Range, in Minerals for industry-Summary of geological survey of 1955-1961, Volume 2-Northern California: San Francisco, Southern Pacific Co., pt. 2, p. 85-196. 1964b, Eastern Mojave and Colorado Deserts, in Minerals for industry-Summary of geological survey of 1955-1961, Volume 3-Southern California: San Francisco, Southern Pacific Co., pt. 3, p. 99-198.

Ogle, B. A., 1953, Geology of Eel River Valley area, Humboldt County, California: California Div. Mines Bull. 164, 128 p.

Osthaus, B. B., 1955, Interpretation of chemical analyses of montmorillonites, in Clays and clay technology: California Div. Mines Bull. 169, p. 95-100.

1956, Kinetic studies on montmorillonites and nontronite by the acid-dissolution technique, in Swineford, Ada, ed., Clays and clay minerals: Natl. Conf. Clays and Clay Minerals, 4th, University Park, Pa., October 10-13, 1955, Proc., Natl. Acad. Sci--Natl. Research Council Pub. 456, v. 7,p. 301-321.

Pabst, Adolf, 1938, Minerals of California: California Div. Mines Bull. $113,344 \mathrm{p}$.

Pack, R. W.. 1914, Ornamental marble near Barstow, California, in Contributions to economic geology, 1912, Part 1, Metals and nonmetals except fuels: U.S. Geol. Survey Bull. 540, p. 363-368.

Pask, J. A., and Turner, M. D., 1952, Geology and ceramic properties of the Ione Formation, Buena Vista area, Amador County, California: California Div. Mines Spec. Rept. 19, 39 p.

Patterson, S. H., 1964, Halloysitic underclay and amorphous inorganic matter in Hawaii, in Clays and clay minerals, 12th Natl. Conf., Atlanta, Georgia, 1963, Proc.: New York, Macmillan Co., p. 153-172.

1971, Investigations of ferruginous bauxite and other mineral resources on Kauai and a reconnaissance of ferruginous bauxite deposits on Maui, Hawaii: U.S. Geol. Survey Prof. Paper $656,74 \mathrm{p}$.

Pearson, R. C., Hayes, P. T., and Fillo, P. V., 1967, Mineral resources of the Ventana primitive area, Monterey County, California: U.S. Geol. Survey Bull. 1261-B, 42 p.

Phalen, W. C., 1911, Potash salts-their uses and occurrence in the United States: U.S. Geol. Survey Mineral Resources U.S., 1910 , pt. 2, p. 747-767.

Powers, H. A., 1932, The lavas of the Modoc Lava Bed quadrangle, California: Am. Mineralogist, v. 17, no. 7, p. 253-294.

Prescott, Basil, 1908, The occurrence and genesis of the magnetite ores of Shasta County, California: Econ. Geology, v. 3, no. 6 , p. $465-480$.

Ralston, O. C., Pike, R. D., and Duschak, L. H., 1925, Plastic magnesia: U.S. Bur. Mines Bull. 236, 111 p.

Richter, D. H., Ault, W. U., Eaton, J. P., and Moore, J. G., 1964, The 1961 eruption of Kilauea Volcano, Hawaii: U.S. Geol. Survey Prof. Paper 474-D, $34 \mathrm{p}$.

Rollins, R. Z., 1951, Agricultural gypsum, in Jenkins, O. P., ed., Minerals useful to California agriculture: California Div. Mines Bull. 155, p. 105-116.

Ross, C. S., and Hendricks, S. B., 1941, Clay minerals of the montmorillonite group, their mineral and chemical relationships, and the factors controlling base exchange: Soil Sci. Soc. America Proc., 1941 , v. 6, p. 58-62.

1945, Minerals of the montmorillonite group, their origin and relation to soils and clays: U.S. Geol. Survey Prof. Paper 205-B pt. 4, p. B23-B79 [1946].

Ross, C. S., and Shannon, E. V., 1926, The minerals of bentonite and related clays and their physical properties: Am. Ceramic Soc. Jour., v. 9, no. 2, p. 77-96.

Rubey, W. W., and Callaghan, Eugene, 1936, Magnesite and brucite. in Hewett, D. F., and others, Mineral resources of the region around Boulder Dam: U.S. Geol. Survey Bull. 871, p. 113-144.

Russel, I. C., 1889, Quaternary history of Mono Valley, California: U.S. Geol. Survey 8th Ann. Rept., 1886-1887, pt. 1, p. 261-394.

Sampson, R. J., 1937, Mineral resources of Los Angeles County: California Jour. Mines and Geology, v. 33, no. 3, p. 173-213.

Sampson, R. J., and Tucker, W. B., 1931, Feldspar, silica, andalusite, and cyanite deposits of California: California Div. Mines Rept. 27, no. 3, p. 407-458.

1942, Mineral resources of Imperial County: California Jour. Mines and Geology, v. 38, no. 2, p. 105-145.

Schaller, W. T., 1930, Borate minerals from the Kramer district, Mojave Desert, Califormia: U.S. Geol. Survey Prof. Paper 158 p. $137-170$.

Schneider, Hyrum, 1927, A study of glau conite: Jour. Geology, v. 35, no. 4 , p. 289-310.

Schroter, G. A., and Campbell, Ian, 1942, Geological features of some deposits of bleaching clay: Am. Inst. Mining Metall. Engineers Trans., v. 148, p. 178-208.

Shelton, J. S., 1955, Glendora volcanic rocks, Los Angeles Basin, California: Geol Soc. America Bull., v. 66, no. 1, p. 45-89.

Sheppard, R. A., and Gude, A. J., 3d, 1965a, Zeolitic authigenesis of tuffs in the Ricardo Formation, Kern County, southern California, in Geological Survey research 1965: U.S. Geol. Survey Prof. Paper 525-D, p. D44-D47.

$1965 \mathrm{~b}$, Potash feldspar of possible economic value in the Barstow Formation, San Bernardino County, California: U.S. Geol. Survey Circ. 500, 7 p.

1969, Diagenesis of tuffs in the Barstow Formation, Mud Hills, San Bernardino County, California: U.S. Geol. Survey Prof. Paper 634, 35 p.

Sherman, G. D., 1950, The genesis and morphology of Hawaiian ferruginous laterite crusts: Pacific Sci., v. 4, no. 4, p. 315-322.

Sherman, G. D., Kanehiro, Yoshinori, and Matsusaka, Yoshito, 1953 , The role of dehydration in the development of laterite [Hawaii]: Pacific Sci., v. 7, no. 4, p. 438-446.

Simpson, E. C., 1935, Geology and mineral deposits of the Elizabeth Lake quadrangle, California Jour. Mines and Geology, v. 30, no. 4 , p. 371-415.

Slaughter, M., and Earley, J. W., 1965, Mineralogy and geological significance of the Mowry bentonites, Wyoming: Geol. Soc. America Spec. Paper 83, 95 p.

Smith, C. A., 1924, The manufacture of an insulating brick from diatomaceous earth: Am. Ceramic Soc. Jour., v. 7, no. 1, p. $52-60$.

Smith, G. I., 1973, Subsurface stratigraphy and composition of saline bodies. Searles Lake, California; a preliminary report: U.S. Geol. Survey open-file rept., 118 p.

Speil, Sidney, Berkelhamer, L. H., Pask, J. A., and Davies, Ben, 1945. Differential thermal analysis, its application to clays and other aluminous minerals: U.S. Bur. Mines Tech. Paper 664, $81 \mathrm{p}$.

Spurck, W. H., 1964, Western Mojave Desert, in Minerals for industry-Summary of geological survey of 1955-1961, Volume 3-Southern California: San Francisco, Southern Pacific Co., pt. 2, p. 37-97.

Stewart, J. H., 1970, Upper Precambrian and Lower Cambrian strata in the southern Great Basin, California and Nevada: U.S. Geol. 
Survey Prof. Paper 620, 206 p.

Sutherland, J. C., 1935, Geological investigation of the clays of Riverside and Orange Counties, southern California: California Jour. Mines and Geology, v. 31, no. 1, p. 51-87.

Takahashi, J. R., 1929, Significance of micro-crystals of carbonates in bituminous shales, a preliminary note: Am. Assoc. Petroleum Geologists Bull., v. 13, no. 10, pt. 2, p. 1377-1386.

Taliaferro, N. L., 1943, Franciscan-Knoxville problem: Am. Assoc. Petroleum Geologists Bull., v. 27, no. 2, p. 109-219.

Taliaferro, N. L., and Hudson, F. S., 1943, Genesis of manganese deposits of the Coast Ranges of California, in Jenkins, O. P., Manganese in California: California Div. Mines Bull. 125, p. 217-275.

Tischler, M. S., 1964, Northern Sierra Nevada, in Minerals for industry-Summary of geological survey of 1955-1961, Volume 2-Northern California: San Francisco, Southern Pacific Co. pt. 1, p. 9-83.

Townsend, R. H., 1933, Method and cost of quarrying limestone at the plant of the Calaveras Cement Company, San Andreas, California: U.S. Bur. Mines Inf. Circ. 6610, 13 p.

Tucker, W. B., 1919, Tulare County: California State Mining Bur. Rept. 15, pt. 4, p. 900-954.

1921, Los Angeles Field Division: California State Mining Bur. Rept. 17, p. 263-390.

1923a, Economic minerals of the Avawitz Mountains: California State Mining Bur. Rept. 18, p. 114-117.

1923b, Limestone deposits of McCloud River, Shasta County, and their possible value for cement material: California State Mining Bur. Rept. 19, no. 2, p. 69-71.

1924, Los Angeles Field Division: California State Mining Bur. Rept. 20, no. 2, p. 87-98.

1925a, Los Angeles Field Division: California State Mining Bur. Rept. 21, no. 1, p. 58-71.

1925b, Los Angeles Field Division: California State Mining Bur. Rept. 21, no. 3, p. 325-382.

1926, Los Angeles Field Division: California State Mining Bur. Rept. 22 , no. 2 , p. 248-285.

1927a, Los Angeles Field Division: California State Mining Bur. Rept. 22 , no. 4, p. 453-530.

1927b, Los Angeles Field Division: California State Mining Bur. Rept. 23, no. 3, p. 287-345.

1929, Los Angeles Field Division: California Div. Mines and Mining Rept. 25, no. 1, p. 20-81.

Tucker, W. B., and Sampson, R. J., 1929, Los Angeles Field Division: California Div. Mines Rept. 25, no. 4, p. 468-526.

1930, Los Angeles Field Division: California Div. Mines Rept. 26 , no. 3, p. 202-325.

1931, Los Angeles Field Division: California Div. Mines Rept. 27 , no. 3 , p. 262-401.

1933, Los Angeles Field Division: California Div. Mines Rept. 28 , nos. 3 and 4 , p. $247-277$.

1938, Mineral resources of Inyo County: California Jour. Mines and Geology, v. 34 , no. 4 , p. 368-500.

1940 , Current mining activity in southern California: California Jour. Mines and Geology, v. 36, no. 1, p. 9-82.

1944, Mineral resources of San Bernardino County: California Jour. Mines and Geology, v. 39, no. 4, p. 427-549.

Turner, H. W., 1891, The geology of Mount Diablo, California, with a supplement on The chemistry of the Mount Diablo rocks, by W. H. Melville: Geol. Soc. America Bull., v. 2, p. 384-414.

1898, Chiastolite-schist, in Diller, J. S., The educational series of rock specimens collected and distributed by the United States Geological Survey: U.S. Geol. Survey Bull. 150, p. $339-343$.

Tyson, P. T., 1851, Reports on the geology and topography of California, communicated to the Senate by the Secretary of War: Ex. Doc. 47, 31st Cong., 1st sess., summarized in Am.
Jour. Sci., ser. 2, v. 11, no. 32, p. 301.

Ver Planck, W. E., Jr., 1955, Mines, mineral resources, and mineral industries of Marin County, California: California Jour. Mines and Geology, v. 51, no. 3, p. 221-289.

1958, Salt in California: California Div. Mines Bull. 175, 168 p.

Vitaliano, C. J., 1950, Needles magnesite deposit, San Bernardino County, California: California Jour. Mines and Geology, v. 46, no. 3, p. 357-372.

Walker, G. W., 1950a, Sierra Blanca Limestone in Santa Barbara County, California: California Div. Mines Spec. Rept. 1-A, 8 p. 1950b, The Calera Limestone in San Mateo and Santa Clara Counties, California: California Div. Mines Spec. Rept. 1-B, $7 \mathrm{p}$.

Walker, J. L., Sherman, G. D., and Katsura, Takashi, 1969, The iron and titanium minerals in the titaniferous ferruginous iatosols of Hawaii; Pacific Sci., v. 23, no. 3, p. 291-304.

Washington, H. S., 1917, Chemical analyses of igneous rocks published from 1884 to 1913 , inclusive, with a critical discussion of the character and use of analyses: U.S. Geol. Survey Prof. Paper 99, $1201 \mathrm{p}$.

Watts, W. L., 1897, Oil and gas yielding formations of Los Angeles, Ventura, and Santa Barbara Counties: California State Mining Bur. Bull. 11, 94 p.

Weaver, C. E., 1949, Geology and mineral deposits of an area north of San Francisco Bay, California: California Div. Mines Bull. $149,135 \mathrm{p}$.

Weber, F. H., Jr., 1963a, Geology and mineral deposits of the Ord Mountain district, San Bernardino County, California: California Div. Mines and Geology Spec. Rept. 77, 45 p. $1963 \mathrm{~b}$, Limestone and dolomite, in Geology and mineral resources of San Diego County: California Div. Mines and Geology County Rept. 3, p. 173-185.

Weigel, W. M., 1927, Technology and uses of silica and sand: U.S. Bur. Mines Bull. 266, 204 p.

Weitz, J. H., 1942, High-grade dolomite deposits in the United States: U.S. Bur. Mines Inf. Circ. 7226, 86 p.

Wells, R. C., 1937, Analyses of rocks and minerals from the laboratory of the United States Geological Survey, 1914-1936: U.S. Geol. Survey Bull. 878, 134 p.

Wentworth, C. K., Wells, R. C., and Allen, V. T., 1940, Ceramic clay in Hawaii: Am. Mineralogist, v. 25, no. 1, p. 1-33.

Whitehouse, U. G., Jeffrey, L. M., and Debbrecht, J. D., 1960, Differential settling tendencies of clay minerals in saline waters, in Swineford, Ada, ed., Clays and clay minerals, Natl. Conf. Clay and Clay Minerals, 7th, Washington, D. C., 1958, Proc.: New York, Pergamon Press, Internat. Earth Sci. Ser. Mon. 5, p. 1-79.

Williams, Howel, 1932, Geology of the Lassen Volcanic National Park, California: California Univ. Pubs. Geol. Sci., v. 21, no. 8, p. 195-385.

Winston, W. B., 1947, Pumice: California Jour Mines and Geology, v. 43 , no. $4,469-479$.

Woodford, A. O., and Harris, T. F., 1928, Geology of Blackhawk Canyon, San Bernardino Mountains, California: California Univ. Pubs. Geol. Sci., v. 17, no. 8, p. 265-304.

Wright, L. A., Stewart, R. M., Gay, T. E., Jr., and Hazenbush, G. C., 1953, Mines and mineral deposits of San Bernardino County, California: California Jour. Mines and Geology, v. 49, nos. 1 and 2, p. 49-259, and $192 \mathrm{p}$. of tables.

Wright, T. L., 1971, Chemistry of Kilauea and Mauna Loa [Hawaii] lava in space and time: U.S. Geol. Survey Prof. Paper 735, 40 p.

Yale, C. G., and Stone, R. W., 1921, Magnesite: U.S. Geol. Survey Mineral Resources U.S., 1918, pt. 2, p. 141-158.

Youngman, E. P., 1930, Deposits of titanium-bearing ores: U.S. Bur. Mines Inf. Circ. 6386, 49 p. 


\section{TABLES 1-17, 23-25}

The stratigraphic nomenclature used in tables 1-9 does not necessarily reflect the latest usage of the U.S. Geological Survey; names and ages were taken from many published sources. 
. 
TABLE 1. - Analyses of samples from California containing 90-100 percent uncombined silica (Group $A_{3}$ ), common- and mixed-rock categories

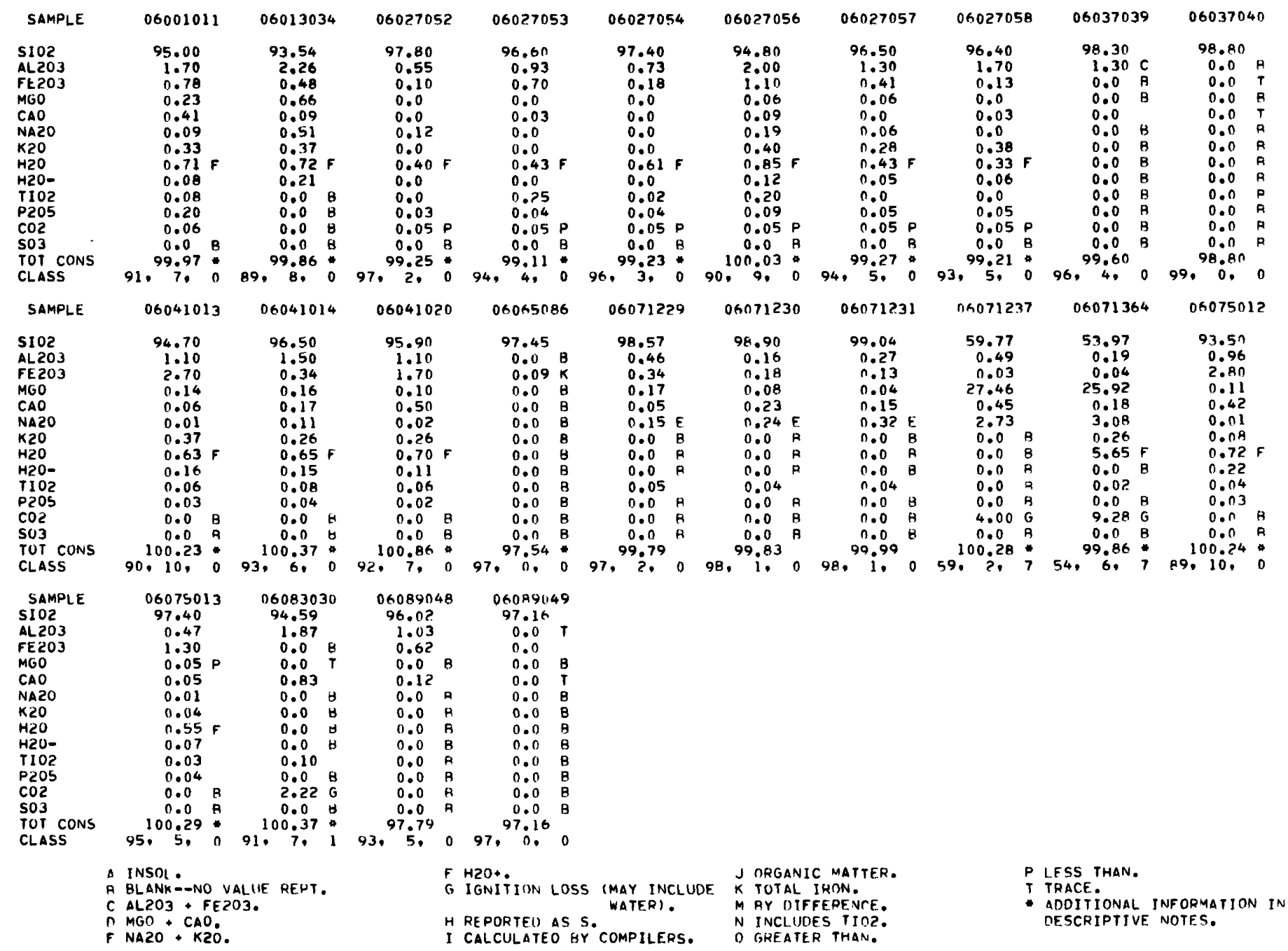

06001011 JURASSIC \& CRETACEDUS, FRANCISCAN FM. "T1S, R4W" OAKLANO. (I31:TR) RADIOLARIAN CHERT. "MMISC: FEO = O.11, MNO = 0.1R,

06013034 JURASSIC 8 CKETACEOUS, FRANCISCAN FM. "TIN.KIE" MOUNT DIABLO. (99:124/ 221:9,A,25,?R) CHERT, RROWNISH-REDI MIN.

06027056 CAMBRIAN, ZABRISKIE (NTZITE. S20,T24N,R6E. $(585: 34,8,114)$ QUARTZITE: MIN. INDEX MAP, MEAS SEC. SPECTRO ANAL. ALSO GIVEN. MISC: FEO $=0.20$, MNO $=0.00$.

06027053 CAMBRIAN, ZAGRISKIE QTZITE. S7,8.TIOS.R40E \& S29-32,T9S,R4OE. (585:35,8,R2) QUARTZITEI MIN. INDEX MAP, MFAS SEC.

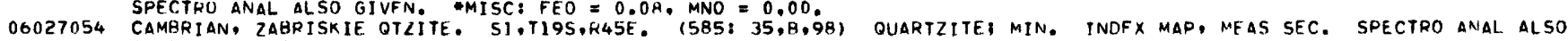
GIVEN. MISC: FEO $=0.20$, MNO $=0.00$.

06027056 PRECAMBRIAN \& CAMBRIAN, WOOD CANYON FM. SI,TI9S.R45E. (585:35,8,98) (DUARTZITE: MIN. INDEX MAD. MEAS SEC.

06027057 PRECAMBRIAN, STIRLING OTZITE. SI,T195,R45E. $(585: 35,100,101)$ QUARTZITE, MIN. INTEX MAP, MEAS SEC. SPECTRO ANAL ALSO PRECAMBRIAN, STIRLING OTZITE. SI,TI9S,R4SE. $(585: 35,100,101)$ QUARTZITE: MIN. INOEX MAP, MEAS SEC. SPECTRO ANAL ALSO
GIVEN. MISC: FEO $=0,08$. MNO $=0.00$. 06027058 PRECAMBRIAN, STIRLING QTZITE. S1.TI9S.R4SE. (585:35,100,101) QUARTZITE, MIN. INDEX MAP. MEAS SEC. SPECTRO ANAL ALSO GIVEN. MISC: FEO $=0.08$, MNO $=0.00$.

06037039 "TSN,R12W" ACTON. $(67: 527)$ SAND, USE: 15.

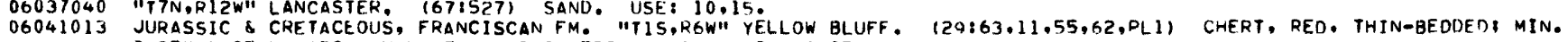

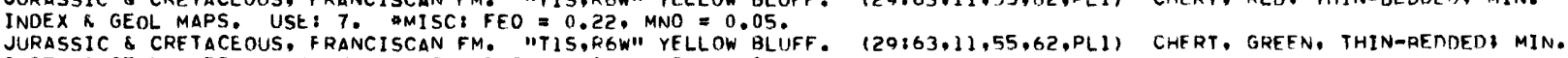

06041014 JURASSIC \& CRETACEOUS, FRANCISCAN FM. "ITIS,RGW" YELLOW BLUFF. $(29: 63,1), 55,62, P L 1)$ CHERT, GREEN, THIN-RENDED MIN.
06041020 INDEX \& GEOL MAPS. USE: 7. MMISC: FEO = 0.38 . MNO = 0.03. 06041020 JURASSIC \& CRETACEOUS, FRANCISCAN FM. "TIS,RGW" SAUSAL

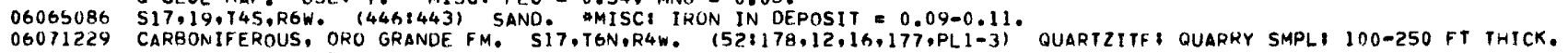

06071229 CARBONIFEROUS, ORO GRANDE FM. SI7,T6N,R4W. (52:178,12,16,177,PL1-3) QUARTZITF: QUARKY SMPLI 100-250 FT THICK.

06071230 CARBONIFEROUS, ORO GRANOE FM. SI7,TGN,R4W. (52:176,12,175,PL1-3) OUARTZITES TONNAGE. INDEX \& GEOL MAPS. GEOL SEC. USE: 4,14 .

06071231 CARBONIFEROUS, ORO GRANDE FM. SII,TGN,R4W. (52:178,PL1-3) QUARTZITE, TONNAGE. INDFX \& GEOL MAP, GEOL SEC. USE:4.14. 06071237 "T8N,ESE" HECTOR. (439:27) HECTORITE. HMISC: F = 5.35, FE203 REPT AS 0.026. DUPLICATE ANAL OF SAMPL. SIDP 55.86.

06071364 "TBN,R5E" HECTOR. (541:12) HECTORITE MAX VALUES OF 6 SMPL: FOR MIN VALUES SEE SIO2 53.91. TMISC: FEO = 0.00. 06075012 JURASSIC \& CRETACEOUS, FRANCISCAN FM. "T2S,R5W" SAN FRANCISCO. $129: 63,11.55,62.0 L 1)$ CHERT, RED, THIN-BEDDED MIN. 06075013 JURASSIC \& CRETACEOUS, FRANCISCAN FM. "T25,R5W" SAN FRANCISCO. 129163.11,55.62.PL11 CHERT, REN1 25 FT THICK: MIN.

06083030 "T8N,R34W" LOMPOC: (464:53) OIATOMACEOUS SHALE, COMPACT:FIRING TESIS. USE: 11. "MISC: FEO = 0.76.

06089048 HOLOCENE. S26,T37N,RZE. (366:166,263-165) OIATOMITE, WHITE: 340 FT EXPOSED. INDEX MAP.

06089049 HOLOCENE. S33,T37N,R2E TO S10,T36N,R4E. (366:166,163-165) DIATOMITE, WHITE: 340 FT EXPOSED. TNDEX MAP. 
TABLE 2. - Analyses of samples from California containing 75-90 percent uncombined silica (Group A4), common-and mixed-rock categories

\begin{tabular}{|c|c|c|c|c|c|c|c|c|c|c|c|c|c|c|c|c|c|c|c|c|}
\hline SAMPLE & \multicolumn{2}{|r|}{06007007} & & \multicolumn{2}{|l|}{06013033} & \multicolumn{2}{|l|}{06013036} & \multicolumn{2}{|c|}{06027055} & \multicolumn{2}{|c|}{06027059} & \multicolumn{2}{|c|}{06037018} & \multicolumn{2}{|l|}{06037020} & \multicolumn{2}{|l|}{$060370 ? 4$} & 06037029 & \multicolumn{2}{|l|}{06053107} \\
\hline $\begin{array}{l}\text { S102 } \\
\text { AL203 } \\
\text { FE203 } \\
\text { MGO } \\
\text { CAO } \\
\text { NA2O } \\
\text { K2O } \\
\text { H2O } \\
\text { H2O- } \\
\text { TIO2 } \\
\text { P2O5 } \\
\text { CO2 } \\
\text { SO3 } \\
\text { TOT CONS } \\
\text { CLASS }\end{array}$ & & $\begin{array}{c}88.70 \\
4.50 \\
0.50 \\
0.36 \\
0.93 \\
0.03 \\
0.0 \\
4.46 \\
0.0 \\
0.0 \\
0.0 \\
0.0 \\
0.0 \\
100.08 \\
80.0 \\
18 .\end{array}$ & 90 & $\begin{array}{c}96.37 \\
2.38 \\
1.70 \\
0.0 \\
0.0 \\
0.0 \\
0.0 \\
0.0 \\
0.0 \\
0.0 \\
0.0 \\
0.0 \\
0.0 \\
100.45 \\
0.10 .\end{array}$ & $\begin{array}{l}B \\
B \\
B \\
B \\
8 \\
B \\
B \\
B \\
B \\
B \\
0\end{array}$ & $\begin{array}{c}95.08 \\
2.17 \\
2.82 \\
0.0 \\
0.0 \\
0.0 \\
0.0 \\
0.0 \\
0.0 \\
0.0 \\
0.0 \\
0.0 \\
0.0 \\
100.07 \\
88,12 .\end{array}$ & $\begin{array}{l}B \\
B \\
B \\
B \\
B \\
B \\
B \\
B \\
B \\
B\end{array}$ & $\begin{array}{r}87.40 \\
5.20 \\
1.80 \\
0.43 \\
0.11 \\
0.20 \\
2.30 \\
1.40 \\
0.15 \\
0.31 \\
0.08 \\
0.05 \\
0.0 \\
99.63 \\
76,20 .\end{array}$ & F & $\begin{array}{r}90.50 \\
3.40 \\
0.32 \\
0.05 \\
0.03 \\
0.0 \\
0.70 \\
0.56 \\
0.04 \\
0.32 \\
0.15 \\
0.05 \\
0.0 \\
96.40 \\
94.11 .\end{array}$ & $\begin{array}{l}P \\
B \\
0 \\
0\end{array}$ & $\begin{array}{c}82.55 \\
4.82 \\
0.90 \\
0.0 \\
1.30 \\
1.09 \\
0.0 \\
2.90 \\
5.53 \\
0.0 \\
0.0 \\
0.0 \\
0.0 \\
100.03 \\
73,24 .\end{array}$ & $\begin{array}{l}\text { B } \\
\text { E } \\
\text { B } \\
\text { F } \\
\text { B } \\
B \\
\text { B } \\
\text { B } \\
\\
\end{array}$ & $\begin{array}{c}8.90 \\
2.28 \\
0.87 \\
0.0 \\
1.05 \\
0.49 \\
0.0 \\
2.80 \\
3.10 \\
0.0 \\
0.0 \\
0.0 \\
0.0 \\
99.96 \\
140\end{array}$ & $\begin{array}{l}B \\
B \\
B \\
B \\
* \\
0\end{array}$ & $\begin{array}{c}84.00 \\
4.50 \\
1.27 \\
0.0 \\
1.10 \\
0.0 \\
0.0 \\
0.0 \\
0.0 \\
0.85 \\
0.0 \\
6.84 \\
0.0 \\
98.79 \\
75.21 .\end{array}$ & $\begin{array}{l}T \\
B \\
B \\
B \\
J \\
B \\
G \\
B \\
\\
2\end{array}$ & $\begin{array}{r}84.45 \\
4.14 \\
1.48 \\
0.52 \\
1.25 \\
0.46 \\
0.64 \\
3.115 \\
3.25 \\
0.20 \mathrm{~J} \\
0.28 \\
0.0 \\
0.18 \\
100.82 \\
75.21 .0\end{array}$ & $\begin{array}{c}83.61 \\
4.70 \\
1.19 \\
0.17 \\
1.43 \\
1.45 \\
0.0 \\
0.0 \\
0.0 \\
0.12 \\
0.0 \\
5.81 \\
0.0 \\
99.90 \\
74.20 .\end{array}$ & $\begin{array}{l}\text { E } \\
\text { Q } \\
8 \\
\text { A } \\
\text { T } \\
\text { G } \\
\\
\end{array}$ \\
\hline SAMPLE & & 06053108 & & 0605310 & & 0607118 & & 0607119 & & 0607129 & & 0607122 & & 06071305 & & 0607303 & & 06073032 & 06075011 & 11 \\
\hline $\begin{array}{l}\text { SIO2 } \\
\text { AL2O3 } \\
\text { FE203 } \\
\text { MGO } \\
\text { CAO } \\
\text { NA2O } \\
\text { K2O } \\
\text { H2O } \\
\text { H2O- } \\
\text { TIO2 } \\
\text { P2O5 } \\
\text { CO2 } \\
\text { SO3 } \\
\text { TOT CONS } \\
\text { CLASS }\end{array}$ & & $\begin{array}{c}84.11 \\
3.55 \\
1.29 \\
0.86 \\
1.24 \\
0.00 \\
0.0 \\
0.0 \\
5.12 \\
0.0 \\
0.00 \\
0.88 \\
0.0 \\
97.05 \\
97.05 \\
76.19 .2\end{array}$ & 81. & $\begin{array}{c}86.89 \\
2.32 \\
1.28 \\
0.0 \\
0.43 \\
2.32 \\
1.26 \\
0.0 \\
0.0 \\
0.0 \\
0.0 \\
4.89 \\
0.0 \\
99.39 \\
14 .\end{array}$ & $\begin{array}{l}\text { B } \\
\text { B } \\
B \\
B \\
G \\
B\end{array}$ & $\begin{array}{c}53.68 \\
0.60 \\
0.0 \\
25.34 \\
0.52 \\
3.00 \\
0.07 \\
0.24 \\
7.28 \\
0.0 \\
0.0 \\
0.0 \\
0.0 \\
100.16 \\
53.17 .\end{array}$ & $\begin{array}{l}F \\
B \\
B \\
B \\
B \\
B \\
W \\
0\end{array}$ & $\begin{array}{r}57.50 \\
0.68 \\
0.24 \\
25.98 \\
0.47 \\
2.48 \\
0.45 \\
6.88 \\
3.52 \\
0.02 \\
0.34 \\
0.0 \\
0.0 \\
98.56 \\
56.13 .\end{array}$ & $\begin{array}{l}8 \\
\\
\\
\end{array}$ & $\begin{array}{c}56.41 \\
0.37 \\
0.0 \\
1.82 \\
0.0 \\
12.78 \\
1.00 \\
9.47 \\
0.0 \\
0.0 \\
0.0 \\
0.0 \\
0.0 \\
100.00 \\
56.10 .\end{array}$ & $\begin{array}{l}B \\
B \\
B \\
B \\
B \\
B \\
B \\
B \\
0\end{array}$ & $\begin{array}{r}55.17 \\
0.33 \\
0.12 \\
24.51 \\
0.90 \\
2.20 \\
0.08 \\
2.84 \\
8.93 \\
0.01 \\
0.05 \\
0.63 \\
0.0 \\
99.83 \\
54.13,\end{array}$ & F & $\begin{array}{c}53.91 \\
0.08 \\
0.0 \\
25.83 \\
0.12 \\
2.97 \\
0.16 \\
5.57 \\
0.0 \\
0.0 \\
0.0 \\
9.28 \\
0.0 \\
99.07 \\
54, \quad 6 .\end{array}$ & $\begin{array}{l}B \\
6 \\
B \\
* \\
7\end{array}$ & $\begin{array}{c}92.00 \\
5.00 \\
0.10 \\
0.0 \\
1.40 \\
0.10 \\
0.0 \\
0.0 \\
0.0 \\
0.0 \\
0.0 \\
0.30 \\
0.0 \\
98.90 \\
14 .\end{array}$ & $\begin{array}{l}E \\
B \\
B \\
B \\
B \\
B \\
G \\
B\end{array}$ & $\begin{array}{rr}94.00 \\
5.20 \\
0.10 \\
0.00 \\
0.02 \\
0.0 \\
0.0 & 8 \\
0.0 & 8 \\
0.0 & 8 \\
0.0 & 8 \\
0.0 & 8 \\
0.0 & 8 \\
0.0 & 8 \\
99.32 & \\
15 . & 0\end{array}$ & $\begin{array}{c}93.00 \\
2.00 \\
2.40 \\
0.13 \\
0.11 \\
0.11 \\
0.41 \\
1.00 \\
0.26 \\
0.12 \\
0.05 \\
0.0 \\
0.0 \\
100.04 \\
12.0\end{array}$ & F \\
\hline SAMPLE & & 06079010 & & 06083015 & & 06083016 & & 0608301 & & 0608301 & & 0608301 & & 06083020 & & $060830 ?$ & & 06083024 & 06083025 & \\
\hline $\begin{array}{l}\text { SIO2 } \\
\text { AL2O3 } \\
\text { FE203 } \\
\text { MGO } \\
\text { CAO } \\
\text { NA2O } \\
\text { K2O } \\
\text { H2O } \\
\text { H2O- } \\
\text { TIO2 } \\
\text { P2O5 } \\
\text { CO2 } \\
\text { SO3 } \\
\text { TOT CONS } \\
\text { CLASS }\end{array}$ & & $\begin{array}{c}84.90 \\
4.80 \\
2.10 \\
1.20 \\
0.46 \\
0.70 \\
0.31 \\
2.00 \\
0.72 \\
0.18 \\
0.02 \\
0.0 \\
0.0 \\
99.65 \\
74, \\
20 .\end{array}$ & 80 . & $\begin{array}{c}86.24 \\
3.05 \\
1.02 \\
0.63 \\
0.18 \\
0.0 \\
0.0 \\
0.0 \\
5.62 \\
0.00 \\
0.0 \\
1.20 \\
0.0 \\
97.94 \\
17 .\end{array}$ & $\begin{array}{l}\text { B } \\
B \\
B\end{array}$ & $\begin{array}{c}88.40 \\
2.05 \\
1.13 \\
0.52 \\
0.34 \\
0.0 \\
0.0 \\
0.0 \\
6.08 \\
0.0 \\
0.0 \\
0.31 \\
0.0 \\
98.83 \\
84.0150\end{array}$ & $\begin{array}{l}\mathrm{B} \\
\mathrm{B} \\
\mathrm{B}\end{array}$ & $\begin{array}{c}88.68 \\
2.68 \\
0.0 \\
1.30 \\
1.61 \\
0.0 \\
0.0 \\
0.0 \\
0.0 \\
0.0 \\
0.0 \\
5.54 \\
0.0 \\
99.81 \\
10 .\end{array}$ & $\begin{array}{l} \\
\\
B \\
B \\
B \\
B \\
B \\
B \\
6 \\
B \\
6\end{array}$ & $\begin{array}{c}88.72 \\
2.68 \\
0.0 \\
1.30 \\
1.61 \\
0.0 \\
0.0 \\
5.54 \\
0.0 \\
0.10 \\
0.0 \\
0.0 \\
0.0 \\
99.95 \\
84.913 .\end{array}$ & $\begin{array}{l}\top \\
B \\
B \\
B \\
B \\
B \\
B \\
B \\
0\end{array}$ & $\begin{array}{c}88.60 \\
3.72 \\
1.50 \\
0.60 \\
0.60 \\
0.50 \\
0.50 \\
0.0 \\
0.0 \\
0.20 \\
0.0 \\
3.75 \\
0.0 \\
99.97 \\
16 .\end{array}$ & $\begin{array}{l}\text { B } \\
\text { B } \\
\text { B } \\
\text { G } \\
\text { B } \\
2\end{array}$ & $\begin{array}{c}89.70 \\
3.72 \\
1.09 \\
0.65 \\
0.35 \\
0.82 \\
0.0 \\
0.0 \\
0.0 \\
0.10 \\
0.0 \\
3.70 \\
0.0 \\
100.13 \\
82,15 .\end{array}$ & $\begin{array}{l}E \\
B \\
B \\
B\end{array}$ & $\begin{array}{c}92.53 \\
1.11 \\
0.78 \\
0.50 \\
0.35 \\
0.0 \\
0.0 \\
3.86 \\
0.0 \\
0.0 \\
0.0 \\
0.0 \\
0.0 \\
99.13 \\
90,9 .\end{array}$ & $\begin{array}{l}B \\
B \\
B \\
B \\
B\end{array}$ & $\begin{array}{ccc}86.9 ? & \\
4.27 & \\
0.0 & B \\
0.0 & T \\
1.60 & \\
2.48 & E \\
0.0 & B \\
0.0 & B \\
0.0 & B \\
0.0 & B \\
0.0 & B \\
5.13 & 6 \\
0.0 & 8 \\
100.40 & \\
16 . & 3\end{array}$ & $\begin{array}{c}92.37 \\
2.46 \\
0.0 \\
0.0 \\
1.70 \\
0.0 \\
0.0 \\
0.0 \\
0.0 \\
0.0 \\
0.0 \\
2.74 \\
0.0 \\
99.27 \\
88, \quad 8 .\end{array}$ & $\begin{array}{l}R \\
R \\
B \\
R \\
B \\
R \\
A \\
R \\
G \\
B \\
3\end{array}$ \\
\hline $\begin{array}{l}\text { SAMPLE } \\
\text { SIO2 } \\
\text { AL2O3 } \\
\text { FE2O3 } \\
\text { MGO } \\
\text { CAO } \\
\text { NA2O } \\
\text { K2O } \\
\text { H2O } \\
\text { H2O- } \\
\text { TIO2 } \\
\text { P2O5 } \\
\text { CO2 } \\
\text { SO3 } \\
\text { TOT CONS } \\
\text { CLASS }\end{array}$ & & $\begin{array}{c}06083029 \\
85.28 \\
5.35 \\
0.0 \\
1.30 \\
1.12 \\
0.0 \\
0.0 \\
0.0 \\
0.0 \\
0.0 \\
0.21 \\
0.0 \\
5.57 \\
0.0 \\
9.0 \\
9.95 \\
18 .\end{array}$ & $\begin{array}{l}B \\
G \\
B \\
* \\
5\end{array}$ & $\begin{array}{c}06087025 \\
87.02 \\
2.97 \\
0.0 \\
0.25 \\
1.12 \\
0.0 \\
0.0 \\
0.0 \\
0.0 \\
0.0 \\
0.0 \\
7.40 \\
0.0 \\
98.76 \\
14 . \\
14\end{array}$ & $\begin{array}{l}B \\
B \\
B \\
B \\
B \\
B \\
6 \\
B \\
3\end{array}$ & $\begin{array}{c}06089044 \\
86.79 \\
6.30 \\
0.0 \\
0.0 \\
0.51 \\
0.0 \\
0.0 \\
6.20 \\
0.0 \\
0.0 \\
0.0 \\
0.0 \\
0.0 \\
99.80 \\
76.0 \\
23 .\end{array}$ & $\begin{array}{l}44 \\
C \\
B \\
B \\
B \\
B \\
B \\
B \\
B \\
B \\
B \\
B \\
0\end{array}$ & $\begin{array}{c}0608904 \\
88.05 \\
6.25 \\
1.08 \\
0.0 \\
1.00 \\
0.0 \\
0.0 \\
0.0 \\
0.0 \\
0.0 \\
0.0 \\
0.0 \\
0.0 \\
96.38 \\
76.21 .\end{array}$ & $\begin{array}{l}45 \\
B \\
B \\
B \\
8 \\
8 \\
8 \\
8 \\
B \\
B \\
2\end{array}$ & $\begin{array}{c}0608904 \\
88.35 \\
3.24 \\
0.84 \\
0.45 \\
0.37 \\
0.0 \\
0.0 \\
0.0 \\
5.74 \\
0.0 \\
0.0 \\
0.88 \\
0.0 \\
99.87 \\
82,17 .\end{array}$ & $\begin{array}{l}\text { B } \\
\text { B } \\
\text { B } \\
\text { B } \\
2\end{array}$ & $\begin{array}{c}0608904 \\
91.33 \\
2.59 \\
0.92 \\
0.00 \\
0.06 \\
0.0 \\
0.0 \\
0.0 \\
0.0 \\
0.0 \\
0.0 \\
0.0 \\
0.0 \\
95.50 \\
86,10 .\end{array}$ & $\begin{array}{l}47 \\
B \\
B \\
B \\
B \\
B \\
B \\
R \\
R \\
B \\
B \\
1\end{array}$ & $\begin{array}{c}06089050 \\
98.05 \\
6.25 \\
1.08 \\
0.0 \\
1.00 \\
0.0 \\
0.0 \\
0.0 \\
0.0 \\
0.0 \\
0.0 \\
0.0 \\
0.0 \\
96.38 \\
96,21 .\end{array}$ & $\begin{array}{l}\text { B } \\
\text { B } \\
8 \\
8 \\
8 \\
B \\
B \\
\text { B } \\
\text { R }\end{array}$ & & & & & \\
\hline & & $\begin{array}{l}\text { INSOL. } \\
\text { BLANK--NO } \\
\text { AL2O3 * FE } \\
\text { MGO * CAO } \\
\text { NAZO * K2O }\end{array}$ & $\begin{array}{l}\text { VALL } \\
\text { E203 } \\
0 .\end{array}$ & E REPT. & & & $\begin{array}{l}F \\
G \\
H \\
I\end{array}$ & $\begin{array}{l}\text { HZO* } \\
\text { IGNITION L } \\
\text { REPORTED A } \\
\text { CALCULATED }\end{array}$ & $\begin{array}{l}\text { AS } 5 \\
0 \text { Br }\end{array}$ & $\begin{array}{l}\text { S (MAY INC } \\
\text { WATER). } \\
\text { Y COMPILER }\end{array}$ & & $\begin{array}{ll}J & \text { ORGA } \\
K & \text { TOTA } \\
M & \text { RY D } \\
N & \text { INCL } \\
O & \text { GREA }\end{array}$ & & $\begin{array}{l}\text { C MATTER, } \\
\text { IRON. } \\
\text { FERENCE. } \\
\text { ES TIOZ. } \\
\text { R THAN. }\end{array}$ & & & $\begin{array}{l}P \\
T \\
\end{array}$ & $\begin{array}{l}\text { LESS THAN. } \\
\text { TRACE. } \\
\text { ADOITIONAL } \\
\text { OESCRIPTIVE }\end{array}$ & $\begin{array}{l}\text { INFORMATION } \\
\text { E NOTES. }\end{array}$ & IN \\
\hline
\end{tabular}


TABLE 2.-Analyses of samples from California containing 75-90 percent uncombined silica (Group $A_{4}$ ), common-and mixed-rock categories -Continued

06007007 "T21.22N,RIEN CHICO. (3071302) CLAY, WHITE, USE: 5 . 06013033 JURASSIC \& CRETACEOUS, FRANCISCAN FM, "TIN,R5W" POINT RICHMOND, $(138: 268,241,254,255 / 221: 9,8,25,28)$ CHERT, RED,
SILICEOUSI THIN-BEDDED IMIN. GEOL MAP. USE: 4 .

06013036 JURASSIC \& CRETACEOUS, FRANCISCAN FM, "TIN,R5W" RED ROCK ISLAND. $(138: 268,254)$ CHERT, RED, FOS: MIN.

06027055 PRECAMBRIAN \& CAMBRIAN, WOOD CANYON FM. S17,18,20,21,T24N,R7F, (585:35,8,11B) QUARTZITE \& CONGLOMERATE, MIN. INDEX MAP. MEAS SEC. SPECTRO ANAL ALSO GIVEN. OMISC: FEO $=0.20$, MNO $=0.00$.

06027059 PRECAMBRIAN, STIRLING OTZITE. SI,T19S,R45F. $1585835,100,1011$ QUARTZITE MIN. INDEX MAP. MEAS SEC. SPECTRO ANAL ALSO 06037018 MIOCENE. MONTEREY FM. "T4S,R14W" PALOS VERDES HILLS, $(61: 13, P L 1)$, SHALE, CHERTY. INDEX MAP. MISC: FEO=0.13, CL=0,81. 06037020 MIOCENE, MONTEREY FM. "T4S,RI4W" PALOS VERDES HILLS, $161113, P L 11$ SHALE, CHERTY. INDEX MAP. MMISC FEO=0.20, CL=0.27. 06037024 "T4S,R14W" TORRENCE. (401:361) DIATOMITE. AMISCI TIO2 = 0.23, ORG MATIER CALC FROM REPT C.

06037029 MIOCENE, MONTEREY FM. "TIN,RI5W" SANTA MONICA MTNS. (61:13,PLI) SHALE, CHERTY. INDEX MAP. \$MISCI FEO = O.51. IIO2 = 0.351 ORG MATTER CALC FROM ORG C.

06053107 S18.T24S.R9E. $(339136,35)$ DIATOMITE. MISCI MNO, TRI NACL $=1.42$.

06053108 MIOCENE, MONTERFY FM. "T24S.RIIE" BRAOLEY. (169:108,99,101) DIATOMITE, WHITE, COMPACT, USE: 7.

06053109 MIOCENE, MONTEREY SH. "T16S,R2W" CARMELO BAY AREA. (356: 25,24,PLI) SHALE, WHITE. CHALKY, FOS: OVER 1,000 FT THICK: 06071189 MIN: B.D., 1,R-2.1. INUEX \& GEOL MAP, GEOL SEC.

06071191 "TBN,R5E" HECTOR, (563:110/411:248) SAPONITE.

06071220 PLIOCENE, RED MOUNTAIN ANUESITE, TBN,R5E, HECTOR, $(6: 31.23-25,29)$ HECTORITE: MIN. INDEX MAP. MMISCI FEO I IR, MNO $=T R, L I 20=1.14, B A O=0.00, R B 20=0.00, F=4.75, C L=0.211$ LESS $0=F, C L .2 .04$.

06071309 "T8N,R5E" HECTOR, (541:TABLE 2,PI2) HECTORITE: MINIMUM VALUFS OF 6 SMPLS, FOR MAXIMUM VALUES SEE SIOZ 53.97. MISC: FEO $=0.00$. MNO $=0.00$, LIZO $=1.151 \mathrm{I} \cdot$ L. $=14.85$, LESS H2O*.

06073031 "T155,R4W" LA JOLLA. (31:1AO) SAND. USEI IO.

06073032 "TIIS,RSW" OCEANSIDE. (311180) BEACH SAND. USE: 10.

06073032 "JURASSIC \& CRETACEOUS, FRANCISCAN FM. "T2S,R5W" SAN FRANCISCO, $129163,11,55,62$, PLL1) CHERT, REO, THIN-BEDDED MIN.

06075011 JURASSIC \& CRETACEDUS, FRANCISCAN FM, "T2S, R5W" SAN FRANCISCO. $129163,11,55,62$, PLI1 CHERT, RED, THIN-BEDDED MIN. 06079010 JURASSIC, KNOXVILLE FM. "TI2N,R32W" STANLEY MTN. (29:63.64,PL1) CHERT, RROWN, THIN-BEDDED MIN. INDEX \& GEOL MAPS. 06083015 MIOCENE, MONTEREY FM. "TBN,R34W" LOMPOC. (169:108,98) DIATOMITE, USE, 6.

06083016 MIOCENE, MONTEREY FM. "T8N,R34W" LOMPOC. (1691108,98) DIATOMITE. USE: 6.

06083017 MIOCENE, MONTEREY FM \& (OR) MIOCENE, PLIOCENE, SISQUOC FM. "T8N,R34W" LOMPOC. (114:313,305) DIATOMACEOUS EARTH.

06083018 "TRN.R34W" LOMPOC. (569:2) DIATOMACEOUS EARTH.

06083019 "T8N,R34W" LOMPOC. (583:43) DIATOMITE.

06083020 MIOCENE. MONTEREY FM. "T8N,R34W" LOMPOC. (570:40/ 131:T6) DIATOMITE.

06083021 "T6N.R34W" WHITE HILLS. (534:193:) DIATOMACEOUS EARTH, WHITE.

06083024 MIOCENE, MONTEREY SH. "T8N,R35W" POINT SAL. $(187: 12,11$, PLI/ 138:290,278) SHALE, WHITE, PORCELAIN-LIKE) 8.0. 2.12.

06083025 MIOCENE, MONTEREY FM. "IBN,R35W, POINT SAL" $(187: 12,10, P L 1)$ FLINT, P.D., 2.54 MIN. INDEX \& GEOL MAP, GEOL SEC.

06083029 "TRN,R34W" LOMPOC. (464:53) DIATOMACEOUS EARTH, BROWN: FIRING TESTS. USE: 11 . \#MISC: FEO = 1.12.

06087025 MIOCENE, MONTEREY SH, "T125,RZE" CHITTENOEN, (305:64.63,PL 17,18) SHALE, WHITE TO RROWN. INDEX \& GEOL MAP, GEOL SEC.

06089044 "T31.32N,R5W" REDDING. (172:131) SANOSTONE.

06089045 HOLOCENE. S33,T37N,R2E TO SIO,T36N,R4E. (26:129,128,PL2) DIATOMITE, WHITE. INDEX MAP. USE: 11.

06089046 MIOCENE, MONTEREY FM. "T35N,R3E" BURNEY. (169:108,98,102) OIATOMITE, FRIABLE. USE: 4.

06089047 HOLOCENE. S25,T37N,R2E. (366:166,163-165) DIATOMITE, WHITE: 340 FT EXPOSED. INDEX MAPS.

06089050 HOLOCENE. S33,T37N,R2E TO S10,T36N,R4E. (366:166,163-165) OIATOMITE, WHITE: 34O FT EXPOSED. INDEX MAP. 
TABLE 3. - Analyses of samples from California and Hawaii containing uncombined silica and clay each less than 75 percent; uncombined silica and clay each greater than carbonate (Group B), common- and mixed-rock categories

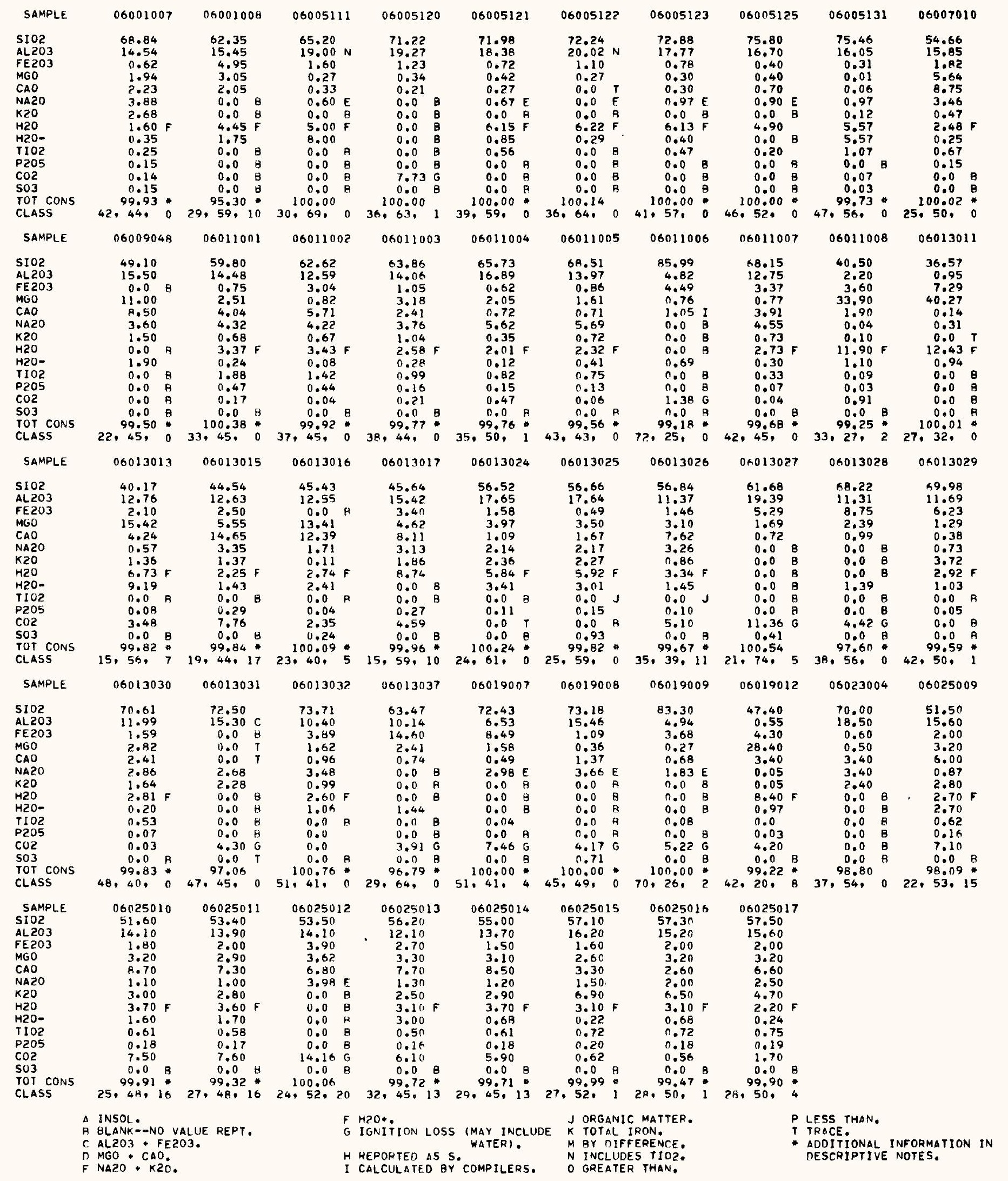


TABLE 3. - Analyses of samples from California and Hawaii containing uncombined silica and clay each less than 75 percent; uncombined silica and clay each greater than carbonate (Group B), common-and mixed-rock categories-Continued

06001007 JURASSIC \& CRETACEOUS, FRANCISCAN FM. "TIS,R3W" PIEDMONT. (137:22.18-21) SANDSTONE1 MINB B.D. = 2.720. "MISC: 06001008 JURASSIC \& CRETACEOUS, FRANCISCAN FM. "TIS.R4W" OAKLAND. $(138: 269,242,266)$ SHALE, GREEN! FINE-GR MIN. \$MISC: 06001008 JURASSIC \& CRETACEOUS, FRANCISCAN FM. "TIS,R4W" OAKLAND. $(138: 269,242,266)$ SHALE, GREEN FINE-GRI
06005111 EOCENE, IONE FM. "T6N,R9E" IONE. $(31378,355,377)$ SAND, FINE-GRI MIN. INDEX \& GEOL MAPS. USE: 3.

06005120 EOCENE. 520,30,31,T7N,R9E. (43:38/ 147:354,261,PL1,6) SAND, WHITE, FINE-GR: MIN: AVG THICKNESS 16 FTI PHYS PROP, 06005121 EOCENE. TESTS. INDEX MAPS. USE: 3,5, $13: 378,355,377)$ SAND, FINE-GR: MIN. INDEX \& GEOL MAPS. USE: 3. 06005122 EOCENE, IONE FM. "TGN,R9E" IONE. $(31378,355,377)$ SAND, FINE-GRI MIN. INDEX \& GEOL MAPS. USE: 3. 06005123 EOCENE, IONE FM, "TGN,R9E" IONE. $(31378,355,377)$ SAND, FINE-GR: MIN. INDEX \& GEOL MAPS. USE: 3. 06005125 EOCENE, IONE FM. "TGN,R9E" IONE. $(31378,355,377)$ SAND, FINE-GR: MIN. INDEX \& GEOL MAPS. USE 3. 06005131 EOCENE, IONE FM. HTGN,R9E" NEAR IONE. (315111,12) CLAY: PHYS PROP. \$MISC: FEO = 0.00, VZO5 = 0.011 HZO REPT AS I.L. 06007010 HOLOCENE. LASSEN PEAK AREA. $(111: 205,204)$ DIABASE TUFF: MIN. $4 M 1 S C:$ FEO $=5.12$, MNO = $0.18,1.120=0.00$, 06009048 PALEOZOIC, CALAVERAS FM, "T3N,R13E" CARSON HILL. (82:14) AUGITE CRYSTAL TUFF. MMISC: FEO = 8.40.

06011001 JURASSIC \& CRETACEOUS, FRANCISCAN FM. "TIGN,R4W" GOAT MTN LOOKOUT, (186:33,31,PL1,2) METASEDIMENT: MIN. INREX \& 06011002 JURASSIC CRETACEOUS, FRANCISCAN FM. "MNIGN,R4W11 GOAT MTN LOOKOUT. $1186: 33.31$, PLI,2) METASEDIMENT MIN. INOEX \& GEOL 06011002 JURASSIC CRETACEOUS, FRANCISCAN FM. "TIGN,R4W

06011003 JURASSIC \& CRETACEOUS, FRANCISCAN FM. "TIGN,R4W" GOAT MTN LOOKOUT, $(186: 23,20,21$, PLL, 1,2$)$ METAGRAYWACKE OR

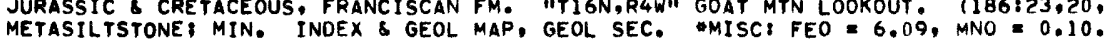

06011004

METASILTSTONE: MIN. INDEX \& GEOL MAP, GEOL SEC. $M I S C$ I FEO $=4.10$, MNO $=0.11$.

06011005 JURASSIC \& CRETACEOUS, FRANCISCAN FM. "TI6N,R4W" GOAT MTN LOOKOUT. $(186: 23,20,21, P L 1,2)$ METAGRAYWACKE OR METASILTSTONE: MIN. INDEX \& GEOL MAP, GEOL SEC. $M$ MISC: FEO $=3.66$, MNO $=0.16$.

06011006 S20,T17N,R4W. (1451361/211122,124) SANDSTONE, GRAY \& BUFF, FINE-GR: 4-6 FT THICK, P.D., 2.558: PHYS PROP. USF: 15, 06011007 JURASSIC \& CRETACEOUS, FRANCISCAN FM. "TIGN,R4W" GOAT MTN LOOKOUT. (186:33,31,PL1,2) METASEDIMENT: MIN. INDEX \& GEOL MAPS, GEOL SEC. $M I S C I$ FEO $=1.90$, MNO $=0.08$.

JURASSIC, KNOXVILLE FM. "TI3N,R4,5,W" TMOMPSON CANYON. (372:925,922) DETRITAL SERPENTINITE. "MISC:FFJm?.9. MNO=0,08.

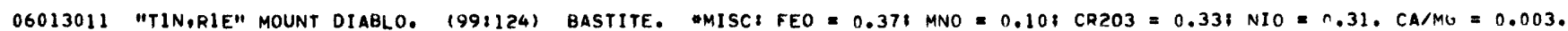

06013013 CRETACEOUS. "TIN,RIE" MOUNT DIABLO. (508:408,403,407.PL15) SHALE, FRIABLE. GEOL MAP, GEOL SEC. \#MISC: FEO = 3.56) 06013015 MIOCENE. "TIN,RIE" MOUNT OIABLO. (508:412,400,411,PL15) SANOSTONE, FRIARLE. GEOL MAP \& SEC. MISCIFEO=3,08: MNO=.44. 06013016 CRETACEOUS, "TIN,RIE" MOUNT OIABLO. (508:404,400,PL15) SHALEY GABBRO, GREEN, FRIABLE. GEOL MAP, GEOL SEC. "MISC: 06013017 JURASSIC, KNOXVILLE FM. "TIN,RIE" MOUNT DIABLO, $1508: 409,400,410$, PLI5I SHALE, BROWN, FRIABLE: MIN. GEOL MAP. GEOL SEC. HMISC: FEO $=3.73$, MNO $=0.33$, CR2O3 $=0.12$.

06013024 CRETACEOUS. "TIN,RIE" MOUNT DIABLO. (50B:408,400,403,PLI5) SHALE, GRAY, FRIABLE. GEOL MAP, GEOL SEC. "MISC: FEO $=5.25$; MNO $=0.321 \mathrm{NIO}=$ TR.

06013025 CRETACEOUS. "TIN,RIE" MOUNT DIABLO. (508: 404,400,403.PL15) SHALE, BLACK. GEOL MAP, GEOL SEC. "MISC: FEO = 5.221 06013026 JURASSIC \& CRETACEOUS, FRANCISCAN FM. "TIN,RIE" BAGLEY CREEK. (508:412,400,PL15/ 29:33,11) SANDSTONE, GREFN. GEOL MAP, GEOL SEC. MMISC: FEO $=4.95 ;$ MNO $=0.22:$ ORGANIC MATTER $=$ TR.

06013027 "TIN,RIW" COWELL. (173:122) CLAY. USE: 4 .

06013028 JURASSIC S CRETACEOUS, FRANCISCAN FM. "TIN,R5W" POINT RICHMONO. (138:269.265,268) SHALE, RED MIN. "MISC: MNO=0.13.

06013029 JURASSIC \& CRETACEOUS. "TIN,RIE" MOUNT OIABLO. (508:411,400,403,410,PL15/ 29:64,11,PL1) SHALE, RED, FRIABLE M MIN, INDEX \& GEOL MAPS, GEOL SEC. HMISC: FEO $=2.08$; MNO $=0.49$.

06013030 JURASSIC \& CRETACEOUS, FRANCISCAN FM. "TIS,R4W" BERKELEY HILLS, (578:493,495/ 29:108,11,92) METAGRAYWACKE) MIN.

06013031 INIS, \& GEOL MAPS. "MISC: FEO 2 2.22, MNO = 0.05 . FAIRMOUNT. (345:328) PUMICE: PHYS PROP. USE, 4.

06013032 CRETACEOUS, CHICO FM. "TIN,RIE" MOUNT DIABLO. (508:412,400,411,PL15) SANOSTONE, BROWN, FINE-GR. GEOL MAP, GEOL SEC. 06013032 CRETACEOUS, CHICO FM. "TIN,RIE" MOUNT DIABLO. 06013037 JURASSIC \& CRETACEOUS, FRANCISCAN FM. "TIN,R5WH REO ROCK ISLAND, (138:269.257,264,265) SHALE, PED, FINE-GD: MIN. MISC: MNO $=0.08$

06019007 S8,T15S,R12E. (345:310,309) DIATOMITEI TONNAGE. USEI 4,11. $\$ M I S C:$ NA2O K20, BY DIF: MN3O4 = 0.00.

06019008 T11S,R2IE, FRIANT. (345:324,323) VOLCANIC ASH, CREAM-COLOREO. USE: 1,4. *MISC: NAZO + K20, REPT AS ALKALIES \& UNDETO. 06019009 S8,T15S,RI2E. (3451310,309) DIATOMITE: TONNAGE. USE: 4.11. \$MISC: MN304 = 0.001 ALKALIES BY DIF.

06019012 MIOCENE, TEMBLOR FMI BIG BLUE SERPENTINOUS MBR. CANTUA CREEK. (372:925,922) DETRITAL SERPENTINITE. "MISC: FEO = 1.4. MNO $=0.07$.

2 FT THICK. INDEX MAPS.

S23,T11S,RI3E. $1394: 170,160,164)$

06025010 MISCI FEO $=2.8$, MNO $=0.04$

S23,TI1S,RI3E. (394:170,160, 06025011 S20.T14S,R15E (394:174,i59,1

0602501

06025012 TERTIARY, S6-8,T16S,R9E. $(4471132, P L 1)$ CLAY. INOEX MAP
06025013 S23.T11S,R13E. $(3941170,160,164)$ SANDSTONE, SILTSTONE: I MISC: FEO $=2.3$, MNO $=0.07$

06025014 MISC: FEO $=1.0$, MNO $=0.06$. S23,TIIS,R13E. (394:170,160,164)

AMISC: FEO $=2.7$, MNO $=0.04$.
06025015 S23,TIIS,R13E. (394:170,160,164 - MISC, FEO $=5.8$, MNO $=0.13$.

06025016 S23.T11S,R13E. (394:170,160,164) MISC: FEO $=5.3$, MNO $=0.13$. 06025017 S23,TI1S,R13E. (394:170,160,164) SANDSTONE, SILTSTONE: INTERBEDDEO: WELL. 5,232 FT SMPL AT 1,310 FT. INDEX MAP. SANDSTONE, SILTSTONE: INTERBEDOED: WELL, 5.232 FT: SMPL AT 1,R8O FT. INDEX MAP. SILTSTONE \& SANOSTONE. FINE-GR: WELL, $13,443 \mathrm{FT}$ : SMPL AT 7,800 FT. INDEX MAP. PLII CLAY. INOEX MAP. SANDSTONE, SILTSTONE: INTERBEODED: WELL, 5.232 FT: SMPL AT 2.220 FT, INIEX MAP. SANOSTONE, SILTSTONE: INTERBEDDED, WELL, 5,232 FT: SMPL AT 4,090 FT. INDEX MAP. SANOSTONE, SILTSTONEः INTERBEDDEO: WELL, 5,232 FT: SMPL AT 4,485 FT. INDEX MAP. SANOSTONE, SILTSTONE: INTERREDDED WELL, 5,232 FT: SMPL AT 3,520 FT. INDEX MAP. 
TABLE 3. - Analyses of samples from California and Hawaii containing uncombined silica and clay each less than 75 percent; uncombined silica and clay each greater than carbonate (Group B), common-and mixed-rock categories-Continued

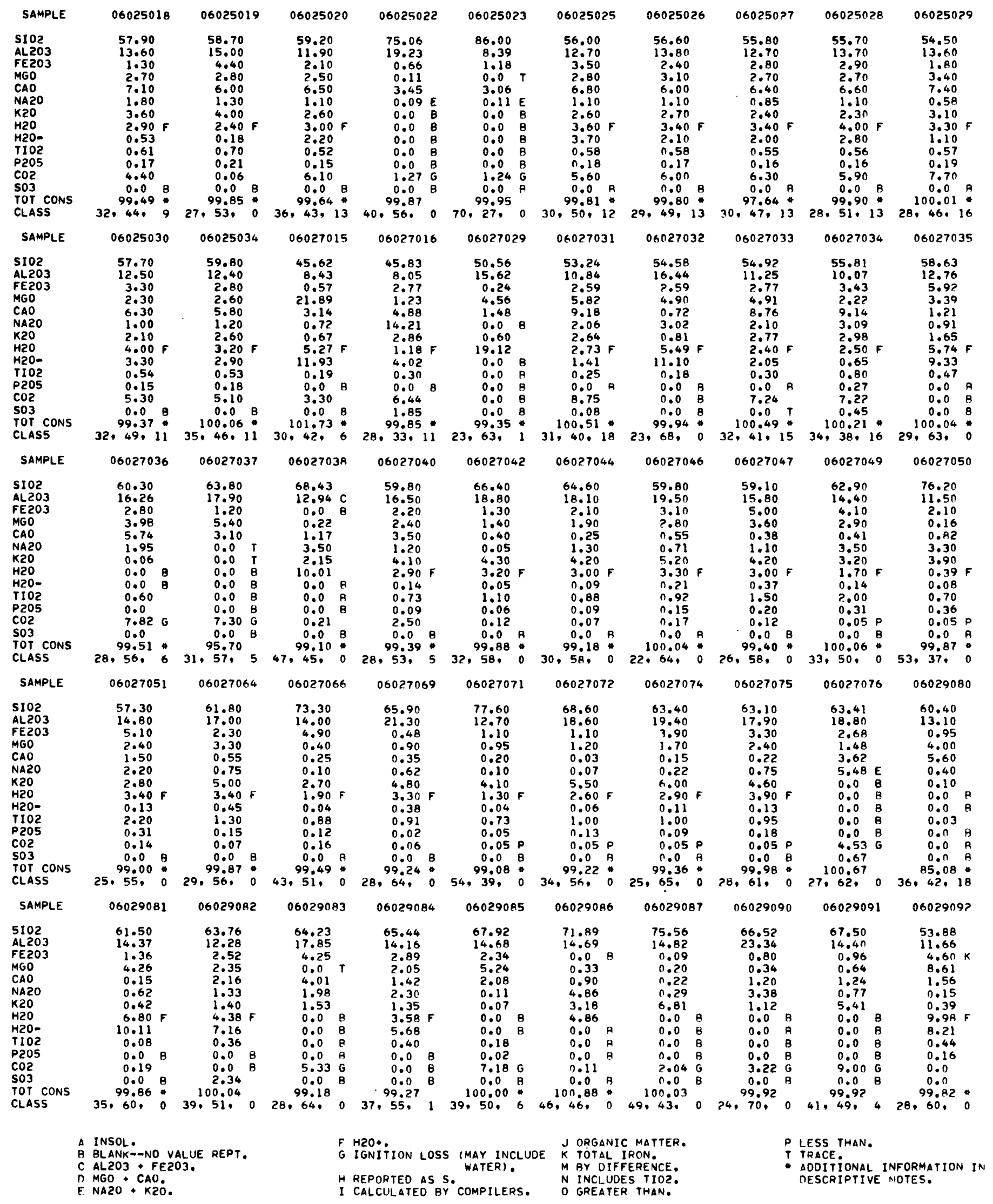


TABLE 3. - Analyses of samples from California and Hawaii containing uncombined silica and clay each less than 75 percent; uncombined silica and clay each greater than carbonate (Group B), common-and mixed-rock categories-Continued

\begin{abstract}
06025018 S23.T11S,R13E, 1394:170,160,164) SANOSTONE, SILTSTONE INTERBEDOED WELL. 5,232 FTI SMPL AT 2,840 FT. INDEX MAP. 06025019 S23,TIIS,RI3E. (394:170,160,164) SANOSTONE, SILTSTONEI INTERBEDNED; WELL, 5.232 FT, SMPL AT 5,210 FT. INDEX MAP. 06025020 S23,TIIS,RI3E. $(394: 170,160,164)$ SANDSTONE, SILTSTONE INTERBEDDED WELL, 5,232 FT: SMPL AT B90 FT. INDEX MAP.

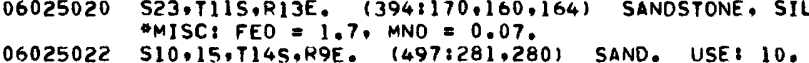

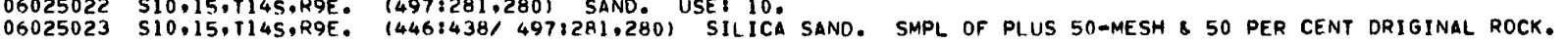
06025025 HOLOCENE. "T13,14S,R19,20E" $13948170,159,174)$ SEDIMENTS: AVG.OF 6 ANAL. INDEX MAP. "MISC: FEO = 0.6: MNO = .05. 06025026 S20,T14S,R15E; $(394: 174,159,170)$ SILTSTONE \& SANOSTONE, FINE-GR: WELL, 13,443 FT; SMPL AT 3.58O FT. INDEX MAP. 06025027 S20,T14S,RI5E. (394:174,159,170) SILTSTONE S SANOSTONE, FINE-GR: WELL, 13.443 FT' SMPL AT G.600 FT. INDEX MAP. 06025028 S20,TI4S,RISE. (394:174,159,170) SILTSTONE \& SANOSTONE, FINE-GR WELL. 13,443 FTI SMPL AT 4.680 FT. INDEX MAP. 06025029 S20,TI4S,R15E. (394:174,159,170) SILTSTONE \& SANOSTONE, FINE-GR WELL, 13.443 FT, SMPL AT 9,42N FT. INDEX MAP. 06025030 S20,T14S,R15E. (394:174,159,170) SILTSTONE \& SANDSTONE. FINE-GR WELL, 13,443 FT: SMPL AT 2,040 FT. INDEX MAP. 06025034 "TI2S,RI2E" SALTON TROUGH. (393:393.385) DETRITUS! MIN MEAN OF 20 SMPL. INDEX MAP. SPECTRO ANAL ALSO GIVEN. 06027015 "MISC: FEO $=0.91$. MNO $=0.04$.
\end{abstract}

06027016 "T17S,R38E" KEFLER, (84:97.98) CLAY, GRAY, FRIABLE: MIN. "MISC: FE0 0.43, MNO=0.12, CL=7.33. ANAL OF SOLUBLE PART ALSO 06027029 "T20.2IN,R4.5E" ARMAGOSA RIVER. (498:514.513) BENTONITE, WHITE, FRIABLE: B.D.. 2.45 . USE: 6. "MISC: NACL = 7.17. 06027031 "T17S,R37E" KEELER. 1565:89/ B4:97) CLAY, SANOY, 12-18 IN THICK, \$MISC1 FEO = 0.77, MNO = 0.10, CL = 0.05.

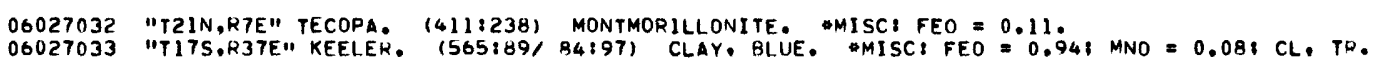
06027034 "TITS,R37E" OWENS LAKE, 184:931 VOLCANIC ASH. GRAY. MISC: FFO $=0.67$, MNO $=0.231$ CL $=0.88,0=0.20$. 06U27035 "T19S,R37E" OLANCHA. 1346:302.315.319) FULLERS EARTH: 8.0.02.52. USE: 6. "MISC: MNO = 0.03.

06027036 HOLOCENE. S13,23,T21S,R38E. $(87363,62, P L 1,3)$ PUMICE, GRAY-WHITE 30 FT THICK, MIN. INOEX \& GEOL MAPS, GEOL SEC. 06027037 "S32.33, T2ZN,R7E" (140:10,5) HALLOYSITE: 1-10 FT THICK, USF: 6. 0602703 PLEISTOCENE. S32.33,T22N,H7E. (498:521) VOLCANIC ASH: 4-6 FT THICK. USF: 1.6 . $\$ M I S C:$ NACL = 0.47.

06027040 CAMBRIAN, HARKLESS FM, S30.T13S.R46E. (585:22,8) PELITEI MIN, INDEX MAP. SPECTRO ANAL ALSO GIVEN. MMISC: 06027042 CAMBRIAN, HARKLESS FM. S14,24,T7S,R38E. $(595: 22,8,77)$ PELITE, MIN. INDEX MAP, MEAS SEC. SPECTRO ANAL ALSO GIVEN. 06027044 CAMERIAN, HARKLESS FM. S1,12,13,24,110S.R35E. $(585: 23,8)$ PELITE: MIN. INDEX MAP. SPECTRO ANAL ALSO GIVEN. MMISC: 06027046 CAMBRIAN. POLETA FM. SI5.T7S.R38E. $1585: 23.8 .74)$ PELITE: MIN. INDEX MAP. MEAS SEC. SPECTRO ANAL ALSO GIVEN. MMISC: 06027047 PRECAMBRIAN. DEEP SPRING FM. S14,24,T7S,R38E, $(585: 23,8)$ PELITE: MIN. INDFX MAP. SPECTRO ANAL ALSO GIVEN. MMISC: 06027049 PRECAMBRIAN \& CAMBRIAN, CAMPITO FM, ANDREWS MOUNTAIN MBR. SI4.24.T7S,R38F. (585834.8) SANDSTONE: MIN. INDEX MAP.

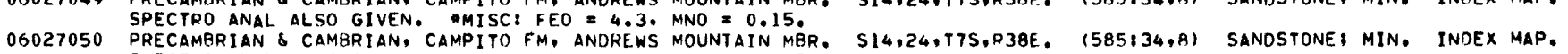
06027051 PRECAMBRIAN S CAMBRIAN, CAMPITO FM, ANDREWS MOUNTAIN MBR. LAT 37 DEG. 191, LONG 117 DEG. 431.4 (585134.8) SANOSTONE, PRECAMBRIAN \& CAMBRIAN, CAMPITO FM, ANDREWS MOUNTAIN MBR, LAT 37 DEG. 19.
MIN. INDEX MAP. SPECTRO ANAL ALSO GIVEN. MMISC: FEO $=6.6$. MNO $=0.12$.

06027064 PRECAMBRIAN \& CAMBRIAN, WOON CANYON FM. S9,10,T20N,R8E. (585:24,8,127) PELITE: MIN. INDEX MAP, MEAS SEC. SPECTRO 06027066 PRECAMBRIAN, STIRLING QTZITE. S11,T27N,R2F. (585:25,8) PELITE, MIN. INDEX MAP. SPECTRO ANAL ALSO GIVEN, MMISC: 06027069 PRECAMBRIAN. JOHNNIE FM. S9-11,T20N,R8E. $(585: 25,8,127)$ PELITE: MIN. INDEX MAP, MEAS SEC. SPECTRO ANAL ALSO GIVEN. 06027071 PRECAMBRIAN, JOHNNIE FM. S20,T21S,R46E. $(585: 25,8)$ PELITE: MIN. INDEX MAP. SPECTRO ANAL ALSO GIVEN. MMISC: 06027072 PRECAMBRIAN, JOHNNIE FM. LAT 36 DEG. 031 1 ONG 117 DEG. 041. $1585: 25,8)$ PELITE: MIN. INDEX MAP. SPECTRO ANAL ALSO 06027074 PRECAMBRIAN, JOHNNIE FM. LAT 36 DEG. 03 , LONG 117 DEG. 041. $1585: 25,8)$ PELITE MIN. INDEX MAP. SPECTRO ANAL ALSO 06027075 PRECAMBRIAN, JOHNNIE FM. LAT 36 DEG. 01 , LONG 117 DEG. $04 \%$ (585:25,8) PELITE: MIN. INDEX MAP. SPECTRO ANAL ALSO

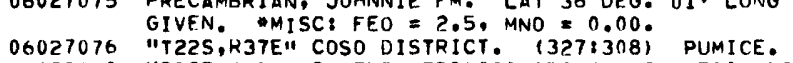

06029080 MIOCENE \& PLIOCENE, TROPICO GROUP. S26,T11N.R8W. $(213: 353,328,350,352, P L 51,52)$ TUFF, WHITE. INDEX \& GEOL MAPS, GEOL. 06029081 MIOCENE \& PLIOCENE. TROPICO GR. S2,3,T11N.R9W. (346:302.319/ 117:74,69,75,PL1,2) FULLERS EARTH. WHITE: 6 FT THICK. 06029082 MIOCENE \& OLIGOCENE, VAQUEROS FM. S18,TION,R22W. $(292: 253,258, P L 31)$ BENTONITE. INDEX \& GEOL MAPS. GEOL SEC. 06029083 OLIGOCENE \& MIOCENE, TEMBLOR FM. 514.T275,R28E, 115:94) VOLCANIC ASHI MIN. 06029084 EOCENE, TEJON FM. S19,T10N,R19W. 1292:253,PL31) HENTONITE, WHITE: 15-20 FT THICK. INOEX \& GEOL MAPS, GEOL. SEC. 06029095 "T30S,R33E" TEHACHAPI. (453:183) CLAY. USE: 6. "MISCI CL $=0.18$. 06029086 PLIOCENE, RICARDO FM. S5, $6, T 295, R 38 E$, (B7:85,68,PL1,4) PUMICITE, WHITE. FINE-GR. COMPACT: LAYERS TO 9 FT THICK. 06029087 TERTIARY. S34,T30S.R36E. (117:80.69.75,82) CLAY, WHITE TO RUFF, FINE-GRI MINI PHYS PROP, FIRING TESTS. INDEX \& GEOL 06029090 "T28S.R38E" EL PASO RANGE. 1327:308) TUFF.

06029091 "T28S,R38E" EL PASO RANGE. (327:308) TUFF. COARSE-GR: 7 FT THICK: MIN. INOEX GEOL MAPS, USE: 6 . MMISC: MNO $=0.18$, CO2 REPT AS CO3, ZRO2 = 0.00 . 
TABLE 3. - Analyses of samples from California and Hawaii containing uncombined silica and clay each less than 75 percent; uncombined silica and clay each greater than carbonate (Group B), common-and mixed-rock categories-Continued

\begin{tabular}{|c|c|c|c|c|c|c|c|c|c|c|c|c|c|c|c|c|c|c|c|c|}
\hline SAMPLE & \multicolumn{3}{|c|}{06029094} & \multicolumn{2}{|c|}{06029095} & \multicolumn{2}{|l|}{06029096} & \multicolumn{2}{|c|}{06029104} & \multicolumn{2}{|l|}{06033002} & \multicolumn{2}{|c|}{06033003} & \multicolumn{2}{|l|}{06033004} & \multicolumn{2}{|l|}{06035001} & 06035002 & \multicolumn{2}{|l|}{06035003} \\
\hline $\begin{array}{l}\text { S102 } \\
\text { AL203 } \\
\text { FE2O3 } \\
\text { MGO } \\
\text { CAO } \\
\text { NA2O } \\
\text { K2O } \\
\text { H2O } \\
\text { H2O- } \\
\text { TIO2 } \\
\text { P2O5 } \\
\text { CO2 } \\
\text { SO3 } \\
\text { TOT CONS } \\
\text { CLASS }\end{array}$ & & $\begin{array}{c}68.92 \\
11.04 \\
0.95 \\
1.51 \\
1.10 \\
1.46 \\
1.54 \\
4.70 \\
2.23 \\
0.14 \\
0.03 \\
0.0 \\
0.0 \\
99.82 \\
48.46 .\end{array}$ & $\begin{array}{l}B \\
0 \\
0\end{array}$ & $\begin{array}{r}68.98 \\
11.10 \\
0.97 \\
1.37 \\
0.94 \\
1.75 \\
1.77 \\
4.83 \\
7.67 \\
0.14 \\
0.03 \\
0.02 \\
0.0 \\
99.80 \\
48,450\end{array}$ & $\begin{array}{l}8 \\
0\end{array}$ & $\begin{array}{c}72.49 \\
11.87 \\
0.63 \\
0.13 \\
0.57 \\
2.46 \\
5.39 \\
5.44 \\
0.52 \\
0.12 \\
0.02 \\
0.01 \\
0.0 \\
99.93 \\
51.40 .\end{array}$ & $\begin{array}{l}\text { B } \\
0\end{array}$ & $\begin{array}{r}58.95 \\
9.92 \\
2.03 \\
4.64 \\
1.09 \\
0.19 \\
0.46 \\
7.11 \\
14.78 \\
0.74 \\
0.03 \\
0.02 \\
0.02 \\
99.76 \\
39.53 .\end{array}$ & F & $\begin{array}{c}72.98 \\
13.21 \\
0.60 \\
0.37 \\
1.19 \\
2.99 \\
4.36 \\
2.76 \\
0.19 \\
0.28 \\
0.02 \\
0.0 \\
0.0 \\
99.77 \\
49,41 .\end{array}$ & $\begin{array}{l}T \\
R \\
0\end{array}$ & $\begin{array}{c}68.50 \\
12.82 \\
1.29 \\
2.21 \\
1.82 \\
6.03 \\
1.26 \\
2.11 \\
0.28 \\
0.60 \\
0.16 \\
0.00 \\
0.0 \\
100.47 \\
44.41 .\end{array}$ & $\begin{array}{l}R \\
B \\
0\end{array}$ & $\begin{array}{r}71.99 \\
10.17 \\
0.99 \\
1.43 \\
2.76 \\
2.32 \\
1.45 \\
2.82 \\
0.09 \\
0.47 \\
0.09 \\
0.34 \\
0.0 \\
100.11 \\
53,33 .\end{array}$ & $\begin{array}{l}\text { B } \\
1 \\
1\end{array}$ & $\begin{array}{c}55.93 \\
17.34 \\
1.50 \\
7.29 \\
8.04 \\
3.32 \\
1.35 \\
0.26 \\
0.0 \\
0.0 \\
0.0 \\
0.0 \\
0.0 \\
100.23 \\
24,51 .\end{array}$ & $\begin{array}{l}8 \\
0\end{array}$ & $\begin{array}{c}56.53 \\
17.50 \\
1.35 \\
5.94 \\
8.07 \\
3.51 \\
1.55 \\
0.27 \\
0.0 \\
0.54 \\
0.15 \\
0.0 \\
0.0 \\
100.56 \\
24.52 .\end{array}$ & $\begin{array}{c}56.70 \\
15.75 \\
1.29 \\
7.16 \\
7.67 \\
3.36 \\
1.56 \\
0.30 \\
0.0 \\
0.65 \\
0.20 \\
0.0 \\
0.0 \\
100.18 \\
27.47 .\end{array}$ & B \\
\hline SAMPLE & & 0603500 & & 0603701 & & 06037014 & & 060.3701 & & 06037017 & & 0603701 & & 06037023 & & $060370 ? 7$ & & 06037028 & 0603703 & \\
\hline $\begin{array}{l}\text { S1O2 } \\
\text { AL2O3 } \\
\text { FE203 } \\
\text { MGO } \\
\text { CAO } \\
\text { NA2O } \\
\text { K2O } \\
\text { H2O } \\
\text { H2O- } \\
\text { T102 } \\
\text { P2O5 } \\
\text { CO2 } \\
\text { SO3 } \\
\text { TOT CONS } \\
\text { CLASS }\end{array}$ & & $\begin{array}{c}70.01 \\
12.61 \\
1.47 \\
0.72 \\
1.06 \\
1.94 \\
5.12 \\
4.68 \\
2.37 \\
0.0 \\
0.04 \\
0.0 \\
0.0 \\
100.52 \\
46.45 .\end{array}$ & $\begin{array}{l}B \\
B \\
- \\
0\end{array}$ & $\begin{array}{r}57.14 \\
14.64 \\
4.29 \\
2.62 \\
2.75 \\
1.89 \\
0.46 \\
4.77 \\
10.30 \\
0.74 \\
0.20 \\
0.01 \\
0.02 \\
100.06 \\
26.65 .\end{array}$ & $\begin{array}{l}H \\
\\
0\end{array}$ & $\begin{array}{c}62.85 \\
16.78 \\
3.71 \\
1.93 \\
5.13 \\
3.27 \\
0.51 \\
2.16 \\
2.59 \\
0.61 \\
0.25 \\
0.0 \\
0.01 \\
100.07 \\
29.59\end{array}$ & $\begin{array}{l}H \\
0 \\
0\end{array}$ & $\begin{array}{r}69.20 \\
8.76 \\
9.64 \\
1.87 \\
2.24 \\
0.78 \\
0.0 \\
0.0 \\
0.0 \\
0.0 \\
0.0 \\
7.40 \\
0.0 \\
99.89 \\
43.49 .\end{array}$ & $\begin{array}{l} \\
E \\
B \\
B \\
B \\
B \\
B \\
G \\
8 \\
6\end{array}$ & $\begin{array}{r}72.16 \\
13.73 \\
0.32 \\
0.10 \\
1.04 \\
3.69 \\
3.95 \\
3.52 \\
0.14 \\
0.04 \\
0.03 \\
0.01 \\
0.02 \\
99.73 \\
48,42 .\end{array}$ & H & $\begin{array}{c}82.80 \\
6.06 \\
1.94 \\
0.92 \\
0.51 \\
0.0 \\
0.0 \\
0.0 \\
5.88 \\
0.0 \\
0.0 \\
0.0 \\
0.0 \\
98.11 \\
70,29 .\end{array}$ & $\begin{array}{l}B \\
B \\
B \\
A \\
A \\
T \\
B \\
0\end{array}$ & 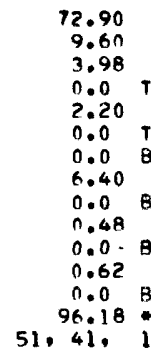 & $\begin{array}{l}8 \\
1 \\
1\end{array}$ & $\begin{array}{c}59.26 \\
15.80 \\
7.54 k \\
3.62 \\
4.10 \\
0.0 \\
0.0 \\
0.0 \\
0.0 \\
0.0 \\
0.0 \\
6.58 \\
1.35 \\
98.25 \\
23,66.2\end{array}$ & $\begin{array}{l}B \\
B \\
B \\
B \\
B \\
B \\
B \\
G\end{array}$ & $\begin{array}{c}78.70 \\
5.83 \\
1.92 \\
0.71 \\
2.59 \\
0.56 \\
1.06 \\
4.26 \\
3.64 \\
0.39 \\
0.34 \\
0.0 \\
0.0 \\
100.97 \\
66,28 .\end{array}$ & 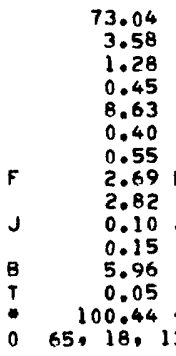 & $\begin{array}{l}5 \\
5 \\
13\end{array}$ \\
\hline SAMPLE & & 0603703 & & 0603703 & & 06041009 & & 0604101 & & 06041011 & & 0604101 & & 06041016 & & 06041017 & 17 & 06041018 & 060 & 19 \\
\hline $\begin{array}{l}\text { SIO2 } \\
\text { AL2O3 } \\
\text { FE2O3 } \\
\text { MGO } \\
\text { CAO } \\
\text { NA2O } \\
\text { K2O } \\
\text { H2O } \\
\text { H2O- } \\
\text { TIO2 } \\
\text { P2O5 } \\
\text { CO2 } \\
\text { SO3 } \\
\text { TOT CONS } \\
\text { CLASS }\end{array}$ & & $\begin{array}{c}73.71 \\
7.25 \\
2.63 \\
1.47 \\
1.72 \\
1.19 \\
1.00 \\
6.94 \\
2.88 \\
0.0 \\
0.24 \\
0.0 \\
0.16 \\
100.13 \\
58,35\end{array}$ & $\begin{array}{l}J \\
T\end{array}$ & $\begin{array}{c}65.66 \\
12.71 \\
3.54 \\
0.71 \\
1.44 \\
1.10 \\
1.72 \\
8.03 \\
4.34 \\
0.40 \\
0.0 \\
0.0 \\
0.0 \\
99.65 \\
39.55 .\end{array}$ & $\begin{array}{l}B \\
B \\
B\end{array}$ & $\begin{array}{r}60.00 \\
18.10 \\
1.00 \\
2.90 \\
1.10 \\
1.80 \\
3.20 \\
4.40 \\
0.64 \\
0.73 \\
0.17 \\
0.10 \\
0.34 \\
99.42 \\
27 . \\
57 .\end{array}$ & $\begin{array}{l}\mathrm{H} \\
\text { * } \\
0\end{array}$ & $\begin{array}{r}67.00 \\
14.10 \\
0.90 \\
2.80 \\
1.30 \\
3.40 \\
1.60 \\
2.80 \\
0.60 \\
0.60 \\
0.10 \\
0.10 \\
0.0 \\
99.40 \\
41,44,\end{array}$ & F & $\begin{array}{c}60.90 \\
13.10 \\
9.20 \\
2.30 \\
0.0 \\
0.08 \\
4.90 \\
4.60 \\
2.00 \\
0.92 \\
0.14 \\
0.0 \\
0.0 \\
99.25 \\
27.02 .\end{array}$ & $\begin{array}{l}R \\
B \\
1 \\
1\end{array}$ & $\begin{array}{r}58.40 \\
14.30 \\
7.40 \\
3.30 \\
2.00 \\
1.70 \\
3.90 \\
3.10 \\
2.00 \\
0.66 \\
1.30 \\
0.0 \\
0.0 \\
99.46 \\
25,60 .\end{array}$ & F & $\begin{array}{c}69.00 \\
11.70 \\
1.00 \\
3.80 \\
1.30 \\
2.00 \\
2.30 \\
3.30 \mathrm{~F} \\
0.30 \\
0.70 \\
0.20 \\
0.0 \\
0.0 \\
99.90 \\
47, \\
38 .\end{array}$ & $\begin{array}{l}8 \\
B \\
0\end{array}$ & $\begin{array}{c}70.04 \\
13.88 \\
0.91 \\
2.11 \\
1.79 \\
4.01 \\
0.97 \\
3.15 \mathrm{~F} \\
0.25 \\
0.14 \\
0.0 \\
0.07 \\
0.0 \\
99.65 \\
44,440\end{array}$ & $\begin{array}{l}\text { B } \\
\text { : }\end{array}$ & $\begin{array}{c}70.31 \\
14.03 \\
0.85 \\
2.07 \\
1.31 \\
4.12 \\
1.19 \\
2.37 \\
0.23 \\
0.36 \\
0.05 \\
0.09 \\
0.0 \\
99.69 \\
45,43 .\end{array}$ & $\begin{array}{c}72.90 \\
11.30 \\
1.10 \\
2.70 \\
0.60 \\
3.80 \\
0.90 \\
2.20 \\
0.40 \\
0.60 \\
0.10 \\
0.10 \\
0.0 \\
99.70 \\
52.36,\end{array}$ & F \\
\hline SAMPLE & & 0604102 & & 0604102 & & 06043004 & & $060430 n$ & & 06047001 & & 0604700 & & 06047004 & & 06047005 & & 06047006 & 0605100 & 01 \\
\hline $\begin{array}{l}\text { SIO2 } \\
\text { AL2O3 } \\
\text { FE2O3 } \\
\text { MGO } \\
\text { CAO } \\
\text { NA2O } \\
\text { K2O } \\
\text { H2O } \\
\text { H2O- } \\
\text { TIO2 } \\
\text { P2OS } \\
\text { CO2 } \\
\text { SO3 } \\
\text { TOT CONS } \\
\text { CLASS }\end{array}$ & & $\begin{array}{c}68.20 \\
13.00 \\
1.60 \\
2.40 \\
2.00 \\
2.20 \\
2.20 \\
3.20 \\
0.50 \\
0.62 \\
0.10 \\
1.00 \\
0.0 \\
99.80 \\
43,43 .\end{array}$ & $\begin{array}{l}B \\
2 \\
2\end{array}$ & $\begin{array}{c}69.77 \\
14.82 \\
1.21 \\
2.38 \\
1.99 \\
3.89 \\
1.01 \\
2.96 \\
0.20 \\
0.41 \\
0.08 \\
0.10 \\
0.0 \\
100.20 \\
42.47 .\end{array}$ & B & $\begin{array}{c}60.35 \\
17.62 \\
5.64 \\
1.04 \\
0.45 \\
1.00 \\
3.16 \\
4.36 \mathrm{~F} \\
1.02 \\
0.75 \\
0.17 \\
0.0 \\
0.05 \\
99.76 \\
23.66 .\end{array}$ & i & $\begin{array}{c}71.70 \\
12.91 \\
4.57 \\
2.06 \\
1.11 \\
0.0 \\
0.0 \\
0.0 \\
0.0 \\
0.0 \\
0.0 \\
3.82 \\
0.0 \\
96.17 \\
44.49 .\end{array}$ & $\begin{array}{l} \\
\\
B \\
B \\
B \\
B \\
B \\
B \\
G \\
B \\
\end{array}$ & $\begin{array}{c}55.18 \\
16.26 \\
4.10 \\
2.54 \\
2.26 \\
0.0 \\
0.0 \\
0.0 \\
0.0 \\
0.0 \\
0.0 \\
16.96 \\
0.0 \\
97.30 \\
2.66 .\end{array}$ & $\begin{array}{l}A \\
A \\
B \\
B \\
A \\
B \\
G \\
B\end{array}$ & $\begin{array}{c}58.90 \\
17.60 \\
5.04 \\
1.35 \\
0.56 \\
0.0 \\
0.0 \\
0.0 \\
0.0 \\
0.0 \\
0.0 \\
15.42 \\
0.0 \\
98.87 \\
2.73 .\end{array}$ & $\begin{array}{l}\text { B } \\
R \\
B \\
R \\
R \\
B \\
G \\
R \\
4\end{array}$ & $\begin{array}{c}68.46 \\
17.60 \\
4.50 \\
0.30 \\
0.0 \\
0.0 \\
0.0 \\
0.0 \\
0.0 \\
0.0 \\
0.0 \\
0.0 \\
7.60 \\
0.00 \\
0.0 \\
98.46 \\
66 . \\
37 .\end{array}$ & $\begin{array}{l}\text { T } \\
B \\
B \\
8 \\
8 \\
8 \\
8 \\
8 \\
6 \\
\text { B }\end{array}$ & $\begin{array}{c}70.16 \\
16.82 \\
4.23 \\
0.0 \\
0.0 \\
0.0 \\
0.0 \\
0.0 \\
0.0 \\
0.0 \\
0.0 \\
7.34 \\
0.0 \\
98.5 \\
36,03 \\
63 .\end{array}$ & $\begin{array}{l}B \\
B \\
B \\
B \\
B \\
B \\
B \\
B \\
G \\
B\end{array}$ & $\begin{array}{c}60.00 \\
15.85 \\
5.95 \\
1.65 \\
2.14 \\
0.0 \\
0.0 \\
0.0 \\
0.0 \\
0.0 \\
0.0 \\
12.54 \\
0.0 \\
98.13 \\
25,66,\end{array}$ & $\begin{array}{c}60.10 \\
13.56 \\
3.30 \\
0.72 \\
4.96 \\
2.00 \\
0.68 \\
12.87 \\
0.0 \\
0.0 \\
0.0 \\
0.0 \\
1.83 \\
100.16 \\
32.57 .\end{array}$ & $\begin{array}{l}\text { R } \\
\text { R } \\
\text { R } \\
\text { R } \\
\text {. }\end{array}$ \\
\hline $\begin{array}{l}\text { SAMPLE } \\
\text { SIO2 } \\
\text { AL2O3 } \\
\text { FE2O3 } \\
\text { MGO } \\
\text { CAO } \\
\text { NA2O } \\
\text { K2O } \\
\text { H2O } \\
\text { H20- } \\
\text { TIO2 } \\
\text { P2O5 } \\
\text { CO2 } \\
\text { SO3 } \\
\text { TOT CONS } \\
\text { CLASS }\end{array}$ & & $\begin{array}{c}0605100 \\
67.39 \\
15.99 \\
0.56 \\
0.77 \\
1.63 \\
4.74 \\
4.80 \\
2.06 \\
0.0 \\
0.0 \\
0.0 \\
0.0 \\
0.0 \\
99.93 \\
38.0 \\
48 .\end{array}$ & $\begin{array}{l}8 \\
8 \\
8 \\
B \\
8 \\
0 \\
0\end{array}$ & $\begin{array}{c}0605100 \\
75.28 \\
12.62 \\
1.76 \\
0.92 \\
0.85 \\
0.0 \\
0.0 \\
2.50 \\
5.50 \\
0.0 \\
0.43 \\
0.0 \\
0.0 \\
99.86 \\
51.47 .\end{array}$ & $\begin{array}{l}03 \\
k \\
k \\
B \\
B \\
F \\
B \\
B \\
B \\
0\end{array}$ & $\begin{array}{c}06051004 \\
75.30 \\
12.60 \\
0.31 \\
0.41 \\
0.52 \\
3.90 \\
4.90 \\
1.00 \\
0.19 \\
0.03 \\
0.0 \\
0.09 \\
0.0 \\
100.04 \\
53,37 .\end{array}$ & $\begin{array}{l}R \\
0 \\
0\end{array}$ & $\begin{array}{c}0605100 \\
75.40 \\
13.50 \\
0.42 \\
0.10 \\
0.42 \\
3.80 \\
4.80 \\
0.71 \\
0.17 \\
0.04 \\
0.0 \\
0.08 \\
0.0 \\
99.99 \\
51.99 .\end{array}$ & $\begin{array}{l}05 \\
F \\
B \\
B \\
B\end{array}$ & $\begin{array}{c}06051006 \\
75.50 \\
12.60 \\
0.37 \\
0.05 \\
0.62 \\
4.00 \\
5.10 \\
0.71 \\
0.16 \\
0.04 \\
0.0 \\
0.06 \\
0.0 \\
100.03 \\
53,37,\end{array}$ & $\begin{array}{l}B \\
0 \\
0\end{array}$ & $\begin{array}{c}0605100 \\
75.50 \\
12.80 \\
0.34 \\
0.05 \\
0.62 \\
3.70 \\
5.10 \\
0.70 \\
0.15 \\
0.05 \\
0.0 \\
0.07 \\
0.0 \\
99.80 \\
52.37 .\end{array}$ & $\begin{array}{l}n 7 \\
\text { F } \\
\text { B } \\
8 \\
0\end{array}$ & $\begin{array}{c}06051008 \\
75.60 \\
13.10 \\
0.80 \\
0.18 \\
0.56 \\
3.80 \\
4.80 \\
0.35 \\
0.0 \\
0.11 \\
0.01 \\
0.16 \\
0.0 \\
99.64 \\
52 . \\
38 .\end{array}$ & $\begin{array}{l}8 \\
0\end{array}$ & $\begin{array}{c}06051009 \\
75.70 \\
13.10 \\
0.62 \\
0.05 \\
0.64 \\
4.00 \\
4.50 \\
0.71 \\
0.09 \\
0.05 \\
0.0 \\
0.05 \\
0.0 \\
100.06 \\
52.38 .\end{array}$ & $\begin{array}{l}P \\
B \\
0 \\
0\end{array}$ & $\begin{array}{c}06051010 \\
76.70 \\
13.00 \\
0.50 \\
0.13 \\
0.52 \\
3.70 \\
4.50 \\
0.34 \\
0.0 \\
0.06 \\
0.0 \\
0.08 \\
0.0 \\
99.82 \\
53,37 .\end{array}$ & $\begin{array}{l}\text { B } \\
\text { * } \\
0\end{array}$ & \\
\hline & & $\begin{array}{l}\text { INSOL } \\
\text { BLANK }=\text { NO } \\
\text { AL2O3 * FE } \\
\text { MGO + CAO } \\
\text { NAZO * K2O }\end{array}$ & & REPT . & & & $\begin{array}{l}H \\
I\end{array}$ & $\begin{array}{l}\text { HZO+ } \\
\text { IGNITION } \\
\text { REPORTEO } \\
\text { CALCULATED }\end{array}$ & $\begin{array}{l}\text { AS } S \\
D_{B Y}\end{array}$ & $\begin{array}{l}\text { (MAY INCL } \\
\text { WATER). } \\
\text { COMPILERS }\end{array}$ & & $\begin{array}{ll}J & \text { ORGA } \\
K & \text { TOTA } \\
M & \text { BY } \\
\text { N } & \text { INCL } \\
O & \text { GREA }\end{array}$ & & $\begin{array}{l}\text { MATTER. } \\
\text { RON. } \\
\text { ERENCE. } \\
\text { S TIOZ. } \\
\text { THAN. }\end{array}$ & & & & $\begin{array}{l}\text { FSS THAN. } \\
\text { TRACE. } \\
\text { ADDITIONAL } \\
\text { OESCRIPTIVE }\end{array}$ & $\begin{array}{l}\text { INFORMATION } \\
\text { IE NOTES. }\end{array}$ & $N$ IN \\
\hline
\end{tabular}


TABLE 3. - Analyses of samples from California and Hawaii containing uncombined silica and clay each less than 75 percent; uncombined silica and clay each greater than carbonate (Group B), common- and mixed-rock categories-Continued

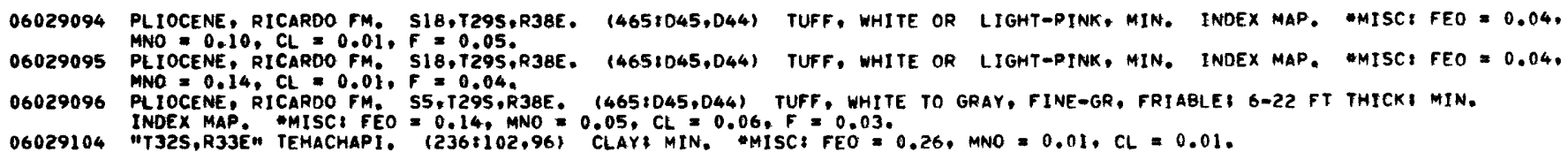


TABLE 3. - Analyses of samples from California and Hawaii containing uncombined silica and clay each less than 75 percent; uncombined silica and clay each greater than carbonate (Group B), common- and mixed-rock categories-Continued

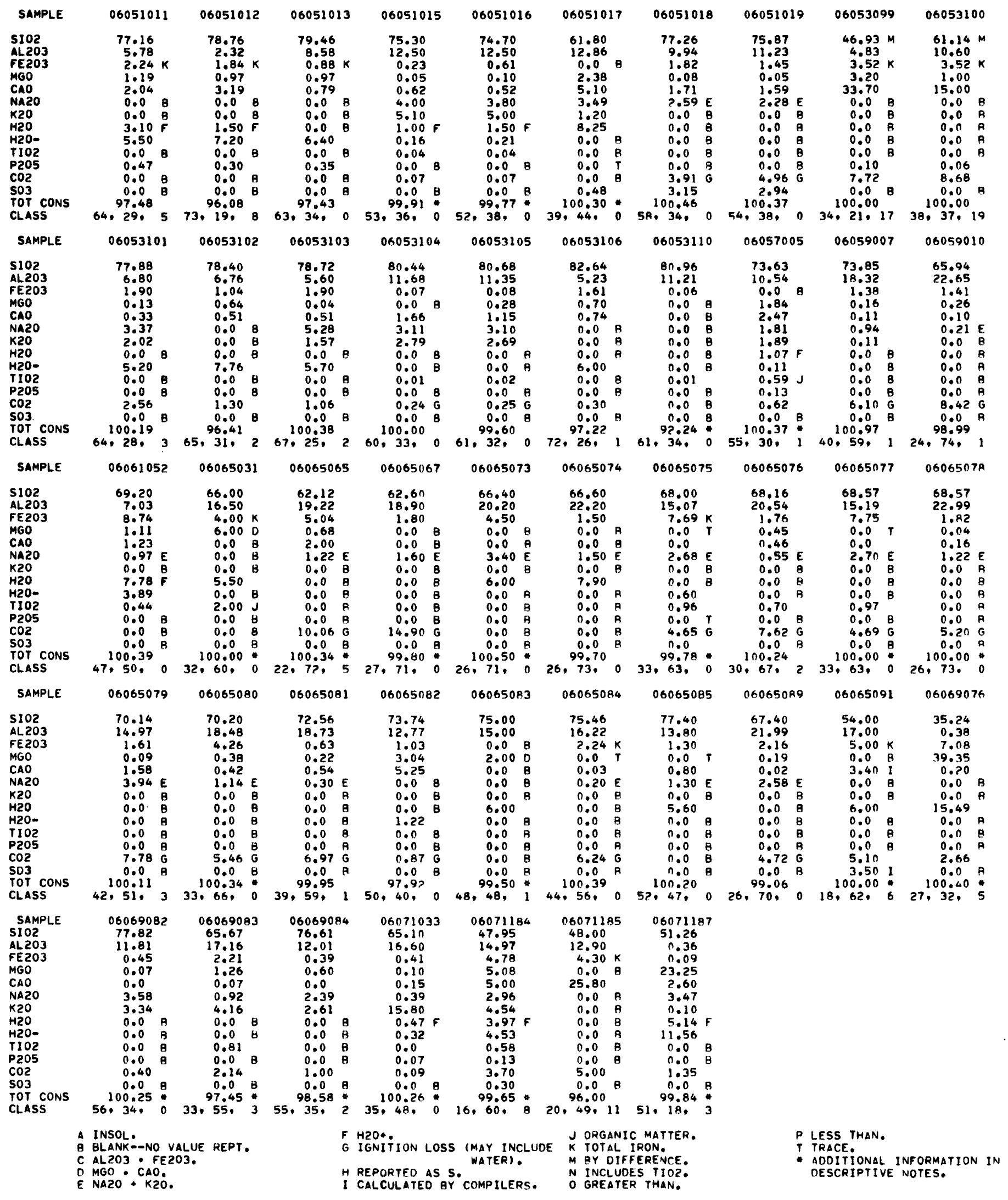


TABLE 3. - Analyses of samples from California and Hawaii containing uncombined silica and clay each less than 75 percent; uncombined silica and clay each greater than carbonate (Group B), common- and mixed-rock categories-Continued

06051011 PLEISTOCENE. S2,T4S,RZ9E. (116:SHEET 1$)$ DIATOMACEOUS EARTH, GRAY\$ 4 FT THICK PHYS PROPI TONNAGE. INOEX \& GEOL MAPS. 06051012 GEOL SEC. USF: 9,11 .

06051013 PLEISTOCENE. S21,T3S,R29E. (116:SHEET 1$)$ DIATOMACEOUS EARTH, WHITE, 8 FT THICK, TONNAGE PHYS PROP. INOEX \& GEOL 06051015 HOLOCENE. "TIS.R27E" SOUTHERN COULEE. (3711426,417,PL1) PUMICE, GRAY. INDEX \& GEDL MAPS, GEOL SEC. "MISC: FEO =

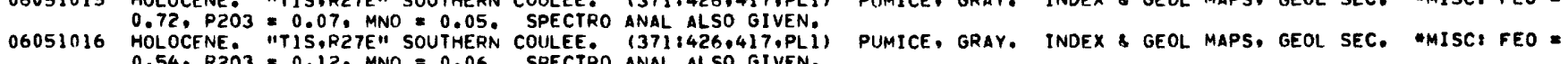
06051017 "TIN,R28E" LAKE MONO. $(4438307$,PLI6) CLAY, DIATOMACEOUS, OUTCROP SMPL. INDEX MAPS. OMISCI FEO = 3.57. NACL = 1.17.

06051018 S27,2R,T3S,R32E. (327:308) PUMICE.

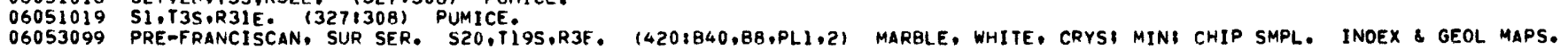

06053100 PRE-FRANCISCAN, SUR SER, S20,T195,R3E. (420:840,PL1,2) MARBLE, WHITE, FINE TO COARSELY CRYSI CHIP SMPL. INOEX \&

06053101 MIOCENE, MONTEREY FM. "T15S,RIE" MONTEREY, (1691108,98,102) DIATOMITEI 300 FT THICK. USE: 4,11.

06053102 MIOCENE, MONTEREY FM. "TI5S,RIE" MONTEREY, (1691108.98,102) DIATOMITE: 300 FT THICK. USE: 4,11.

06053103 MIOCENE, MONTEREY FM. "TISS,RIE" MONTEREY. (1698108,98,102) DIATOMITE, 300 FT THICK. USE: 4,11.

06053104 S22,T15S,RIW. (265:99,98,PLI) SAND, WHITE, MED-GR: MIN, INDEX \& GEOL MAPS. USE 10 .

06053105 S33,T15S,RIW. (265195,94,PL1) SAND: MIN. INDEX \& GEOL MAPS, USE15,11.

06053106 MIOCENE, MONTEREY FM. "T24S,RIIE" BRAOLEY, (169:108,99,101) DIATOMITE, WHITE, COMPACT. USE: 7.

06053110 "T15S,RIE" DEL MONTE. (564:39) BEACH SAND: TYPICAL SMPL, USE, 14,15. $4 M I S C:$ CR203 = <0.001.

06057005 PALEOZOIC, CALAVERAS FM. "T16N,RRE" NEVAOA CITY. $(361881,80)$ ARGILLITEI MIN, UMISC: FEO = 1.87, SRO = TR. BAO = 0.12,

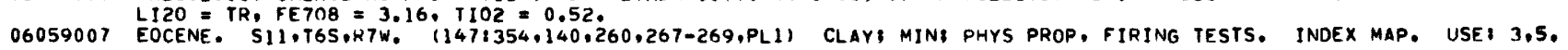

06059010 PALEOCENE, MARTINEZ FM. TSS,R7W, SERRANO MINE, $1478: 85,81,83, P L 1 / 147: 356, P I 1)$ CLAY, WHITE OR PINK, PLASTIC MINE

06061052 "TIIN,R6E" LINCOLN. $13: 414,351.363 .413)$ CLAY. SANDYI MIN. INDEX MAP, MEAS SEC. USE: 7.

06065031 TERTIARY, "T55,R5W" ALBEHHILL. (43:84,75-82) CLAY, WHITE, SANOY, PLASTICI PHYS PROP, FIRING TESTS. "MISCI ORG CALC

06065065 PALEOCENE, SILVERADO FM, S21,22,T5S,R5W, $(147: 355,162-173,323-332, P L 1 / 185: 88,77-86, P L 1-6)$ CLAY,SANOY, 20 FT THICK, PALEOCENE, SILVERADO FM, S21,22,T5S,R5W, $(147: 355,162-173,323-332, P L 1 / 185: 88,77-86, P L 1-6)$
PHYS PROP, FIRING TESTS. INDEX MAPS, MEAS SEC, GEOL SEC. USE: 3. MISCI NAPO - K20 BY DIF.

06065067 PALEOCENE, SILVERADO FM. 522,T5S,R5W. (185:88,77-81,86,PL1-6) CLAY, WHITE TO GRAYI 30 FT THICK PHYS PROP, FIRING TESTS. INDEX \& GEOL MAPS, GEOL SEC. USE: 3,5 . MISC: ALKALIES INCL CA \& MG.

06065073 PALEOCENE. S23,T5S,R5W. (185:89,77-79,86,PL1-6) CLAY, RED, PLASTIC: 30 FT THICK PHYS PROP. FIRING TESTS. INDEX \& GEOL MAPS, GEOL SEC, IISE: 3. MISC: NAZO $\mathrm{K} 20$, INCL MGO \& CAO.

06065074 PALEOCENE, SILVERADO FM, S22,T5S,R5W. (195:89,77-81,86,PLI-6) CLAY, WHITE TO GRAYI 30 FT THICK, PHYS PROP, FIRING TESTS. INDEX \& GEOL MAPS, GEDL SEC. USE: 3,5 .

06065075 PALEOCENE. S22,T5S,R5W $(285: 208 \% 185: 88,77-79,86, P L 1-6)$ CLAY, PINK, MOTL. PLASTICI 150 FT THICK PHYS PROP.

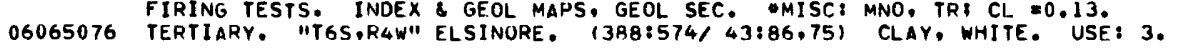

06065077 EOCENE. T5S,R5W, ALBERHILL. $(147: 355,163,328-334$, PLI) CLAY, PINK-YELLOW MOTL, FINE-GR, PLASTICI PHYS PROP, FIRING TESTS. INDEX MAPS. MEAS SEC, GEOL SEC. USE: 3. MISC: NAZO K KO BY DIFI MNO = TRICL = O.13.

06065076 EOCENE. S21,22,T5S,R5W. (147:355,162-173,317-319,PL1) CLAY, SANDYI 8 FT THICK: PHYS PROP, FIRING TESTS. INOEX MAPS, 06065079 PALEOCENE, MARTINEZ FM. T7S,R3W. WILDOMAR. K20 BY DIF. $147: 356,180$, PLI/ 478:74,52,75,PL1) CLAYI MINI 30-42 IN THICK. INDEX MAP. PALEOCENE, MARTINEZ FM. T7S,R3W, WILDOMAR, $1147: 356,180, P L 1 / 478: 74,52,75, P L 1)$ CLAYI MINI 30-42 IN THICK,
USE: 5.
EOCENE. S22,T5S,R5W, $(147: 355,162,176,177,315-320, P L 1)$ CLAY, MEO-GR, PLASTIC: 50-70 FT THICK PHYS PROP,

06065080 EOCENE: S22, T5S,R5W. (147:355,162,176,177,315-320,PL1) CLAY, MED-GR,

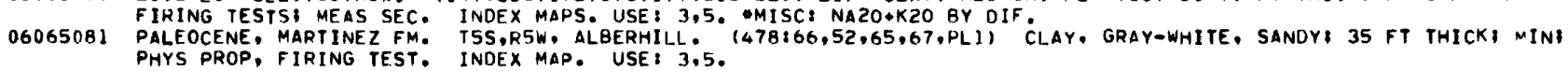

06065082 OLIGOCENE(P) \& MIOCENF, VAQUEROS SS \& EOCENE \& OLIGOCENE, SESPE FM. S29,T4S,R6W. (446:444/ 228:103,PL1.2) SAND. INDEX \& GEOL MAP, GEOL SEC.

06065083 TERTIARY. "T5S.R5W" ALBERHILL. (43884,75,76) SAND, WHITE, COARSE CONTAINS CLAY: PHYS PRDP, FIRING TESTS. USE: 7.

06065084

06065085

06065089

AMISC: FES2 = 1.5. "T5S,R5W" ALBERHILL. (285:201,185,186,196,199/ 147:354,162,163,168,264,PLS1,10) CLAY, GRAY: BEO 20 FT THICK, PHYS PROP, FIRING TESTS. INDEX MAP, MEAS SEC, GEOL SEC. USE: 3,5 .
PAY

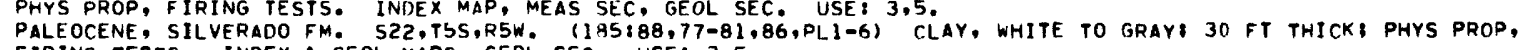
FIRING TESTS. INDEX \& GEOL MAPS. GEDL SEC. USE: 3,5 .

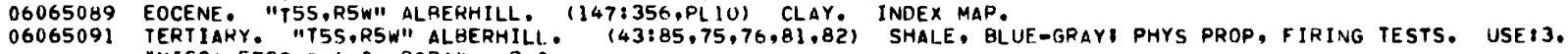

06069076 S35.TI7S,RIIE. (21):508.504) WEATHERED SERPENTINE. GREEN, INUEX MAP. HMISC: HZO REPT AS I.L. LESS CO2.

06069082 JURASSIC \& CKETACEOUS, FRANCISCAN FM. S32,T17S,RI2E, $(170: 100,82,88-90,99,112, P L 8,9)$ SANNSTONE, CHIP SMPL: MIN. INDEX \& GEOL MAP. GEOL SEC. UMISC: FEO = 0.36. ALTERED: FOR UNALTERED SMPL, SEE SIO2 76.61.

06069083 JURASSIC \& CRETACEOUS, FRANCISCAN FM. S32,T17S,R12E. (170:100,88,90,PL8,9) SHALE, GRAY: BEOS 1-6 IN THICK CHIP SMPL, MIN. INOEX \& GEOL MAPS, GEOL SEC. MISC: TOTAL FE $=5.26$.

06069084 JURASSIC \& CRETACEOUS, FRANCISCAN FM. S32,T17S,RI2E. $(170: 100,82,88-90,99,112, P L 8,9)$ SANOSTONE: CHIP SMPL: MIN.

06071033 MIOCENE, BARSTOW GM. S20,TIIN,RIW. $(460: 5,1-4)$ TUFF, WHITE TO GRAY MIN: $1.5-7$ FT THICK. INOEX \& GEDL MAPS. USE: 5,10 . MMISC: FEO $=0.04$ I MNO $=0.04: B 203=0.68$, DETD BY SPECTRO ANAL.

06071184 MIOCENE, BARSTOW FM. S24,T1IN,R2W. $(46188,4,7)$ MUDSTONE: TYPICAL SMPL; MIN. INDEX \& GEOL MAPS. \#MISC: FEO = 0.90,

06071185 MNO $=0.11$, BAO $=0.05, C L=0.03, F=0.14$.

06071187 TERTIARY. S27,TBN,RSE. $(573: 241,238-240 / 563: 110)$ BENTONITIC CLAYI PHYS PROP. INDEX MAP, GEOL SEC. AMISC: TERTIARY, S27, TBN,R5E, $\quad(573: 241,238-240 /$
FEO $=0.06, F=0.00, L I 20=0.60, S R=T R$. 
TABLE 3. - Analyses of samples from California and Hawail containing uncombined silica and clay each less than 75 percent; uncombined silica and clay each greater than carbonate (Group B), common-and mixed-rock categories-Continued

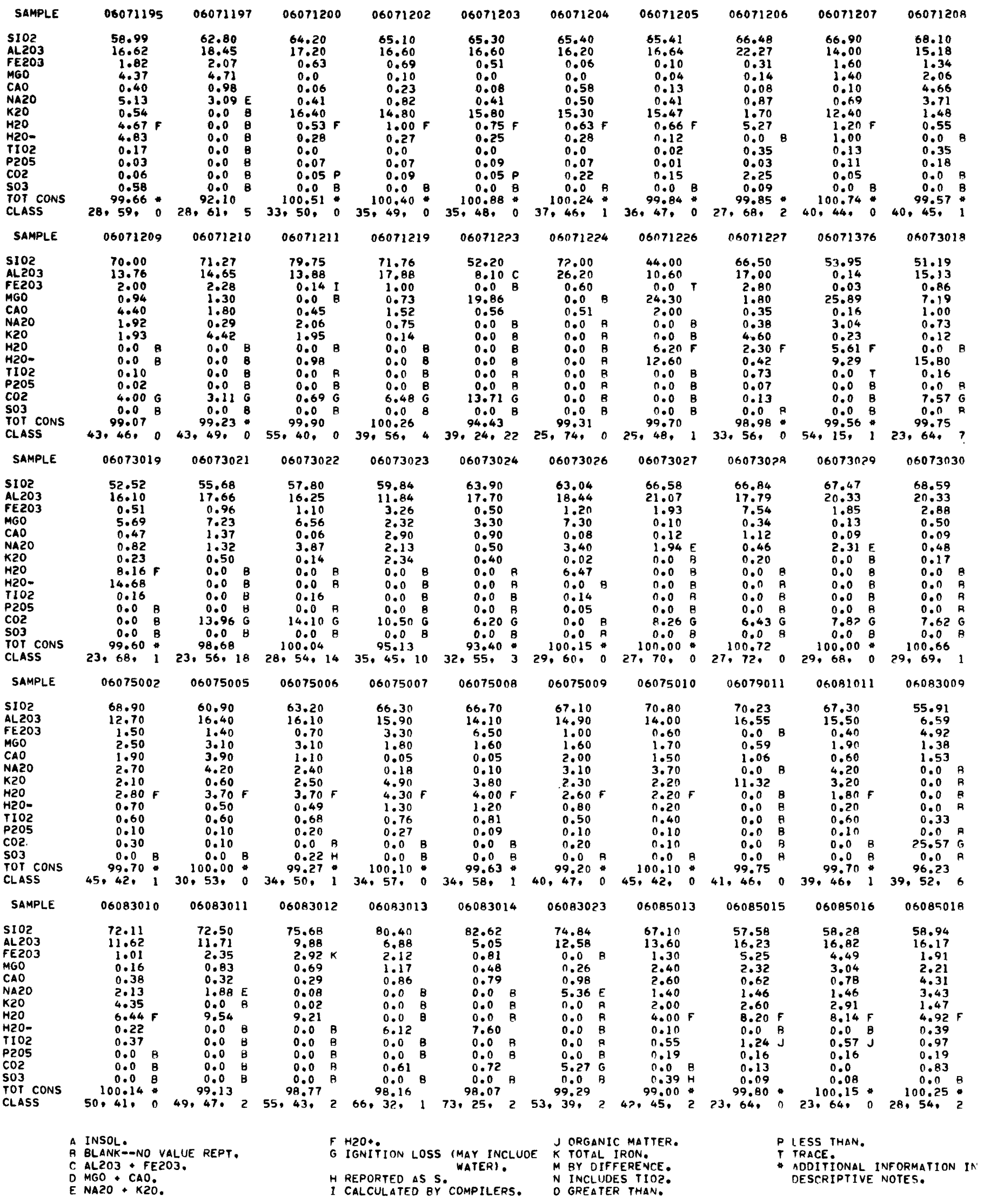


TABLE 3. - Analyses of samples from California and Hawaii containing uncombined silica and clay each less than 75 percent; uncombined.silica and clay each greater than carbonate (Group B), common-and mixed-rock categories-Continued

06071195 PLIOCENE, RED MOUNTAIN ANDESITE. TB,9N,R5E, HECTOR, $(6: 31.23-30)$ BENTONITE. INDEX MAP, GEOL SEC. MNO $=0.011$ LI2O $=0.24: B A O=0.021 \mathrm{RB20}=0.001 \mathrm{~F}=1.141 \mathrm{CL}=0.671 \mathrm{LESS} 0=\mathrm{F}, \mathrm{CL}$. 0.63 .

06071197 "TIIN,R9E" BALCH. (502:305) RENTONITE, BEOS 10 FT THICK.

06071200 MIOCENE, BARSTOW FM. S24,TIIN,R2W. (460:5,1-4) TUFF, WHITE TO GRAY: $1.5-7$ FT THICK: MIN. INDEX \& GEOL MAPS. USE: 5. MMISC: FEO $=0.08$, MNO $=0.0218203=0.58$ BY SPECTRO ANAL.

06071202 MIOCENE, BARSTOW FM, S24,TIIN,R2W. $(460: 5,1-4)$ TUFF, WHITE TO GRAY; MIN, $1.5-7$ FT THICK. INDEX \& GEOL MAPS. USE: 5.10. MISC: FEO $=0.041$ MNO $=0.041$ B203 $=0.55$. DETO AS B BY SPECTRO ANAL.

06071203 MIOCENE, BARSTOW FM. S24,TIIN,R2W. $(460: 5,1-4)$ TUFF, WHITE TO GRAY 1.5-7 FT THICK: MIN. INDEX \& GEOL MAPS. USE: 5. 06071204 MIOCENE, BARSTOW FM. S19,T11N,RIW. $(460.5,1-4)$ TUFF, WHITE TO GRAYI $1.5-7$ FT THICK MIN. INDEX \& GEOL MAPS. USE: 5. 06071205 MIOCENE, BARSTOW FM. S24. TIIN, B2W. $=0.93$ BY SPECTRO ANAL.

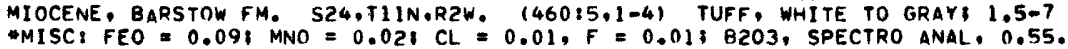

06071206 MESOZOIC \& PERMIAN, HODGE VOLCANIC SER, S36.T9N,R4W. (52:143,142,PL1-3) CLAY, WHITE\& COMPOS SMPL. INOEX \& GEOL MAP, 06071207 MIOCENE, BARSTOW FM. S24,TIIN,R2W. $(460: 5,1=4)$ TUFF, WHITE TO GRAY $1.5-7$ FT THICK 8 MIN, INDEX \& GEOL MAPS. USE: 5 . 06071208 "TINQR4W" ONO. (99:123) SANDSTONE DIKE. MMISC: FEO $=1.70$, MNO $=0.20$, RAO $=0.06$.

06071209 S19.TIIN.R2W \& S24,TIIN,R3W. 1503:391) PUMICITEI 50-100 FT THICK. USEI 4.

06071210 S34.35.T7N.R4W. (507:512.511) CLAY, WHITE TO GRAY AVG THICKNESS 25 FTI TONNAGE. USE: 9. QMISC SOL CL. O.11. 06071211 TRIASSIC OR OLDER, S16,T7N,R2E. (532:11,44,PL1) QUARTZITE, GRAY, FELDSPATHIC, FINE- TO MED-GR MIN. INDEX \& GEOL 06071219 "TI4N,R1BE" HART, (147:354,196,197,265,PL1) CLAYI MIN, 60-70 FT THICKI PHYS PROP, FIRING TESTS. INOEX MAP. USE: 9. 06071223 TERTIARY. S27,TBN,RSE. 1573:243,238,239,2421 TUFF, BROWNA MIN. PHYS PROP. INDEX MAP. SPECTRO ANAL ALSO GIVEN.

06071224 06071226 06071227

06071376 06073018

06073019

06073021

06073022 06073023 06073024

06073026

06073027

06073028

06073029

06073030

06075002

06075005

06075006

06075007

06075008

06075009

06075010

06079011

06081011

06083009

06083010

06083011

06083012

06083013

06083014

06083023

06085013

06085015

06085016

06085018 JURASSIC \& CRETACEOUS, FRANCISCAN FM, "T9S,R5,
MAP, GEOL SEC. MISC: FEO $=4.37$, MNO $=0.14$.

S6.T4N,RIIE. $(255: 242,241)$ VOLCANIC ASH. USE: 15.

PRECAMBRIAN, STIRLING QTZITE. S6.TI7N,R7E. (585:25.8.141) PELITE: MIN, INDEX MAP, MEAS SEC. SPECTRO ANAL ALSO

PRECAMBRIAN, STIRLING OTZITE. S6.TIT

"TIVEN, "MISC: FEO = 1.9, MNO = 0000 .

PLIOCENE, SAN DIEGO FM, S15,T18S,R2W. (467:11/115:10,5.7,8,11,15,PL1) BENTONITE, REDS 4 FT THICK, TONNAGE, INDEX \& GEOL MAPS, MEAS SEC. USE

PLIOCENE, SAN TIEGOFM. S25,TI8S,R2W. (115:10,5-15,PL1) BENTONITE: MIN: BENS 4 FT THICKI TONNAGE. INTEX G GEOL MAPS, MEAS SEC. USE: 6.8 . MISC: FEO $=0.261$ MNO $=0.0031 \mathrm{C}=T R$.

PLIOCENE, SAN DIEGO FM. SI7,18,T18S,RIW. (453:183/115:10,8,PL1/ 118:61,60,PLI) BENTONITEI FEW IN TO 4 FT THICK,

TONNAGE. INDEX \& GEOL MAP, MEAS SEC. USE: 6.9.

"TI8S,R2W" OTAY. (457:20/6121304) MONTMORILLONITE.

"TIBS,R2W" OTAY, (140:10,5) MONTMORILLONITE. MISC: H2O = 24,12, H2O- = 20,253 NOT INCLUDED IN TOTAL.

PLIOCENE, SAN DIEGO FM. S17-20,T18S,RIW. (410:96/115110,7,9,15,PLI) BENTONITE: BEDS 4 FT THICK: TONNAGE.

INDEX \& GEOL MAPS, MEAS SEC. IISE: 6.11. $M I S C$ : MNO $=0.01$.

S3,T13S,R3W. (559:690,689) PINITE, GRAY, BEDDED, USE: 9,13. MISC: NAZO K20, BY DIF.

EOCENE. "TI35,R5W" CARDIFF, (147:355,202,330-332,PL1) CLAY, MEN-GRI PHYS PROP, FIRING TESTS. INDEX MAP. USE: 3.

S3,TI3S,R3W. (559:690,689) PINITE, WHITE, BEDOED, USE: 9,13. \$MISC: NAPO * K2O, BY DIF.

PLEISTOCENE. "T135,R5W" CARDIFF, (147:354,287-295,PL1) CLAY, SANDY, 10-12 FT THICK PHYS PROP, FIRING TESTS. INDEX MUP, USE: 3, MMISC: FEO = 2.8 . MNO = 0.1. MISC: FEO = 4.4, MNO = 0.1.

JURASSIC \& CRETACEOUS, FRANCISCAN FM. "T2S.R5W" SAN FRANCISCO. (29:3R.11.PLI) SILTSTONE: MIN. INDEX \& GEOL MAPS. MISC: FEO $=4.9$, MNO $=0.093$ LESS $0=5$, 1.11 .

JURASSIC CRETACEOUS, FRANCISCAN FM, "T2S, RSW" SAN FRANCISCO, $(29: 64,11$,PLI) SHALE, GREENI I-FT BEDI MIN. INDEX \& GEOL MAPS. $M I S C:$ FEO $=0.96$, MNO $=0.08$. JURASSIC CRETACEOUS, FRANCISCAN FM. "T2S.R5W" SAN FRANCISCO. MISC: FEO $=0.58$, MNO $=0.10$. JURASSIC C CRETACEOUS, FRANCISCAN FM. "T2S,R5W" SAN FRANCISCO. "MISC: FEO $=2.9$, MNO = 0.1. MISC: FEO $=2.5$, MNO $=0.1$ (29:64,11,PL1) SHALE, REDI MIN. INDEX 8 GEOL MAPS. (29:33,11,32,PL1) GRAYWACKE: MIN. INDEX \& GEOL MAPS. S23.T26S,RIOE. (17:583) UIATOMACEOUS EARTH: 6 FT THICK. GEOL MAP. USF: 1.

JURASSIC \& CRETACEOUS, FRANCISCAN FM. "T3S,RSW" SAN BRUNO MTN, $(29: 33,11,32, P L 1)$ ARKOSIC GRAYWACKE MIN. INDEX \& GEOL MAPS. MISC? FEO $=3.8$, MNO $=0.1$.

MIOCENE, MONTEREY SH. "TION,R34W" SANTA MARIA. (480:1381) OIL-SHALE.

MIOCENE, MONTEREY FM. "TBN,R34W" LOMPOC. $(61: 24,14, P L 1,2)$ VOLCANIC ASH. INDEX MAP. "MISC: FEO = 1.19, MNO = 0.01,

BAO = 0.15.

"TBN,R34W" (534:193) DIATOMACEOUS EARTH.
MIOCENE. MONTEREY FM. "TBN,R34W" LOMPOC. (169:108.98) DIATOMITE.

MIOCENE, MONTEREY FM. "T8N,R34W" LOMPOC: (169:108,98) DIATOMITE: USE: 7.

MIOCENE. "TBN,R35W" POINT SAL RIDGE. (187:18,16,17,PL1) VOLCANIC ASH MIN: B.0., 2.402 . INDEX \& GEOL MAP, GEOL SEC. JURASSIC \& CRETACEOUS. FRANCISCAN FM. "TIOS,RGE" PACHECO PASS. (29:3R,11.PLI) SHALF, DARK-GRAY OR BLACK MIN.

MMISC: FEO $=3.5$, MNO = 0.06. 6 GEOL MAPS. USE: 11. MISC: FEO $=3.16:$ TIOZ $=0.76$. SPECTRO ANAL ALSO GIVEN.

CRETACEOU5, BERRYESSA FM. SI2.T10S,R4E, 166323.18 .24 .31 .PLI) SHALE, GREEN, BEDDEDI 200 FT THICK M MINI B.D., 2.55: PHYS PROP. INDEX \& GEOL MAPS. USE: 11: MMISC: FEO=2.66; TIOZ=0.76. SPECTRO ANAL ALSO GIVEN. 
TABLE 3. - Analyses of samples from California and Hawaii containing uncombined silica and clay each less than 75 percent; uncombined silica and clay each greater than carbonate (Group B), common-and mixed-rock categories-Continued

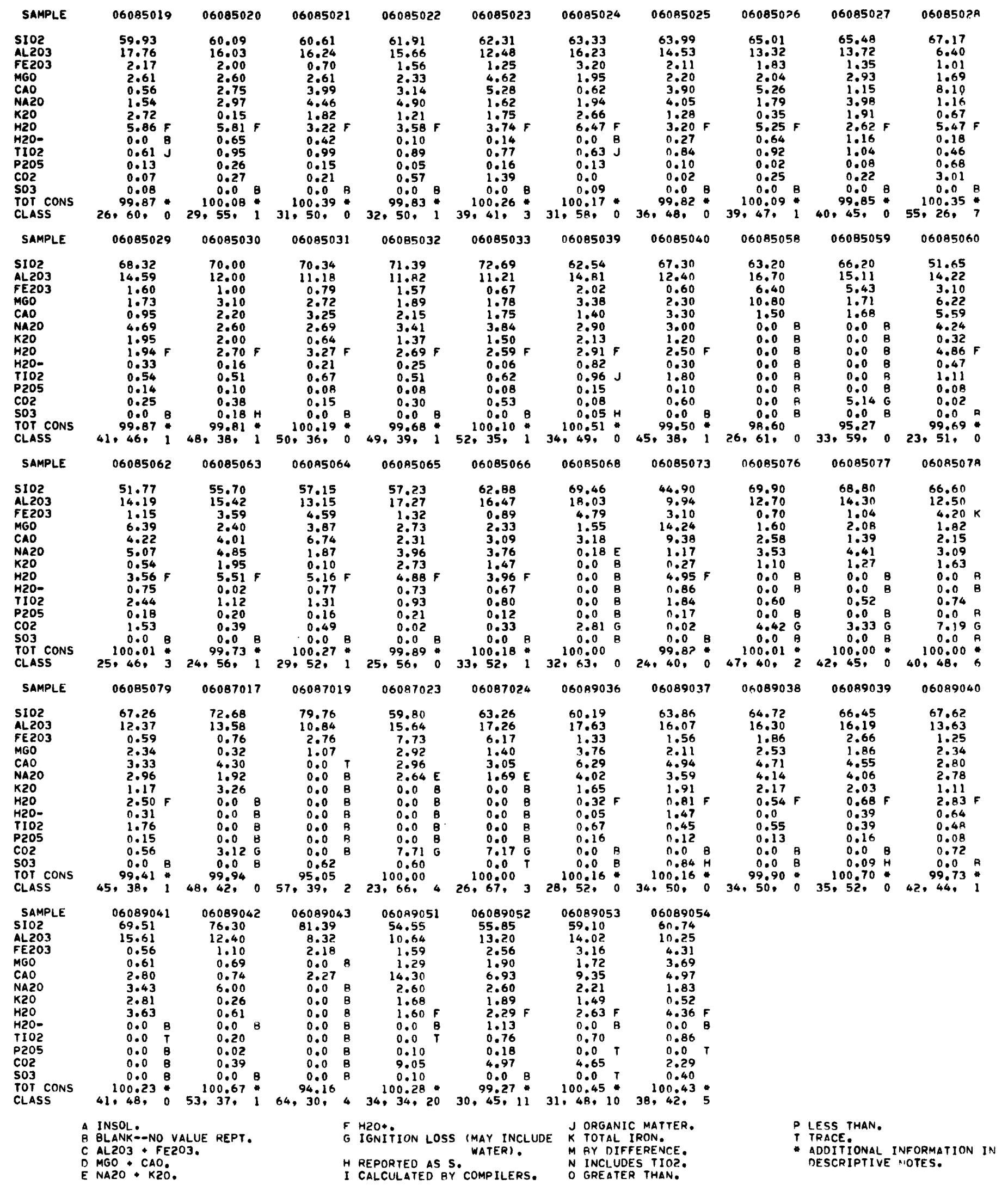


TABLE 3. - Analyses of samples from California and Hawaii containing uncombined silica and clay each less than 75 percent; uncombined silica and clay each greater than carbonate (Group B), common- and mixed-rock categories-Continued

06085019 CRETACEOUS, BERRYESSA FM. S18,T9S,R4E, (66:23,18,22-24,PL1) SHALE, RLACK, REODED: 120 FT THICK: MIN! B.D., 2.63, PHYS PROP. INDEX \& GEOL MAP. USE:I9. "MISC: FEO=5.09: TIO2 = 0.74. SPECTRO ANAL ALSO GIVEN.

06085020 JURASSIC \& CRETACEOUS, FRANCISCAN FM. "T9S,R5,6E" PACHECO PASS. (186:51,45-50,PL3) METASHALY ROCK, MIN. INOEX \& GEOL 060B5021 JURASSIC \& CRETACEOUS, FRANCISCAN FM. "T9S,R5,6E" PACHECO PASS. (186:51.45-50,PL3) METAGRAYWACKE MIN. INDEX \& GEOL 06085022 JURASSIC S CRETACEOUS. FRANCISCAN FM. "T9S,R5,6E" PACHECO PASS. (186852,45-50,PL3) METAGRAYWACKE) MIN. INDEX \& GEOL 06085023 JURASSIC \& CRETACEOUS, FRANCISCAN FM. "T9S,R5,6E" PACHECO PASS. (186:52,45-50,PL3) METAGRAYWACKE: MIN. INDEX GEOL 06085023 MAP, GEOL SEC. MISC: FEO $=4.36$, MNO $=0.39$.

06085024 CRETACEOUS, BERRYESSA FM, S22,78S,R3E, (66,23,15,18,24,31,PL1) SHALE, GREEN, MASSIVE: MIN, B.D., 2.631 PHYS PROP. INDEX \& GEOL MAPS. \#MISC: FEO $=2.161^{1}$ TIO2 $=0.76$. SPECTRO ANAL ALSO GIVEN.

06085025 JURASSIC C CRETACEOUS, FRANCISCAN FM. "T9S.R5,6E" PACHECO PASS. MAP, GEOL SEC. MMISC: FEO $=3.26$, MNO 00.07.
06085026 JURASSIC \& CRETACEOUS, FRANCISCAN FM. "TOS,R5, GE" PACHECO PASS.

06085027 JURASSIC SCRETACEOUS, FRANCISCAN FM, "ToS, MAP OSIC \& CRETACEOUS, FRANCISCAN FM, 06085028 JURASSIC SCRETACEOUS, FRANCISCAN FM. "T9S,R5.6E" PACHECO PASS.

06085029 JURASSIC SRETACEOUS, FRANCISCAN FM, "TOS,R5,6E" PACHECO PASS. JURASSIC "CRETACEOUS, FRANCISCAN FM, "T9S,R5,6E" PAC,

06085030 JURASSIC S CRETACEOUS, FRANCISCAN FM. "T9S.R5.6E" PACHECO PASS.

06085031 GEOL MAPS: MISC: FEO $=2.8$, MNO $=0.08$.

06085032 JURASSIC SEC. MMISC, FEO $=4.14$, MNO $=0.06$. MAP, GEOL SEC, \$MISC, FEO $=2.17$, MNO $=0,0 R_{0}$ 06085033 JURASSIC \& CRETACEOUS. FRANCISCAN FM. "T9S,R5,6E" PACHECO PASS. (186:51,45-50,PL3) METAGRAYWACKE: MIN. INDEX \& GEOL MAP, GEOL SEC, \#MISC: FEO $=2,73$. MNO $=0,05$.

06085039 JURASSIC SRETACEOUS. FRANCISCAN FM. "T9S.RIE" FERN HILL. (29:38,11,PL1) SILTSTONE, BLACK: MIN. INDEX \& GEOL MAPS. JMISC: FEO $=5.47$, MNO $=0.05$, TIO2 $=0.871$ LESS $0=5,0.03$.

06085040 JURASSIC CRFTACEOUS. FRANCISCAN FM. "T9S,RIE" NEW ALMADEN DIST. (29133,11,32,PLI) GRAYWACKE: MIN. INOEX G GEOL

06085058 JURASSIC \& CRETACEOUS, FRANCISCAN FM. S17,18,T7S,R2W. $(331: 391,374)$ CLAY.

06085059 JURASSIC \& CRETACEOUS, FRANCISCAN FM. S17.18,T7S,R2W. (331:377.374) CLAY AVG COMPOS OF FOUR NEPOSITS. USE: 4.

06085060 JURASSIC \& CRETACEOUS, FRANCISCAN FM. "T9S,R5,6E" PACHECO PASS, (186:52,45-50,PL3) TUFF \& TUFFACEOUS GRAYWACKE) MIN. INDEX \& GEOL MAP, GEOL SEC. UMISC: FEO $=7.67$, MNO = 0.14 .
06085062 JURASSIC CRFTACEOUS. FRANCISCAN FM. "T9S,R5,6E" PACHECO PASS INDEX \& GEOL MAP, GEOL SEC. MMISC: FEO $=8.06$. MNO $=0.16$.

06085063 JURASSIC CRETACEOUS, FRANCISCAN FM. "T9S,R5,6E" PACHECO PASS. MAP, GEOL SEC. MMISC: FEO $=4.50$, MNO $=0.07$.

06085064 JURASSIC CRETACEOUS, FRANCISCAN FM. "T9S,R5, $6 E "$ PACHECO PASS

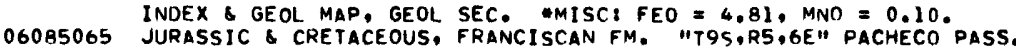
MAP, GEOL SEC. MMISCI FEO = 5.38. MNO $=0.19$. 06085066 JURASSIC OCRETACEOUS, FRANCISCAN FM, "T9S,R5, $6 E "$ PACHECN PASS.

06085068 "TBS, R2W" SARATOGA. (67:527) 3.34 SAND. MNO E 0.07 .

06085073 JURASSIC S CRETACEOUS, FRANCISCAN FM. S24,T9S,RIE. $(40: 37,4$, PLI) TUFF MIN. INDEX 8 GEOL MAP, GEOL SEC.

06085076 JURASSIC \& CRETACEOUS, FRANCISCAN FM. HALLS VALLEY. (31R:359.354,355) METAGRAYWACKE; MIN. INDEX \& GEOL MAP. JURASSIC CRETACEOUS, FRANCISCAN FM. HALLS VALLEY, (3IR:359.354.355) METAGRAYWACKE
MISC: FEO $=2.79$, MNO $=0.05$, SRO $=0.035$ I IGNITION LOSS REPT AS H20 * CO2, RY DIF.

06085077

JURASSIC \& CRETACEOUS, FRANCISCAN FM. HALLS VALLEY. (318:359,354,355) METAGRAYWACKE: MIN. INDEX \& GEOL MAP. AMISC: FEO $=? .80$. MNO $=0.05$, SRO $=0.0111$ IGNITION LOSS REPT AS HZO + COP. BY OIF.

06085078 JURASSIC \& CRETACEOUS, FRANCISCAN FM. HALLS VALLEY, (318:359,354,355) METAGRAYWACKE 1 MIN. INDEX \& GEOL MAP. -MISC: MNO $=0.06$, SRO $=0.021$ IGNITION LOSS REPT AS H2O * CO2, BY DIF.

06085079 JURASSIC \& CRETACEOUS, FRAINCISCAN FM. S2,T9S,RIE. (40:17,4,PL1) GRAYWACKE. INDEX \& GEOL MAP, GEOL SEC. MISC: FEO $=4.03$, MNO $=0.08$.

06087017 TIOS,RIW, SOQUEL. (342:89.88) SANDSTONE.

06087019 "T10S,R3W" DAVENPORT, $(173: 123,122)$ SHALE, OIATOMACEOUS, USE: 4.

06087023 "TIZS,R2E" CHITTENDEN. (305:78,PLSI7,1B) CLAY, BLUE, UNIFORM TEX: 40 FT THICK. INDEX \& GEOL MAPS, GEOL SEC, USE: 4. 06087024 "TI2S,R2E" CHITTENDEN. (305:78,PL17,18) CLAY, BLUE, UNIFORM TEXTURE: 40 FT THICK. INDEX \& GEOL MAP, GEOL SEC. USE:4. 06089036 HOLOCENE. "T3IN,R5E" LASSEN PEAK. $1379: 477$, PL1) PUMICE, DARK. "MISC: FEO = 4.001 MNO = 0.09.

06089037 HOLOCENE. S34,T31N,R4E. (5298258/ 543:335,311,FIGI) VOLCANIC ASH, ANOESITICI MIN. INDEX \& GEOL MAPS, GEOL SEC.

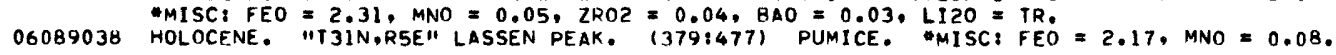

06089039 HOLOCENE. "T3IN,R5E" LASSEN PEAK. $(141: 41,42,55)$ VOLCANIC ASH, ANDESITICE MIN, PHYS PROP. INOEX MAP.

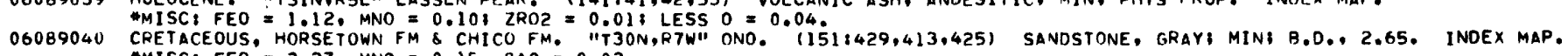
MISC: $F E O=3.27$. MNO $=0.15, B A O=0.03$.

06089041 PLIOCENE, TEHAMA FM. T32,33N,R4W, REDDING. (111:197/ 13:247,222,243,246,PL31 TUFF1 MIN. INDEX \& GEOL MAPS.

06089042 "T3IN,R6W" SHASTA. (568:263) ALTEREO TUFF. INDEX MAP. $M I S C I F F O=1.9,4 N O=0.06$.

06089043 HOLOCENE, S12,T36N,R3E. (366:166,163-165) DIATOMACEOUS EARTH, WHITE: 340 FT EXPOSED. INDEX MAP.

06089051 CRETACEOUS, HORSETOWN FM. "S11.T28N,R7W" (151:429.413.425.430) SANOSTONE DIKE. GRAYI MIN: B.D.. 2.7346. INOEX MAP. 06089052 MISC: FEO $=1.16$, MNO $=1.531 \mathrm{LI}$ I $=0.00 . \mathrm{CL}=0.09$.

52 CRETACEOUS, HORSETOWN FM \& CHICO FM. "S23,T29N,R7W" $(151: 429,430,434)$

HISC: FEO $=4.77$, MNO $=0.24$.

9053 CRETACEOUS, HORSETOWN FM \& CHICO FM. "S11,T28N,RTW" (151:429,413,425)

FEO $=1.42$, MNO = TR, $L I 20=0.00, C L=T R$.

06089054 CRETACEOUS, HORSETOWN FM \& CHICO FM. "SIO;

$F E O=6.21$, MNO $=T R, L I 20=0.00, C L=T R$.

SANDSTONE, GRAY; MIN. R.0.1 2.69. INDEX MAP. SANDSTONE DIKE, GRAY MIN, INDEX MAP. MMISC: GANOSTONE, GRAYI MIN. INDEX MAP. "MISC: 
TABLE 3. - Analyses of samples from California and Hawaii containing uncombined silica and clay each less than 75 percent; uncombined silica and clay each greater than carbonate (Group B), common-and mixed-rock categories-Continued

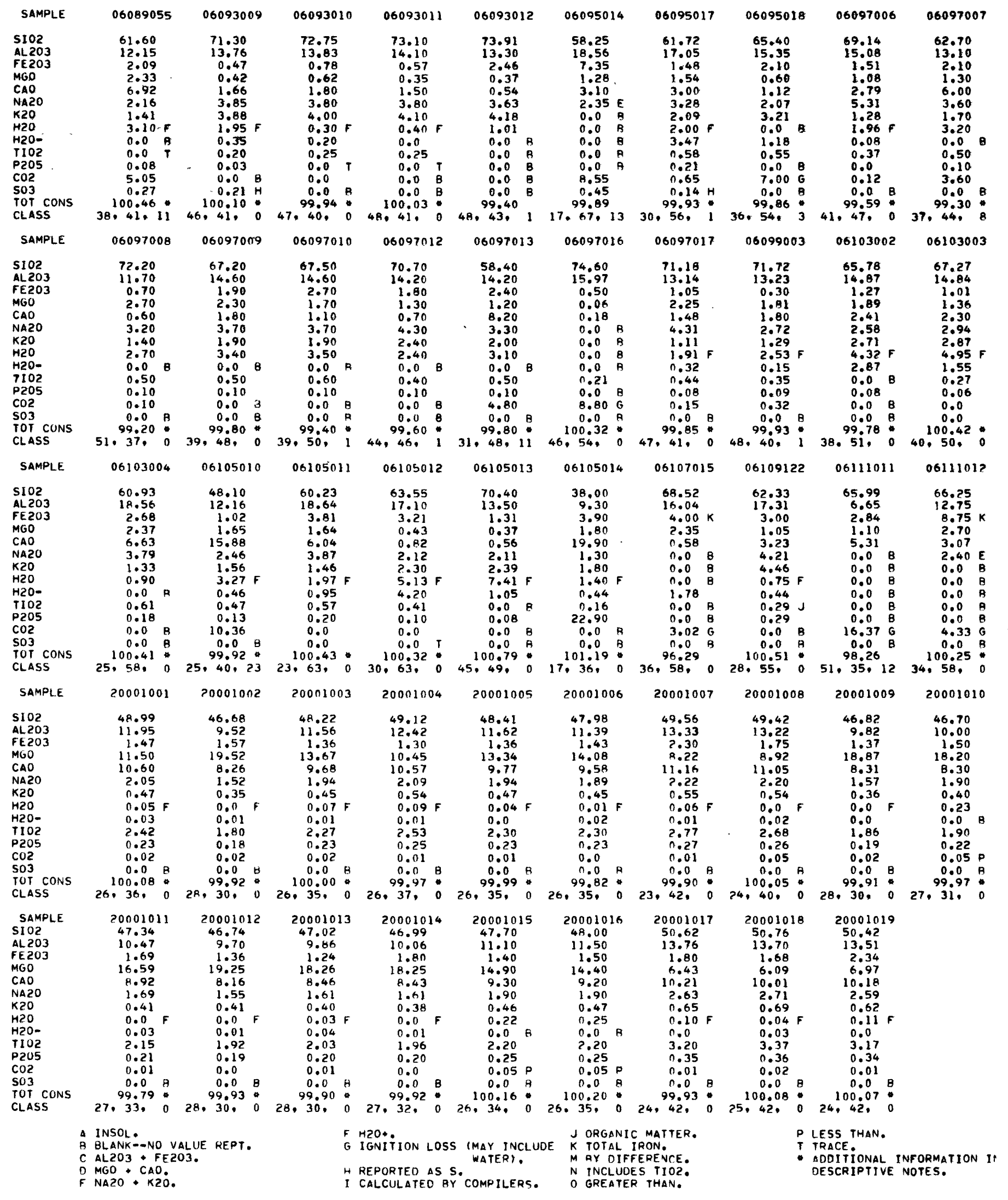


TABLE 3. - Analyses of samples from California and Hawaii containing uncombined silica and clay each less than 75 percent; uncombined silica and clay each greater than carbonate (Group B), common-and mixed-rock categories-Continued

06089055 CRETACEOUS, HORSETOWN FM \& CHICO FM. "S11,T28N,R7W" (151:429,413.425) SANDSTONE DIKE, GRAYI MIN. INOEX MAP. "MISC: 06093009 HOLOCENE. "T43N,R4E" PUMICE STONE MTN. (4231292,294/ 548:473,476,PL43) PUMICE, WHITE TO GRAY. INDEX MAP. USE, 4 .

06093010 HOLOCENE. T43N,R4E, GLASS MTN, $17: 502,487,4931$ PUMICE, RHYOLITIC. INDEX \& GEOL MAPS. $M$ MISC: FEO = 1.61 , MNO = 0.001

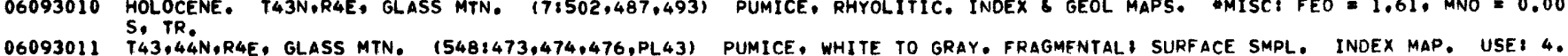
T43, $44 N, R 4 E$, GLASS MTN. $1548,473,474,476$
$M I S C$ FEO $=1.86$, MNO $=0.00,5=T R$

06093012 "TISC $1.42 N, R 3.4 W "$ MOUNT SHASTA. $(3381408)$ PUMICE.

06095014 "TSN,R2W" SUISAN. (5551120,121) CLAY, USE, 4 .

06095017 EOCENE. TEJON FM. "TSN.R2W" SUISUN. $151: 436,433$, SHALE, BANDED, MINI DH SMPL. $M M I S C$, FEO = 2.64 . MNO = 0.06, $B A O=0.07$, CR2O3 $=$ TR, LESS $0=0.05$

06095018 PLIOCENE, PINOLE TUFF. T3N,R2W, CORDELIA. (575:12) TUFF, $4 M I S C:$ FEO $=1.22, \mathrm{MNO}=0.05, \mathrm{CL}=0.01$.

06097006 JURASSIC \& CRETACEOUS, FRANCISCAN FM. "T6N,R9W" VALLEY FORD. (578:493/ 576:559.558/ 29:108.90) METAGRAYWACKEI MIN. 06097007 JURASSIC \$ CRETACEOUS, FRANCISCAN FM. "S31.TION.RIOW" $129: 33,11.32$,PL1) GRAYWACKE MIN. INOEX GEOL MAPS, 06097008 JURASSIC \& CRETACEOUS. FRANCISCAN FM. "S24,TION,RIIW" 129833,11 .32,PLI) GRAYWACKE: MIN. INDEX \& GEOL MAPS. \#MISC:

06097009

06097010

06097012

06097013

06097016

JURASSIC \& CRETACEOUS, FRANCISCAN FM. "TGN,R9W" VALLEY FORD, (580:98,96) GRAYWACKE: MIN: B.D., 2.70. INDEX \& GEOL MAP. MISCI FEO $=2.37$, MNO $=0.06$.

JURASSIC \& CRETACEOUS, FRANCISCAN FM. "S3,T5S,R5E" $14811136,29: 33,11,31$, PLI) GRAYWACKE MIN. INDEX \& GEOL MAPS. MISC: FEO $=3.58$, MNO $=0.00$, ZRO2 $=0.04$.

PLIOCENE, TEHAMA FM. T24N,R5W. PASKENTA. (111:194/ 13:247,243,246,PL3) TUFF, DACITICI MIN. INDEX \& GEOL MAPS. MEAS SEC. $M I S C$ FEO $=1.008$ MNO = TR.

06103004

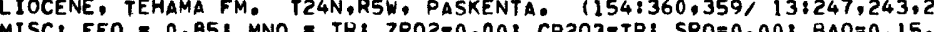
PLIOCENE, TUSCAN FM. "T2TN,R2W" RED BLUF, (8:232,216.231, FIG3) VOLCANIC BRECCIA, PALE BUFFI MIN, INDEX \& GFOL MAPS GEOL SEC. MISC: MNO $=0.101$ SRO $=0.121$ BAO $=0.02$

CRETACEOUS, HORSETOWN FM. "T3ON,R7W" ONO. $(151: 429,413,430)$ SANDSTONE OIKE, GRAYI MIN. INDEX MAP. "MISC: FEO = 2.14. 06105011 PLIOCENE. "T32N,R5W" REDDING CREEK BASIN, $(154: 360,359)$ TUFF. \#MISC: FEO =0.88: MNO = TRI SRO =0.051 ZRO2 = $0.01:$ 06105012 MIOCENE. "T32N,R6E" HAY FORK. (157:23,24,PLI) TUFF, INDEX \& GEOL MAP. WMISC: FEO = 0.92 , MNO = O.03, SO3 REPT AS S. 06105013 OLIGOCENE, WEAVERVILLE FM. S23,T3N,R6E. (111:228/ 376:68,65) TUFF, INDEX \& GEOL MAPS. \#MISC: FEO = 1.K1; MNO, TR.

06105014 OLIGOCENE(?), WEAVERVILLE FM, S13,T3N,RGE, (376:68,65-67,70-72) PHOSPHORITF, PIJRPLISH-RED, FINE-GR: I FT THICK, MIN. INDEX \& GEOL MAPS. USE: 2. MMISC: FEO $=0.18$, MNO $=0.11$. SPECTRO ANAL ALSO GIVEN.

06107015 S34.T2IN,RZRE $(489: 906)$ SHALE. USE: 3.

06109122 "T3N,RIBE" NEAR CLOVER MEADOW. (111:217\% 607:196) TUFF, MIN. $M I S C:$ FEO $=1.63$, MNO $=0.08$, FES2 = $0.06,2 R O 2=0.04$, SRO $=0.05, B A O=0.24$, LI2O $=T R$, TIOZ $=1.05 \%$ ORG MATTER CALC FROM REPT C.

06111011 "T3N,R23W" VENTURA. (480:1381) OIL SHALE, DARK, CHERTY.

06111012 "T3N,R23W" VENTURA. $(493: 98,97)$ SAND, FINE-GR, USE: 12, "MISC: I.L.. INCL UNOETD.

20001001 HOLOCENE, KILAUEA VOLCANO, $15: A 5, A 3, A 4)$ PUMICE. NORM, $\triangle M I S C: F E 0=10.08, M N D=0.18, C L=0.02, F=0.03: L E S S 0=0.01$.

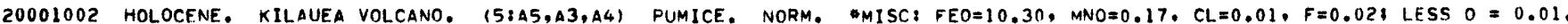

20001003 HOLOCENE. KILAUEA VOLCANO. $(5: A 5, A 3, A 4)$ PUMICE. NORM. $\quad M I S C: F E 0=10.31, M N 0=0.18, C L=0.01, F=0.03:$ LESS $0=0.01$.

20001004 HOLOCENE. KILAUEA VOLCANO. HOLOCENE, KILAUEA VOLCANO. LESS $0=0.02$

20001005 HOLOCENE. KILAUEA VOLCANo.

20001006 hOLOCENE. KILLUEA VOLCANo.

20001007 HOLOCENE. KILAUEA VOLCANO.

20001008 HOLOCENE. KILAUEA VOLCANO.

20001009 hOLOCENE. KILAUEa VOLCANO.

20001010 hOLOCENE, KILAUEa volcano.

$(5: A 5, A 3, A 4)$

PUMICE. NORM.

$(5: A 5, A 3, A 4)$

PUMICE. NORM.

MISC: FEO $=10.17, M N O=0.18, C L=0.01, F=0.04, C R 203=0.211$

20001011 holocene. KIlauea volcano.

(5:A5,A3,A4) PUMICE. NORM. $\triangle M I S C$ FEO $=9.23$, YNO $=0.17, C L=0.02, F=0.03 ;$ LESS $0=0.01$.

20001012 hOLOCENE. KILAUEA VOLCANO.

(58A5,A3,A4) PUMICE. NORM, AMISC: FEO=9.72, MNO=0.18, CL=0.02, F=0.03; LESS $0=0.01$.

20001013

holocene, killauea volcano.

20001014

HOLOCENE.

KILAUEA VOLCANO.

(51A5,A3,A4) PUMICE.

NORM. MISC: $F E O=10.53$, $M N O=0.17, C L=0.01, F=0.02$ LESS $0=0.01$.

20001015 HOLOCENE. kilauea volcano. 
TABLE 3. - Analyses of samples from California and Hawaii containing uncombined silica and clay each less than 75 percent; uncombined silica and clay each greater than carbonate (Group B), common-and mixed-rock categories-Continued

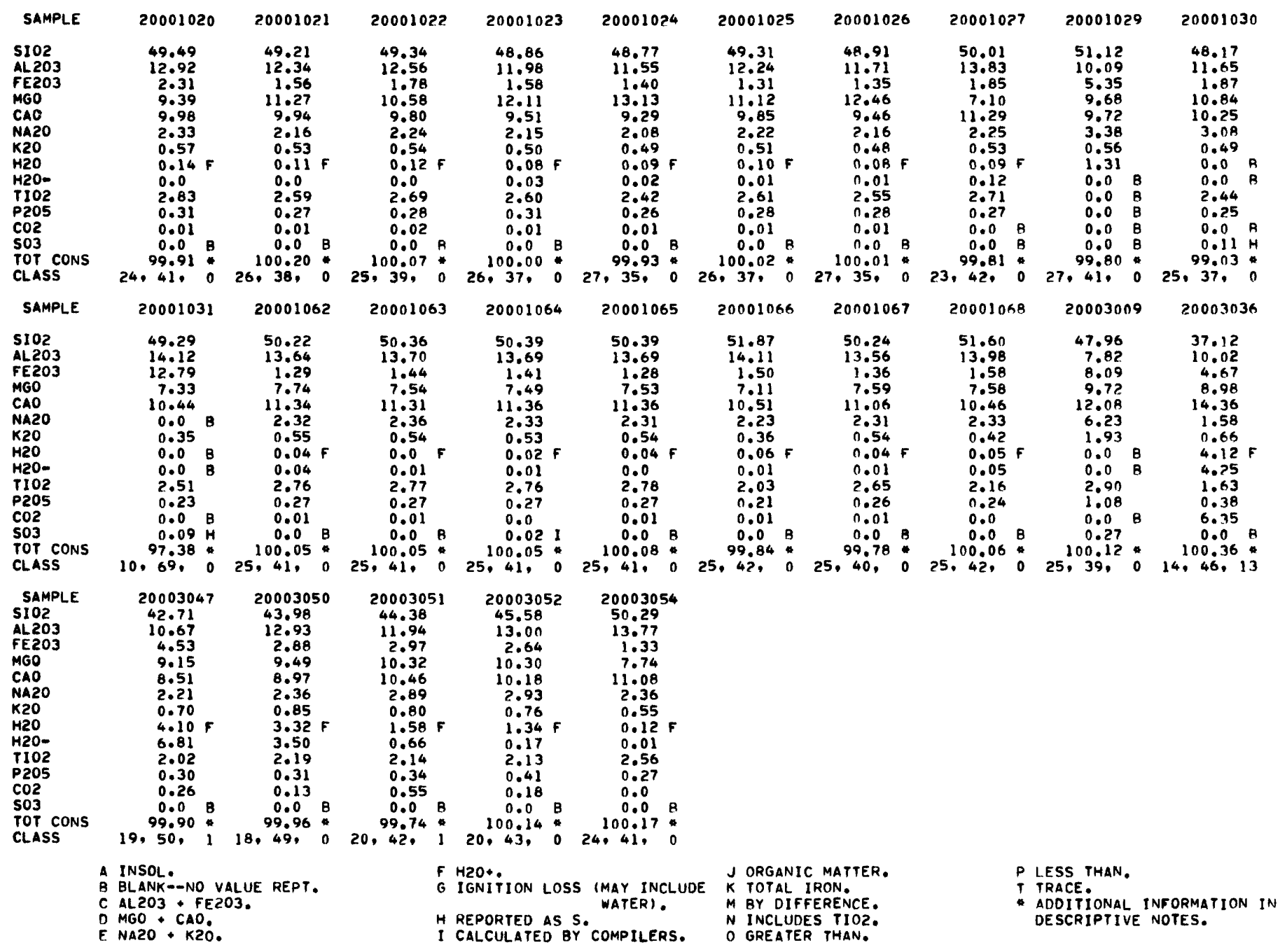


TABLE 3. - Analyses of samples from California and Hawaii containing uncombined silica and clay each less than 75 percent; uncombined silica and clay each greater than carbonate (Group B), common-and mixed-rock categories-Continued

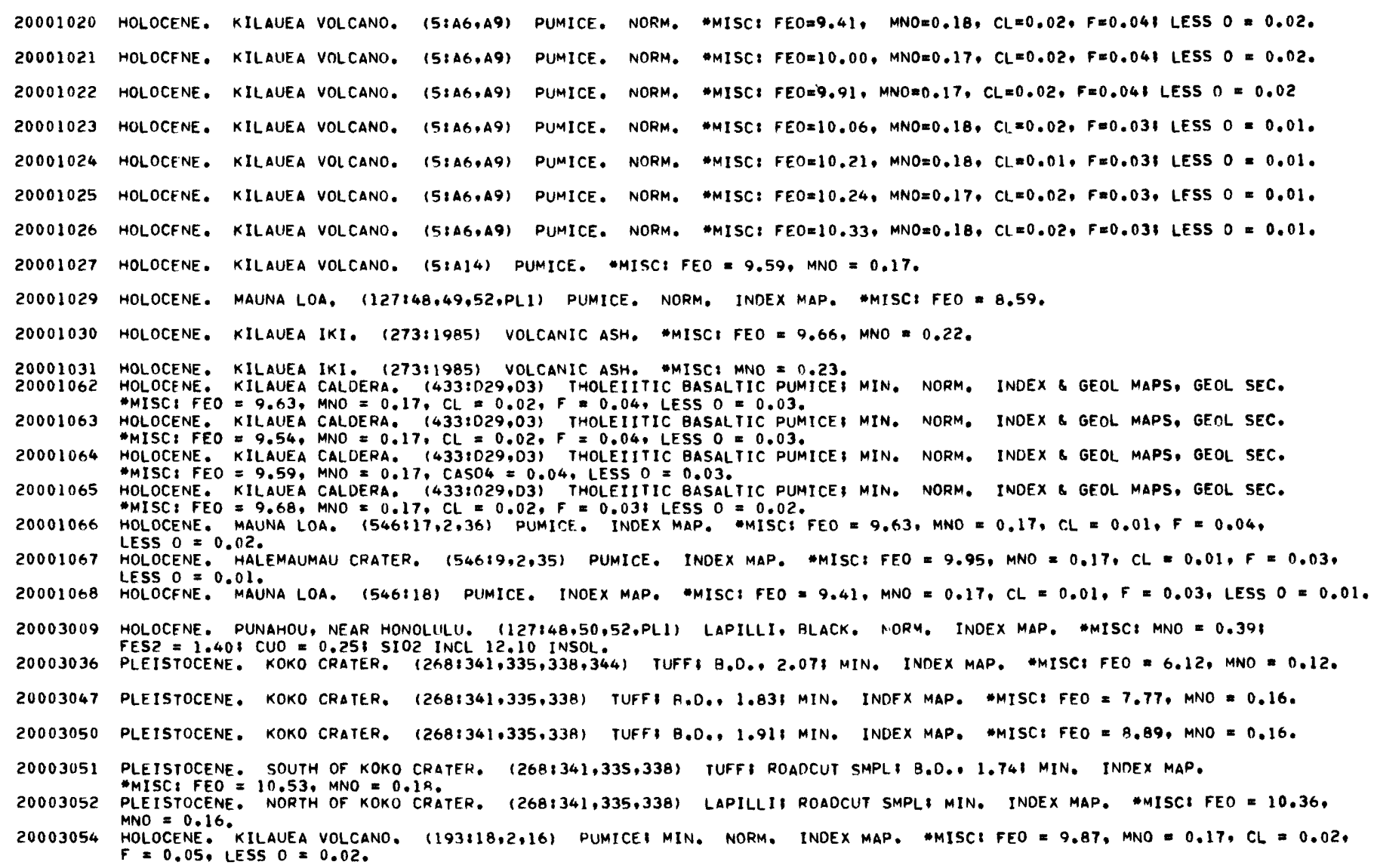


TABLE 4. - Analyses of samples from California containing uncombined silica and carbonate each less than 75 percent; each greater than clay (Group C), common- and mixed-rock categories

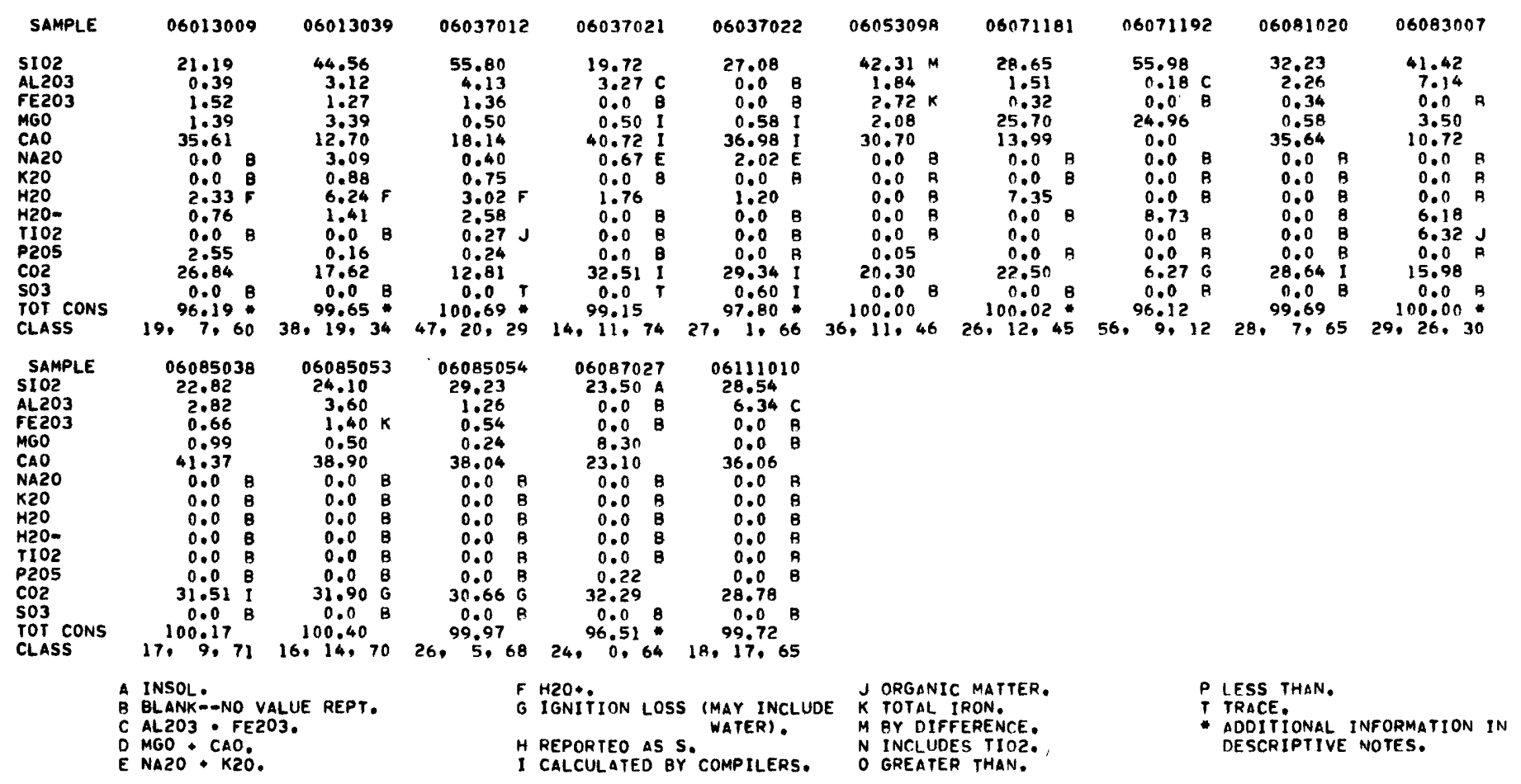

06013009 JURASSIC, KNOXVILLE FM. "TIN,RIE" MT DIABLO 1508:409.400,PL15) LIMESTONF, GRAY, FRIABLE. GEOL MAP, GEOL SEC. (111.286) SHALE, DARK, CALCAREOUS, COMPACT, UMISC, FEO $=5.21$, MNO $=$ TR. 9 CRETACEOUS, "TIS.RIW" MOUNT DIABLO. 06037012 MIOCENE, MODELO FM. "T2S,RISW" MULHOLLAND HIGHWAY, $(293,108,109, P L 16,17)$ SHALE, WHITE, FOS, MIN, INDEX \& GEOL MAP, 06037021 GEOL SEC. $M M Y S C$ : FEO $=0.44$, T1O2 $=0.25$; ORG MATTER CALC FROM ORG C.

O6037022 "TIN,R9W" GLENDORA. (72:880) LIMESTONE, SHELL. "MISC: SIO2 INCLUDES FE \& AL203. CA/MG a 63.8.

06053098 PRE-FRANCISCAN, SUR SER. S32,T195.R3E. 14208B40,B8,PL1,2) MARBLE. WHITE. FINE TO COARSELY CRYSI SELECTED SMPL. 0607118I TERTIARY, S2?, TBN, R2IE. (524:364,357-360,PL62-64) SHALE, WHITE, SOFT, THIN-BEDDEDI 1.95 FT THICK, TONNAGE. INDEX \& GEOL MAPS, MEAS SEC, GEOL SEC. MISC: CR203 $=0.00$. CA/MG $=0.544$.

06071192 "S35,T8N,R5E" (467:11) MONTMORILLONITE.

06081020 JURASSIC \& CRETACEOUS, FRANCISCAN FM. "T4S,R6W" KEN ROYCE QUARRY. (52617.4,PL1) LIMESTONE. INDEX * GEOL MAPS. USE: 4.7. $C A / M G=61.4$.

06083007 MIOCENE. "T4N,R25W" CARPINTERIA. (530:49,FIG35) SHALE. INDEX \& GEOL MAP. "MISC: FEO= 8.74, FEO \& AL203, ESTIMATED.

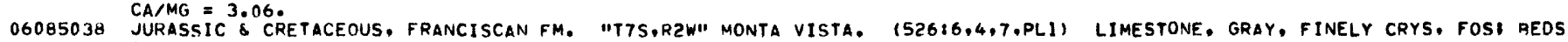
3-18 IN THICK: TYPICAL ANAL FROM QUARRY. INDEX \& GEOL MAPS, USE:4.7. CA/MG $=41.8$.

06085053 JURASSIC \& CRETACEOUS. FRANCISCAN FM. S17,18,T7S,R2W. $(331: 376,374)$ LIMF5TONE: TONNAGE: SCREEN SIZE, C0.5 INI PHYS

O6085054 JURASSIC \& CRETACEOUS, FRANCISCAN FM. $517,18, T 75, R 2 W . \quad(331: 377.374 .376)$ LIMESTONE AVG COMPOS OF MANY SMPLI TONNAGE. USE: 4. CA/MG $>100$. 06111010 EOCENE. S22,27,T5N,R25W. (504:268,267/ 556:349,PL37) LIMESTONE, FINE-GRI 250 FT THICK. INDEX MAP. UCE 4.18. 

TABLE 5. - Analyses of samples from California and Hawaii containing 75-90 percent clay (Group $\left.D_{5}\right)$, common- and mixed-rock categories

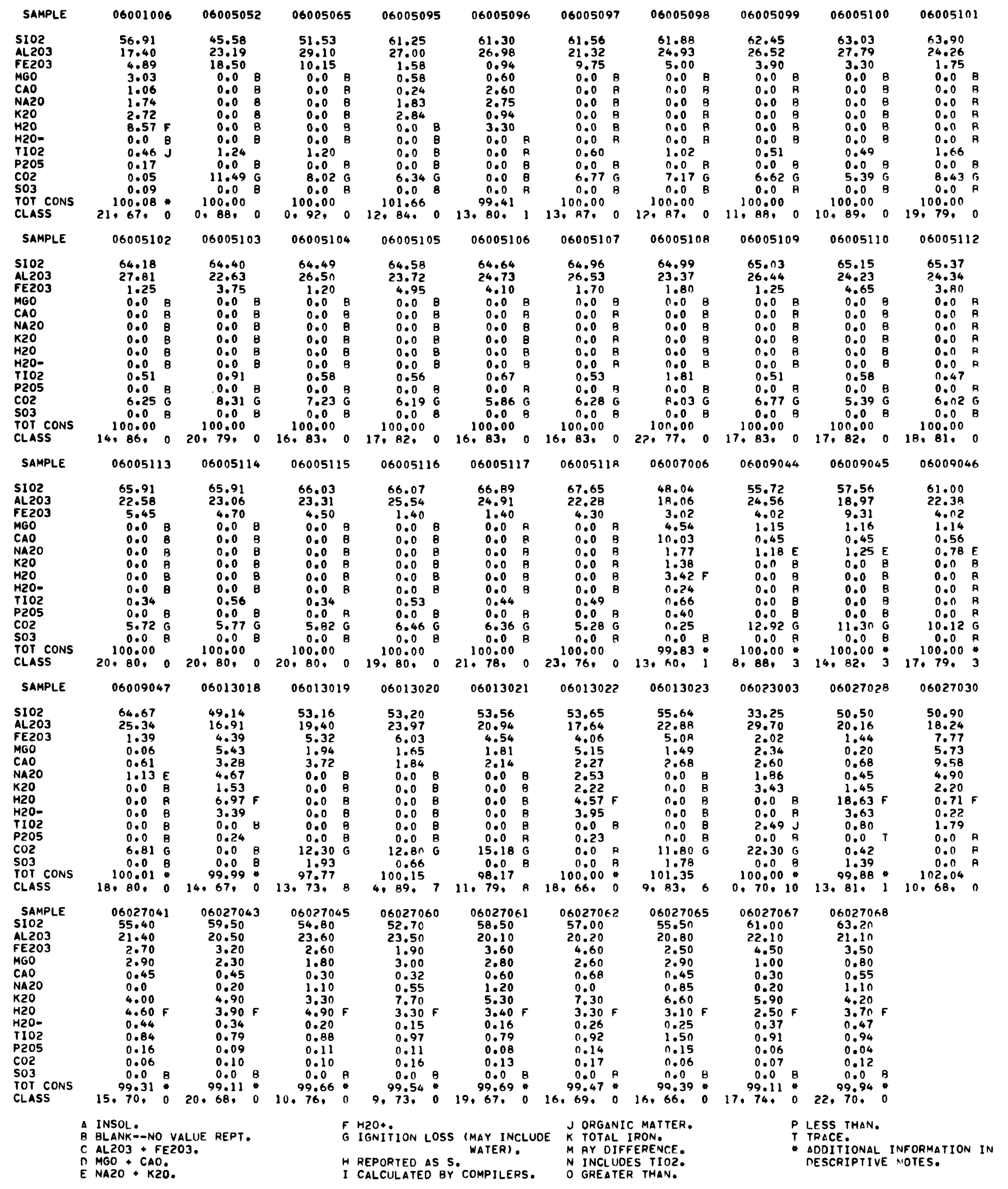


TABLE 5. - Analyses of samples from California andHawaii containing 75-90 percent clay (Group $D_{5}$ ), common- and mixed-rock categoriesContinued

06001006 CRETACEOUS, BERRYESSA FM. S3,T5S,R1E, $(66123,18,22-24,31$, PLI) SHALE, GREEN, BEDDEDI MIN, B.D., 2.50 PHYS PROP. INDEX \& GEOL MAPS. MMISC: FEO $=2.30 ;$ TIO2 $=0.69$. SPECTRO ANAL ALSO GIVEN.

06005052 EOCENE, IONE FM. "SI8,T5N,RIOE" (417:39,17,PL1-3) CLAY, RED WITH IRON NODULES DH, 234-235 FTI MIN. INDEX GEOL 06005065 EOCENE, IONE FM. "S24,T5N,R9E" (417139,35,PL1-3) CLAY, BUFF; DH, 157-1S8 FT. INDEX \& GEOL MAPS, GEOL SEC.

06005095 EOCENF. T6N,R9E, IONE, (1471354,18,52,273-276,PL1) CLAY, WHITE, FINF-GR 12 FT EXPOSED, AVG THICKNESS 16 FTI PHYS 06005096 PROP, FIRING TESTS. INDEX MAP, USE: 3,5 .

06005097 EOCENE, IONE FM. S13,TSN,R9E, (417338,3-5,PL1-4) CLAY, YELLOW, SANDY, DH, 290-291 FI, MIN. INDEX \& GEOL MAPS, GEOL. 06005098 EOCENE. S7,TSN,RIOE. (417:38,27,PL1-3) ARGILLACEOUS SILTSTONE: DH, $232-233$ FT. INDEX \& GEOL MAPS, GEOL SEC.

06005099 EOCENE, IONE FM. S13,T5N,R9E. (417:39,3-5,17,PLI-4) CLAY, RED \& WHITE: OH, 305-306 FT, MIN. INDEX \& GEOL MAPS, 06005100 EOCENE, IONE FM. S13,T5N,R9E. (417:38,3-5,17,PL1-4) SILTSTONE, YELLOW \& WHTE, ARGILLACEOUSI DH, 294-295 FT, MIN. INDEX \& GEOL MAPS, GEOL SEC. USE: 14.

06005101 EOCENF, IONE FM. S7,T5N,RIOE. (417:38,17,PL1-3) CLAY, BROWN: DH, 41-42 FT: MIN. INDEX \& GEOL MAPS, GEOL SEC. USE 114. 06005102 EOCENE, IONE FM. S13.15N.R9E. (417:38,3-5,PL1-4) CONGLOMERATE: OH, 296-297 FT: MIN, INDEX \& GEOL MAPS, GEOL SEC. 06005103 EOCENE, IONE FM. S24,T5N,R9E. (417139,3-5,17,32,PL1,3) CLAY, WHITE, DH, 167-168 FT, MIN. INDEX \& GEOL MAPS, GEOL 06005104 EOCENE, IONF FM. S13,T5N,R9E, (417:39,3-5,38,PL1-4) CONGLOMERATE. WHITE: DH, 302-303 FT, MIN. INDEX \& GEOL MAPS, 06005105 EOCENE, IONE FM. S13,TSN,K9E. (417139,3-5,38,PL1-4) CLAY, RED: OH, 306-307 FT1 MIN. INDEX GEOL. MAPS. GEOL SEC. USE: 14.

06005106 EOCENE, IONE FM. S13,T5N,R9E. GEOL SEC. IISE: 14.

06005107 EOCENE, IONE FM. S13,TSN,R9E.
06005108 USE: 14 .

06005107 EOCENE, IONE FM. S13,TSN,R9E
USE: 14.
06005108 EOCENE, IONE FM. S7,TSN,R1OE. $\{417: 38,3-5,17, P L 1-4)$ CLAY, RED, SANDY, DH, 278-279 FTI MIN, INDEX \& GEOL MAPS, (417:38,3-5,PL1-4) CONGLOMERATE, DH, 295-296 FT: MIN. INDEX \& GEOL MAPS, GEDL SEC. $(417: 38,17, P(1-3)$ CLAY, BROWN DH, 35-36 FT: MIN. INDEX \& GEOL MAPS, GEOL SEC. USE:14. 06005109 EOCENE, IONE FM. S13.T5N,R9E.

06005110 EOCENE. IONE FM. S13,T5N,R9E. (417:39,3-5,38,DL1-4) CLAY, REDI DH, 308-309 FTI MIN. INDEX \& GEOL MAPS. GFOL SEC.

06005112 EOCENE. S13,T5N,R9E. (417:38.5,29.PL1-4) CLAY, WHITE, YELLOW STAINS, DH, 373-374 FT. INDEX \& GEOL MAPS, GEOL SEC. 06005113 EOCENE. S13.TSN,R9E. $(417138,29, P L 1-4)$ CLAY, RED \& BUFF, SILTY: DH, $311-313$ FT. TNDEX \& GEOL MAPS, GEOL SEC.

06005114 EOCENE, IONE FM. S13,T5N,R9E. (417:39,3-5,38,PLI-4) CLAY, REO OH, 307-308 FT: MIN. INDEX \& GEDL MAPS, GEOL SEC. EOCENE, IONE FM. S13,T5N,R9E. (417:39,3-5,38,PL1-4) CLAY, RED: DH, 310-311 FTI MIN. INDEX \& GEOL MAPS, GEOL SEC. USE: 14. IONE FM. S13,T5N,R9E. (417:39,3-5,38,PL1-4) CLAY, WHITEI DH, 303-304 FT: MIN. INDEX \& GEOL MAPS. GEOL SEC.

O6005116 EOCENE, IONE FM. S13,T5N.R9E. USE: 14. EOCENE, IONE FM, S13,T5N,R9E. (417:39,3-5,38,PL1-4) CLAY, WHITE, DH, 304-305 FT: MIN. INDEX \& GEOL MAPS. GEOL SEC. USE: 14, IONE FM. S13,T5N,K9E. (417139,3-5,38,PL1-4) CLAY, RED: DH, 309-310 FT: MIN. INDEX \& TEOL MAPS, GEOL SEC. USE: 14 .

0600511

06005118

06007006

06009044

06009045

EOCENE, IONE FM. "T3N,K9E" HELISMA. (147:355,69,305-310,PL1) CLAY, FINE GRI 8-15 FT THICKI PHYS PRDP, FIRING TESTS. INOEX MAP. USE: 3. MISC: NAZO K KO, BY DIF.

06009046 MAP. MISC: NAPO K KOO BY OIF.

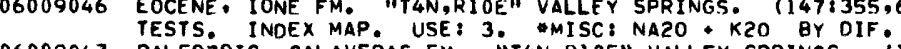

06009047 PALEOZOIC, CALAVERAS FM. "T4N,RIOE" VALLEY SPRINGS. (147:354,68,258,270,PL1) KAOLIN, WHITEI 15-25 FT THICK: PHYS 06013018 CRETACEOUS. "TIN,RIE" MOUNT DIAQLD, (508:406,400,PL15) SHALE. GEOL MAP \& SEC. *MISC: FEO = 3.82, MNO =0.22, NI0= TR.

06013019 "TIN.RIW" COWELL. (173:122) SHALE CLAY. USE: 4.

06013020 "TIN,RIW" COWFLL: (173:122) SHALE CLAY: USE: 4.

06013022 "CRETACEOUS. "TIN,RIE" MOUNT DIABLO, (508:406,384.400,403.PL15) SHALE, SLATE-COLORED, FRIABLE. GEOL MAP, GEOL SEC.

06013022 CRETACEOUS. "TIN.RIE" MOUNT DIABLO. 1508

06013023 "TIN,RIW" CONCORD. (173:122) SHALE CLAY. USE: 4.

06023003 PLEISTOCENE, HOOKTON FM. "T4N,RIW" EUREKA. 1340:302/ 409:84,8,10,PL1,21 CLAY, BLUE, PLASTICI AVG, 22 FT THICK. INDEX \& GEOL MAPS, GEOL SEC. USE: 3. MMISC: ANAL RECALCULATED TO INCLUDE I. L.

06027028 HOLOCENE. S4,T22S,R39E. 1498:519/202:230,223-229,PL4) CLAY, WHITE, FINF-GRI MIN. INDEX \& GEOL MAPS. "MISC: $A S 203=0.001, \mathrm{CL}=0.13, \mathrm{B203}=0.00$

06027030 S30,T22S,R38E. (402:i1):PL1) VOLCANIC CINDERS, REDI MIN. INDEX MAP.

06027041 CAMRRIAN, HAKKLESS FM. S14.24.T7S,R38E. $(585,22,8,77)$ PELITE: MIN. INDEX MAP, MEAS SEC. SPECTRO ANAL ALSO GIVEN. -MISC: FEO $=6.2$. MNO $=0.16$.

06027043 CAMBRIAN. HARKLESS FM. SI5,T7S,R38E. $1585123,8,73)$ PELITE, MIN. INDEX MAP, MEAS SFC. SPECTRO ANAL ALSO GIVEN. AMISC: FEO = 2.8. MNO $=0.04$.

06027045 CAMBRIAN, HARKLESS FM, SI,12,13,24,TIOS,R35E. (58S:23,8) PELITE: MIN. INDEX MAP. SPECTRO ANAL ALSO GIVEN. "MISC, FEO $=5.9$, MNO $=0.07$.

06027060 PRECAMBRIAN \& CAMBRIAN, WOOD CANYON FM. S7,R,T1OS,R40E \& S29-32,T9S,R40E. (585:24,8,82) PELITE: MIN. INDEX MAP, MEAS SEC. SPECTHO ANAL ALSO GIVEN. MISC: FEO $=5.1$, MNO $=0.08$

06027061 PRECAMBRIAN CAMBRIAN, WOOD CANYON FM, S7,8,TIOS,R40E \& S29-32,T9S,R40E. (585:24,8,82) PELITE, MIN. INDEX MAP, MEAS

SEC. SPECTRO ANAL ALSO GIVEN. WMISC: FEO $=3.0$, MNO = 0.03 .

06027062 ALSO GIVEN ALSO GIVEN, MISC: FEO $=2.3$. MNO $=0.00$ SI,TLS.R45E. (585224.8,99) PELITE: MIN. INDEX MAP, MEAS SEC. SPECTRO ANAL. ALSO GIVEN. $M$ MISC: FFO $=4.7$. MNO $=0.03$. 06027067 PRECAMBRIAN. JDHNNIE FM. S9-11,T2ON,RBE. MISC: FEO $=0.20$, MNO $=0.00$. 
TABLE 5. - Analyses of samples from California and Hawaii containing 75-90 percent clay (Group $\left.D_{5}\right)$, common- and mixed-rock categoriesContinued

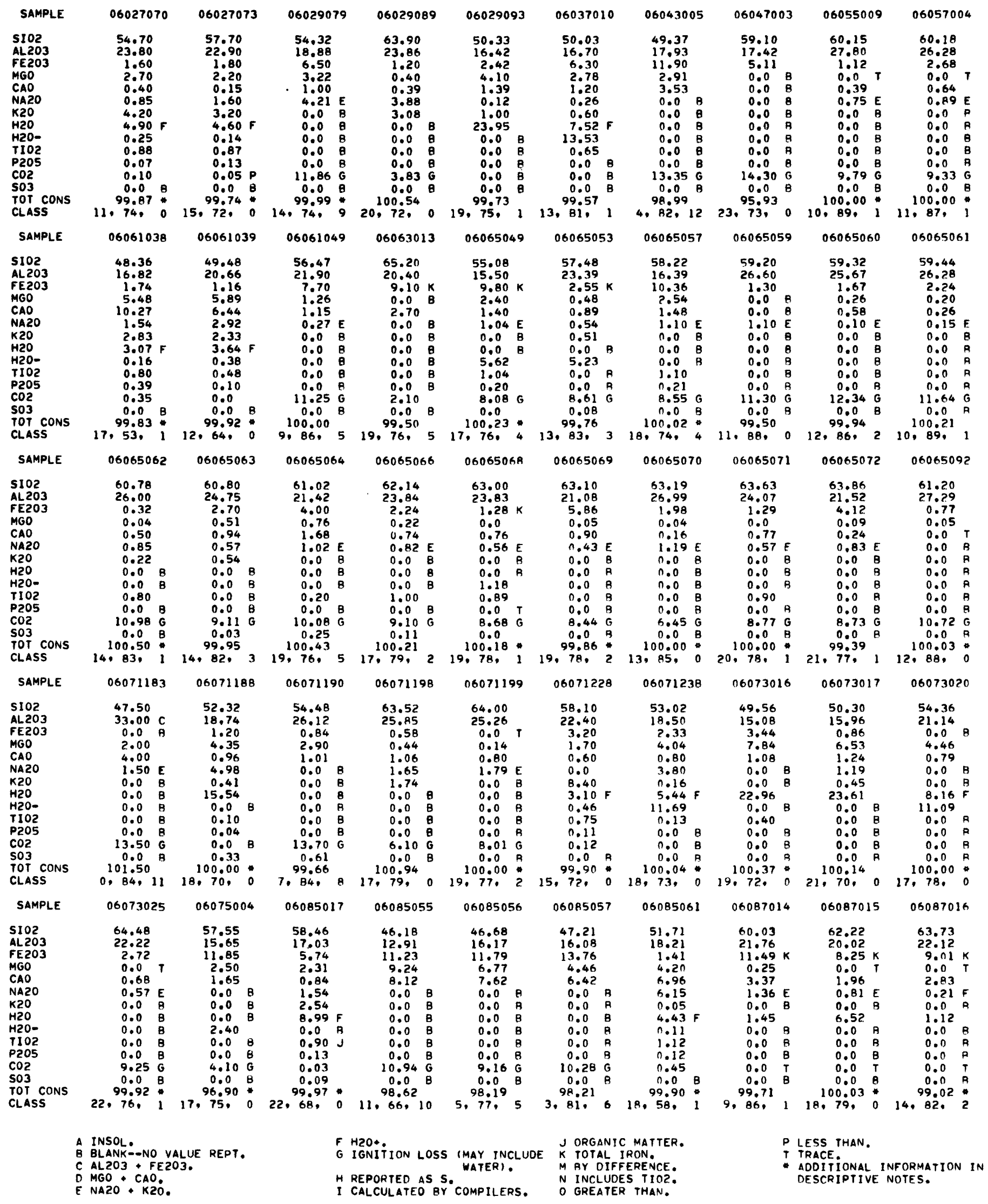


TABLE 5. - Analyses of samples from California and Hawaï containing 75-90 percent clay (Group $\left.D_{5}\right)$, common- and mixed-rock categoriesContinued

06027070 PRECAMBRIAN, JOHNNIE FM. S17,18,T205,R46E. (585:25,8,105) PELITE, MIN, INDEX MAP, MEAS SEC. SPECTRO ANAL ALSO GIVEN. 06027073 PRECAMBRIAN, JOHNNIE FM. LAT 36 DEG, $22 \%$ LONG 117 DEG. 061. $(585: 25, B)$ PELITE: MIN. INDEX MAP. SPECTRO ANAL ALSO 06029079 OLIGOCENE \& MIOCENE, TEMBLOR FM. S14,T27S,R28E. $(218275,274 / 15193)$ FULLERS EARTHI 15-50 FT THICK. USE: 6. - MISC: NA2O * K2O, OY DIF.

06029089 "T2BS,R38E" EL PASO RANGE: (327:308) PUMICE.

06029093 "TIIN.R24W" MARICOPA. (440889,95) BENTONITE.

06037010 "TIS,RI6-1 BW" SANTA MONICA MTN, (403:183,182) BENTONITE.

06043005 "SIO,T4S,RIBE" BRICEBURG. (147:356.PLI) CLAY. INDEX MAP.

06047003 "TSS,R15E" MERCEO FALLS. (147:356,128,PL1) CLAY, WHITE. INDEX MAP, USE: 5.

06055009 S12,13,TBN,R7W: S7,18,TBN,R6W. $(147: 354,135,258-270, P L 1)$ KAOLIN, WHITE: PHYS PROP, FIRING TESTS. INDEX MAP. USE:3,5. 06057004 TERTIARY. S13,T14N,R7E. (147:354,135,137,261,267-269,PL1) KAOLINI SMPL OF 10 FTI PHYS PROP, FIRING TESTS. INOEX MAP. 06061038 PALEOZOIC, CALAVERAS FM. S19,TIAN,R1OE, (B2:14,PLI-3) TUFF, FINE-GR. INDEX 8 GEOL MAPS, GEDL SEC. UMISCI FEO = 7.68 , 06061039 PALEOZOIC, CALAVERAS FM, S12,T14N,R9E. (B2:14,PLI-4) TUFF, FINE-GR. INDEX \& GEDL MAPS, GEOL SEC. $4 M I S C$. FEO = 6.27, 06061039 PALEOZOIC, CALAVERAS FM. S12,TI4N,R9E, $(82: 14, P L 1-4)$ TUFF, FINE-GR. INDEX \& GEDL MAPS, GEOL SEC.

06063013 TRIASSIC, CEDAR FM. S6-8,T25N,RBE. $(23: 311,310)$ SLATE, USE: 4.

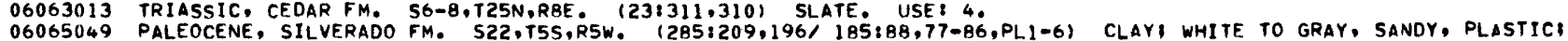

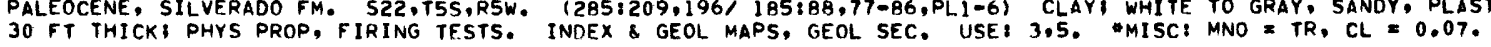

06065053 PALEOCENE, SILVERADO FM. S23,T5S,R5W. (285:205,186,196/ 185:89,77-81,PL1-6) CLAY, WHITE, SANDY, PLASTIC, 10 FT THICK, PHYS PROP, FIRING TESTS. INDEX \& GEOL MAPS, GEOL SEC, USE: 3,5 ;
EOCENE. T5S,RSW. TEMFSCAL VALLEY $1147: 355,162,168,311-319$, PLI) CLAY, MED-GR B FT THICK PHYS PROP, FIRING TFSTS.

\section{INDEX MAPS, MEAS SEC, GEOL SEC. USE :3. AMISC: NAZO * K2O BY DIF, MNO $=$ TR: CL $=0.07$. \\ 06065057}

06065059

06065060

06065061

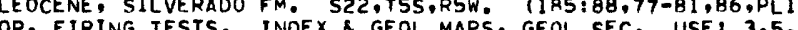

PROP, FIRING TESTS. INDEX \& GEOL MAPS, GEOL SEC, USE: 3,5 .

PROP, FIRING TESTS. INDEX MAP. USE: 3,5 .

(478:66,52,65,67,PLI) CLAY, GRAY-WHITEI MINI ABOUT 35 FT THICKI PHYS

PALEOCENE, SILVERADO FM. S22,T5S,RSW. $1285: 201,199 / 18$

CLAY, WHITE, 7 FT THICK, PHYS PROP, FIRING TESTS, INDEX MAPI MEAS SE S3S, TSS,R6W. $1147,354,162,169,272,274$

06065063 EOCENE. TSS,RSW, ALBERHILL. (1471354,162,168,258,287,293-295,PL1) CLAY, WHITE, FINE-GR, PLASTICI B FT THICKI PHYS PROP, FIRING TESTS. INDEX MAP, MEAS SEC, GEOL SEC, USE:5.

06065064 TERTIARY. "T6S,R4W" ELSINORE. (388:574/43:86,75) CLAY, GRAY, USE: 14.

06065066 TERTIARY. "TAS,R4W" ELSINORE, (388:574/ 43:86,75) FULLERS EARTH, GRAY.

06065068 TERTIARY. "TSS,RSW" ALBERHILL. $(285: 199,185,186,196)$ CLAY, GRAY, PLASTICI PHYS PROP, FIRING TESTS. INDEX MAP, 06065069 EOCENE. S26,T5S,R5W. (147:355,162,178,324-332,PLi) CLAY, BLUE, SANDY, MED-GR, PLASTICI 40 FT THICKI PHYS PROP. FIRING

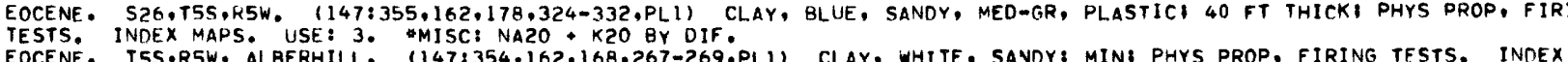

06065070 EOCENE. TSS,R5W, ALBERHILL. $(147: 354,162,168,267-269$, PLI) CLAY, WHITE, SAVDY: MIN: PHYS PROP, FIRING TESTS. INOEX

06065071 EOCENE. TSS,R5W, ALBERHILL. (147:354,162,264-271,PL1) CLAY, PLASTICI PHYS PROP, FIRING TESTS. INDEX MAPS, MEAS SECE GEOL SEC. USE: 5. \#MISC: NAZO $+K 20$ BY DIF: MNO $=0.00$. 06065072 PALEOCENE, SILVERADO FM. S3,10,T4S,R7W. $1478: 78,52,77, P L 1 / 228: 77,74, P L 1,21$ SHALE, RED OR PINK: MIN: PHYS PROP,
FIRING TESTS. INDEX \& GEOL MAP, GFOL SEC. USE: 3.

06065092 EOCENE. "TSS,RSW" ALBERHILL. (566:1169)" CLAY, WHITE WITH BLACK SPOTS: 4 FT THICK, PHYS PROP. MEAS SEC. USE: 14. *MISC: NAZO + KZO = TR.

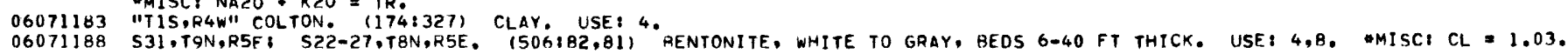

06071190 T8N,R5E. HECTOR. (503:380,262) RENTONITE, GEDS 2-15 FT THICK. INDEX MAP.

06071198 S13,24,T14N,RI7E. (147:354,194,258-271,PL1) CLAY: MINI PHYS PROP, FIRING TESTS. INDEX MAP. USE: 14.

06071199 S13.24,114N,K17E, (147:354,194,258-271,PL1) CLAY: MIN: PHYS PROP. FIRING TESTS. INNEX MAP. USE: 14. "MISC: NA20 +

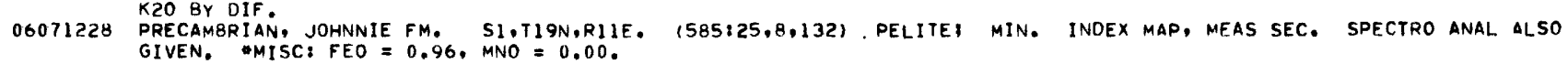

06071238 "TBN,RSE" HECTOR, $(439: 34,30)$ BENTONITE, PALE GRAY. AMISC: FEO =0.13.

06073016 PLIOCENE, SAN OIEGO FM. S17,T185,R1W. (440188, 115110,5-15,PL1) BENTONITE, TONNAGE. INDEX \& GEOL MAPS, MEAS SEC.

06073017 PLIOCENE, SAN DIEGO FM. S30,TI8S,RIW \& S25,T18S,R2W. $(440888,115: 10,5,7,11,15, P L 1)$ BENTONITE: 0-4 FT THICK.

06073020 "TI7S,R2W" NATIONAL CITY. (563:106) BENTONITE. "MISC: MGO INCL ALKALIES.

06073025 PLEISTOCENE, "T135,R5W" CARDIFF. (147:354,287-295.PL1) CLAY, SANOYI 10-12 FT THICK: PHYS PROP, FIRING TESTS, INHEX

06075004 JURASSIC \& CRETACEOUS, FRANCISCAN FM. "T2S,R5W" SAN FRANCISC0. $(138: 269.242,264,265)$ SHALE, RED, FINE-GR: MIN.

06075004 JURASSIC \& CRETACEOUS

06085017 CRETACEOUS, BERRYESSA FM. S36,T9S,R4E, $(66: 23,18,22-24,31, P L 1)$ SHALE, GREENI MINI B.D., 2.36 , PHYS PROP. INDEX \& 06085055 GEOL MAPS. MMISCI FEO $=0.721$ TIO2 $=0.65$. SPECTRO ANAL ALSO GIVEN.

06085056 JURASSIC S CRETACEOUS, FRANCISCAN FM. 517,18,T7S,R2W. $(331: 377,374)$ CLAYI AVG COMPOS OF FOUR DEPOSITS. USE, 4.

06085057 JURASSIC \& CRETACEOUS, FRANCISCAN FM. S17,18,T7S,R2W. $(331: 377,374)$ CLAY AVG COMPOS OF FOUR NEPOSITS. USE: 4 .

06085061 JURASSIC \& CRETACEOUS. FRANCISCAN FM. "T9S,R5,6E" PACHECO PASS, (186151,45-50,PL3) TUFF \& TUFFACEOUS GRAYWACKE3 MIN. 06087014 "THE \& GEOL MAP, GEOL SEC. \#MISC: FEO = 4.86, MNO $=0.12$.

06087015 "TIIS,R2W" SANTA CRUZ. (72:881.880) SHALE. USE: 4: MMISC: SAND = 0.251 H20 INCL CARBONACEOUS MATTER.

06087016 "TIIS,R2W" SANTA CRUZ. (72:881,880) CLAY, FINE TEXTURE, USE: 4. "MISC: HZO INCL CARBONACEOUS MATTER. 
TABLE 5. - Analyses of samples from California and Hawaii containing 75-90 percent clay $\left(\right.$ Group $\left.D_{5}\right)$, common- and mixed-rock categoriesContinued

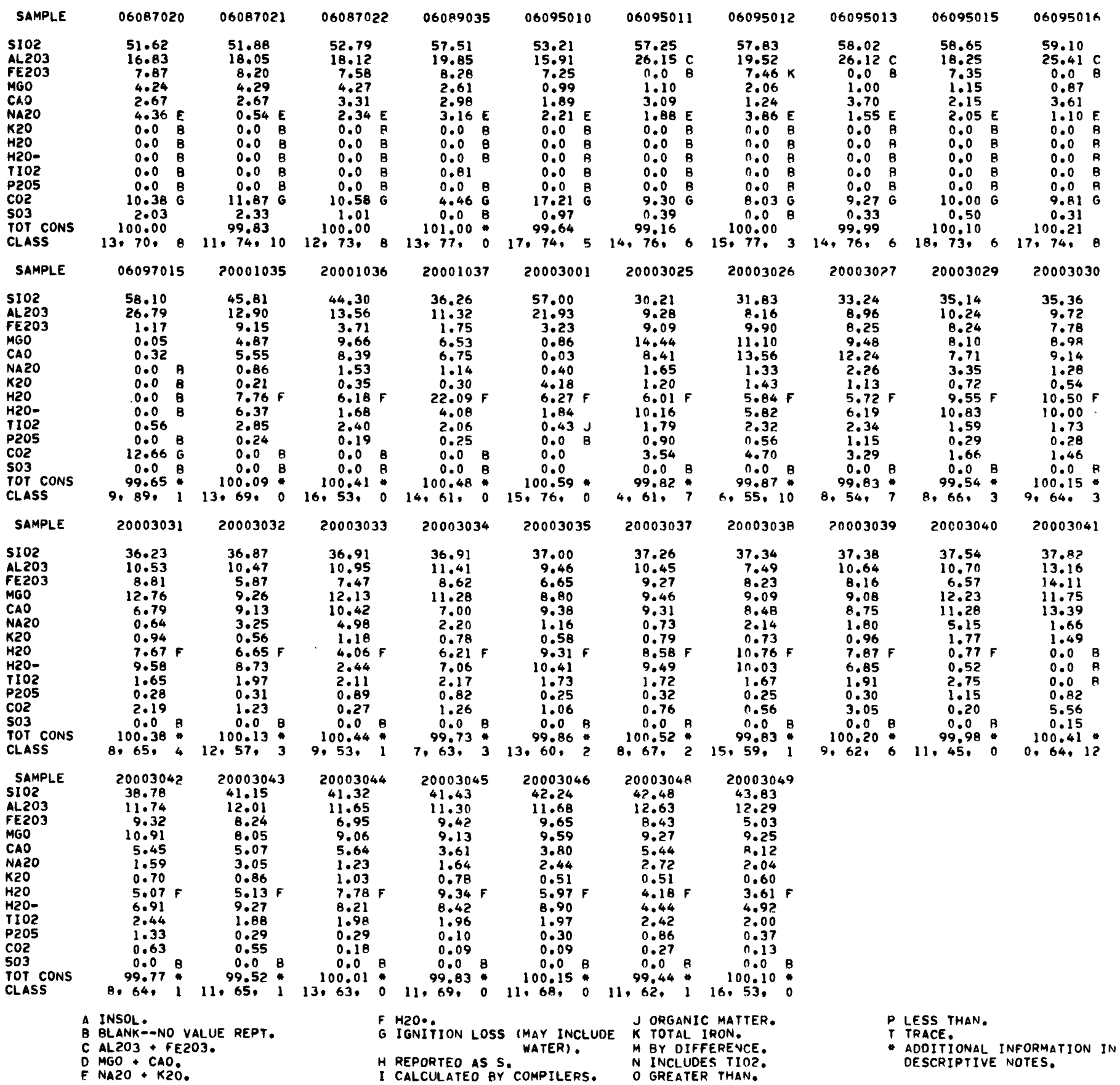


TABLE 5. - Analyses of samples from California and Hawaii containing 75-90 percent clay (Group $D_{5}$ ), common- and mixed-rock categoriesContinued

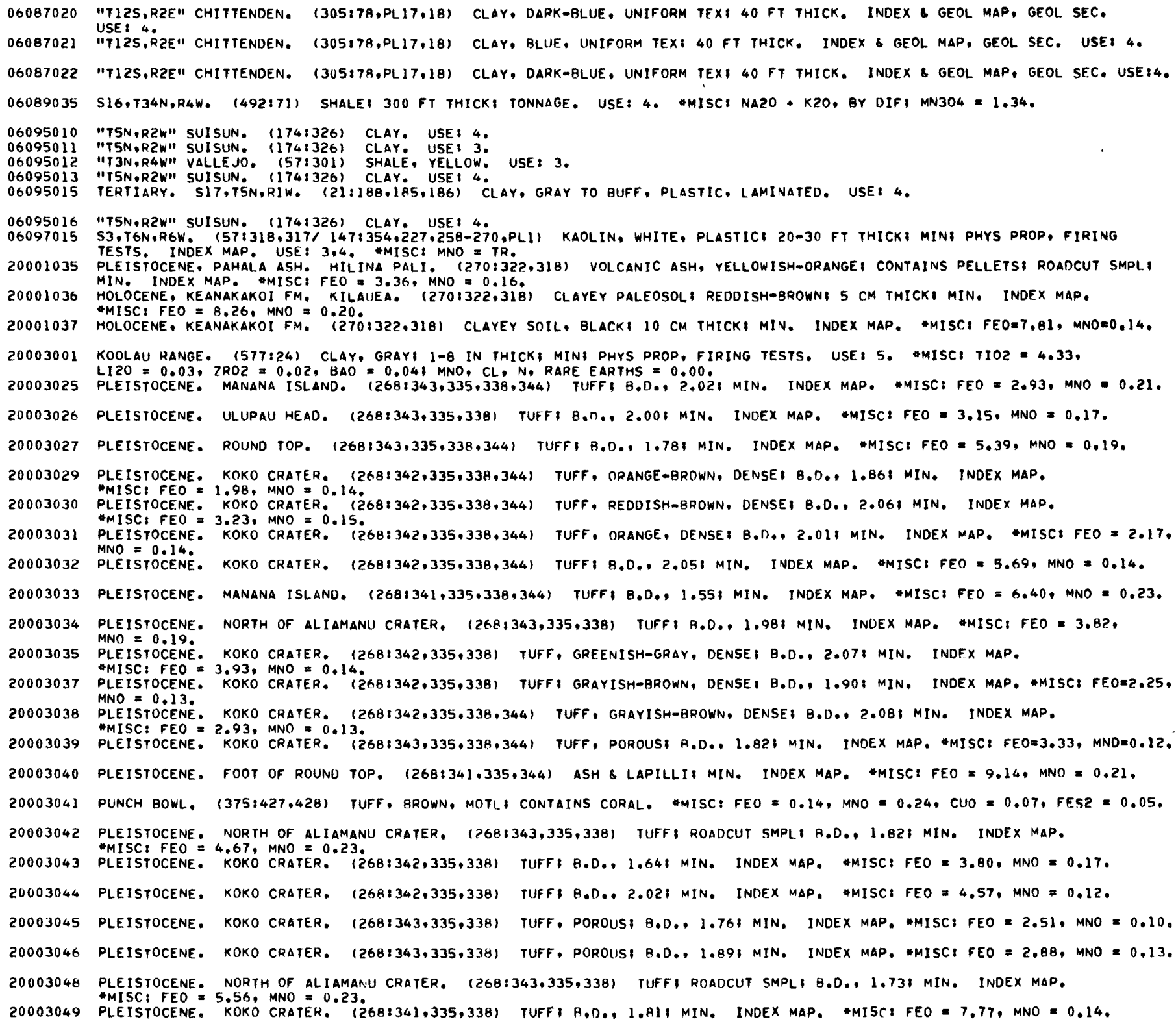


TABLE 6. - Analyses of samples from California and Hawaii containing 90-100 percent clay (Group $D_{6}$ ), from all rock categories

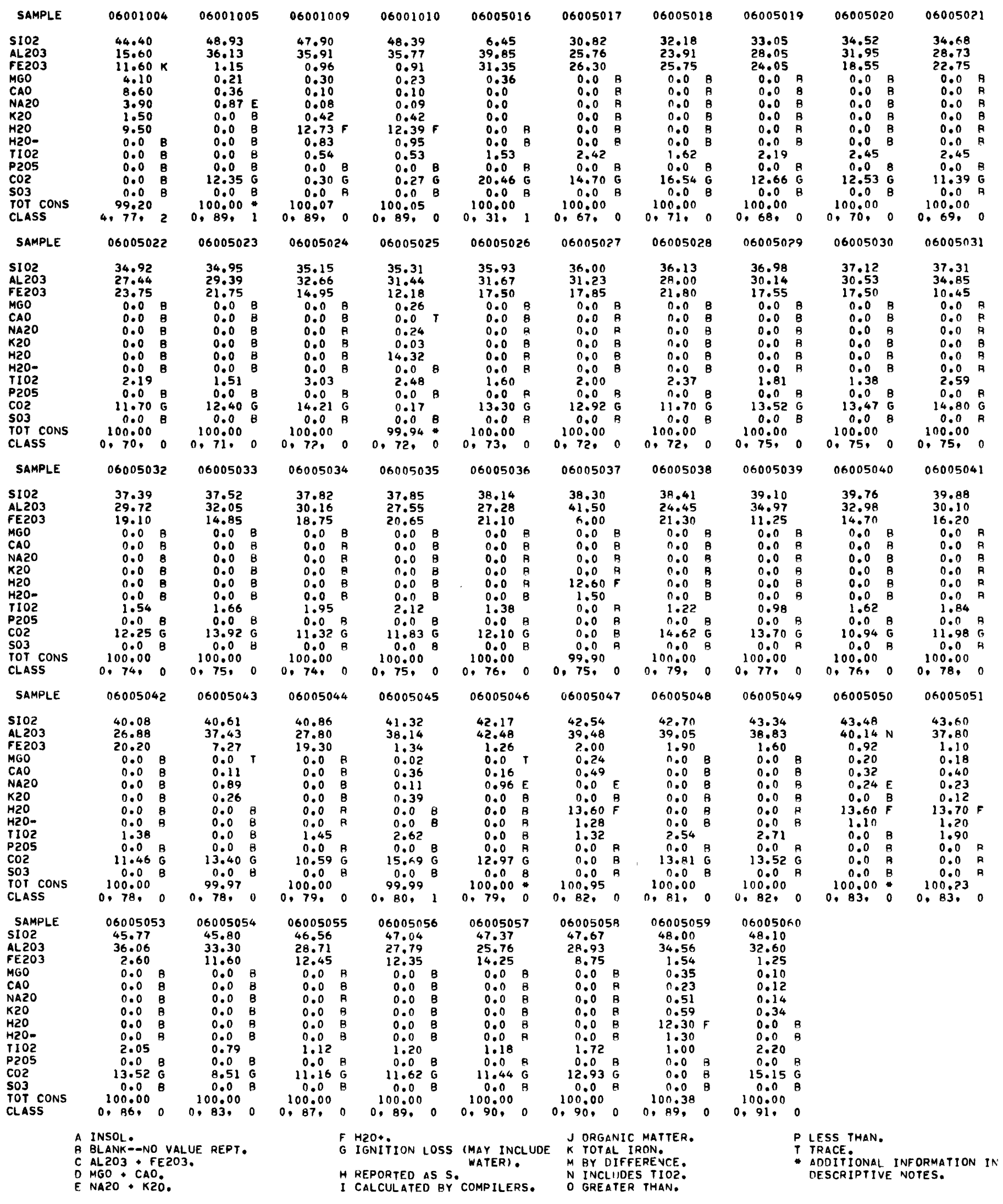


TABLE 6. - Analyses of samples from California and Hawaii containing 90-100 percent clay (Group $D_{6}$ ), from all rock categories - Continued

06001004 "TIS,R4W" BERKELEY. (2551102,101) BRECCIATED ROCK, LIGHT-COLORED.

06001005 EOCENE, TESLA FM. S26,T3S,R3E. (147:354,43-45,258-270,PL1/ 113:135,138) CLAY, WHITE, PLASTICI OUTCROP 6-8 FT THICK, PHYS PROP. FIRING TESTS. INDEX MAPS, GEOL SEC. USE: 3.5. "MISC: ALKALIES, BY OIF.

06001009 EOCENE. "S25,T3S,R3E" (4:277,273-275) CLAY, WHITE; MIN, INDEX MAP, MEAS SEC,

06001010 EOCENE. "S25,T3S,R3E" (4:277,273-275) CLAY, WHITE: MIN. INDEX MAP, MEAS SEC. USE: 5.

06005016 EOCENE, IONE FM. "TGN,R9E" IONE, $(37: 15,2,22$,PL1,2) LATERITE, MINI FIRING TEST. INTEX \& GEOL MAP, GEOL SEC. USE:5,14.

06005017 EOCENE, IONE FM. "S24,T5N,R9E" (417:39,3,17,33,PL1-3) CLAY, REN, BUFF, MOTL: OH, 1R4-194 FT: MIN. INDEX G GEOL MAPS,

06005018 GEOL SEC, USE : 14 .

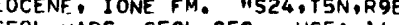
06005019 EEOL MAPS, GEOL SEC. USE: 14 .

06005020 EOCENE, IONE FM. S13,TSN,R9E.

06005021 EEOL SEC. USE: 14. "S24,TSN,R9E" GEOL MAPS, GEOL SEC. USE: 14 .

06005022 EOCENE, IONE FM. "S24,TSN, RQE" GEOL SEC, USE: 14 .

06005023 EOCENE, IONE FM. "S24, TSN,R9E"

06005024 GEOL MAPS, GEOL SEC. USE: 14." MAPS, GEOL SEC. USE: 14 .

06005025 EOCENF, IONE FM. S24,TSN,R9E. MISC: $F$ EO $=2.33$, I MISC: $F E O=2.33,1 . L=1.18$

06005026 EOCENE, IONE FM. "S24,TSN, R9E"

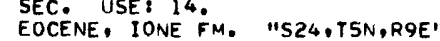

06005027 EOCENE, IONE FM. "S24.T5N, R9E" EOCENE , IONE FM. "S24, TSN, R9E" GEOL MAPS, GEOL SEC. USE: 14. EOCENE, IONE FM. "S24, TSN, RQE" GEOL MAPS, GEOL SEC. USE: 14 ." GEOL MAPS, GEOL SEC. USE: 14. EOCENE, IONE FM. "S24,TSN, R9E" MAPS. GEOL SEC. USE? 14.

06005028

06005029

06005030

06005031

06005032 EOCENE, IONE FM. "S24,TSN, R9E" GEOL SEC, USF: 14.

06005033 EOCENE, IONE FM. "S24,TSN, R9E" GEOL MAPS, GEOL SEC. USE: 14.

06005034 EOCENE, IONE FM. "S24,TSN, R9E" GEOL SEC, USE: 14 .

06005035 EOCENE, IONE FM." "S24,TSN,R9E"

SEC. USE: 14. 06005036 EOCENE, IONE FM. "S24, TSN, R9E"

$(417: 39,3,17,33, P L 1-3)$ CLAY, REN, BUFF, MOTL: OH, 1R4-194 FT MIN, INDEX \& GEOL MAPS
$(417: 39,3,17,33, \mathrm{PL} 1-3)$ CLAY, RED, WHITE, BUFF, MOTL, DH, 182-183 FT: MIN. INNEX \& (417:39,3-5,17,35.PL1-3) CLAY, RED, BUFF, MOTL; DH, 5-6 FTI MIN. INDEX \& GEOL MAPS. (417:38,17,28,PL1-3) CLAY, BUFF, RED,SANOYS DH, 169-169.5 FTI MIN. INDEX \& GEOL MAPS, (417:39,3,17,33,PL1-3) CLAY, RED, WHITE, BUFF, MOTL OH, 178-179 FTI MIN, INDEX \& (417:39,3,17,33,PL1-3) CLAY, RED \& WHITE: DH, 201-202 FT, MIN. INDEX \& GEOL MAPS, (417:39,3-5,17,35,PL1-3) CLAY, RED, YELLOW, PURPLE, MOTLI DH, 40-41 FT: MIN. INDEX \& (417:39.3.17,33,PL1-3) CLAY, REO, WHITE, MOTL: DH, 156-157 FT: MIN. INDEX \& GEOL (37:22,2,PLL.2) CLAY, RED MIN. INOEX MAPS, GEOL MAPS, GEOL SEC. USE: 3,14 . 1417:39,3-5,17,35,PL1-3) CLAY, RED, MOTL: DH, 31-32 FT MIN, INOEX \& GEOL MAPS, GEOL (417:39,3-5,17,35,PL1-3) CLAY, RED, BUFF, MOTL' DH, 20-11 FT: MIN. INDEX \& GEOL MAPS, (417:39,3,17,33.PL1-3) ClAY, RED, WHITE, AUFF, MOTL, DH, 176-177 FT; MIN, INDEX \& (417139,3,17,33,PL1-3) CLAY, RED, WHITE, BUFF, MOTLI DH, 180-181 FT, MIN, INDEX \& $(417839,3,17,33, P L 1-3)$ CIAY, REO, WHITE, BUFF, MOTL, OH, 196-197 FT, MIN. INDEX \& $(417: 39,3,17,33, P L 1-3)$ CLAY, BUFF, GRAY, MOTL; OH, 144-145 FT: MIN. INDEX \& GEOL 1417:39,3,17,33,PL1-3) CLAY, RED \& WHITE: DH, 199-200 FTI MIN. INOEX \& GEOL MAPS, (417:39,3-5,17,35,PL1-3) CLAY, RED, BUFF, PURPLE, MOTL: OH, 15-16 FT, MIN. INOEX \& (417:39,3,17,33,PL1-3) CLAY, YELLOW, RED: DH, 178-179 FT: MINI INOEX \& GEOL MAPS, (417:39,3,17,33,PL1-3) CLAY, RED, WHITE; DH, 218-219 FT: MIN. INOEX \& GEOL MAPS, GEDL

06005037 TERTIARY. "TGN,R9E" IONE. (3:383,355,357,384) LATERITE, RED, BUFF, MASSIVE. INOEX S GEOL MAPS, MEAS SEC, GEOL SEC. 06005038 EOCENE, IONE FM. S7,T5N,R10E. $1417: 38,17,27, P L 1-31$ CLAY, RED, BUFF, MOTII DH, 226-227 FTI MIN. INDEX \& GEOL MAPS, 06005039 EEOL SEC. USE: 14. IONE FM. "S24, T5N, R9E" EOCENE, IONE FM.

06005040 EOCENE, IONE. FM. "S24,TSN,R9E" GEOL SEC. USF: 14 .

06005041 EOCENE, IONE FM. "S24,TSN,R9E" USE: 14 .

06005042 EOCENF, IONE FM. "S24, TSN, R9E"

06005043 EOCEENE. TGN.R9E, IONF. $(1471355,18.53, P L 1,6)$ LATERITES PHYS PROP, FIRING TESTS. INDEX MAP. USE: 19

06005044 EOCENF, IONE FM. "S24,T5N,R9E" (417:39,3,17,33,PL1-3) CLAY, BUFF, PURPLE, MOTL, DH, 180-181 FT\& MIN. INDEX \& GEOL 06005045 MAPENE. IONE FM. "TGN,H9E" IONE. $(37: 22,2,6 . P L 1,2)$ CLAY: AVG OF 4 ANAL: MIN: FIRING TESTS. INDEX \& GEOL MAPS, GEOL 06005046 EOCENE, IONE FM. T6N, K9E, IONF, $1147: 354,53,272-276, P L 1 / 7113,2)$ CLAY, MED-GR, PLASIICI PHYS PROP, FIRING TFSTSI 06005047 EOCENE, IONE FM. "TGN,R9E" IONE. $(3: 381,355,384)$ CLAY, WHITE: MIN, INDEX \& GEOL MAPS, MEAS SEC, GEOL SEC.

06005048 EOCENE, IONE FM. "S24,TSN,R9E" $(417: 39,3,17,33, P L 1-3)$ CLAY, WHITE, SILTY DH, 129-130 FT MIN. INDEX \& GEOL MAPS,

06005049 EOCENE. IONE FM. "S24,TSN,R9E" $(417: 39,3,17,33$, PLI-3) CLAY, WHITE, SANOY OH, 124-125 FT: MIN. INDEX \& GEOL MAPS,

06005050 EOCENE, IONE FM. "TGN,R9E" IONE. $(38381,355,384)$ CLAY, WHITEI MIN. INDEX \& GEOL MAP, MEAS SEC, GEOL SEC. *MISC: NAZO + K2O BY DIF.

06005051 EOCENE, IONE FM. "T6N,R9E" IONE. (3:381,355,384) CLAY, WHITE\& MIN. INNEX \& GEOL MAPS, MEAS SFC, GEOL SEC.

06005053 EOCENE, IONE FM. S13,TSN,R9E. (417:39,17,29,PL1-4) CLAY, GRAY, DH, 154-155 FTI MIN. INOEX \& GEOL MAPS, GEOL SEC. 06005054 USERASSIC. AMADOR GR. S7,T5N.R10E. (417838,13,27,PL1-3) GREENSTONE, WEATHEREDI DH. 263-264 FT. INDEX \& GEOL MAPS, GEOL SEC.

06005055 EOCENE, IONE FM. "S24,TSN,R9E" (417:39,3-5,17,35.PL1-3) CLAY, RED, YELLOW, PURPLE, MOTL, SANOY, OH, 45-46 FTI MIN. INDEX \& GEOL MAPS, GEOL SEC. USE: 14 .

06005056 EOCENE, IONE FM. S7,TSN,RIOE, (417:38,17.27,PL1-3) CLAY, REO, BUFF, MOTLI DH, 228-229 FTI MIN. INDEX \& GEOL MAPS. GEOL SEC. USE: 14 . 06005057 EOCENE, IONE FM. S18,T5N,R1OE. (417:39,17,3n,PL1-3) CLAY, RED, RUFF, MOTL\& CONTAINS IRON NODULES; OH, $228-229$ FT:

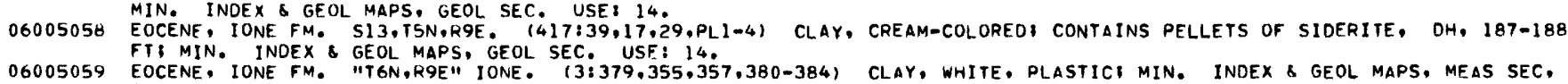

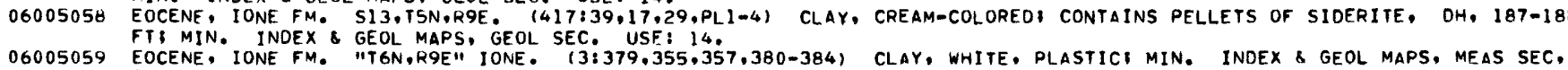
06005059 EOCENE, IONE FM. 06005060 EOCENE. IONE FM. "TGN,R9E" IONE. (37:22,4,25,26) CLAY, REDI MIN\& FIRING TESTS. INDEX \& GEOL MAPS. USE: 3,5. 
TABLE 6. - Analyses of samples from California and Hawaii containing 90-100 percent clay (Group $D_{6}$ ), from all rock categories- Continued

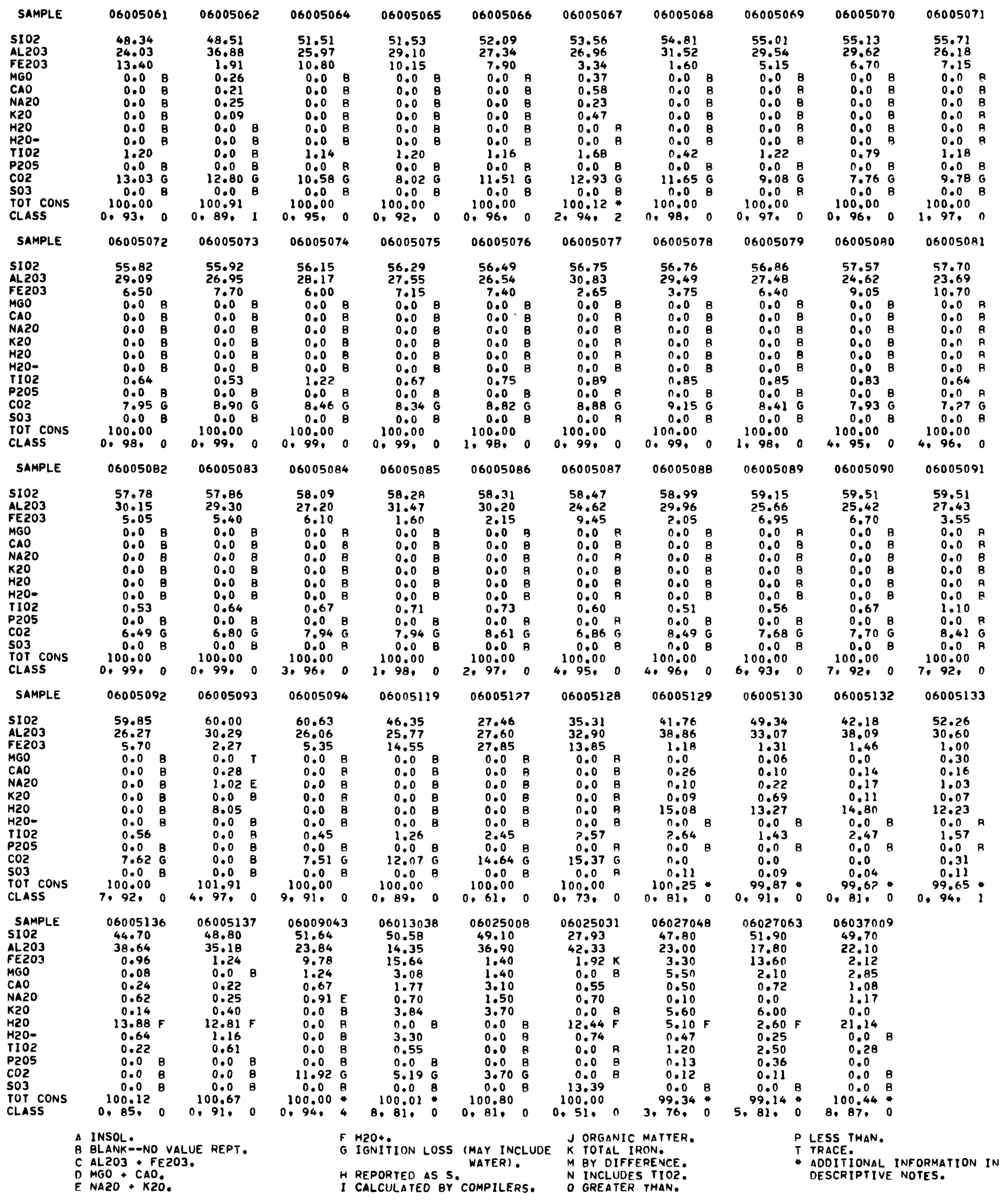


TABLE 6. - Analyses of samples from California and Hawaii containing 90-100 percent clay (Group $\left.D_{6}\right)$, from all rock categories- Continued

06005061 EOCENE, IONE FM. S7,T5N,RIOE. (417:38,24,27,PL1-3) CLAY, RED, RUFF, MOTL. SANDY OH, 220-221 FT: MIN. INDEX \& GEOL 06005062 EOCENE. T6N,RIOE, BUENA VISTA. (147:354,18.59,280-285,PL1) CLAY, YELLOW, MEO-GRI 6-10 FT THICK PHYS PROP, FIRING 06005064 EOCENE, IONE FM. S7,T5N,RIOE, $(417138,17.27$, PL1-3) CLAY, RED, BUFF, MOTLI DH, $222-223$ FT: MIN. INDEX \& GEOL MAPS, 06005065 EOCENE, IONE FM. "S24,T5N,R9E" $1417: 39,35, \mathrm{PL} 1-3)$ CLAY, BUFF: DH, 157-158 FT. INDEX \& GEOL MAPS, GEOL SEC.

06005066 EOCENE, IONE FM. S7,T5N,RIOE. (417838,17.27,PL1-3) CLAY, BUFF, SILTYI CONTAINS SIDERITE NODULES DH, 215-216 FT, MIN. 06005067 EOCENE, IONE FM. "IGN,R9E" IONE, (306:497,491-495) CLAY DH SMPL BEDS 13-196 FT THICK. INDEX \& GEOL MAP. USE: 3.5. 06005068 EOCENE, TIONE INCL ZRO2. 06005069 EOCENE, IONE FM. S7.T5N,RIOE. 1417138,17, PLI-3) CLAY, BUFF, DH. 230-231 FTI MIN. INDEX \& GEOL MAPS, GEOL SEC. 06005070 EOCENE. IONE FM. S13,T5N,R9E. (417:38,3-5,17,PLI-4) CLAY, RED, SANDY, DH. $277-278$ FT, MIN. INDEX \& GEOL MAPS, 06005071 EOCENE. S18.T5N,RIOE. (417:38,30,PLI-3) CLAY, BUFF, SILTY, OH, 253-254 FT. INDEX \&EOL MAPS, GEOL SEC. 06005072 EOCENE, IONE FM. S13,T5N,R9E. (417:38,3-5,17,PL1-4) CLAY, REO B BUFI OH, 283-284 FT, MIN. INDEX \& GEOL MAPS,

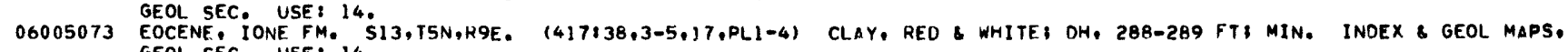
06005074 EOCENE. IONE FM. S7,T5N,RIOE. (417:38,17.27,PL1-3) CLAY, RED, BUFF DH, $139-140$ FT, MIN. INDEX \& GEOL MAPS, GEOL. 06005075 EOCENE. IONE FM. S13,T5N,R9E. (417:38,3-5,17,PL1-4) CLAY. RED \& BUFF: OH. $281-282$ FT: MIN. INDEX \& GEOL MAPS, 06005076 EOCENE, IONE FM. S13,T5N,R9E. $(417838,3-5,17, P L 1-4)$ CLAY, RED \& BUFF, DH. $282-283$ FT: MIN. INDEX \& GEOL MAPS, 06005077 EOCENE, IONE FM. S13,TSN,R9E. (417:38,3-5.PL1-4) CONGLOMERATE, DH, 298-299 FTI MIN. INDEX \& GEOL MAPS. GEOL SEC. 06005078 EOCENE, IONE FM. S24,T5N,R9E. (417:39. PLI-3) CLAY, GREENISH-BIFF, DH, 114-115 FT. INDEX \& GEOL MAPS, GEOL SEC. 06005079 EOCENE, IONE FM. S13,T5N,R9E. (417:38,3-5,17,PLI-4) CLAY, RED, SANDY: OH, 276-277 FTI MIN. INDEX GEOL MAPS, 06005080 EOCENE, IONE FM. S13,T5N,R9E. (417:38,3-5,17,PL1-4) CLAY, RED, YELLOW, WHITE. MOTL. SANDY DH, 285-286 FT: MIN. 06005081 EOCENE, IONE FM. S13,TSN,R9E. (417138,3-5,PL1-4) CLAY, YELLOW, SANDY, DH, $291-292$ FT: MIN. INDEX \& GEOL MAPS. GEOL 06005082 EOCENE. IONE FM. S13,T5N,R9E. (417:38,3-5,17,PL1-4) CLAY, RED \& BUFF, DH, $284-285$ FTI MIN. INDEX \& GEOL MAPS, 06005083 EOCENE. IONE FM. ${ }^{2}$ S13,T5N,R9E. $(417338,3-5,17$, PLI-4) CLAY, RED, SANOY DH, 279-280 FT, MIN. INDEX GEDL MAPS, 06005084 EOCENE, IONE FM. S13,T5N,R9E. $(417138,3-5,17, P L 1-4)$ CLAY, RED \& BUFF: DH, 280-281 FT: MIN. INDEX \& GEOL MAPS, 06005085 EOCENE, IONE FM. S13,T5N,R9E. (417:38,3-5.PLI-4) CONGLOMERATE; DH, 300-301 FT: MIN. INDEX \& GEOL MAPS. GEOL SEC. 06005086 EOCENE, IONE FM. S13,T5N,R9E. (417:38,3-5,PL1-4) CONGLOMERATE\& DH, 299-300 FT: MIN. INDEX GEOL MAPS. GEOL SEC. 06005087 EOCENE, IONE FM, S13,TSN,R9E. $(417138,3-5, P L 1-4)$ CLAY, YELLOW, SANDY DH, 292-293 FT3 MIN. INDEX \& GEOL MAPS, GEOL 06005088 EOCENE, IONE FM. SI3,T5N,R9E, (417:38,3-5,PL1-4) CONGLOMERATE; DH, 297-298 FT; MIN. INDEX \& GEOL MAPS, GEOL SEC. 06005089 USE: 14. 06005090 EOCENE, IONE FM. S13,15N,H9E, $(417: 38,3-5,17, P L 1-4)$ CLAY, RED. YELLOW. WHITE. MOTL, SANOY OH, 286-287 FT, MIN. 06005091 EOCENE. ST,TSN,RIOE. (417:38,27,PLI-3) ARGILLACEOUS SILTSTONE OH, $235-236$ FT. INDEX G GEOL MAPS. GEOL SEC. 06005092 EOCENE, IONE FM. S13,T5N,R9E. (417:38,3-5,17,PL1-4) CLAY, RED, YELLOW, WHITE, MOTL. SANDY, DH, 287-288 FT, MIN. 06005093 "TTNERIOE" CARBONDALE. $(21: 207,206)$ CLAY. 06005094 EOCENE, IONE FM. SI3,T5N,R9E. (417138,3-5,17,PLI-4) CLAY, WHITE, SANDY, DH, $289-290$ FT: MIN. INDEX \& GEOL MAPS. 06005119 EOCENE, IONE FM. S7,T5N,R10E. (417:38,17,27,PL1-3) CLAY, RED, BUFF, MOTL) DH, $224-225$ FT MIN. INDEX \& GEOL MAPS. 06005127 EOCENE, IONE FM. "S24,TSN,R9E". $(417139,3,5,26,33, P L 1-3)$ CLAY, REO \& WHITE MOTL: CONTAINS IRON NODULESI

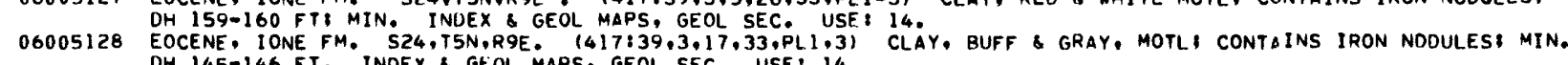
06005129 EOCENE, IONE FM. "T6N,R9E" NEAR IONE. (315111,12) CLAY PHYS PROP. "MISC: FEO = 0.15, VZOS = O.023 HZO REPT AS 1.L. 06005130 EOCENE, IONE FM. "TGN,R9E" NEAR IONE, $(315: 11,12)$ CLAY PHYS PROP. "MISC: FEO = 0.28, VZO5 = O.01: H20 REPT AS I.L. 06005132 EOCENE, IONE FM. "TGN,R9E" NEAR IONE. $(315: 11,12)$ CLAYI PHYS PROP. "MISC: FEO = 0.14, VZOS = 0.02 H2O REPT AS I.L. 06005133 EOCENE, IONE FM. "TGN,R9E" NEAR IONE, $(315111,12)$ CLAY PHYS PROP. "MISC: FEO = 0.00. VZO5 = 0.011 HZO REPT AS 1.L. 06005136 "T6N,R9E" IONE. (400:56) KAOLINITE. 06005137 "TGN,R9E" IONE, (400:56) ANAUXITE.
06009043 EOCENE, IONE FM, "T6N,R9E" VALLEY SPRINGS, $(147: 355,68,337-346, P L 1)$ CLAY, PINK, MOTL, FINE-GR, PLASTIC, PHYS PROP.

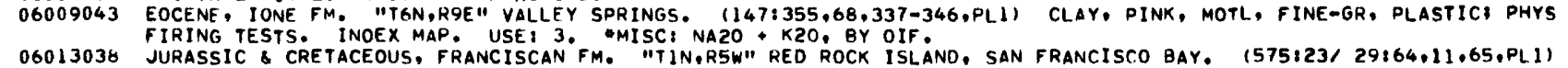
06013038 JURASSIC \& CRETACEOUS, FRANCISCAN FM. "TIN,R5W" RED ROCK ISLAND, SAN FRANCISCO
SHALE, RED MIN. INOEX \& GEOL MAPS. USE: 13 . "MISC: FEO $=0.65$, MNO 0.36 .

06025008 S24,T125,R19E. (447:131,PL1) CLAY, WHITE: 100 FT THICK. INDEX MAP, USE: 14.

06025031 TERTIARY, "TIOSPRI6E" IRIS. (43:47.48/ 497:269.268) CLAY, WHITE.

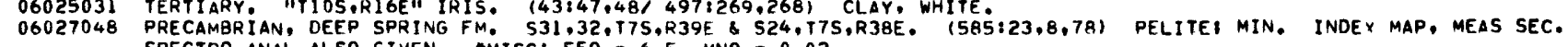

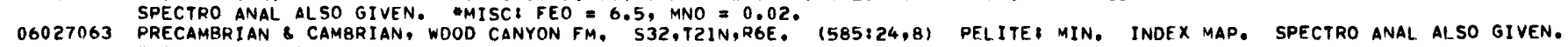

06037009 "TISQR8W" CLAREMONT. $(3541263)$ MONTMORILLONITE SELECTEO SMPL. "MISC: MNO =TR. 
TABLE 6. - Analyses of samples from California and Hawaii containing 90-100 percent clay (Group $\left.D_{6}\right)$, from all rock categories - Continued

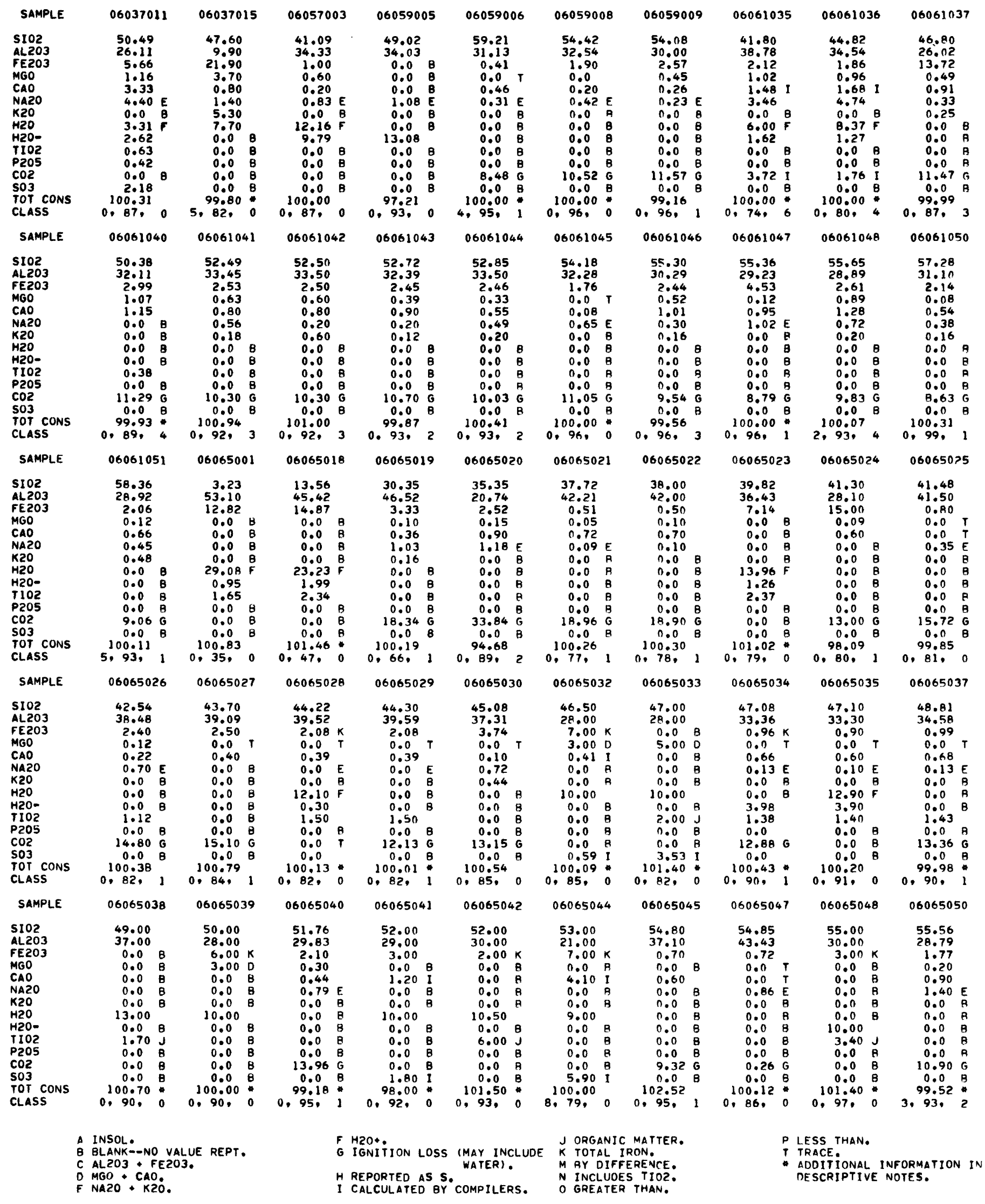


TABLE 6. - Analyses of samples from California and Hawaii containing 90-100 percent clay (Group $D_{6}$ ), from all rock categories - Continued

06037011 MIOCENE, MONTEREY FM. "TlS,RI2W" LOS ANGELES. (43:56) SHALE, BLUE, THIN-BEDded, USE: 3.

06037015 PLEISTOCENE. "T5S,R14W" SAN PEDRO. (108296) SHELL MARL. UNCONSOLIDATED. "MISC: FEO $=1.5$.

06057003 "T16N,RBE" NEVADA CITY. (3:386) HALLOYSITE, MICACEOUS.

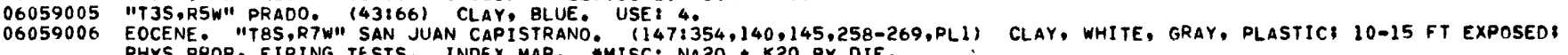

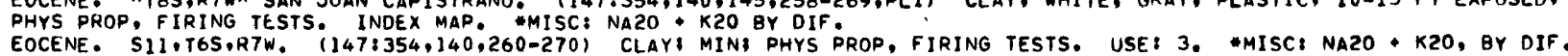

06059008 EOCENE. S11,T6S,R7W. $(147: 354,140,260-270)$ CLAY MIN, PHYS PROP, FIRING TESTS, USE: 3. *MISC: NA20 + K20, OY DIF,
06059009 PALEOCENE, MARTINEZ FM. T5S,R7W, SERRANO MINE. $(478185,81,83$,PL1) CLAY, WHITE OR PINK, PLASTIC: MIN. INDEX MAP.

06061035 "TI2N,R6E" LINCOLN, (256:109) CLAY, WHITE. "MISC, CO2 INCL I.L.. 2.56.

06061036 "TI2N,R6E" LINCOLN. (256:109) CLAY, BLUE, PLASTIC. *MISC: CO2 INCL I.L.. 0.44.

06061037 EOCENE, IONE FM. S4,TI2N, R6E. $\{1478355,147,149,158,336-346$, PLI $)$ CLAYI 6 FT THICK, PHYS PROP, FIRING TESTS. INDEX

06061040 EOCENE, IONE FM, S4,9,10,T12N,R6E. (43:68,67) CLAY, WHITE, PHYS PROP, FIRING TESTS: MEAS SEC. USE, 3,5,

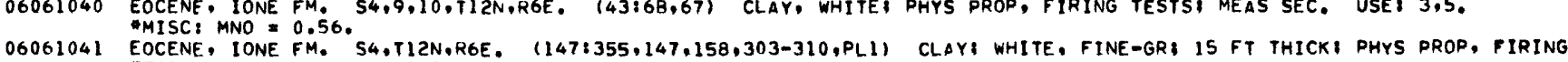

06061041 EOCENE, IONE FM. S4,TILN,R6E, (147:355,147,158,303-310,PL1) CLAY WHITE, FINE-GR

06061042 "TIZN,R6W" LINCOLN DIST. (148:204,205) CLAY, PLASTICI FIRING TESTS. USE: 3,5.

06061043 EOCENE, IONE FM. S4,T12N,R6E. (1471355,147,149,158,291-296,PL1) CLAY: 22 FT THICK: PHYS PROP, FIRING TESTC. INDEX

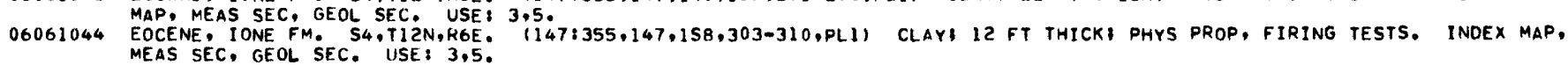

06061045 SI2,24,TI3N,RGE. (147:355,159,292-296,PL1) KAOLIN, WHITE, PLASTIC, PHYS PROP, FIRING TESTS. INDEX MAP. USEI 3.5. MISC: NAZO K KZO, BY DIF.

06061046 EOCENE, IONE FM. S4,TI2N,R6E. (147:355,147,149,158,298-308,PL1) CLAY: 8 FT THICK PHYS PROP, FIRING TESTS. INDEX MAP, MEAS SEC, GEOL SEC. USE: 3.5 .

06061047 EOCENE. IONE. FM. S4,T12N, R6E. $1147: 355,147,149,158,291-296, P L 1)$ CLAY, SANDY, PLASTIC: B FT THICK: PHYS PROP, FIRING TESTS. INDEX MAP, MEAS SEC, GEOL SEC. USE: 3.5. MISC: NAZO + KZO, QY DIF.

06061048 EOCENE, IONE FM. S4,TI2N,R6E. (147:355,147,149,158,291-296,PL1) CLAY, SANDY, PLASTICI 8 FT THICK, PHYS PROP, FIRING

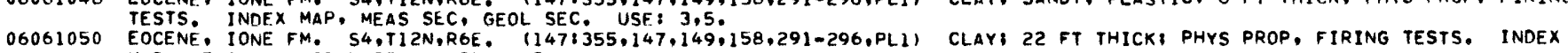
06061050 EOCENE. IONE FM. S4, T12N, R6E; 114713

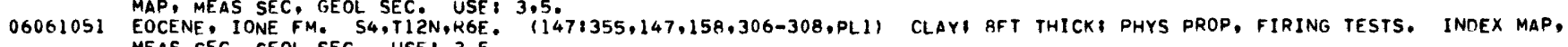

06065001 MEAS SEC, GEOL SEC. USE: 305. (165:121) BAUXITE, RED, PISOLITIC.

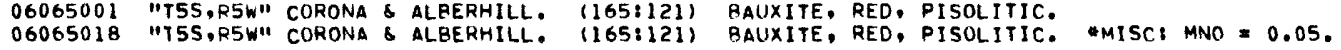

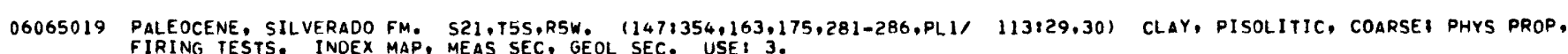

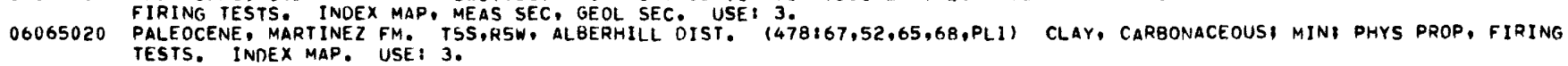

06065021 PALEOCENE, SILVERADO FM. S22, T5S, R5W. (185:89,77-86,PL1-6/ 566:1173) CLAY, CARBONACEOUS. PLASTIC: R FT THICK:

06065022 "TSS, R5W" ALBERHILL. (I48:204,205) CLAY, PLASTIC: FIRING TESTS.

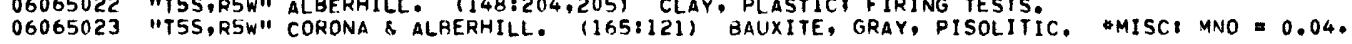

06065024 TERTIARY. "T65,R4W" ELSINORE. 1388:574/43:86,75,85) CLAY, RED.

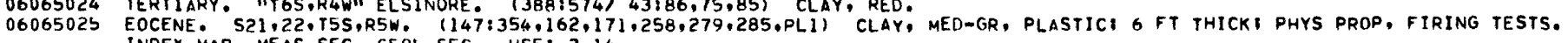

06065026 INDEX MAP, MEAS SEC. GEOL SEC. USE: $3,14$.

06065026 TERTIARY. "TGS,R4W" ELSINORE. (3883574/43886,75) CLAY, WHITE, USE: 5.

06065027 TERTIARY, "T6S,R4W" ELSINORE, (388:574/43:86,75) CLAY, WHITE, PISOLITIC.

06065028 PALEOCENE: S22, TSS,R5W. $(285: 203,186,196,185: 89,77-86,941-6)$ CLAYI PHYS PROP. FIRING TESTS. INDEX \& GEOL MAPS, GEOL 06065029 EOCENE. T5S,R6W, ALBERHILL. $(147: 354,136,163,277-285, P L 1)$ CLAY, MEU GR: 4 FT THICK. PHYS PROP, FIRING TESTS. INDEX

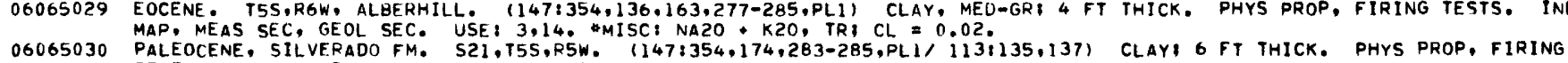
06065030 PALEOCENE. SILVERADO FM. S21, T5S,R5W. $(147: 354,174,283-285, P L 1 / 113: 135,137)$ CLAY 6 FT THICK. PHYS PROP, FIRING
06065032 TESTS. INDEX MAP, MEAS SEC, GEOL SEC, USE: 3,5.

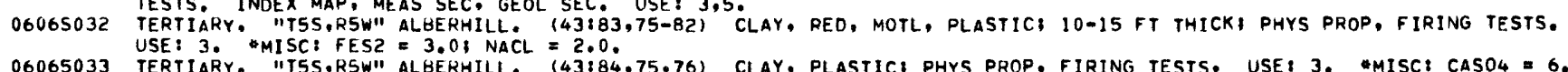
06065033 TERTIARY. "TSS,RSW" ALBERHILL. $(43884,75,76)$ CLAY, PLASTIC: PHYS PROP, FIRING TESTS. USE: 3. "MISC: CASO4 = 6.0, 06065034 TERTIARY. "T5S,R5W" ALBERHILL. (285:198,185,186,196) CLAY, GRAY, PLASTICI PHYS PROP. FIRING TESTS. INDEX MAP. GFOL

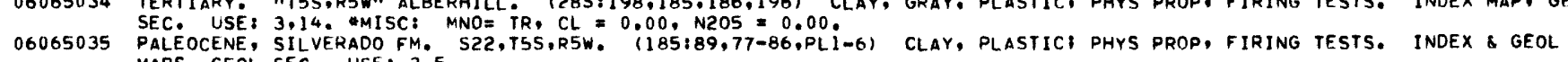
PALEOCENE, SILVERADO FM. S22,TSS,R5W. $(185: 89,77-86, P L 1-6)$ CLAY, PLASTIC PHYS PROP, FIRING TESTS. INOEX
06065037 EOCENE. TSS,R6W, AL8ERHILL. (147:354,136,162,163,168,PL1) CLAY: PHYS PROP, FIRING TESTS. INDEX MAP. GEOL SEC, MEAS 06065037 EOCENE. TSS,R6W, AL8ERHILL. (147:354,136,162,163,168,PLI) CLAY: PHYS PROP, FIRING TESTS. INDEX MAP, GEOL SEC, MEAS
06065038 TERTIASE: 5, "MISC: MNO = TR. ALKALIES BY DIF. 06065038 TERTIARY, "TSS,RSW" ALBERHILL. $(43883,75-82)$ CLAY, GRAY: 4 FT THICK PHYS PROP, FIRING TESTS.
06065039 PALEOCENE. S22,T5S,R5W. $(43883,77) 185: 88,77,79, P L 1-6)$ CLAY, RED, MOTL, PLASTIC; 10-15 FT THICK: PHYS PROP, FIRING 06065039 PALEOCENE. S22,T5S,RSW. $143883,77 / 185: 88,77,79, P L 1-6)$ CLAY, RED
TESTS. INDEX \& GEOL MAPS, GEOL SEC. USE: 3. HMISC: FES2 3.0 . 06065040 EOCENE. S2?,T5S,R5W. $11471355,162,176,298,306-308$, PLI) CLAY, FINE-GR, PLASTICI 7 FT THICKI PHYS PROP, FIRING TESTS. 06065041 IERTIARY, "ISS,R5W" ALBERHILL. $(43: 84,75,76,80)$ CLAY, PLASTIC, 5-6 FT THICK PHYS PROP, FIRING TESTS. USE: 3,5. 06065042 "TERTIARY. "TSS,RSW" ALBERHILL. $(43: 83,75.76 .82)$ CLAY, DARK-GRAY, PLASTIC PHYS PROP. FIRING TESTS. "MISC: FES2 = 1.0, 06065044 TERTIARY, "TSS,RSW" ALBERHILL. $(43: 84,76)$ CLAY. PLASTIC: 12-18 FT THICK: PHYS PROP, FIRING TESTS.

06065045 TERTIARY. "TSS,R5W" ALBERHILL. (43:86,75,85) CLAY, SANDY.

06065045 TERTIARY. "TSS,R5W" ALBERHILL: $(43186,75,85)$ CLAY, SANDY,
06065047 EOCENE. S21,22, 15S,R5W. $(147: 354,162,163,171,272-276, P L 1)$ CLAY, PHYS PROP, FIRING TESTS. INDEX MAPS, GEOL SEC. 06065048 UERTIARY. "TSS,RSW" ALGERHILL. (43184,76,82) CLAY, GRAY, PLASTICI PHYS PROP, FIRING TESTS. \&MISC: ORG MATTER CALC 06065050 FROM C.

EOCENE. S21,22,T5S,R5W. (147:355,162.163.168-173.298-308,PL1) CLAY,WHITF, FINE-GR: PHYS PROP, FIRING TESTS. INDEX MAPS, MEAS SEC, GEOL SEC. USE: 3. MISC: NA20. K20, BY DIF. 
TABLE 6. - Analyses of samples from California and Hawaii containing 90-100 percent clay $\left(\right.$ Group $\left.D_{6}\right)$, from all rock categories -Continued

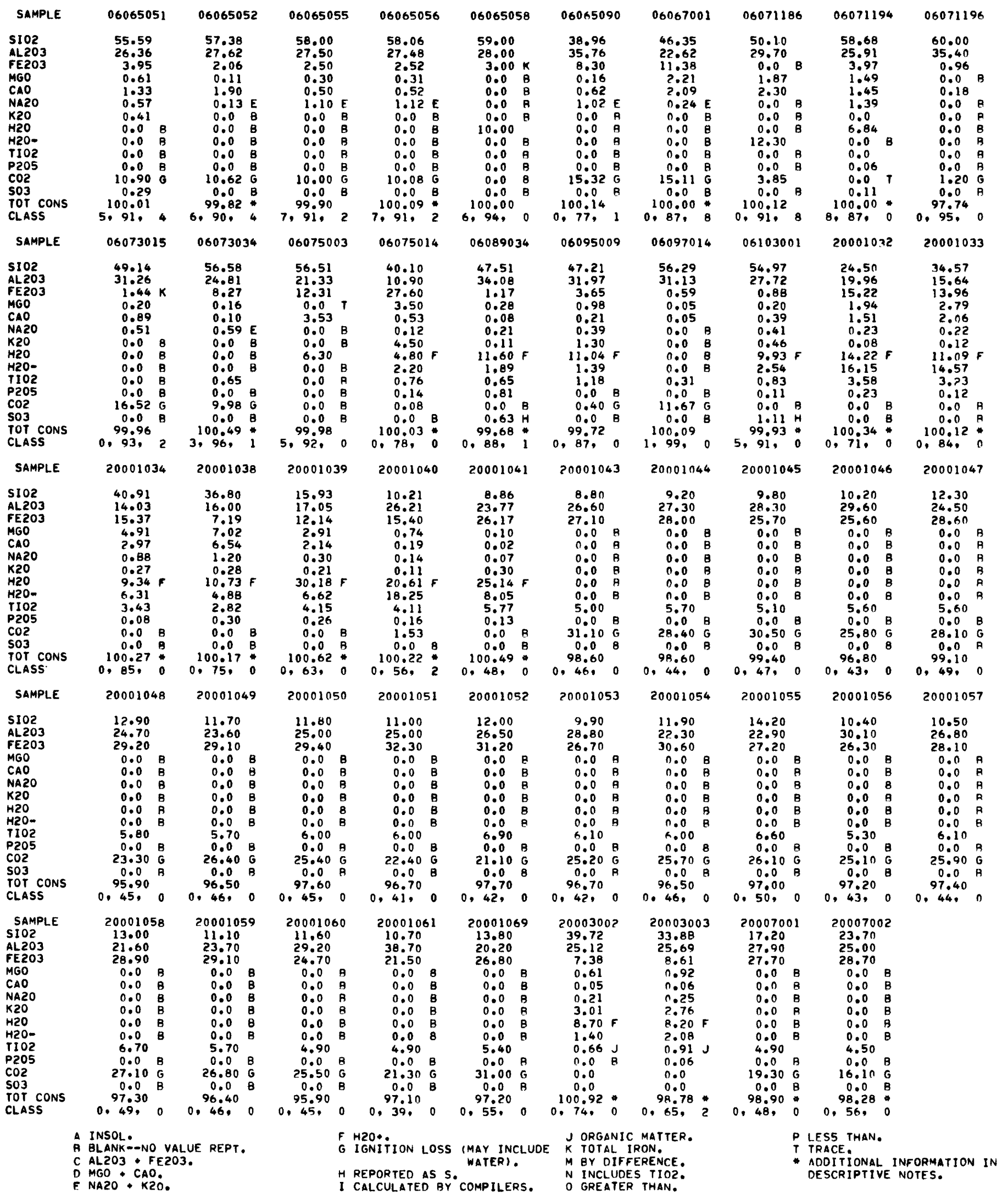


TABLE 6. - Analyses of samples from California and Hawaii containing 90-100 percent clay (Group $\left.D_{6}\right)$, from all rock categories - Continued

06065051 EOCENE. T5S,R5W, ALAERHILL, (147:854,162-169,293-295,PL1,10) CLAY, WHITE, FINE-GR, PLASTIC, 8 FT THICK, PHYS PROP,

06065052 PIRING TESTS, INDEX MAPS. MEAS SEC, GEOL SEC. USE: 5. PHYS PROP, FIRING TESTS. INDEX \& GEOL MAP. GEOL SEC, USE: 3, AMISC: NAZO K20, BY DIF.

06065055 PALEOCENE, SILVERADO FM. S22,T5S,R5W. (185:88,77-81,PL1-6) CLAY; PHYS PROP, FIRING TESTS: 20 FT THICK. INDEX \& GEOL 06065056 EOCENE. S21,22,T5S,R5W. $(1471354,162,163.171,272-276, P L 1)$ CLAY: PHYS PROP, FIRING TESTS. INDEX MAPS, GEOL SEC. EOCENE. S21,22,T5S,R5W, $(1471354,162,163 \cdot 171,272-276, P L 1)$ CLAY: PHYS PROP, FIRING TESTS
USE: 3. MISC: NA20 + K20. BY DIF.

06065058 TERTIARY. "T5S,R5W" ALBERHILL. (43:83,75,76) CLAY, PLASTICI PHYS PROP, FIRING TESTS.

06065090 EDCENE. "TSS.R5W" ALPERHILL. (S66:1173) BAUXITE: 2 FT THICK: FIRING TESTS. USE: 14.

06067001 "S7,TSN,RIOE" (147:355,186,337-346) CLAY, FINE-GR: PHYS PROP, FIRING TESTS. USE: 3. "MISC: ALKALIES BY DIF.

06071186 "T1S,R4W" COLTON. (21:175) CLAY. USE: 4.

06071194 "TION,R2W" GARSTOW. (567:S) RENTONITE, WHITE, MISC, CL $=0.10$.

06071196 S4.T6N.R9E. (507:512) CLAY BEDS 50 TO 100 FT THICK.

06073015 S29,T145,R2E. (4961354, 1471354,201,267-269,PLI) KAOLIN, WHITE: MIN, 50 FT THICK PHYS PROP, FIRING TESTS. INDEX MAP. USE: 5 .

06073034 TERTIARY. "TI2S,R3W" CARLSBAD, (147:355/ 364:776) CLAY, YELLOW: FINE-GR, DENSE, PLASTICI PHYS PROP, FIRING TESTS.

06075003 INDEX MAP. USE: 3. "MISC: NAZO + K2O, BY DIF.

06075014 JURASSIC \& CRETACEOUS, FRANCISCAN FM. "S31.T2S,RSW" $(29164,65$, PLI) GHALE, RED? MIN, ROADCUT SMPL. INDEX \& GEOL MAPS.

$060 R 9034$ S26,27,T30N,RSE. (141:119,55,R8/543:285,200,282-284) SOLFATARIC MUD, CREAM, WHITEI MIN. INDEX G GEOL MAPS, GEOL

06095009 EOCENE. "T4N,RZE" RIO VISTA. (4:284,273) CLAY. GRAY: MIN: AT 4,309-FT DEPTH.

06097014 S3,T6N,R6W. 157:318,317/ 147:354,227,258-270,PL1) KAOLIN, WHITE, PLASTIC, 20-30 FT THICK: MIN, PHYS PROP, FIRING

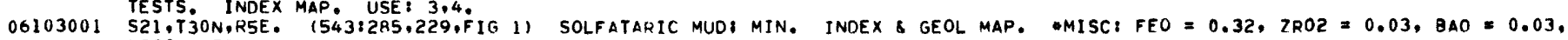
20001032 PLEISTOCENE. PAHALA ASH. NEAR JUNCTION OF WRIGHT RD \& HWY 11. (270:322,318) PALAGDNITIC TUFF: MIN. INDEX MAP. 20001033 PLEISTOCENE, PAHALA ASH. NEAR PAHALA. $(270: 322,318)$ PALAGONITIC TUFF: MIN. INDEX MAP. 20001034 PLEISTOCENE. PAHALA ASH. NEAR HONUAPO. $(270: 322,318)$ VOLCANIC ASH, YELLOWISH-ORANGF CONTAINS PELLETS, ROAOCUT SMPL, 20001038 PLEISTOCENE. PAHALA ASH. NEAR JUNCTION OF WRIGHT RD 8 HWY 11 . $1270: 322,3181$ CLAYEY ASH, WEATHERED 10 CM THICK MIN. 20001039 PLEISTOCENE, PAHALA ASH. NEAR JUNCTION OF HWY 11 \& TWENTY-TWO MILE RD. (270:322,318) CLAYEY ASH: WEATHERED MIN. 20001040 PLEISTOCENE. PAHALA ASH. NEAR JUNCTION OF HWY 11 \& TWENTY-TWO MILE RD. (270:322,318) CLAY ROADCUT SMPL: 200010411.5 M THICK: MIN. INDEX MAP. JMISC: FEO $=2.50$, MNO $=0.06$. 20001041 PLEISTOCENE, PAHALA ASH. AKAKA FALLS, EAST SLOPE OF MAUNA KEA. $(2708322,318)$ CLAY, 1.5 M THICK, MIN. INDEX MAP. 20001043 WEST OF HILO BAY. (75:21-23) FERRUGINOUS BAUXITE. CHANNEL SMPL FROM ROADCUT: MIN. INDEX MAP. SPECTRO DATA ALSO GIVEN. 20001044 WEST 16 . HILO BAY. (75:21-23) FERPUGINOUS BAUXITE, CHANNEL SMPL FROM ROADCUT: MIN. INDEX MAP. SPECTRO DATA ALSO GIVEN. 20001045 WEST OF PEPEEKEO POINT. $(75,21-23)$ FERRUGINOUS GAUXITE, CHANNEL SMPL FROM ROADCUTI MIN. INDEX MAP. SPECTRO DATA 20001046 WEST OF PEPEEKEO POINT. (75:21-23) FERRUGINOUS BAUXITE, CHANNEL SMPL FROM ROAOCUTI MIN. INDEX MAP. SPECTRO DATA 20001047 HAKALAU BAY. (75:21-23) FERRUGINOUS BAUXITE. CHANNEL SMPL FROM ROADCUT: MIN. INDEX MAP. SPECTRO DATA ALSO GIVEN. 20001047 HAKALAU BAY. (75:21-23) FERRUGINOUS BAUXITE. CHANNEL SMPL FROM ROADCUT: MIN. INDEX MAP. SPECTRO DATA ALSO GIVEN.
20001048 HSE:16.
HAKALAU BAY. (75:21-23) FERRUGINOUS GAUXITE, CHANNEL SMPL FROM RDADCUT; MIN. INDEX MAP. SPECTRO DATA ALSO GIVEN. 20001048 HAKALAU BAY, $175: 21-23)$ FERRUGINOUS BAUXITE, CHANNEL SMPL FROM RDADCUT: MIN. INDEX MAP. SPECTRO DATA ALSO GIVEN.
20001049 WEE:16. DUP ANAL, SEE SMPL 20001047 . HILO RAY. $175: 41,22,4$ ? $)$ FERRUGINOUS BAUXITE. CHANNEL SMPL: DEPTH, B.0 FT. INDEX MAP. SPECTRO DATA ALSO GIVEN. 20001050 WEST OF HILO BAY. (75:41,22,42) FERRUGINOUS BAUXITE, CHANNEL SMPL; DEPTH, 7.3 FT. INDEX MAP. SPECTRO DATA ALSO GIVEN. 20001051 HILO BAY. $(75: 41,22,42)$ FERRUGINOUS BAUXITE, CHANNEL SMPL, DEPTH, 11.0 FT. INDEX MAP. SPECTRO DATA ALSO GIVEN. USE:16. 20001052 PAPAIKOU. (75:41.22,42) FERRUGINOUS BAUXITE. CHANNEL SMPL: DEPTH, 3.5 FT. INDEX MAP. SPECTRO DATA ALSO GIVEN. USE:16. 20001053 WEST OF PEPEEKEO POINT. $175: 41,22,42)$ FERRUGINOUS BAUXITE, CHANNEL SMPL: DEPTH. 9.0 FT. INDEX MAP. SPECTRO DATA ALSO 20001054 WEST OF PEPEEKEO POINT. $175: 41,22,42)$ FERRUGINOUS BAUXITE, CHANNEL SMPLI DEPTH, 5.5 FT. INDEX MAP. SPECTRO DATA ALSD 20001055 SOUTHWEST OF HAKALAU BAY. $(75: 41,22,42)$ FERRUGINOUS BAUXITE, CHANNEL SMPL, DEPIH, 10.0 FT. INDEX MAP. SPECTRO DATA 20001056 HAKALAU BAY. $(75: 41,22,42)$ FERRUGINOUS BAUXITE, CHANNEL SMPL, DEPTH, 4,8 FT. INDEX MAP. SPECTRO DATA ALSO GIVEN. 20001056 HAKALAU BAY, $(75: 41,22,42)$ FERRUGINOUS BAUXITE, CHANNEL SMPLI DEPTH, 4,8 FT. INDEX MAP. SPECTRO DATA ALSO GIVEN.
USE: 16. 20001057 HANOHINA. $(75141,22,42)$ FERRUGINOUS BAUXITE, CHANNEL SMPL DEPTH, 1, B FT. INDEX MAP. SPECTRO DATA ALSO GIVEN, USE 116. 20001056 SOUTHWEST OF HANOHINA. $(75341,22.42)$ FERRUGINOUS BAUXITE, CHANNEL SMPL, DEPTH, 3.0 FT. INDEX MAP. SPECTRO DATA ALSO 20001059 SOUTHWEST OF MAULUA BAY. $(75841.22,42)$ FERRUGINOUS BAUXITE, CHANNEL SMPL, DEPTH, 3.0 FT. INDEX MAP. SPECTRO DATA 20001060 MLULUA. $(75: 41,22,42)$ FERRUGINOUS BAUXITE, CHANNEL SMPLI DEPTH, 3.0 FT. INDEX MAP. SPECTRO DATA ALSO GIVEN. USE, 16. 20001061 SOUTH OF KOHOLALELE LANDING, $175: 41.22,42)$ FERRUGINOUS BAUXITE, CHANNEL SMPL; DEPTH, 4.0 FT. INDEX MAP. 20001069 PPECTRO DATA ALSO GIVEN. USE: 16. WALUTU RIVER. $(611140,8,33)$ BAUXITIC CLAY, RED OR REO-BROWN, VERY FINE GR,
COMPOS SMPLI MIN: TONNAGE. INOEX MAP, MEAS SEC. SPECTRO ANAL ALSO GIVEN.

20003002 KOOLAU RANGE, (577:24) CLAY, GRAY: 1-8 IN THICK: MINI PHYS PROP, FIRING TESTS. USE: 5. "MISC: TIO2 = 14.061 20003003 KOOLAU RANGE. 1577:24) CLAY. GRAY 1-8 IN THICK: MIN PHYS PROP, FIRING TESTS. USF, 5. 20007001 LIZO $=0.04$, ZROZ $=0.04:$ MNO, CL, N, RARE EARTHS $=0.00$.

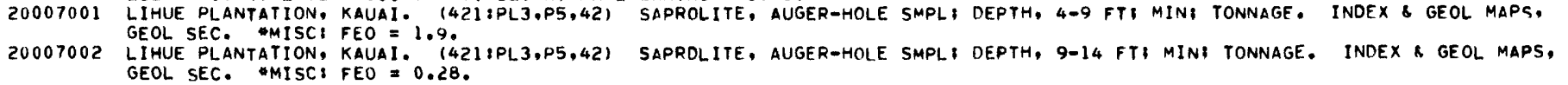


TABLE 6. - Analyses of samples from California and Hawaii containing 90-100 percent clay (Group $D_{6}$ ), from all rock categories - Continued

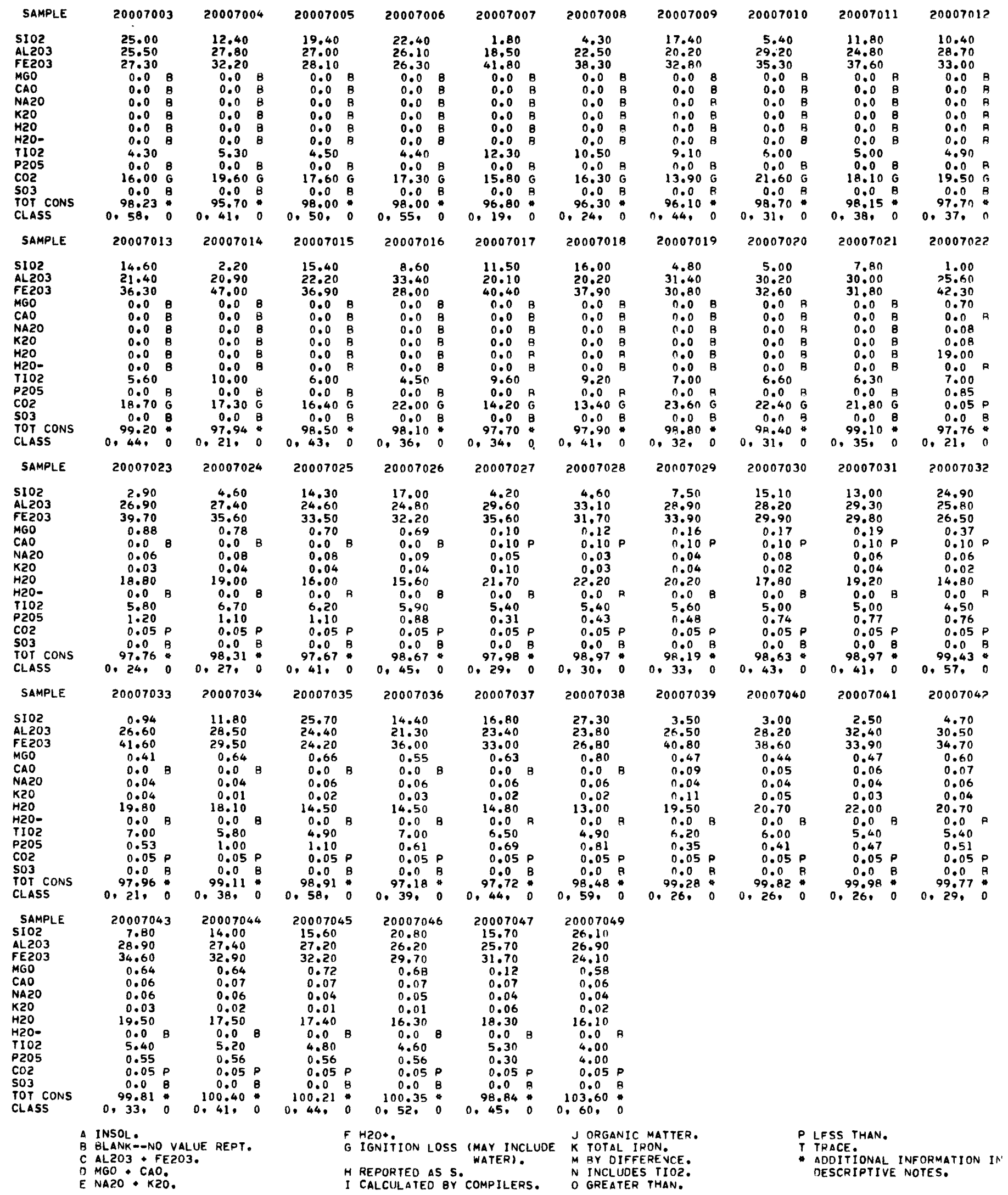


TABLE 6. - Analyses of samples from California and Hawaii containing 90-100 percent clay (Group $\left.D_{6}\right)$, from all rock categories - Continued

20007003 LIHUE PLANTATION, KAUAI, (421:PL3,P5,42) SAPROLITE, AUGER-HOLE SMPL: DEPTH, 14-19 FTI MINI TONNAGE. INDEX GEOL MAP,

GEOL SEC. \#MISC: FEO $=0.13$.

GEOL SEC. $\triangle M I S C:$ FEO $=1.40$ LIHUE PLANTATION, KAUAI LEUUE PLANTATION, KAUAI 14 GEOL SEC. HMISC: FEO $=1.5$.

20007007

20007008

20007009

20007010

20007011

20007012

20007013

20007014

20007015

20007016

20007017

20007018

20007019

20007020

2000702

20007022

20007023

20007024

20007025

20007026

20007027

20007028

20007029

20007030

20007031

20007032

20007033

20007034

20007035

20007036

20007037

20007038

20007039

20007040

2000704

20007042

20007043

20007044

20007045

20007046

20007047

20007049

LIHUE PLANTATION, KAUAI I GEOL SEC. *MISC: FEO $=2.7$ GEOL SEC. MISC: FEO $=0.85$. GEOL SEC, MISC: FEO $=1.2$.

GEOL SEC. AMISC: FEO = 2.0

PRINCE VILLE RANCH, KAUAI, GEOL SEC. MISC: FEO $=1.6$. GEOL SEC. MISC: FEO $=i .9$,

HMISC: FEO $=3.0$, MNO $=0.32$

KAUAI, $(421111,5,42, P L 3)$ SAPR
MISC: FEO $=0.62$, MNO $=0.32$. KAUAI: $(421: 11,5,41,42, P L 3)$

MISCI FEO $=1.6$, MNO $=0.40$.

KAUAI: $1421: 11,5,41,42, P L 31$

MISC, FEO $=1,2, M N O=0,36$.
KAUAI. $(421: 11,5,41,42, P L 3)$

HMISC: FEO $=1.0$, MNO $=0.33$

KAUAI. $1421: 11,5,42,52, P L 3)$
INDEX \& GEOL MAPS, GEOL SEC.

INDEX \& GEOL MAPS, GEOL SEC,
KAUA1. (421:11,5,42,52,PL3)

INDEX \& GEOL MAPS, GEOL SEC. AMISC: FEO $=6.6$.

INDEX PLANTATION, KAUAI.

INDEX \& GEOL MAPS, GEOL SEC, \#MISC: FEO $=4.4$.

LIHUE PLANTATION, KAUAI. (421:PL3,P5,42) FERRUGINOUS BAUXITE, AUGER-HOLE SMPL, DEPTH, 5-10 FT: MINI TONNAGE. INDEX \& GEOL MAPS, GEOL SEC. MMISC: FEO $=1.2$.

LIHUE PLANTATION, KAUAI. (421:PL3,P5,42) SAPROLITE, AUGER-HOLE SMPL: DEPTH, 10-15 FT: MINI TONNAGE. INDEX \& GEOL MAP,

LIHUE PLANTATION, KAUAI. (421:PL3,P5,42) SAPROLITE, AUGER-HOLE SMPL: DEPTH, 15-20 FT: MINI TONNAGE. INDEX G GEOL MAP,

PRINCEVILLE RANCH, KAUAI, (421:PLI,P5.42) FERRUGINOUS BAUXITE, AUGER-HOLE SMPL: DEPTH, $4-9$ FT. MIN\& TONNAGE.

INDEX \& GEOL MAPS, GEOL SEC. MISC: FEO $=0.54$.

PRINCEVILLE RANCH, KAUAT: (421:PL1,P5,42) FERRUGINOUS BAUXITE, AUGER-HOLE SMPL: DEPTH, 14-19 FT: MIN: TONNAGE,

INDEX \& GEOL MAPS. GEOL SEC. MISC: FEO $=1.6$

PRINCEVILLE RANCH, KAUAI, (421:PLI,P5,42) SAPROLITE, AUGER-HOLE SMPL, DEPTH, 9-14 FT: MIN: TONNAGE. INDEX \& GEOL MAP,

(421:PL1,P5,42) SAPROLITE, AUGER-HOLE SMPL; DEPTH, 14-19 FT: MINI TONNAGE。

INDEX \& GEOL MAPS, GEOL SEC. AMISC: FEO = 1.2

PRINCEVILLE RANCH, KAUAI. (421:PL1,P5,42) FERRUGINOUS BAUXITE, AUGER-HOLE SMPL, DEPTH, 4=9 FT: MIN: TONNAGE. INDEX AND GEOL MAPS, GEOL SEC, HISC: FEO $=1.2$.

PRINCE VILLE RANCH, KAUAI, (421:PL1,P5,42) FERRUGINOUS BAUXITEI AUGER-HOLE SMPL: DEPTH, 9-14 FT: MINI TONNAGE.

INDEX \& GEOL MAPS, GEDL SEC. HMISC: FEO $=1.6$.

INDEX \& GEOL MAPS, GEOL SEC. MISC: FEO $=1.4$

PRINCEVILLE RANCH, KALIAI. (421:11,5,42,52,PLI) FERRUGINOUS BAUXITE, AUGER-HOLE SMPL, DEPTH, 4-9 FT: MIN: TONNAGE:

FIRING TESTS. INDEX G GEOL MAPS, GEOL SEC. AMISCI FEO $=1.0$. MNO=0.10.

FIRING TESTS. INDEX \& GEOL MAPS, GEOL SEC, HMISC: FEO $=1.1$, MNO $=0,34$.

PRINCEVILLE RANCH, KAUAI. (421111,5,42,52,PL1) FERRUGINOUS BAUXITE, AUGER-HOLE SMPL: DEPTH, 14-19 FT: MIN! TONNAGEI

FIRING TESTS. INDEX GEOL MAPS, GEOL SEC MMISC: FEO=2.7. MNO=0,26.

PRINCEVILLE RANCH, KAUAI. (421:11,5,42,PLi) SAPROLITE, AUGER-HOLE SMPL, OEPTH, 19-29 FT: MIN: TONNAGE,

INDEX \& GEOL MAPS, GEOL SEC. HMISC: FEO $=1.1:$ MNO $=0.32$.

PRINCEVILLE RANCH, KAUAI. (421:11,5,42,PL) SAPROLITE, AUGER-HOLE SMPL, DEPTH, 29-39 FTI MINI TONNAGE.

INDEX \& GEOL MAPS: GEOL SEC. MISC: FEO = 1.1: MNO $=0.32$.

INDEX \& GEOL MAPS, GEOL SEC, $\$ M I S C 1$ FEO $=1,1$ MNO $=0,32$,

FIRING TESTS. INDEX \& GEOL MAPS, GEOL SEC. MISC: FEO $=0.71$. MNO $=0.061$ SMPL CONTAINS ORGANIC MATTER.

PRINCEVILLE RANCH, KAUAI. $(421: 11,5,40,42,52$, PLI) FERRUGINOUS BAUXITEI LOG OF AUGER HOLE: DEPTH, 9-14 FT: MIN: TONNAGE: FIRING TESTS, INDEX \& GEOL MAPS, GEOL SEC. "MISCI FEO $=1.0$, MNO $=0.11$.

PRINCEVILLE RANCH, KALIAI, $(421: 11,5,40,42,52$, PL1) FERRUGINOUS BAUXITE: LOG OF AUGER HOLEI DEPTH, 14-22 FT: MINI INDEX \& GEOL MAPS, GEOL SEC. MMISC: FEO $=1.01$ MNO $=0.121$ SMPL CONTAINS ORGANIC MATTER.

PRINCEVILLE RANCH, KAUA1, (421:11,5,40,42,52,PL1) SAPROLITE: LOG OF AUGER HOLEI DEPTH, 22-24 FT: MIN: TDNNAGE. INDEX \& GEOL MAPS, GEOL SEC. MMISC: FEO $=1.31$ MNO $=0.17 ;$ SMPL CONTAINS ORGANIC MATTER.

PRINCFVILLE RANCH, KAUAI. (421:11,5,40,42,52,PL1) SAPROLITE: LOG OF AUGER HOLE: DEPTH, 24-34 FT: MIN: TONNAGE, INDEX \& GEOL MAPS, GEOL SEC. MISC: FEO $=1.33$ MNO $=0,16$. PRINCEVILLE RANCH, KAllAI. $(421: 11,5,40,42,52$, PLI) SAPROLITE LOG DF AUGER HOLE: DEPTH. 34-54 FT: MIN: TONNAGE, INDEX \& GEOL MAPS, GEOL SEC. HMISC: FEO $=1.31$ MNO $=0.27$.

KAUAI. (421:11,5,42,52,PL3) FERRUGINOUS BAUXITE, AUGER-HOLE SMPL: DEPTH, $4-9$ FT, MINI TONNAGE: FIRING TESTS.

INDEX \& GEOL MAPS, GEOL SEC. AMISC: FEO $=0.91$. MNO $=0.04$.

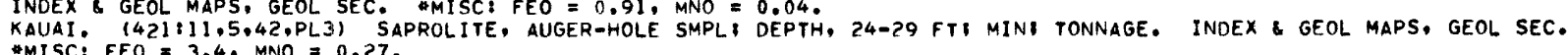

KAUAI. (421:11,5,42,PL3) SAPROLITE, AUGER-HOLE SMPL; DEPTH, 39-44FT: MIN: TONNAGE. INDEX G GEOL MAPS, GEOL SEC,

KAUAI. $(421111,5,42,52, P L 3)$ SAPROLITE, AUGER-HOLE SMPL, DEPTH, 4-9 FT: MIN, TONNAGE: FIRING TESTS.

INDEX \& GEOL MAPS, GEOL SEC. $M I S C$ I FEO $=2.5$, MNO $=0.18$.

INDEX (421:11,5,42,52,PL3) SAPROLITE. AUGER-HOLE SMPL DEPT

INDEX \& GEOL MAPS, GEOL SEC. $M I S C: F E O=1.6$. MNO $=0.17$.

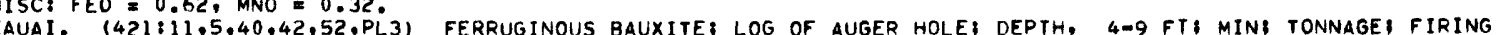

INDEX \& GEOL MAPS, GEOL SEC. $\$ M I S C$ FEO $=1.5$, MNO $=0.17$

KAUAI. (421:11,5,40,42,52,PL3) FERRUGINOUS BAUXITE; LOG OF AUGER HOLEI DEPTH, 9-14 FTI MIN: TONNAGE: FIRING TESTS. INDEX \& GEOL MAPS, GEOL SEC. MMISC: FEO $=2.1$. MNO $=0.18$

KAUAI. (421:11,5,40,42,52,PL3) FERRUGINOUS BAUXITE: LOG OF AUGER HOLE: DEPTH, 14-19 FT? MINI TONNAGE: FIRING TESTS. INDEX \& GEOL MAPS, GEOL SEC. MISC: FEO $=2.5$. MNO $=0.16$

KAUAI. (42, $111,5,40,42$, PL3) FERRUGINOUS RAUXITE: LOG OF AUGER HOLE: DEPTH, 19-24 FT: MIN: TONNAGE. INDEX A GEOL MAPS, GEOL SEC, MISC: FEO $=2.0$, MNO $=0.44$.

KAUAI (421:11,5,40,42,PL3) FERRUGINOUS BAUXITE; LOG OF AUGER HOLE: DEPTH, 24-29 FT: MIN: TONNAGE. INDEX \& GEOL MAPS,

GEOL SEC MISC: FEO = 1.8 , MNO = 0.42.

INDEX \& GEOL MAPS, GEOL SEC.

SAPROLITE, LOG OF AUGER HOLE: DEPTH, 39-49 FT: MIN: TONNAGE.

INDEX \& GEOL MAPS, GEOL SEC.

SAPROLITE, LOG OF AUGER HOLE: DEPTH, 49-54 FT: MIN: TONNAGE. INDEX \& GEOL MAPS, GEOL SEC.

SAPROLITE, AUGER-HOLE SMPL: DEPTH, 4-9 FTI MINI TONNAGE: FIRING TESTS.

AMISC: FEO = 1.4 , MNO $=0.10$.

MISC: FEO $=1.2$, MNO $=0.45$. 
TABLE 6. - Analyses of samples from California and Hawail containing 90-100 percent clay (Group $\left.D_{6}\right)$, from all rock categories - Continued

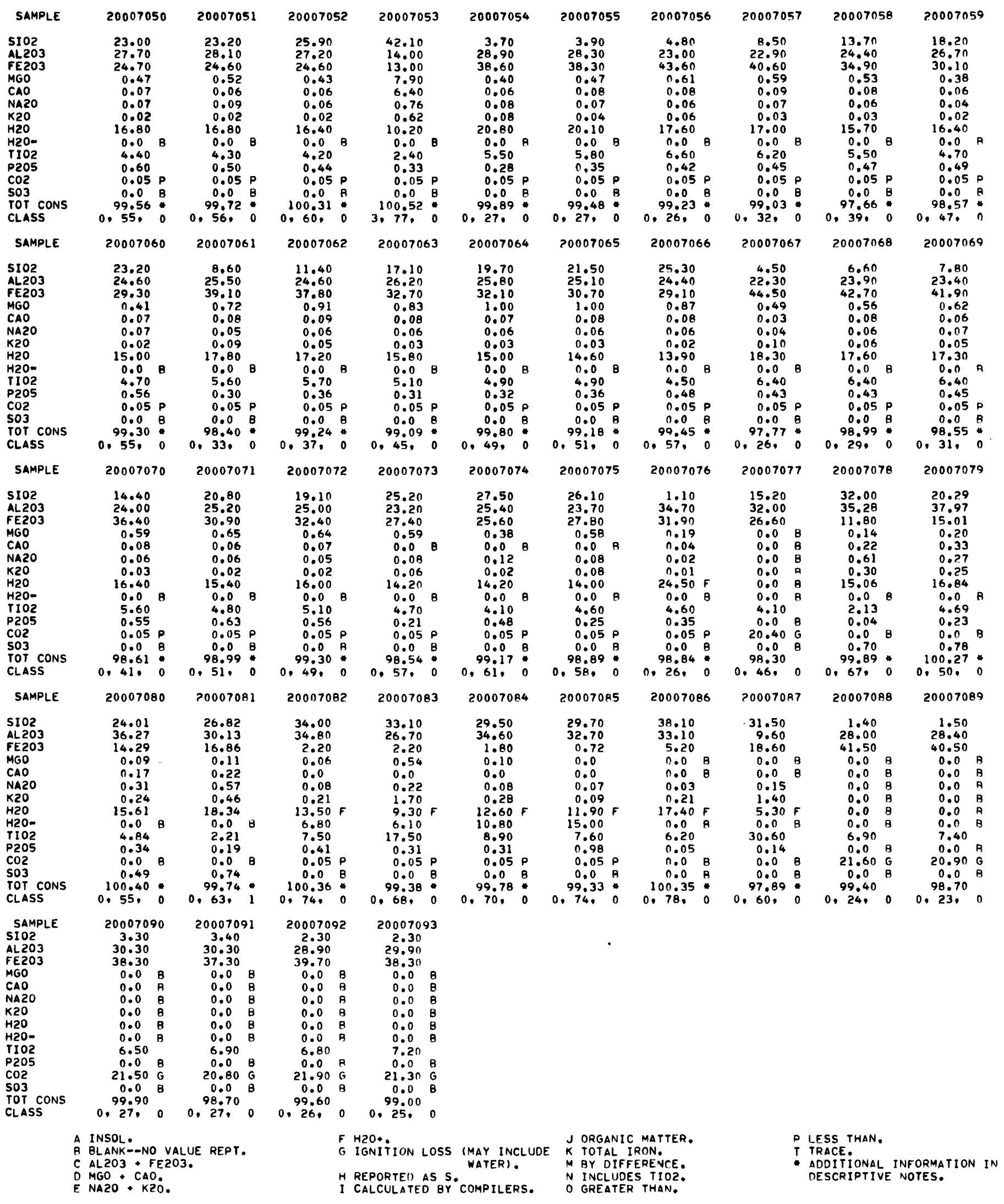


TABLE 6. - Analyses of samples from California and Hawaii containing 90-100 percent clay (Group $D_{6}$ ), from all rock categories - Continued

20007050 KAUAI. (421:11,5,42,PL3) SAPROLITE, AUGER-HOLE SMPL: DEPTH, 19-24 FT: MIN: TONNAGE. INDEX \& GEOL MAPS, GEOL SEC. 20007051 KAUAI. $(421: 11,5,42, P L 3)$ SAPROLITE, AUGER-HOLE SMPL: DEPTH, $24-29$ FTI MINI TONNAGE. INDEX \& GEOL MAPS. GEOL SEC. 20007051 KAUAI: $421: 1,5,42, P L 3)$ SAPROLITE, AUGEP-HOLE SMPL: DEPTH, $24-29$ FT MINI TONNAGE. INDEX \& GEOL MAPS, GEOL SEC.
20007052 KAUAI: $1421: 11,5,42, P L 3) .28$ SAPROLITE. AUGER-HOLE SMPL DEPTH, $29-35$ FT: MINI TONNAGE. INDEX \& GEOL MAPS. GEOL SEC. 20007053 KAUAI. 1421:11,5,42,PL3) SAPROLITE, AUGER-HOLE SMPL, DEPTH, 35-35.5 FT: MIN: TONNAGE. INDEX \& GEOL MAPS. GEOL SEC. 20007054 KAUAI. (421:11,5,41,52,PL3) FERRUGINOUS BAUXITE; LOG OF AUGER HOLE, DEPTH, $4-7$ FT: MINI TONNAGEI FIRING TESTS. 20007055 KAUAI. (421,11,5,41,52,PL3) FERRUGINOUS BAUXITEI LOG OF AUGER HOLE, DEPTH, 7-9 FT: MINI TONNAGEI FIRING TESTS.

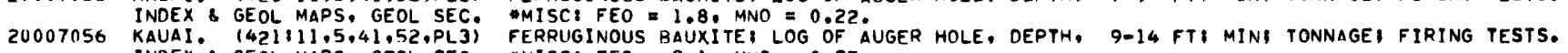

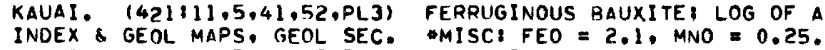


TABLE 6. - Analyses of samples from California and Hawaii containing 90-100 percent clay (Group $D_{6}$ ), from all rock categories -Continued

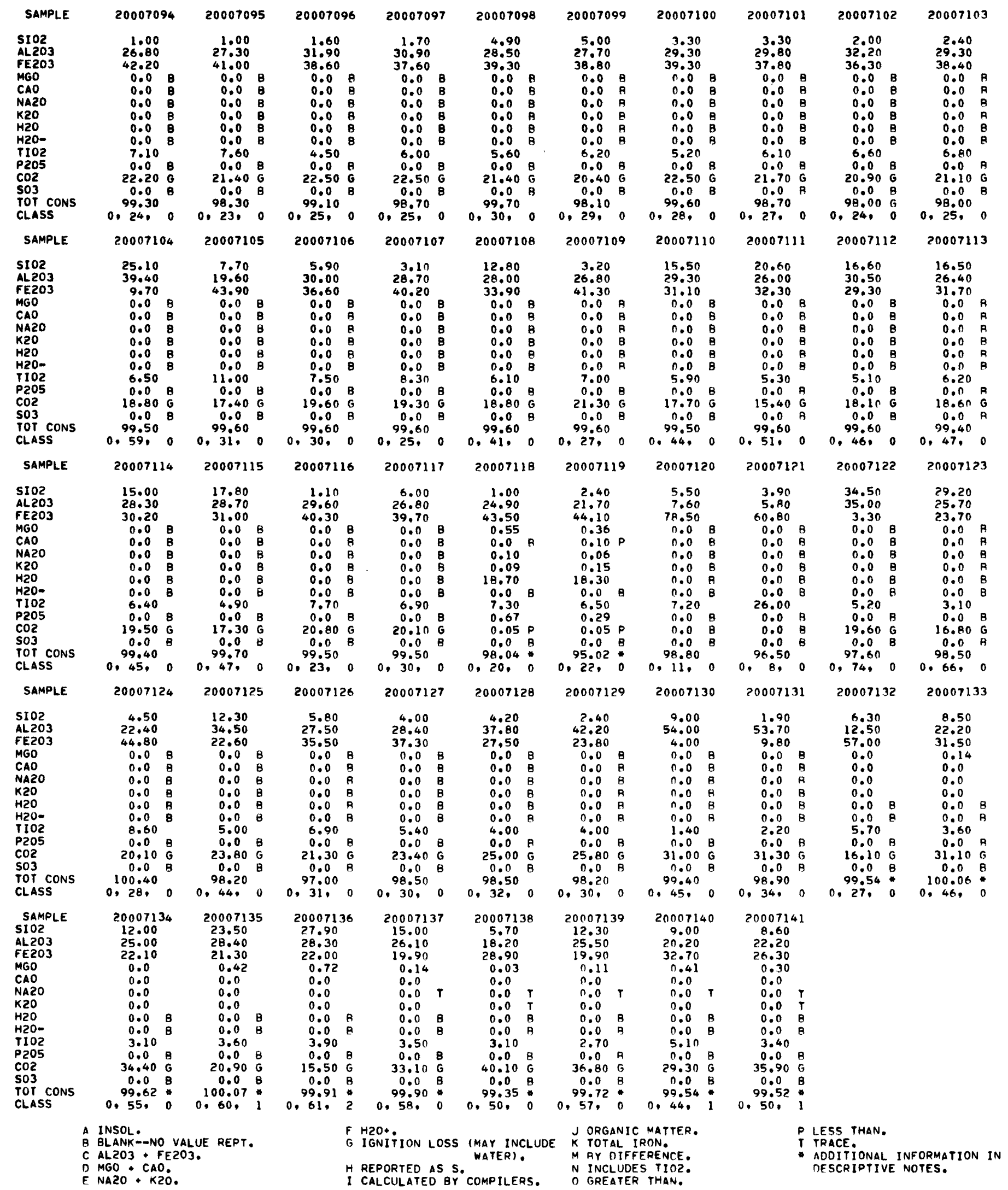


TABLE 6. - Analyses of samples from California and Hawaii containing 90-100 percent clay (Group $\left.D_{6}\right)$, from all rock categories - Continued

20007094 SOUTHWEST OF KILAUEA, $(75: 7,5,6)$ FERRUGINOUS BAUXITE, CHANNEL SMPL, MIN. INDEX MAP. SPECTRO DATA ALSO GIVEN. USF 116. 20007095 SOUTHWEST OF KILAUEA. $(75: 7.5,6)$ FERRUGINOUS BAUXITE. CHANNEL SMPL: MIN. INDEX MAP. SPECTRO DATA ALSO GIVEN. USE 16. 20007096 KILOHANA CRATER. (75:7.5,6) FERRUGINOU5 BAUXITE, CHANNEL SMPL: MIN. INDEX MAP. SPECTRO DATA ALSO GIVEN. USE: 16. 20007097 KILOHANA CRATER. (75:7.5.6) FERRUGINOUS BAUXITE, CHANNEL SMPL: MIN. INDFX MAP. SPECTRO DATA ALSO GIVEN. USE: 16. 20007098 KILOHANA CRATER. $175: 7,5,6)$ FERRUGINOUS BAUXITE, CHANNEL SMPL, MIN. INDEX MAP. SPECTRO DATA ALSO GIVEN. USE: 16. 20007099 KILOHANA CRATER, $(75: 7,5,6)$ FERRUGINOUS BAUXITE. CHANNEL SMPL MIN. INDEX MAP. SPECTRO DATA ALSO GIVEN. USE: 16. 20007100 KILOHANA CRATER. $(75: 7,5,6)$ FERRUGINOUS BAUXITE, COMPOSITE SMPL: MIN. INDEX MAP. SPECTRO DATA ALSO GIVEN, USE: 16. 20007101 KILOHANA CRATER. $(75: 7.5,6)$ FERRUGINOUS BAUXITE, COMPOSITE SMPL, MIN. INOEX MAP. SPECTRO DATA ALSO GIVEN. USE, 16. 20007102 WEST OF KAPAA. $(75: 30,5)$ FERRUGINOUS BAUXITE, AUGER SMPL, DEPTH, 5 FT. INDEX MAP. USE: 16. 20007103 WEST OF KAPAA. $(75: 30,5)$ FERRUGINOUS BAUXITE, CHANNEL SMPL, DEPTH, 7.1 FT. INDEX MAP. USE: 16. 20007104 KANEHA RESERVOIR. (75:30,5.34) FERRUGINOUS BAUXITE: DEPTH, 1.0 FT. INDEX MAP. SPECTRO DATA AISO GIVEN. USE: 16. 20007105 KILAUEA AREA. 175830.5 .29$)$ FERRUGINOUS BAUXITE: 1-FT GRAB SMPL: DEPTH, 1.0 FT. INDEX MAP. SPECTRO DATA ALSO GIVEN.

20007106 SOUTHWEST OF KILAUEA. $175: 30,5,34)$ FERRUGINOUS BAUXITE. CHANNEL SMPL DEPTH, 4.8 FT. INOEX MAP. SPECTRO DATA ALSO GIVEN, USE: 16 .

20007107 ROAD NEAR ANINI STREAM. $175: 30,5,33)$ FERRUGINOUS BAUXITE, CHANNEL SMPL, DEPTH. 7.0 FT. INDEX MAP. SPECTRO DATA ALSO GIVEN, USE: 16

20007108 KILOHANA CRATER. USE: 16 .

20007109 KILOHANA CRATER, GIVEN, USE: 16. (75:30.5.34) FERRUGINOUS BAUXITE, CHANNEL SMPL: DEPTH, 9.4 FT. INDEX MAP. SPECTRO DATA ALSO GIVEN.

20007110 KILOHANA CRATER. 20007112 KILOHANA CRATER.

(75:30,5,33) FERRUGINOUS EAUXITE, COMPOSITE SMPL: DEPTH, 5.2 FT: MIN. INDEX MAP. SPECTRO DATA ALSO

20007112 KILOHANA CRATE (75:30,5,33) FERRUGINOUS BAUXITE, CHANNEL SMPL: DEPTH, 8.0 FT. INREX MAP. SPECTRD DATA ALSO GIVEN. KILOHANA CRATER。 $\quad(75: 30,5,34)$ FERPUGINOUS BAUXITE, CHANNEL SMPL DEPTH, 500 FT. INDEX MAP. SPECTRO DATA ALSO GIVEN. 20007113 KAPAA AREA. (75:30,5.33) FERRUGINOUS BAUXITE. CHANNEL SMPL: DEPTH, 9.0 FT. INDEX MAP. SPECTRO DATA ALSO GIVEN. USE:16. 20007114 PRINCEVILLE RANCH. $(75: 30.5 .34)$ FERRUGINOUS BAUXITE, CHANNEL SMPL: DEPTH. 3.2 FT. INDEX MAP. SPECTRO DATA ALSO GIVEN. 20007115 UNINI STREAM. $(75: 30,5,33)$ FERRUGINOUS BAUXITE. CHANNEL SMPL: DEPTH, 8.0 FT. INDEX MAP. SPECTRO DATA ALSO GIVEN.

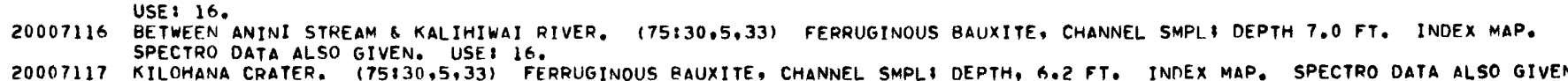

20007116 BETWEEN ANINI STREAM KALIHIWAI RIVER, $(75: 30,5,33)$ FERRUGINOUS BAUXITE, CHANNEL SMPL, DEPTH 7.0 FT. INDEX MAP.
20007117 KPECTRO DATA ALSO GIVEN. USE: 16.

20007116 BETWEEN ANINI STREAM KALIHIWAI RIVER, $(75: 30,5,33)$ FERRUGINOUS BAUXITE, CHANNEL SMPL, DEPTH 7.0 FT. INDEX MAP.
20007117 KPECTRO DATA ALSO GIVEN. USE: 16. USE! 16.

20007118 KAUAI. (609:156) SAPKOLITE: MIN. $M I S C:$ FEO $=1.1$, P205 REPT AS P203, MNO $=0.08$.

20007119 KAUAI. (6098156) SAPROLITE: MIN. MISC: FEO $=0.93$, P205 REPT AS P203, MNO = 0.08, SMPL CONTAINS ORG MATTER.

20007120 KAU1, OFF KOKEE RD. (176:317) FFRRUGINOUS LATERITE: FRIABLES MIN; DEPTH, 11-27 FT.

20007121 KAUAI, WAIMEA CANYON. 1176:3181 FERRUGINOUS LATERITE: MIN.

$200071225.5 \mathrm{MI}$ NW OF LIHUE. (611:TABLE 9) KAOLINITIC FRAGMENTS: CREAM-COLORED ROADCUT SMPL MINI TONNAGE. INOEX MAP.

200071233 MI NW OF KAPAA. (611:TAGLE 9) KAOLINITIC CRUSTI ROAOCITT SMPL: MIN\& TONNAGE. INDEX MAP. SPECTRO ANAL ALSO GIVEN.

200071242 MI SE OF KILOUEA. (611:TABLE 9$)$ BAUXITE NOOULES: ROADCUT SMPL: MIN: TONNAGE. INOEX MAP. SPECTRO ANAL ALSO GIVEN.

200071252 MI $S$ OF KILOUEA. 1611:TABLE 9) BAUXITIC FRAGMENTS, REO-BROWN ROAOCUT SMPLI MINI TONNAGE. INOEX MAP.

$200071206 \mathrm{MI} N W$ OF LIHUE. (611:TABLE 9) RAUXITIC FRAGMENTS, RED-BROWN, ROADCUT SMPLI MINB TONNAGE. INDEX MAP.

200071274 MI NW OF KAPAA. (611:TABLE9) BALIITIC FRAGMENTS, RED-BROWN: ROADCUT SMPL: MINI TONNAGE. INDEX MAP.

200071281 MI W OF LIHUE. (611:TABLE 9) BAUXITIC FRAGMENTS, RED-BROWN ROADCUT SMPLI MIN: TONNAGE. INDEX MAP.

200071294 MI NW OF WAILUA. (611:TABLE 9) BAUXITIC CRUSTI ROADCUT SMPLI MIN TONNAGE. INDEX MAP. SPECTRO ANAL ALSO GIVEN.

200071305 MI NW OF KAPAA. (61):TABLE 9) BAUXITIC FPAGMENTS, WHITE: ROADCUT SMPL: MIN! TONNAGE. INDEX MAP. SPECTRO ANAL ALSO

200071314 MI NW OF LIHUE. (61):TABLE 91 RAUXITIC FRAGMENTS, YELLOW; ROADCUT SMPL: MIN, TONNAGE. INDEX MAP. SPECTRO ANAL ALSO

20007132 KAUI, KOKEE CANYON ROAD, WAIMEA DIST. KAUI, KOKEE CANYON ROAD,
MNO $=0.04$, FEO $=1.9$.

20007133 KAUI, KOKEE CANYON ROAU, WAIMEA OIST. MNO $=0.02, F E O=3.0$

20007134 KAUI, KOKEE CANYON ROAD, WAIMEA OIST. MNO $=0.02$, FEO $=3.0$.

20007135 KAUI, KOKEE CANYON ROAD, WAIMEA DIST. MNO $=0.05$, FEO $=1,9$. 20007136 KAUI, KOKEE CANYON ROAD, WAIMEA DIST.

20007137 KAUI, KOKEE CANYON ROAO, WAIMEA DIST.

(48:295.293) CLAY: MIN: 10 - 12 IN BELOW SURFACE: B.D.. 1.4. AMISC:

(48:?95,293) CLAYI MIN: 12 - 15 IN BELOW SURFACEI B.D.. 0.9. *MISC:

(48:295.293) CLAY MIN 15 - 19 IN BELOW SURFACEI B.D.. 0.9. AMISC:

(48:295,293) CLAYI MIN 19- 25 IN BELOW SURFACE: 8.0., 1.7. MMISC:

(48:295.293) CLAY: MIN: 25. IN BELOW SURFACE: B.D.. 1.7. *MISC: MNO $=0.09$,

20007138 KAUI, KOKEE CANYON ROAD, WAIMEA DIST.

(48:300) CLAY. MIN. MISC: MNO $=0.06$, FEO $=2.1$.

20007139 KAUI, KOKEE CANYON ROAD, WAIMEA DIST.

(48:300) CLAY, MIN, AMISC\& MNO $=0.02$, FEO $=3.3$.

20007140 KaUI, KOKEE CANYON ROAD, WAIMEA DIST.

(481300) CLAY, MIN. MISC, MNO $=0.01$, FEO $=2.4$.

20007141 KAUI, KOKEE CANYON ROAD, WAIMEA DICT.

(48:300) CLAY, MIN, MISC: MNO $=0.03$, FEO $=2.8$.

148:300) CLAY, MIN. $M I S C$ : MNO $=0.02, F E O=2.8$. 
TABLE 6. - Analyses of samples from California and Hawaii containing 90-100 percent clay (Group $\left.D_{6}\right)$, from all rock categories - Continued

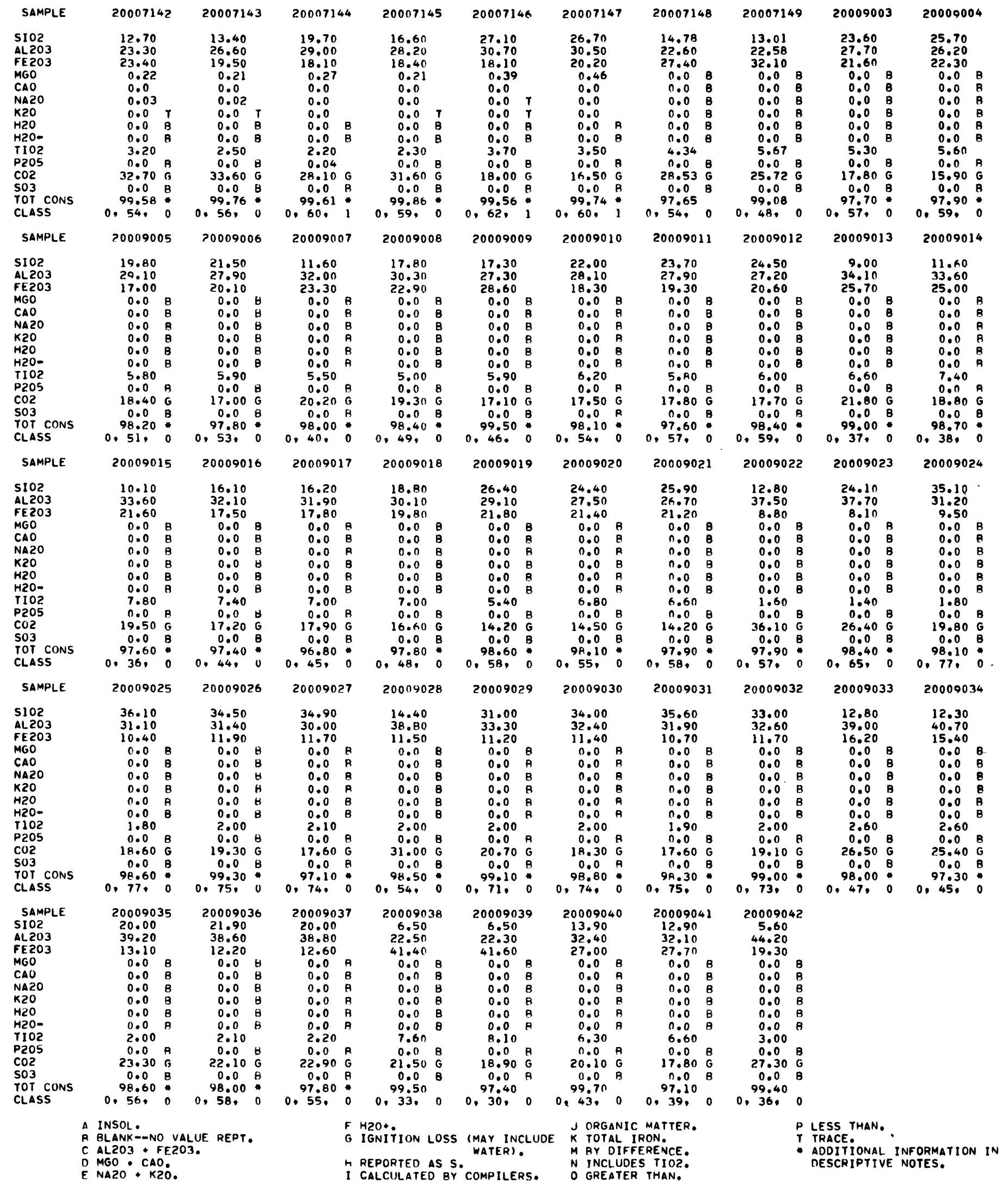


TABLE 6. - Analyses of samples from California and Hawaii containing 90-100 percent clav (Group $\left.D_{6}\right)$, from all rock categories - Continued

20007142 KAUI, KOKEE CANYON ROAD, WAIMEA DIST. 20007143 KEO $=4.0$. KOKE CANYON ROAD, WAIMEA DIST.

20007144 KAUI, KOKEE CANYON ROAD, WAIMEA DIST. MNO

20007145 KAUI, KOKEE CANYON ROAD, WAIMEA DIST.

20007145 KAUI, KOKEE CANYON ROAD, WAIMEA DIST.

20007146 KAUI, KOKEE CANYON ROAD, WAIMEA DIST.
MNO $=0.07$, FEO $=1.5$.

20007147 KAUI, KOKEE CANYON ROAD, WAIMEA DIST.

20007148 KAUI, WAIMEA CANYON.

20007149

20009003

20009004

20009005

20009006

20009007

20009008

20009009

20009010

20009011

20009012

20009013

20009014

20009015

20009016

20009017

20009018

20009019

20009020

2000902

20009022

20009023

20009024

20009025

20009026

20009027

20009028

20009029

20009030

20009031

20009032

20009033

20009034

20009035

20009036

20009037

20009038

20009039

20009040

20009041

20009042

GEOL SEC. MMISC, FEO $=2.2$.

NEAR HAIKU. MAUI. (421:PL7,P1,5)

GEOL SEC. MISCI FEO $=8.1$.

GEOL SEC. WMISC, FEO $=5.4$.

GEOL SEC. \#MISC: FEO $=5.40$

NEAR ULUMALU, MAUI (421 1 PL7, P1,5)

GEOL SEC $M I S C$ FEO $=3$.

NEAR ULUMALU. MAUI (421:PL 7,P1,

GEOL SEC

GEOL SEC. \#MISC: FEO $=3.3$.

NEAR ULUMALU, MAUI $1421: P L$

GEOL SEC. $M I S C:$ FEO $=6.0$.

NEAR ULUMALU, MAUI $1421: P L$

GEOL SEC. MISC: FEO $=3.1$.

NEAR ULUMALU, MAUI. $(421 ; \mathrm{PL} 7, \mathrm{P1}, 5$

GEOL SEC, $M I I S C 8$ FEO $=2.4$

INDEX \& GEOL MAPS, GEOL SEC. MISC FEO $=1.8$. GEOL SEC. MISCI FEO $=2.3$.

NEAR WAIPIO BAY, MAUI - $(421: P L 7, P 1,5)$

GEOL SEC. MISC: FEO $=5.0$.

NEAR WAIPIO BAY, MAUI. $14218 P L 7, P 1,5$

GEOL SEC. MMISC: FEO $=7.1$

NEAR WAIPIO BAY, MAUI. (42i $I P L 7, P 1,5)$

GEOL SEC. HISCI FEO $=6.0$.

NEAR WAIPIO BAY, MAUI 1421 \&PL7,PI, 5 )

GEOL SEC. MISC, FEO $=5.5$

NEAR WAIPIO BAY, MAUI. $142 i$ :PL7,P1,5)

NEAR WAIPIO BAY, MAUI 1421 .

NEAR WAIPIO BAY, MAUI. (421 1 PL7,P1,5)

GEOL SEC. \#MISCI FEO $=3.5$

NEAR WAIPIO BAY, MAUI. (421:PL7,P),5)

GEOL SEC. $M I S C: F E O=3.3$.

NEAR HONOKAHUA, MAUI. (42liPL6,P5,42)

GEOL SEC. \#MISC: FEO $=1.1$.

NEAR HONOKAHUA, MAUI (421 $($ PL $6, P 5,42)$

GEOL SEC. \#MISC: FEO = 0.7 .

NEAR hONOKAHUA. MAU1. (42) iPL6, P5,42)

GEOL SEC. MISC: FEO $=0.7$.

NEAR HONOKAHUA. MAUI. 1421 iPL6,P5,42

GEOL SEC. \#MISCI FEO $=0.6$.

NEAR HONOKAHUA, MAUI. (4218PL $6,95,42)$

GEOL SEC. $M I S C$ FEO $=0.2$.

NEAR HONOKAHUA, MAUI, $(421$ iPL $6, P 5,42)$

GEOL SEC. $M I S C$ : FEO $=0.8$.

NEAR HONOKAHUA, MAUI. $(421:$ PL6,P5,42)

GEOL SEC. MMISC: FEO $=0.8$

GEOL SEC. MMISCI FEO $=0.8$,
NEAR HONOKAHUA, MAUI. $1421: P L 6, P 5,42)$
GEOL SEC. MMISC

GEOL SEC. HMISC FEO $=0.99$
NEAR HONOKAHUA, MAUI. $(421: P L 6, P 5,42)$

GEOL SEC. MISC: FEO $=0.7$

NEAR HONOKAHUA, MAUI. $(421$ :PL6,P5,42)

GEOL SEC. "MISC: FEO $=0.6$.

NEAR HONOKAHUA, MAUI. $(421$ iPL6,P5,42)

GEOL SEC. HMISC: FEO $=0.6$.

NEAR KAHAKULOA, MAUI. $(421$ :PL6,P5,42)

GEOL SEC. MISC: FEO $=0.9$.

NEAR KAHAKULOA, MAUI. 1421 iPL6,P5,42

GEOL SEC. MISC: FEO $=0.9$.

NEAR KAHAKULOA, MAUI. 1421 \% 1 , 6,PS, 42

GEOL SEC. \#MISC: FEO $=1.0$.

NEAR KAHAKULOA, MAUI. $(421$ (PL6,P5,42)

GEOL SEC. MISC: FEO $=1.1$

NEAR KAHAKULOA, MAUI, 1421 :

GEOL SEC. MISC: FEO $=1.3$ DUP ANAL, SEE SMPL 20009038 .
(48:295,293) CLAY: MINI, -14 IN BELOW SURFACEI B.D., 0.8. MISC: MNO $=0.03$.

(48:295,293) CLAY: MIN! 14 - 20 IN BELOW SURFACE: 8.0., 0.9. "MISC:

(481295,293) CLAY MINI 20 - 25 IN BELOW SURFACE: B.0.. 0.8. \#MISC:

(48:295,293) CLAYI MINI 25 - 31 IN BELOW SURFACE, B.D.. 0.9. MISC:

(48:295,293) CLAYI MIN 31 - 38 IN BELOW SURFACE: B.0., 1.7. \#MISC:

(48:295,293) CLAY: MIN? 38* IN BELOW SURFACE: B.D., 1.7. \#MISC: MNO = 0.08,

(351442.441) LATERITE CRUST: 10 - 15 IN BELOW SURFACE; MIN, B.D., 0.9,

(35:442,441) LATERITE CRUST, 10 - 15 IN BELOW SURFACE: MIN. R.D., 2.1 .

NEAR HAIKU, MAUI - (421:PL7,P1,5) SAPROLITE: AUGER-HOLE SMPL, DEPTH, 4-9 FT: MIN: TONNAGE. INDEX \& GEOL MAP, GEOL SEC.

NMISC: FEO = 1.7: $1421: P L 7, P 1.5)$ SAPROLITE: AUGER-HOLE SMPL, DEPTH, 9-10.5 FTI MIN: TONNAGE. INDEX \& GEOL MAPS,

NEAR HAIKU, MAUI. (421:PL7,P1,5) SAPROLITE: AUGER-HOLE SMPL: DEPTH, $10.5-14$ FT: MIN: TONNAGE. INDEX \& GEOL MAPS,

NEAR ULUMALU, MAUI. $(421: P L 7, P 1,5)$ SAPROLITE, AUGER-HOLE SMPL; DEPTH, 4-9 FT: MIN\& TONNAGE. INDEX \& GEOL MAPS,

SAPROLITE, AUGER-HOLE SMPL, DEPTH, 9-16 FT: MIN: TONNAGE. INDEX \& GEOL MAPS,

SAPROLITE, AUGER-HOLE SMPL; DEPTH, 16-20 FT: MIN: TONNAGE. INDEX \& GEOL MAPS,

SAPROLITE, AUGER-HOLE SMPL, NEPTH, 20-24 FTI MINI TONNAGE. INDEX \& GEDL MAPS,

SAPROLITE, AUGER-HOLE SMPL, DEPTH, 24-34 FTI MIN: TONNAGE, INDEX \& GEOL MAPS,

NEAR WAIPIO BAY, MAUI. 1421,8 PL7,P1,5) FERRUGINOUS 8AUXITE: AUGER-HOLE SMPL, DEPTH, $4=9$ FTI MINI TONNAGE.

NEAR WAIPIO BAY, MAUI. (42I IPL7,P1,5) SAPROLITE, AUGER-HOLE SMPL, DEPTH, 9-14 FT: MIN, TONNAGE. INDEX \& GEOL MAPE,

SAPROLITE; AUGER-HOLE SMPL: DEPTH, 14-19 FTI MINI TONNAGF.

SAPRDLITE: AUGER-HOLE SMPL: DEPTH, $19.24 \mathrm{FTI} M I N I$ TONNAGE.

INDEX \& GEOL MAPS,

SAPROLITE: AUGER-HOLE SMPL; DEPTH, 24-27 FT; MIN: TONNAGE.

INDEX \& GEOL MAPS,

SAPROLITE: AUGER-HOLE SMPL; DEPTH, $27-29 \mathrm{FTI}$ MINI TONNAGE.

INDEX \& GEOL MAPS,

SAPRDLITE: AUGER-HOLE SMPL: DEPTH, 29-33 FTI MINI TONNAGF.

INDEX \& GEOL MAPG,

SAPROLITE: AUGER-HOLE SMPL: DEPTH, 33-38 FT: MIN: TONNAGE.

INDEX $\&$ GEOL MAPS.

SAPROLITE: AUGERMHOLE SMPL: DEPTH, 38-45 FTI MINI TONNAGE, INDEX \& GEOL MAPS,

SAPROLITE, AUGER-HOLE SMPL 1 DEPTH, 4-9 FT, MIN, TONNAGE, INDEX \& GEOL MAPS,

SAPROLITE, AUGER-HOLE SMPL, DEPTH, 9-14 FT: MINI TONNAGE. INDEX \& GEOL MAPS,

SAPROLITE, AUGER-HOLE SMPL: DEPTH, 14-19 FT: MIN: TONNAGE.

INDEX \& GEOL MAPS,

SAPROLITE, AUGER-HOLE SMPL: DEPTH, 19-24 FT: MINI TONNAGE.

INDEX \& GEOL MAPS,

SAPROLITE, AUGER HOLE SMPL: DEPTH, 24-30 FT: MIN: TONNAGE. INDEX \& GEOL MAPS,

SAPRDLITE, AUGER-HOLE SMPL; DEPTH, 30-33 FTI MIN; TONNAGE. INDEX \& GEOL MAPS,

SAPROLITE, AUGER-HOLE SMPL, DEPTH, 7-9 FT: MIN TONNAGE. INDEX \& GEOL MAPS,

SAPROLITE, AUGER-HOLE SMPL, DEPTH, 9-19 FT, MIN, TONNAGE. INDEX \& GEDL MAPS,

SAPROLITE, AUGER-HOLE SMPL; DEPTH, 19-29 FTI MIN: TONNAGE. INDEX 8 GEOL MAPS,

SAPROLITE, AUGER-HOLE SMPL; DEPTH, 29-39 FTI MIN; TONNAGE. INDEX \& GEOL MAPS,

SAPROLITE, AUGER-HOLE SMPL: DEPTH, 39-47 FT: MIN, TONNAGF. INDEX \& GEOL MAPS,

SAPROLITE, AUGER-HOLE SMPL, DEPTH, 4-9 FT: MIN, TONNAGE, INDEX \& GEOL MAPS,

SAPROLITE, AUGER-HOLE SMPL: DEPTH, 9-14 FT: MIN, TONNAGE. INDEX \& GEOL MAPS,

SAPROLITE, AUGER-HOLE SMPL: DEPTH, 14-19 FT: MIN: TONNAGF. INDEX \& GEOL MAPS,

SAPROLITE, AUGER-HOLE SMPL; DEPTH, 19-24 FT: MIN; TONNAGE. INDEX \& GEOL MAPS,

SAPROLITE, AUGER-HOLE SMPL: DEPTH, 24-29 FT; NIN: TONNAGE. INDEX \& GEOL MAPS,

WEST OF HONOPOU STREAM. $(75: 15-17)$ FERRUGINOUS BAUXITE, CHANNEL SMPL, MIN. INDEX MAP. SPECTRO DATA ALSO GIVEN, USE: 16.

WEST OF HONOPOU STREAM, (75:15-17) FERRUGINOUS BAUXITE, CHANNEL SMPL: MIN. INDEX MAP. SPECTRO DATA ALSO GIVEN. USE:16.

SOUTH OF OPANA POINT. $(75: 15-17)$ FERRUGINOUS BAUXITE, CHANNEL SMPL: MIN. INDEX MAP. SPECTRO DATA ALSO GIVEN. USE:I6.

SOUTH OF OPANA POINT, (75:15-17) FERRUGINOUS BAUXITE, CHANNEL SMPL: MIN. INDEX MAP. SPECTRO DATA ALSO GIVEN, USE:16. DUP ANAL, SEE SMPL 20009040 .
KAHAKULOA. (75:15-17) FERRUGINOUS BAUXITE, CHANNEL SMPL, MIN. INDEX MAP, SPECTRO DATA ALSO GIVEN. USE: 16. 
TABLE 6. - Analyses of samples from California and Hawaii containing 90-100 percent clay (Group $\left.D_{6}\right)$, from all rock categories-Continued

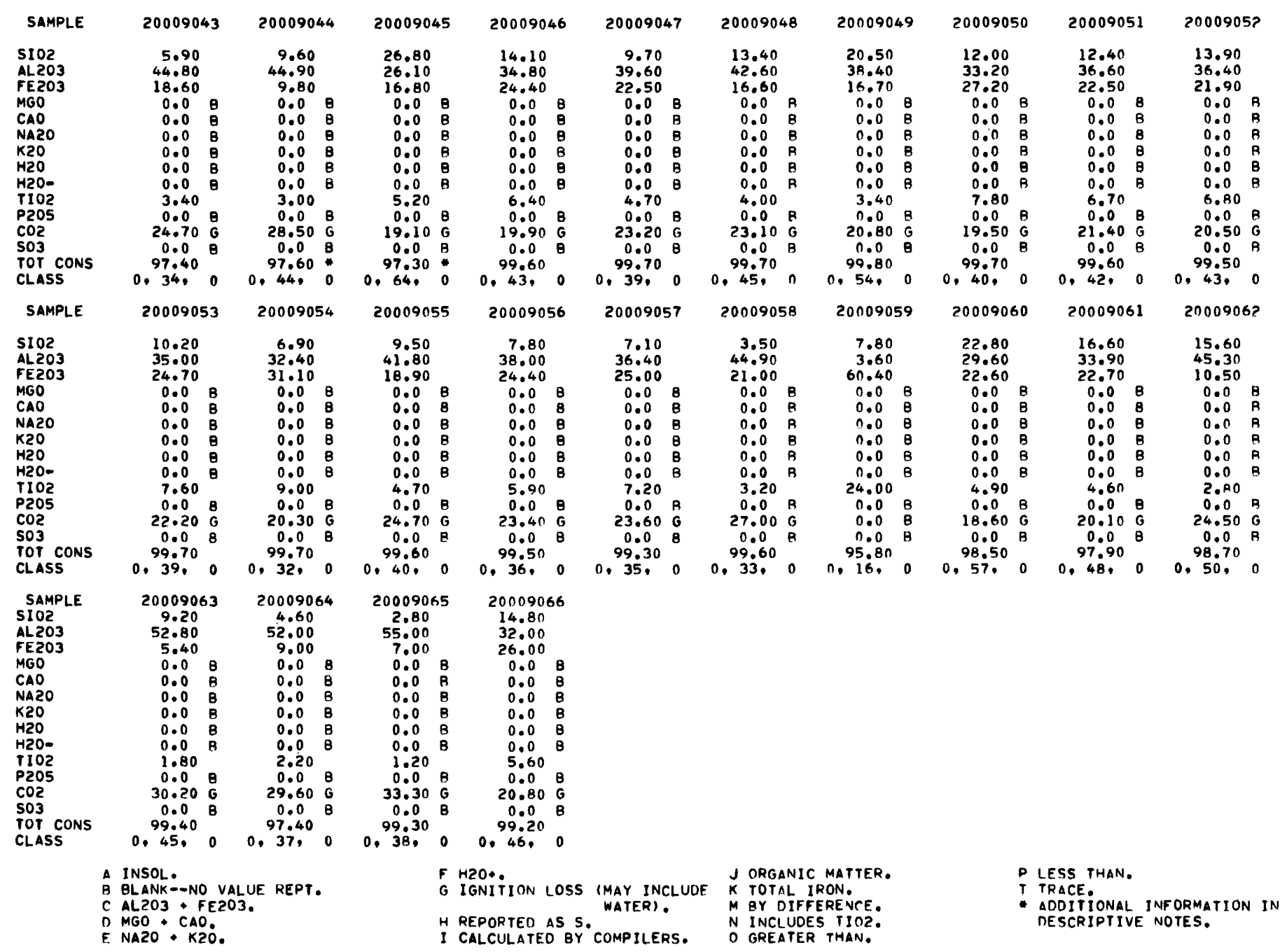


TABLE 6. - Analyses of samples from California and Hawaii containing 90-100 percent clay (Group $D_{6}$ ), from all rock categories-Continued

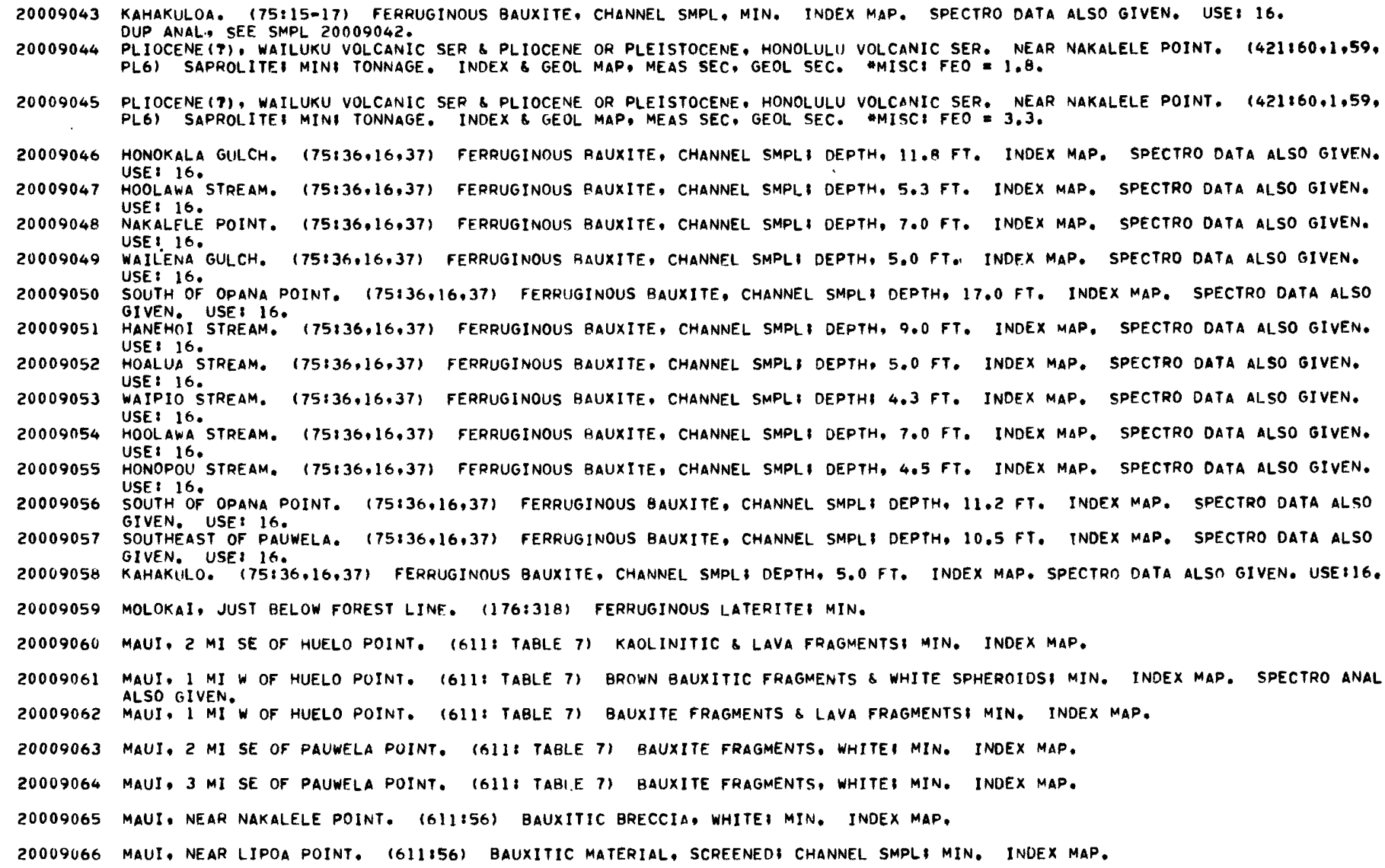


TABLE 7. - Analyses of samples from California and Hawaii containing clay and carbonate each less than 75 percent; clay and carbonate each greater than uncombined silica (Group E), common- and mixed-rock categories

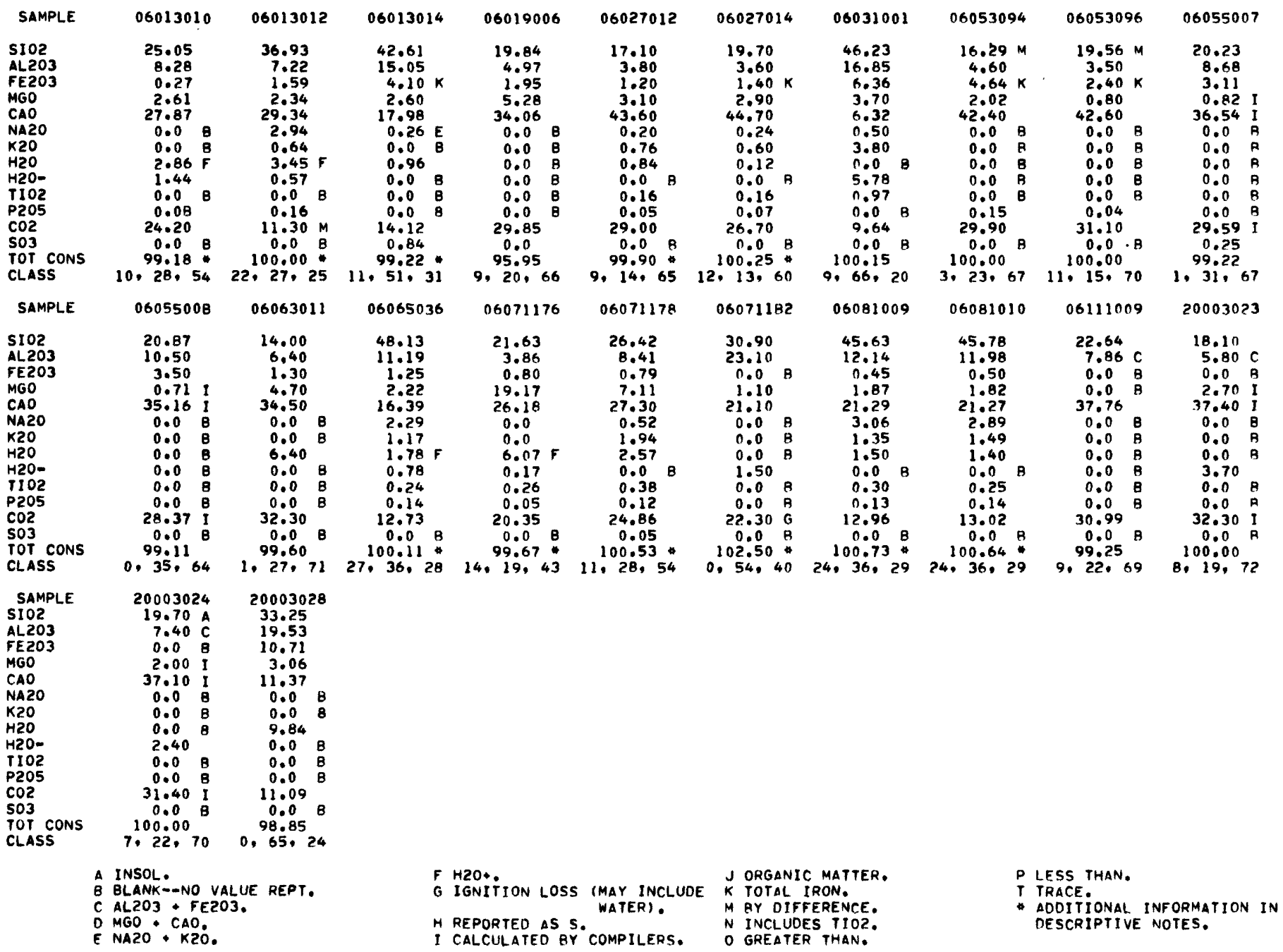


TABLE 7. - Analyses of samples from California and Hawaii containing clay and carbonate each less than 75 percent; clay and carbonate each greater than uncombined silica (Group E), common- and mixed-rock categories - Continued

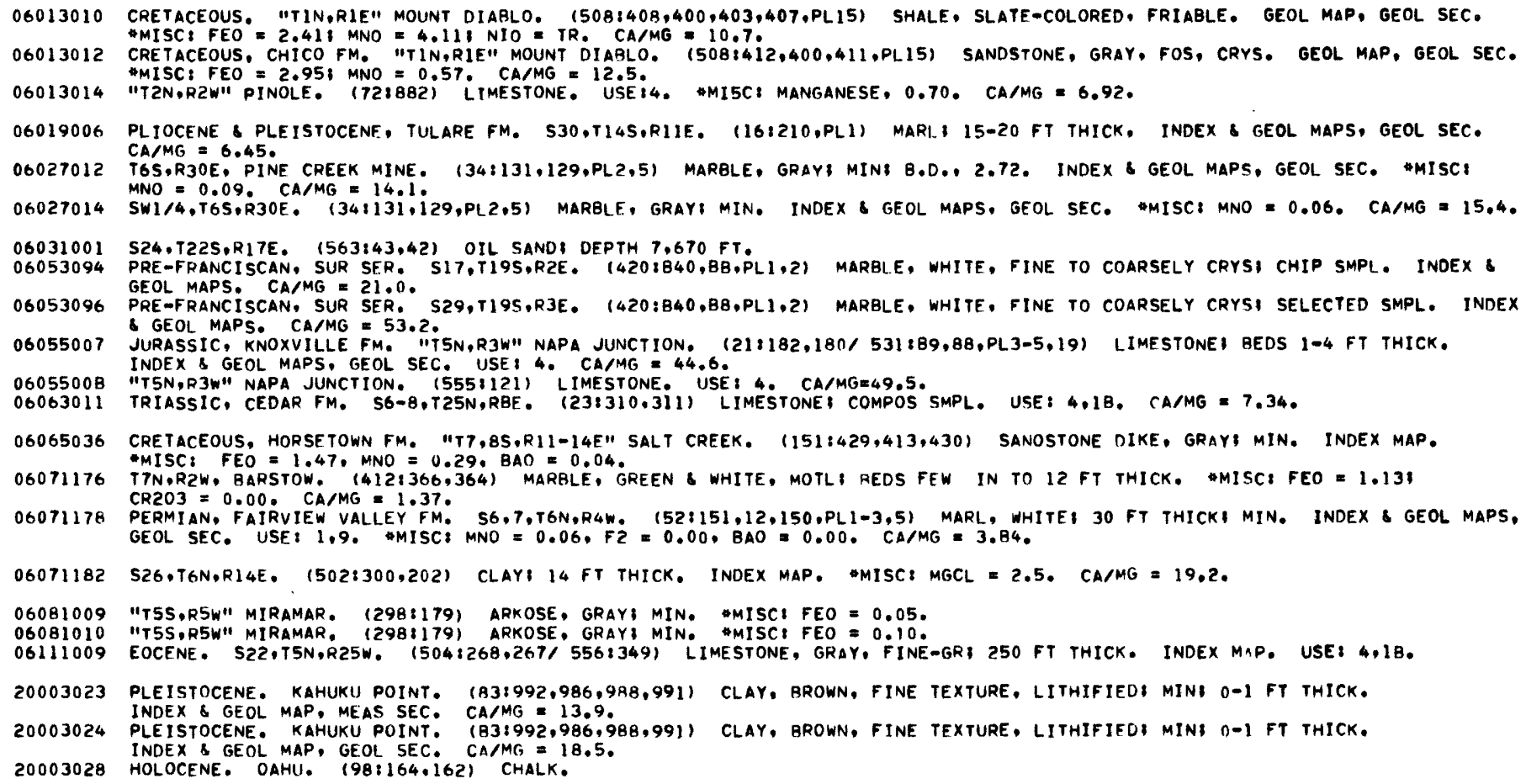




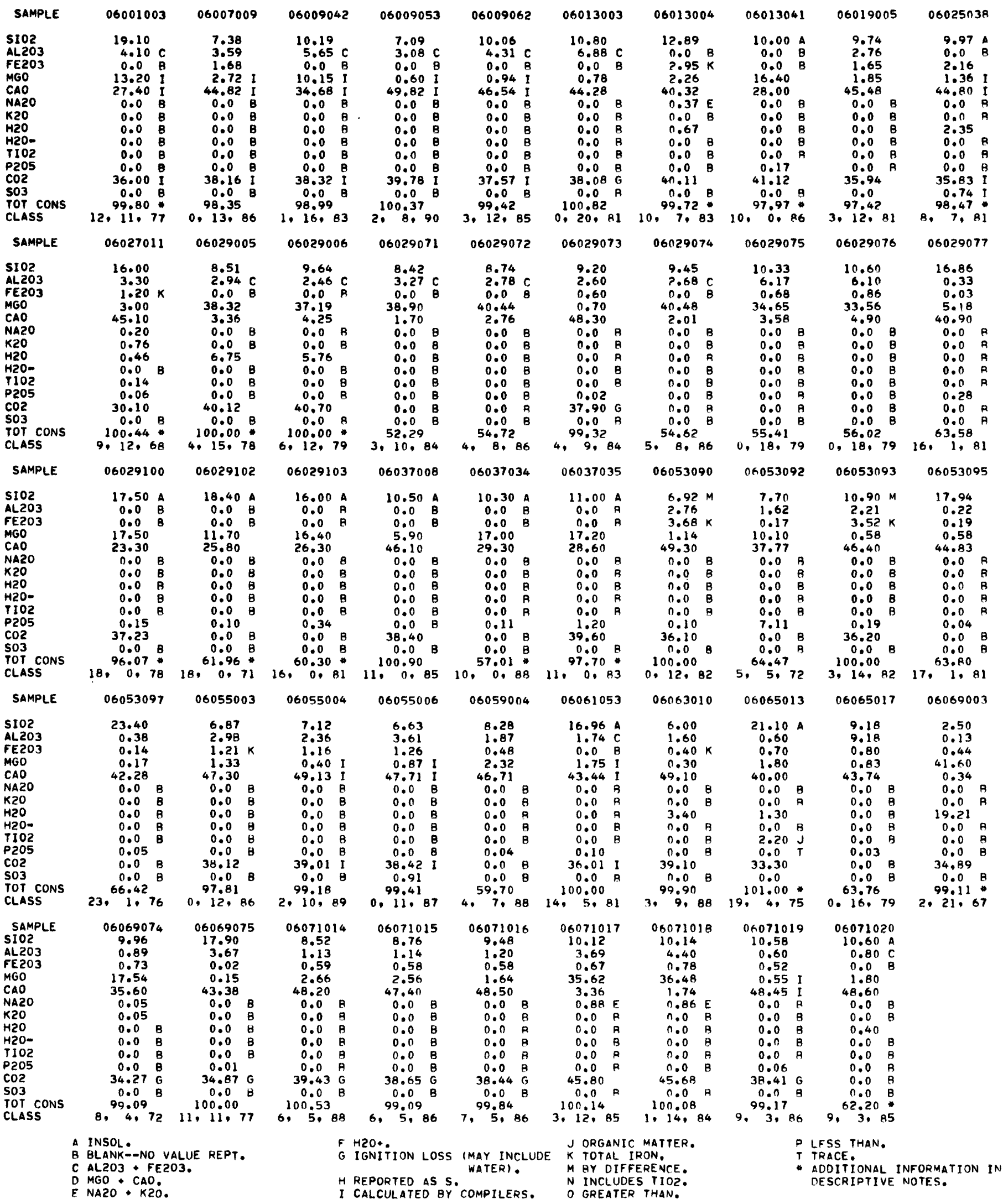


TABLE 8. - Analyses of samples from California containing 75-90 percent carbonate (Group $\left.F_{1}\right)$, common- and mixed-rock categories - Continued

06001003 "T4,5S,RIW" NILFS. 172:881" LIMESTONE, ARGILLACEOUS, USE: 4, "WMISC: SIO2 REPT AS SILICA \& CLAY. CA/MG = 2.08. 06007009 S6.T21N.R4E. (571867/5561213.PL37) MARBLE, QUARRY SMPLI TONNAGE. INDEX MAP. CA/MG = 16.6.

06009042 PALEOZOIC, CALAVERAS FM. T3N,R14E, VALLECITO. (96:103,94,PL A.D) DOLOMITIC LIMESTONEI DH OF I,352 FT. INHEX \& GEOL 06009053 PALEOZOIC. CALAVERAS FM. "T4N,RI2E" SAN ANIREAS. $(485: 3,2)$ LIMESTONE: D-H SMPL. CA/MG = 83.0.

06009062 PALEOZOIC, CALAVERAS FM. "T4N,RIZE" SAN ANDREAS. $(485: 3.2)$ LIMESTONE\$ D-H SMPL. CA/MG a 49.5 .

06013003 "TIN,RIW" COWELL. (1731122,PL4) TRAVERTINE. INDEX MAP. USE: 4. CA/MG $=56.8$.

06013004 "T2N,R3W" MARTINEZ, 172:882) LIMESTONE, USE: 4. "MISC: MN $=0.15$. CA/MG $=17.8$.

06013041 MIOCENE, CLAREMONT SH. "TIS,R3W" BERKELEY HILLS. (386:C6) DOLOMITE. OMISC, FEOM2.2, MNO=0.04, 5ROM0.036. CA/MG=1.71. 06019005 PL IOCENE \& PLEISTOCENE, TULARE FM. S30,T145,RI1E, (16:210,PL1) LIMESTONE, THIN BEDS. INDEX \& GEOL MAPS, GEOL SEC. 06025038 "SIS.TIIS.RI3E" MUD VOLCANOS. $(2531236,234)$ TRAVERTINEI I IN TO 2 FT THICK. MMISCI NACL 1.26 . CA/MG = 32.2. 06027011 T6S,R30E, PINE CREEK MINE. 134:131.129.PL2,5) MARBLE, GRAYI MINI B.0., 2.7B. INDEX \& GEOL MAPS, GEOL SEC. "MISC: 06029005 MIOCENE. S11.TION,RI1W. (20711118,1115-11171 4361116.114,117) MAGNESITE, WHITE. THIN BEDS: TONNAGE. INOEX \& GEOL

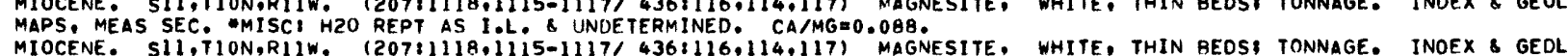
06029006 MIOCENE. SIl, T1ON,RIIW. (20711118,1115-11171 436:116,114,117) MAGNESITE, WHITE, THIN BEOS, TONNAGE. INOEX \& GEDL

06029071 MIOCENE. S11,TIONORIIW. (436:116,114-117) MAGNESITE. WHITE, THIN BEDSI TONNAGE. INDEX \& GEDL MAPS. MEAS SEC. 06029072 MIOCENE. S11,TION,R11W. $(436: 116,114-117)$ MAGNESITE, WHITE, THIN BEDS, TONNAGE. GFOL MAP, MEAS SEC. CA/MG = O.068.

06029073 PALEOZOIC, BEAN CANYON FM. "T32S,R33E" TEHACHAPI, (55:7,5) LIMESTONE, GRAY TO WHITF, COARSELY CRYS. USE, 4 . 06029074 MIOCENE. SII,TION,RIIW, (436:116.114-117) MAGNESITE, WHITE. THIN REDS, 1 FT THICK, TONNAGE. GEOL MAP, MEAS SEC.

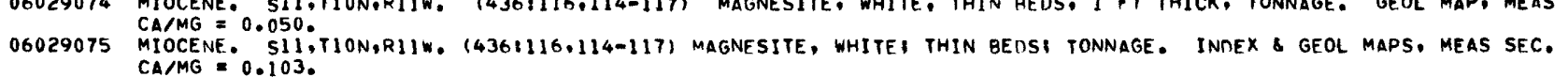
06029076 MIOCENE. S11.T1ON,RIIW. $1436: 116,114-117)$ MAGNESITE, WHITE THIN BEDS TONNAGE. GFOL MAP. MEAS SEC. CA/MG = O.146. 06029077 PRE-CPETACEOUS. S25.T30S,R34E. (229:216,233) LIMESTONE, WHITE TO GRAY, FINE TO COARSELY CRYS. INDEX \& GEOL MAPS. 06029100 MIOCENE. MONTEREY FM. CHICO MARTINEZ CHERT MBR, "T28S,R2OE" CHICO MARTINEZ CREEK. (386:C6) DOLOMITE. AMISCI 06029102 MIOCENE. "T31S,R26E" PALOMA OIL-FIELD. (386:C6,C5) DOLOMITE DEPTH, 12.060 FT. "MISC FFO=5.7, MNO=0.24, SRO=0.021.

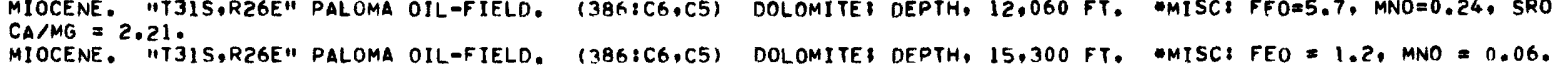

06029103 MIOCENE. "T31S,R26E" PALOMA OIL-FIELD. (386:C6,C5) DOLOMITE DEPTH, $15.300 \mathrm{FT}$. $4 M I S C:$ FEO = 1.2 , MNO = 0.06. 06037008 PRE-CRETACEOUS, PLACERITA FM. S3-6,T3N,R15W. $(4042221.218,220)$ LIMESTONE, WHITE, CRYSI MIN. INOEX MAP.

06037034 MIOCENE, MONTEREY SH, MALAGA MUDSTONE MBR. "T4S,R14W" PALOS VERDES HILLS. (386IC6) DOLOMITE. "MISC, FEO = O.28,

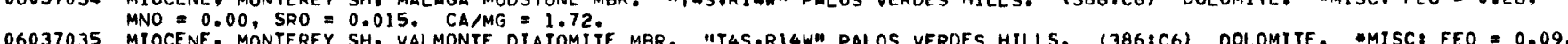
MIOCENE. MONTEREY SH, VALMONTE DIATOMITE MBR, "TAS,RI4W" PALOS VERDES HILLS. (386:C6) DOLOMITE. "MISC: FEO = O.09. MNO $=0.01$ CAIMG $=1.57$. PRE-FRANCISCAN, SUR SER, S20,T19S,RZE. (420:840,B8,PL1,2) MARBLE, WHITE, FINE TO COARSELY CRYS CHIP SMPL. INDEX \&. 06053090 06053092 06053093 06053095 06053097 06055003 06055004 PRE-FRANCISCAN, SUR SER O S36,T20S R4E。

$(265: 66,5, P L 1)$ DOLOMITE. INDEX \& GFOL MAPS. CAIMG $=2.81$.

06055006

06059004

06061053

06063010

06065013

06065017

06069003

06069074

06069075

06071014

06071015

06071016

06071017

0607101

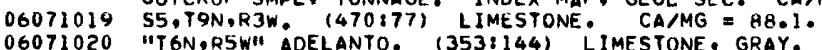

06071019 S5,T9N,R3W. $(470: 77)$ LIMESTONE. CAMMG = A8.1.
06071020 "TGN,R5W" ADELANTO. (353:144) LIMESTONE, GRAY.

PRE-FRANCISCAN, SUR SER. S20,TI9S.R2E. PRE-FRANCISCAN, SUR SER. SI,T19S,RIE, $(265: 65,5,63,64, P L 1)$ LIMESTONE, WHITE, COARSELY CRYSI MIN TONNAGE. INDEX \&

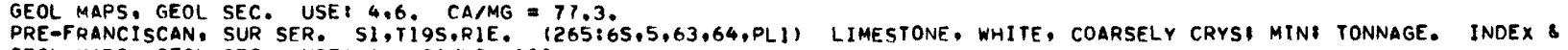
GEOL MAPS. GEOL SEC. USE: 4. CA/MG $>100$.

"TSN,R4W" NAPA JUNCTION. (231310) LIMESTONE. CA/MG $=35.6$. "TAN,R4W" NAPA JUNCTION. $(555: 32,121)$ LIMESTONE. USE: 4. CA/MG $>100$.

JURASSIC, KNOXVILLE FM. "T4N,R4W" NAPA JUNCTION. (21:182/ 531:89,88,PL3-5,19) LIMESTONE, CRYS, FOSI BEDS 1-4 FT THICK. INOEX \& GEOL MAPS, GEOL SEC. USE: 4. CA/MG $=54.8$. TRIASSIC \& JURASSIC, BEDFORD CANYON FM. S34,T4S,R7W. (228:87,86,PLI,?) LIMESTONE, GRAY TO BLACK, FINE-GR, CRYS. INDEX \& GEOL MAP. USE: 4. CA/MG $=20.1$.

PALEOZOIC. CALAVERAS FM. S30,TI3N,R9E: (94:464,460,461,PL3) LIMESTONE. INDEX \& GEOL MAP. CA/MG = 24.8.

TRIASSIC, CEDAR FM. S6-8,T25N,R8E, (23:310,311) LIMESTONE; COMPOS OF 11 SMPL. USE: 4.18. CA/MG >100.

"T3S,R4E" GARNET HILL. (353:142) CALICHE: AVG OF SEVERAL SMPL. OMISC: ALKALIES=TR, LI=TR, NITRATES=NONE. CA/MGaZ2.2. TRIASSIC \& JURASSIC, BEDFORD CANYON FM. S32,T4S,R6W. (228:117,PLI,2) LIMESTONE, GRAY. FINE-GR. INDEX \& GEOL MAPS. CAMMG = 52.?. (211:508,504) HYOROMAGNESITE, WHITE, POWDERY. INDEX MAP. $M$ MISC: M2O = I.L. LFSS CO2, CA/MG = 0.008. PRE-FRANCISCAN, SUR SER. SW1/4,T135,R5E, (54:32,11,31,PL1) DDLOMITE: TONNAGE. INDEX \& GEOL MAPS, GEOL SEC. $C A M G=2,03$.

PRE-FRANCISCAN, SUR SER, SW1/4,T135,R5E. 154:32,11,31,PL1) LIMESTONE; TONNAGE. INDEX \& GEOL MAPS, GEOL SEC. CA/MG $>100$.

"T5N,R4W" VICTORVILLE, $170: 91$ LIMESTDNE CONGLOMERATE; CONTAINS CHERT, USE: 4. CA/MG a 18.1.

"T5N,R4W" VICTORVILLE. (70:9) LIMESTONE CONGLOMERATE: CONTAINS CHERT. USE: 4. CA/MG = 18.5.

"TSN.R4W" VICTORVILLE, (70:9) LIMESTONE CONGLOMERATE CONTAINS CHERT. USE: 4 . CA/MG $=$ ?9.6.

TERTIARY. S21,28,TIIN,R6E, (58:74,72,PL1/ 436:118,117) MAGNESITE, WHITE, PINK, FINF-GR, BEDDEDI 30-40 FT THICK: TONNAGE. INDEX MAP. GEOL SEC. CA/MG $=0.094$.

TERTIARY. S21,28,TIIN,K6E. (58:74,72,PLI/436:118,117) MAGNESITE, WHITE, PINK, FINEMGR, BEDDEDI 30-40 FT THICK

OUTCROP SMPL TONNAGE. INUEX MAP, GEOL SEC. CA/MG $=0.048$.

"TGN,R5W" ADELANTO. (353:144) LIMESTONE, GRAY. "MISC: INSOL = MUSCOVITE. QTZ, ETC. CAMG a 27.0. 
TABLE 8. - Analyses of samples from California containing 75-90 percent carbonate (Group $F_{1}$ ), common- and mixea'-rock categories - Continued

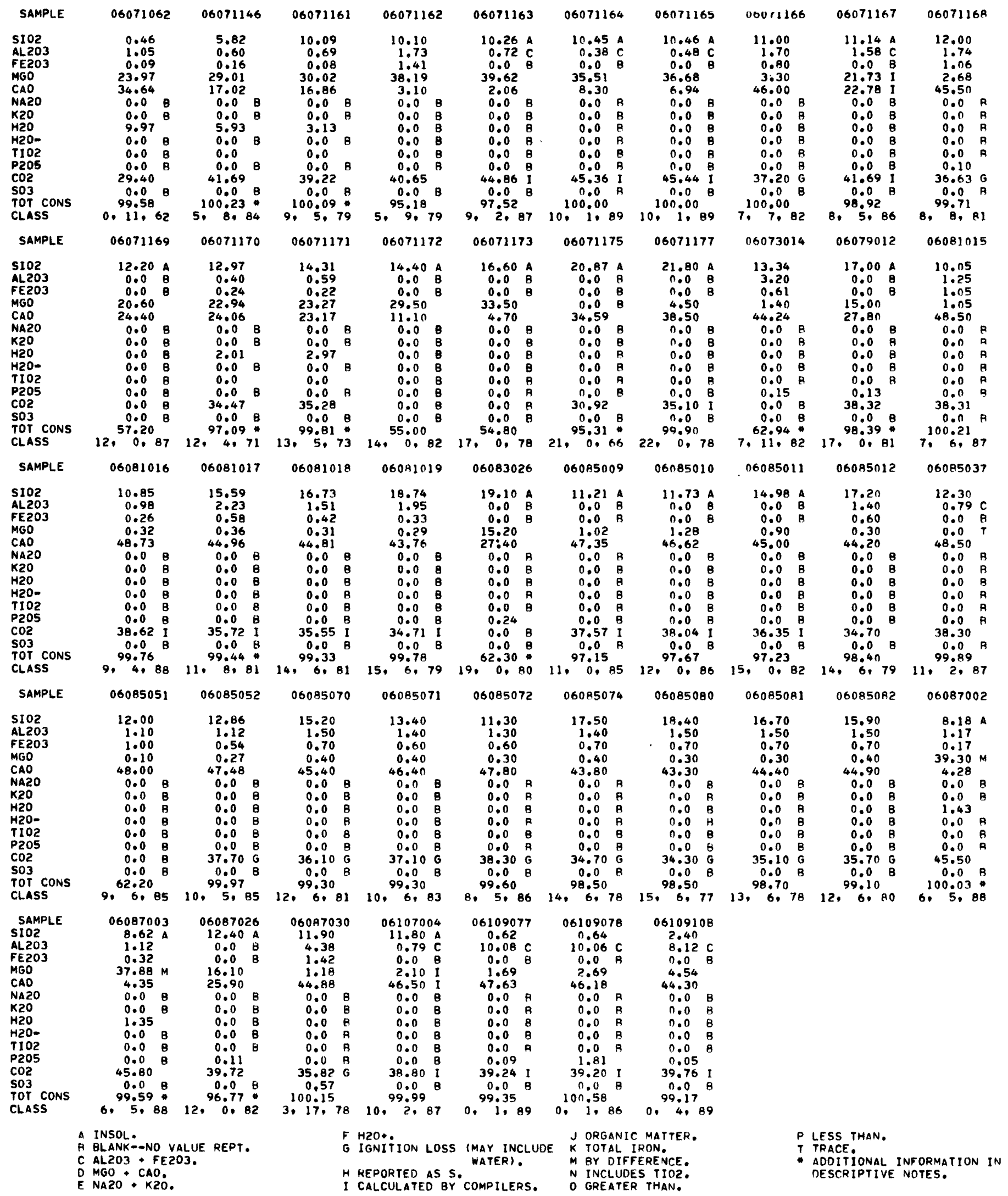


TABLE 8. - Analyses of samples from California containing 75-90 percent carbonate (Group $F_{1}$ ), common- and mixed-rock categories - Continued

06071062 S15.TGN,RIW. (507:524) DOLOMITE, WHITE TO BROWN, FINE- TO MED-CRYS. CA/MG = 1.45 .

06071146 TERTIARY. S22,T8N,R21E, 1524:364.357-360.PL62-64) MAGNESITE, WHITE, THIN BEDDED, 5.50 FT THICK, TONNAGE. INDEX \&

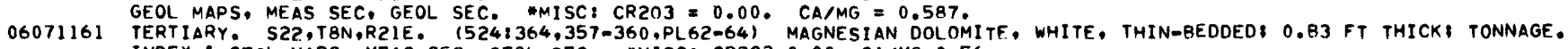
06071162 INDEX \& GEOL MAPS, MEAS SEC, GEOL SEC. MISC, CR203=0,00, CA/MG=0,56.

06071163 TERTIARY. S22,T8N,R21E. (524,368.357-363,PL62-64) MAGNESITE, BROWN, FINE-GR, THIN-REDDED: 2.3-FT CHANNEL SMPL:

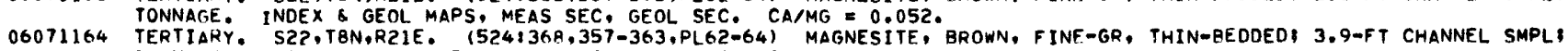

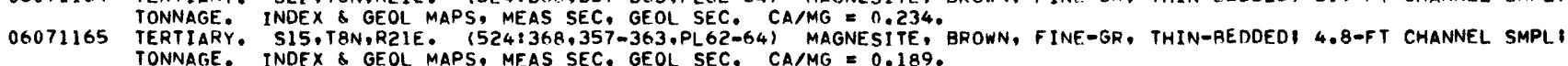

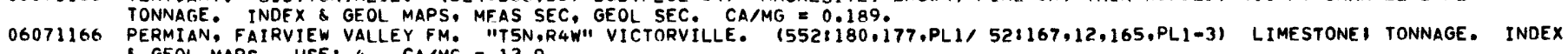

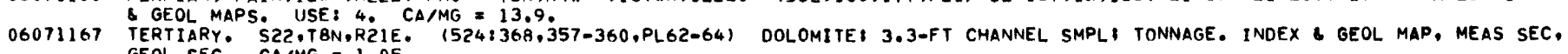
GEOL SEC. CA/MG $=1.05$.

06071168 CARBONIFEROUS, ORO GRANDE FM. "TSN,R4W" VICTORVILLE. (55:7.8) LIMESTONE, CONGLOMERATE, GRAY \& WHITE, CRYSI 1,350 FT

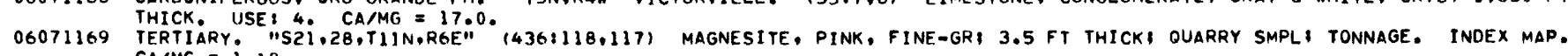

06071170 TERTIARY, S22,TBN,R21E. 1524:364,357-360,PL62-64) DOLOMITE, WHITE, THIN-BEDDEDI O.11 FT THICK: TONNAGE. INDEX \&

GEOL MAPS. MEAS SEC, GEOL SEC, MISC: CR203 $=0.00$. CAMM $=1.05$.

06071171 TERTIARY. S22,T8N,R2IE. (524:364,357-360,PL62-64) DOLOMITE, BUFF, GRAY, BROWN: 4.70 FT THICK. TONNAGE. INDEX \&

06071172 TERIIARY. "S21,28,T1IN,RGE" (436:118,117) MAGNESITE, WHITE, PINK, 5.0 FT THICK, QUARRY SMPLI TONNAGE. INOEX MAP.

06071173 TERTIARY. "S21,28,TI1N,RGE" $(436: 118,117)$ MAGNESITE, WHITE FINE-GR, 2.8 FT THICK QIIARRY SMPLI TONNAGE. INDEX MAP. 06071175 S30,TIIN,RIW. $(160: 34,24,36, P L 2-4)$ STRONTIANITE ROCK, BROWN, POROUSI TONNAGE. INDEX \& GEOL MAPS, MEAS SEC, GEOL SEC. 06071177 PALEOZOIC. FURNACE LS. T3N,RIE, MONARCH FLAT, (550:268,267-270,PL36) LIMESTONE, COMPOS SMPLI MIN. INOEX \& GEOL MAPS. 06073014 S27,T17S,R1E. (118:180,179,PL1) LIMESTONE. CREAM-COLORED, POROUS: 1 -B FT THICK IONNAGE. INDEX \& GEOL MAPS. USE: 4.

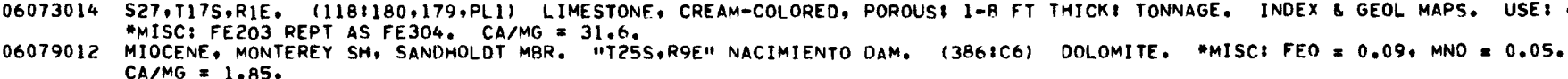

06081015 JURASSIC \& CRETACEOUS, FRANCISCAN FM. "T4S.R6W" CALERA. $(1751358.354)$ LIMESTONE3 TONNAGE. CAIMG . 46.2.

06081016 JURASSIC \& CRETACEOUS, FRANCISCAN FM. "T4S,R6W" ROCKAWAY OUARRY. (52617,4,PL1) LIMESTONEI REPR 35 FT SEC. INDEX \&

06081017 JURASSIC \& CRETACEOUS, FRANCISCAN FM. "T5S, R4W" HILL TOP OUARRY, $1526: 7,4,6, P L 1)$ LIMESTONE, GRAY TO BLACK: BEDS 1-8 IN THICK: SMPL OF 35-FT SEC. INDEX \& GEOL MAPS. USE: 7. "MISC: ORG PRESENT. CAMG >1O0.

06081018 JURASSIC \& CRETACEOUS, FRANCISCAN FM. "T4S,R6W" ROCKAWAY QUARRY, $1526: 7,4,6, P L 11$ LIMESTONE. OARK -BLUE: TAKEN ACROSS

40-FT SEC. INDEX \& GEOL MAPS. USE: 4,7 " CA/MG $>100$.
06081019 JURASSIC \& CRETACEOUS, FRANCISCAN FM. "T5S,R4W" SKYLINE QUARRY, $1526: 7,4, P L 1)$ LIMESTONEI COMPOS SMPL OF 70 FT. INDEX 06081019 JURASSIC \& CRETACEOUS, FRANCISCAN FM. "T5S, R4W" SKYLINE QUARRY, $(526: 7,4, P L 1)$
06083026 MIOCENE, MONTEREY SH. "TIN,R27W" SAN RAFAEL MTNS. $(386: C 6)$ DOLOMITE. "MISC: FEO $0.30, M N O=0.03$, SRO = 0.027.

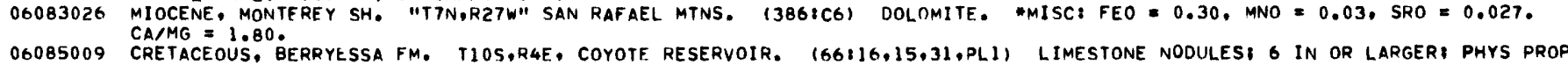

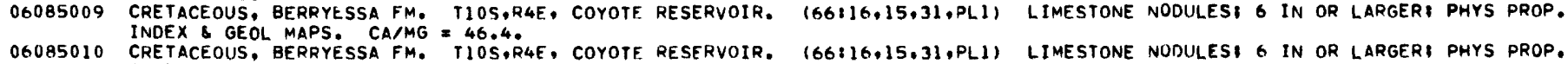

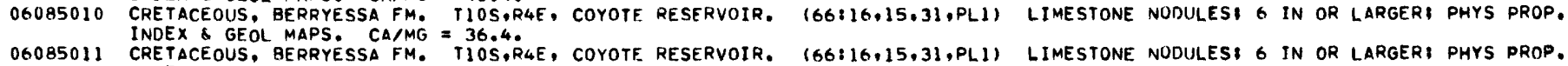
06085011 CRETACEOUS, BERRYESSA FM. TIOS,R4E, COYOTE RESERVOIR. (66:16,15.31,PL1) LIMESTONE NODULES 6 IN OR LARGER: PHYS PROP,
06085012 JURE \& GEOL MAPS. CAMG = 50.0. 06085012 JURASSIC \& CRETACEOUS, FRANCISCAN FM. "TGS, R2W" LOS ALTOS, $129176,11,68-70, P L 1$
MASSIVE: AVG OF B ANAL. INDEX \& GEOL MAPS. MEAS SEC. USE: $4,7.9$ CAMG $>100$.

06085037 JURASSIC \& CRETACEOUS, FRANCISCAN FM. "T5S,R3W" EAGLES, (175:358) LIMESTONE: QUARRY SMPL: TONNAGE. USE: 7.

06085051 JURASSIC \& CRETACEOUS, FRANCISCAN FM. S17,18,T7S,R2W. $(331: 391,374,376)$ LIMESTONE: TONNAGE. CA/MG >100.

06085052 JURASSIC \& CRETACEOUS, FRANCISCAN FM. S17,18,T75,R2W. $(331: 377,374,376)$ LIMESTONE 1 AVG COMPOS OF MANY SMPL TONNAGE.

0608507 USE: 4 . CAIMG $>100$

USERASSIC \& CRETACEOUS FRANCISCAN FM. CALERA LS MBR. S17,18,T7S,R2W. (40:24,21) LIMESTONE $\$$ INCL CHERT LENSES

0608507 QUARRY SMPL. USE : 4 . CAIMG $>100$

JURASSIC \& CRETACEOUS: FRANCISCAN FM, CALERA LS MBR, S17,18,T7S,R2W. $(40: 24,21)$ LIMESTONE: INCL CHERT LENSES

0608507

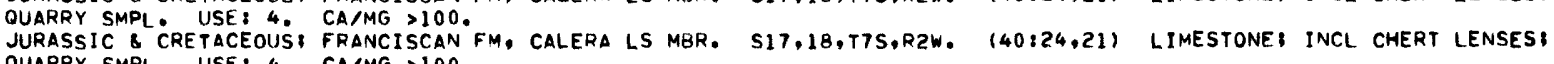

06085074 QUARAY SMPL. USE 24 . CAMMG $>100$. JURASSIC \& CRETACEOUS, FRANCISCAN FM, CALERA LS MBR, S17,18,T7S,R2W. $140: 24,21)$ LIMESTONE INCL CHERT LENSESI

06085080 QUARRY SMPL. USE: 4 , CAMMG $>100$. JURASSIC \& CRETACEOUS, FRANCISCAN FM, CALERA LS MBR, S17,18,T7S,R2W. $(40: 24,21)$ LIMESTONE: INCL CHERT LENSES: 06085081 QUARRY SMPL. USE: 4. CAIMG >100. QUARRY SMPL USE: 4 : CAMMG 06085082 QUARRY SMPL. USE: 4. CA/MG $>100$. 06087002 "IIIS,RIW" CLIFFSIDE. (428:11,10) MAGNESITE. *MISC: H2O = $1 . L$. LESS CO2. CAMG = 0.109.

06087003 "TI1S,RIW" CLIFFSIDE. (428:11.8.10) MAGNESITE, USE: 10. *MISC: NACL $=0.15$. CA/MG =0.115.

06087026 OLIGOCENE \& (OR) MIOCENE, MINDEGO BASALT. "T9S,RZW" SANTA CRUZ MTNS. (386ICG) DOLOMITE. "MISC: FEO = 2.S, MNOE0.04. 06087030 JURASSIC \& CKETACEOUS. FRANCISCAN FM, "TIOS,R3W" DAVENPORT. (291:81) LIMESTONE, GRAY TO WHITE, COMPACT, TONNAGE. 06107004 S35.36.T17S,R27E, (556:339,PL37) LIMESTONE, WHITE TO BLACK, INDEX MAP. CA/MG = 22.1 .

06109077 PALEOZOIC, CALAVERAS FM. S35,TIN,R15E. (264:20,7-9,PL1.3) LIMFSTONE: OUTCROP SMPL, MIN, INDEX \& GEOL MAP. 06109078 PALEOZOIC, CALAVERAS FM. S35.TIN,R15E. (264:20,7-9,PL1.3) LIMESTONE: OUTCROP SMPL, MIN, INDEX \& GEOL MAP. 06109108 CALMG $=17.2$.
MALEZOIC, CALAVERAS FM. S35,TIN.R15E. (264:20,7-9,19,PL1.3) DOLOMITIC LIMESTONEI OUTCROP SMPL, MIN, INDEX \& GEOL
MAPS, CAMG 9.76 . 
TABLE 8. - Analyses of samples from California containing 75-90 percent carbonate (Group $\left.F_{1}\right)$, common- and mixed-rock categories - Continued

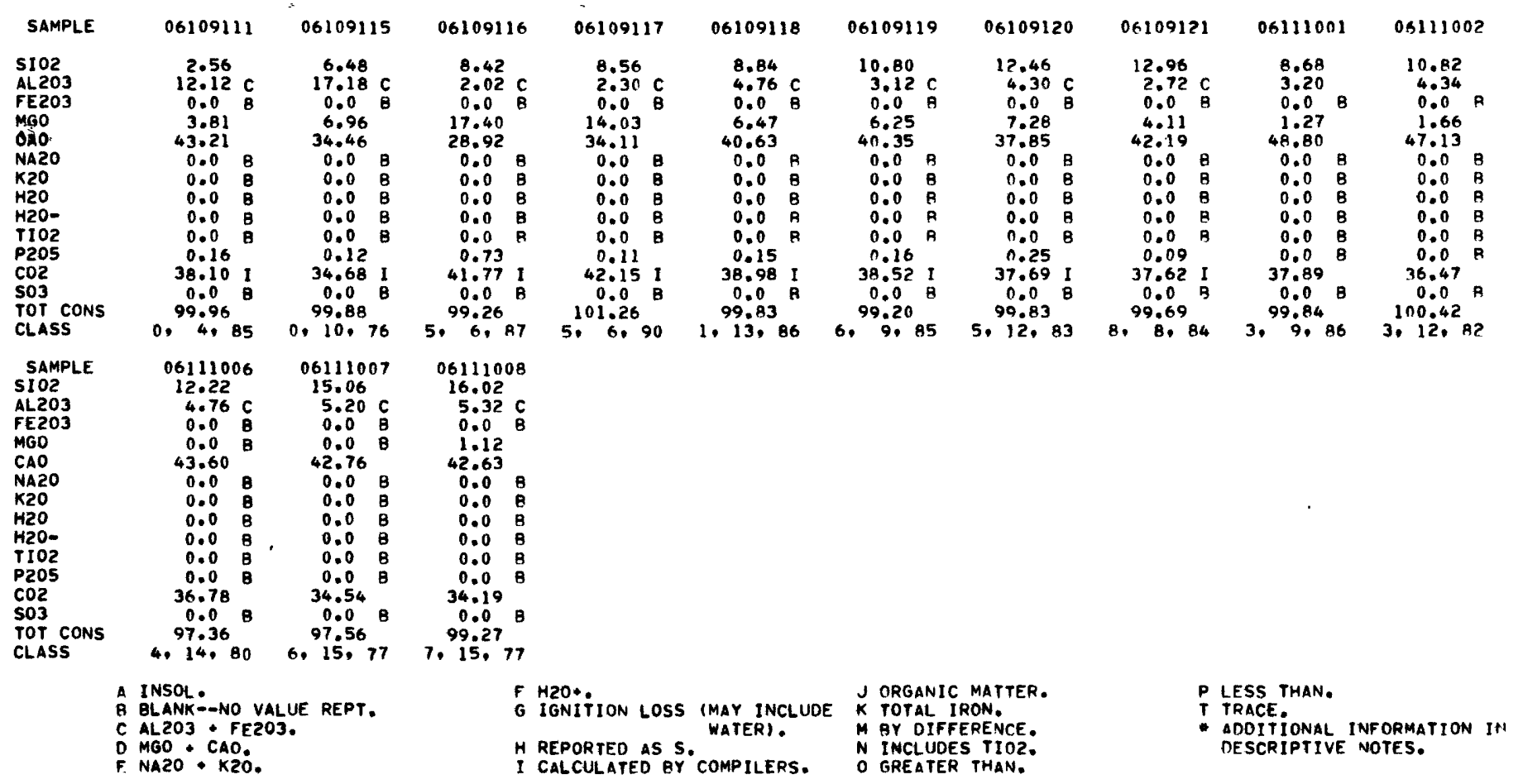


TABLE 8. - Analyses of samples from California containing 75-90 percent carbonate (Group $F_{1}$ ), common- and mixed-rock categories - Continued

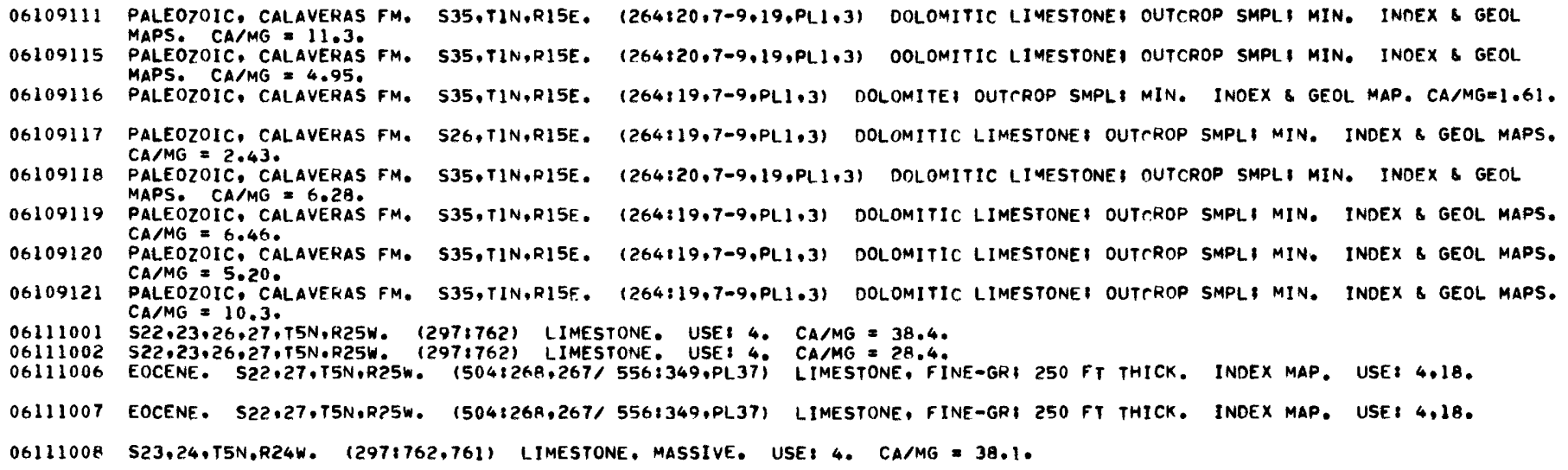


TABLE 9. - Analyses of samples from California and Hawaii containing more than 90 percent carbonate (Group $\left.F_{2}\right)$, common-rock category

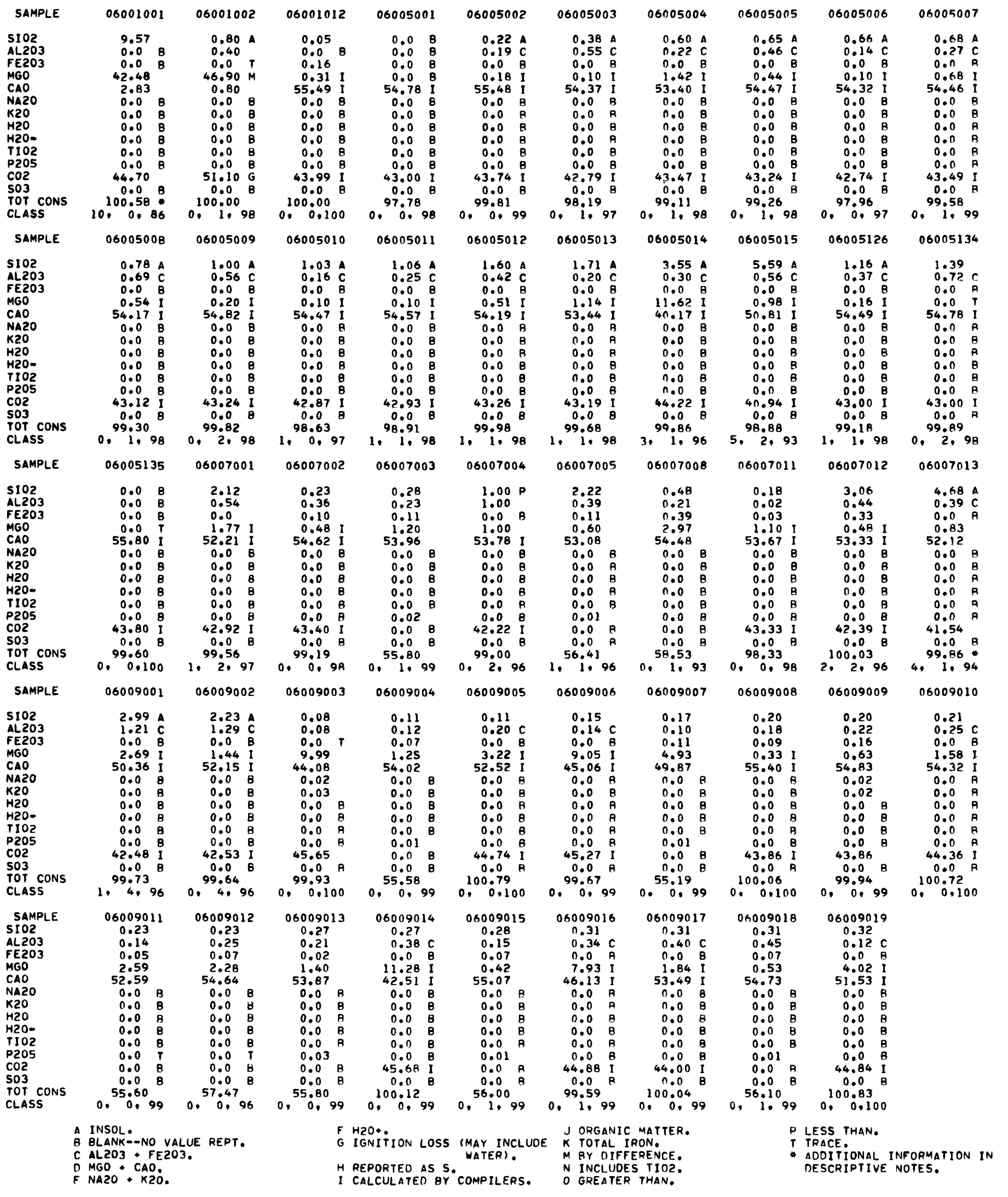


TABLE 9. - Analyses of samples from California andHawaii containing more than 90 percent carbonate (Group $F_{2}$ ), common-rock category Continued

06001001 S27.T4S.R3E. (553:151/58:15,16,41,PL1) MAGNESITE. INDEX MAP, USE: 16, MISC: FEO 1.00 . CA/MG = 0.067.

06001002 "T3S,R2E" LIVERMORE, $(428: 10,9)$ MAGNESITE. CA/MG $=0.017$.

06001012 "T4S,R2W" WASHINGTON CORNERS. (256:107) LIMESTONE, USE: 4. CA/MG > 100.

06005001 PALEOZOIC, CALAVERAS FM. S16,T6N,R10E. (556:208,207,PL37) LIMESTONE, FINELY CRYS. INDEX MAP. USE: 18.

06005002 PALEOZOIC. CALAVERAS FM. "T7N.R12E" VOLCANO. (556:212,207,211,PL37) LIMESTONE: DH. $230-235$ FT. INOEX MAP. USE: 4. 0600S003 PALEOZOIC, CALAVERAS FM. S23,T7N,RI2E, 1801213,212, PLI) LIMESTONE, GRAY; SURFACE SMPL. INDEX \& GEOL MAP, GEOL SEC. 06005003 PALEOZOIC, CALAVERAS FM. S23,T7N,RI2E. $180: 213,212$, PLI) LIMESTONE, GRAY; SURFACE SMPL. INDEX \& GEOL MAP, GEOL SEC. 06005004 PALEOZOIC, CALAVERAS FM. S5,T7N,RI2E. (556:210,207,PL37) LIMESTONE, GRAY TO WHITE: TONNAGE. INDEX MAP. CA/MG=37.6. 06005005 PALEOZOIC, CALAVERAS FM. S23,T7N,RIZE. (80:213.212,PL1) LIMESTONE, GRAY SURFACE SMPL. INDEX \& GEOL MAP, GEOL SEC. 06005006 PALEOZOIC, CALAVERAS FM. S23,T7N,RI2E. $180: 213.212$,PLI) LIMESTONE, GRAY: SURFACE SMPL. INDEX \& GEOL MAP, GEOL SEC. 06005007 PALEOZOIC, CALAVERAS FM. S23,T7N,RI2E. (80:213,212,PL1) LIMESTONE, GRAY: SURFACE SMPL. INDEX \& GEOL MAP, GEOL SEC. 06005008 PALEOZOIC, CALAVERAS FM, S23,T7N,R12E. (80:213,212,PL1) LIMESTONE, GRAYI SURFACE SMPL. INDEX \& GEOL MAP, GEOL SEC. 06005009 PALEOZOIC, CALAVERAS FM. S5,8,T7N,R1OE. (556:211,207,PL37) LIMESTONE, GRAY, FINELY CRYS. INDEX MAP. CA/MG >100. 06005010 PALEOZOIC, CALAVERAS FM. S23,T7N,RI2E. (801213.212.PL1) LIMESTONE, GRAYI SURFACE SMPL. INDEX \& GEOL MAPS, GEOL SEC. 06005011 PALEOZOIC, CALAVERAS FM. S23,T7N,P12E. $(80: 213,212$, PL1) LIMESTONE, GRAYI SURFACE SMPL. INOEX \& GEOL MAP. GEOL SEC. 06005012 PALEOZOIC, CALAVERAS FM, S3,TGN,RIOE. (556:208,207,PL37) LIMESTONE, GRAY, MED-GR. INDEX MAP. CA/MG \$100.

06005013 PALEOZOIC, CALAVERAS FM. S23,T7N,R12E. (80:213.212,PL1) LIMESTONE, GRAY, SURFACE SMPL. INDEX \& GEOL MAP, GEOL SEC. 06005014 PALEOZOIC, CALAVERAS FM. S33,T7N,RIOE. $15562209,207,208, P L 37)$ MAGNESIAN LIMESTONE, GRAY, INDEX MAP. CA/MG = 3.46. 06005015 PALEOZOIC, CALAVERAS FM. S6,T7N,P11E. (556:209,207,PL37) MARBLE, WHITE TO GRAY, QUARRY SMPL. INDEX MAP. USE:15. 06005126 PALEOZOIC. CALAVERAS FM. "T7N,RI2E" VOLCANO, $1556: 212.207 .211$.PL37) LIMESTONEI SURFACE SMPL. INDEX MAP. USE: 4. $C A / M G>100$.

06005134 "TGN,R9E" IONE. $168: 660)$ LIMFSTONE, USE ? 2.

06005135 "S28.29,T7N,RI2E" (144:241) MARRLE, WHITE WITH BLUISH \& BLACK VEINS: QUARRY SMPL.

06007001 S8.T21N,R4E. (571:69/5561214,PL37) MARBLE: TONNAGE. INDEX MAP. CA/MG 29.5 .

06007002 S18.T21N,R4E. (571:69/556:215.214.PL37) MARBLE: TONNAGE. INDEX MAP. CA/MG >100.

06007003 PALEOZOIC, CALAVERAS FM. S8,T2IN,R4E. $1130: 19,17,18, P L 1,2)$ LIMFSTONE, GRAY TO BLACK, DENSE: MIN. INDEX \& GEOL MAPS, 06007004 SB,T21N,R4E. (556:214,PL37) LIMESTONE. INOEX MAP. TONNAGE. CA/MG $=53.8$.

06007005 PALEOZOIC, CALAVERAS FM. S17,T2IN,R4E, (130119.6.17,18,PL1) LIMESTONE, GRAY TO BLACK, DENSF: MIN. INDEX \& GEOL MAP, 06007008 SB,T21N,R4E. (571:67, 556:213,PL37) MARBLE: QUARRY SMPL: TONNAGE. INOEX MAP. USE: 2 . CA/MG = 18.9.

06007011 S11,T2ZN,RGE. (571:68) MARBLE: COARSE-GRI QUARRY SMPLI COMPOS OF 4 SMPLSI TONNAGE. INDEX MAP. CA/MG = 48.8.

$06007012535,122 N, R 6 E$. (571:68) MARBLE; QUARRY SMPL, COMPOS OF 4 SMPLS: TONNAGE. INDEX MAP. CAMMG >100.

06007013 CARBONIFEROUS TO JURASSIC. S2,T21N,R6E. (2838576,567,PL1/571:69,MAP 21 LIMESTONEI MIN. INDEX 8 GEOL MAPS.

06009001 PALEOZOIC, CALAVERAS FM. S32, T5N,RIIE. $(556: 218,96: 104,94$, PL A.D) LIMESTONE. WHITE TO GRAY, FINE-GR. INDEX \& GEOL

06009002 PALEOZOIC. CALAVERAS FM. S4,T4N,RIIE. $1556: 218 / 96: 104.94 .211 . P L$ A.0) LIMESTONE, WHITE TO GRAY, FINE-GR. INOEX \& 06009003 PALEOZOIC. CALAVERAS FM. SI0,T4N,R13E. $(93: 14,4,7, P L 1,2)$ LIMESTONE, WHITE TO GRAY. COARSE- TO FINE-GR CHIP SMPL REPR 06009004 PALEOZOIC. CALAVERAS FM, S31,T4N,RI4E. $(96: 104,94, P L, A, D)$ LIMESTONE. INDEX \& GEOL MAPS, GEOL SEC. CA/MG E 43.2. 06009005 PALE0ZOIC, CALAVERAS FM. S3,10,T4N,R13E. (96:102,94,PL,A,D) DOLOMITIC LIMESTONE, WHITE TO GRAY, 600-1,500 FT THICK, 06009006 SMPL TAKEN ACROSS $100 \mathrm{FT}$. INDEX \& GEOL MAPS, GEOL SEC. CA/MG $=16.3$.

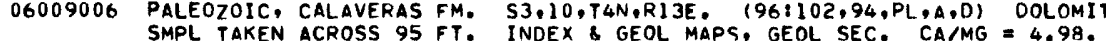
06009007 PALEOZOIC. CALAVERAS FM. S31.T4N,R14E. $196: 104,94, P L$.A.D) MAGNESIAN LIMESTONE. INHEX \& GEOL MAPS, GEOL SEC. 06009008 PALEOZOIC, CALAVERAS FM. S25,35,T4N,R12E, $(96198,94,97$, PL A,D) LIMESTONF, WHITE TO GRAY, MASSIVE, COARSELY CRYS DH OF 164 FT. INDEX \& GEOL MAPS, GEOL SEC. USE: 4. CAMMG $>100$.

06009009 PALEOZOIC. CALAVERAS FM, S11,T4N,RI3E. (93:14,4,7.PL1.2) LIMESTONE, WHITE, GRAY, COARSE- TO FINE-GRI CHIP SMPL OF $100 \mathrm{FT}$. INDEX \& GEOL MAPS. CARMG $=87.0$.

06009010 PALEOZOIC. CALAVERAS FM. S3,10,T4N,R13E. 196:102,94,PL,A.DI LIMESTONE, WHITE TO GRAY, 600-1,500 FT THICK SMPL TAKEN

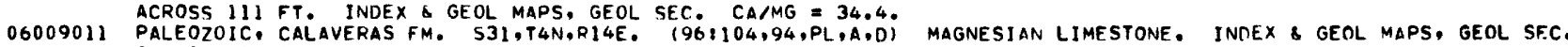
06009012 PALEOZOIC, CALAVERAS FM. S31,T4N,RI4E. $1961104,94, P L, A, D)$ MAGNESIAN LIMESTONE. INDEX \& GEOL MAPS, GEOL SEC. 06009013 PALEOZOIC, CALAVERAS FM. S31,T4N,R14E, $(96: 104,94, P L, A, D)$ LIMESTONE. INDEX \& GEOL MAPS, GEOL SEC. CA/MG = 38,5.

06009014 PALEOZOIC, CALAVERAS FM, S3,10,T4N,RI3E. (96:102,94,PL,A,D) DOLOMITIC LIMESTONE, WHITE TO GRAY, 600-1,500 FT THICK

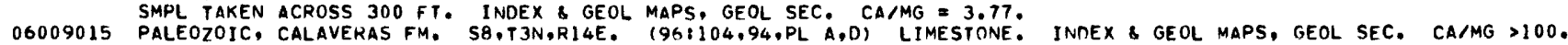
06009016 PALEOZOIC, CALAVERAS FM, S3,10,T4N,RI3E. (96:102,94,PL,A,D) DOLOMITIC LIMESTONE, WHITE TO GRAY, 600-1,500 FT THICK 06009017 PMPL TAKEN ACROSS 951 FT. INDEX \& GEOL MAPS, GEOL SEC. CA/MG = 5.82. 06009018 ACROSS 300 FT. INDEX O GEUL MAPS, GEOL SEC. CAIMG $=29.1$.

06009019 PALEOZOIC, CALAVERAS FM. S3,10,T4N,R13E, (96:102,94,PL,A,D) DOLOMITIC LIMESTONE, WHITE TO GRAY, G00-1,500 FT THICK: SMPL TAKEN ACROSS $94 \mathrm{FT}$. INDEX \& GEOL MAPS, GEOL SEC. CA/MG $=12.8$. 
TABLE 9. - Analyses of samples from California and Hawaii containing more than 90 percent carbonate (Group $\left.F_{2}\right)$, common-rock category Continued

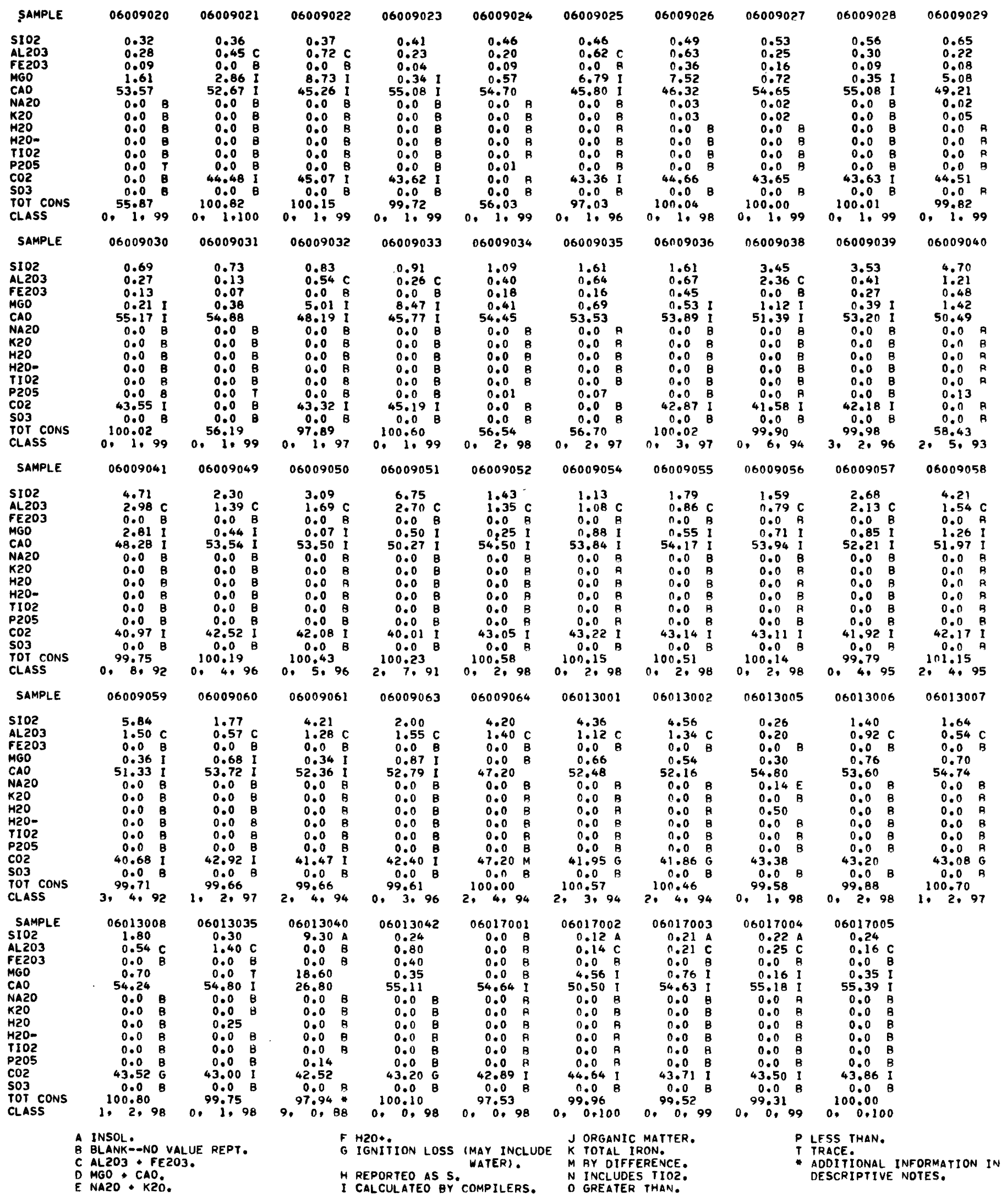


TABLE 9. - Analyses of samples from California and Hawaii containing more than 90 percent carbonate (Group $\left.F_{2}\right)$, common-rock category Continued

06009020 PALEOZOIC, CALAVERAS FM. S31,T4N,R14E, (96:104,94,PL,A.0) LIMESTONE. INDEX \& GEOL MAPS, GEOL SEC. CA/MG = 33.3. 06009021 PALEOZOIC, CALAVERAS FM. S3,10,T4N,R13E, (96:102,94,PL,A,D) LIMESTONE, WHITE TO GRAY, 600-1,500 FT THICK SMPL TAKEN 06009022 ACROSS 121 FT. INOEX \& GFOL MAPS, GEOL SEC. CAMG = 18.4 .

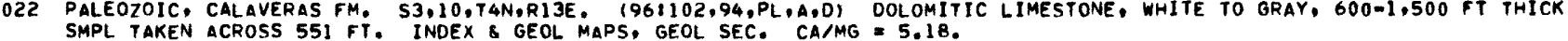
06009023 PALEOZIC. CALAVERAS FM. S4,T2N,R14E. $196: 103,94, P L$ A.01 LAMG 5 . 5.18. WHONE, WHITE, COARSELY CRYSI TONNAGE. INDEX \& GEOL 06009024 PALEOZOIC. CALAVERAS FM. S8,T3N,RI4E. 196:104,94,PL A,O) LIMESTONE, INDEX GEOL MAPS, GEOL SEC. CA/MG = 9R.0. 06009025 PALEOZOIC, CALAVERAS FM. S3,10,T4N,R13E. (96:102,94,PL,A,D) DOLOMITIC LIMESTONE, WHITE TO GRAY, 600-1,500 FT THICK, SMPL TAKEN ACROSS $1.042 \mathrm{FT}$. INDEX \& GEOL MAPS, GEOL SEC. CA/MG $=6.75$. 06009026 PALEOZOIC. CALAVERAS FM. S11,T4N,RI3E. (93114,4.7,PLI,2) LIMESTONE; WHITE TO GRAY, COARSE- TO FINE-GR; 700-FT CHIP 060 SMPL. INDEX G GEOL MAPS. CAMG $=6.16$.

06009027 PALEOZOIC. CALAVERAS FM. S28,T4N,R13E. (93114,4,17,PL1,2) LIMESTONE. WHITE TO GRAY, COARSE-GR 80-FT CHIP SMPL. INDEX \& GEOL MAPS. CAIMG $=75.9$.

06009028 PALEOZOIC. CALAVERAS FM. S25,35,T4N,R12E. (96:98,94,97,PL A,D) LIMESTONE, WHITE TO GRAY, MASSIVE, COARSELY CRYS

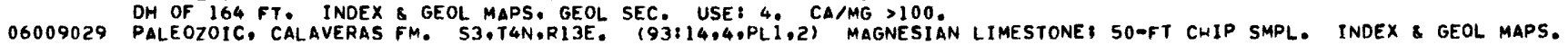
06009030 PALEOZOIC, CALAVERAS FM. S25,35,T4N,R12E. $196: 98,94,97, \mathrm{PL} A, 01$ LIMESTONE, WHITE TO GRAY, MASSTVE, COARSELY CRYSI 06009031 PH OF 164 FT. INDEX \& GEOL MAPS, GEOL SEC, USE: 4. CA/MG $>100$.

06009032 PALE0ZOIC, CALAVERAS FM. S3,10,T4N,R13E. 196:102,94,PL,A,D) DOLOMITIC LIMESTONE, WHITE TO GRAY, 600-1,500 FT THICKI SMPL TAKEN ACROSS 1 , 239 FT. INOEX 8 GEOL MAPS, GEOL SEC. CA MMG $=9.62$.

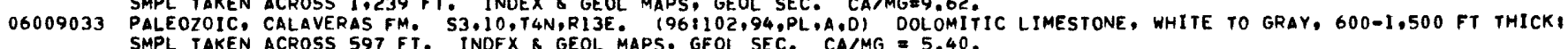
06009034 PMPL TAKEN ACROSS S97 FT. INDEX \& GEOL MAPS, GEOL SEC. CAAMG 25 .40. 06009035 PALE0ZOIC, CaLAVERAS FM. SB,T3N,R14E. (96:104,94,PL A,D) LIMESTONE. INNEX \& GEOL MAPS, GEOL SEC. CA/MG = 77.6. 06009036 PALEOZOIC, CALAVERAS FM. S25,35,T4N,R12E. $196: 98,94,97$, PL A.D) LIMESTONE, WHITE TO GRAY, MASSIVE, COARSELY CRYS

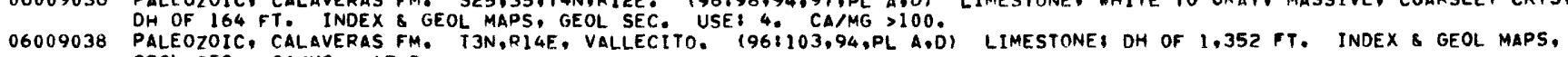
06009038 PALEOZOIC, CALAVERAS FM. T3N,R14E, VALLECITO. (96:103.94,PL A.D) LIMESTONE, DH OF 1,352 FT. INDEX 8 GEOL MAPS, 06009039 PALEOZOIC, CALAVERAS FM. S25,35,T4N,RI2E. (96:98,94,97,PL A,D) LIMESTONE, WHITE TO GRAY. MASSIVE, COARSELY CRYSI 06009040 PALEOZOIC, CALAVERAS FM. S36,T6N, RI2E. $961103,94^{\circ}, \mathrm{PL}$ CAMG $\$$ LIM0. LIMESTONF, WHITE TO GRAY, COARSE-GR. INDEX \& GEOL MAPS, 06009041 PALEOZOIC, CALAVERAS FM. T3N,R14E, VALLECITO. (96:103,94,PL A.D) LIMESTONE, DH OF 1,352 FT. INDEX \& GEOL MAPS, 06009049 PELLEOZOIC, CALAVERAS FM. "T4N,RIZE" SAN ANDREAS. $(485: 3,2)$ LIMESTONE. USE: 4 . CA/MG $>100$.

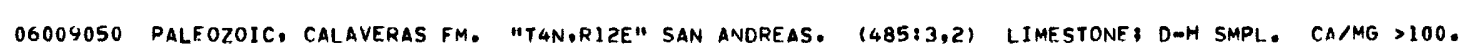

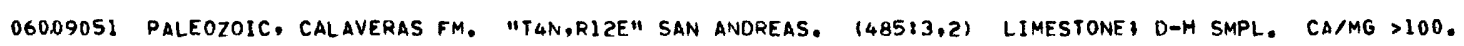

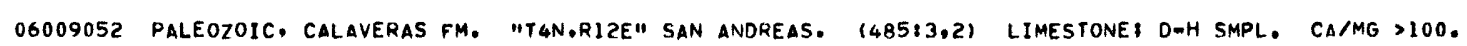
06009054 PALEOZOIC, CALAVERAS FM. "T4N,RIZE" SAN ANOREAS. $(485: 3,2)$ LIMESTONE: D-H SMPL. CA/MG $=61.2$. 06009055 PALE0Z0IC, CalaVERAS FM. "T4N,RlzE" SAN ANIREAS, (485:3,2) LIMESTONES D-H SMPL. CA/MG $=98.5$. 06009056 PALEOZOIC, CALAVERAS FM. "T4N.RIZE" SAN ANOREAS. $(485: 3.2)$ LIMESTONE: D-H SMPL. CA/MG $=76.0$. 06009057 PALEOZOIC, CalAVERAS FM. "T4N.R12E" SAN ANDREAS. (485:3,2) LIMESTONE: D-H SMPL. CA/MG $=61.4$. 06009058 PALEOZOIC, CaLAVERAS FM. "T4N,RIZE" SAN ANOREAS. $(48583.2)$ LIMESTONEI D-H SMPL. CA/MG $=41.2$. 06009059 PALEOZOIC, CALAVERAS FM. "T4N,R12E" SAN ANDREAS. $(485: 3,2)$ LIMESTONE\$ D-M SMPL. CA/MG $>100$. 06009060 PaLEOZOIC, CALAVERAS FM. "T4M,R1ZE" SAN ANDREAS. $(485: 3,2)$ LIMESTONE: O-M SMPL. CAMMG $=79.0$. 06009061 PALE0ZOIC, CalaVERAS FM. "T4N,RIZE" SAN ANDREAS. (485:3,2) LIMESTONE: D-H SMPL. CA/MG $>100$. 06009063 PALEOZOIC, CALAVERAS FM. "T4N,RIZE" SAN ANDREAS, $1485: 3,21$ LIMESTONE

\section{4 "TAN,R10E" CAMPO SECO. 168:660) LIMESTONE.}

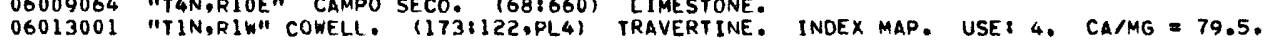
06013002 "TIN,RIW" COWELL. (1731122,PL4) TRAVERTINE. INDEX MAP. USE: 4. CA/MG = 96.6. 06013005 "T2N,R3W" PORT COSTA. (303:311,310/173: 119,PL4) TRAVERTINE. INDEX MAP. USE 4,18. CA/MG $>100$. 06013006 PLEISTOCENE TO RECENT. "TIN,RIW" CONCORD. $(175: 360,359)$ TRAVERTINE. USEI 4 . CA/MG = 70.5 . 06013007 "TIN,RIW" COWELL. (173:122,PL4) TRAVERTINE, QUARRY SMPL. INDEX MAP. USE: 4. CA/MG = 78.2. 06013008 "TIN,RIW" COWELL. (173:122,PL4) TRAVERTINE, FLOAT. INDEX MAP. USE:4. CA/MG = 77.5.

06013035 "TIS.RIW" MOUNT DIABLO. (512:301) LIMESTONE, COMPACT: B.D. 2.63.

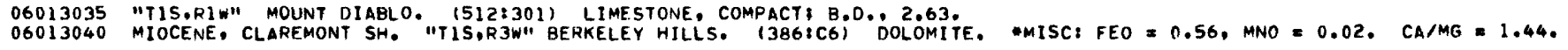

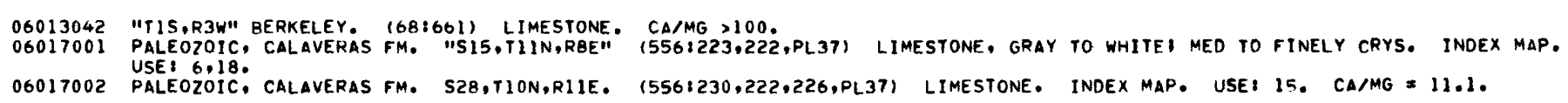
06017003 PALEOZOIC, CALAVERAS FM. "T9N.R9E" SHINGLE SPRINGS, (5561231,222,230,PL37) LIMESTONE, WHITE. INOEX MAP. USE: $12,18$.

06017004 PALEOZOIC. CALAVERAS FM. S6,18,TIZN,K9E, (556:225,222,224,PL37) LIMESTONE, GRAY TO BLACK, FINELY CRYS CHIP SMPL OF

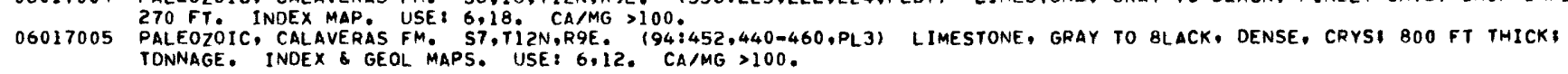


TABLE 9. - Analyses of samples from California and Hawaii containing more than 90 percent carbonate (Group $F_{2}$ ), common-rock category Continued

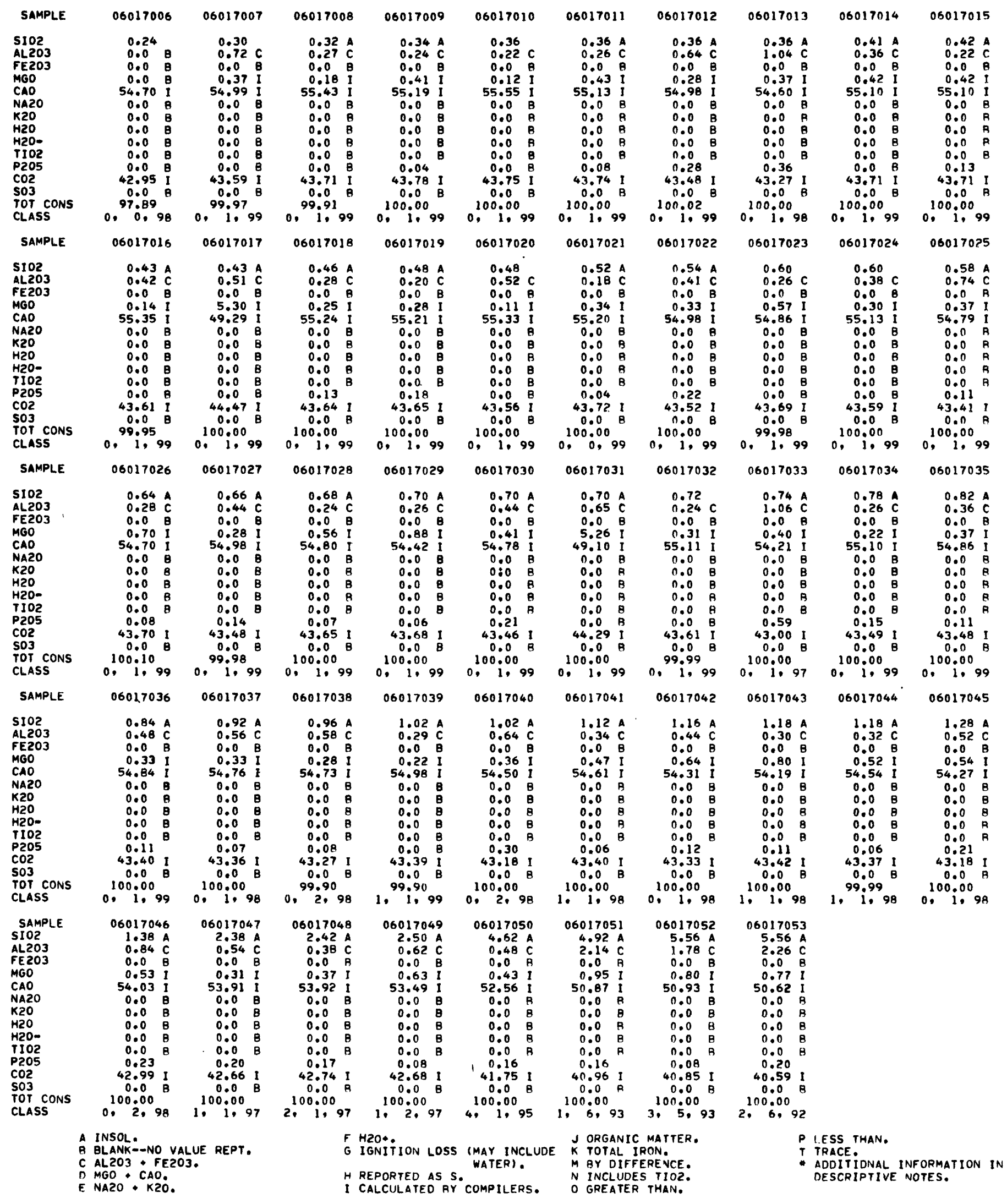


TABLE 9. - Analyses of samples from California and Hawaii containing more than 90 percent carbonate (Group $\left.F_{2}\right)$, common-rock category Continued

06017006 PALE07OIC, CALAVERAS FM. S15,22,T9N,R9E, (556:231.222,PL37, 95:447.PL9,10) LIMESTONE, WHITE, MED- TO COARSE-GR. 06017007 PALEOZOIC. CALAVERAS FM. S7,TI2N, 49E. $194: 452,440-460$, PL3) LIMESTONE, GRAY TO BLACK, DENSE, CRYS; 800 FT THICK

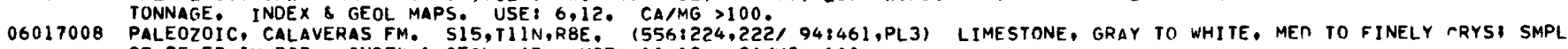
06017009 OF 25 FT IN PIT INDEX \& GEOL MAP. USE: 10.18 . CAMMG $>100$

06017009 PALEOZOIC, CALAVERAS FM. S7.T12N,R9E. $(94: 454.440-460$. PL3) LIMESTONE, GRAY TO BLACK, DENSE. CRYS BOO FT THICK TONNAGE. INDEX \& GEOL MAPS. USF: 6,12 . CA/MG $>100$.

06017010 PALEOZOIC, CALAVERAS FM. S7,T12N,R9E. (94:452,440-460,PL3) LIMESTONE, GRAY TO BLACK, DENSE, CRYS BOO FT THICK, 06017011 PALEOZOIC, CALAVERAS FM. S7,TI2N,R9E. $194: 454,440-460, P L 3)$ LIMESTONE. GRAY TO BLACK, DENSE, CRYS: BOO FT THICK:

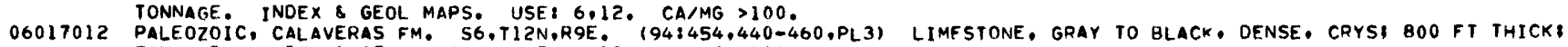
06017013 PALEOTOIC, CALAVERAS FM. S7,TI2N, 69E. $(94: 454,440-460, P L 3)$ LIMESTONE, GRAY TO BLACK. DENSE, CRYS: BOO FT THICK 06017014 PALEOZOIC, CALAVERAS FM. "T9N.R9E" SHINGLF SPRINGS. (556:231, 22?,230,PL37) LIMESTONE, WHITE. INDEX MAP. USE: 12, 18. 06017015 PALEOZOIC. CALAVERAS FM. S6,TI2N,R9E. (94:454,440-460,PL3) LIMESTONE, GRAY TO BLACK, DENSE, CRYSI 800 FT THICK: 06017016 PALEOZOIC. CALAVERAS FM. SB.17,T9N,R9E. (556:232,222,PL37) LIMFSTONE, GRAY TO WHITFI QUARRY SMPL. INDEX MAP. 06017017 PALEOZOIC, CALAVERAS FM. S28,TION,R11E. (556:230.222,226,PL37) LIMESTONF. INDEX MAP. USE: 15. CA/MG = 9.30. 06017018 PALEOZOIC. CALAVERAS FM. S6,TIZN,R9E, (94:454,440-460,PL3) LIMESTONE, GRAY TO BLACK, DENSE, CRYSI 800 FT THICK, 06017019 PALEOZOIC. CALAVERAS FM. S6,T12N, 69E. $(94: 454,440-460$, PL 3) LIMESTONE, GRAY TO BLACK. DENSE. CRYSI BOO FT THICK, TONNAGE. INDEX \& GEOL MAPS. USE: 6.12. CA/MG $>100$.

06017020 PALEOZOIC. CALAVERAS FM. S7,TI2N.R9E. $194: 452,440-460, P L 3)$ LIMESTONE, GRAY TO BLACK, DENSE, CISYSI 800 FT THICK 06017021 PALEOZOIC, CALAVERAS FM. S18,TI2N,R9E, $194: 454,440-460, P L 3)$ LIMFSTONE. GRAY TO BLACK. DENSE, CRYS 800 FT THICK 06017022 PALEOZOIC, CALAVERAS FM. S7,TI2N,R9E. $(94: 454,440-460, P L 3)$ LIMESTONE, GRAY TO BLACK, DENSE, CRYSI BOO FT THICK 06017023 PALEOZOIC, CALAVERAS FM. "T9N.R9E" SHINGLE SPRINGS. $1556: 231,222,230$, PL 37) LIMESTONE, WHITE. INOEX MAP. USE: 12,18. 06017024 PALEOZOIC, CALAVERAS FM. "T9N,R9E" SHINGLE SPRINGS, $(556: 231,222,230$, PL37) LIMESTONE, WHITE. INDEX MAP. USE: $12,18$. 06017025 PALEOZOIC, CALAVERAS FM. S7,TIIN,R9E. $1949454,440-460$, PL3) LIMESTONE, GRAY TO BLACK. DENSE, CRYS 800 FT THICK 1 06017026 PALEOZOIC, CALAVERAS FM. S7,TI2N,R9E. $(94: 454,440-460$, PL3) LIMESTONE, GRAY TO BLACK. DENSE, CRYSI 800 FT THICK: 06017027 PALEOZOIC. CALAVERAS FM. S6, TI2N, 69E, $194: 454,440-460$, PL3) LIMESTONE. GRAY TO BLACK, DENSE, CRYSI 800 FT THICK:

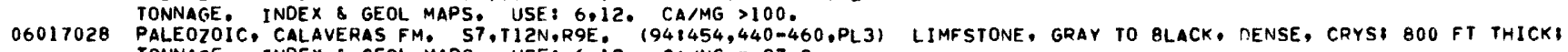

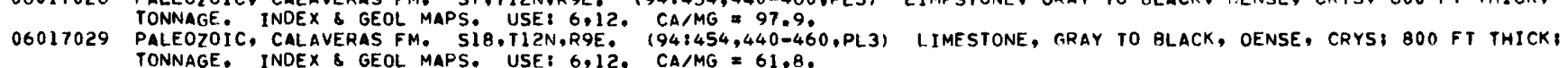

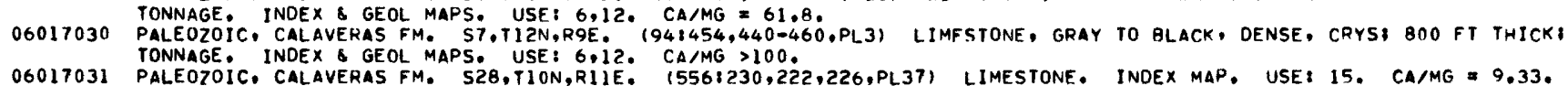

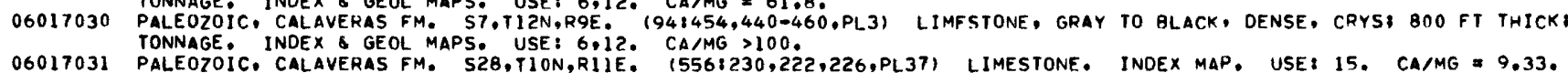

06017032 PALEOZOIC, CALAVERAS FM, "TON,R9E" SHINGLE SPRINGS, (556:231.222,230,PL37) LIMESTONE, WHITE. INDEX MAP. USE: 12,19.

06017033 PALEOZOIC, CALAVERAS FM. S7,T12N,R9E. $(94: 454,440-460$, PL3) LIMFSTONE, GRAY TO BLACK, DENSE, CRYS 3 COMPOS CHIP SMPL;

06017033 PALEOZOIC, CALAVERAS FM. S7,T12N,R9E. $194: 454,440-460, P L 3)$ LIMFSTON
800FT THICK TONNAGE. INDEX GEOL MAPS. USE: 6,12 . CAMG $>100$.

06017034 PALEOZOIC, CALAVERAS FM. S6,TI2N,R9E, $(94: 454,440-460$, PL 3$)$ LIMESTONE, GRAY TO 8 LACK, DENSE, CRYS BO FT THICK

06017035 PALEOZOIC, CALAVERAS FM. S7,TI2N, G9E. $(94: 454,440-460$, PL3) LIMESTONE, GRAY TO BLACK, DENSE, CRYS BO FI THICK 06017036 PALEOZOIC, CALAVERAS FM. S6,T12N,R9E. $(94: 454,440-460$, PLI3) LIMESTONE, GRAY TO BLACK, DENSE, CRYS 800 FT THICK 06017037 PALEOZOIC, CALAVERAS FM. S6,TI2N,R9E. $194: 454.440-460$. PL 3$)$ LIMFSTONE. GRAY TO BLACK, DENSE. CRYS 800 FT THICK

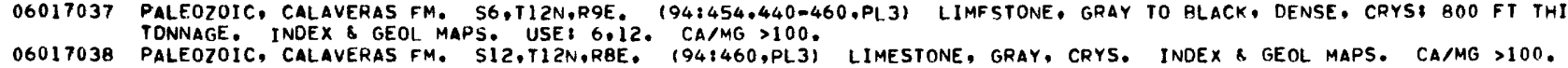

06017039 PALEOZOIC, CALAVERAS FM. S6,18,TI2N,R9E. (556:225,222,224,PL37) LIMESTONE, GRAY TO BLACK, FINELY CRYS CHIP SMPL OF 06017040 PALEOZOIC. CALAVERAS FM. 57,18 , CAMM $>100$.

06017040 PALEOZOIC, CALAVERAS FM, S7,TI IN,R9E, (94:454,440-460,PL3) LIMESTONE. GRAY TO BLACK, DENSE, CRYS: COMPOS CHIP SMPL 06017041 PALEOZOIC, CALAVERAS FM. S7,TI2N,R9E. $(94: 454,440-460$. PL 3$)$ LIMESTONE. GRAY TO BLACK, DENSE, CRYSI BOO FT THICK:

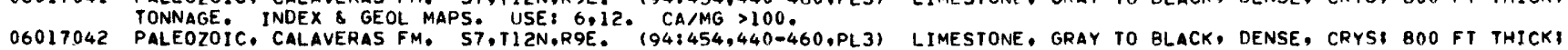
06017042 PALEOZOIC. CALAVERAS FM. S7,TI2N,R9E. $194: 454,440-460$,

06017043 PALEOZOIC, CALAVERAS FM. S7,TIZN,R9E, $(94: 454,440-460$, PL3) LIMESTONE, GRAY TO BLACK, DENSE, CRYSI 800 FT THICK: 06017044 PALEOZOIC, CALAVERAS FM. SIB,TI2N, R9E. $194: 454,440-460$, PL3) LIMESTONE. GRAY TO BLACK, DENSE, CRYSI B00 FT THICK 06017045 PALEOZOIC, CALAVERAS FM. S6,TI2N, R9E. $(94: 454,440-460$, PL3) LIMESTONE, GRAY TO BLACK. DENSE, CRYSI BOO FT THICK: 06017046 PALEOZOIC, CALAVERAS FM. S7,TI2N,R9E. (94:454,440-460,PL3) LIMESTONE, GRAY TO BLACK, DENSE, CRYS: 800 FT THICK: 06017047 PALEOZOIC, CALAVERAS FM, S7,TI2N,R9E. $(94: 454,440-460$, PLI) LIMESTONE, GRAY TO BLACK, DENSE, CRYS COMPOS CHIP SMPL,

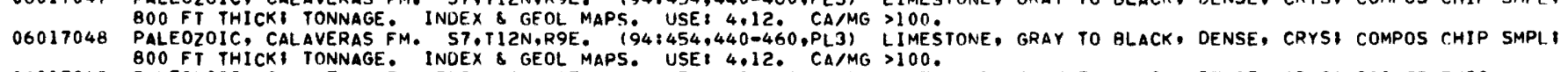

06017049 PALEOZOIC. CALAVERAS FM. SI8,TIZN,R9E. $(94: 454,440-460$. PL3) LIMESTONE, GRAY TO BLACK, DENSE, CRYSI BOO FT THICK,

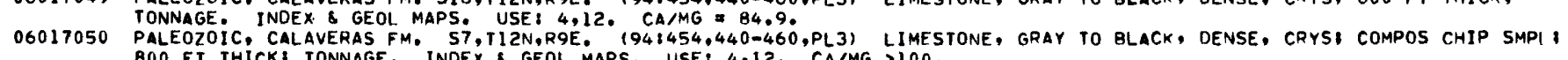
06017051 PALEOZOIC, CALAVERAS FM. S7,T12N,R9E, $(94: 454,440-460$, PL3) LIMESTONE, GRAY TO BLACK, DENSE, CRYS ROO FT THICK, 06017052 PALEOZOIC. CALAVERAS FM. S6,TI2N,R9E. $(941454,440-460$, PL 3$)$ LIMESTONE, GRAY TO BLACK, DENSE, CRYS 800 FT THICK,

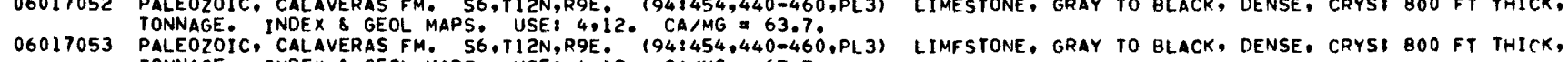
06017053 PALEOZOIC, CALAVERAS FM. S6, T12N, R9E. $194: 454,440=460$,
TONNAGE. INDEX \& GEOL MAPS. USE: 4,12 . CA/MG $=65.7$. 
TABLE 9. - Analyses of samples from California and Hawaii containing more than 90 percent carbonate (Group $\left.F_{2}\right)$, common-rock category Continued

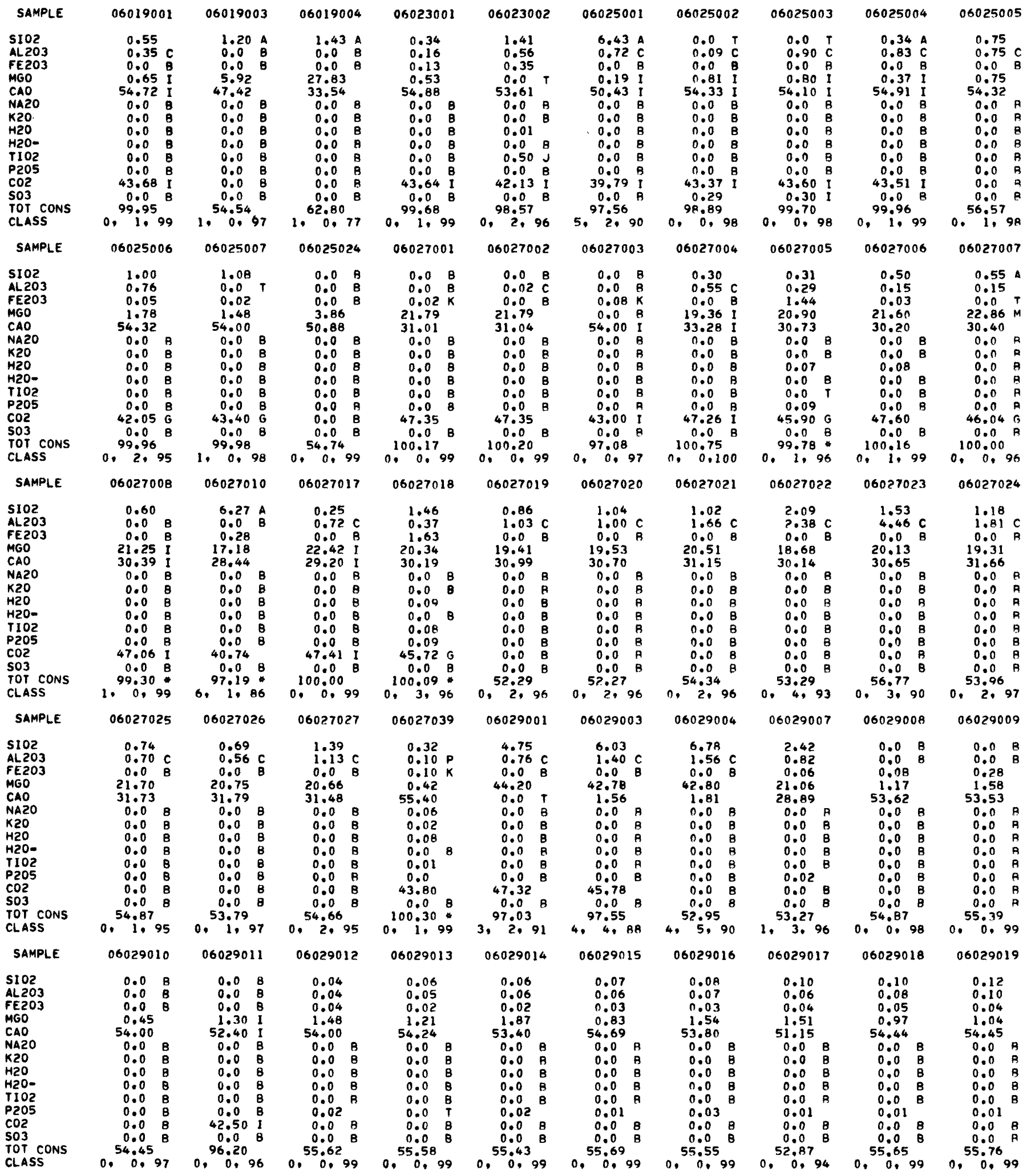

A INSOL.
R BLANK--NO VALUE REPT.
C AL2O3 + FEZO3.
D MGO + CAO.
E NAZO + K2O.
F HZOP.

6 IGNITION LOSS (MAY INCLUDE K TOTAL IRON.

H REPORTED AS S. WATERT.
I CALCULATED BY COMPILERS. N INCLUDES TIOZ:
O GREATER THAN.
P I ESS THAN.

- adoitional information in. DESCRIPTIVE NOTES. 
TABLE 9. - Analyses of samples from California and Hawaii containing more than 90 percent carbonate (Group $F_{2}$ ), common-rock category Continued

06019001 S15,T11S.R24E. (556:236,PL37) LIMESTONE. INDEX MAP. USE: 2.12, CA/MG = 84.2.

06019003 PRE-CRETACEOUS. S29.30.T7S.R26E. (86:253,244.PL7) LIMESTONE, GRAY, MED-GR, CRYSI MINi B.D.,2.69. INDEX A GEOL MAPS.

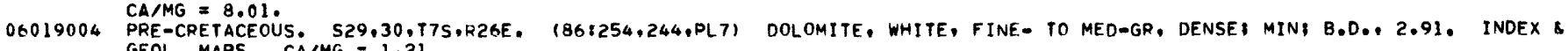
06023001 DEVONIAN. SIB,19,T6N,RSE. (27:518,517, 556:239,PL37) LIMESTONE. INDEX MAP. CA/MG >100.

06023002 JURASSIC \& CRETACEOUS, FRANCISCAN FM, S13,14,T5N,RIE, (3741394,393/ 271516) LIMESTONE. USEI ?,12.

06025001 S31.32,7135,R9E. (497:277) 556:241,PL37) LIMESTONE. INDEX MAP. CA/MG $\$ 100$.

06025002 "T25,165,R9,10E" COYOTE MTN. (557:101,PL 16) LIMESTONE, CRYS. INDEX MAP. CA/MG = 67.1.

06025003 S22,T15S,R9E -SI.T165,R9E. (559:736/556:240,PL37) LIMESTONE, GRAY: TONNAGE. INDEX MAP. USE: 4. CA/MG = 67.6.

06025004 S1,12,T14S,R9E. (497:277) LIMESTONE, GRAY, CRYS, CA/MG $>100$.

06025005 "TI5.165.R9,10E" COYOTE MTNS. (557:15,101.PLi16) LIMESTONEI CRYS. INDEX MAP. CA/MG = 72.4 .

06025006 TERTIARY. S22,T15S,R9E. (497:277.276/556:240,PL37) LIMESTONE, WHITE: TONNAGE. GEOL MAP. USE: 4. CA/MG = 30.5.

06025007 TERTIARY, S22,T15S,R9E. (497:277,276/556:240,PL37) LIMESTONE, BLUE: TONNAGE. GEOI MAP. USE: 4 . CA/MG = 36.5.

06025024 PALEOZOIC OR MESOZOIC. "S22,TI5S,R9E" $1230: 5,3)$ MAGNESIAN LIMESTONE, CRYSI AVG OF 26 SMPL. INDEX MAP. USE: 4. $C A / M G=13,2$

06027001 CAMBRIAN, INYO MARBLE. "T17-195,R36-38E" INYO MARBLE CO. (145:359) MARBLE: QUARRY SMPL. CA/MG = 1.42.

06027002 "TI7S,R37E" KEELER. 1535177.811 DOLOMITE. CA/MG $=1.42$.

06027003 CARBONIFEROUS, "TI7,185,R41,42E" REVENUE CANYON. (231:5) LIMESTONE, GRAY, MED-GR, CRYSi SELECTED SMPL. USE: 6.18.

06027004 "T17S,R37E" KEELER, (535:77,81) DOLOMITE, CA/MG $=1.72$.

06027005 PRECAMBRIAN, NOONDAY DOL. T2ON,RRE. NOPAH MTN. $(269: 302,274.300)$ ALGAL DOLOMITE, CREAMI 1.500 FT THICK. INDEX MAPS.

06027006 "TISS: MNO $=0.05$. CARE" CARTAGO. (428:15.8.10) DOLOMITE. USE: 15. CA/MG $=1.40$.

06027007 "TI9S.R36E" CARTAGO. (428111.10) DOLOMITE. USE: 15. CAMG $=1.33$

06027008 "TI7S,R37E" KEELER, (222:218) DOLOMITE. WHITE, USE; 15, *MISC: SIO2 INCL IRON. CA/MG I. I.43.

06027010 CAMRRIAN, PROSPECT MOUNTAIN OTZ, T2ON,RIOF, HORSE SPRING. (276:30,PL1) NOLOMITE, GRAY, FINE-GR: REDS 10 FT THICK. INDEX \& GEOL MAPS, GEOL SEC. MISC: FEO 3.25 . MNO 1.03 . CAMMG 1.66 .

06027017 "TOS,R34E" (535:77) DOLOMITE. CA/MG $=1.30$.

06027018 PRECAMBRIAN, REEO DOL. "T6S,R34E" HEAD OF WYMAN CREEK, $(269: 302)$ DOLOMITE. $4 M I S C I$ MNO $=0.12$. CA/MG = 1.48.

06027019 "T6S.R35.36E" WYMAN CREEK. (535:77,81) DOLOMITE. CA/MG $=1.60$.

06027020 "T6S.R35.36E" WYMAN CREEK, (535:77,81) DOLOMITE. CAMMG $=1.57$.

06027021 "T6S,R35,36E" WYMAN CREEK. (535177.81) DOLOMITE. CA/MG = 1.52.

06027022 "T6S,R35,36E" WYMAN CREEK, $1535: 78,81)$ DOLOMITE. CAMG $=1.61$.

06027023 "TSS,R35,36E" WYMAN CREEK, $1535: 78,81)$ DOLOMITE, CAMMG $=1.52$.

06027024 "T6S.R35.36E" WYMAN CREEK. (535:77,81) DOLOMITE. CAMMG $=1.64$.

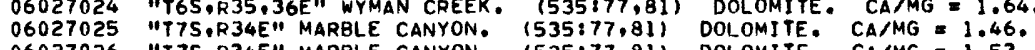

06027026 "T7S.R34E" MARBLE CANYON. $(535: 77,81)$ DOLOMITE. CAMGG $=1.53$.

06027027 "I7S,R35E" ANTELOPE CREEK. (535:77,81) DOLOMITE, CA/MG = 1.52.
06027039 MISSISSIPPIAN \& PENNSYVANIAN, LEE FLAT LS. "S20,T195,R42E" (251:38,PLI,2) LIMESTONE, GRAY TO WHITE, MED-GR: 750 FT THICK: COMPOS SMPL. INDEX \& GEOL MAPS. GEOL SEC. "MISC: MNO $=0.00$, CA/MG $>100$.

06029001 MIOCENE. S11,T1ON.R11W. (207:1118,1115/4361116,114-117) MAGNESITE, WHITE THIN-BEOS TONNAGE. INDEX G GEOL MAPS,

06029003 SII,TION,R11W. (20711118,1115-1117/5531152) MAGNESITE. INDEX MAP, MEAS SEC. CA/MG = 0.036.

06029004 MIOCENE, S11,T1ON,RI1W. (436:116,114-117) MAGNESITE, WHITE, THIN BEDS: TONNAGE. INOEX \& GEOL MAPS, MEAS SEC. 06029007 S34.TION,RI7W. $\quad$ 229:216.232) DOLOMITE, WHITE, FINE-GR, SUGARY, TONNAGE. INDEX $\$$ GEOL MAPS. CA/MG = 1.37.

06029008 "T9N,RZOW" FRAZIER PARK. (230:4.3) LIMESTONE. WHITE TO GRAY, COARSELY CRYS 06029009 PALEOZOIC, BEAN CANYON FM. "TIIN,RI4W" CREAL. AREA. (231:6) LIMESTONE: AVG OF 13 SMPL. USE: 4. CA/MG = 33.9.

06029010 "TSN,R2OW" (230:4,3) LIMESTONE, WHITE TO GRAY, COARSELY CRYSI MIN, INDEX MAP. USE: 4. CA/MG $>100$.

06029011 S5-7.T1ON,R14W. (470877) LIMESTONE, GRAY, WHITE, BANDED, CA/MGE40.3.

O6029012 PALEOZOIC, BEAN CANYON FM. S5-7.TION,R14W. (229:216.215; LIMESTONE, GRAY, WHITE, BANDED, CRYSI RANDOM SMPL. INDEX GEOL MAPS. USE: 4 . CAMMG $=36.5$. PALEOZOIC. S22,T9N,RI8W. (229:217,225) LIMESTONE, GRAY, WHITE, MOTL. FINE TO COARSELY CRYS. INDEX \& GEOL MAPS.

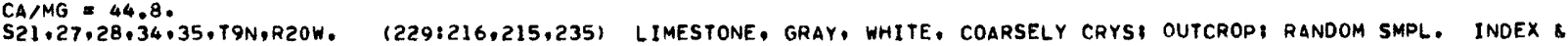

06029013

06029014

06029015 GEOL MAPS. USE: 4 . CA/MG $=28.6$

06029016 SAMG S21,27,28,34,35,T9N,R20W, (229:216,215,223) LIMESTONE, GRAY, WHITE, COARSELY CRYS; OUTCROP, RANDOM SMPL. INDEX \& GEOL MAPS. USE: 4 . CAMG $=34,9$.

06029017 PALEOZOIC. BEAN CANYON FM. S5-7,T1ON,R14W. $(229: 216,233)$ LIMESTONE, GRAY, WHITE, BANDED, CRYSI RANDOM SMPL. INDEX \&

06029018 PALEOZOIC: S22, T9N.RI8W. $(229: 217.224)$ LIMESTONE, WHITE, GRAY, MOTL. FINE TO COARSELY CRYS. INDEX \& GEOL MAPS.

06029019 CAMMG $=56.1$.

PALEOTOIC. S22,T9N,R18W. (229:217.224) LIMESTONE, WHITE, GRAY, MOTL, FINE TO COARSELY CRYS. INOEX GEOL MAPS.

CAIMG $=52.4$. 
TABLE 9. - Analyses of samples from California and Hawaii containing more than 90 percent carbonate (Group $F_{2}$ ), common-rock category Continued

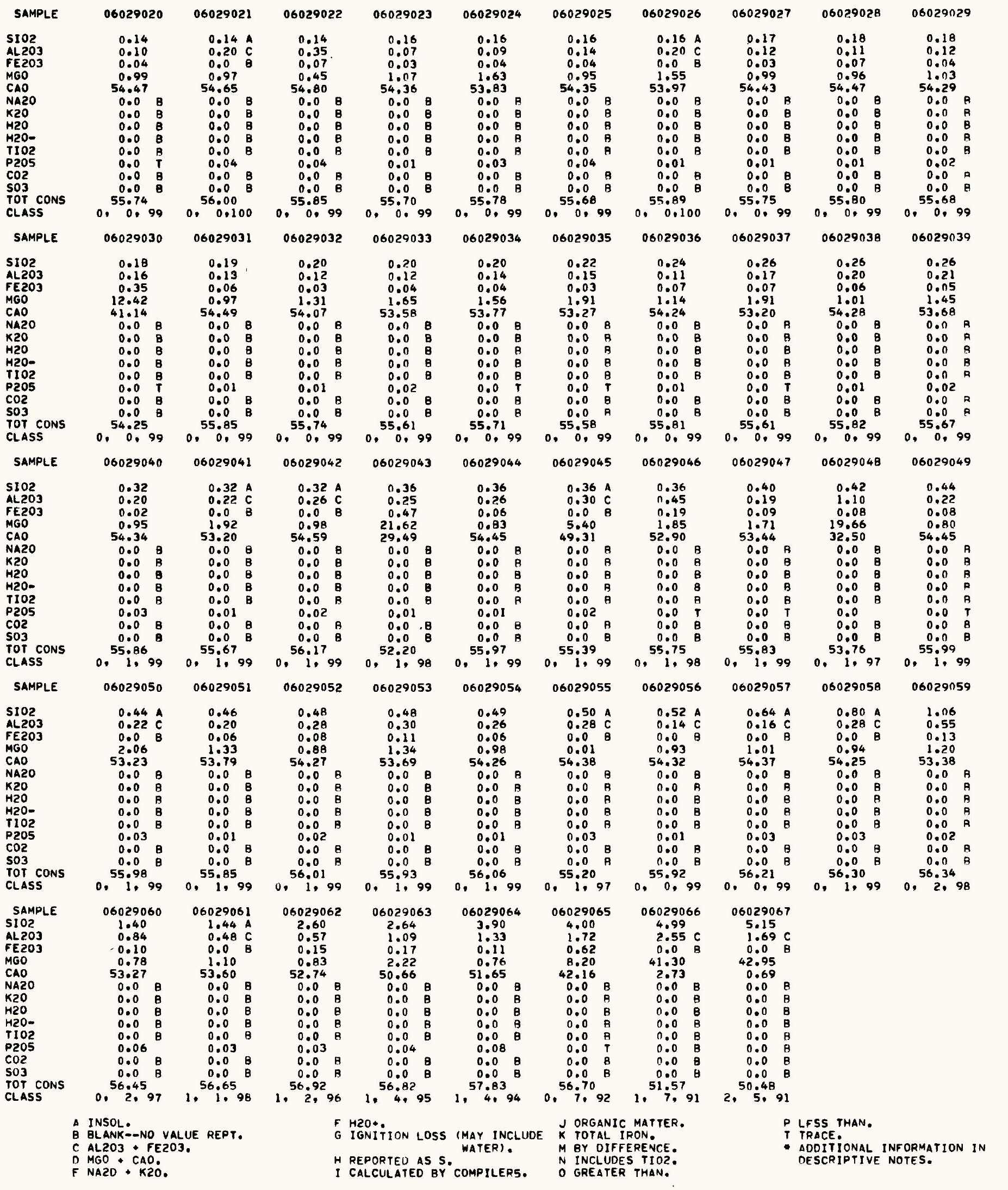


TABLE 9. - Analyses of samples from California and Hawaii containing more than 90 percent carbonate (Group $F_{2}$ ), common-rock category Continued

06029020 PALEOZOIC. S22,TON,RIAW.

06029021 PALEOZOIC, BEAN CANYON FM. $C A / M G=56.3$.

06029022 S21,27,28,34,35,T9N,R2OW. CA/MG $>100$.

06029023 PALEOZOIC. S22.T9N,RIBW. $C A / M G=50.8$.

06029024 PRE-CRETACEOUS, S26.T9N.R2ON

06029025 PALEOZOIC. S26.TON.RIBW. GEOL MAPS, CAMMG $=57.2$. GEOL MAPS. CAMMG $=57.2$. PALEOZOIC, BEAN CANYON FM.
CAMMG $=34,8$. PALEOZOIC. S27,TON,RLBW. GEOL MAPS. CA/MG $=55.0$ PALEOZOIC. SR2, TSN.RIBW.
CA PALEOZOIC. S22,T9N.RIBW. PALEOZOIC. S22, T9N.RIBW. $C_{A} / M G=3.31$.

\section{2}

06029027

0602902

06029029

06029030

06029031 PALEOZOIC. S22,TIN,RIBW.

06029033 PALEOZOIC. ${ }^{2}$ S22.T9N,RIBW.

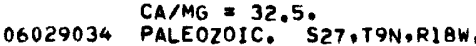
GEOL MAPS. CA/MG $=34.5$.

06029035 PALEOZOIC. S22.T9N.R1BW.

06029035 PALEOZOIC. S22.T9N,R1BW.

06029036 PALEOZOIC. S22,TSN,RIBW.

CAMMG $=47.6$.

06029037

06029038

06029039

06029040

06029041

06029042

06029043

06029044

06029045

06029046

06029047

06029048

06029049

06029050

06029051

06029052

06029053

06029054

06029055

06029056

06029057

06029058

06029059

06029060

06029061

06029062

06029063

06029064

06029065

06029066

06029067

PALEOZOIC, BEAN CANYON FM.

PALEOZOIC (P) S26.TSN,R18W. GEOL MAPS. CA/MG $=53.7$. PALEOZOIC. S26,T9N,RIUW. GEOL MAPS. CA/MG $=37.0$. INOEX \& GEOL MAPS. CA/MG $=57.2$. PRE-CRETACEOUS, S25,TON,RZOW. USE: $2 .$, CAMMG $=1.36$. $C A / M G=65.6$. 6. CALMG $=9,13$.

CAYMG $=9.13$. CA/MG $=31.3$. $C A / M G=68.1$. PALEOzOIC. BEAN CANYON FM. CA/MG $=25.8$. PALEOZOIC. SZ2, T9N,RIUW. $C A / M G=40.4$. PALEOZOIC. SZ2, TON.RIBW. $\mathrm{CA} / \mathrm{MG}=61.7$. PALEOZOIC, 'S22.T9N,RIBW. $C A / M G=40,1$. PALEOZOIC. S22,TON.RIBW. $C A / M G=55.40$ CACMG $=55.4^{\circ}$. CA MAZ $>100$. CALEO 100 . PALEZOIC. BEAN CAMMG = 58.40 PALEOZOIC, BEAN CANYON FM. CA $/ M G=53.8$ PALEOZOIC, BEAN CANYON FM. CAMMG $=57.7$ S21.27,28,34.35, T TN,R2OW. GEOL MAPS. USE: 4 . CA/MG PALEOZOIC. S2Z.TAN,RIBW.
CAMG $=68.3$. CAIMG $=63.5$. PALEOZOIC. S22,23,26,27,TYN,R18W. (229:217,224) LIMESTONE, GRAY, WHITE, MOTL, FINE TO COARSELY CRYSI RANDOM SMPL.

(229:217,224) LIMESTONE, WHITE, GRAY, MOTL, FINE TO COARSELY CRYS. INDEX \& GEOL MAPS. S27,TIIN,RI4W. (229:216,221) LIMESTONE: BEDS 2-20 FT THICK. INOEX \& GEOL MAPS, USE: 4. (229:216,223.235) LIMESTONE, WHITE, GRAY, COARSELY CRYS: RANDOM SMPL, OUTCROP, USEI 4. (229:217.224) LIMESTONE, GRAY, WHITE, MOTL, FINE TO COARSELY CRYS. INDEX \& GEOL MAPS.

(229:216,223) LIMESTONE, WHITE, COARSELY CRYS, INDEX \& GEOL MAPS. USE 14. CA/MG=33.0, (229:217.224) LIMESTONE, GRAY, WHITE, MOTL, FINE TO COARSELY CRYSI RANDOM SMPL. INOEX S27,TIIN.RI4W. (229:216,221) LIMESTONE, BEDS 2-20 FT THICK. INDEX \& GEOL MAPS. USEI 4 . (229:217,224) LIMESTONE, GRAY, WHITE, MOTL, FINE TO COARSELY CRYSI RANDOM SMPL. INDEX \& (229:217.224) LIMESTONE, WHITE, GRAY, MOTL, FINE TO COARSELY CRYS. INDEX O GEOL MAPS. (229:217,224) LIMESTONE, GRAY, WHITE, MOTL, FINE TO COARSELY CRYS. INDEX O GEOL MAPS. (229:217,224) LIMESTONE, GRAY, WHITE, MOTL, FINE TO COARSELY CRYS. INDEX \& GEOL MAPS. (229:217.224) LIMESTONE, GRAY. WHITE, MOTL, FINE TO COARSELY CRYS. INDEX \& GEOL MAPS. (2291217,224) LIMESTONE, GRAY, WHITE, MOTL, FINE TO COARSELY CRYS, INDEX \& GEOL MAPS. (229:217.224) LIMESTONE, GRAY, WHITE, MOTL, FINE TO COARSELY CRYS. INDEX O GEOL MAPS. (229:217,224) LIMESTONE, GRAY, WHITE, MOTL, FINE TO COARSELY CRYS3 RANDOM SMPL. INDEX \& (229:217,214) LIMESTONE, GRAY, WHITE, MOTL, FINE TO COARSELY CRYS. INDEX \& GEOL MAPS. (229:217.224) LIMESTONE, GRAY, WHITE, MOTL, FINE TO COARSELY CRYS. INDEX \& GEOL MAPS. S26.TIIN,R14W. (229:216.221) LIMESTONE, INOEX S GEOL MAPS. USE: 4. CA/MG 27.9 . 1229:217,224) LIMESTONE, gRAY, WHITE, MOTL, FINE TO COARSELY CRYSi RANDOM SMPL, INDEX \& 229:217.224) LIMESTONE, GRAY. WHITE, MOTL, FINE TO COARSELY CRYS; RANDOM SMPL. INDEX \& T.2. TI1N.R14W.

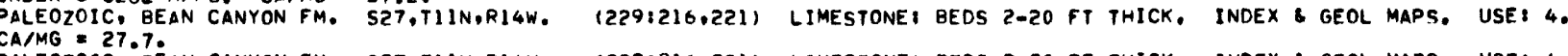
作

(5) 1229:216.221) LIMESTONE! BEOS 2-20 FT THICK. INDEX \& GEOL MAPS. USE: 4. 1229:217.224) LIMESTONE, GRAY, WHITE, MOTL, FINE TO COARSELY CRYS. INDEX \& GEOL MAPS. S27.TIIN.R14W. (2291216.221) LIMESTONE: BEDS 2-20 FT THICK. INDEX \& GEOL MAPS, USE: 4. S26.TIIN,RI4W. 1229:216,221) LIMESTONE. INOEX GEOL MAPS. USE: 4. CA/MG $=28.6$. PALEOZOIC. S22,T9N,R18W. (229:217.224) LIMESTONE, GRAY, WHITE, MOTL, FINE TO COARSELY CRYS. INDEX \& GEOL MAPS. INDEX TACEOUS, S31,TION,R16W. (229:216,236) DOLOMITE, WHITE, MED-GR, SUGARY, MASSIVEI QUARRY RANDOM SMPLI MIN.

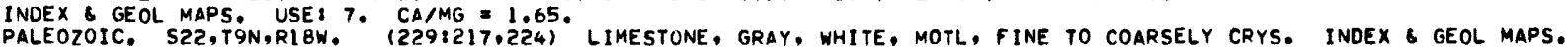
PALEOZOIC. BEAN CANYON FM. S27,TIIN,RI4W. 1229:216.2211 LIMESTONE: BEDS 2-20 FT THICK. INDEX \& GEOL MAPS. USE: 4.

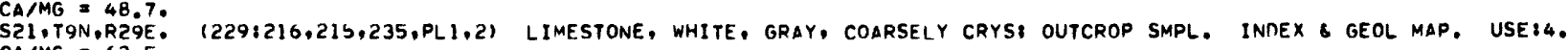

S2T.TIIN.RIAW. (229:216,221) LIMESTONE: BEDS 2-20 FT THICK. INDEX \& GEOL MAPS. USE: 4. (229:217,224) LIMESTONE, GRAY, WHITE, MOTL, FINE TO COARSELY CRYS. INDEX \& GEOL MAPS. (229:217,224) LIMESTONE, GRAY, WHITE, MOTL, FINE TO COARSELY CRYS. INDEX G GEOL MAPS. (229:217,224) LIMESTONE, GRAY, WHITE, MOTL, FINE TO COARSELY CRYS. INDEX \& GEOL MAPS. (2291217.224) L.JMESTONE, GRAY, WHITE, MOTL, FINE TO COARSELY CRYS. INDEX G GEOL MAPS. S27.TIIN,R14W. (229:216.221) LIMESTONE, BEDS 2-20 FT THICK. INDEX 8 GEOL MAPS. USE 4. S27.TIIN,R14W. (229:216.221) LIMESTONE: BEOS 2-20 FT THICK. INDEX \& GEOL MAPS. USE? 4 S27,TIIN,RI4W. 1229:216.221) LIMESTONE BEDS 2-20 FT THICK. INOEX \& GEOL MAPS. USE: 4. S27,TIIN,RI4W. (229:216,221) LIMESTONE, BEDS 2-20 FT THICK. INOEX \& GEOL MAPS. USE: 4 . (229:216,223.235) LIMESTONE, GRAY, WHITE, COAPSELY CRYSI OUTCROP: RANOOM SMPL. INDEX \& $=44.5$

229:217,2241 LIMESTONE, GRAY, WHITE, MOTL, FINE TO COARSELY CRYS. INDEX \& GEOL MAPS. PALEOZOIC, S22,T9N,RI8W. (229:217,224.225,PLI,Z) LIMESTONE, FINE- TO COARSE-GR, CRYS. INDEX \& GEOL MAPS. CA/MG=68.0. PALEOZOIC, S22,T9N,RIBW. (229:217,224,225,PLI,2) LIMESTONE, FINE- TO COARSE-GR, CRYS. INDEX \& GEOL MAPS. CA/MG 5.14 . MIOCENE. S11.T1ON,R11W. (436:116.114-117) MAGNESITE, WHITE, THIN BEDSi TONNAGE. INDEX G GEOL MAPS, MEAS SEC. MIOCENE. SI1,TION,RIIW. (436:116,114-117) MAGNESITE, WHITE, THIN BEDS: TONNAGE. INDEX G GEOL MAPS, MEAS SEC. CAMMG = 0.016 . 
TABLE 9. - Analyses of samples from California and Hawaii containing more than 90 percent carbonate (Group $\left.F_{2}\right)$, common-rock category Continued

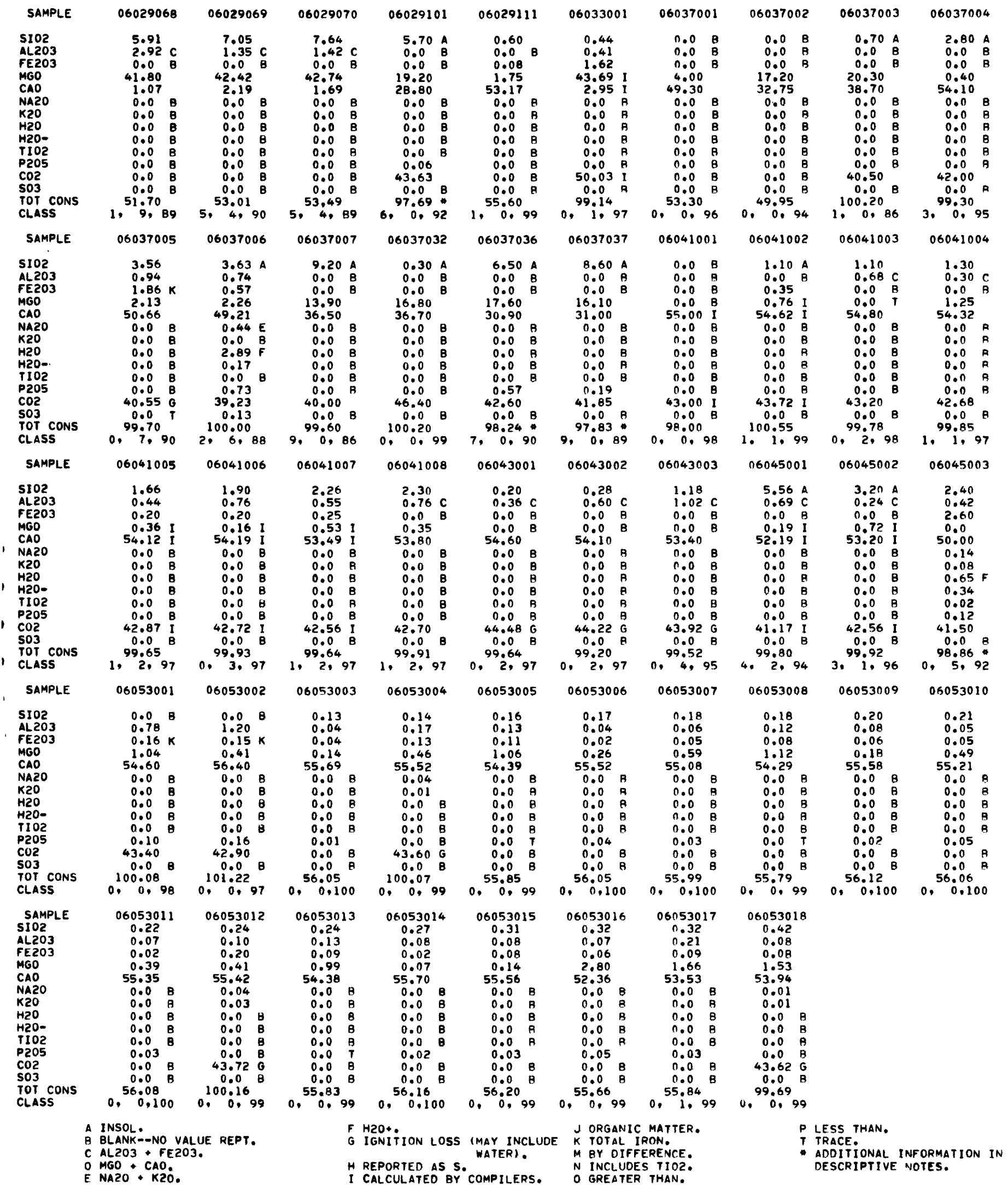


TABLE 9. - Analyses of samples from California and Hawaii containing more than 90 percent carbonate (Group $F_{2}$ ), common-rock category Continued

06029068 MIOCENE. S11,TION,RI1W. (436:116,114-117) MAGNESITE, WHITE, THIN BEDSI TONNAGE. INDEX G GEOL MAPS, MEAS SEC. 06029069 MIOCENE. S11.T1ON,R11W. (436:116.114-117) MAGNESITE, WHITE, THIN BEDSI TONNAGE. INDEX \& GEOL MAPS, MEAS SEC. 06029070 MIOCENE. S11,TION,R11W. (4361116.114-117) MAGNESITE, WHITE, THIN BEOS TONNAGE. INDEX \& GEOL MAPS, MEAS SEC. 06029101 MIOCENE: MONTEREY FM. CHICO MARTINEZ CHERT MBR. "T28S,R20E" CHICO MARTINEZ CREEK. (386ICG) DOLOMITE. "MISCI 06029111 "T9N,R18W" EAST OF LEBEC. (231:51 LIMESTONE, TAKEN ACROSS WIDTH OF 1.000 FT. USE: 4. CA/MG $=30.4$.

06033001 S12.T14N.R7W. (28:21.PLT) MAGNESITE. INDEX MAP. CA/MG $=0.068$.

06037001 PRE-CRETACEOUS. PLACERITA FM. S28,T3N.R14W. (445:203/404:219.244.249) DOLOMITIC LIMESTONE, WHITE, CRYS. INDEX \&

06037002 PRE-CRETACEOUS, PLACERITA FM. S28,T3N,R14W. $14458203,174 / 4048219,2491$ DOLOMITEI OUARRY SMPL 20 FT THICK. INDEX 8 06037003 PRE-CRETACEOUS, PLACERITA FM. "S3-6,T3N,R15W" (404:221,218-220) DOLOMITE. WHITE, CRYS MIN. INOEX MAP. CA/MG: 1.91. 06037004 PRE-CRETACEOUS, PLACERITA FM. S3-6.T3N,R15W. (404:221,218-220) LIMESTONE, WHITE, CRYSI MIN. INDEX MAP. CA/MG >100. 06037005 PALEOCENE. MARTINEZ FM. "TIS,R17W" CASTELLAMARE. (293:134,133,PL16) LIMESTONE, AGAL, COMPOS SMPL OF 96 FTI TONNAGE. 06037006 PLIOCENE. "T4S,R14W"LOMITA. (499:328/556:249,PL37) LIMESTONE. FOS, 30 FT THICK. INDEX MAP. USE:2.7. CA/MG=21.3. 06037007 PRE-CRETACEOUS, PLACERITA FM. S3-6.T3N.R15W. (404:221,218-220) DOLOMITE, WHITE, CRYSI TYPICAL SMPL, MIN. INDEX MAP. 06037032 PRE-CRETACEOUS, PLACERITA FM. S3-6,T3N,R15W. $(404: 221,218-220)$ DOLOMITE, WHITE, CRYSI MIN. INDEX MAP. CA/MG = 2.18. 06037036 MIOCENE, MONTEREY SH, ALTAMIRA SH MBR. "T4S,RI4W" PALOS VERDES HILLS, (386:C6) DOLOMITE. "MISC: FEO=0.07, MNO =0.00. 06037037 MIOCENE, MONTEREY SH. SAN CLEMENTE ISLAND. $13863(66)$ DOLOMITE. $\bullet M I S C:$ FEO =0.05, MNO = 0.04 . CA/MG = 1.93 .

06041001 PALEOZOIC. "T3N,R9W" POINT REYES STATION. (531116,89,PL9,10,19) LIMESTONE: MIN. INDEX 8 GEOL MAPS, GEOL SEC. USE:18. 06041002 PALEOZOIC, "T2N,RIOW" POINT REYES STATION. (14:131,133,PL4) LIMESTONE, WHITE, CRYS. INOEX \& GEOL MAP. USE, 18. 06041003 JURASSIC 8 CRETACEOUS, FRANCISCAN FM. "T 3N,R8W" OLEMA. (175:358,356) LIMESTONE: 50-100 FT THICK: TONNAGE. USE: 18. 06041004 PRE-FRANCISCAN, GABILAN LS. "T3N,R9W" INVERNESS PARK. $(175: 353 / 521: 260,259$, PL4) LIMESTONE, WHITE, COARSELY CRYS. 06041005 PRE-FRANCISCAN, GABILAN LS. "T3N,R9W" TOMALES BAY. $1175,353 / 531: 89,16, P(9,10,19)$ LIMESTONE. WHITE TO BROWN, CRYS

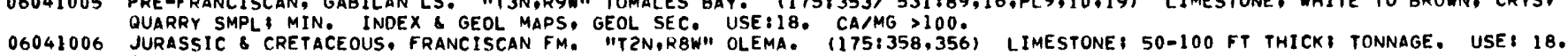
06041006 JURASSIC 6 CRETACEOUS, FRANCISCAN FM. "T2N,R8W" OLEMA. $(175: 358,356)$ LIMESTONE: 50-100 FT THICK: TONNAGE. USE: 18. 06041007 PRE-FRANCISCAN, GABILAN LS. "T3N,R9W" TOMALFS BAY, (1751353/531:89,16,PL9,10,19) LIMESTONE, WHITE TO RROWN, CRYS1 06041008 PRE-FRANCISCAN, GABILAN LS. "T3N,R9W" TOMALES BAY. (175;353/ 531:89,16,PL9,10,191 LIMESTONE, WHITE TO BROWN, CRYS1

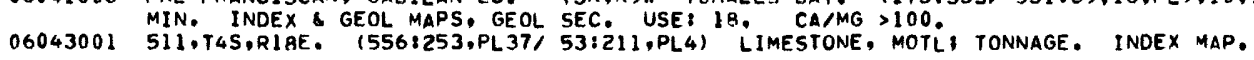
06043002 S11,T4S,R1BE. (556:253,PL37/ 53:211,PL4) LIMESTONE, WHITE: TONNAGE. INDEX MAP. $06043003511 . T 45, R 18 E$. (556:253,PL37/ 53:211,PL4) LIMESTONE, BLACK, TONNAGE. INDEX MAP.

06045001 JURASSIC \& CRETACEOUS, FRANCISCAN FM. S36,T22N,R15W. (556:254,253,PL37/ 406:361,383,PLI0A) LIMESTONE, PINK TO 06045002 JURASSIC \& CRETACEOUS, FRANCISCAN FM. S26.TI7N,RI3W. (556:254,PL37/ 406:361,351,PL10B) TRAVERTINEI 6-8 FT THICK. 06045003 JURASSIC \& CRETACEOUS, FRANCISCAN FM. "TIIN,R13W" ROCK PILE PEAK. $129176.11 .69,74$, PLI) LIMESTONE, RED. INOEX \& GEOL MAPS. MISC: FEO $=0.00$, MNO $=0.59$. "TIIN,RI3W" ROCK PILE PEAK. 129176,11.69,74,PLII LIMESTONE, REO. INOEX 6 GEOL 06053001 PRE-FRANCISCAN, SUR SERIES. S22,T20S,R3E. 1420:B40,B8,PLI,2) MARBLE, WHITE. FINE TO COARSELY CRYS. INDEX GEOL MAP. 06053002 PRE-FRANCISCAN, SUR SERIES. SZ2,T20S,R3E. $1420: B 40,88, P L 1,21$ MARBLE, WHITE, FINE TO COARSELY CRYS. INOEX \& GEOL MAP. 06053003 PRE-FRANCISCAN, SUR SFR, S36,T185,RIE, (265:65,5,64,PL1) LIMESTONE, WHITE, COARSELY CRYS I MIN: TONNAGE. INDEX \& GEOL 06053004 PRE-FRANCISCAN, SUR SER. 535,T13S,R4E. (54131,11,PL1) LIMESTONE, GRAY, MED TO COARSELY CRYS, TONNAGE. INIEX \& GEOL 06053005 PRE-FRANCISCAN, SUR SER. S17,18,20,T18S,R4E. (265:67.5.66,PL1) LIMESTONE. GRAY, MEO TO COARSELY CRYS. INDEX \& GEOL 06053005 PRE-FRANCISCAN, SUR SER. S17,18,20,T185,R4E. $(265: 67,5.66, P L 1)$ LIMESTONE. GRAY, MEO TO COARSELY CRYS. INDEX \& GEOL
06053006 PAPS. USE 19. CAMG = 51.3. 06053006 PRE-FRANCISCAN, SUR SER, S36,TIRS,RIE.
MAPS, GEOL SEC. USE 6,12 CA CMG >100.
06053007 PRE-FRANCISCAN, SUR SER. S36,TIBS,RIE. 06053007 PRE-FRANCISCAN, SUR SER, S36,TIBS,RIE: $(265165.5,64$, PLI) LIMESTONE, WHITE, COARSELY CRYSI MIN TONNAGE. INDEX \& GEOL 06053008 PRE-FRANCISCAN, SUR SER, S17,18,20,T185,R4E, $(265: 67,5,66, P L 1)$ LIMESTONE. GRAY, MED TO COARSELY CRYS. INDEX \& GEOL 06053009 PRE-FRANCISCAN, SUR SER. S36,T18S,RIE. $(265865,5,64$, PLI) LIMESTONE, WHITE, COARSELY CRYS: MIN 3 TONNAGE. INDEX \& GEOL MAPS, GEOL SEC. USE: 6,12. CA/MG >100. 06053010 PRE-FPANCISCAN, SUR SER. S36,TIRS,RIE. (265:65,5,64,PL) LIMESTONE, WHITE, COARSELY CRYSI MINI TONNAGE. INOEX \& GEOL 06053011 PRE-FRANCISCAN, SUR SER. S25,TIRS,RIE. (265:65,5,64,PLI) LIMESTONE, WHITE, COARSELY CRYS: MIN TONNAGE. INOEX G GEOL 06053012 PRE-FRANCISCAN, SUR SER. S35,T135,R4E. (54:31,11,PL1) LIMESTONE: TONNAGE. INDEX GEOL MAPS, GEOL SEC. CA/MG >100. 06053013 PRE-FRANCISCAN, SUR SER. S17,18,20,T185,R4E. (265:67,5,66,PLI) LIMESTONE, GRAY, MED TO COARSELY CRYS. INDEX \& GEOL 06053014 PRE-FRANCISCAN, SUR SER. S36,T1BS,RIE. $(265: 65,5.64$,PLI) LIMESTONE, WHITE, COARSELY CRYSI MINI TONNAGE. INDEX \& GEOL 06053015 PRE-FRANCISCAN, SUR SFR. S36,T185, 100 . $1265165,5,64$, PLI) LIMESTONE, WHITE, COARSELY CRYSI MINI TONNAGE. INDEX \& GEOL

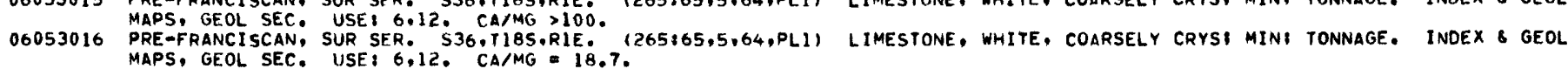
06053017 PRE-FRANCISCAN, SUR SER, S28.T165,R4E. $1265: 67,5$. PL1) LIMESTONE, CRYS. INDEX \& GEOL MAPS. USE: 19. CA/MG = 32.2. 06053018 PRE-FRANCISCAN, SUR SER. S35,T13S,R4E, $154: 31,11$, PL1) LIMESTONE, GRAY, MEO TO COARSELY CRYS, TONNAGE. INOEX \& GEOL MAPS, GEOL SEC, CAIMG $=35.3$. 
TABLE 9. - Analyses of samples from California and Hawaï containing more than 90 percent carbonate (Group $F_{2}$ ), common-rock category Continued

\begin{tabular}{|c|c|c|c|c|c|c|c|c|c|c|}
\hline SAMPLE & 06053019 & 06053020 & 06053021 & 06053022 & 06053023 & 06053024 & 06053025 & 06053026 & 06053027 & 06053028 \\
\hline $\begin{array}{l}\text { S102 } \\
\text { AL203 } \\
\text { FE203 } \\
\text { MGO } \\
\text { CAO } \\
\text { NA2O } \\
\text { K20 } \\
\text { H2O } \\
\text { H2O- } \\
\text { TIO2 } \\
\text { P205 } \\
\text { CO2 } \\
\text { SO3 } \\
\text { TOT CONS } \\
\text { CLASS }\end{array}$ & 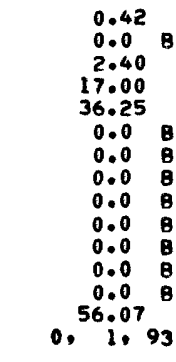 & $\begin{array}{c}0.44 \\
0.11 \\
0.03 \\
1.08 \\
54.31 \\
0.08 \\
0.08 \\
0.08 \\
0.08 \\
0.08 \\
0.19 \\
0.0 \\
0.08 \\
56.16 \\
0,0.99\end{array}$ & 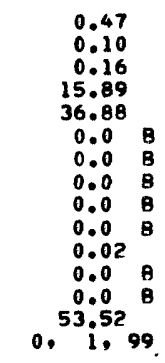 & $\begin{array}{cc}0.50 \\
0.12 \\
0.07 \\
0.24 \\
55.30 \\
0.0 & 8 \\
0.0 & 8 \\
0.0 & 8 \\
0.0 & 8 \\
0.0 & 8 \\
0.04 & 0 \\
0.0 & 8 \\
0.0 & 8 \\
56.27 & \\
0, & 1.99\end{array}$ & 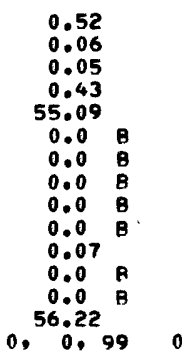 & $\begin{array}{cc}0.54 \\
0.30 \\
0.06 \\
2.07 \\
52.81 \\
0.0 & B \\
0.0 & 8 \\
0.0 & B \\
0.0 & 8 \\
0.0 & B \\
0.02 & \\
0.0 & 8 \\
0.0 & 8 \\
55.80 & 1.99 \\
0.9 & 1.99\end{array}$ & $\begin{array}{cc}0.55 \\
0.14 \\
0.13 \\
0.83 \\
54.54 \\
0.0 & 8 \\
0.0 & B \\
0.00 & 8 \\
0.0 & 8 \\
0.0 & 8 \\
0.01 & \\
0.0 & 8 \\
0.0 & 8 \\
56.20 & 8 \\
0.09 & 1.99\end{array}$ & 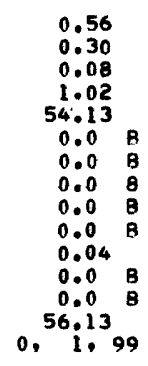 & 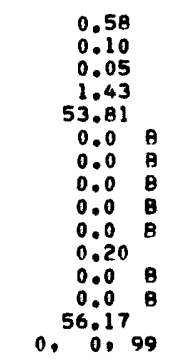 & $\begin{array}{c}0.62 \\
0.13 \\
0.02 \\
0.37 \\
55.00 \\
0.0 \\
0.0 \\
0.0 \\
0.0 \\
0.0 \\
0.0 \\
0.05 \\
0.0 \\
0.0 \\
56.19 \\
0,0.99\end{array}$ \\
\hline SAMPLE & 06053029 & 06053030 & 06053031 & 06053032 & 06053033 & 06053034 & 06053035 & 06053036 & 06053037 & 06053038 \\
\hline $\begin{array}{l}\text { S102 } \\
\text { AL203 } \\
\text { FE203 } \\
\text { MGO } \\
\text { CAO } \\
\text { NA2O } \\
\text { K20 } \\
\text { H2O } \\
\text { H2O- } \\
\text { TIO2 } \\
\text { P205 } \\
\text { CO2 } \\
\text { SO3 } \\
\text { TOT CONS } \\
\text { CLASS }\end{array}$ & 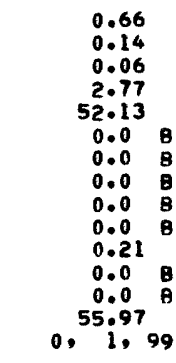 & $\begin{array}{c}0.66 \\
0.21 \\
0.05 \\
0.52 \\
54.78 \\
0.00 \\
0.00 \\
0.08 \\
0.00 \\
0.008 \\
0.03 \\
0.08 \\
0.00 \\
56.25 \\
0.09 \\
0.09\end{array}$ & 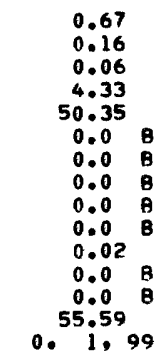 & $\begin{array}{cc}0.68 \\
0.30 \\
0.57 & 1 \\
20.67 \\
33.00 \\
0.0 & 8 \\
0.0 & 8 \\
0.0 & 8 \\
0.0 & 8 \\
0.0 & 8 \\
0.02 & 1 \\
45.10 & 6 \\
0.0 & 8 \\
100.39 & 8 \\
0.01,95\end{array}$ & $\begin{array}{cc}0.69 \\
0.17 \\
0.04 \\
0.48 \\
54.92 \\
0.0 & B \\
0.0 & B \\
0.0 & 8 \\
0.0 & B \\
0.0 & 8 \\
0.02 & \\
0.0 & 8 \\
0.0 & 8 \\
56.32 & \\
0, & 1.99\end{array}$ & 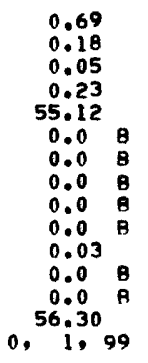 & $\begin{array}{cc}0.70 \\
0.14 \\
0.08 \\
0.33 \\
55.04 \\
0.0 \\
0.0 \\
0.0 & B \\
0.0 & B \\
0.0 & B \\
0.0 & B \\
0.20 & \\
0.0 & B \\
0.0 & B \\
56.49 & B \\
0 . & 1.99\end{array}$ & $\begin{array}{cc}0.70 \\
0.16 \\
0.08 \\
0.64 \\
54.71 \\
0.0 & B \\
0.0 & B \\
0.0 & B \\
0.0 & B \\
0.0 & B \\
0.20 & \\
0.0 & 8 \\
0.0 & B \\
56.49 & \\
0 . & 1.99\end{array}$ & $\begin{array}{cc}0.70 \\
0.20 \\
0.05 \\
0.48 \\
54.82 \\
0.0 \\
0.0 & B \\
0.0 & 8 \\
0.0 & 8 \\
0.0 & 8 \\
0.0 & 8 \\
0.02 & \\
0.0 & 8 \\
0.0 & B \\
56.27 & \\
0.09 & 1.99\end{array}$ & $\begin{array}{c}0.72 \\
0.15 \\
0.07 \\
1.19 \\
53.96 \\
0.0 \\
0.0 \\
0.0 \\
0.0 \\
0.0 \\
0.0 \\
0.21 \\
0.0 \\
0.0 \\
56.30 \\
0.0 \\
0.1099\end{array}$ \\
\hline SAMPLE & 06053039 & 06053040 & 06053041 & 06053042 & 06053043 & 06053044 & 06053045 & 06053046 & 06053047 . & 06053048 \\
\hline $\begin{array}{l}\text { S102 } \\
\text { AL203 } \\
\text { FE203 } \\
\text { MGO } \\
\text { CAO } \\
\text { NA2O } \\
\text { K20 } \\
\text { H2O } \\
\text { H2O- } \\
\text { T1O2 } \\
\text { P205 } \\
\text { CO2 } \\
\text { SO3 } \\
\text { TOT CONS } \\
\text { CLASS }\end{array}$ & 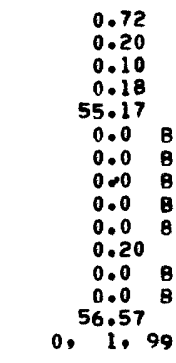 & $\begin{array}{c}0.74 \mathrm{M} \\
0.97 \\
0.11 \mathrm{~K} \\
0.36 \\
55.30 \\
0.00 \\
0.00 \\
0.08 \\
0.00 \\
0.008 \\
0.12 \\
42.40 \\
0.008 \\
100.00 \\
0.1096\end{array}$ & $\begin{array}{cc}0.76 \\
0.13 \\
0.08 \\
3.66 \\
51.14 \\
0.0 & B \\
0.0 & B \\
0.0 & B \\
0.0 & B \\
0.0 & B \\
0.05 & \\
0.0 & B \\
0.0 & B \\
55.82 & \\
0 . & 1.99\end{array}$ & 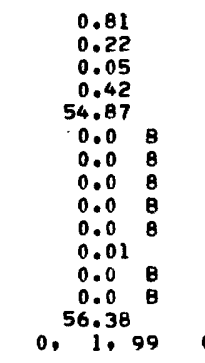 & $\begin{array}{cc}0.82 \\
0.13 \\
0.13 \\
0.99 \\
54.02 \\
0.0 & 8 \\
0.0 & B \\
0.0 & B \\
0.0 & 8 \\
0.0 & B \\
0.02 & \\
43.89 & G \\
0.0 & B \\
100.00 & 1.08 \\
0.01098\end{array}$ & 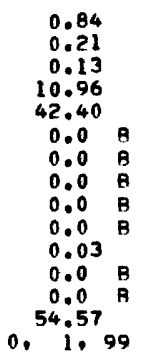 & $\begin{array}{ccc}0.84 \\
0.21 \\
0.29 \\
20.35 \\
32.00 \\
0.00 & 8 \\
0.0 & 8 \\
0.0 & 8 \\
0.0 & 8 \\
0.0 & 8 \\
0.01 & 1 \\
46.53 & 6 \\
0.0 & \\
100.29 & 0 \\
0.01 .98 & 1.98\end{array}$ & 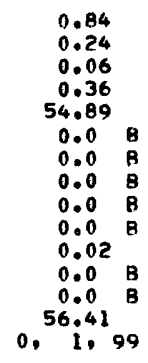 & 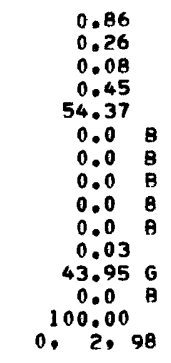 & $\begin{array}{c}0.85 \mathrm{~m} \\
0.60 \\
0.15 \mathrm{~K} \\
0.58 \\
54.40 \\
0.0 \mathrm{~B} \\
0.0 \mathrm{R} \\
0.0 \mathrm{~B} \\
0.0 \mathrm{~B} \\
0.0 \\
0.12 \\
43.30 \\
0.0 \mathrm{~B} \\
100.00 \\
0.1 .98\end{array}$ \\
\hline $\begin{array}{l}\text { SAMPLE } \\
\text { S102 } \\
\text { AL203 } \\
\text { FEE203 } \\
\text { MGO } \\
\text { CAO } \\
\text { NA2O } \\
\text { K20 } \\
\text { H20 } \\
\text { H20- } \\
\text { 1102 } \\
\text { P205 } \\
\text { CO2 } \\
\text { SO3 } \\
\text { TOT CONS } \\
\text { CLASS }\end{array}$ & 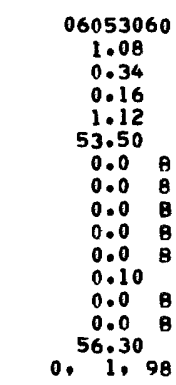 & 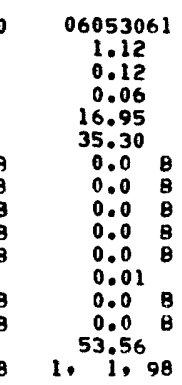 & 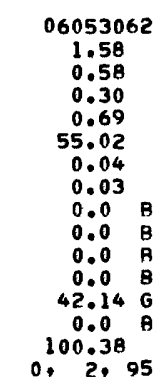 & 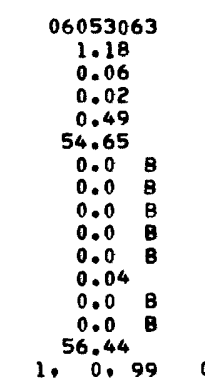 & $\begin{array}{c}06053064 \\
1.24 \mathrm{M} \\
1.29 \mathrm{~K} \\
0.12 \mathrm{~K} \\
0.26 \\
54.00 \\
0.0 \mathrm{~B} \\
0.0 \mathrm{~B} \\
0.0 \mathrm{~B} \\
0.0 \mathrm{~B} \\
0.0 \mathrm{~B} \\
0.09 \\
43.00 \\
0.0 \mathrm{~B} \\
100.00 \\
0.07\end{array}$ & 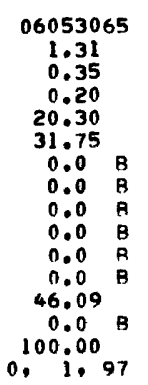 & 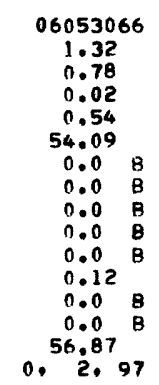 & 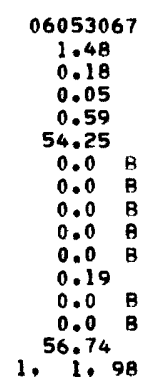 & & \\
\hline & $\begin{array}{l}\text { A INSOL. } \\
\text { B BLANK:-NC } \\
\text { C } \\
\text { AL2O3 } \\
\text { O MGO } \\
\text { E NAZO }\end{array}$ & REPT. & & TEO & LERS & $\begin{array}{ll}J & \text { ORG } \\
K & \text { TOT } \\
M & \text { BY } \\
N & \text { INC } \\
O & \text { GRE }\end{array}$ & $\begin{array}{l}\text { TER. } \\
\text { CE. } \\
\text { O2. } \\
N_{0}\end{array}$ & & ESCR!PTIVE & \\
\hline
\end{tabular}


TABLE 9. - Analyses of samples from California and Hawaii containing more than 90 percent carbonate (Group $F_{2}$ ), common-rock categoryContinued

06053019 PRE-FRANCISCAN, SUR SER, S7,T15S,RSE, (265:60.5,PL1) DOLOMITE, WHITE, FINE TO COARSELY CRYS. INDEX \& GEOL MAPS.

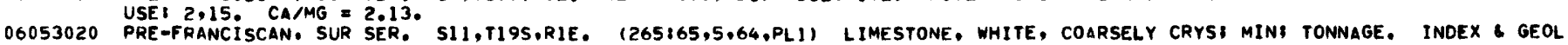
06053021 PRE-FRANCISCAN, SUR SER, S36.TIBS,RIE. (265:65.5.64.PL, CALCAREOUS DOLDMITE MIN; TONNAGE. INDEX \& GEOL MAPS. GEOL 06053021 PRE-FRANCISCAN, SUR SER, S36,TI8S,RIE. 1265:65.5.64,PL1) CALCAREOUS DOLDMITE: MIN; TONNAGE. INDEX \& GEOL MAPS, GEOL 06053022 PRE-FRANCISCAN, SUR SER, S36,TI8S,RIE, (265:65,5,64,PL1) LIMESTONE, WHITE, COARSELY CRYSI MINI TONNAGE. INDEX \& GEOL 06053023 PRE-FRANCISCAN. SUR SER, S2,T19S,RIE. (265:65,5,63,64,PL1) LIMESTONE. WHITE, COARSELY CRYS: MINI TONNAGE. INOEX \& 06053024 PRE-FRANCISCAN, SUR SER. S28,T16S,R4E. (265:67,5,PL1) LIMESTONE, CRYS. INDEX G GEOL MAPS. USE: 19. CA/MG . 25.5.

06053025 PRE-FRANCISCAN, SUR SER, S36,T18S,RIE. (265:65,5,64,PL1) LIMESTONE, WHITE. COARSELY CRYS\$ MINI TDNNAGE. INDEX \& GEOL 06053026 PRE-FRANCISCAN, SUR SER, SI/2,TI8S,RIE. (265:66,5,PL1) LIMESTONE, WHITE, FINE TO COARSELY CRYS. INDEX \& GEDL MAPS.

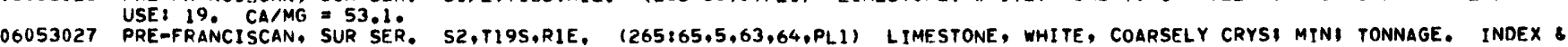

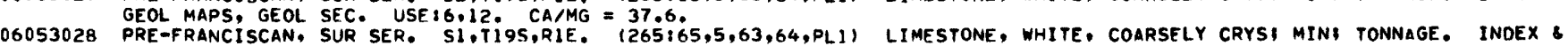
06053029 PRE-FRANCISCAN, SUR SER. S2,T195,RIE. $(265: 65,5,63,64$, PLI) LIMESTONE. WHITE, COARSELY CRYSI MINE TONNAGE. INDEX \& 06053029 PRE-FRANCISCAN, SUR SER, S2,T19S,RIE, $1265: 65,5,63,64$, PLII LIMESTONE. WHITE, COARSELY CRYS MIN 06053030 PRE-FRANCISCAN, SUR SER, S36,TI8S,RIE. (265:65,5.64,PLI) LIMESTONE, WHITE, COARSELY CRYSI MIN TONNAGE. INDEX GEDL 06053031 PAPS, GEOL SEC. USE 6,12 CA CAMG >100. $265,65,5,64$, PLI) LIMESTONE, WHITE, COARSELY CRYS 3 MIN 1 TONNAGE. INDEX \& GEOL

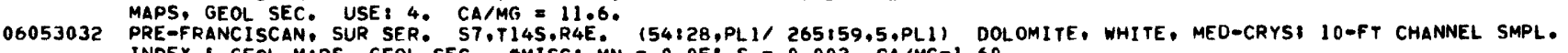

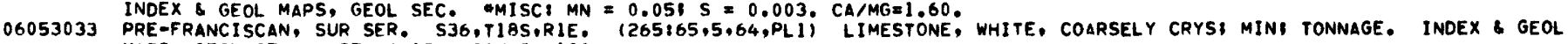
06053034 MAPS, GEOL SEC. USE: 6.12. CA/MG >100. MAPS. GEOL SEC. USE: 6,12. CA/MG $>100$. $265,65,5,64$, PLI) LIMESTONE, WHITE, COARSEL CRYSO MINI TONNAGE. INDEX G GEOL 06053035 PRE-FRANCISCAN, SUR SER. S2,TI9S,RIE. (265:65.5.63,64,PL1) LIMESTONE, WHITE, COARSELY CRYSI MIN3 TONNAGE. INDEX \& GEOL MAPS, GEOL SEC. USE 36,12 . CA/MG $>100$.

06053036 PRE-FRANCISCAN, SUR SER. S11, T19S,RIE, (265:65,5,64,PL1) LIMESTONE, WHITE, COARSELY CRYS 1 MINI TONNAGE. INDEX GEOL MAPS, GEOL SEC. USE: 6.12 . CA/MG = 85.5.

06053037 PRE-FRANCISCAN, SUR SER, S36,T1BS,RIE. (265:65,5,64,PL1) LIMESTONE, WHITE, COARSELY CRYSI MINI TONNAGE. INDEX \& GEOL

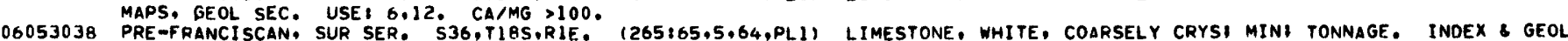

06053039 PRE-FRANCISCAN, SUR SER, S36,TI8S,RIE, (265:65,5,64,PLI) LIMESTONE, WHITE, COARSELY CRYSI MIN TONNAGE. INDEX \& GEOL 06053040 PRE-FRANCISCAN, SUR SER. S21,TI9S,R2E. PRE-FRANCISCAN, SUR SER, S21,TI9S,R2E.
INDEX GEOL MAPS. CA/MG >100.

06053041 PRE-FRANCISCAN, SUR SER. S36,T18S,RIE. MAPS, GEOL SEC. USE: 4. CA/MG = 14.0.
06053042 PRE $-F R A N C I S C A N$, SUR SER, S36,TI8S,RIE:
MAPS, GEOL SEC, USE: 6.12 CA CAMG >100. (265:65,5,64,PL1) LIMESTONE, WHITE, COARSELY CRYSI MIN TONNAGE, INDEX
$(420: B 40,88, P L 1,2)$ MARBLE, WHITE, FINE TO COARSELY CRYS SELECTED SMPL. (265:65,5.64,PL1) LIMESTONE, WHITE, COARSELY CRYSI MIN: TONNAGE, INDEX \& GEOL (265:65,5,64,PL1) LIMESTONE, WHITE, COARSELY CRYSI MINI TONNAGE, INOEX G GEOL 06053043 PRE-FRANCISCAN, SUR SER, S35.36,T135.R4E 06053044 PRE-FRANCISCAN, SUR SER. S36.T18S,RIE. (265:65.5,64,PL1) CALCAREOUS DOLOMITE; MINI TONNAGE. INDEX GEOL MAPS, 06053045 PRE-FRANCISCAN, SUR SER. S7,T145,R4E, 154:28,PL1/ 265:59,5,PL1) DOLOMITE, WHITE, MEO-CRYSI 10-FT CHIP SMPL; TONNAGE,

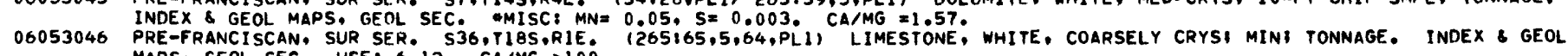
MAPS, GEOL SEC. USE: 6,12 CAMG $>100$. 06053048 PRE-FRANCISCAN, SUR SER. S22,T2OS,R3E. INDEX GEOL MAPS. CA/MG F 93,8
06053050 PRE-FRANCISCAN, SUR SER. S35,TI3S,R4E. (54:31,11,PL1) LIMESTONE, TONNAGE, INDEX \& GEOL MAPS. GEOL SEC. CA/MG >100.

06053051

06053052 PRE-FRANCISCAN, SUR SER. S36, TI8S,RIE.

06053053 MAPS, GEOL SEC. USE: 6.12 . CA MMG $>100$
PRE-FRANCISCAN, SUR SER, S35,TI3S, R4E.

$(4208840,88, P L I, 2)$ MARBLE, WHITE, FINE TO COARSELY CRYS SELECTEO SMPL. (54:31,11,PL1) LIMESTONE: TONNAGE. INDEX \& GEOL MAPS, GEOL SEC. CA/MG $>100$. PRE-FRANCISCAN, SUR SER, S35, TI3S,R4E. (265:65,5.64,PL1) LIMESTONE, WHITE, COARSELY CRYS MINI TONNAGE, INDEX \& GEOL 154:31,11,PL1) LIMESTONE: TONNAGE. INOEX \& GEOL MAPS, GEOL SEC. CA/MG $>100$. PRE-FRANCISCAN. SUR SER. S36,T18S,RIE, (265:65,5,64.PL1) LIMESTONE, WHITE, COARSELY CRYS I MIN\& TONNAGE. INDEX \& GEOL 06053055 MAPS, GEOL SEC, USE: 6,12 . CA/MG $=41.1$. 06053056 PRE-FRANCISCAN, SUR SER, S25, T18S,RIE, $(265165,5,64$, PLI) LIMESTONE, WHITE, COARSELY CRYS 1 MIN T TONNAGE. INDEX \& GEOL 06053057 MAPS, GEOL SEC. USE: 6,12 . CA/MG $>100$ PRE-FRANCISCAN, SUR 06053058 PRE-FRANCISCAN, SUR SER. TIBS,RIE, BIXBY MTN, $(265363,5,62, P L 1)$ LIMESTONE, WHITE, MED- TO COARSE-GR. INDEX \& GEOL MAPS. CA/MG $=79,7$.

06053059 PRE-FRANCISCAN, SUR SER, SB,TI9S,R2E. (420:B40,B8,PL1,2) MARBLE, WHITE, FINE TO COARSELY CRYS SELECTED SMPL. INDEX 06053060 S GEOL MAPS. CAMMG >100.

06053060 PRE-FRANCISCAN, SUR SER. S6,T21S,RSE. MAPS. CA/MG $=47.8$.

06053061

06053062 PRE-FRANCISCAN, SUR SER. S35,T135,R4E. (54:31.22.PL1/ 265:55.5,PL1) LIMESTONE, GRAY. MED TO COARSELY CRYS TONNAGE. 06053063 INDEX GEOL MAPS, GEOL SEC. CAMMG $=79.7$

PRE-FRANCISCAN, SUR SER, S36,T18S,RIE. $(265: 65,5,64$, PLI) LIMESTONE, WHITE, COARSELY CRYSI MINI TONNAGE. INDEX \& GEOL
MAPS, GEOL SEC. USE: 6,12. CAMMG $>100$. 06053064 PREMFRANCISCAN, SUR SER. S21,TISS,R2E. S GEOL MAPS. CAMMG >100.

06053065 PRE-FRANCISCAN, SUR SEK. S1.2,T145,R3E. (265157,5.56,PL1) DOLOMITE. WHITE, MED TO COARSELY CRYS. INDEX S GEOL MAPS. 06053066 PRE-FRANCISCAN, SUR SER. T18S,RIE, BIXBY MTN. $1265: 63,5,62, P L 1)$ LIMESTONE, WHITE, MED- TO COARSE-GR. INOEX \& GEOL 06053067 PRE-FRANCISCAN, SUR SER, S36,T18S,RIE, (265:65,5,64,PL1) LIMESTONE, WHITE, COARSELY CRYS, MINB TONNAGE. INDEX \& GEOL MAPS. GEOL SEC. USE: 6,12. CA/MG $=91.9$. 
TABLE 9. - Analyses of samples from California and Hawaii containing more than 90 percent carbonate (Group $\left.F_{2}\right)$, common- rock category Continued

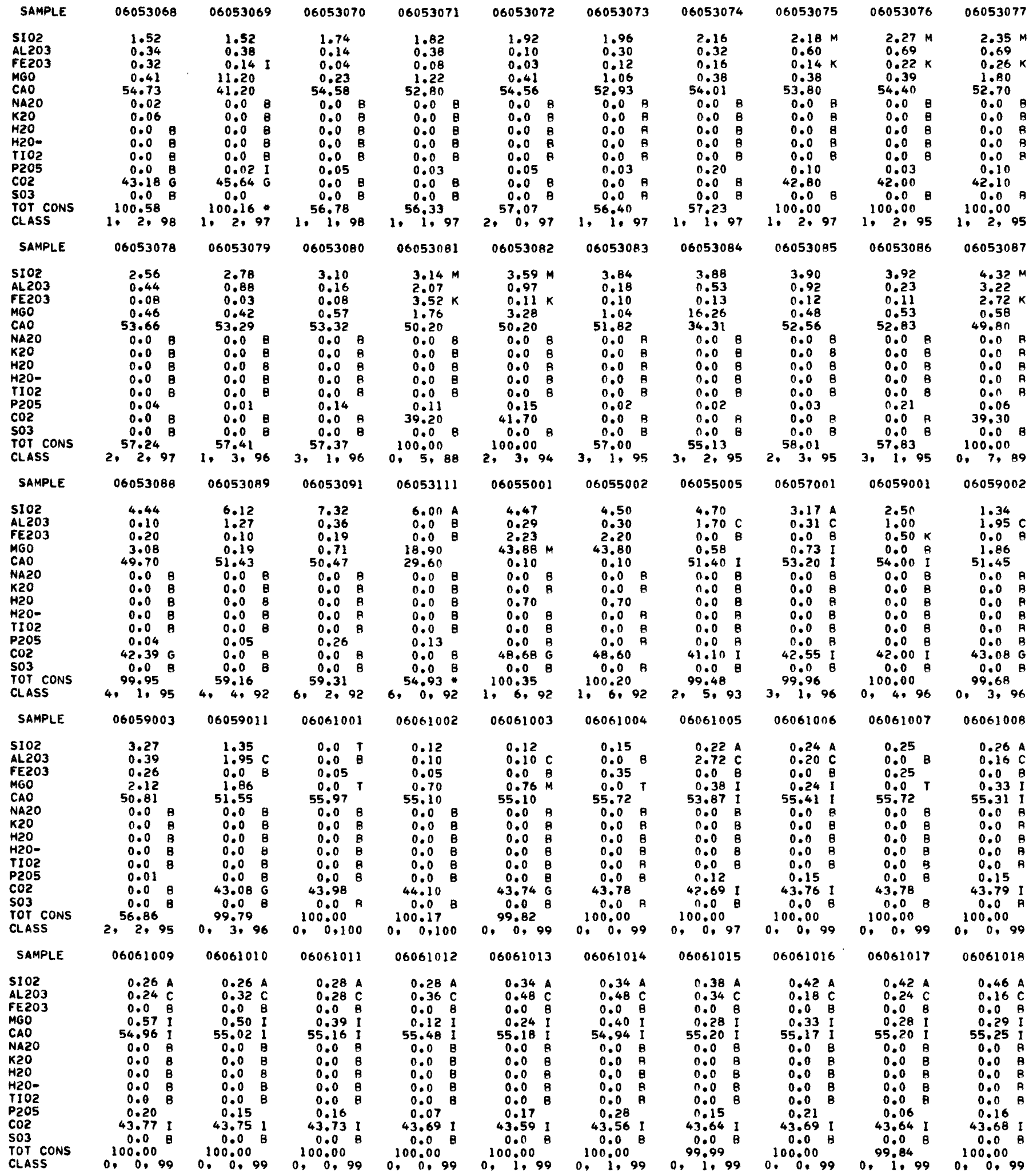

A INSOL.

B BLANK--NO VALUE REPT.

C AL203 - FE203

MGO CAO.

E NA2O + K2O
G HZOث: IGNIION LOSS (MAY INCLUDE

H REPORTEO AS S.

I CALCULATED BY COMPILERS.
$J$ ORGANIC MATTER.

$K$ TOTAL IRON.

$M$ BY DIFFERENCE.

$N$ INCLUOES TIO2
P LESS THAN.

T TRACE, ADOITIONAL INFORMATION IN DESCRIPTIVE NOTES. 
TABLE 9. - Analyses of samples from California and Hawaii containing more than 90 percent carbonate (Group $F_{2}$ ), common-rock category Continued

\begin{abstract}
06053068 PRE-FRANCISCAN, SUR SER, S36,T135,R4E, (54:31,29,PLI) LIMESTONE TONNAGE. INDEX GEOL MAPS, GEOL SEC, CA/MG >100.
06053069 PRE-FRANCISCAN, SUR SER. S7,T14S,R3E. (54:28,PL1/ $265: 59,5, P L 1)$ DOLOMITE, WHITE, MED-CRYSI IO-FT CHIP SMPL. TONNAGE. 06053070 PRE-FRANCISCAN, SUR SER. S12,T19S,RIE. $(265165,5,64$, PLI) LIMESTONE, WHITE, COARSELY CRYSI MIN, TONNAGE. INDEX \& GEOL 06053071 MAPS. GEOL SEC. USE: 6.12 . CA/MG $>100$.


TABLE 9. - Analyses of samples from California and Hawaii containing more than 90 percent carbonate (Group $F_{2}$ ), common-rock category Continued

06061019 PALEOZOIC, CALAVERAS FM, S30,T13N,R9E. (556:265,222,PL37) LIMESTONE, GRAY, FINE-GR, FOS, SMPL OF 50 FT, TONNAGE. 06061020 PALEOZOIC, CALAVERAS FM, S6,TI2N,R9E, $194: 454,440-460, P L 31$ LIMESTONE, GRAY TO BLACK, DENSE, CRYSI 800 FT THICK, 06061021 PALEOZOIC, CALAVERAS FM. S30,T13N,R9E. $(94,1464,460$, PL3) LIMESTONE COMPOS CHIP SMPL. INDEX G GEOL MAP. CA/MG >100. 06061022 PALEOZOIC, CALAVERAS FM. S22,T13N,RBE, (556:264,222,PL37) LIMESTONE, GRAY. INDEX MAP. USE: 18. CA/MG , 100. 06061023 PALEOZOIC, CALAVERAS FM. S30,T13N,R9E. $194: 464,460$, PL3) LIMESTONEे. INDEX \& GEOL MAP. CA/ME >100. 06061024 PALEOZOIC, CALAVERAS FM, S30,TI3N,R9E, $194: 464,460, P L 3)$ LIMESTONE: COMPOS CHIP SMPL. INDEX \& GEOL MAP. CA/MG >100. 06061025 PALE0Z01C, CALAVERAS FM. "TI2N,R8E" AUBURN, (556:266,PL37) LIMESTONE, GRAY, MED-GRI SMPL OF 110 FT. INDEX MAP. 06061026 PALEOZOIC, CALAVERAS FM. S30,T13N,R9E. $(941464,460$, PL3) LIMESTONE. INDEX \& GEOL MAP. CA/MG >100. 06061027 PALEOZOIC, CALAVERAS FM. S3,T12N,R8E, (5561263,222,PL37/ 94:460,PL3) LIMESTONE, GRAY, FINE- TO MED-CRYS. INDEX

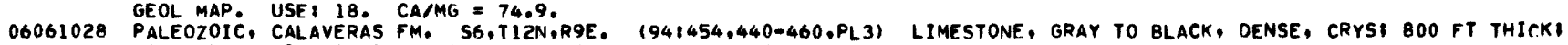

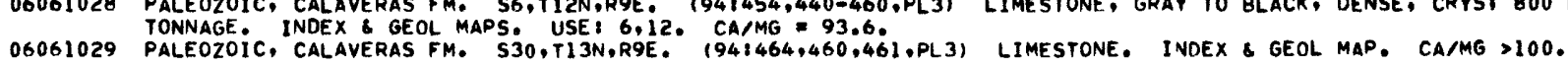
06061030 PALEOZOIC, CALAVERAS FM. S30,T13N.R9E. $(949464,460,461 . P L 3)$ LIMESTONEI COMPOS CHIP SMPL. INDEX \& GEOL MAP.

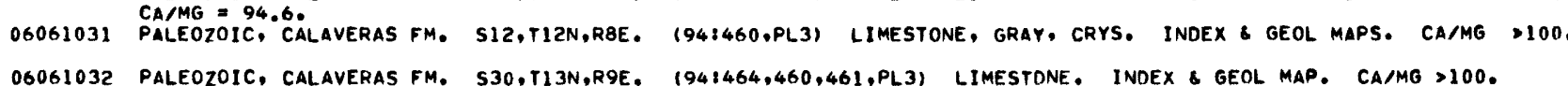
06061033 PALEOZOIC, CALAVERAS FM, S30,T13N,R9E, (5S68264,222,PL37) LIMESTONE, GRAY, INDEX MAP. USE: 18. CA/MG = 65.6. 06061034 PALEOZOIC, CALAVERAS FM. S30,T13N,R9E. $(94: 464,460,461, P L 3)$ LIMESTONE: COMPOS CHIP SMPL. INDEX GEOL MAP. 06061054 "TAMG $=24$. 8 ,

06063001 SI,12,T23N,RIIE. (556:269,PL37) LIMESTONE, WHITE: TONNAGE. INDEX MAP.

06063002 S6-8,16,17,21,28,T25N,R8E. (377:179,178) LIMESTONE, $M I S C$ : CASO4 = TR. CA/MG $>100$.

0606300353,10, T25N,R9E. (25:142,PL2) TRAVERTINE, BANDED; 20 FT THICK. INDEX \& GEOL MAP. USE: 6,15. CA/MG >100. $0606300453,10, T 25 N, R 9 E$. (25:142,PL2) TRAVERTINE, BANDED, 20 FT THICK. INDEX \& GEOL MAP. USE: 6,15. CA/MG >100. 06063005 S3.10,T25N,R9E. (25:142,PL2) TRAVERTINE, BANDED: 20 FT THICK. INDEX G GEOL MAPS. USE: 6,15. CA/MG = 88.6. 06063006 S1,12.T23N,R11E, (5561269,PL37) LIMESTONE, GRAY: TONNAGE, INDEX MAP, CA/MG >100.

06063007 PALE020IC, CALAVERAS FM. S30,T13N,R9E. $(94: 464,460$, PL3) LIMESTONE. INDEX \& GEOL MAP. CA/MG >100.

$0606300851,12, T 23 N, R 11 E$, (556:269,PL37) LIMESTONE, GRAY, TONNAGE, INDEX MAP.

06063009 TRIASSIC, CEDAR FM. S6-8,T25N,R8E, $(23: 311,310)$ LIMESTONE, USE: 4,18, CA/MG >100.

06065002 "T2S,P5W" CRESTMORE, (164:331,328,330) LIMESTONE, WHITE, USE: 4, CA/MG 19.7.

06065003 "T2S,RSW" CRESTMORE. (164:331,328,330) LIMFSTONE, WHITE, USE: 4. CA/MG $=20.3$.

06065004 "T2S,R5W" CRESTMORE. $(164: 331,328,330)$ LIMESTONE, WHITE, USE 4. CA/MG = 22.6 .

06065005 "T2S,R5W" CRESTMORE, (164:331,328,330) LIMESTONE, WHITE, USE: 4 . CA/MG = 19.4 .

06065006 PALEOZOIC, JURUPA SER, S2,3,T2S,RSW, (21:75/ 55:7,10) LIMESTONE, WHITE, BLUE, COARSELY CRYSI 200-300 FT THICK.

06065007 PALEOZO1C. "T3S,R18E" BLYTHE. (23117) DOLOMITE. CA/MG $=1.39$.

06065008 PALEOZOIC: S23,T35,R3E. (556:273,270,274,PL37) LIMESTONE, WHITE, COARSELY CRYS 100-200 FT THICK. INDEX MAP.

06065009 "T2S,R5W" RIVERSIDE. (65:886,881-804,PL 1) MARELE, COMPOS SMPL, DH. MIN. INDEX \& GEOL MAP. USE $4,7$. -MISC: FEO $=0.22$, MNO $=0.01, \mathrm{~S}=0.01, \mathrm{~F}=0.02, \mathrm{CL}=0.01$, BAO $=0.02$. CA/MG $=9.45$

06065010 "T2S,R5W" RIVERSIDE, 165:886,B81-884,PL 11 MARBLE, COMPOS SMPL, DH, MIN, INDEX \& GEOL MAP. USE: 4,7. -MISC: $F E O=0.14$, MNO $=0.01, S=0.02, F=0.02, C L=0.00$, BAO $=0.02$, CA/MG $=6.03$.

06065011 "T35,R4E" GARNET HILL. (353:143) DOLOMITIC LIMESTONE, WHITE, CRYS\$ MIN. CA/MG 11.0.

06065012 "T3S,RIAE" EAGLE MTN DIST. (261:38,PLג) DOLOMITE, WHITE, COARSELY CRYS, INDEX \& GEOL MAPS. AMISC\& TOTAL IRON BY DIF. 06065014 CARBONIFEROUS. S22,23.T3S,R3E, (501:516,515) LIMESTONE: MIN3 8-20 FT THICK, TONNAGE. CA/MG = 22.3.

06065015 PALEOZOIC, "T4S,R4E" PALM SPRINGS. (556:272,270,PL37) LIMESTONE. INDEX MAP. CA/MG = 30.5.

06065016 PALE0201C. "T2S,R20E" BLYTHE. (232:7) LIMESTONE, AVG OF 7 RANDOM SMPL. CA/MG = 38.6.

06065087 "S13,T3S,R13E" (261:38,PL1) DOLOMITE, WHITE, COARSELY CRYS. INDEX \& GEOL MAPS. UMISCI FEOEO,78 BY DIF. CA/MG =1.64. 06065088 "S23,T3S,R13E". (261:38, PLI) LIMESTONE, GRAY, COARSELY CRYS. INDEX \& GEOL MAPS. CA/MG a 30.6.

06069001 "T12S,R5E" HOLLISTER, (142:383/555:118/1731118,PL4) LIMESTONE, INDEX MAP. USE: 18. "MISCI GYPSUM I TR,

06069002 "T12S.R5E" HOLLISTER, (535277.81) DOLOMITE. CA/MG = 1.43.

06069004 PRE-FRANCISCAN, SUR SER, SWI/4,T13S,R5E, (54:32,11.31,PL1) LIMESTONE: TONNAGE. INDEX \& GEOL MAPS, GEOL SEC.

PRE -FRANCISCAN, SUR SER. SWI/4.T13S,R5E, (556:275,PL37/54:31,11,PL1) LIMESTONE, WHITE, GRAY, VARIEGATED, COARSELY CRYS: TONNAGE. INDEX GEO MAPS GEOL

06069006 PRE-FRANCISCAN, SUR SER, SWI/4,TI3S,R5E, (54:32,11,PL1) DOLOMITE, TONNAGE. INDEX \& GEOL MAPS. GEOL SEC. CA/MGE1.97.

06069007 PRE-FRANCISCAN, SUR SER, S23,TI4S,R5E, 1556:275,PL37/ 54:38,PL1) LIMESTONE, TONNAGE. INDEX \& GEOL MAPS, GEOL SEC. USE: 18. CARMG $=64.5$. 
TABLE 9. - Analyses of samples from California and Hawaï containing more than 90 percent carbonate (Group $F_{2}$ ), common-rock category Continued

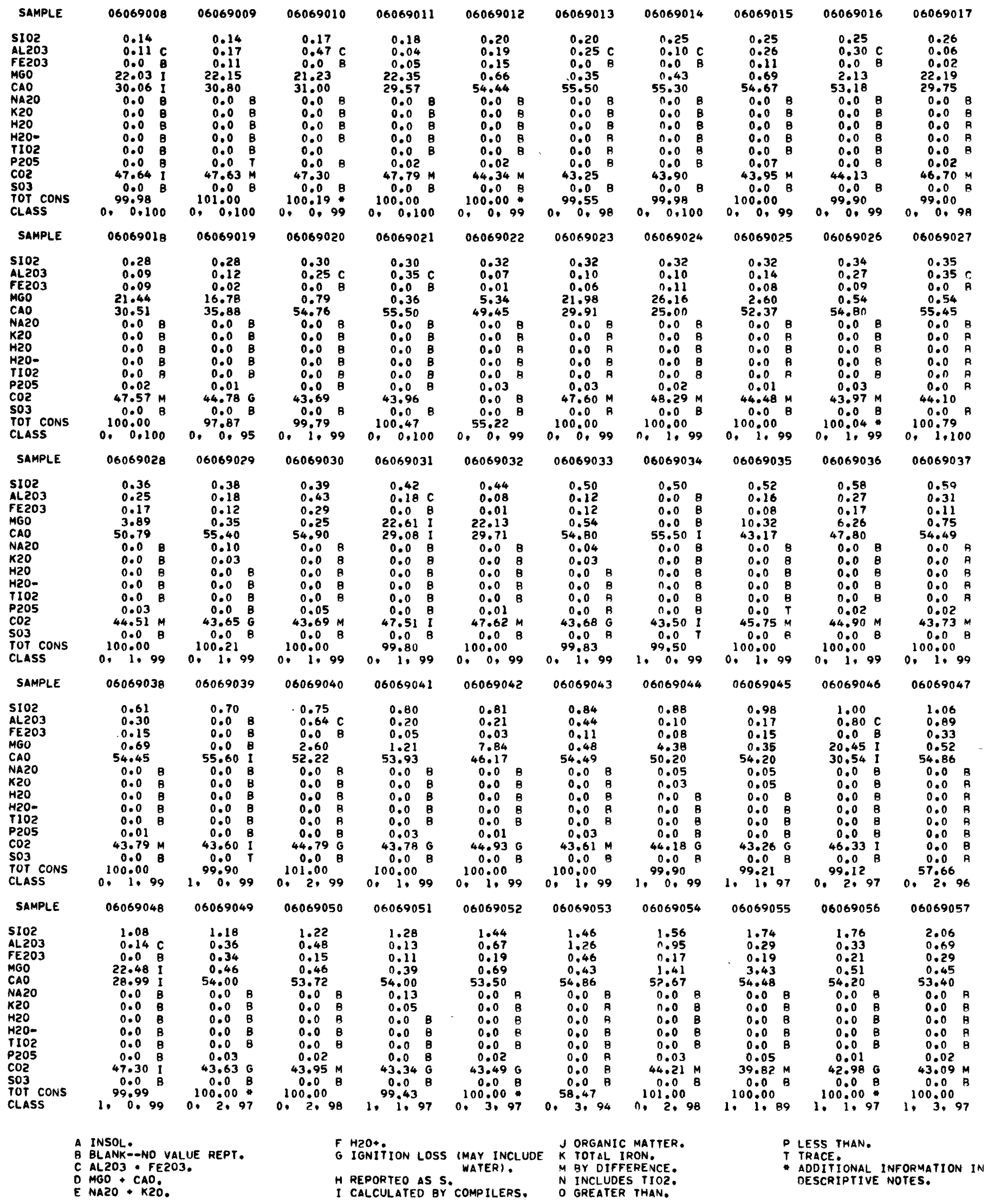


TABLE 9. - Analyses of samples from California and Hawaii containing more than 90 percent carbonate (Group $\left.F_{2}\right)$, common-rock category Continued

06069008 "TI2S,R5E" HOLLISTER. (535:77.81) DOLOMITE. CA/MG $=1.36$.

06069009 PRE-FRANCISCAN, SUR SER, NE1/4,T145,R5E. (54:35,11,PLi) DOLOMITE, WHITE, COARSELY CRYS, TONNAGE. INDEX \& GEOL MAPS, GEOL SEC. CA/MG $=1.39$.

06069010 PRE-FRANCISCAN, SUR SER, NE1/4,T14S,RSE, (535:77/ 54:37,11,PL1/ 556:278) DOLOMITE, WHITE, MEDMCRYS? TONNAGE. INDEX \&

06069011 PRE-FRANCISCAN, SUR SER. S34,T13S,R5E. $(54: 35,11$, PLI) DOLOMITE. WHITE, COARSELY CRYS. INDEX \& GEOL MAP, GEDL SEC.

06069012 PRE-FRANCISCAN, SUR SER. SE1/4.T135,RSE. (54:36,11.22,PL1) LIMESTONE. INDEX \& GEOL MAPS, GEDI. SEC.

06069013 PRE-FRANCISCAN. SUR SER. SWI/4,TI3S,RSE. (556:275,PL37/ 54:31,22,PLI) LIMESTONE, WHITE, GRAY, VARIEGATED, COARSELY

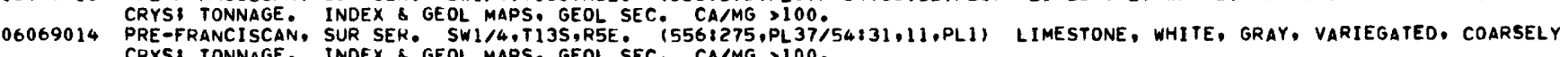

06069015 PRE-FRANCISCAN, SUR SER, SE 1/4,TI3S,R5E, (54:36,11,PLi) LIMESTONE, GRAY, BANDED: MED TO COARSELY CRYS TONNAGE. 06069016 PRE-FRANCISCAN, SUR SER. SWI/4,T13S,R5E. (556:275,PL37/54:31,11,PLI) LIMESTONE, WHITE, GRAY, VARIEGATEO, COARSELY 0606916 PRE-FRANCISCAN, SUR SER, SW1/4,T13S,R5E, (556:275, PL 37/54:31, 11, PLI) LIMESTONE, WHITE, GRAY, VARIEGATEO, COARSELY 06069017 PRE-FRANCISCAN, SUR SER. S34,T13S.R5E. (54,35,1),PL1) DOLOMITE, WHITE, COARSELY CRYS. INDEX \& GEOL MAP, GEOL SEC. 06069018 PRE-FRANCISCAN, SUR SER, SE1/4,T135,R5E. $(54: 36,11$, PL1) DOLOMITE. INDEX \& GEDL MAPS, GEOL SEC. CA/MG =, 42. 06069019 PRE-FRANCISCAN, SUR SER. SW1/4,T13S,RSE. $(54132,11,31$, PLI) DOLOMITE; TONNAGE. INDEX \& GEOL MAPS, GEOL SEC. 06069020 PRE-FRANCISCAN, SUR SER, SW1/4,713S,R5E, (556:275,PL37/54131,11,PL1) LIMESTONE, WHITE, GRAY, VARIEGATED, CDARSELY CRYS I TONNAGE. INDEX \& GEOL MAPS, GEOL SEC. CAMMG $=69.3$.

06069021 PRE-FRANCISCAN, SUR SER. SW1/4,TI3S,R5E, (556:275, PL 37154:31,11,PL1) LIMESTONE, WHITE, GRAY, VARIEGATED, CDARSELY CRYS TONNAGE. INDEX \& GEOL MAPS, GEOL SEC. CAIMG $>100$.

06069022 PRE -FRANCISCAN, SUR SER. SW1/4,TI3S,RSE. (54:32,11,31,PL1) LIMESTONEI TONNAGE. INDEX \& GEOL MAPS, GEOL SEC.

06069023 PRE-FRANCISCAN, SUR SER. S34,T13S,R5E. 154:35,11,PL1) DOLOMITE, WHITE, COARSELY CRYS. INDEX \& GEOL MAP, GEOL SEC. 06069024 PRE-FRANCISCAN, SUR SER, SE1/4,TI3S,R5E, $(54: 33,11$, PL1) DOLOMITE. INDEX \& GEOL MAPS, GEOL SEC. CA/MG = O.956.

06069025 PRE-FRANCISCAN, SUR SER, S20,T13S,RSE, 154:25,11,PL1) LIMESTONE, WHITE, COARSELY CRYSI SURFACE SMPL: TONNAGE. INDEX \& GEOL MAPS, GEOL SEC. CA/MG $=20.1$.

06069026 PRE-FRANCISCAN, SUR SER. SEI/4,T13S,RSE. (54136,11,22,PL1) LIMFSTONE. INDEX \& GEOL MAPS, GEOL SEC. *MISC: I.L. BY DIF. CAIMG >100:

06069027 PRE-FRANCISCAN, SUR SER. SWI/4,T13S,R5E, (5561275,PL37) 54:31,22,PL1) LIMESTONE, WHITE, GRAY, VARIEGATED, COARSELY 06069028 PRE 1 FRANCISCAN, SUR SER, SWI/4,TI3S,R5E, (54:31,11,22,PL1) LIMESTONE, WHITE, GRAY, VARIEGATED, COARSELY CRYS

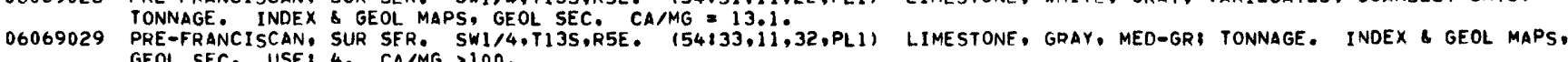
GEOL SEC. USF: 4 . CA/MG $>100$.

06069030 PRE-FRANCISCAN, SUR SER. SEI/4,T135,R5E. $(54: 36,11$, PLI) LIMESTONE, GRAY, BANDED, MED TO COARSELY CRYS $)$ TONNAGE. INDEX \& GEOL MAPS, GEOL SEC. CAMG $>100$

06069031 "TI2S,R5E" HOLLISTER, (535:77,81) DOLOMITE, CA/MG $=1.29$

06069032 PRE-FRANCISCAN. SUR SER. S34,T135,R5E, (54:35,11,PL1), DOLOMITE, WHITE, COARSELY CRYS. INDEX \& GEOL MAP, GEOL SEC.

06069033 PRE-FRANCISCAN, SUR SER. SW1/4,T13S,R5E. (54,33,11,32,PL1) LIMESTONE, GRAY, MEO-GR TONNAGE. INDEX \& GEOL MAPS, 06069034 "TI2S,RSE" HOLLISTER. (142:383/ 1731118,PL4) LIMESTONE, INDEX MAP. USE: 18.

06069035 PRE-FRANCISCAN, SUR SER. SW1/4,T135,R5E, (54132,11,31,PL1) LIMESTONE, TDNNAGE. INDEX \& GEOL MAPS, GEOL SEC. 06069036 PRE-FRANCISCAN, SUR SER. SW1/4,T135,R5E. 154:31,11,22,PL1) LIMESTONE, WHITE, GRAY, VARIEGATED, COARSELY CRYS,

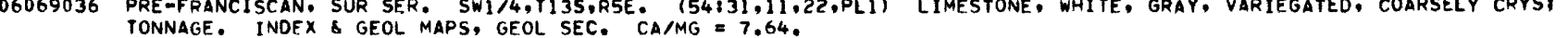
06069037 PRE -FRANCISCAN, SUR SER. SE1/4,T135,R5E. (54:36,11, PL1) LIMESTONE, GRAY, BANDED, MED TO COARSELY CRYS TONNAGE. 06069038 PRE-FRANCISCAN, SUR SER. SE1/4,T13S.R5E. (54:36.11,PL1) LIMESTONE. GRAY, BANDED. MED TO CDARSELY CRYS IONNAGE.

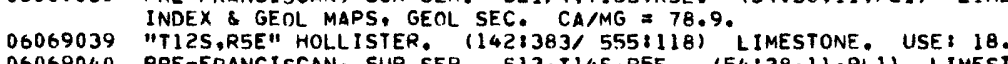

06069040 PRE-FRANCISCAN, SUR SER. S13,T14S,R5E. (54:38,11,PLi) LIMESTONEI COMPOS OF 33 SMPL TONNAGE. INOEX \& GEOL MAPS, 06069041 GEOL SEC. CA PRG $=20.1$. PRE-FRANCISCAN, SUR SER: SW1/4,T13S,R5E, $154: 32,11,31$, PL1) LIMESTONE, TONNAGE. INDEX \& GEOL MAPS, GEOL SEC. 06069042 PRE-FRANCISCAN, SUR SER. SWI/4,T13S,R5E. 06069043 PRE-FRANCISCAN, SUR SER. SE1/4,TI3S,R5E.

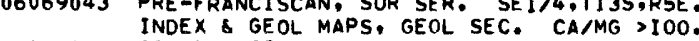
06069044 PRE-FRANCISCAN, SUR SER. SWI/4,T13S.R5E. GEOL SEC. USE: 4. CA/MG $=11.5$.

06069045 PRE-FRANCISCAN, SUR SER. SW1/4,T135.R5E. $154: 32,11,31$, PL1) LIMESTONE, TONNAGE. INOEX \& GEOL MAPS, GEOL SEC. (54:36,11,PL1) LIMESTONE, GRAY, BANDED, MED TO COARSELY CRYSI TDNNAGE. (54:33,11,32,PL1) LIMESTONE, GRAY, MED-GR 1 TONNAGE. INDEX \& GEOL MAPS, 06069046 PRE-FRANCISCAN, "IIRS,R5E" HOLLISTER, $160: 636 / 556: 278,274, P L 37)$ DOLOMITE. INDEX MAP. CA/MG = 1.49.

06069047 MIOCENE \& OLIGOCENE, VAQUEROS FM. S9.T135.R4E. (54:23,PL1) LIMESTONE, CRYS. INDEX \& GEOL MAPS, GEOL SEC. CA/MG>100.

06069048 "TI2S,R5E" HOLLISTER. (535:77.81) DOLOMITE. CA/MG $=1.29$.

06069049 PRE FRANCISCAN, SUR SER. SE $1 / 4, T 135, R 5 E$. $154: 33,11,22$, PLi) LIMESTONE. TONNAGE. INDEX \& GEOL MAPS, GEOL SEC. 06069050 PRE-FRANCISCAN. SUR SER. SEI/4,T13S,R5E. PRE-FRANCISCAN, SUR SER. SE1/4,T13S,R5E.

06069051 PRE-FRANCISCAN, SUR SER. SWI/4,T13S,R5E.

GEOL SEC. USE: 4. CA/MG >100.
06069052 PRE-FRANCISCAN, SUR SER, SE1/4, T135,R5E. $(54: 33,11,22, P L 1)$ LIMESTONE. TONNAGE. INDEX \& GEOL MAPS, GEOL SEC.
SMISC: I.L. BY DIF. CA/MG 77.5 . 06069053 MIOCENE \& OLIGOCENE, VAUUEROS FM. S9,T135,R4E. 154:23,PLI) LIMESTONE, CRYS. INDEX \& GEOL MAPS, GEOL SEC. CA/MG 1 100.

06069054 PRE-FRANCISCAN, SUR SER. S34,T13S,R5E. $(54,36,11$,PL1) LIMESTONE, GRAY, MEO TO COARSELY CRYS, TONNAGE. INDEX \& GEOL 06069055 PAPS, GEOL SEC. CA/MG $=37.4$.

PRE-FRANCISCAN, SUR SER. SW1/4,T13S,R5E, (54:31,11,22,PL1) LIMESTONE, WHITE, GRAY, VARIEGATED, COARSELY CRYS

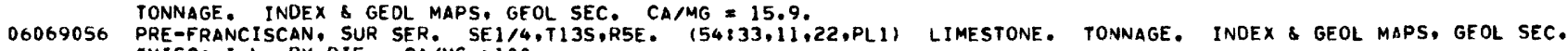
06069057 PRE -FRANCISCAN, SUR SER. SEI/4,T13S,R5E. $154: 36,11$,PL1) LIMESTONE, GRAY, BANDED, MED TO COARSELY CRYS 1 TONNAGE. INDEX \& GEOL MAPS, GFOL SEC. CA/MG $>100$. 
TABLE 9. - Analyses of samples from California and Hawaii containing more than 90 percent carbonate (Group $\left.F_{2}\right)$, common-rock category Continued

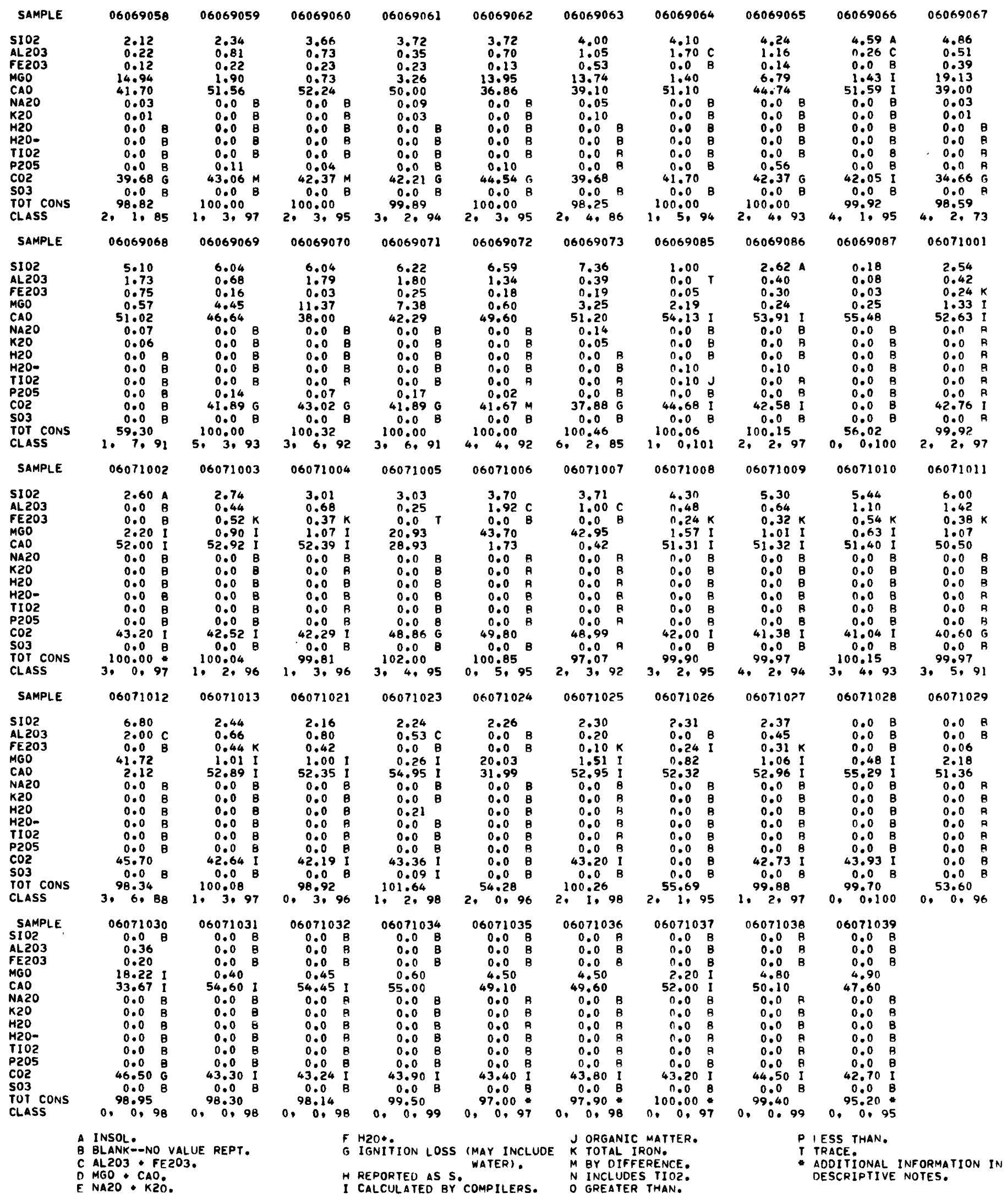


TABLE 9. - Analyses of samples from California and Hawaii containing more than 90 percent carbonate (Group $\left.F_{2}\right)$, common-rock category Continued

06069058 PRE-FRANCISCAN, SUR SFR, SW1/4,T135,R5E. (54:32,11,31,PL1) DOLOMITE: TONNAGE. INDEX G GEOL MAPS, GEOL SEC. 06069059 PRE-FRANCISCAN, SUR SER. SE1/4,T135,R5E. (54:36,11,PL1) LIMESTONE, GRAY, BANDED, MED TO COARSELY CRYS: TONNAGE, INDEX \& GEOL MAPS. GEOL SEC. CAIMG $=27.1$. 06069060 PRE-FRANCISCAN, SUR SER. SWI/4,T13S,R5E. (54131,11,22,PL1) LIMESTONE, WHITE, GRAY, VARIEGATED, COARSELY CRYS 06069061 PRE-FRANCISCAN, SUR SER. SWI/4,T13S,RSE. (54:32,11,31,PL1) LIMESTONE, TONNAGE. INDEX \& GEOL MAPS, GEOL SEC.

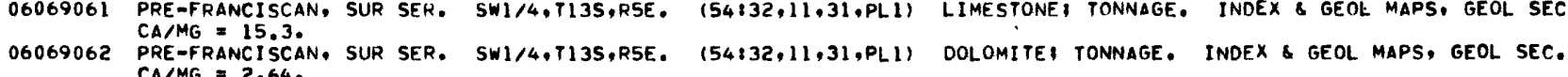

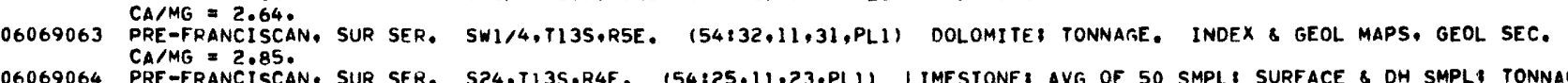
06069064 PRE -FRANCISCAN, SUR SER. S24,T13S.R4E. 154:25.11.23,PL1) LIMESTONE AVG OF 50 SMPL SURFACE \& DH SMPL TONNAGE. 06069065 PRE-FRANCISCAN, SUR SER. SW1/4,T135,R5E. (54:32,11.31,PL1) LIMESTONE, TONNAGE. INDEX \& GEOL MAPS, GEOL SEC. 06069066 PRE-FRANCISCAN, SUR SER, S27,T15S,R7E. (556:275,PL37, 54139,11, PL1) LIMESTONE, GRAY TO WHITE3 COMPOS SMPL OF 75 FT.

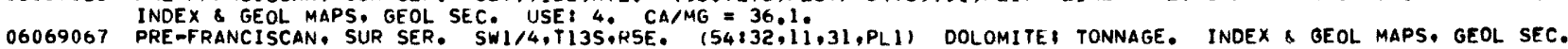

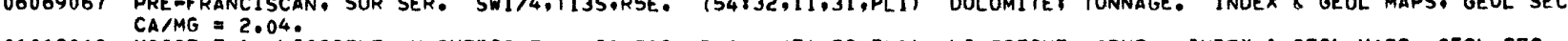
06069068 MIOCENE \& OLIGOCENE, VAQUEROS FM. S9,T135,R4E. (54:23,PLI) LIMESTONE, CRYS. INDEX \& GEOL MAPS, GEOL SEC. 06069069 PRE-FRANCISCAN, SUR SER. SW1/4,T135,R5E. (54132,11,31,PL1) LIMESTONE: TONNAGE. INDEX \& GEOL MAPS, GEOL SEC. 06069070 PRE-FRANCISCAN, SUR SER, SWI/4,T135,R5E. $154132,11,31$, PL1) DOLOMITE, TONNAGE. INDEX \& GEOL MAPS, GEOL SEC. 06069071 PRE-FRANCISCAN, SUR SER. SW1/4,T135,RSE. $(54: 32,11,31$, PL1) LIMESTONE, TONNAGE. INDEX \& GEOL MAPS, GEOL SEC.

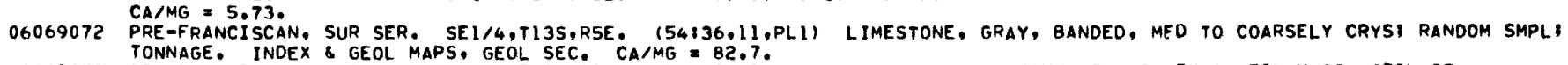

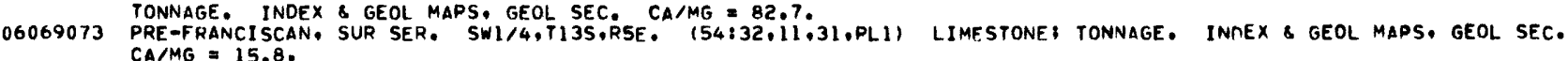

06069085 PRE -FRANCISCAN. "T135,R4E" CHITTENDEN, 1305158 ) LIMESTONE, CRYS, USE: 4. CA/MG = 24.7.

06069086 PRE-FRANCISCAN. "TI35,R4E" CHITTENDEN, (305:58) LIMESTONE, BLUE TO WHITE, CRYS. USE: 4 . CA/MG 1100.

06069087 PRE-FRANCISCAN, SUR SER. "T135,R3,4E" LOS VERGELES DIST. (265:55,PL1) LIMESTONE, WHITE, CRYS. INDEX \& GEOL MAPS. USE: 7.12. CA/MG >100

06071001 S4,T3N,R7W. (556:291,290,PL37) LIMESTONE, WHITE, CRYSI TONNAGE, INDEX MAP. USE: 4 . CA/MG = 39.6.

06071002 "T1S,R4W" COLTON. (145:360) MARBLE, PHYS PROP. USE: 15. OMISCI INSOL = BIOTITE \& PYROLUSITE. CA/MG = 23.6.

06071003 S4,T3N,R7W. (556:291,290,PL37) LIMESTONE, WHITE, CRYSI TONNAGE, INDEX MAP. USE: 4 . CA/MG = 58.B.

06071004 S4,T3N.R7W. (556:291,290,PL37) LIMESTONE. WHITE, CRYSI TONNAGE. INOEX MAP. USE: 4 . CA/MG = 49.0.

06071005 S11.12,T9N,R4W. 1507:5231 DOLOMITE, WHITE TO GRAY, USE: 12 . CA/MG $=1.39$.

06071006 S14.T9N,R6W. $\quad(507: 533,532)$ MAGNESITEI $300 \mathrm{FT}$ THICK. CA/MG $=0.040$.

06071007 S14,T9N,RGW. $(5071533,532)$ MAGNESITE: 300 FT THICK. CAIMG $=0.010$.

06071008 S4,T3N.R7W. (556:291,290,PL37) LIMESTONE, WHITE, CRYSI TONNAGE. INDEX MAP. USE: 4. CA/MG $=32.7$.

06071009 S4,T3N.R7W. (556:291,290,PL37) LIMESTONE, WHITE, CRYS TONNAGE. INDEX MAP. USEI 4 . CA/MG = 50.0.

06071010 S4,T3N,R7W. 1556:291,290,PL37) LIMESTONE, WHITE, CRYS, TONNAGE. INDEX MAP. USE: 4. CA/MG = 81.6.

06071011 S4,T3N.R7W. 1556:290,291,PL371 LIMESTONE, WHITE, CRYS: TONNAGE, INDEX MAP. USE: 4. CA/MG = 47.2.

06071012 S14.T9N.R6W. 15078533,532$)$ MAGNESITE: 300 FT THICK. USE: 18 . CA/MG $=0.051$.

06071013 S4,T3N,R7W. 1556:291,290,PL37) LIMESTONE, WHITE, CRYSI TONNAGE. INDEX MAP. USE: 4 . CA/MG = 52.4.

06071021 S4,T3N.R7W. (556:291,290,PL37) LIMESTONE. WHITE, CRYSI TONNAGE. INDEX MAP. USE: 4. CA/MG = 52.4.

06071023 PRECAMBRIAN. S12,TIIN,R6E. (120:873,872,876/557115,103) LIMESTONE, PINK, CRYS. INDEX MAP. USE, 6. CA/MG , I00.

06071024 S17,20.21,T6N,R14E. (556:286,284,287,PL37) DOLOMITE, WHITE, CRYS3 30-110 FT THICK, TONNAGE. INDEX MAP. CA/MG = 1.60.

06071025 S4,T3N,R7W. 1556:291,290,PL37) LIMESTONE, WHITE, CRYS TONNAGE. INDEX MAP. USE: 4. CA/MG = 35.1.

$06071026517,20,21, T 6 N, R 14 E$. (556:286,284-287,PL37) LIMESTONE, GRAY, FINELY CRYS\$ TONNAGE. INDEX MAP. CA/MG = 63.8.

06071027 S4,T3N.R7W. (556:291,290,PL37) LIMESTONE, WHITE, CRYSI TONNAGE. INDEX MAP. USE: 4. CA/MG = 50.0.

06071028 PALEOZOIC, FURNACE LS, S14,T3N,RIE, $(556: 299,300$, PL37/ 550:26B,267,269,PL36) LIMESTONE, WHITE, SUGARY. CA/MG >100.

06071029 PLEISTOCENE, BLACKHAWK GRECCIA. "S14,T3N.RIE" (231:6) LIMESTONE, BRECCIATED: AVG OF 6 RANDOM SMPL: 100-600 FT THICK. 06071030 PALEOZOIC. S29.T6N.RI3E. (40RI171.170) MAFBLE, OOLOMITICI TYPICAL SMPLI TONNAGE. CA/MG 1 .85.

06071031 S12,T15N,RI3E. (503:385,384) LIMESTONE, FINE-GR TO COARSELY CRYS; BEDS 10-300 FT THICK, AVG OF 4 ANAL. CA/MG >100.

06071032 CARBONIFEROUS. S12,T15N,RI3E. (556:287,PL37) LIMESTONE, GRAY TO WHITE, FINE TO COARSELY CRYSI REDS 300 FT THICKI 06071034 PALEOZOIC, FURNACE LS. T3N,PIE, CUSHENBURY CANYON. $(550: 268,267,269$, PL36) LIMESTONE. WHITE, COMPOS SMPLI MIN. INDEX

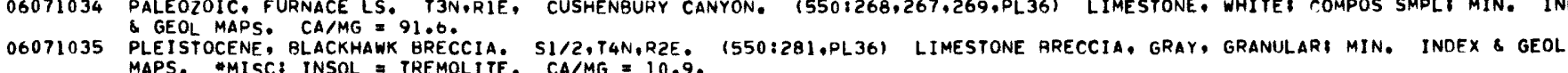
06071036 PLEISTOCENE, BLACKHAWK BRECCIA. SII2,T4N, 102E, (550:281,PL36) LIMESTONE BRECCIA. GRAY, GRANULAR; MIN. INDEX \& GEOL 00071037 "TIS,R4W" COLTON. $1144: 240)$ MARBLE: PHYS PROP. USE:15. "MISC: BIOTITE \& PYROLUSITE = 2.6 . CA/MG = 23.6 .

06071038 PALEOZOIC, FURNACE LS. S12.T3N.RIE. (550:268.269.PL36) LIMESTONE, WHITEI MIN. INDEX \& GEOL MAPS. CA/MG = 10.4.

06071039 PLEISTOCENE, BLACKHAWK BRECCIA. S1/2,T4N,R2E. (550:281,PL36) LIMESTONE RRECCIA, GRAY, GRANULAR; MIN. INDEX GEOL MAPS. "MISC: INSOL TREMOLITE, CA/MG $=9.71$. 
TABLE 9. - Analyses of samples from California and Hawaii containing more than 90 percent carbonate (Group $F_{2}$ ), common-rock category Continued

\begin{tabular}{|c|c|c|c|c|c|c|c|c|c|c|c|c|c|c|c|c|c|c|c|}
\hline SAMPLE & 06071040 & 06071041 & & 0607104 & & 0607104 & & 06071045 & & 0607104 & & 0607104 & & 0607104 & & 0607104 & & 06071050 & \\
\hline $\begin{array}{l}\text { SIO2 } \\
\text { AL2O3 } \\
\text { FE2O3 } \\
\text { MGO } \\
\text { CAO } \\
\text { NA2O } \\
\text { K2O } \\
\text { H2O } \\
\text { H2O- } \\
\text { TIO2 } \\
\text { P2O5 } \\
\text { CO2 } \\
\text { SO3 } \\
\text { TOT CONS } \\
\text { CLASS }\end{array}$ & $\begin{array}{cc}0.0 & 8 \\
0.0 & B \\
0.0 & B \\
5.00 \\
47.30 \\
0.0 \\
0.0 \\
0.0 \\
0.0 \\
0.0 \\
0.0 \\
0.0 \\
0.0 \\
0.0 \\
52.30 \\
0.0 .95\end{array}$ & $\begin{array}{c}0.0 \\
0.0 \\
0.0 \\
19.70 \\
31.20 \\
0.0 \\
0.0 \\
0.0 \\
0.0 \\
0.0 \\
0.0 \\
0.0 \\
0.0 \\
50.90 \\
0.00 \\
0.07\end{array}$ & $\begin{array}{l}\mathrm{B} \\
\mathrm{B} \\
\mathrm{B} \\
\mathrm{B} \\
\mathrm{B} \\
\mathrm{B} \\
\mathrm{B} \\
\mathrm{B}\end{array}$ & $\begin{array}{c}0.0 \\
0.0 \\
0.0 \\
5.70 \\
48.10 \\
0.0 \\
0.0 \\
0.0 \\
0.0 \\
0.0 \\
0.0 \\
44.00 \\
0.00 \\
97.80 \\
0.0 .9\end{array}$ & $\begin{array}{l}\text { B } \\
B \\
B \\
\\
B \\
B \\
B \\
B \\
B \\
B \\
1 \\
B \\
B \\
98\end{array}$ & $\begin{array}{c}0.0 \\
0.0 \\
0.0 \\
7.50 \\
45.60 \\
0.0 \\
0.0 \\
0.0 \\
0.0 \\
0.0 \\
0.0 \\
44.00 \\
0.0 \\
97.10 \\
0.90 .9\end{array}$ & $\begin{array}{l}B \\
B \\
B \\
B \\
B \\
B \\
I \\
I \\
B \\
7 \\
7\end{array}$ & $\begin{array}{c}0.0 \\
0.0 \\
0.0 \\
22.00 \\
30.00 \\
0.0 \\
0.0 \\
0.0 \\
0.0 \\
0.0 \\
0.0 \\
43.30 \\
0.0 \\
95.30 \\
0 . \quad 0.9\end{array}$ & $\begin{array}{l}B \\
B \\
B \\
1 \\
1 \\
B \\
B \\
B \\
B \\
B \\
B \\
I \\
B\end{array}$ & $\begin{array}{c}0.0 \\
0.0 \\
0.0 \\
0.0 \\
55.00 \\
0.0 \\
0.0 \\
0.0 \\
0.0 \\
0.0 \\
0.0 \\
43.00 \\
0.0 \\
98.00 \\
0.0 .9\end{array}$ & $\begin{array}{l}B \\
B \\
B \\
B \\
I \\
B \\
B \\
B \\
B \\
B \\
B \\
I \\
B\end{array}$ & $\begin{array}{c}0.0 \\
0.0 \\
0.0 \\
0.0 \\
55.20 \\
0.0 \\
0.0 \\
0.0 \\
0.0 \\
0.0 \\
0.0 \\
43.30 \\
0.0 \\
98.50 \\
0.0 .5\end{array}$ & $\begin{array}{l}8 \\
8 \\
8 \\
8 \\
1 \\
1 \\
8 \\
8 \\
B \\
B \\
B \\
8 \\
I \\
B \\
9\end{array}$ & $\begin{array}{c}0.0 \\
0.0 \\
0.0 \\
0.0 \\
55.20 \\
0.0 \\
0.0 \\
0.0 \\
0.0 \\
0.0 \\
0.0 \\
43.30 \\
0.0 \\
100.00 \\
0.0 .0\end{array}$ & $\begin{array}{l}B \\
B \\
B \\
B \\
I \\
B \\
B \\
B \\
B \\
B \\
B \\
I \\
B \\
\\
98\end{array}$ & $\begin{array}{c}0.0 \\
0.0 \\
0.0 \\
0.0 \\
55.40 \\
0.0 \\
0.0 \\
0.0 \\
0.0 \\
0.0 \\
0.0 \\
43.40 \\
0.00 \\
98.80 \\
0.0 .9\end{array}$ & $\begin{array}{l}B \\
B \\
B \\
B \\
1 \\
B \\
B \\
B \\
8 \\
B \\
B \\
B \\
I \\
B \\
\end{array}$ & $\begin{array}{c}0.0 \\
0.0 \\
0.0 \\
0.0 \\
56.00 \\
0.0 \\
0.0 \\
0.0 \\
0.0 \\
0.0 \\
0.0 \\
43.00 \\
0.0 \\
99.00 \\
0.009\end{array}$ & $\begin{array}{l}\mathrm{D} \\
\mathrm{B} \\
\mathrm{A} \\
\mathrm{B} \\
\mathrm{B} \\
\mathrm{A} \\
\mathrm{I} \\
\mathrm{B}\end{array}$ \\
\hline SAMPLE & 06071051 & 06071052 & & 0607105 & & 0607105 & & 0607105 & & 0607105 & & 0607105 & & 0607105 & & 0607106 & & 06071061 & \\
\hline $\begin{array}{l}5102 \\
\text { AL2O3 } \\
\text { FE2O3 } \\
\text { MGO } \\
\text { CAO } \\
\text { NA2O } \\
\text { K2O } \\
\text { H2O } \\
\text { H2O- } \\
\text { T1O2 } \\
\text { P2O5 } \\
\text { CO2 } \\
\text { SO3 } \\
\text { TOT CONS } \\
\text { CLASS }\end{array}$ & 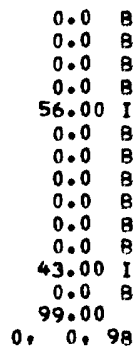 & $\begin{array}{c}0.0 \\
0.0 \\
0.0 \\
0.0 \\
55.00 \\
0.0 \\
0.0 \\
0.0 \\
0.0 \\
0.0 \\
0.0 \\
44.00 \\
0.0 \\
99.00 \\
0.009\end{array}$ & $\begin{array}{l}\text { B } \\
\text { B } \\
\text { B } \\
\text { B } \\
1 \\
\text { B } \\
\text { B } \\
8 \\
B \\
B \\
B \\
1 \\
B\end{array}$ & $\begin{array}{c}0.10 \\
0.72 \\
0.36 \\
1.45 \\
52.55 \\
0.0 \\
0.0 \\
0.00 \\
0.0 \\
0.0 \\
0.0 \\
42.84 \\
0.0 \\
98.02 \\
0.0 .9\end{array}$ & $\begin{array}{l}K \\
I \\
I \\
B \\
B \\
B \\
B \\
A \\
B \\
I \\
B \\
\\
97\end{array}$ & $\begin{array}{c}0.20 \\
0.30 \\
0.0 \\
0.0 \\
56.00 \\
0.0 \\
0.0 \\
0.0 \\
0.0 \\
0.0 \\
0.0 \\
43.30 \\
0.0 \\
99.80 \\
0.0 .9\end{array}$ & $\begin{array}{l}B \\
B \\
B \\
B \\
B \\
B \\
G \\
B \\
\\
\\
\end{array}$ & $\begin{array}{c}0.32 \\
0.12 \\
0.10 \\
0.76 \\
54.81 \\
0.0 \\
0.0 \\
0.0 \\
0.0 \\
0.0 \\
0.0 \\
43.87 \\
0.0 \\
99.98 \\
0,109\end{array}$ & $\begin{array}{l}K \\
I \\
I \\
B \\
B \\
B \\
B \\
B \\
B \\
I \\
B \\
\\
99\end{array}$ & $\begin{array}{c}0.40 \\
0.0 \\
0.0 \\
0.40 \\
54.70 \\
0.0 \\
0.0 \\
0.0 \\
0.0 \\
0.0 \\
0.0 \\
43.30 \\
0.0 \\
98.80 \\
0.0 .9\end{array}$ & $\begin{array}{l}\text { A } \\
B \\
B\end{array}$ & $\begin{array}{c}0.40 \\
0.0 \\
0.40 \\
21.70 \\
32.10 \\
0.0 \\
0.0 \\
0.0 \\
0.0 \\
0.0 \\
0.0 \\
0.0 \\
0.0 \\
54.60 \\
0.0 \\
1.09\end{array}$ & $\begin{array}{l}B \\
\\
8 \\
8 \\
8 \\
B \\
B \\
8 \\
B \\
B \\
95\end{array}$ & $\begin{array}{r}0.40 \\
0.50 \\
0.0 \\
21.10 \\
31.00 \\
0.0 \\
0.0 \\
0.0 \\
0.0 \\
0.0 \\
0.0 \\
0.0 \\
0.0 \\
53.00 \\
0 . \quad 1.9\end{array}$ & $\begin{array}{l}C \\
B \\
\\
B \\
B \\
B \\
B \\
B \\
B \\
B \\
B \\
B \\
9\end{array}$ & $\begin{array}{c}0.43 \\
0.24 \\
0.0 \\
17.19 \\
35.34 \\
0.0 \\
0.0 \\
0.0 \\
0.0 \\
0.0 \\
0.0 \\
46.52 \\
0.0 \\
99.72 \\
0.7 .9\end{array}$ & $\begin{array}{l}A \\
C \\
B \\
1 \\
I \\
I \\
B \\
B \\
B \\
B \\
B \\
B \\
I \\
B \\
\\
99\end{array}$ & $\begin{array}{c}0.46 \\
0.48 \\
0.0 \\
43.08 \\
4.46 \\
0.0 \\
0.0 \\
0.0 \\
0.0 \\
0.0 \\
0.0 \\
50.54 \\
0.0 \\
99.02 \\
0.1 .98\end{array}$ & $\begin{array}{l}A \\
C \\
B \\
I \\
I \\
R \\
R \\
R \\
R \\
R \\
R \\
I \\
B\end{array}$ \\
\hline SAMPLE & 06071063 & 06071064 & & 0607106 & & 06071066 & & 06071067 & & 0607106 & & 0607106 & & 0607107 & & 0607107 & & 06071072 & \\
\hline $\begin{array}{l}\mathrm{SIOZ} \\
\text { AL2O3 } \\
\text { FE2O3 } \\
\text { MGO } \\
\text { CAO } \\
\text { NA2O } \\
\text { K2O } \\
\text { H2O } \\
\text { H2O- } \\
\text { TIO2 } \\
\text { P2O5 } \\
\text { CO2 } \\
\text { SO3 } \\
\text { TOT CONS } \\
\text { CLASS }\end{array}$ & $\begin{array}{c}0.50 \\
0.0 \\
0.20 \\
1.70 \\
54.68 \\
0.0 \\
0.0 \\
0.0 \\
0.0 \\
0.0 \\
0.0 \\
42.0 \\
0.08 \\
1000 \\
0.00 \\
0.0 .097\end{array}$ & $\begin{array}{c}0.50 \\
0.28 \\
0.02 \\
0.08 \\
55.50 \\
0.0 \\
0.0 \\
0.0 \\
0.0 \\
0.0 \\
0.0 \\
43.61 \\
0.0 \\
99.99 \\
0 . \quad 1.99\end{array}$ & $\begin{array}{l}B \\
B \\
B \\
B \\
B \\
B \\
G \\
B\end{array}$ & $\begin{array}{c}0.52 \\
0.15 \\
0.45 \\
3.94 \\
50.85 \\
0.0 \\
0.0 \\
0.0 \\
0.0 \\
0.0 \\
0.05 \\
0.0 \\
0.0 \\
55.96 \\
0.91 .9\end{array}$ & $\begin{array}{l} \\
B \\
B \\
B \\
B \\
B \\
B \\
B \\
B \\
B \\
98\end{array}$ & $\begin{array}{c}0.55 \\
0.85 \\
0.0 \\
0.0 \\
55.22 \\
0.0 \\
0.0 \\
0.0 \\
0.0 \\
0.0 \\
0.0 \\
43.38 \\
0.0 \\
100.00 \\
0, \quad 1.09\end{array}$ & $\begin{array}{l}C \\
B \\
\\
B \\
B \\
B \\
B \\
B \\
B \\
B \\
9\end{array}$ & $\begin{array}{c}0.60 \\
0.45 \\
0.0 \\
20.20 \\
31.04 \\
0.0 \\
0.0 \\
0.0 \\
0.0 \\
0.0 \\
0.0 \\
0.0 \\
0.0 \\
52.29 \\
0 . \quad 1.98\end{array}$ & $\begin{array}{l}C \\
B \\
\\
B \\
B \\
B \\
B \\
B \\
B \\
B \\
B \\
B \\
\\
98\end{array}$ & $\begin{array}{c}0.60 \\
0.50 \\
0.0 \\
21.03 \\
30.81 \\
0.0 \\
0.0 \\
0.0 \\
0.0 \\
0.0 \\
0.0 \\
47.16 \\
0.0 \\
100.10 \\
0 . \quad 1.9\end{array}$ & $\begin{array}{l}\text { C } \\
\text { B } \\
\text { I } \\
\text { I } \\
B \\
B \\
B \\
B \\
B \\
B \\
I \\
B\end{array}$ & $\begin{array}{c}0.63 \\
0.59 \\
0.32 \\
4.14 \\
51.00 \\
0.0 \\
0.0 \\
0.0 \\
0.0 \\
0.0 \\
0.05 \\
0.0 \\
0.0 \\
56.73 \\
0.01 .9\end{array}$ & $\begin{array}{l}\text { B } \\
B \\
B \\
B \\
B \\
B \\
B \\
\\
97\end{array}$ & $\begin{array}{c}0.70 \\
0.38 \\
0.20 \\
1.15 \\
53.96 \\
0.0 \\
0.0 \\
0.0 \\
0.0 \\
0.0 \\
0.0 \\
43.61 \\
0.0 \\
100.00 \\
0.01 .9\end{array}$ & $\begin{array}{l}K \\
1 \\
1 \\
1 \\
B \\
B \\
B \\
B \\
B \\
B \\
1 \\
B\end{array}$ & $\begin{array}{c}0.78 \\
0.24 \\
0.0 \\
41.12 \\
6.06 \\
0.0 \\
0.0 \\
0.0 \\
0.0 \\
0.0 \\
0.0 \\
49.66 \\
0.0 \\
97.86 \\
0.91 .9\end{array}$ & $\begin{array}{l}A \\
C \\
B \\
\\
B \\
B \\
B \\
B \\
B \\
B \\
1 \\
B \\
97\end{array}$ & $\begin{array}{c}0.84 \\
2.77 \\
0.43 \\
43.00 \\
1.94 \\
0.0 \\
0.0 \\
0.0 \\
0.0 \\
0.0 \\
0.0 \\
50.80 \\
0.0 \\
99.78 \\
0 . \quad 1.96\end{array}$ & $\begin{array}{l}\text { A } \\
\text { A } \\
\text { A } \\
\text { B } \\
\text { B } \\
\text { B }\end{array}$ \\
\hline SAMPLE & 06071074 & 06071075 & & 0607107 & & 0607107 & & 0607107 & & 0607108 & & 0607108 & & 0607108 & & 0607108 & & 06071084 & \\
\hline $\begin{array}{l}\text { SIO2 } \\
\text { AL2O3 } \\
\text { FE2O3 } \\
\text { MGO } \\
\text { CAO } \\
\text { NA2O } \\
\text { K2O } \\
\text { H2O } \\
\text { H2O- } \\
\text { TIO2 } \\
\text { P2O5 } \\
\text { CO2 } \\
\text { SO3 } \\
\text { TOT CONS } \\
\text { CLASS }\end{array}$ & 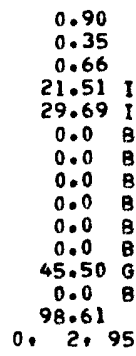 & $\begin{array}{c}0.97 \\
0.11 \\
0.75 \\
4.98 \\
50.24 \\
0.0 \\
0.0 \\
0.0 \\
0.0 \\
0.0 \\
0.11 \\
0.0 \\
0.0 \\
57.16 \\
0.0 \\
0.09\end{array}$ & $\begin{array}{l}B \\
B \\
B \\
B \\
B\end{array}$ & $\begin{array}{c}0.99 \\
0.20 \\
0.38 \\
3.49 \\
51.29 \\
0.0 \\
0.0 \\
0.0 \\
0.0 \\
0.0 \\
0.04 \\
0.0 \\
0.0 \\
56.39 \\
0.2 .9\end{array}$ & $\begin{array}{l} \\
B \\
B \\
B \\
8 \\
B \\
B \\
B \\
B \\
97\end{array}$ & $\begin{array}{c}1.00 \\
0.36 \\
0.20 \\
18.23 \\
33.68 \\
0.0 \\
0.0 \\
0.0 \\
0.0 \\
0.0 \\
0.0 \\
46.50 \\
0.0 \\
99.97 \\
0,2.98\end{array}$ & $\begin{array}{l} \\
B \\
B \\
B \\
B \\
B \\
B \\
B \\
6 \\
B \\
98\end{array}$ & $\begin{array}{c}1.01 \\
0.54 \\
0.0 \\
20.35 \\
31.46 \\
0.0 \\
0.0 \\
0.0 \\
0.0 \\
0.0 \\
0.0 \\
46.64 \\
0.0 \\
100.00 \\
0,2.98\end{array}$ & $\begin{array}{l}C \\
B \\
I \\
I \\
B \\
B \\
B \\
B \\
B \\
B \\
G \\
B \\
B\end{array}$ & $\begin{array}{c}1.09 \\
0.0 \\
0.0 \\
0.45 \\
54.45 \\
0.0 \\
0.0 \\
0.0 \\
0.0 \\
0.0 \\
0.0 \\
43.24 \\
0.0 \\
99.23 \\
1.0 .29\end{array}$ & $\begin{array}{l}B \\
B \\
\\
I \\
B \\
B \\
B \\
B \\
B \\
B \\
I \\
B\end{array}$ & $\begin{array}{c}1.10 \\
0.03 \\
0.11 \\
1.93 \\
52.88 \\
0.0 \\
0.0 \\
0.0 \\
0.0 \\
0.0 \\
0.0 \\
43.94 \\
0.0 \\
99.99 \\
1, \quad 1.98\end{array}$ & $\begin{array}{l} \\
B \\
B \\
B \\
B \\
B \\
1 \\
G \\
B \\
98\end{array}$ & $\begin{array}{c}1.10 \\
0.25 \\
0.19 \\
2.98 \\
51.18 \\
0.0 \\
0.0 \\
0.0 \\
0.0 \\
0.0 \\
0.08 \\
0.0 \\
0.0 \\
55.78 \\
0.1 .9\end{array}$ & $\begin{array}{l} \\
B \\
B \\
B \\
B \\
B \\
B \\
B \\
98\end{array}$ & $\begin{array}{c}1.16 \\
0.24 \\
0.0 \\
20.69 \\
30.99 \\
0.0 \\
0.0 \\
0.0 \\
0.0 \\
0.0 \\
0.0 \\
46.92 \\
0.0 \\
100.00 \\
101.9\end{array}$ & $\begin{array}{l}A \\
C \\
B \\
I \\
I \\
B \\
B \\
B \\
B \\
B \\
B \\
I \\
B\end{array}$ & $\begin{array}{c}1.17 \\
0.13 \\
0.0 \\
0.13 \\
55.14 \\
0.0 \\
0.0 \\
0.0 \\
0.0 \\
0.0 \\
0.0 \\
43.43 \\
0.0 \\
100.00 \\
100.09\end{array}$ & $\begin{array}{l}\text { C } \\
\text { B } \\
I \\
I \\
\text { B } \\
\text { B } \\
B \\
B \\
\text { B } \\
\text { B } \\
I \\
\text { B }\end{array}$ \\
\hline SAMPLE & 06071086 & 06071087 & & 0607108 & & 0607108 & & 0607109 & & 0607109 & & 0607109 & & 06071096 & & 0607109 & & 06071096 & \\
\hline $\begin{array}{l}\mathrm{SIO} 2 \\
\text { AL2O3 } \\
\text { FE2O3 } \\
\text { MGO } \\
\text { CAO } \\
\text { NA2O } \\
\text { K2O } \\
\text { H2O } \\
\text { H2O- } \\
\text { TIO2 } \\
\text { P2O5 } \\
\text { CO2 } \\
\text { SO3 } \\
\text { TOT CONS } \\
\text { CLASS }\end{array}$ & $\begin{array}{cc}1.34 & A \\
0.18 & C \\
0.0 & B \\
43.84 \\
0.78 \\
0.0 \\
0.0 \\
0.0 \\
0.0 \\
0.0 \\
0.0 \\
0.0 \\
48.47 \\
0.0 \\
94.61 \\
94 \\
1.99\end{array}$ & $\begin{array}{c}1.34 \\
0.88 \\
0.0 \\
39.41 \\
7.16 \\
0.0 \\
0.0 \\
0.0 \\
0.0 \\
0.0 \\
0.0 \\
48.67 \\
0.0 \\
97.46 \\
98 \\
2.95\end{array}$ & $\begin{array}{l}\mathrm{B} \\
\mathrm{B} \\
\mathrm{B} \\
\mathrm{B} \\
\mathrm{B} \\
\mathrm{B} \\
\mathbf{I} \\
\mathrm{B}\end{array}$ & $\begin{array}{c}1.38 \\
0.40 \\
0.0 \\
46.32 \\
1.10 \\
0.0 \\
0.0 \\
0.0 \\
0.0 \\
0.0 \\
0.0 \\
50.74 \\
0.0 \\
99.94 \\
1.9\end{array}$ & $\begin{array}{l}A \\
C \\
B \\
\\
B \\
B \\
B \\
B \\
B \\
B \\
B \\
I \\
B \\
98\end{array}$ & $\begin{array}{c}1.40 \\
0.40 \\
0.0 \\
19.70 \\
31.20 \\
0.0 \\
0.0 \\
0.0 \\
0.0 \\
0.0 \\
0.0 \\
46.20 \\
0.0 \\
98.90 \\
1.97\end{array}$ & $\begin{array}{l} \\
B \\
B \\
8 \\
8 \\
B \\
8 \\
1 \\
B \\
8 \\
97\end{array}$ & $\begin{array}{c}1.40 \\
0.0 \\
0.0 \\
19.70 \\
31.20 \\
0.0 \\
0.0 \\
0.0 \\
0.0 \\
0.0 \\
0.0 \\
46.00 \\
0.0 \\
98.90 \\
0.9\end{array}$ & $\begin{array}{l}A \\
\text { B } \\
\text { B } \\
\text { I } \\
\text { I } \\
\text { B } \\
\text { B } \\
\text { B } \\
\text { B } \\
\text { B } \\
\text { B } \\
\text { I } \\
\text { B } \\
4 \\
7\end{array}$ & $\begin{array}{c}1.45 \\
0.28 \\
0.24 \\
4.58 \\
50.65 \\
0.0 \\
0.0 \\
0.0 \\
0.0 \\
0.0 \\
0.03 \\
0.0 \\
0.0 \\
57.23 \\
1,95\end{array}$ & $\begin{array}{l} \\
B \\
B \\
B \\
B \\
B \\
B \\
B\end{array}$ & $\begin{array}{c}1.52 \\
0.32 \\
0.0 \\
44.62 \\
0.86 \\
0.0 \\
0.0 \\
0.0 \\
0.0 \\
0.0 \\
0.0 \\
49.39 \\
0.0 \\
96.71 \\
1.95\end{array}$ & $\begin{array}{l}A \\
C \\
B\end{array}$ & $\begin{array}{c}1.55 \\
0.96 \\
0.14 \\
3.00 \\
51.14 \\
0.0 \\
0.0 \\
0.0 \\
0.0 \\
0.0 \\
0.19 \\
0.0 \\
0.0 \\
56.98 \\
0,3.9\end{array}$ & $\begin{array}{l} \\
\text { B } \\
\text { B } \\
\text { B } \\
\text { B } \\
\text { B } \\
\text { B } \\
\text { B }\end{array}$ & $\begin{array}{c}1.56 \\
0.73 \\
0.94 \\
20.23 \\
31.22 \\
0.0 \\
0.0 \\
0.0 \\
0.0 \\
0.0 \\
0.0 \\
0.0 \\
0.0 \\
54.68 \\
0.39\end{array}$ & $\begin{array}{l}\text { I } \\
\text { B } \\
8 \\
8 \\
8 \\
B \\
B \\
8 \\
B\end{array}$ & $\begin{array}{c}1.57 \\
0.15 \\
0.43 \\
1.98 \\
53.27 \\
0.0 \\
0.0 \\
0.0 \\
0.0 \\
0.0 \\
0.04 \\
0.0 \\
0.0 \\
57.44 \\
1.06\end{array}$ & $\begin{array}{l}\text { A } \\
B \\
\text { B } \\
\text { B } \\
\text { R }\end{array}$ \\
\hline
\end{tabular}

A INSOL.

R BLANK=-NO VALUE REPT. C AL203 - FE203.

D MGO CAO.

E NA2O K K20.
G HEO+ IGNION LOSS IMAY INCLUDE H REPORTED AS S. WATERI.
I CALCULATED BY" COMPILERS.
$J$ ORGANIC MATTER. TOTAL IRON. $M$ BY DIFFERENCE. $N$ INCLUDES TIOZ.
$O$ GREATER THAN.
P LESS THAN.

T TRACE. DESCRIPTIVE NOTES. 
TABLE 9. - Analyses of samples from California and Hawaii containing more than 90 percent carbonate (Group $F_{2}$ ), common-rock category Continued

06071040 PALEOZOIC, FURNACE LS, T3N,R2F, RLACKHAWK CANYON, (550:282,PL36) LIMESTONE. INDEX \& GEOL MAPS. "MISC: INSOL =

06071041 SAN BERNAROINO MTN., ALACKHAWK CANYON. $1404: 2211$ DOLOMITE. CA/MG $=1.58$.

06071042 PLEISTOCENE, BLACKHAWK BRECCIA. SI/2,T4N,R2E. (550:281,PL36) LIMESTONE BRECCIA, GRAY, GRANULAR: MIN. INDEX \& GEOL

06071043 PLEISTOCENE, BLACKHAWK BRECCIA. S1/2,T4N,R2E. (550:281,PL36) LIMESTONE RRECCIA, GRAY, GRANULAR MIN. INDEX \& GEOL D6071045 MAPS. MMISC: INSOL = TREMOLITF. CAIMG $=6.08$.

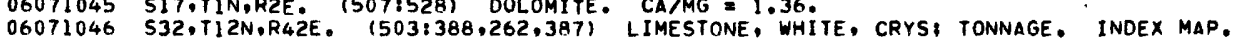

06071047 S19,TIS,R4W. (21:77) LIMESTONE, COARSELY CRYS. USE: 18.

06071048 S20:TIIN,RIW. (160:32,23-33,PL3-7) LIMESTONE, 8ROWN, GRANULAR, MINI TONNAGE. INOEX GEOL MAPS, MEAS SEC, GEOL SEC. USE: 16. AMISC: SRCO3=1.5.

USE 16. OMISCI SRCO3=1.5.

06071049 S5.TIN,R4W. (507:521) LIMESTONE. WHITE, CRYS. USE: 4.

06071050 S19,TIS,R4W. (21:77) LIMESTONE, COARSELY CRYS, USE 18.

06071051 CARBONIFEROUS, ORO GRANDE FM. S25,T6N,R4W. (52:169.PL1-3) LIMESTONE, GRAY TO WHITE, MED TO COARSELY CRYSI TONNAGE. INDEX \& GEOL MAPS. GEOL SEC. USE: 9,10 .

06071052 IISN,RISE, IVANPAH. (503:389.262) LIMESTONE, WHITE, GRAY, CRYS. INDEX MAP.

06071053 S4,T3N,R7W. (556:290,291,PL37) LIMESTONE, WHITE, CRYS, TONNAGE. INDEX MAP. USE: 4. CA/MG = 73.2 .

06071054 PRECAMBRIAN. ESSEX SER. S10,T3N,R16E. (507:521,518/556:284,283,PL37) (IMESTONE, COARSELY CRYS. INDEX MAP. USE 19,12.

06071055 54,T3M.R7W. (556:291,290,PL37) LIMESTONE, WHITE, CRYSI TONNAGE, INDEX MAP. CA/MG = 72.1.

06071056 PALEOZ01C, FURNACE LIMESTONE, S8,17,T3N,RIE. (550:268,270,PL36) LIMESTONE, WHITE: COMPOS SMPLI MIN. INDEX B GEOL

06071057 S27.28,33,T4N,R3W. (507:523.521) DOLOMITE, WHITE TO BROWN: 700 FT THICK, TONNAGE. CA/MG = 1.48 .

$06071058 \quad 517,20,21, T 6 N, R 14 E$. (507:518) OOLOMITE. CA/MG $=1.47$.

06071060 "T9N,RI4E" PROVIDENCE MTN. (309:317.316) DOLOMITE. INDEX \& GEOL MAP. CA/MG a 2.06.

06071061 TERTIARY. S22,T8N,R21E, (524:368,357-363,PL62-64) MAGNESITE, BROWN, FINE-GR, THIN-BEDDED, 1.9-FT CHANNEL SMPL,

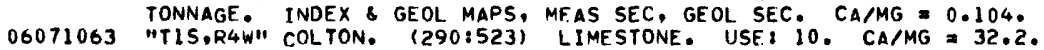

06071064 SI7,TGN,RI4E, (507:518) LIMESTONE, WHITE, FINE-GR 750 FT THICK, TONNAGE. CA/MG $>100$.

06071065 CARBONIFEROUS, ORO GRANDE FM. S15,T6N,R4W. $(56: 34,8,9,33$, PL 1, 2) LIMESTONE, GRAY, BANDEO, MED-CRYS, MASSIVE 3 250 FT THICK: SPOT SMPLI TONNAGE. INIEX \& GEOL MAP, GEOL SEC. USE: 4. CA/MG = 12.9.

06071066 PALEO7OIC, JURIPA SER, S19,30,T1S,R4W. (337:24/55:7,10/21:183) LIMESTONE. WHITE, BLUE-GRAY, VARIGATED, COARSELY

06071067 S20,21,T6N,RI4E. (507:518) DOLOMITE. CARMG $=1.54$.

06071068 PALEOZOIC. S17,20,21.T6N.RI4E. (408:175) DOLOMITE; TONNAGE. CA/MG 1.46 .

06071069 CARBONIFEROUS, ORO GRANDE FM. S15,T6N,R4W. $(56: 34,9,33$, PLI,2) LIMESTONE, GRAY, BANOED: SPOT SMPL; 250 FT THICK:

06071070 S4,T3N,R7W. (556:291,290,PL37) LIMESTONE, WHITE, CRYSI TONNAGE. INOEX MAP. CA/MG $=46.9$.

06071071 TERTIARY, S22,T8N,R21E. (524:368,357-363,PL62-65) MAGNESITE, BROWN, FINE-GR, THIN-BEDDEOI 3-FT CHANNEL SMPL, TONNAGE. TERTIARY S22,T8N,R21E. $(524: 368,357-363, P L 62-65)$
INDEX \& GEOL MAPS, MEAS SEC. GEOL SEC. CA MAG $=0.147$.

06071072 S15.T6N,RIW. (507:534.533) MAGNESITE, WHITE. CA/MG $=0.045$

06071074 PALEOZOIC. S21.T6N.R14E. (40811711 DOLOMITE, GRAY, FINELY CRYS TONNAGE. CAMGG = 1.38 .

06071075 CARBONIFEROUS, ORO GRANDE FM. S15,TGN,R4W. $156: 34,9,33, P L 1,2)$ LIMESTONE, GRAY, MED-CRYS SPOT SMPL, 250 FT THICK: TONNAGE. INDEX \& GEOL MAPS, GEOL SEC. USE: 4. CA/MG = 10.1 .

06071076 CARBONIFERDUS, ORO GRANDE FM. S15.T6N,R4W. $156,34,9,33$, PL 1,21

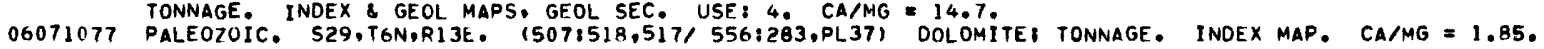

$0607107858,9, T 14 N, R 16 E$. (556:294,293,PL37) DOLOMITE, INOEX MAP, CA/MG 1.55.

$06071080 \quad 512,13,24, T 15 N, R 13 E$ \& S7,T15N,R14E. (507:521) LIMESTONE. CA/MG $\$ 100$

06071081 PALEOZOIC, FURNACE LS, "S14,T3N,RIE" (55:7,9) LIMESTONE, GRAY, WHITE, VARIEGATED, COARSELY CRYS. USE: 4,12. 06071082 CARBONIFEROUS, ORO GRANDE FM. S8,9,T6N,R4W. $(56: 35,8,9, P L 1,2)$ LIMESTONE. GRAY, MED-CRYS, MASSIVE TD BRECCIATEO.

06071083 "TIS.R2OE" CHUBAUCK, (558:333,315,332) DOLOMITE, WHITE, COARSELY CRYS: TONVAGE. INDEX MAP. USE: 18. CA/MG = 1.50.

06071084 "TIS,RZOE" CHUBBUCK, (558:333,315,332) LIMESTONE, WHITE, COARSELY CRYS, TONNAGE. INDEX MAP. USE, 18. CA/MG >100.

06071086 TERTIARY, S22,T8N,R21E, (524:368.357-363,PL62-64) MAGNESITE, BROWN, FINE-GR, THIN-8EDDED: 3.5-FT CHANNEL SMPL) TONNAGE. INDEX \& GEOL MAPS. MEAS SEC, GEOL SEC. CA/MG $=0.018$

06071087 TERTIARY, S22,T8N,R21E. (524:368,358,369,PL62-64) DOLOMITIC MAGNESITE, 1.2-FT CHANNEL SMPL, TONNAGE. INDEX \& GEOL

06071088 TERTIARY. S2?,TBN,R2IE. (524:368,358-363,PL62-64) MAGNESITE, BROWN, FINE-GR, THIN-BEDDED $2.5-F T$ CHANNEL SMPL, TONNAGE. INDEX \& GEOL MAPS. MEAS SEC. GEOL SEC. CA/MG $=0.024$.

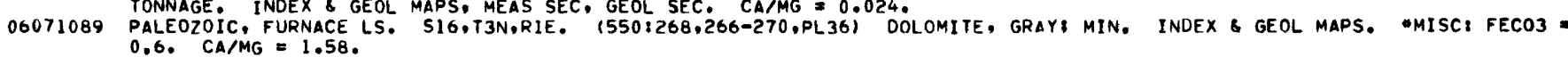

06071091 PALEOZOIC, FURNACE LS. S16,T3N,RIE. (556:299,300,PL37) DOLOMITE, GRAY MIN. INOEX MAP. \#MISCI FECO3=0.6. CA/MG=1.58.

06071092 CARBONIFEROUS, ORO GRANDE FM. S15,T6N,R4W. (56:34,9,PLI,2) LIMESTONE, GRAY, MED-GRI SPOT SMPL, TONNAGE. INOEX \& 06071093 TERTIARY. S15,T8N,R2IE, (524:368,357-363,PL62-64) MAGNESITE, BROWN, FINE-GR, THIN-BEDDED 2-FT CHANNEL SMPL TONNAGE, INDEX \& GEOL MAPS, MEAS SEC. GEOL. SEC. CA/MG $=0.019$.

06071094 CARBONIFEROUS, ORO GRANDE FM. S9,T6N,R4W, $\left(56: 33,9, \mathrm{PL}_{1,2)}\right.$ LIMESTONE, GRAY, MEDMCRYS. MASSIVE) TYPICAL SMPL. INDEX \&

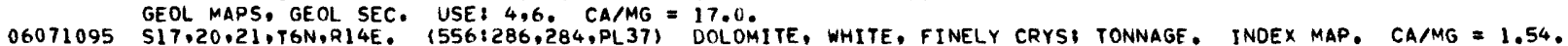

06071096 CARBONIFEROUS, ORO GRANDE FM, S15,T6N,R4W. (56:34,9,33,PL1,2) LIMESTONE, GRAY, MED-CRYS, MASSIVE, 250 FT THICK, TONNAGE. INDEX \& GEOL MAPS, GEOL SEC. USE: 4. CA/MG $=26.9$. 
TABLE 9. - Analyses of samples from California and Hawaii containing more than 90 percent carbonate (Group $\left.F_{2}\right)$, common-rock categoryContinued

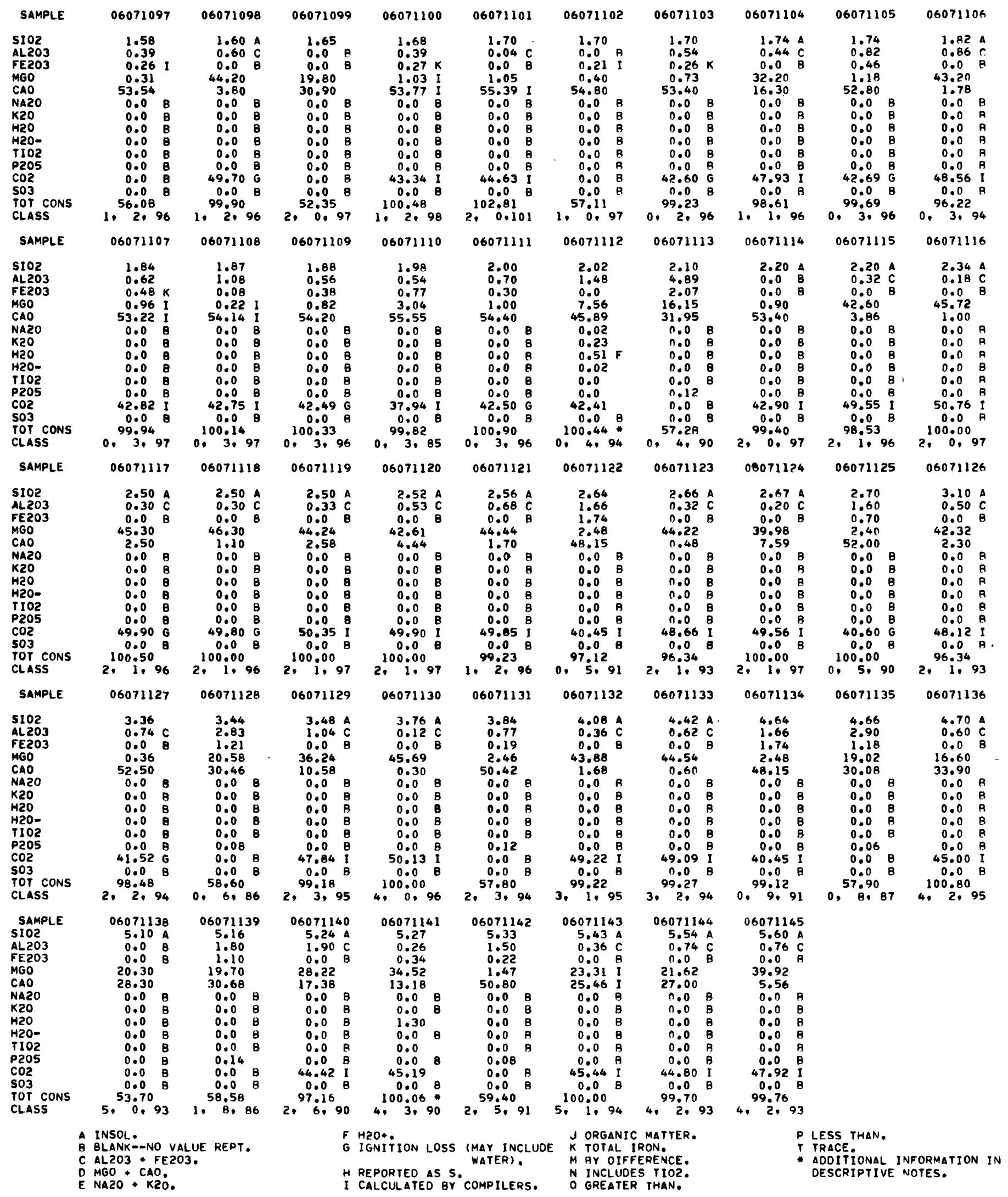


TABLE 9. - Analyses of samples from California and Hawaii containing more than 90 percent carbonate (Group $F_{2}$ ), common-rock category Continued

06071097 S17.20,21,T6N,R14E. (5561286,284,285,PL37) LIMESTONE, WHITE TO BROWN. MASSIVE, FINELY CRYSI TONNAGE. INDEX MAP. 06071098 TERTIARY, TEN,R2IE, HOMER SIDING. (524:371) MAGNESITE, WHITE, FINE-GR, POROUSI BEDS B FT THICK. INDEX \& GEOL MAPS.

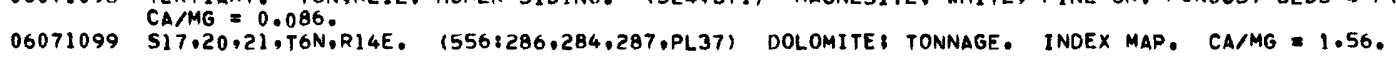
06071100 S4,T3N,R7W. (556:291.290,PL37) LIMESTONE, WHITE, CRYSI TONNAGE, INDEX MAP. CA/MG = 52.2.

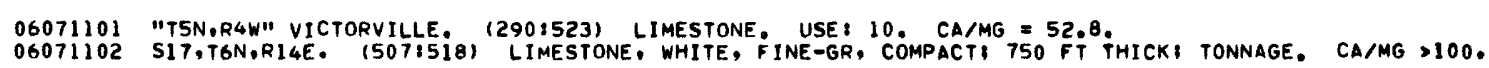
06071103 S4.T3N.R7W. (556:290.291.PL37) LIMESTONE, WHITE, CRYSI TONNAGE. INDEX MAP. USE: 4. CA/MG = 73.2.

06071104 TERTIARY, S22,T8N,R21E, (524:368,357-369,PL62-65) MAGNESITIC DOLOMITEI 1-FT CHANNEL SMPL TONNAGE. INDEX \& GEOL MAPS, MEAS SEC, GEOL SEC, CAIMG $=0.506$.
06071105 CARBONIFEROUS, ORO GRANDE FM. S13,T9S,R4W. (52:162,161.PL1-3) LIMESTONE, WHITE 365 FT THICK, SPOT SMPL, TONNAGE.
INDEX \& GEOL MAPS, GEOL SEC. CA/MG $=44.7$.

06071106 TERTIARY. S22,T8N,R2IE. $(524,368,357-363$, PL62-64) MAGNESITE, 8ROWN, FINE-GR, THIN-BEDDED 3.0-FT CHANNEL SMPL 06071107 S4.T3N,R7W. (556:291.290.PL37) LIMESTONE, WHITE, CRYS; TONNAGE. INDEX MAP. CA/MG 55.4 .

$0607110858,9, T 14 N, R 16 E$. (5561294,293,PL37) LIMESTONE. INDEX MAP. USE: 10,12 . CA/MG $>100$.

06071109 CARBONIFEROUS, ORO GRANDE FM. SI3.T9S,R4W. (52:162,161,PL1-3), LIMESTONE, WHITEI SPOT SMPLI 365 FT THICK TONNAGE. 06071110 CAMBRIAN. S26.T6N,R14E. (507:531.529) LIMESTONE, FINE-GR: BEDS 10 FT THICK. USE: 15 . CAMMG = 18.3.

06071111 CARBONIFEROUS, ORO GRANDE FM. S2,T6N,R6W: S35,T7W,R6W. $(521169,16,165$, PLI-3) LIMESTONE, GRAY, MASSIVE, TONNAGE. 06071112 TTN.R2W, BARSTOW. (412:366.365) LIMESTONE, GRAY, CRYS. MISC: FEO =0.30, CR203 = 0.00. CA/MG = 6.07.

06071113 CARBONIFEROUS, ORO GRANDE FM, S15,T6N,R4W. $(56: 35,8-10, P L 1,2)$ DOLOMITE, WHITE, MEO TO COARSELY CRYS, MASSIVEI

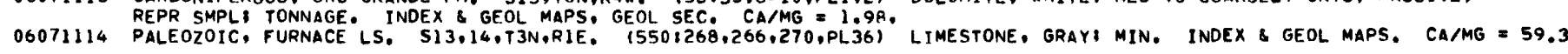

06071115 TERTIARY, S2?,T8N,R21E, (524:368,357-363,PL62-64) MAGNESITE, BROWN, FINE-GR, THIN-BEDOED 1,7-FT CHANNEL SMPL, TONNAGE. INDEX \& GEOL MAPS. MEAS SEC. GEOL SEC. CAMMG $=0.091$.

06071116 TERTIARY. S22.T8N,R21E. 1524:368.357-363,369,PL62-64) MAGNESITE, WHITE, THIN-BEDDEDI 4-FT CHANNEL SMPL, TONNAGE.

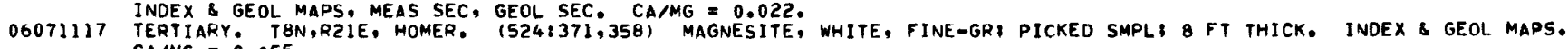
06071118 TERTIARY T8N,R21E. HOMER, $(524: 371.358)$ MAGNESITE, WHITE, FINF - GRI PICKED SMPL B FT THICK. INDEX \& GEOL MAPS.

06071119 TERTIARY, S2?,T8N,R21E. (524:368,357-363,PL62-64) MAGNESITE, BROWN, FINE-GR. THIN-BEDOED: 1.7-FT CHANNEL SMPL, 06071120 TONNAGE, INDEX \& GEOL MAPS, MEAS SEC, GEOL SEC. CAIMG $=0.058$.

06071120 TERTIARY. S22,T8N,R21E. (524:368.357-363,PL62-64) MAGNESITE, BROWN, FINE-GR, THIN-AEDOEDI I.3-FT CHANNEL SMPLI 06071121 TONNAGE. INDEX 8 GEOL MAPS, MEAS SEC, GEOL SEC. CAIMG = 0.104.

06071121 TERTIARY, S15,TBN,R21E, (524:368,357-363,PL62-64) MAGNESITE, BROWN, FINE-GR, THIN-AEDDEDI 2.3-FT CHANNEL SMPLI TONNAGE. INDEX \& GEOL MAPS, MEAS SEC, GEOL SEC. CA/MG 0.038 ,
06071122 CAMBRIAN. S2,11,TSN,RI4E. (556:297.298,PL37) LIMESTONE, VARIGATEDI BEOS 20 FT THICK. INDEX MAP. USE:15. CA/MG=19.4. 06071123 TERTIARY. S22,T8N,R21E. (524:368.357-363,PL62-64) MAGNESITE, WHITE, FINE-GR. THIN-BEDDEOI 2.0-FT CHANNEL SMPLI

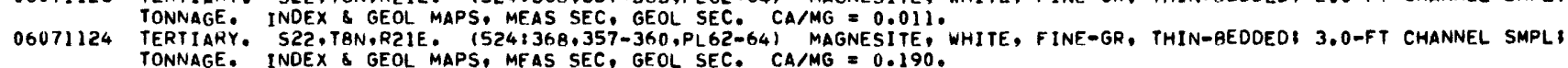
06071125 CARBONIFEROUS, ORO GRANDE FM. S2,11,14-16,T6N,R4W. (552:177,PL1/ 52:167,165,PL1-3) LIMESTONE, GRAYI MED-GR, CRYS. 06071126 TERTIARY, S15,TBN,R21E. (524:368,357-363,PL62-64) MAGNESITE, BROWN, FINE-GR, THIN-BEDDEOI 3.5-FT CHANNEL SMPL: TONNAGE. INDEX \& GEOL MAPS, MEAS SEC, GEOL SEC, CA/MG $=0.054$.
06071127 CARBONIFEROUS, ORO GRANDE FM. S2,TGN,R6W. (556:280,PL37) LIMESTONE. GRAY TO WHITE. FINE TO MED CRYS TONNAGE. INDEX 06071128 CARBONIFEROUS, ORO GRANDE FM. S16.T6N,R4W. $156: 35,9, P L 1,21$ DOLOMITE, WHITE, MED-CRYS, 200 FT THICK. INDEX \& GEOL 06071128 CARBONIFEROUS. ORO GRANDE FM.

06071129 TERTIARY, S22,T8N,R21E. $1524: 368,357-363, P L 62-64)$ MAGNESITE, 8ROWN, FINE-GR, THIN-AEDDED: $2.5-F T$ CHANNEL SMPL: TONNAGE. INDEX \& GEOL MAPS. MEAS SEC. GEOL SEC. CAMG $=0.292$.

06071130 TERTIARY, S22,T8N,R21E, (524:368,357-363,PL62-64) MAGNESITE, 8ROWN, FINE-GR, THIN-BEDOED: 2.7-FT CHANNEL SMPL,

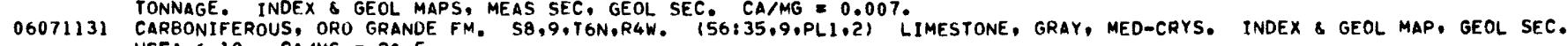
06071132 TERTIARY. SI5,T8N,R21E. (524:368,357-363.PL62-64) MAGNESITE, BROWN, FINE-GR, THIN-BEDOEDI 1.7-FT CHANNEL SMPL,

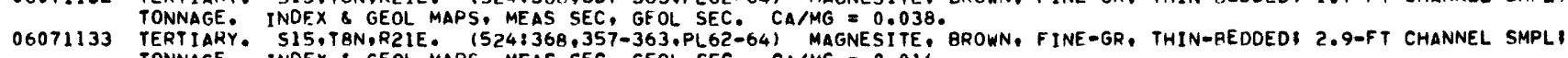
06071133 TERTIARY. S15,TBN,R21E. $(524: 368,357-363$, PL62-64) MAGNESITE,
06071134 TONNAGE. INDEX 8 GEOL MAPS, MEAS SEC, GEOL SEC. CAIMG E 0.014. 06071134 CAMBRIAN. SII.TSN.RI4E. (507:529.53i) LIMFSTONE, VARIEGATED. USE: 15. CAMG = 19.4.

06071135 CARBONIFEROUS, ORO GRANDE FM. S16,T6N,R4W. (56:35,9,PL1,2) DOLOMITE, WHITE, MED-CRYS: 200 FT THICK, INDEX \& GEOL 06071136 PALEOZOIC, FURNACE LS. S8,T3N,RIE. (550:268-270,PL36) DOLOMITE, WHITE, FINE-GR, MIN. INDEX \& GEOL MAPS. CA/MG=2.04. 06071138 TERTIARY, "TIIN,RGE" AFTON. $(436: 118,117)$ MAGNESITE, WHITE: LUMP SMPL; TONNAGE. INDEX MAP. CA/MG = 1.39.

06071139 CARBONIFEROUS, ORO GRANDE FM. S10,15,T6N,R4W. (56:35,9.PLI.2) DOLOMITE, WHITE, MED TO COARSELY CRYS. MASSIVE TONNAGE. INDEX \& GEOL. MAPS, GEOL SEC. CAMMG $=1.56$.

06071140 TERTIARY. S22,TBN,R21E. (524:368,357-360,PL62-64) DOLOMITE; 3.4-FT CHANNEL SMPLI TONNAGE. INDEX \& GEOL MAP. MEAS SEC, 06071141 TERTIARY. S22,T8N,R21E. (5241364,357-360,PL62-64) DOLOMITIC MAGNESITE, WHITE, THIN-BEDDEDI 4.90 FT THICK, TONNAGE.

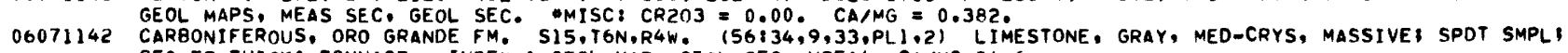
06071143250 FT THICK TONNAGE, INDEX \& GF OL MAP, GEOL SEC; USE: 4. CA/MG 34.6.

06071143 TERTIARY, S22,TBN,R2IE. (524:368,357-360.PL62-64) DOLOMITE: 3.2-FT CHANNEL SMPLI TONNAGE. INDEX \& GEOL MAP. MEAS SEC, 06071144 GEOL SEC. CA/MG = 1.09.

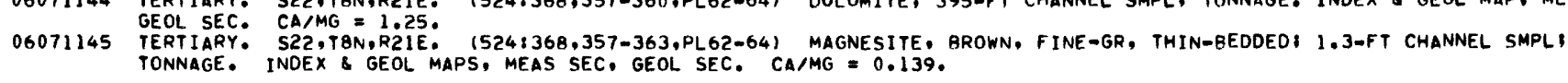


TABLE 9. - Analyses of samples from California and Hawaii containing more than 90 percent carbonate (Group $\left.F_{2}\right)$, common-rock category Continued

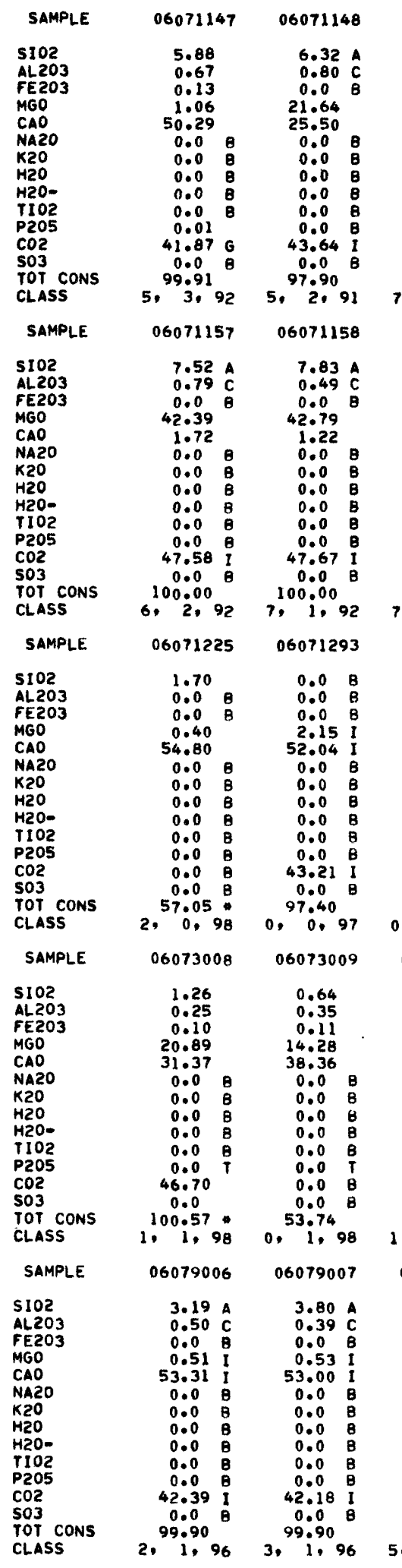

A INSOL.

C AL203 $\mathrm{FE2O3}$.

E NAZO
06071149

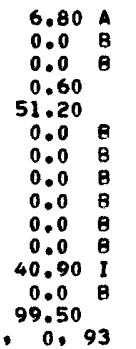

06071159

$7.9 A$
0.70

0.0 B

37.68

0.0 B

$\begin{array}{ll}0.0 & B \\ 0.0 & B\end{array}$

$\begin{array}{ll}0.0 & \text { B } \\ 0.0 & B\end{array}$

0.0 B

$\begin{array}{cc}0.0 & R \\ 46.35 & I\end{array}$

46.35 I

99.35

06071377

0.33 A

$0.13 \mathrm{C}$

1.031
54

54.441

0.0 B

0.0 B

0.0 B

0.0 B

0.0 B

44.171
0.0

100,38

$0,0,100$

06073010

1.51
0.39

0.39

6.44
47.16

$0.0 \mathrm{~B}$

0.0 B

$\begin{array}{ll}0.0 & B \\ 0.0 & 8\end{array}$

0.0 B

0.02

0.0 B

0.0
55.69

1. 2,98

06079008

\subsection{7}

$\begin{array}{ll}0.0 & 8 \\ 0.0 & 8\end{array}$

$\begin{array}{ccc}0.0 & B\end{array}$

51.16 I

$\begin{array}{ll}0.0 & 8 \\ 0.0 & B\end{array}$

0.08

$0.0 \quad B$

0.0 B

40.161

9.08

5. 0.91

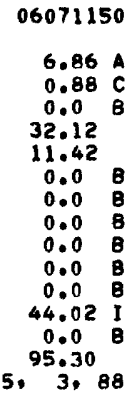

06071151

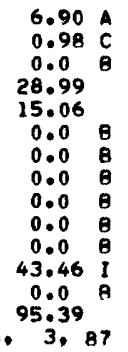

06071160

$\begin{array}{ll}8.62 & A \\ 0.80 & C \\ 0.0 & B \\ 31.86 & \end{array}$

13.86

13.008
0.08

$\begin{array}{ll}0.0 & 8 \\ 0.0 & \mathrm{~B}\end{array}$

$\begin{array}{ll}0.0 & 8 \\ 0.0 & 8\end{array}$

$\begin{array}{ll}0.0 & 8 \\ 0.0 & 8\end{array}$

0.0
44.98
0.98

0.08

7. 2,2690

06073001

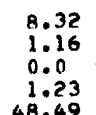

1.23
48.49

$0.0 \quad 8$

0.08

0.000

0.08

0.02

$0.0 \quad B$

$\begin{array}{cc}0.0 & 8 \\ 59.50 & \end{array}$

6. 4.89

06073011

1.86

$\begin{array}{ll}1.10 \\ 0.0 & 8\end{array}$

0.57 I

$1.15 \mathrm{E}$

0.0 B

$\begin{array}{ll}0.0 & 8 \\ 0.0 & 8\end{array}$

0.0 B

$\begin{array}{cc}0.0 & 8 \\ 42.08 & 1\end{array}$

$\begin{array}{cc}42.08 & 1 \\ 0.0 & B\end{array}$

9.008

$0,3,95$

06079009

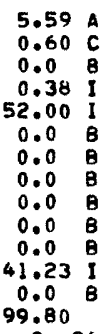

$\begin{array}{cc}5.59 & A \\ 0.60 & C \\ 0.0 & 8 \\ 0.38 & 1 \\ 52.00 & 1 \\ 0.0 & B \\ 0.0 & 8 \\ 0.0 & 8 \\ 0.0 & B \\ 0.0 & B \\ 0.0 & B \\ 41.23 & 1 \\ 0.0 & B \\ 99.80 & \end{array}$

06071212

0.0 B

0.0 B

55.901

$\begin{array}{ccc}5.0 & B \\ 0 & \end{array}$

$\begin{array}{ll}0.0 & 8 \\ 0.0 & B\end{array}$

$\begin{array}{lll}0.0 & B \\ 0.0 & B\end{array}$

$\begin{array}{ll}0.0 & 8 \\ 0.0 & 8\end{array}$

43.90 I

0.0
99.80

06073002

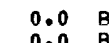

0.0
0.0

54.0

0.08

0.0 B

$0.0 \quad B$

0.0 B

0.0 B

$0.0 \quad B$

0.0
54.44

0. 0,97

06073012

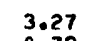

$$
\begin{array}{r}
3.27 \\
0.79 \\
0.19 \\
7.35
\end{array}
$$

46.51

0.08

$0.0 \quad 8$

0.0 B

0.0 B

0.0 B

0.0
58.40

2. 38.40

06079013

$6.00 \mathrm{~A}$

$\begin{array}{lll}0.0 & B \\ 0.0 & B\end{array}$

18.70

29.30

$0.0 \quad 8$

0.0 B

$0.0 \quad \mathrm{~B}$

0.0

0.10
43.95

$0.0 \mathrm{~B}$
$0 . \quad 0.100$

$6, \quad 0,92$
06071152
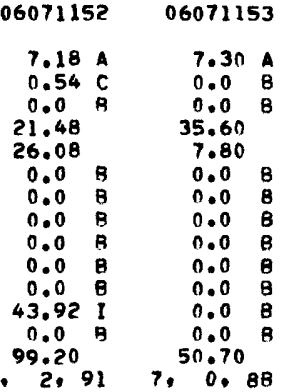

06071213

$\begin{array}{ll}0.0 & B \\ 0.0 & B \\ 0.0 & B\end{array}$

6.42

0.06

$\begin{array}{ll}0.0 & 8 \\ 0.0 & 8\end{array}$

0.0 B

$\begin{array}{ll}0.0 & B \\ 0.0 & B\end{array}$

$0.0 \quad B$

0.08

50.0

$0, \quad 0.98$

06073003

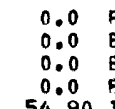

54.901

$0.0 \quad 8$

0.0 B

0.0

$0.0 \quad 8$

$\begin{array}{ll}0.0 & B \\ 0.0 & B\end{array}$

43.10 I

0.0
98.00

0 .

06073013
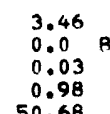

0.98
50.68

$0.0 \mathrm{~B}$

0.0 B

0.0 B

0.0 B

$0.0 \quad B$

$0.0 \mathrm{~B}$

55.15

3. 0.93

06079014

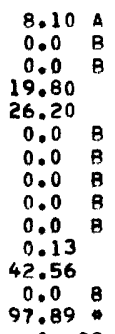

97.89
06071214

0.20

0.0

0.10

$\begin{array}{cc}55.77 & 1 \\ 0.0 & A\end{array}$

$\begin{array}{ll}0.0 & \text { B } \\ 0.0 & B\end{array}$

$0.0 \quad B$

$0.0 \quad B$

0.0

43.791
0.0

100.06

$0, \quad 0.100$

06073004

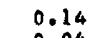

0.04
0.04

0.33
55.18

0.0

$0.0 \quad B$

$\begin{array}{ll}0.0 & B \\ 0.0 & B\end{array}$

$\begin{array}{ll}0.0 & B \\ 0.0 & B\end{array}$

0.0
56.24

0 . 0,98

06075001

0.09 A

0.11

18.65
35.00

18.05
0.0

$0.0 \quad 8$

$\begin{array}{ll}0.0 & B \\ 0.0 & B\end{array}$

0.0 B

$0.0 \quad B$

0.0
46.77

0.0

100.79

06079015

$3.00 \mathrm{~A}$

$\begin{array}{lll}0.0 & B \\ 0.0 & 8\end{array}$

0.0
20.40

30.00

$\begin{array}{ll}0.0 & B \\ 0.0 & 8\end{array}$

0.08

$0.0 \quad B$

0.06

$0.0 \quad 8$

0.0
53.61

3. 0.96
0.0 B

06071154

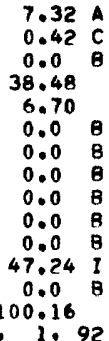

06071215

0.30

0.0
0.40

0.30

55.351

$0.0 \mathrm{~B}$

$\begin{array}{ll}0.0 & B \\ 0.0 & B\end{array}$

0.0 B

$0.0 \mathrm{~B}$

0.0
43.45

43.45
0.0

$0,99,80$

06073005

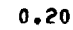

0.20
0.05

0.05

0.27
55.42

$0.0 B$

$0.0 \quad \mathrm{~B}$

0.0 B

0.0 B

0.0

$0.0 \mathrm{~B}$

0.0 B

56.13.

06079001

$0.0 \quad B$

0.0 B

$\begin{array}{ll}0.0 & B \\ 0.68 & 1\end{array}$

0.68
52.58

0.0 B

0.0 B

0.0 B

0.08

0.011

42.17 I

0.0 B

$0,99.53$

06081001

\section{0}

0.16

0.0
54.16

$\begin{array}{cc}54.16 & 1 \\ 0.0 & B\end{array}$

$0.0 \quad B$

$\begin{array}{ll}0.0 & 8 \\ 0.0 & B\end{array}$

$0.0 \quad B$

42.531 
TABLE 9. - Analvses of samples from California and Hawaii containing more than 90 percent carbonate (Group $\left.F_{2}\right)$, common-rock category Continued

06071147 CARBONJFEROUS, ORO GRANDE FM. "TGN,R5W" ORO GRANDE, (55:7.A) LIMESTONE, GRAY, MED-GR, CRYS. USE: 4 , CA/MG = 47.4.

06071148 TERTIARY, S22,T8N,R21E. (524:368,357-360,PL62-64) DOLOMITEI 4.0-FT CHANNEL SMPL: TONNAGE. INDEX GEOL MAP, MEAS SEC, 06071149 PALEOZOIC, FURNACE LS. SI4,T3N,RIE. (550:268,266-270,PL36) LIMFSTONE, COMPOS SMPL, MIN. INDEX B GEOL MAP.

06071150 TERTIARY, SI5,T8N,R21E. (524:368,357-363,PL62-64) MAGNESITE, BROWN, FINE-GR, THIN-REDDEO 2.3-FT CHANNEL SMPL TONNAGE. INDEX 8 GEOL MAPS, MEAS SEC, GEOL SEC. CA/MG $=0.356$.

06071151 TERTIARY. S22,T8N,R2IE. (524:368,357-360.PL62-64) DOLOMITE: 0.R-FT CHANNEL SMPL TONNAGE. INDEX \& GEOL MAP, MEAS SEC, 06071152 TERTIARY. S22,T8N,R21E. (524:368,357-360,PL62-64) DOLOMITE: 4.5-FT CHANNEL SMPL, TONNAGE. INDEX \&. GEOL MAP, MEAS SEC, 06071153 TERTIARY, "TIIN,RGE" AFTON, (436:118,117) MAGNESITE, WHITE: CHANNEL SMPL: 1.0 FT THICK: TONNAGE. CA/MG = 0.219.

06071154 TERTIARY, S22,T8N,R21E, (524:36R,357-363,PL62-64) MAGNESITE, BROWN, FINE-GR, THIN-PEDOED, 5.1-FT CHANNEL SMPL,

06071154 TERTIARY, S22,T8N,R21E. (524:36R,357-363,PL62-64) MAGNESITE,

06071155 TERTIARY, S22,T8N,R21E. (524:368,357-363,PL62-64) MAGNESITE, BROWN, FINE-GR, THIN-BEDOEDI 3,9-FT CHANNEL SMPL,

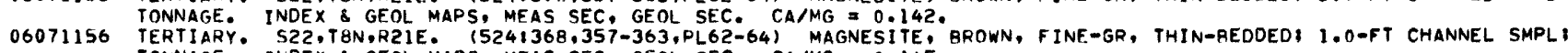

06071157 TERTIARY, S22,T8N,R21E. (524:368,357-363,PL62-64) MAGNESITE, BROWN, FINE-GR, THIN-AEDDED, $2.8-F T$ CHANNEL SMPL, 06071158 TONNAGE. INDEX \& GEOL MAPS, MEAS SEC, GEOL SEC. CAIMG $=0.041$.

TERTIARY. S22,TBN,R2IE. (524:368,357-363,PL62-64) MAGNESITE, BROWN, FINE-GR, THIN-AEDOED 3.5-FT CHANNEL SMPL 06071159 TONNAGE. INDEX \& GEOL MAPS. MEAS SEC, GEOL SEC. CATMG $=0.028$.

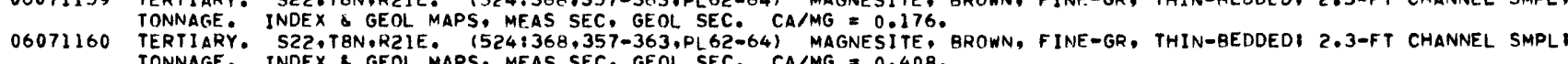

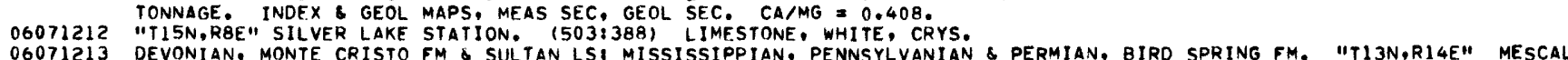
RANGE. (231:7) LIMESTONE; AVG OF 20 SUPFACE SMPLS OF $600 \mathrm{FT}$. USE: 4. CA/MG $=7.40$

06071214 "T3N,R6W" PINE LODGE. (290:523) LIMESTONE. USE: 10. CA/MG $>100$.

06071215 "T3N,R6W" PINE LODGE. (290:523) LIMESTONE. USE: 10: CA/MG >100.

06071216 "T3N,R6W" PINE LODGE. (2908523) LIMESTONE. USE: 10: CAMG >100

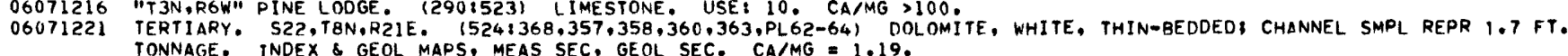

06071225 S17.20,T6N,R14E. (507:518) LIMESTONE, \$ISC: FEO $=0.15$. CA/MG $>100$.

06071293 "T1S,R5W" COLTON. (172:169) MARBLE. CA/MG $=24.2$.

06071377 "TIS,R4W" COLTON. (6B:660) LIMESTONE. CA/MG $=41.6$.

06073001 S27,T175,RIE. (118:180,13,179,PLI) LIMESTONE, CREAM-COLORED, POROUS, POORLY INDURATEDI 1-8 FT THICK IONNAGE.

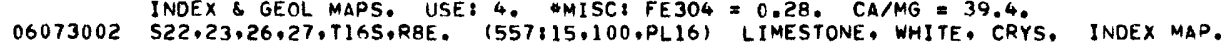

06073003 CARBONIFEROUS, S22,T165,RBE, (556:301,300,PL37) LIMESTONE, WHITE, COARSELY CRYS BFDS 20-100 FT THICK. INDEX MAP.

06073004 S10.T11S,R4E. (118:181,180,PL1) LIMESTONE, WHITE TO GRAY, OENSE, COARSELY CRYSI 25-30 FT THICK. INDEX \& GEOL MAPS. 06073005 SIO,TI1S,R4E. (118,1B1,180,PLI) LIMESTONE, WHITE. GRAY, DENSE, COARSELY CRYSI 30 FT THICK. INDEX S GEOL MAPS.

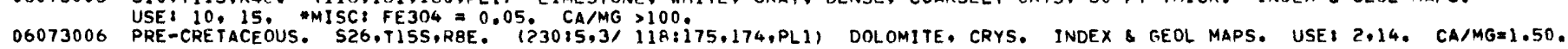

06073007 "T17S,R7E" LIVE OAK SPRINGS. $(535: 77,81)$ DOLOMITE, CA/MG $=1.50$.

06073008 CARBONIFEROUS. S26,TISS,R8E. $(556: 301,300, P L 37)$ DOLOMITE. INDEX MAP. $\$ M I S C$, SIOL $=0.43$, INSOL $=0.83$. CA/MG 1.50 .

06073009 TRIASSIC DR JURASSIC. SI1.TI8S,R8E, (230:5.3/ 118:185,181,PL1) MAGNESIAN LIMESTONE, WHITE, GRAY, BANDED, COARSELY

06073010 S10,TIIS,R INDEX (118:181,180,PL1) LIMESTONE, WHITE, DENSE, COARSELY CRYS: $25-30$ FT THICK. INDEX \& GEOL MAP. USE $110,15$. 06073011 CARBONIFEROUS. "T17S,RIE" JAMUL. (556:302,300,PL37/ 1731117,116,PL4) LIMESTONE, CHALKLIKE, FINE-GR. INDEX MAP.

06073012 CRETACEOUS, S23,T16S,R8E. (118:177,175,176,PLI) MAGNESIAN LIMESTONE, CREAU, FINE- TO MEO-GR, THIN- TO THICK-BEODED. INDEX \& GEOL MAPS. USE: 4,15 . CA/MG $=6.33$

06073013 PRE-CRETACEOUS. S27,T16S,R8E: $(230: 5,3,118: 178,176,177$, PLI) LIMESTONE, CRYS. MASSIVE: AVG OF 4 SMPL. INDEX MAP.

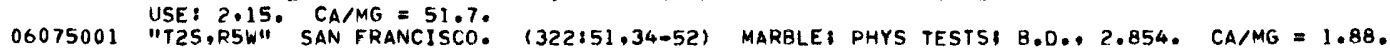

06079001 HOLOCENE. S9,16,T31S,R15E. $(21: 112,111)$ MARBLE, WHITE, MASSIVE: 15 FT THICK. \&MISC: FECO3 = 3.93, MNCO3 = 0.06.

06079002 S9,16,T31S,RI5E. (73:641) ONYX: 22 IN THICK, USE:15. $M$ ISC: MNO $=0.04$, FED $=2.441$ CALCULATED BY COMPILERS.

06079003 SA/MG $=90.8$. 30 . 1313 . $(3411513)$ DOLOMITE, WHITE, CA/MG $=1.55$

06079003
06079006
MIOCENE, SANTA MARGARITA FM. S9,10,15,T26S,R9E. (556:305.304,PL37) LIMESTONE, BUFF, FOS TONNAGE. INDEX MAP.

06079007 MSE: 6,18. CAMMG >100. SANTA MARGARITA FM. S9,10,15,1265,R9E. (556:305,304,PL37) LIMESTONE, BUFF, FOSI TONNAGE. INDEX MAP.

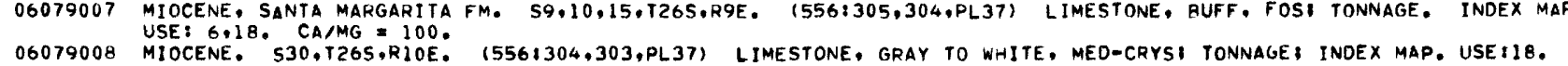

06079009 MIOCENE. S30,T265,R10E. (556:304,303,PL37) LIMESTONE, GRAY TO WHITE, MEDMCRYS: TONNAGE INDEX MAP. USE:1R. CA/MG>100.

06079013 MIOCENE, MONTEREY SH, SANDHOLDT M8R. "T25S,R9E" NACIMIENTO DAM, (386:C6) DOLOMITE. \#MISC: FEO = 0.02, MNO = 0.00. 06079014 MIOCENE, MONTEREY SH. "TIOS,R27W" TEMBLOR RANGE. (386:C6) DOLOMITE. "MISC: FEO = 1.0 . MNO = 0.05 , SRO = 0.054.

06079015 MIOCENE, MONTEREY SH. "T315,14E" PISMO SYNCLINE AREA. (386:C6) DOLOMITE. MISC: FEO = 0.11,MNO = 0.02, SRO = 0.025. CA/MG $=1.47$

06081001 HOLOCENE." "T6S,RIW" ALVISO, $(175: 360,556: 308)$ SHELL LIMESTONE, TONNAGE. INDEX MAP. USE: $2,4$.

06081002 HOLOCENE. "TGS,RIW" ALVISO. (556:308,307,PL37) SHELL LIMESTONE: TONNAGE. INDEX MAP. USE, 2,4.

06081003 HOLOCENE. "TGS,RIW" ALVISO. (556:308,307,PL37) SHELL LIMESTONE, TONNAGE. INDEX MAP. USE: 2,4 . 
TABLE 9. - Analyses of samples from California and Hawaii containing more than 90 percent carbonate (Group $F_{2}$ ), common-rock category Continued

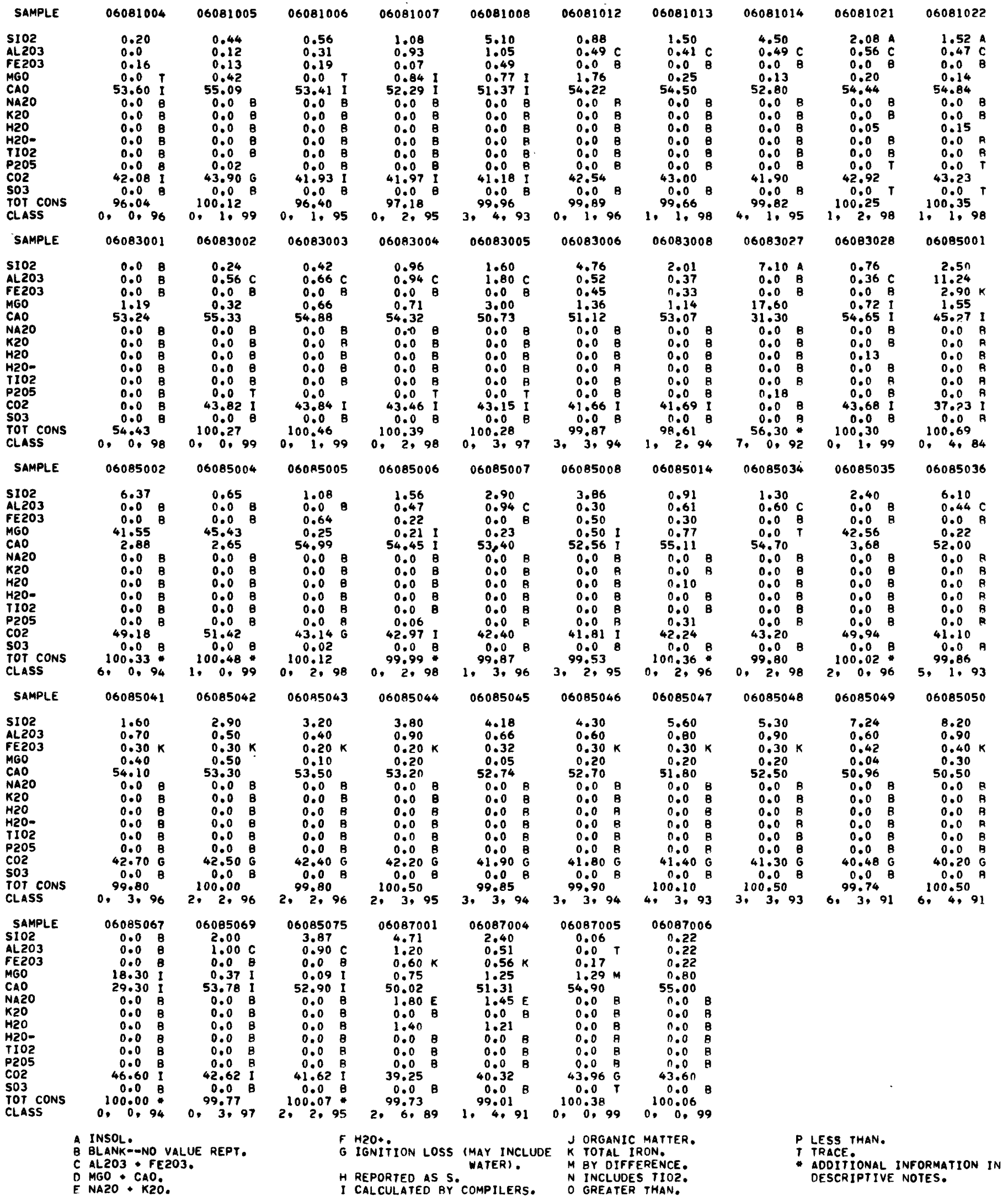


TABLE 9. - Analyses of samples from California and Hawaii containing more than 90 percent carbonate (Group $F_{2}$ ), common-rock category Continued

06081004 HOLOCENE, "T4S,R4W" MILBRAE TO ALVISO. (175:360/556:308,307,PL37) SHELL LIMESTONEI TONNAGE. INDEX MAP. USE: $2,4$.

06081005 PLEISTOCENE. "T5S,R3W" REDWOOD CITY. (55:6,2) SHELLS; 5-20 FT THICK: TONNAGE. USE: 4. CA/MG >100.

06081006 HOLOCENE, S11,12,T45,R4W (175:360/556:308,307,PL37) SHELL LIMESTONE: AVG OF 10 ANAL, TONNAGE. INDEX MAP.

06081007 HOLOCENE. "T4S,R4W" MILLBRAE TO ALVISO, (175:360/5561308,307,PL37) SHELL LIMESTONE) TONNAGE. INDEX MAP. USE: $2,4$. 06081008 JURASSIC 8 CRETACEOUS, FRANCISCAN FM. S11,T4S,R6W. (556:308,PL37/ 139835,433,PL5) LIMESTONE, GRAY, OENSE. INDEX 4 06081008 JURASSIC \& CRETACEOUS, FRANCISCAN FM. SII,T4S,R6W. (556:308,PL37/ 1398435,433,PL5) LIMESTONE, GRAY, DENSE. INDEX 06081012 JURASSIC \& CRETACEOUS, FRANCISCAN FM. "T4S,R6W" CALERA. (175:358) LIMESTONE QUARRY SMPLI TONNAGE. CAMMG = 30.8. 06081013 JURASSIC \& CRETACEOUS, FRANCISCAN FM. "T5S,R4W" CRYSTAL SPRINGS LAKE. (175:358,354) LIMESTONE8 OUARRY SMPLI TONNAGE. 06081014 JURASSIC 8 CRETACEOUS, FRANCISCAN FM. "T4S,R6W" CALERA. (175:358) LIMESTONE! TONNAGE. CAMMG >1O0.

06081021 JURASSIC \& CRETACEOUS, FRANCISCAN FM. T4S,R4W. PERMANTE CANYON. $(57515 / 29176,11,12,14,68-70)$ LIMESTONE, GRAY, COMPACT, STRATIFIED, FOS: CONTAINS CHERTI 60 FT THICK, INDEX \& GEOL MAPS, MEAS SEC. USE: 6.4. CA/MG $>100$.

06081022 JURASSIC \& CRETACEOUS, FRANCISCAN FM. T4S,R4W, PERMANTE CANYON. $1575: 5 / 29: 76,11,12,14,68-70)$ LIMESTONE, GRAY, COMPACT, STRATIFIED, FOS: CONTAINS CHERTI 60 FT THICK. INDEX \& GEOL MAPS, MEAS SEC. USE: 6.4. CA/MG >100.

06083001 EOCENE, SIERRA BLANCA FM. "T7,8N,R25,26W" INDIAN CREEK, (23014,3) LIMESTONE, ALGAL 250 FT THICK AVG OF 6 SMPL: 06083002 EOCENE, SIERRA BLANCA IS, "T7,8N,R25,26W" INDIAN CREEK, (312:70,62,66-68) LIMESTONE) BUFF, WHITE, DENSE, FINE-GR, FOS. INDEX \& GEOL MAPS, MEAS SEC. GEOL SEC. CA/MG >100.

06083003 EOCENE, SIERRA BLANCA LS. "T7,BN,R25,26W" INDIAN CREEK, $(312170,62,66-68,78)$ LIMESTONE, BUFF TO WHITE, FINE-GR, FOS. INDEX GEOL MAPS, MEAS SEC, GEOL SEC, CA/MG $=83.2$. 06083004 EOCENE, SIERRA BLANCA LS. "T7,8N,R25,26W" INDIAN CREEK, $(312170,62,66-68)$ LIMESTONE, BUFF TO WHITE, FINE-GR, FOS 06083005 EOCENE, SIERRA BLANCA LS. "TT,BN,R,25, C6W"INOIAN CREEK, (312:70.62,66-68) LIMESTONE, BUFF TO WHITE, FINE-GR, FOS. INDEX : GEOL MAPS, MEAS SEC, GEOL SEC. CAMG $=16.9$.

06083006 EOCENE, SIERRA BLANCA LS, "TGN,R27W" SANTA BARBARA." (52515,4,PL1) LIMESTONE, WHITE TO GRAY, FINELY CRYS, FOS, 35-40 FT CHANNEL SMPL, BEOS 4 IN - 5 FT THICK: MIN. INDEX \& GEOL MAPS. CA/MG $=37.6$.

06083008 EOCENE, SIERRA BLANCA LS. "TGN,R27W" BIG PINE MTN RD. (525:5,4,PL1) LIMESTONE, WHITE TO GRAY, FOS, CRYSI COMPOS GRAB SMPLI MINI 70-80 FT THICK. INDEX \& GEOL MAPS. CAIMG $=46.6$.

06083027 MIOCENE. "TBN,R3IW" SANTA YNEZ RIVER AREA. (386:C6) REEF ROCK, 40 FT THICK. "MISC: FEO = 0.07 , MNO = 0.03 ,

06083028 "TION,R3SW" BETTERAVIA. $168: 660)$. LIMESTONE, CA/MG $=76.0$

06085001 "TOS,RIE" EDENVALE. (299:185,186) MARL, FOS, BEDS 200 FT THICK, USE: 2. CAMG = 29.2.

06085002 "T9S,R3E" MADRONE, (5531151/58:15,16,79,PLI) MAGNESITE, BUFF. TNDEX MAP. "MISCI FEO = 0.35. CA/MG = 0.069.

06085004

"TOS,R3E" MADRONE, (553:LSI) MAGNESITE. "MISCI FEO $=0.33$. CA/MG $=0.058$.

06085005

06085006

06085007

JURASSIC \& CRETACEOUS, FRANCISCAN FM. S14,T75,R3W. (199:9, 5568312, PL37) LIMESTONE. INDEX MAP. CA/MG >1O0.

06085008

06085014

JURASSIC \& CRETACEOUS, FRANCISCAN FM. S13,T7S,R3W. (2991185/29:76,11,68,PL-1) LIMESIONE, BLUE TO BLACK, FINE-GR. INDEX \& GEOL MAPS. USE: 4.6 . CA/MG >100, MISC: MN304 $=0.05$

JURASSIC \& CRETACEOUS, FRANCISCAN FM. "T9S,RI,2E" LONGWALL CANYON. (29:76,11,69,72,PL1) OOLITIC LIMESTONE, FOS: BEDS 50 FT THICK. INDEX \& GEOL MAPS. USE: 4,7. MMISCI MNO $=0.01$. CA/MG $=71.6$.

06085034 JURASSIC \& CRETACEOUS, FRANCISCAN FM. "T9S,RIW" LYNDON SWITCH. (175:358,356) LIMESTONE, BEDDEDI TONNAGE.

06085035 "T9S.R3E" MORGAN HILL. 1553:151) MAGNESITE. MISC: FEO $=1.44$. CA/MG 0.086 .

06085036 JURASSIC \& CRETACEOUS, FRANCISCAN FM. "TION,R35W" GUADALUPE. (175:358) LIMESTONE: TONNAGE. CA/MG >IO0.

06085041 JURASSIC \& CRETACEOUS, FRANCISCAN FM. S17,18,T75,R2W. (331:376,374) LIMFSTONEI TONNAGE 1 SCREEN SIZE, \&6 IN: PHYS

06085042

TESTS, USE: 4 , CA M $>100$.

TESTS. USE: 4 , CATMG $>100$.

TESTSO 8 CRETACEOUS, FRANCIS

06085043 JURASSIC \& CRETACEOUS, FRANCISCAN FM. S17.18,T75,R2W.

TESTS USE: 4 . CAMG $>100$.
JURASSIC \& CRETACEOUS. FRANCISCAN FM. S17,18,TTS,R2W.

06085044 JURASSIC 8 CRETACEOUS, FRANCISCAN FM. S17,18,TTS,R2W.

06085045 JURASSIC \& CRETACEOUS, FRANCISCAN FM. S17.18.T7S,R2W. USE: 4. CA/MG $>100$.

06085046 JURASSIC \& CRETACEOUS, FRANCISCAN FM. S17,18,T75,RZW. TESTS. USE: 4. CAIMG $>100$.

06085047 JURASSIC \& CRETACEOUS, FRANCISCAN FM. S17.18.T7S,R2W.

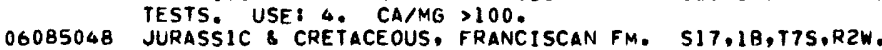
TESTS USE: 4 O CA/MG $>100$

06085049 JURASSIC \& CRETACEOUS, FRANCISCAN FM, S17,1B,T7S,RZW. USE: 4 . CAMG $>100$.

06085050 JURASSIC \& CRETACEOUS, FRANCISCAN FM. S17,18,T7S,RZW.

$(331: 376.374)$ LIMESTONE, TONNAGE SCREEN SIZE, +3 IN: PHYS

$(331: 376,374)$ LIMFSTONE: TONNAGE, SCREEN SIZE, \$4 IN: PHYS

(331:376,374) LIMESTONE, TONNAGE: SCREEN SIZE, 2.5 IN PHYS

$(331: 377,374,376)$ LIMESTONE AVG COMPOS OF MANY SMPL, TONNAGE.

(331:376.374) LIMESTONE: TONNAGE: SCREEN SIZE, -5 INI PHYS

(331:376,374) LIMFSTONE: TONNAGE: SCREEN SIZE, +2 INI PHYS

$(331: 376,374)$ LIMESTONE: TONNAGE: SCREEN SIZE, +1.5 INI PHYS

TESTS. USE: 4 . CAMGG $>100$.

06085067 "T75.

06085069 "T8S,RIW" LOS GATOS. (68:660) LIMESTONE. CA/MG >100.

06085075 "T6S,R2W" MOUNTAIN VIFW. (681660) LIMESTONE. "MISCI ASPHALT $=0.69$ C CAMG >100,

06087001 "T11S,R2W" SANTA CRUZ. (72:881,880) LIMESTONE, SOFT, CONCRETIONARY. USE: 4 . CA/MG = 66.7.

06087004 "TI1S,R2W" SANTA CRUZ. (72:881.880) LIMESTONE, SOFT, CONCRETIONARY. USE: 4. CA/MG = 41.0.

06087005 "T11S,R2W" SANTA CRUZ. (428:10) LIMESTONE. CA/MG $=42.6$.

O6087006 PRE-FRANCISCAN, GABILAN LS. "TIOS,R2W" FELTON, (175:353,350) LIMESTONE, WHITE TO BLUE, COARSELY CRYS: 300-600 FT THICK. USE: 6,12. CAMG $=68.8$. 
TABLE 9. - Analyses of samples from California and Hawaii containing more than 90 percent carbonate (Group $F_{2}$ ), common-rock category Continued

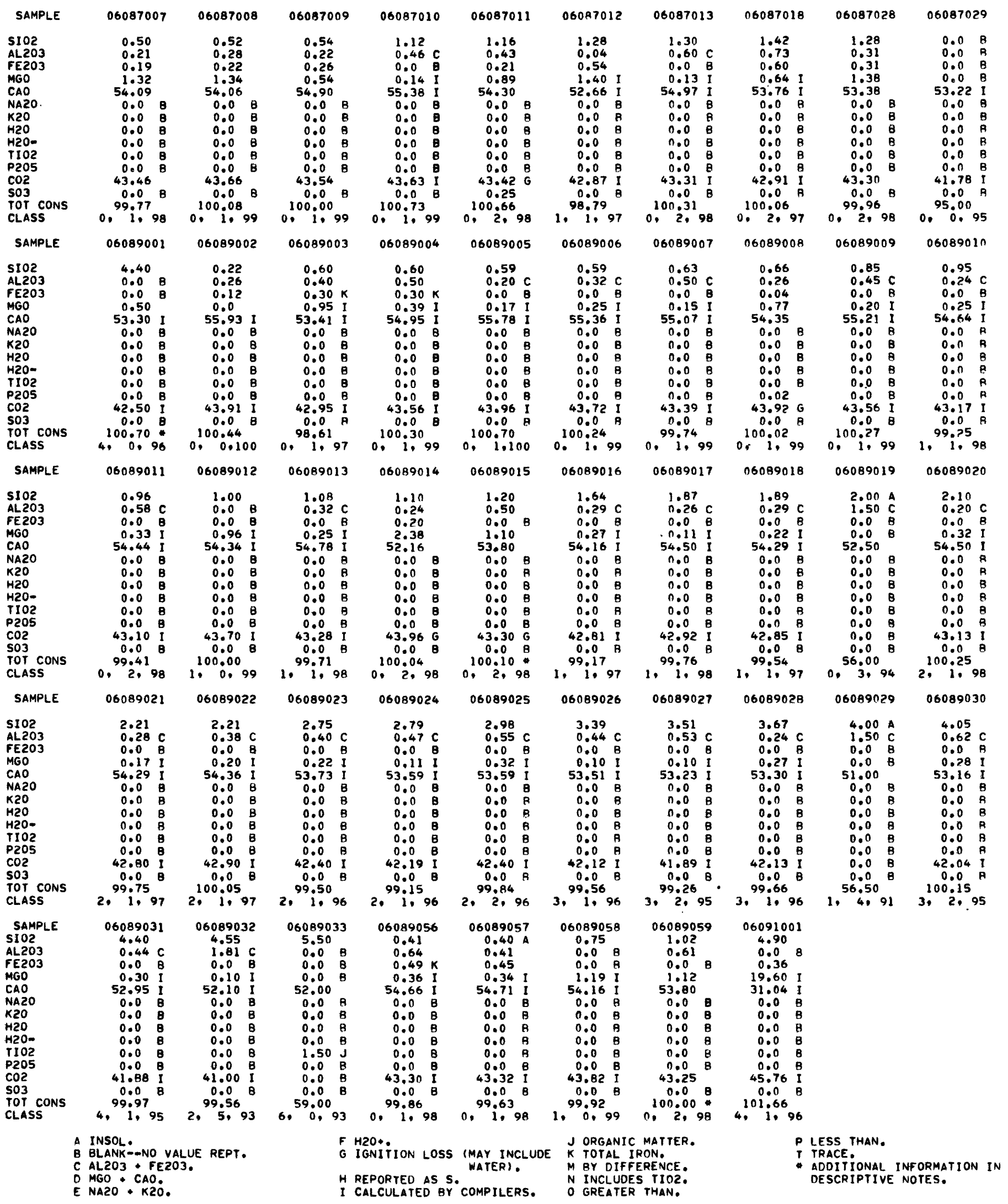


TABLE 9. - Analyses of samples from California and Hawail containing more than 90 percent carbonate (Group $F_{2}$ ), common-rock category Continued

06087007 PRE-FRANCISCAN, GABILAN LS. "TIOS,R2W" FELTON, (1751353.350) LIMESTONE, WHITE TO BLUE, COARSELY CRYSI 300AOAO FT 06087008 PRE-FRANCISCAN, GABILAN LS, "1905,R2W" BONNIE DOON, (1751353,350) LIMESTONE, WHITE TO BLUE, COARSELY CRYS 300-600 FT THICK, INDEX MAP, CA/MG $=40.3$

06087009 PRE-FRANCISCAN, GABILAN LS. "TIOS, R2W" FELTON. (175:353,350) LIMESTONE, WHITE TO BIUE, COARSELY CRYSI 300-600 FT 06087010 "TIOS,R3W" DAVENPORT. (173:123,122,PL4) LIMESTONE, CRYS. INDEX MAP. USE: 4. CA/MG >100.

06087011 JURASSIC \& CRETACEOUS, FRANCISCAN FM. "TIOS,R3W" DAVENPORT. (2911116) LIMESTONE. GRAY TO WHITE: COMPACT: TONNAGE. 06087012 PRE-FRANCISCAN, GABILAN LS. "TIOS,RZW" SANTA CRUZ. $(175: 353,350)$ LIMESTONE. INDEX MAP. CA/MG = 37.6.

06087013 "TIOS,R2W" DAVENPORT, $(1731123,122$, PL4) LIMESTONE, CRYS, INDEX MAP. USE: 4. CA/MG $>100$.

06087018 PRE-FRANCISCAN, SUR SER, S15,T10S,R3W. $1556: 321,320$,PL37/ 55:6,4) LIMESTONE, COARSELY CRYS. INDEX MAP. USE: 4. 06087028 PRE-FRANCISCAN, GABILAN LS. "S20,T10S,R2W" $(1751353,350,351)$ LIMESTONEI QUARRY SMPL. INDEX MAP. CA/MG . 38.7.

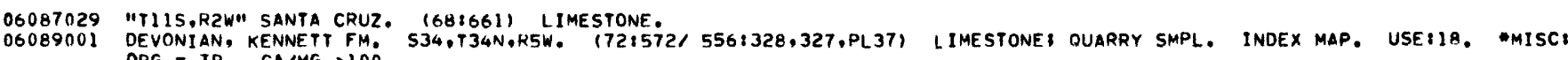
06089002 DEVONIAN, KENNETT FM. S4,T33N,R5W. (329:37,5,PLL1-4) LIMESTONE, GRAY, FINE-GR, THIN- TO THICK-REDDED. INDEX \& GEOL MAPS, GEOL SEC.

06089003 PERMIAN, MCCLOUD LS, S13,134N,R4W. $(26: 166,165$, PL2, 556:328,PL37) LIMESTONE. INDEX MAP. USE: 4. CA/MG = 56.2. 06089004 PERMIAN, MCCLOUD LS, S13,734N,R4W. $(26: 166,165, P L 2 / 556: 328, P L 37)$ LIMESTONE. INDEX MAP. USE, 4. CA/MG $>100$.

06089005 TRIASSIC, MOSSELKUS LS. S1,T33N,R2W. (556:325,323,324,PL37) LIMESTONE, DARK, FOS, BEDS 50-20O FT THICK. INDEX MAP. USE: 12,18 . CA/MG $>100$. (5561325,323,324,PL37) LIMESTDNE, DARK, FOS; AEDS 50-200 FT THICK, INDEX MAP USE: 12,18 TRA

06089007 TRIASSIC, MOSSELKUS LS. S6,T33N,RIW. (556:327,326.PL37) LIMESTONE: SPOT SMPL. INDEX MAP. USE: 18. CA/MG >1O0. 06089008 PERMIAN, MCCLOUD LS. "T33N,R4W" MOUNTAIN GATE. (55:6,1) LIMESTONE, GRAY, FINE-GR, MASSIVE, CRYS. USEI4. CA/MG:70.6. 06089009 TRIASSIC, HOSSELKUS LS. S1,T33N,R2W. $1556: 325,323,324, P L 37)$ LIMESTONE, DARK, FOS, BEDS 50-200 FT THICK. INDEX MAP,

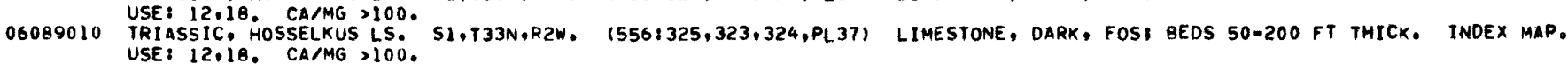

06089010 TRIASSIC, HOSSELKUS LS. S1,T33N,R2W. $(556,325,323,324$, PL 37) LIMESTONE, DARK, FOS: BEDS 50-200 FT THICK. INDEX MAP. 06089011 TRIASSIC, HOSSELKUS LS. S12,T33N,R4W. (556:325,323,PL37) LIMESTONE: 50-200 FT THICKI INDEX MAP. USE 12. CA/MG>100. 06089012 DEVONIAN, KENNETT FM. S34,T34N,R5W. (5561327,PL37) LIMESTONE, QUARRY SMPL. INDEX MAP. USE: 12,18. CA/MG = 56.6. 06089013 TRIASSIC, HOSSELKUS LS, S6,T33N,RIW. (556:327,326,PL37) LIMESTONE: SPOT SMPL. INDEX MAP. USE: 18. CA/MG >100. 06089014 PERMIAN. MCCLOUD LS, S13,T34N,R4E, (492:70,71) LIMESTONE, GRAY, FOS, MASSIVEI 2,000 FT THICK. USE, 4 . CA/MG = 21.9 , 06089015 PERMIAN, MCCLOUD LS, S26,T34N,R4W. (424:477,465-470/347:151,140,143) LIMESTONE, GRAY, FINE-GR, FOS. INDEX MAPS, *MISC: FEO $=0.2$. CA/MG $=48.9$. TRIASSIC. HOSSELKUS LS. USE: 12. CAMG >100.

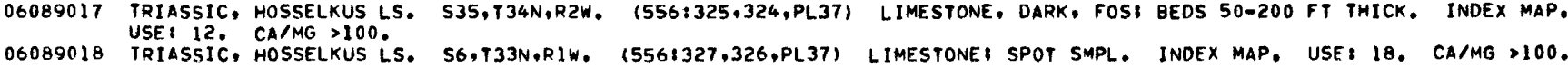
06089019 PERMIAN, MCCLOUD LS. "T33N,RIW" BAIRD, $1155: 176 / 156: 141$ LIMESTONE. INDEX \& GEOL MAP. USE: 12,18. 06089020 TRIASSIC, HOSSFLKUS LS. S1,T33N,R2W. 1556:325,323,324,PL37) LIMESTONE, OARK, FOSI BEOS 50-20O FT THICK. INDEX MAP. USE: 12,18 . CAIMG $\$ 100$.

06089021 TRIASSIC, HOSSELKUS LS, S6,T33N,RIW.

06089022 TRIASSIC, HOSSELKUS LS, SI,T33N.R2W. USE : 12,18 , CA/MG $>100$.

06089023 TRIASSIC, HOSSELKUS LS. S6,T33N,RIW.

06089024 TRIASSIC, HOSSELKUS LS. SI,T33N,R2W.

USE : 12,18 . CA/MG $>100$. TRIASSIC, HOSSELKUS LS, SI,T33N,R2W. USE: 12,18 . CA/MG $>100$.

06089027 TRIASSIC, HOSSELKUS LS, SI,T33N,R2W. USE: 12,18 , CA/MG $>100$ 06089028 DEVONIAN, KENNETT FM. S3I,T3IN,R5W.

06089029 TRIASSIC, HOSSELKUS LS, SB,T34N,R2W.

06089030 TRIASSIC, HOSSELKUS LS. S1,T33N,R2W.

06089031 USE: 12,18 CAIMG $>100$. USE: 12.18 . CA/MG $>100$.

06089032 TRIASSIC, HOSSELKUS LS. S1,T33N,R2W. USE: 12,18 , CA/MG $>100$. (556:327,326,PL37) LIMESTONE: SPOT SMPL. INDEX MAP, USE: 18, CA/MG $>100$. (556:325,323,324,PL37) LIMESTONE, DARK, FOS: BEDS 50-200 FT THICK. INDEX MAP. (556:327,326,PL37) LIMESTONE! SPOT SMPL. INDEX MAP, USE: 18, CA/MG $>100$. (556:325,323,324,PL37) LIMESTONE, DARK, FOSI AEOS 50-200 FT THICK. INDEX MAP. (556:325,323,324,PL.37) LIMESTONE, DARK, FOS: EEDS 50-200 FT THICK, INDEX MAP. (556:327,326,PL37) LIMESTONE: SPOT SMPL. INDEX MAP. USE: 18. CA/MG $>100$. (556:325,323.324,PL37) LIMESTONE, DARK, FOSI BEDS 50-200 FT THICK. INDEX MAP. (556:326,PL37) LIMESTONE, 100 FT THICK, INOEX MAP, USE 2,18 . CA/MG $>100$. $(155: 176 / 556: 325,326, P L 37)$ LIMESTONE, GRAY, FOS: 200 FT THICK, INDEX MAP. (556:325,323,324,PL37) LIMESTONE, DARK. FOSI BEDS 50-200 FT THICK, INDEX MAP. (556:325,323.324,PL37) LIMESTONE, DARK, FOS3 AEDS 50-200 FT THICK. INDEX MAP. $(556: 325,323,324$, PL 37) LIMESTONE, DARK, FOSI AEDS 50-200 FT THICK. INOEX MAP. 06089033 TRIASSIC, HOSSELKUS LS. $57,8, T 34 \mathrm{~N}, \mathrm{R} 2 \mathrm{~W}$ (21:89) LIMESTONE, GRAY, FOS, THIN-AEDOEO, USE: 12.

06089056 PERMIAN, MCCLOUD LS. S13,24,T34N,R4W. (68:661/556:330,PL37) LIMESTONE. INDEX MAP. USE, 4,12. CA/MG >100.

06089057 PERMIAN, MCCLOUD LS. S26,T34N,R4W. $1556: 330,329$, PL37/ 68:661) LIMESTONE. INOEX MAP. USES 12. CA/MG >100.

06089058 PERMIAN, MCCLOUD LS. S7,31.T35N,R3W. (12:183,182) LIMESTONE: TONNAGE. INOEX MAP. CA/MG * 45.5.

06089059 PERMIAN, MCCLOUD LS. S26,T34N.R4W. (366:197,193,195) LIMESTONE, GRAY: THIN-BEDDED TO MASSIVE: FOS: CONTAINS CHERT.

06091001 USE: 12. $530, T 20 N, R I I E$. FE: $(571,69)$ DOLOMITIC MARBLE. SELECTED SMPL. INDEX MAP. CA/MG $=1.53$. 
TABLE 9. - Analyses of samples from California and Hawaii containing more than 90 percent carbonate (Group $\left.F_{2}\right)$, common-rock category Continued

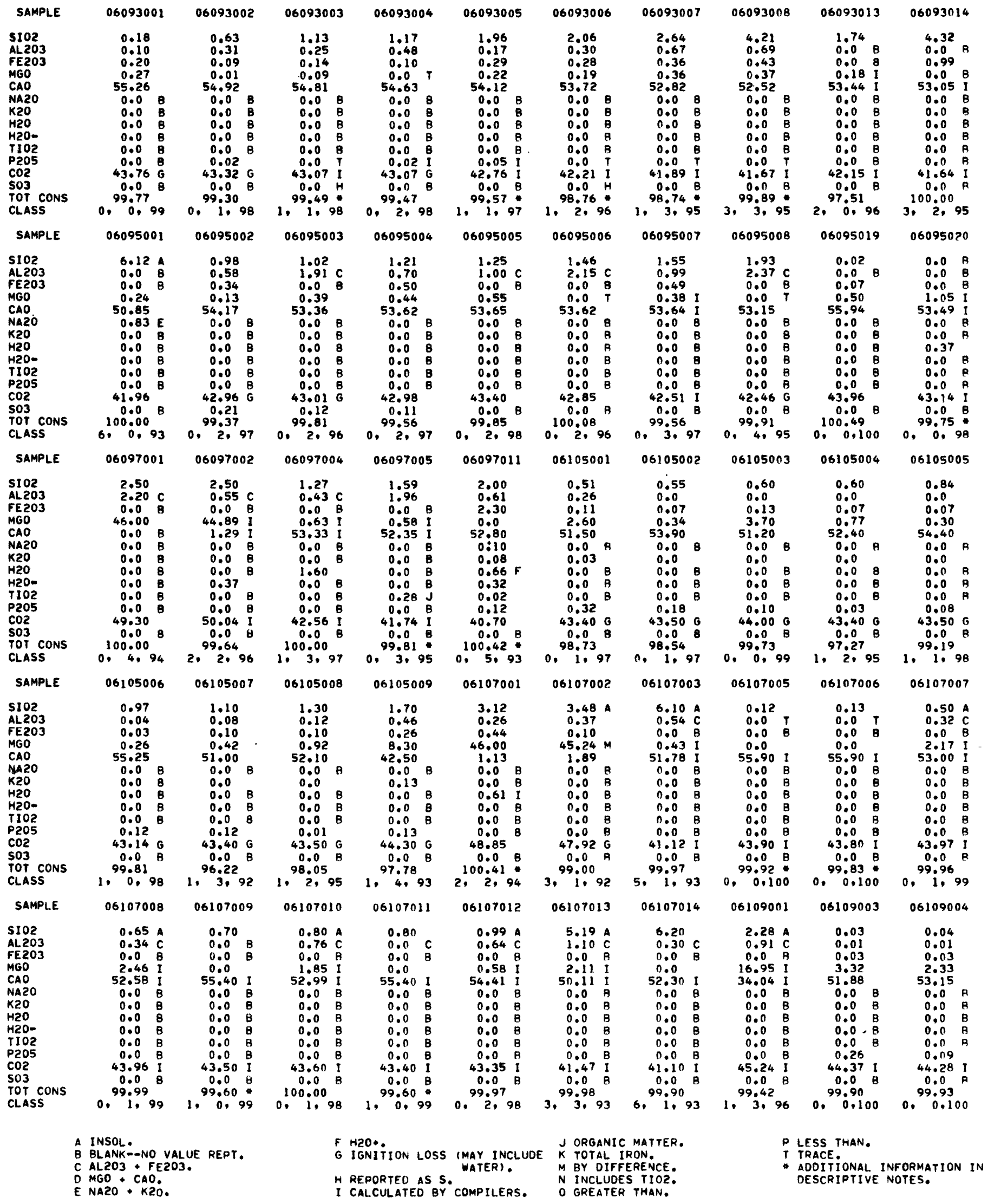


TABLE 9. - Analyses of samples from California and Hawaii containing more than 90 percent carbonate (Group $\left.F_{2}\right)$, common-rock category Continued

06093001 SILURIAN \& DEVONIAN, GAZELLE FM. ST,T42N,R6WI S12,T42N,RTW. (12:181/ 24:331) LIMESTONE, GRAY, MASSIVE TO THIN-BEDDED, 06093002 PALEOZOIC. ST,T42N,R6W. (556:331.330.PL37) LIMESTONE. INDEX MAP. USEI 6.18. CA/MG $>100$.

06093003 PALEOZOIC. S8,T42N,R6W. (279:520,519,PL26) LIMESTONE, GRAY, FINE-GR, COMPACT, THICK-BEDDEDI 200 FT THICK SAMPL OF 35

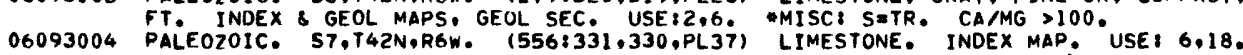

06093005 PALEOZOIC. S8,T42N,R6W. (2791520,519,PL26) LIMESTONE, GRAY, FINE-GR, COMPACT, THICK-BEDDEDI 200 FT THICK. INOEX \&

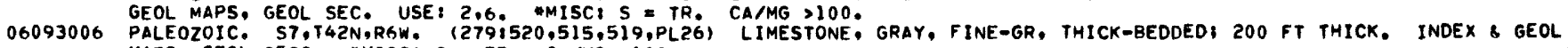
06093007 PALEOZOIC. S18,T42N,R6W. $(279: 520,515$, PLZ6) CONGLOMERATE CARBONATE CEMENT: COMPOS GRAB SMPL. INDEX GEOL MAP, GEOL SEC. HMISC: $S=T R$. CARMG $>100$

06093008 PALEOZOIC. S12,T42N,R7W. (279:520,515-519,PL26) LIMESTONE, GRAY, FINE-GR, THICK-BEODEDI 200 FT THICK SMPL TAKEN ACROSS 50 FT. INDEX \& GEOL MAP, GEOL SEC. MMISC: S=TR, CAMG >100.
06093013 SILURIAN \& DEVONIAN, GAZELLE FM. S5,T42N,R6W. $(12: 183,182)$ LIMESTONE OUTCROP SMPLI TONNAGE. INDEX MAP. CAMG >100.

06093014 "TH2N,RGW" GAZELLE. (68:661) LIMESTONE.

06095001 "T3N,R3W" BENICIA. (72:8B2) LIMESTONE. USE: 4. CA/MG $>100$.

06095002 "TSN,R2W" SUISUN. (1742282) LIMESTONE: USE: 40 CAMG $>100$.

06095003 "TSN,R2W" SUISUN, 1174:2821 LIMESTONE, USE: 4 . CA/MG $>100$.

06095004 "TSN,R2W" SUISAN. (555:121,120) TRAVERTINE. USE: 4 , CA/MG >100,
06095005 CRETACEOUS, CHICO FM. SI7,TSN,RIW. $(21: 185,531: 88,26, P L 4,19)$ TRAVERTINE, POROUS: TYPICAL ANAL. INDEX G GEL MAPS,

06095006 "TSN,R2W" SUISUN. (I74:282) LIMESTONE. USE: 4.

06095007 PLEISTOCENE TO HOLOCENE. "TSN,RZW" SUISUN. (175:360,359) TRAVERTINE, USE: 4. CA/MG >100.

06095008 "TSN,R2W" SUISUN. 1174:282) LIMESTONE, USE 4 .

06095019 "TSN,R2W" SUISUN. (47:17) ARAGONITE. CAMG $>100$.

06095020 "TGN,RZW" SUISUN, 1733641) ONYX, WHITE, GRAY, USE 115. MISC: SRCO3 = 1.59, BAC03 = 0.11. CA/MG . 50.9.

06097001 SIT,TON,RIIW. 1578331,328-330) MAGNESITEI 25 FT THICK. INOEX MAP. USE: 16.

06097002 S17,T9N,RIIW. (57:331.328-330) MAGNESITE, 25 FT THICK, INDEX MAP, USE: 16. CA/MG = 0.029.

06097004 "TIIN,R9W" GEYSERVILLE, (72:633) LIMESTONE, FLOAT, USE: 18. CA/MG = 84.7.

06097005 "T9N,R9W" HEALDSBURG, (5561334,PL37) LIMESTONE. INDEX MAP. "MISC: MNO = 0.181 FECO3 $=1.13$. CA/MG = 90.3.

06097011 JURASSIC \& CRETACEOUS, FRANCISCAN FM. "TBN,RI2W" BLACK MTN. $129: 76,11,12,14$, PLI) LIMESTONE, RED. INDEX \& GEOL MAPS. 06105001 PENNSYLVANIAN OR PERMIAN. S6,7.T30N,RIOW, $(22,42,40$, PLI, 1 , $)$ LIMESTONE, GRAY, DENSE: COMPOS SMPL: 200 FT THICK:

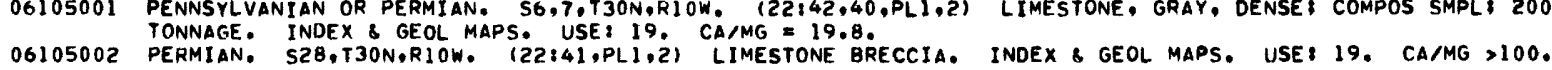

06105003 PENNSYLVANIAN OR PERMIAN, S22,27,T31N,RIOW. (22:41,40,PL1,2) LIMESTONE, GRAY, MASSIVE: 200 FT THICK: TONNAGE. INDEX

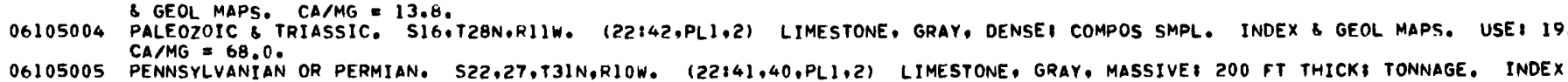

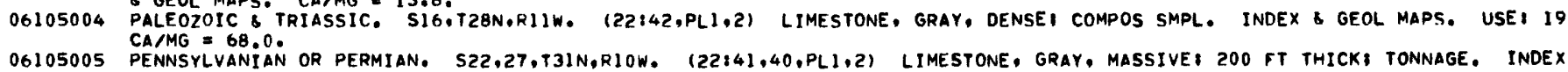
\& GEOL MAPS. CAMMG $>100$.

06105006 PERMIAN. S34,T3IN,RIIW. $(22: 41,40, P L 1,2)$ LIMESTONE. INDEX \& GEOL MAPS. USE: 19. CA/MG $>100$.

06105007 TRIASSIC. S36,T3N,RGE \& S1,T2N,R6E. (22:42,40,41,PL1,2) LIMESTONE, GRAY. INDEX \& GEOL MAPS. USEI 19. CA/MG >100.

06105008 DEVONIAN, S18,30,T7N,R7E. (22142,40,PL1,2) LIMESTONE, GRAY, FINELY CRYSI COMPOS SMPL, 200 FT THICK, INDEX \& GEOL MAP. 06105009 PENNSYLVANIAN OR PERMIAN. S16,17,T32N,RIIW. $(22: 40, P L 1,2)$ LIMESTONEI 200 FT THICK TYPICAL SMPL. INOEX \& GEOL MAPS. 06107001 "T2ZS,R27E" PORTERVILLE. $(36: 535,529-531,555)$ MAGNESITE, AMORPHOUS. USE 15,16. MISCI MNO, NIL HZO = I.L. LESS CO2.

06107002 "T22S,R27E" PORTERVILLE. (428:10) MAGNESITE. CA/MG $=0.042$.

06107003 S25,TI7S,R28E. (556:336.335,PL37) LIMESTONE, GRAY, CRYSi TONNAGE. INDEX MAP. CAMMG $>100$.

06107005 S25,30.T17S,R28E. (200:445) LIMESTONE. MMISC: AL203+FEOETR.

06107006 S25.30,TI7S,R28E: (200:445) LIMESTONE: MISC: AL203+FEO=TR.

06107007 S13.215,R28E. (556:338,PL37) LIMESTONE, WHITE TO BLACK, MOTL, COARSELY CRYS TONNAGE, INDEX MAP. CA/MG - 24.4.

06107008 CARBONIFEROUS, S30,T20S,R3IE, (5568340,PL37) LIMESTONE, WHITE, GRAY, BANDED. INDEX MAP. CA/MG a 21.3.

06107009 S25.30,T175,R28E. (200:455) LIMESTONE. AMISC: AL203 . FEO = TR.

06107010 56,T205,R28E. (556:341,340,PL37) LIMESTONE, GRAY TO BLACK, FINE-GR, INDEX MAP. USE: 6.12. CAIMG = 28,6.

06107011 S25,30.T17S,R28E, (200:445) LIMESTONE, USE: 18, HMISC: AL203+FEO= TR.

06107012 S5,T205,R2BE. (556:337,PL37) LIMESTONE, GRAY TO BLACK, FINELY CRYS. INDEX MAP. CA/MG = 93.8.

06107013 CAR8ONIFEROUS, T20S,R3IE, SPRINGVILLE, (556:340,PL37) LIMESTONE, GRAY, MEO TO FINELY CRYSI SUGARYI GRAB SMPL. INDEX 06107014 S25,30.T17S,R28E. (2008445) LIMESTONE.

06109001 S33.34.TIN,RISE. (5561345) OOLOMITE. GRANULARI 300-FT CHIP SHPL. CA/MG 2.01.

06109003 PALEOZOIC, CALAVERAS FM. S28,TIN,RISE, (264:20,7-9,22,PLI,3) LIMESTONE, WHITE, GRAY, MOTL, COARSE-GR DUTCROP SMPLI

06109004 MINI TONNAGE. INDEX \& GEOL MAPS. USE: 4. CA/MG $=15.6$.

PALEOZOIC, CALAVERAS FM. S28,TIN,RISE, $\left(264120,7-9,22, P_{L} 1,3\right)$ LIMESTONE, WHITE, GRAY, MOTL, COARSE-GRI OUTCROP SMPLI

MIN TONNAGE. INDEX \& GEOL MAPS. USE: 4. CA/MG $=22.8$. 
TABLE 9. - Analyses of samples from California andHawaii containing more than 90 percent carbonate (Group $\left.F_{2}\right)$, common-rock category Continued

\begin{tabular}{|c|c|c|c|c|c|c|c|c|c|c|}
\hline SAMPLE & 06109005 & 06109006 & 06109007 & 06109008 & 06109009 & 06109010 & 06109011 & 06109012 & 06109013 & 6109014 \\
\hline $\begin{array}{l}\text { S102 } \\
\text { AL203 } \\
\text { FE203 } \\
\text { MGO } \\
\text { CAO } \\
\text { NA2O } \\
\text { K20 } \\
\text { H2O } \\
\text { H20- } \\
\text { T102 } \\
\text { P2O5 } \\
\text { CO2 } \\
\text { SO3 } \\
\text { TOT CONS } \\
\text { CLASS }\end{array}$ & 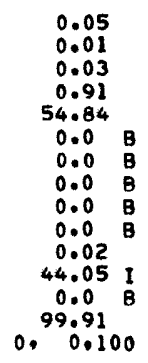 & 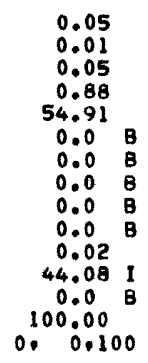 & $\begin{array}{cc}0.05 \\
0.02 \\
0.13 \\
0.92 \\
54.73 \\
0.0 \\
0.0 & 8 \\
0.0 & B \\
0.0 & B \\
0.0 & B \\
0.02 & \\
43.98 & 1 \\
0.0 & B \\
99.85 & \\
0.000 & 0.100\end{array}$ & $\begin{array}{cc}0.06 \\
0.02 \\
0.02 \\
1.02 \\
53.09 \\
0.00 \\
0.0 & 8 \\
0.00 & B \\
0.00 & B \\
0.00 & B \\
0.02 & \\
44.25 & 1 \\
0.00 & B \\
99.98 & \\
0,9.1100\end{array}$ & 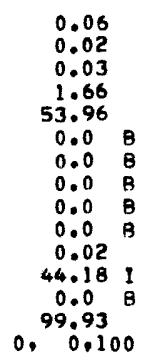 & 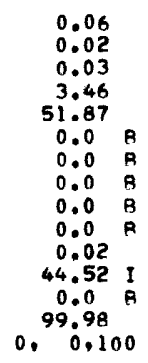 & $\begin{array}{ccc}0.06 \\
0.02 \\
0.05 \\
0.36 \\
55.42 \\
0.0 & B \\
0.0 & 8 \\
0.00 & 8 \\
0.00 & B \\
0.0 & B \\
0.17 & \\
43.90 & 1 \\
0.00 & B \\
99.99 & \\
0.900 & 0.100\end{array}$ & $\begin{array}{ccc}0.07 \\
0.02 \\
0.10 \\
0.31 \\
55.51 \\
0.0 & 8 \\
0.00 & 8 \\
0.00 & B \\
0.00 & 8 \\
0.0 & \text { A } \\
0.05 & \\
43.92 & 1 \\
0.00 & 8 \\
99.98 & \\
0.90 & 0.100\end{array}$ & $\begin{array}{cc}0.10 \\
0.02 \\
0.05 \\
2.51 \\
52.89 \\
0.0 & B \\
0.0 & 8 \\
0.0 & 8 \\
0.0 & B \\
0.0 & B \\
0.02 & \\
44.27 & 1 \\
0.0 & B \\
99.86 & \\
0, \quad 0.100\end{array}$ & $\begin{array}{c}0.11 \\
0.03 \\
0.06 \\
2.01 \\
53.49 \\
0.0 \\
0.0 \\
0.0 \\
0.0 \\
0.0 \\
0.06 \\
44.19 \\
0.0 \\
99.95 \\
0.09 \\
0.100\end{array}$ \\
\hline SAMPLE & 06109015 & 06109016 & 06109017 & 06109018 & 06109019 & 06109020 & 06109021 & 06109022 & 06109023 & 06109074 \\
\hline $\begin{array}{l}\text { S102 } \\
\text { AL203 } \\
\text { TE203 } \\
\text { MGO } \\
\text { CAO } \\
\text { NA2O } \\
\text { K20 } \\
\text { H2O } \\
\text { H2O- } \\
\text { TIO2 } \\
\text { P205 } \\
\text { CO2 } \\
\text { SO3 } \\
\text { TOT CONS } \\
\text { CLASS }\end{array}$ & 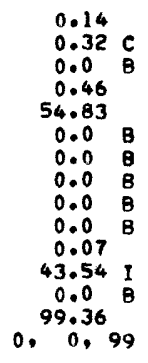 & 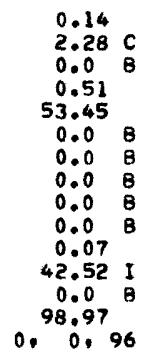 & $\begin{array}{ccc}0.15 \\
0.03 \\
0.05 \\
1.84 \\
53.62 \\
0.0 & 8 \\
0.0 & 8 \\
0.0 & 8 \\
0.0 & 8 \\
0.0 & 8 \\
0.14 & \\
44.11 & 1 \\
0.0 & 8 \\
99.94 & \\
0.90 & 0.100\end{array}$ & $\begin{array}{c}0.15 \\
0.90 \\
0.70 \mathrm{~K} \\
13.70 \\
38.90 \\
0.00 \mathrm{~B} \\
0.00 \mathrm{~B} \\
0.20 \mathrm{~B} \\
0.00 \mathrm{~B} \\
0.00 \mathrm{~B} \\
0.00 \mathrm{~B} \\
45.40 \\
0.00 \\
99.95 \\
0.9898\end{array}$ & $\begin{array}{ccc}0.16 & \\
0.24 & \mathrm{C} \\
0.0 & \mathrm{~B} \\
0.72 & \\
54.91 & \\
0.0 & \mathrm{~B} \\
0.0 & \mathrm{~B} \\
0.00 & \mathrm{~B} \\
0.0 & \mathrm{~B} \\
0.0 & \mathrm{~B} \\
0.09 & \\
43.89 & 1 \\
0.0 & \mathrm{~B} \\
100.01 & \\
0.0 .100\end{array}$ & 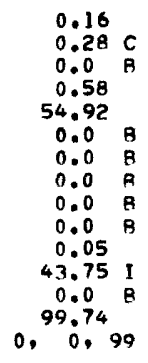 & $\begin{array}{ccc}0.16 & \\
0.34 & c \\
0.0 & 8 \\
1.00 & \\
54.42 & \\
0.0 & 8 \\
0.0 & 8 \\
0.00 & 8 \\
0.0 & 8 \\
0.0 & 8 \\
0.09 & \\
43.89 & 1 \\
0.0 & B \\
99.90 & 09 \\
0 . & 0.99\end{array}$ & 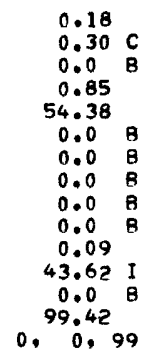 & $\begin{array}{ccc}0.18 & \\
0.48 & C \\
0.0 & 8 \\
0.80 & \\
54.47 & \\
0.0 & 8 \\
0.0 & 8 \\
0.0 & 8 \\
0.0 & 8 \\
0.0 & 8 \\
0.08 & \\
43.64 & 1 \\
0.0 & 8 \\
99.65 & \\
0.99 & 0.99\end{array}$ & $\begin{array}{c}0.18 \\
2.58 \\
0.0 \\
0.91 \\
53.25 \\
0.0 \\
0.0 \\
0.0 \\
0.0 \\
0.0 \\
0.0 \\
0.16 \\
42.80 \\
0.0 \\
99.88 \\
99 \\
0.0 .97\end{array}$ \\
\hline SAMPLE & 06109025 & 06109026 & 06109027 & 06109028 & 06109029 & 06109030 & 06109031 & 06109032 & 06109033 & $0610903 a$ \\
\hline $\begin{array}{l}\text { SIO2 } \\
\text { AL203 } \\
\text { FE203 } \\
\text { MGO } \\
\text { CAO } \\
\text { NA2O } \\
\text { K2O } \\
\text { H2O } \\
\text { H20- } \\
\text { TIO2 } \\
\text { P205 } \\
\text { CO2 } \\
\text { SO3 } \\
\text { TOT CONS } \\
\text { CLASS }\end{array}$ & 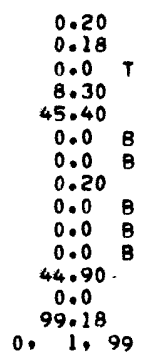 & $\begin{array}{ll}0.20 \\
0.28 \mathrm{C} \\
0.0 & \mathrm{~B} \\
1.85 & \\
53.25 & \\
0.0 & 8 \\
0.0 & 8 \\
0.0 & 8 \\
0.00 & 8 \\
0.0 & \mathrm{~B} \\
0.05 & \\
43.83 & 1 \\
0.0 & 8 \\
99.46 & \\
0, & 0.99\end{array}$ & 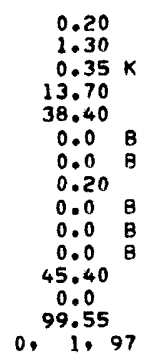 & 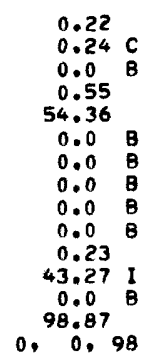 & $\begin{array}{ccc}0.22 & \\
3.58 & \mathrm{C} \\
0.00 & 8 \\
0.46 & \\
53.24 & \\
0.00 & \mathrm{~B} \\
0.00 & 8 \\
0.00 & 8 \\
0.00 & 8 \\
0.00 & 8 \\
0.04 & \\
42.29 & 1 \\
0.00 & 8 \\
99.83 & \\
0.0 .96\end{array}$ & $\begin{array}{ccc}0.24 & \\
0.38 & \mathrm{C} \\
0.0 & \mathrm{~B} \\
0.77 & \\
54.46 & \\
0.0 & \mathrm{~B} \\
0.0 & 8 \\
0.0 & 8 \\
0.0 & 8 \\
0.0 & 8 \\
0.10 & \\
43.59 & \mathrm{I} \\
0.0 & 8 \\
99.54 & \\
0.0 .99\end{array}$ & $\begin{array}{ccc}0.24 & \\
0.42 & \mathrm{C} \\
0.0 & \mathrm{~B} \\
2.17 & \\
52.74 & \\
0.0 & 8 \\
0.0 & \mathrm{~B} \\
0.00 & 8 \\
0.0 & 8 \\
0.0 & \mathrm{~B} \\
0.07 & \\
43.75 & 1 \\
0.00 & \mathrm{~B} \\
99.39 & \\
0, & 0.99\end{array}$ & $\begin{array}{ccc}0.24 & \\
2.40 & \mathrm{C} \\
0.00 & \mathrm{~B} \\
0.49 & \\
53.81 & \\
0.0 & \mathrm{~B} \\
0.0 & \mathrm{~B} \\
0.00 & 8 \\
0.00 & 8 \\
0.00 & 8 \\
0.04 & \\
42.78 & \mathrm{I} \\
0.00 & 8 \\
99.76 & \\
0, & 0.97\end{array}$ & $\begin{array}{ccc}0.24 & \\
4.96 & \mathrm{C} \\
0.00 & \mathrm{~B} \\
0.62 & \\
52.13 & \\
0.0 & \mathrm{~B} \\
0.00 & \mathrm{~B} \\
0.00 & 8 \\
0.00 & \mathrm{~B} \\
0.0 & \mathrm{~B} \\
0.07 & \\
41.60 & 1 \\
0.0 & \mathrm{~B} \\
99.62 & \\
0, & 0.94\end{array}$ & $\begin{array}{c}0.24 \\
9.46 \\
0.0 \\
0.61 \\
49.38 \\
0.08 \\
0.08 \\
0.08 \\
0.08 \\
0.08 \\
1.061 \\
39.43 \\
0.0 \\
100.73 \\
0.008\end{array}$ \\
\hline SAMPLE & 06109035 & 06109036 & 06109037 & 06109038 & 06109039 & 06109040 & 06109041 & 06109042 & 06109043 & 06109044 \\
\hline $\begin{array}{l}\text { SIO2 } \\
\text { AL203 } \\
\text { FE203 } \\
\text { MGO } \\
\text { CAO } \\
\text { NA2O } \\
\text { K2O } \\
\text { H2O } \\
\text { H20- } \\
\text { TIO2 } \\
\text { P205 } \\
\text { CO2 } \\
\text { SO3 } \\
\text { TOT CONS } \\
\text { CLASS }\end{array}$ & $\begin{array}{cc}0.26 \\
0.06 \\
0.11 \\
17.70 \\
34.96 \\
0.00 \\
0.08 \\
0.08 \\
0.08 \\
0.08 \\
0.08 \\
0.03 \\
46.85 & \text { B } \\
0.0 & B \\
99.97 & \\
99.100 & 0.100\end{array}$ & $\begin{array}{ccc}0.26 & \\
0.22 & \mathrm{C} \\
0.0 & \mathrm{~B} \\
3.51 & \\
51.58 \\
0.0 & \mathrm{~B} \\
0.0 & 8 \\
0.0 & 8 \\
0.0 & 8 \\
0.0 & \mathrm{~B} \\
0.06 & \\
44.34 & \mathrm{I} \\
0.0 & 8 \\
99.97 & \\
0.0 .89\end{array}$ & $\begin{array}{ccc}0.26 & \\
2.32 & \mathrm{C} \\
0.0 & \mathrm{~B} \\
0.58 & \\
53.74 & \\
0.0 & 8 \\
0.0 & \mathrm{~B} \\
0.0 & 8 \\
0.0 & \mathrm{~B} \\
0.0 & \mathrm{~B} \\
0.09 & \\
42.84 & 1 \\
0.0 & \mathrm{~B} \\
99.83 & \\
0.0 .97 & 0.97\end{array}$ & $\begin{array}{ccc}0.26 & \\
3.80 & \mathrm{C} \\
0.0 & \mathrm{~B} \\
0.41 & \\
52.74 & \\
0.0 & 8 \\
0.0 & 8 \\
0.0 & 8 \\
0.0 & 8 \\
0.0 & 8 \\
0.06 & \\
41.85 & 1 \\
0.0 & 8 \\
99.12 & \\
0.909 & 0.95\end{array}$ & $\begin{array}{ccc}0.28 & \\
0.26 & \mathrm{C} \\
0.00 & \mathrm{~B} \\
18.33 & \\
33.69 & \\
0.0 & 8 \\
0.0 & 8 \\
0.0 & \mathrm{~B} \\
0.00 & \mathrm{~B} \\
0.0 & 8 \\
0.05 & \\
46.54 & 1 \\
0.0 & \mathrm{~B} \\
99.15 & \\
0.09 & 0.99\end{array}$ & 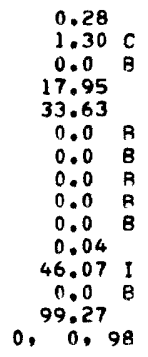 & $\begin{array}{cc}0.28 \\
3.04 \\
0.0 \\
0.0 \\
0.37 \\
53.40 \\
0.0 & 8 \\
0.0 & 8 \\
0.0 & B \\
0.00 & 8 \\
0.0 & B \\
0.05 & \\
43.07 & 1 \\
0.0 & B \\
100.21 & \\
0, \quad 0.97\end{array}$ & $\begin{array}{ccc}0.30 & \\
0.30 & \mathrm{C} \\
0.0 & \mathrm{~B} \\
18.18 & \\
34.21 & \\
0.0 & 8 \\
0.0 & 8 \\
0.0 & 8 \\
0.00 & \mathrm{~B} \\
0.0 & 8 \\
0.11 & \\
46.78 & 1 \\
0.0 & \mathrm{~B} \\
99.88 & \\
0.99 & 1.99\end{array}$ & $\begin{array}{ccc}0.30 & \\
0.32 & c \\
0.0 & 8 \\
0.43 & \\
54.69 & \\
0.0 & 8 \\
0.0 & 8 \\
0.0 & 8 \\
0.00 & 8 \\
0.0 & 8 \\
0.09 & \\
43.40 & 1 \\
0.00 & B \\
99.23 & \\
0.909 & 1.99\end{array}$ & 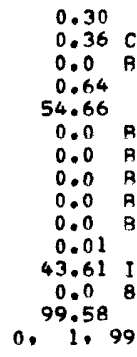 \\
\hline $\begin{array}{l}\text { SAMPLE } \\
\text { S102 } \\
\text { AL203 } \\
\text { FEE203 } \\
\text { MGO } \\
\text { CAD } \\
\text { NA2O } \\
\text { K20 } \\
\text { H2D } \\
\text { H20- } \\
\text { TIO2 } \\
\text { P205 } \\
\text { CO2 } \\
\text { SO3 } \\
\text { TOT CONS } \\
\text { CLASS }\end{array}$ & 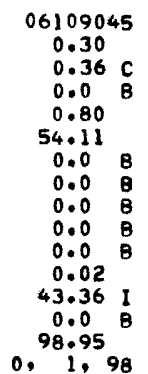 & 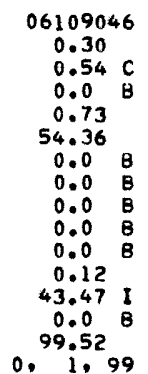 & $\begin{array}{ccc}06109047 \\
0.30 \\
0.66 & \mathrm{C} \\
0.0 & \mathrm{~B} \\
14.10 & \\
38.84 & \\
0.0 & 8 \\
0.0 & B \\
0.0 & 8 \\
0.0 & 8 \\
0.0 & B \\
0.06 & \\
45.94 & 1 \\
0.0 & 8 \\
99.90 & 90\end{array}$ & 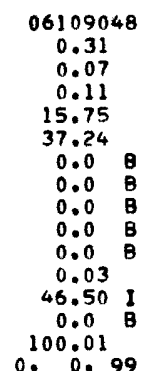 & $\begin{array}{cc}06109049 \\
0.32 \\
0.40 \mathrm{C} \\
0.0 & \mathrm{~B} \\
0.46 \\
54.66 \\
0.0 & \mathrm{~B} \\
0.0 & \mathrm{~B} \\
0.0 & 8 \\
0.0 & 8 \\
0.0 & 8 \\
0.07 & \\
43.35 & 1 \\
0.0 & 8 \\
99.26 & \end{array}$ & 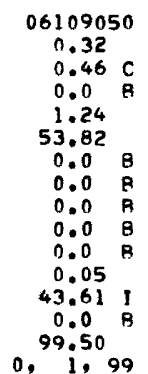 & 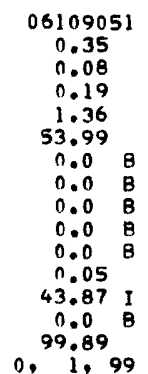 & $\begin{array}{cc}0.36 \\
0.08 \\
0.09 \\
10.15 \\
43.71 \\
0.0 & 8 \\
0.0 & 8 \\
0.0 & 8 \\
0.0 & 8 \\
0.0 & 8 \\
0.02 & \\
45.44 & 1 \\
0.0 & 8 \\
99.85 & 9.99 \\
0.9 & 90\end{array}$ & & \\
\hline
\end{tabular}

A INSOL:-NO VALUE REPT.

C AL203 - FE203.

D MGO CAO.

E NA2O C K2D.
G IGNITION LOSS IMAY INCLUDE

H REPORTEO AS $S$.

I CALCULATED BY COMPILERS.
$J$ ORGANIC MATTER.

$K$ TOTAL IRON.

$M$ BY DIFFERENCE.

N INCLUDES TIOZ.
P LESS THAN.

- TRACEITIONAL INFORMATION IN DESCRIPTIVE NOTES. 
TABLE 9. - Analyses of samples from California and Hawaii containing more than 90 percent carbonate (Group $F_{2}$ ), common-rock category Continued

06109005 PALEOZOIC, CALAVERAS FM. S31,TIN,R16F. GEOL MAPS. USE: 4.6. CA/MG $=60.3$. 06109006 PALEOZOIC, CALAVERAS FM. S31,TIN,RIGE. 06109007 GEOL MAPS. USE 14.6 . CA/MG $=62.4$. 06109007 PALEOZOIC, CALAVERAS FM, S31, TIN,RI6E. 06109008 PALEOZOIC, CALAVERAS FM, S31, TIN,RIGE. GEOL MAPS. USE: 4 . CA/MG $=28.0$. 06109009 PALEOZOIC, CALAVERAS FM. S3I,TIN,RIGE. 06109010 PALEOZOIC, CALAVERAS FM. S31,TIN,R16E. PALEOZOIC, CALAVERAS FM. S31, TIN,RIGE.
GEOL MAPS. USE: 4. CA/MG $=15,0$ 06109011 PALEOZOR, CALAVERAS FM. S28,TIN,R15E, $(264120,7-9,22, P L 1,3)$ 06109012 MINI TONNAGE. INDEX GEOL MAPS. USE 14.6 . CA/MG $>100$. 06109012 PALEOZOIC, CALAVERAS FM. S28,TIN,R15E, $(264: 20,7-9,22, P(1,3)$ 06109013 PALEOZOIC, CALAVERAS FM. S31,TIN,RI6E, (264:20,7-9,23,PL1-3) GEOL MAPS. USE : 6.18. CA/MG $=21.1$.

06109014 PALEOZOIC, CALAVERAS FM. S28,TIN,P15E, $1264: 20,7-9,22, P L 1,31$ MINI TONNAGE. INDEX \& GEOL MAPS. USE; 4. CA/MG $=26.6$.

06109015 PALEOZOIC, CALAVERAS FM. S35,TIN,R15E, (264:19,7=9,23,PL1,3) MINI TONNAGE. INDEX \& GEOL MAPS. CA/MG $>100$

06109016 PALEOZOIC, CALAVERAS FM. S35,TIN,RI5E. $(264119,7-9,23, \mathrm{PL} 1,3)$ MIN: TONNAGE. INDEX \& GEOL MAPS. CA/MG $>100$

06109017 PALEOZOIC, CALAVERAS FM. S28,TIN,R15E, $(264 i 20,7-9,22, P L 1,3)$ INDEX \& GEOL MAPS. USE: 4 CAIMG $=29.1$.

06109018 "T2N,R14E" COLUMBIA. (556:343,342,PL37) MARBLE, WHITE, BLUE, USE: 15. CA/MG = 2.83.

06109019 PALEOZOIC. CALAVERAS FM. 535,TIN,R15E, (264:20,7-9,23,PL1,3) MIN: TONNAGE. INDEX \& GEOL MAPS. USE: 4.6, CA/MG $=76.3$.

06109020 PALEOZOIC, CALAVERAS FM. S35,TIN,RI5E: $(264: 20,7-9,23, \mathrm{PL} \mathrm{i}, 3)$ 06109021 MINI TONNAGE. INDEX \& GEOL MAPS. USE: 4,6 . CA/MG $=94.7$. 06109021 PALEOZOIC, CALAVERAS FM. S35,TIN,RISE: $1264: 19,7-9,23, P L 1,3)$ 06109022 PALEOZOIC, CALAVERAS FM. S35,TIN.R15E: $\left(264,19,7-9,23\right.$, PLI $\left._{1}, 3\right)$ 06109023 MIN: TONNAGE. INDEX \& GEOL MAPS, USE: 4,6 . CA/MG $=64.0$. 06109023 PALEOZOIC, CALAVERAS FM. 535, TIN,RISE: (264:20,7-9,23,PLi,3) MINI TONNAGE. INOEX \& GEOL MAPS. USE: 4,6 . CA/MG $=68,1,1,3)$
06109024 PALEOZOIC, CALAVERAS FM. S35,TIN,RI5E, $(264: 20,7-9,23, \mathrm{PL}, 3)$ MINI TONNAGE. INDEX \& GEOL MAPS, USE: 4,6. CA/MG $=58.5$.
06109025 "T2N,RI4E" COLUMBIA. (556:343.342,PL37) MARBLE, WHITE, BLUE, 06109026 PALEOZOIC, CALAVERAS FM. S35.TIN,RISE. $(264: 19,7-9.23, P L 1,3)$ MINI TONNAGE. INDEX \& GEOL MAPS. USE: 4.6 . CA/MG $=28.8$. 06109027 "T2N,R14E" COLUMBIA. (556:343,342,PL37) MARBLE, WHITE, BLUE, 06109028 PALEOZOIC, CALAVERAS FM. S35,TIN,R15E. (264:19,7-9,23,PL1,3) MINI TONNAGE. INOEX \& GEOL MAPS. CA/MG $=98.8$.

06109029 PALEOZO1C, CALAVERAS FM. S35,T1N,R15E. $(264: 20,7-9,23, \mathrm{PL} 1,3)$ MINI TONNAGE. INDEX \& GEOL MAPS. USE; 4,6 . CA/MG $>100$

06109030 PALEOZOIC. CALAVERAS FM. S35,TIN,R1SE: $(264: 19,7-9,23, P L 1,3)$ MINi TONNAGE. INDEX \& GEOL MAPS. USE: 4,6, CAMG $=70.7$. 06109031 PALEOZOIC, CALAVERAS FM. S35.TIN,RI5E, $(264: 19,7-9,23, \mathrm{PL} 1,31$ 06109032 MINI TONNAGE. INDEX \& GEOL MAPS, USE: 4.6 . CA/MG $=24.3$. 06109032 PALEOZOIC, CALAVERAS FM. 535,TIN,RI5E, $1264: 20,7-9,23, P L 1,31$, MINI TONNAGE. INDEX \& GEOL MAPS. USE: 4,6. CA/MG $>100$,
06109033 PALEOZOIC, CALAVERAS FM. S35,TIN,RI5E, $1264: 20,7-9$, PL 1,3

06109034 PALEOZOIC, CALAVERAS FM, S35,TIN,R15E, (264:20,7-9,23,PL1,3) LIMESTONE, WHITE TO GRAY, MED-COARSE GR OUTCROP SMPL 06109035 MINI TONNAGE. INDEX \& GEOL MAPS. USE: 4,6 , CA/MG $=77.5$. 06109035 PALEOZOIC, CALAVERAS FM. S31,TIN,RI6E, $1264: 20,7-9, P L 1,3)$ DOLOMITE: OUTCROP SMPL: MIN. INDEX \& GEOL MAP. 06109036 PALEOZOIC, CALAVERAS FM. S35.TIN,RISE: $1264120,7-9,23, P L 1,3)$
MIN: TONNAGE. INDEX \& GEOL MAPS. USE: 4.6, CA/MG $=14,7$.

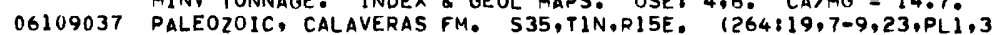

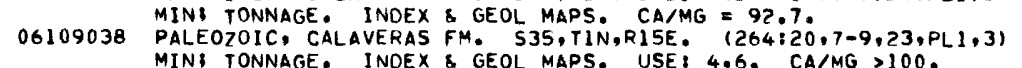

06109039 PALEOZOIC, CALAVERAS FM. S35,TIN,RISE.

06109040 PALEOZOIC, CALAVERAS FM. S35,TIN,RISE.

06109041 PALEOZOIC, CALAVERAS FM. S35.TIN,RI5E. 06109042 PALEOZOIC, CALAVERAS FM. 535,TIN,RI5E.

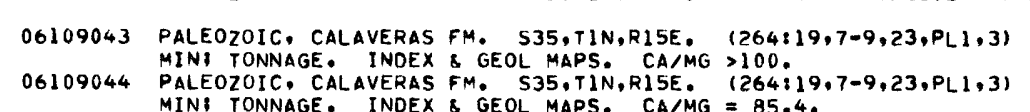

06109045 PALEOZOIC, CALAVERAS FM. S35,TIN,R15E. $(264: 19,7-9,24, P L 1,3)$

LIMESTONE, WHITE TO GRAY, MED-COARSE GR: OUTCROP SMPLI

LIMESTONE, WHITE TO GRAY, MEO-COARSE GRI OUTCROP SMPL;

LIMESTONE, WHITE TO GRAY, MED-COARSE GR: OUTCROP SMPL: 264:19,7-9,PL1,3) DOLOMITE; OUTCROP SMPLI MIN, INDEX \& GEOL MAP. CA/MG 1.84 . 264:19,7-9,PL1,3) DOLOMITE: OUTCROP SMPL; MIN. INDEX \& GEOL MAP. CA/MG=1.87. 264:20,7-9,23,PL1,3) LIMESTONE, WHITE TO GRAY; MEO-COARSE GR OUTCROP SMPL; 6. CA/MG $>100$.
$264: 19.7-9, P L 1,3)$ DOLOMITE: OUTCROP SMPL MIN. INOEX \& GEOL MAP. CA/MGE1.88.

\section{6}

\section{7}

06109048

\section{9}

06109050

06109051

06109052

LIMESTONE, WHITE: OUTRROP SMPLI MIN: TONNAGE. INDEX \& LIMESTONE, WHITE: OUTCROP SMPLI MINI TONNAGE, INDEX \& LIMESTONE, WHITE: OUTCROP SMPL, MIN: TONNAGE, INDEX \& LIMESTONE, WHITE, OUTCROP SMPL, MINi TONNAGE, INDEX \& LIMESTONE, WHITE: OUTCROP SMPL: MIN\& TONNAGE, INDEX \& LIMESTONE, WHITE OUTCROP SMPLI MINI TONNAGE, INDEX \& LIMESTONE, WHITE, GRAY, MOTL, COARSE-GRI OUTCROP SMPL, LIMESTONE, WHITE\& OUTCROP SMPL: MIN\& TONNAGE, INDEX \& LIMESTONE, WHITE, GRAY, MOTL, COARSE-GRI OUTCROP SMPL'? LIMESTONE, WHITE TO GRAY, MED-COARSE GRI OUTCROP SMPL, LIMESTONE, WHITE TO GRAY, MEO-COARSE GRI OUTCROP SMPL, LIMESTONE, WHITE, BANDED, MED-CRYS I OUTCROP SMPL: MIN. , FINE- TO MED-CRYS, PHYS PROP, INDEX MAP. LIMESTONE, WHITE TO GRAY, MED-COARSE GR OUTCROP SMPL: LIMESTONE, WHITE TO GRAY, MED-COARSE GRI OUTCROP SMPL, LIMESTONE, WHITE TO GRAY, MED-COARSE GR OUTCROP SMPLI LIMESTONE, WHITE TO GRAY, MED-COARSE GR: OUTCROP SMPLI LIMESTONE, WHITE TO GPAY, MED-COARSE GRI OUTCROP SMPLI EINED, FINE - TO MED-CRYS, PHYS PROP, INDEX MAP.

IMESTONE, WHITE TO GRAY, MED-COARSE GR OUTCROP SMPLI LIMESTONE, WHITE TO GRAY, MED-COARSE GRI OUTCROP SMPLI IMESTONE, WHITE TO GRAY, MED-COARSE GR OUTCROP SMPLI LIMESTONE, WHITE TO GRAY, MED-COARSE GR OUTCROP SMPL, LIMESTONE, WHITE TO GRAY, MED-COARSE GRI OUTCROP SMPL, LIMESTONE, WHITE TO GRAY, MED-COARSE GRI OUTCROP SMPL: IESTONE: OUTCROP SMPL + MIN, INDEX \& GEOL MAP. PALEOZOIC, CALAVERAS FM. S35,TIN,R1SE, (264:19,7-9,23,PL1,3) MINI TONNAGE. INDEX \& GEOL MAPS. USE: 4,6 . CA/MG $=74.5$ PALEOZOIC, CALAVERAS FM. PALEOTOIC. CALAVERAS FM. S30,TIN,RI6E. (264120,8,19,PL1,3) DOLOMITIC LIMESTONE: OUTCROP SMPL, MIN. INDEX \& GEOL MAPS, CA/MG = 2.36. PALEOZOIC, CALAVERAS FM. S35,TIN,RISE. (264:19,7-9,23,PL1,3) LIMESTONE, WHITE TO GRAY, MED-COARSE GR OUTCROP SMPL

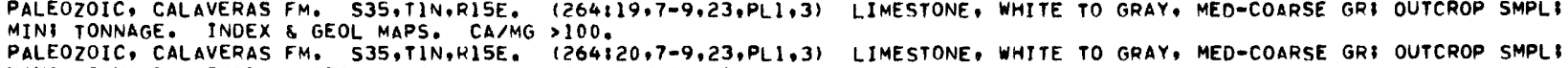

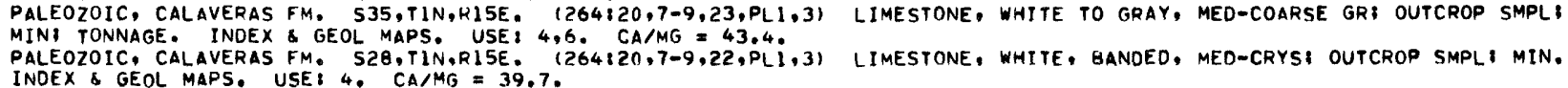

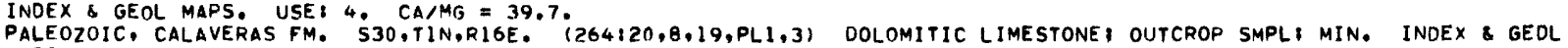
MAPS. CAIMG $=4.31$.
LIMESTONE, WHITE TO GRAY, MEO-COARSE GR: OUTCROP SMPL: LIMESTONE, WHITE TO GRAY, MED-COARSE GR; OUTCROP SMPL, LIMESTONE: OUTCROP: MIN. INOEX \& GEOL MAP. CA/MG=67.3. 
TABLE 9. - Analyses of samples from California and Hawaii containing more than 90 percent carbonate (Group $\left.F_{2}\right)$, common-rock category Continued

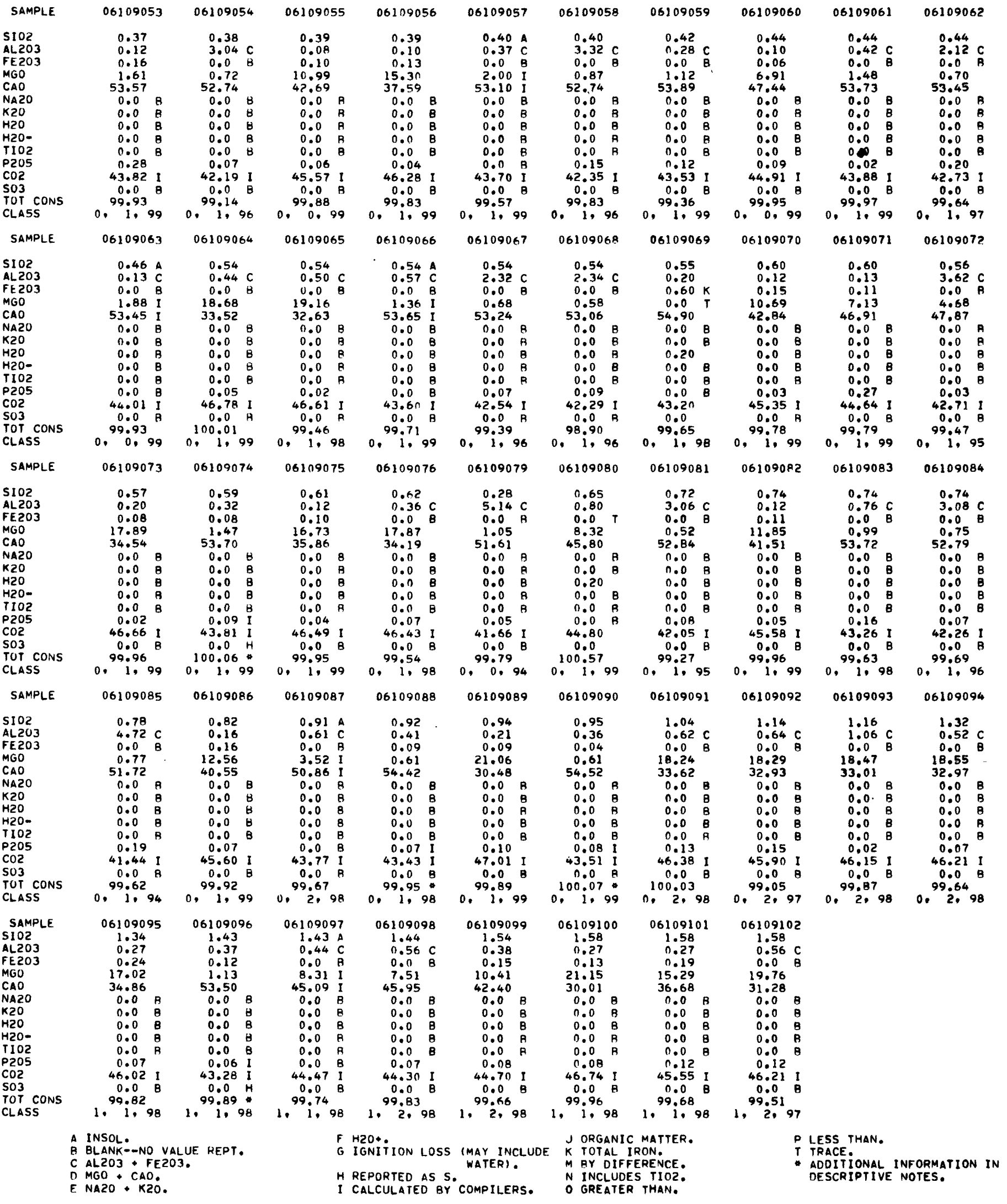


TABLE 9. - Analyses of samples from California and Hawaii containing more than 90 percent carbonate (Group $\left.F_{2}\right)$, common-rock category Continued

06109054

06109055

06109056

06109057

0610905

0610905

06109060

0610906

0610906

06109063

06109064

06109065

06109066

0610906

0610906

0610906

06109070

0610907

0610907

06109073

06109074

06109075

06109076

06109079

06109080

06109081

06109082

06109083

0610908

0610908

06109086

06109087

06109088

0610908

06109090

06109091

06109092

06109093

06109094

06109095

06109096

06109097 06109098

06109099

06109100

0610910

06109102
06109053 PALEOZOIC, CALAVERAS FM. S17,T1N,R15E. (264:20,7-9,22,PL1,3) LIMESTONE, WHITE, BANDED, MED-CRYSI OUTCROP SMPL: MIN. PEOL MAPS. CAMG $=33.3$.

TE. CALAVERAS FM, S35,TIN,PI5E。 PINI TONNAGE. INDEX \& GEOL MAPS. USE: S3I TINORIGE. MAPS. CA/MG $=3.88$. PALEOZOIC. CALAVERAS FM. S34,TIN,R15E. (264:20,8,22,PL1,3) DDLOMITE, WHITE, FINE-GR: OUTCROP SMPLI MIN: TONNAGE. INDEX \& GEOL MAPS. CA/MG 2.46 . "T2N,R14E" COLUMBIA. (556:343.PL37) MARBLE, GRAY. MEO TO COARSELY CRYSI SMPL OF 300 FTI PHYS PROP. INDEX MAP. USE: 15. CAMMG $=26.6$.

PALEOZOIC, CALAVERAS FM. S35,TIN,R15E. (264:20,7-9,23,PL1,3) LIMESTONE, WHITE TO GRAY, MED-COARSE GR: OUTCROP SMPL: MINI TONNAGE. INDEX \& GEOL MAPS, USE: 4,6 . CA/MG $=60.6$.

(264:19,7-9.23,PLI.3) LIMESTONE, WHITE TO GRAY, MED-COBRSE GRI OUTCROP SMPL, PINI TONNAGE. INDEX \& GEOL MAPS, USE $4,6.6$. CA/MG $=48,1$. PALEOZOIC. CALAVERAS FM. S31,TIN,RIGE. (264:20,8,19,PLI,3) DOLOMITIC LIMESTONE: OUTCROP SMPL: MIN. INDEX G GEOL MAPS. CA/MG $=6.87$

PALEOZOIC, CALAVERAS FM. S35,TIN,RI5E, (264:19,7-9,23,PL1,3) LIMESTONE, WHITE TO GRAY, MED-COARSE GRI OUTCROP SMPL, MINI TONNAGE. INDEX \& GEOL MAPS. CA/MG $=36.3$.

PALEOZOIC. CALAVERAS FM. S35,TIN,RISE, (264:20,7-9,PL1,3) LIMESTONE OUTCROP SMPL MIN. INDEX \& GEOL MAP. S20.29,TIN,RISE. (556:347.346.PL37) LIMESTONE, WHITE TD GRAY. FINE- TO MED-CRYS3 SMPL OF 540 FT. INDEX MAP.

PALEOZOIC, CALAVERAS FM. S35,TIN,R15E. (264:19,7-9,23,PL1,3) DOLOMITE, GRAY TO BLACK, WHITE, MOTL, FINE-GRI OUT-

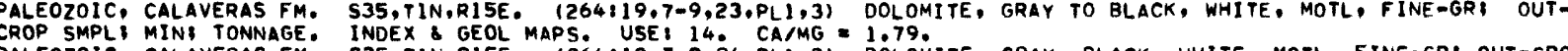

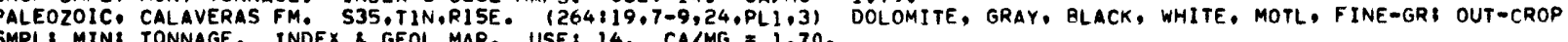
SMPL MIN\& TONNAGE, INDEX \& GEOL MAP, USE: 14 CA/MG $=1.70$

PALEOZOIC, CALAVERAS FM. S35,TIN,R15E, (264:19,7-9,23,PLI.3) LIMESTONE, WHITE TO GRAY, MED-COARSE GR OUTCROP SMPL, MIN: TONNAGE. INDEX \& GEOL MAPS. CA/MG $=78.3$ PALEOZOIC, CALAVERAS FM. S35,TIN,RISE, $1264 i 19,7-9,23, P L 1,3)$ LIMESTONE, WHITE TO GPAY, MED-COARSE GR: OUTCROP SMPL,
MINI TONNAGE. INDEX \& GEOL MAPS. CA/MG $=91.5$.

"T2N,RI4E" COLUMBIA. (556:343.342,PL37) MARBLE. WHITE, BLUE, VEINED, FINE- TO MED-CRYS, PHYS PROP. INDEX MAP. USE: 15.

PALEDZOIC. CALAVERAS FM. $\$ 34, T I N, R 15 E$ INDEX \& GEOL MAPS. CA/MG $=4.01$

PALEOZOIC. CALAVERAS FM. S19,TIN,RIGE.

MAPS. CA/MG $=6.58$.

MAEOZOIC, CALAVERAS FM, S35,TIN,R15E, (264:20,7-9,23,PL1,3) LIMESTONE, WHITE TO GRAY, MED-COARSE GRF OUTCROP SMPL PALEOZOIC, CAL INERAS FM. S2S,TIN,R15E, $(264: 20,7-9, P L 1,3)$ DOLOMITE, GRAY, MED-FINE GR: OUTCROP SMPLI MINI TONNAGE.

PALEOZOIC, CALAVERAS FM. S2S,TIN,R15E. $(264: 20,7-9, P L 1,3)$ DOLOMITE, GRAY, MED $F$ INE GR: OUTCROP SMPL MINI TONNAGE. GEOL SEC. USE: 10 . "MISC: $5=$ TR. CAMG $=36.5$

PALEOZOIC. CALAVERAS FM. S3I.TIN,RI6E. $(264: 20,8,19, P L 1,3)$ DOLOMITIC LIMESTONE OUTCROP SMPL MIN. INOEX \& GEOL PALEOZOIC, CALAVERAS FM. S31,TIN,RI6E. $(264: 20,8,19, P L 1,3)$ DOLOMITIC LIMESTONE OUTCROP SMPL MIN. INOEX \& GEOL
MAPS. CAIMG $=2,14$.

PALEOZOIC, CALAVERAS FM. S35,TIN,RI5E. (264:19,7-9,24,PL1,3) DOLOMITE, GRAY, BLACK, WHITE, MOTL, FINE-GRI OUT-CROP SMPL MIN TONNAGE. INDEX G GEOL MAP, USE: 14, CA/MG $=1.91$.

PALEOZOIC. CALAVERAS FM. S35,TIN.RI5E, (264120,7-9,23,PLi:3) LIMESTONE, WHITE TO GRAY, MED-COARSE GR: OUTCROP SMPLI MINI TONNAGE. INDEX \& GEOL MAPS. USE: 4.6 . CA/MG $=49.2$.

"T2N,RIAE" COLUMBIA. (556:343,342, PL37) "MARBLE, WHITE, BLUE, VEINED, FINE- TO MEO-CRYS, PHYS PROP. INOEX MAP. USE: 15. CA/MG $=5.50$.

PALEOZDIC, CALAVERAS FM. S35,TIN,R15E. (264:20,7-9,23,PL1.3) LIMESTONE, WHITE TO GRAY, MED-COARSE GR: OUTCROP SMPL MIN: TONNAGE. INDEX \& GEOL MAPS. USE : 4,6. CA/MG $>100$.

PALEOZOIC, CALAVERAS FM. S31,TIN,RI6E. $(264120,8,19, P L i, 3)$ DOLOMITIC LIMESTONE, OUTCROP SMPLI MIN. INDEX \& GEOL

MAPS. CA/MG $=3.50$.
PALEOZOIC, CALAVERAS FM. S35,TIN,R15E, (264:19,7-9,23,PL 1,3$)$ LIMESTONE, WHITE TO GRAY, MED-COARSE GR: OUTCROP SMPL MIN: TONNAGE. INDEX \& GEOL MAPS. CA/MG $=54.3$.

PALEOZOIC, CALAVERAS FM. S35,TIN,RISE. (264:20,7-9.23,PL1,3) LIMESTONE. WHITE TO GRAY, MED-COARSE GR: OUTCROP SMPL

MIN: TONNAGE. INDEX \& GEOL MAPS. USE: 4,6 C CAMMG $=70.4$; 31 LIMESTONE, WHITE TO GRAY, MED-COARSE GR: OUTCROP SMPL PALEOZOIC, CALAVERAS FM. S35,TIN,R15E, $1264: 20,7-9,23, P L 1.3$

PALEOZOIC, CALAVERAS FM. S31,TIN,RI6E. $(264: 20,8,19,971,3)$ DOLOMITIC LIMESTONE, OUTCROP SMPL: MIN. INDEX \& GEOL MALEOZOIC, CALAVERAS

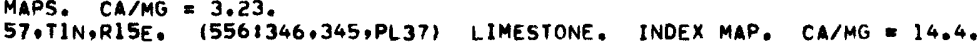

PALEOZOIC. "ST,TIN,RISE" (280:S12,PL24) LIMESTONE. GRAY. WHITE, BANDED, CRYSI OUTCROP SMPL OF 1O5 FT. INIEX \& GEOL MAP. GEOL SEC. MISC: S. TR. CA/MG $=89.2$

PALEOZOIC, CALAVERAS FM. S25,TIN,RI5E. (264:20,7-9,PL1.3) DOLOMITE, GRAY. MED-FINE GR, OUTCROP SMPL: MINE TONNAGE. INDEX \& GEOL MAPS. CAIMG $=1.45$. PALEOZOIC. "S7.TIN,RISE" (280:512,510,PL24) LIMESTONE, GRAY, WHITE, BANDED, CRYSI 51-FT SMPL. INDEX \& GEOL MAPS, GEOL
SEC. *MISC: S. TR. CA/MG $=89.4$.

PALEOZOIC, CALAVERAS FM. S3S,TIN,RISE, (264:19,7-9,PLJ,3) DOLOMITE, OUTCROP SMPL, MIN. INDEX \& GEOL MAP. CAJMG I. A4 PALEOZOIC, CALAVERAS FM. S35,TIN,R15E, 1264:19,7-9,24,PL1,3) DOLOMITE, GRAY, BLACK, WHITE, MOTL, FINE-GR: OUT-CROP SMPL: MINi TONNAGE. INDEX \& GEOL MAP, USE: 140 CA/MG $=1.80$

PALEOZOIC. CALAVERAS FM. S35.TIN,RI5E. (264:19,7-9,PL1,3) DOLOMITE: OUTCROP SMPL: MIN. INDEX \& GEOL MAP. CA/MG= 1.79

PALEOZOIC, CALAVERAS FM, S35.TIN,R15E, (264119,7-9,24,PL1,3) DOLOMITE, GRAY, BLACK, WHITE, MOTL, FINE-GR: OUT-CROP SMPL: MIN: TONNAGE. INDEX \& GEOL MAP, USE: 14. CA/MG $=1.78$

PALEOZOIC, CALAVERAS FM. S34,TIN,R15E. $(264: 20.8,22, P L 1,3 ;$ DOLOMITE. WHITE, FINE-GR: OUTCROP SMPL, MINI TONNAGE. PALEOZOIC, CALAVERAS FM. $534 . T 1 N$.
INDEX \& GEOL MAPS. CAMG $=2.05$.

INDEX \& GEOL MAPS. CA/MG 2.05 .
PALEOZOIC. S6,TIN,RISE. $(280: 512,510, P L 24)$ LIMESTONE, GRAY, WHITE, BANDED, CRYSI $110-F Y$ SMPL. INDEX \& GEOL

MAP, GEOL SEC. USE: 10 . MMISCIS TR. CAMMG $=47.3$.

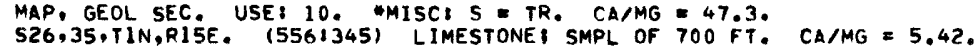

PALEOZOIC, CALAVERAS FM. S35,TIN,RISE, $1264119,7-9,9 L 1.31$ DOLOMITIC LIMESTONE OUTCROP SMPLI MIN. INOEX \& GEOL MAPS. CAIMG $=6.12$.

PALEOZOIC, CALAVERAS FM. S19.TIN.RIGE.

MAPS. CA/MG $=4.07$.

PALEOZOIC. CALAVERAS FM. S25,TIN,RI5E.

INDEX \& GEOL MAPS. CAMG $=1.42$.

PALEOZOIC, CALAVERAS FM. S3I,TIN,RIGE.

MAPS. CA/MG $=2.40$.

PALEOZOIC. CALAVERAS FM. S35.TIN,RISE.

CA/MG $=1.58$.

(264:20,8,19,PL1,3) DOLDMITIC LIMESTONE: OUTCROP SMPL: MIN. INDEX \& GEOL

(264:20,7-9,PL1,3) DOLOMITE, GRAY, MED-FINE GRI OUTCROP SMPL: MIN: TONNAGE.

$(264: 20,8,19, P L 1,3)$ DOLOMITIC LIMESTONE; OUTCROP SMPL: MIN, INOEX \& GEOL

(264119,7-9,PL3) OOLOMITEI OUTCROP SMPLI MIN. INDEX \& GEOL MAPS. 
TABLE 9. - Analyses of samples from California and Hawaii containing more than 90 percent carbonate (Group $\left.F_{2}\right)$, common-rock category Continued

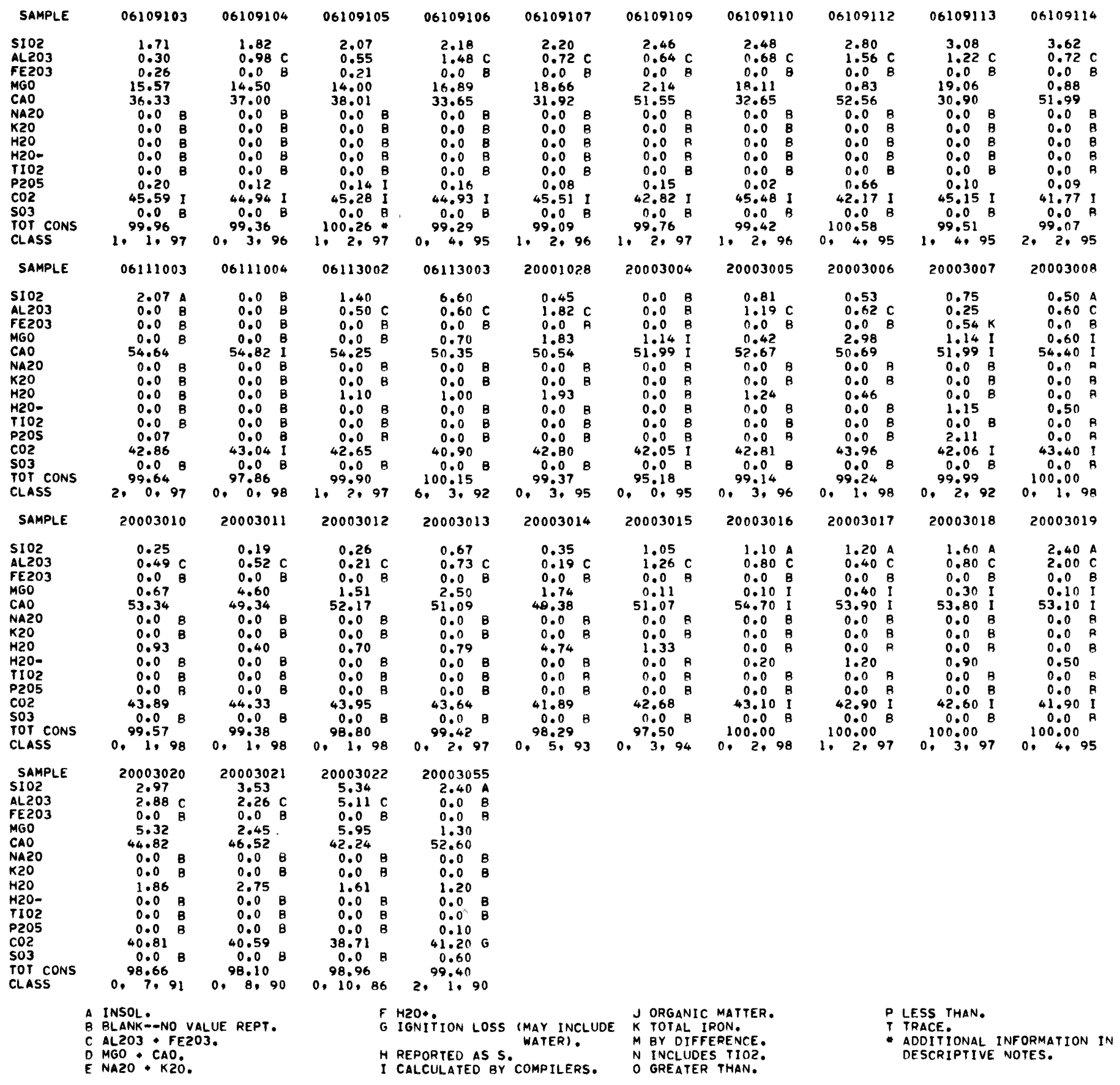


TABLE 9. - Analyses of samples from California and Hawaii containing more than 90 percent carbonate (Group $\left.F_{2}\right)$, common-rock category Continued

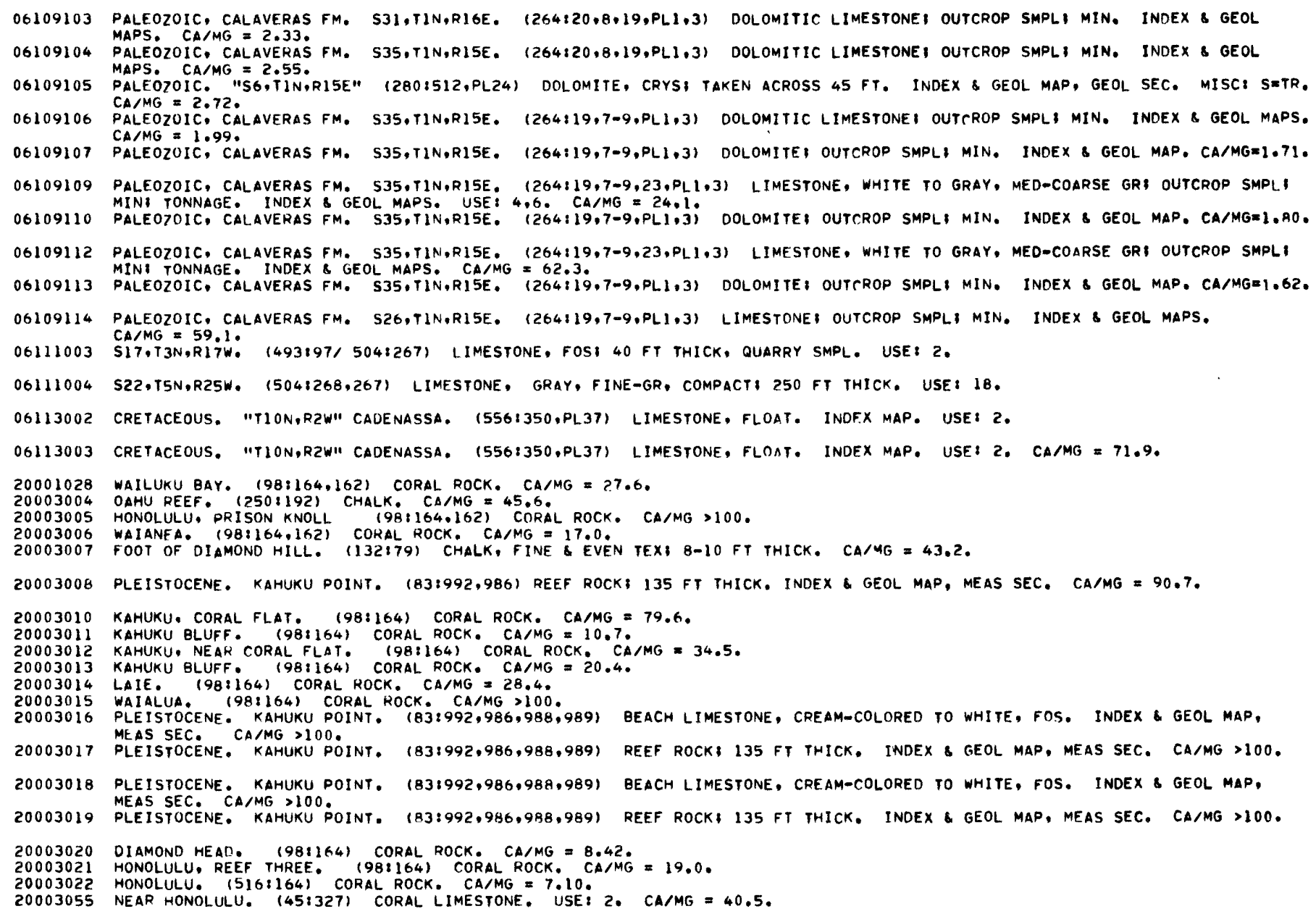


TABLE 10. - Analyses of samples from California 50 percent or more sodium chloride (Group $\mathrm{Na}$ ), special rock category [Amounts in parentheses are not included in total]

\begin{tabular}{|c|c|c|c|c|c|c|c|c|c|c|}
\hline Sample & 06027081 & 06027083 & 06027085 & 06027086 & 06027092 & 06027093 & 06027094 & 06027095 & 06065094 & 06065095 \\
\hline Insoluble & 0.17 & 0.03 & $\ldots$ & 0.50 & --.- &.--- & -... & -..- & -... & 0.20 \\
\hline KCl & $\cdots$ & $\ldots$ & $\ldots$ & .31 & -..- & -..- & -... & $\ldots$ & $\ldots$ & $\ldots$ \\
\hline $\mathrm{MgCl}_{2}$ & $\cdots$ & $\cdots$ & $.00 \mathrm{~T}$ & $\cdots$ & $\cdots$ & $-\cdots$ & $\cdots$ & .60 & $\ldots$ & $\ldots$ \\
\hline $\mathrm{NaCl}$ & 98.52 & 98.71 & 95.49 & 94.54 & 84.0 & 49.1 & 47.9 & 95.51 & 94.68 & 97.76 \\
\hline $\mathrm{CaSO}_{4}$ & -- & .00 & .27 & -... & $\cdots$ & $\ldots$ & 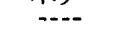 & 1.01 & .77 & .38 \\
\hline $\mathrm{CaSO}_{4} \cdot 2 \mathrm{H}_{2} \mathrm{O}$ & --- & 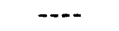 & $\ldots$ & .79 & $\ldots$ & -..- & --.- & $\cdots$ & $\cdots$ & $\cdots$ \\
\hline $\mathrm{CO}_{3}$ & .00 & $\cdots$ & $\ldots$ & --- & --- & $-\cdots$ & $\cdots$ & -..- & -..- & $\ldots$ \\
\hline $\mathrm{HCO}_{3}$ & .00 & -.- & $\cdots$ & $\cdots$ & $\ldots$ & -.- & -..- & $\cdots$ & -..- & $\ldots .$. \\
\hline $\mathrm{K}_{2} \mathrm{SO}_{4}$ & .37 & , - - & $\cdots$ & --- & $\cdots$ & $\cdots$ & $\cdots$ & $\cdots$ & -..- & $\ldots$ \\
\hline $\mathrm{MgCO}_{3}$ & $\ldots$ & .00 & -... & -... & $\cdots$ & $\cdots$ & $+\cdots$ & $\ldots$ & -..- & -..- \\
\hline $\mathrm{MgSO}_{4}$ & --- & $\cdot .00$ & $\cdots$ & $\cdots$ & $\cdots$ & --- & $-\cdot-$ & -..- & 3.12 & -..- \\
\hline $\mathrm{NaBO}_{2}$ & $\cdots$ & .00 & $-\cdots$ & --- & --- & $=--$ & $\cdots$ & -...- & $\cdots$ & $\cdots$ \\
\hline $\mathrm{NaNO}_{3}$ & $\cdots$ & $\cdots$ & $\cdots$ & $--\cdot$ & 12.5 & 48.5 & 50.6 & $-\cdots$ & $\cdots$ & .... \\
\hline $\mathrm{Na}_{2} \mathrm{SO}_{4}$ & 1.02 & 1.26 & 2.78 & 3.53 & $\ldots$ & -... & $\ldots$ & 2.44 & .68 & .70 \\
\hline $\mathrm{Ca}^{-}$ & .00 & $\cdots$ & $\cdots$ & $\cdots$ & $\cdots$ & $--\cdot$ & $\cdots$ & $-\ldots$ & $\cdots$ & $\cdots$ \\
\hline $\mathrm{Mg}$ & .00 & $\cdots$ & +- & -- & $\cdots$ & --- & $\cdots$ & $-\cdots$ & 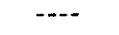 & $\cdots$ \\
\hline $\begin{array}{l}\mathrm{H}_{2} \mathrm{O}+ \\
\mathrm{H}_{2} \mathrm{O}-\end{array}$ & $j .12$ & $\cdots$ & $\cdots$ & $\{.14$ & $\cdots$ & $\cdots$ & $\cdots$ & $\cdots$ & 3.75 & .96 \\
\hline Total & 100.20 & 100.00 & 98.54 & 99.81 & 96.5 & 97.6 & 98.5 & 99.56 & 100.00 & 100.00 \\
\hline Sermple & 06071090 & 06071239 & 06071240 & 06071241 & 06071242 & 06071243 & 06071244 & 06071245 & 06071246 & \\
\hline Insoluble & 1.4 & 1.81 & 1.46 & 3.76 & 1.5 & 5.9 & 5.8 & 4.8 & 0.08 & \\
\hline $\mathrm{CaO}$ & $\cdots$ & $-\cdots$ & --- & $\cdots$ & 1.2 & 3.8 & 6.4 & 2.9 & .05 & \\
\hline $\mathrm{CO}_{2}$ & $\cdots$ & $\cdots$ & $\cdots$ & $\cdots$ & 10.4 & 16.3 & 18.7 & 14.2 & 3.8 & \\
\hline $\mathrm{K}_{2} \mathrm{O}$ & $\cdots$ & 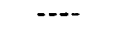 & $\cdots$ & $\ldots$ & .43 & 1.0 & 1.1 & .89 & .27 & \\
\hline $\mathrm{MgO}$ & -- & $-\cdots$ & $\cdots$ & $-\cdots$ & .64 & 2.1 & 1.6 & 1.0 & .03 & \\
\hline NaCl & 44.0 & $\cdots$ & $\cdots$ & $\cdots$ & $-\cdots$ & $\cdots$ & -... & $\ldots$ & -.. & \\
\hline $\mathrm{Na}_{2} \mathrm{O}$ & -.. & --- & $\cdots$ & -..- & 47.5 & 36.9 & 35.8 & 41.0 & 50.7 & \\
\hline $\mathrm{B}_{2} \bar{O}_{3}$ & $\cdots$ & $\cdots$ & $\cdots$ & $\cdots$ & .19 & 2.0 & .85 & .78 & .41 & \\
\hline$B_{4} 0_{7}$ & $\cdots$ & .12 & .02 & .02 & $--\cdot$ & $\cdots$ & $\cdots$ & $\cdots$ & --- & \\
\hline $\mathrm{CO}_{3}$ & $\cdots$ & 2.4 & .9 & 1.0 & $\cdots$ & $\cdots$ & $\cdots$ & $\cdots$ & $\cdots$ & \\
\hline $\mathrm{HCO}_{3}$ & $\cdots$ & 1.0 & 1.4 & .08 & $\cdots$ & -..- & $\cdots$ & $\cdots$ & $\cdots$ & \\
\hline $\mathrm{NaB}_{4} \mathrm{O}_{7}$ & 1.0 & & & $\cdots$ & $\cdots$ & $\cdots$ & $\cdots$ & $\cdots$ & $\cdots$ & \\
\hline $\begin{array}{l}\mathrm{Na}_{2} \mathrm{CO}_{3} \\
\mathrm{NaRCO}_{3}\end{array}$ & $\begin{array}{r}14.8 \\
2.5\end{array}$ & $\ldots$ & --- & $\cdots$ & $\cdots$ & -...- & $\cdots$ & $\cdots$ & $--\cdot$ & \\
\hline $\begin{array}{l}\mathrm{NaBCO}_{3} \\
\mathrm{Na}_{2} \mathrm{SO}_{4}\end{array}$ & $\begin{array}{r}2.5 \\
30.5\end{array}$ & $\ldots$ &.-- & $\cdots$ & $\cdots$ & -..- & $-\cdots$ & $\cdots$ & -..- & \\
\hline $\mathrm{SO}_{\mathrm{s}}$ & -... & $-\cdots$ & $\ldots$ & $\cdots$ & 1.0 & 1.3 & 3.7 & 5.3 & 6.2 & \\
\hline $\mathrm{SO}_{4}$ & $\cdots$ & 8.9 & .5 & .3 & --- & --- & $\ldots$ & $\ldots$ & $\cdots$ & \\
\hline $\mathrm{Cl}$ & $\cdots$ & 47.4 & 56.9 & 56.5 & 40.5 & 26.0 & 21.5 & 27.3 & 46.5 & \\
\hline $\mathbf{K}$ & $\cdots$ & .17 & .02 & .04 & $\ldots$ & -.. & $\cdots$ & -- & $\cdots$ & \\
\hline \multirow{2}{*}{$\begin{array}{l}\mathrm{Na} \\
\mathrm{H}_{2} \mathrm{O} \\
\text { Less } \mathrm{O}=\mathrm{Cl}\end{array}$} & $=-$ & 38.2 & 38.8 & 38.3 & ---- & --- & -- & ---- & $=-$ & \\
\hline & $\begin{array}{l}5.8 \\
-\cdots\end{array}$ & -- & $\ldots$ & $\cdots$ & $\begin{array}{c}5.7 \\
(9.14)\end{array}$ & $\begin{array}{c}9.6 \\
(5.87)\end{array}$ & $\begin{array}{l}10.1 \\
(4.85)\end{array}$ & $\begin{array}{l}7.6 \\
(6.16)\end{array}$ & $\begin{array}{c}1.7 \\
(10.49)\end{array}$ & \\
\hline Total & 100.0 & 100.00 & 100.00 & 100.00 & 99.92 & 99.03 & 100.70 & 99.61 & 99.25 & \\
\hline$\cdots-\cdots$ & & & - & & & & & & & \\
\hline Sample & 06071247 & 06071252 & 06071253 & 06071254 & 06071255 & 06071256 & 06071260 & 06071261 & 06071263 & 06071264 \\
\hline \multirow{9}{*}{$\begin{array}{l}\text { Insoluble } \\
\mathrm{CaO} \\
\mathrm{CO}_{2} \\
\mathrm{~K}_{2} \mathrm{O} \\
\mathrm{MgO} \\
\mathrm{Na}_{2} \mathrm{O} \\
\mathrm{B}_{2} \mathrm{O}_{3} \\
\mathrm{SO}_{3} \\
\mathrm{Cl} \\
\mathrm{H}_{2} \mathrm{O} \quad \\
\text { Less } \mathrm{O}=\mathrm{Cl} \\
\quad \text { Total }\end{array}$} & 1.6 & 3.8 & 3.4 & 8.3 & 0.12 & 2.1 & 0.23 & 6.6 & 0.09 & \\
\hline & $\begin{array}{l}1.5 \\
9.4\end{array}$ & $\begin{array}{r}3.2 \\
20.1\end{array}$ & $\begin{array}{c}.34 \\
11.3\end{array}$ & $\begin{array}{r}7.2 \\
17.0\end{array}$ & $\begin{array}{l}.16 \\
7.6\end{array}$ & $\begin{array}{r}2.1 \\
12.4\end{array}$ & 26.2 & $\begin{array}{r}5.3 \\
13.2\end{array}$ & 9.8 & $\begin{array}{r}7.0 \\
17.1\end{array}$ \\
\hline & .44 & .72 & .33 & .95 & .20 & .33 & .38 & .99 & .35 & $\begin{array}{l}1.3 \\
2.2\end{array}$ \\
\hline & .40 & .92 & 47.44 & $\begin{array}{r}2.0 \\
33.6\end{array}$ & .04 & .58 & .08 & $\begin{array}{r}1.8 \\
35.8\end{array}$ &. .12 & $\begin{array}{r}2.2 \\
31.6\end{array}$ \\
\hline & $\begin{array}{c}46.2 \\
.29\end{array}$ & $\begin{array}{r}38.8 \\
.34\end{array}$ & $\begin{array}{c}47.2 \\
.18\end{array}$ & $\begin{array}{r}33.6 \\
1.5\end{array}$ & $\begin{array}{r}48.6 \\
1.5\end{array}$ & $\begin{array}{r}42.5 \\
1.1\end{array}$ & $\begin{array}{c}43.5 \\
.15\end{array}$ & $\begin{array}{r}35.8 \\
1.3\end{array}$ & $\begin{array}{l}48.7 \\
.10\end{array}$ & $\begin{array}{r}31.6 \\
1.5\end{array}$ \\
\hline & 9.2 & 10.0 & 3.3 & 6.5 & 8.3 & $14 . \overline{6}$ & 1.6 & 3.4 & 1.0 & 2.3 \\
\hline & 33.2 & 12.4 & 39.1 & 15.3 & 37.1 & 20.4 & 17.1 & 25.3 & 44.0 & 18.6 \\
\hline & $\begin{array}{l}5.1 \\
(7.49)\end{array}$ & $\begin{array}{l}10.0 \\
(2.80)\end{array}$ & $\begin{array}{l}5.8 \\
(8.82)\end{array}$ & $\begin{array}{c}9.6 \\
(3.45)\end{array}$ & $\begin{array}{l}4.0 \\
(8.37)\end{array}$ & $\begin{array}{c}7.0 \\
(4.60)\end{array}$ & $\begin{array}{l}13.4 \\
(3.86)\end{array}$ & $\begin{array}{c}9.6 \\
(5.71)\end{array}$ & $\begin{array}{c}4.8 \\
(9.93)\end{array}$ & $\begin{array}{l}10.2 \\
(4.20)\end{array}$ \\
\hline & 99.84 & 97.48 & 102.57 & 98.50 & 99.25 & 98.51 & 98.98 & 97.58 & 99.19 & 97.60 \\
\hline \multirow{11}{*}{$\begin{array}{l}\text { Sample } \\
\text { Insoluble } \\
\mathrm{CaO} \\
\mathrm{CO}_{2} \\
\mathrm{~K}_{2} \mathrm{O} \\
\mathrm{MgO} \\
\mathrm{Na}_{2} \mathrm{O} \\
\mathrm{B}_{2} \mathrm{O}_{3} \\
\mathrm{SO}_{3} \\
\mathrm{Cl} \\
\mathrm{H}_{2} \mathrm{O} \\
\text { Less } \mathrm{O}=\mathrm{Cl}\end{array}$} & 06071265 & 06071266 & 06071271 & 06071272 & 06071273 & 06071274 & 06071276 & 06071277 & 06071278 & 06071280 \\
\hline & 0.18 & 0.20 & 7.6 & 2.7 & 0.84 & 0.22 & 5.4 & 0.51 & 0.08 & 7.6 \\
\hline & .22 & .46 & 1.4 & 1.5 & .55 & .09 & 5.4 & .63 & .10 & 1.4 \\
\hline & 11.0 & 9.8 & 3.2 & 5.2 & 7.8 & 7.6 & 9.2 & 8.2 & 9.3 & 3.2 \\
\hline & & .23 & 1.1 & 1.1 & 1.2 & .47 & .59 & .35 & 2.0 & 1.0 \\
\hline & .08 & .14 & .49 & .35 & .16 & .04 & 1.1 & .16 & .03 & .32 \\
\hline & 47.4 & 46.2 & 43.8 & 45.6 & 46.3 & 49.0 & 40.4 & 47.8 & 45.2 & 43.4 \\
\hline & 1.7 & 2.2 & .23 & .48 & 1.2 & .08 & .15 & .10 & .10 & .12 \\
\hline & 13.0 & 16.2 & 6.8 & $\begin{array}{r}9.9 \\
38.8\end{array}$ & 9.6 & 8.3 & .71 & 9.6 & $\begin{array}{l}23.9 \\
18.8\end{array}$ & $\begin{array}{r}9.4 \\
38.3\end{array}$ \\
\hline & 26.9 & 24.6 & 41.2 & 38.8 & 36.1 & 40.6 & $37 \cdot 9$ & 38.4 & 18.8 & 38.3 \\
\hline & $\begin{array}{l}5.2 \\
(6.07)\end{array}$ & $\begin{array}{l}4.8 \\
(5.55)\end{array}$ & $\begin{array}{l}2.5 \\
(9.30)\end{array}$ & $\begin{array}{l}2.9 \\
(8.76)\end{array}$ & $\begin{array}{c}4.4 \\
(8.15)\end{array}$ & $\begin{array}{l}3.3 \\
(9.16)\end{array}$ & $\begin{array}{c}5.1 \\
(8.56)\end{array}$ & $\begin{array}{l}3.4 \\
(8.67)\end{array}$ & $\begin{array}{c}3.6 \\
(4.24)\end{array}$ & $\begin{array}{l}2.0 \\
(8.65)\end{array}$ \\
\hline Total & 99.86 & 99.28 & 99.02 & 99.77 & 100.00 & 100.54 & 97.39 & 100.48 & 98.87 & $98.09^{-}$ \\
\hline
\end{tabular}


TABLE 10. - Analyses of samples from California 50 percent or more sodium chloride (Group $\mathrm{Na}$ ), special-rock category-Continued

06027081 HOLOCENE. T145,R38E, SALINE VALLEY. $(210: 418,417)$ SALT CRUST, WHITE. INDEX MAP.

06027083 T145,R38E, SALINE VALLEY, (505:498,PL3) SALT, 30 FT THICK. INDEX MAP.

06027085 "T25N,R5E" DEATH VALLEY. (209:410,408,409) SALT I-3 IN THICK. INDEX MAP.

06027086 TERTIARY. "T25,26N,RIE" DEATH VALLEY. (79:18,17,PL1) SALT, BROWNI CRUST, I FT THICK. INDEX MAP.

06027092 "T22N,R2E" AMARgOSA RIVER, UPPER CANYON. $(30: 182,174)$ NITER. INDEX MAP.

06027093 "T22N,R2E" AMARgOSA RIVER, UPPER CANYON. (308182,174) NITER, INOEX MAP.

06027094 "TZZN,RZE" AMARGOSA RIVER, UPPER CANYON. $(301182,174)$ NITER. INDEX MAP.

06027095 "T2IS,R42E" PANAMINT VALLEY, (613:176) SALT, 1-2 FT THICK.

06065094 "TBS,RIOE" SALTON SEA. (30:124,126) SALT CRUSTI 10-20 IN THICK. INDEX MAPS, MEAS SEC.

06065095 "T8S.P1OE" SALTON SEA. (301124,126) SALT CRUST/ 10-20 IN THICK. INDEX MAPS.

06065098 "TBS,RIOE" SALTON. (607:289) ROCK SALT.

06071090 S25,T25S,R43E. (601:278,275,PL5) SALT: DEPTH, 18-25 FT. INDEX MAPS.

06071239 PLEISTOCENE, S25,T25S,R43E, SEARLES LAKE. (466:28,11) HALITE, CORE SMPL, DEPTH, 580.0-583.0 FT, MIN: ANAL OF SOLUBLE 06071240 PLEISTOCENE. S25,T25S.R43E. SEARLES LAKE. PLEISTOCENE. S25,T25S
PORTION. INDEX MAP.

06071241 PLEISTOCENE. S25,T255,R43E, SEARLES LAKE. PORTION. INDEX MAP.

06071242 PLEISTOCENE. S30,T255,R44E, SEARLES LAKE. INDEX MAP.

06071243 PLEISTOCENE. S30,T25S,R44E, SEARLES LAKE. INDEX MAP.

06071244 PLEISTOCENE, S30,T25S,R44E, SEARLES LAKE. INDEX MAP.

06071245 PLEISTOCENE. S30,T25S,R44E, SEARLES LAKE.

06071245 PLEISTOCENE, S30,T25S,R44E, SEARLES LAKE.

PLEISTOCENE. S30, T25S,R44E, SEARLES LAKE. INDEX MAP.

06071247 PLEISTOCENE. S30.T255,R44E, SEARLES LAKE.

06071247 PLEISTOCENE. S30,T25S,R44E, SEARLES LAKE.
06071252 PLEISTOCENE. $53, T 265, R 43 E$, SEARLES LAKE. 06071253 PLEISTOCENE. S3.T26S,R43E, SEARLES LAKE. 06071254 PLEISTOCENE. S3.T265,R43E, SEARLES LAKE. INDEX MAP.

06071255 PLEISTOCENE. S3,T26S,R43E, SEARLES LAKE. INDEX MAP.

06071256 PLEISTOCENE. S3,T265,R43E. SEARLES LAKE. 06071260 PLEISTOCENE. S27,T25S,R43E, SEARLES LAKE. (466128.11) MALITE, CORE SMPL DEPTH, 640.3-650.0 FT: MINI ANAL OF SOLUBLE (466128,11) HALITE, CORE SMPL; OEPTH, 722.8-730.0 FTI MIN: ANAL OF SOLUBLE (466:73,11) HALITE, CORE SMPL, DEPTH, 83.5-87.5 FT: MINI TONNAGE. (466:73,11) HALITE, CORE SMPLI DEPTH, 87.5-92.0 FTI MIN, TONNAGE. (466173,11) HALITE, CORE SMPL, DFPTH, 92.0-94.2 FTI MINI TONNAGE, (466:73,11) MALITE, CORE SMPLI DEPTH, 94.2-100.0 FT: MIN: TONNAGE, (466:73,11) HALITE, CORE SMPL: OEPTH, 100.0-104.8 FT: MINI TONNAGE. (466173,11) HALITE, CORE SMPL: DEPTH, 104.8-109.7 FTI MINI TONNAGE. (466173,11) TRONA, CORE SMPL, DEPTH, 88.1-94.9 FTI MIN: TONNAGE, INDEX MAP. (466173.11) HALITE, CORE SMPL DEPTH, 94.9-98.0 FT, MINI TONNAGE. INDEX MAP. (466:73.11) HALITE, CORE SMPL, OEPTH, 98.0-102.4 FT, MIN, TONNAGE. $(466: 73,11)$ HALITE, CORE SMPL, DEPTH, 102.4-110.0 FT MIN, TONNAGE. (466173.11) HALITE, CORE SMPLI DEPTH, 110.0-114.3 FT? MINI TONNAGE.

(466:73,11) TRONA, CORE SMPL: DEPTH, 83.7-86.8 FT, MINI TONNAGE. INDEX MAP. 06071261 PLEISTOCENE. S27.T25S,R43E, SEARLES LAKE. INDEX MAP.

PLEISTOCENE, S27,T25S,R43E, SEARLES LAKE。 INDEX MAP.

INDEX MAP. PLEISTOCENE. S27, T25S,R43E, SEARLES LAKE.
INDEX MAP. INDEISTOCENE. S27, T25S.R43E, SEARLES LAKE。 INDEX MAP. PLEISTOCENE. S27,T25S.K43E, SEARLES LAKE. INDEX MAP.

$(466: 73,11)$ HALITE, CORE SMPL, DEPTH, 85.8-91.4 FTI MTNI TONNAGE.

(466:73,11) HALITE, CORE SMPL: DEPTH, 92.6-97.1 FT, MINI TONNAGE.

(466:73,11) PIRSSONITE, CORE SMPL DEPTH, 97.1-101.9 FTI MIN: TONNAGE.

(466:73,11) HALITE, CORE SMPLI DEPTH, 101.9-105.6 FT: MINI TONNAGE.

(466:73.11) HALITE, CORE SMPL, DEPTH, 105.6-111.3 FT: MINI TONNAGE.

(466:105,11) HALITE, CORE SMPL; DEPTH, 0-17.1 FT: MIN; TONNAGE. INDEX MAP. 06071271 HOLOCENE. S30.T25S,R44E, SEARLES LAKE. 06071272 HOLOCENE. S30,T25S,R44E, SEARLES LAKE. 06071273 HOLOCENE. S30,T25S,R44E, SEARLES LAKE. 06071274 HOLOCENE, S30,T25S,R44E, SEARLES LAKE. 06071276 HOLOCENE. S3,T265,R43E. SEARLES LAKE. 1466:105,11) HALITE, CORE SMPL, DEPTH, 17.1-30.7 FT: MIN, TONNAGE. INOEX MAP. (466:105,11) HALITE, CORE SMPL, DEPTH, 30.7-55.6 FT: MIN: TONNAGE. INDEX MAP. (466:105,11) HALITE, CORE SMPL: DEPTH, 55.6-68.6 FT: MINI TONNAGE. INDEX MAP. 06071277 HOLOCENE. S3,T265,R43E, SEARLES LAKE. (466:105,11) HALITE, CORE SMPL: DEPTH, 26.9-33.3 FTI MIN! TONNAGE. INDEX MAP. 06071278 HOLOCENE. S3,TZ6S,R43E, SEARLES LAKE. $1466: 105,11$ HALITE, CORF SMPL: DEPTH, 33.3-60.7 FT: MIN, TONNAGE, INDEX MAP. 
TABLE 10. - Analyses of samples from California 50 percent or more sodium chloride (Group Na), special-rock category-Continued

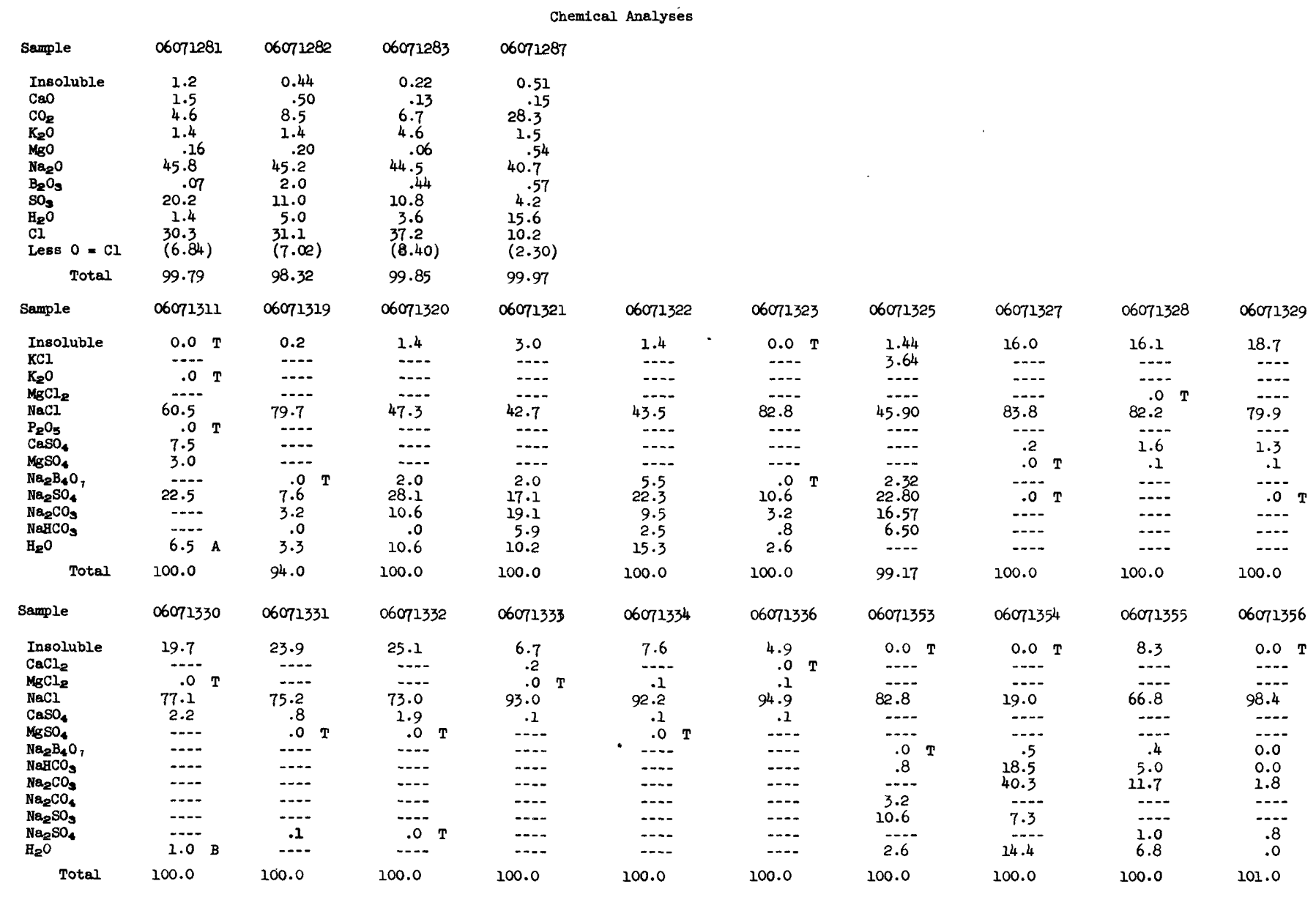

\begin{tabular}{|c|c|c|c|c|c|c|c|c|}
\hline Sample & 06071358 & 06071365 & 06071366 & 06071373 & 06071374 & 06073035 & 06107018 & 06107019 \\
\hline Insoluble & 15.0 & -..- & ...- & --.- & -... & 0.20 & -..- & $\cdots$ \\
\hline $\mathrm{CO}_{2}$ & .... & -..- & -.-- & $\ldots$ & $\ldots$ & $\cdots$ & 11.62 & --- \\
\hline ICI & $\cdots$ & $\ldots$ & $\ldots$ & $\cdots$ & $\cdots$ & .27 & $--\bar{x}$ & $\cdots$ \\
\hline $\mathrm{K}_{\mathbf{2}} \mathrm{O}$ & $\cdots$ & $-\cdots$ & $\cdots$ & $\cdots$ & $\cdots--$ & & 1.76 & --- \\
\hline $\mathrm{NaCl}$ & 39.6 & $>95$. & $\cdots$ & $\cdots$ & $\cdots$ & $97 \cdot 76$ & $\cdots$ & 12.21 \\
\hline $\mathrm{Na}_{2} \mathrm{O}$ & $\cdots$ & -..- & $\cdots$ & $\cdots$ & $\cdots$ & $\cdots$ & $\begin{array}{l}38.39 \\
10.50\end{array}$ & $\cdots$ \\
\hline $\begin{array}{l}\mathrm{N}_{2} \mathrm{O}_{5} \\
\mathrm{~B}_{4} \mathrm{O}\end{array}$ & $\ldots$ & $\ldots$ & .... & .02 & .12 & -... & 10.50 & --.- \\
\hline $\mathrm{CO}_{3}$ & .... & ....- & ...- & 1.0 & 2.4 & -...- & $-\ldots$ & $-\ldots$ \\
\hline $\mathrm{HCO}_{3}$ & --- & --.- & ---- & .08 & 1.0 & ---- & --. & $\cdots-$ \\
\hline $\mathrm{K}_{2} \mathrm{SO}_{4}$ & & $-\cdots$ & $-\cdots$ & $-\cdots-$ & $\cdots$ & $-\cdots$ & $\cdots$ & 3.25 \\
\hline $\mathrm{Na}_{2} \mathrm{~B}_{4} \mathrm{O}_{7}$ & 5.56 & $\cdots$ & $\cdots$ & $\cdots$ & $\cdots$ & $\cdots$ & $\ldots$ & 27.02 \\
\hline NeHCO & 3.4 &.-- & . & $\ldots$ & -... & $\ldots$ & $\ldots$ & \\
\hline $\mathrm{NaNO}_{3}$ & $\cdots$ & $\cdots$ & 95.7 & $\cdots$ & $-\cdots-$ & $-\ldots$ & $\cdots$ & 16.40 \\
\hline $\mathrm{Na}_{3} \mathrm{PO}_{4}$ & & $-\cdots$ & ---- & $-\cdots$ & $\cdots$ & $\cdots$ & $\cdots$ & 1.87 \\
\hline $\mathrm{Na}_{2} \mathrm{SO}_{4}$ & 2.3 & $\cdots$ & $\cdots$ & $\cdots$ & $\cdots$ & .70 & $\cdots$ & 20.91 \\
\hline$\left(\mathrm{NH}_{4}\right)_{2} \mathrm{CO}_{3}$ & & $\cdots$ & $\cdots$ & $-\cdots$ & $\cdots$ & -..- & $\cdots$ & 1.27 \\
\hline $\mathrm{P}_{2} \mathrm{O}_{5}$ & ---- &.--- & --- & $-\cdots$ & $\cdots$ & $--\cdot$ & 1.05 & $\cdots$ \\
\hline $\mathrm{SO}_{4}$ & $\cdots$ &.-- & $\cdots-$ & .3 & 8.9 & $\cdots$ & $13.20 \mathrm{C}$ & $\cdots$ \\
\hline $\mathrm{Cl}^{\circ}$ & --- & --- & -.-- & 56.5 & 47.4 & $-\cdots-$ & 7.40 & $\cdots$ \\
\hline K & $\ldots-$ & $--\cdot$ & --- & .04 & .17 & --- & $\cdots$ & -.. \\
\hline $\mathbf{N a}$ & $\cdots$ & $\cdots$ & $\cdots$ & 38.3 & 38.2 & $-\cdots$ & $\cdots$ & ...- \\
\hline $\mathrm{NH}$ & & $-\cdots$ & $\cdots$ & $\ldots$ & $\cdots$ & $\cdots$ & .42 & $\cdots$ \\
\hline & 12.74 & $\cdots$ & $\cdots$ & $\cdots$ & $\cdots$ & $.96 \mathrm{~B}$ & $17.32 \mathrm{D}$ & $17.07 \mathrm{~A}$ \\
\hline Less $0=\mathrm{Cl}$ & $\cdots$ & $\cdots-$ & 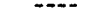 & $\cdots$ & 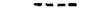 & $\cdots$ & 1.66 & \\
\hline Total & 95.00 & $>95$. & 95.7 & 96.24 & 98.19 & 99.89 & 100.00 & 100.00 \\
\hline
\end{tabular}

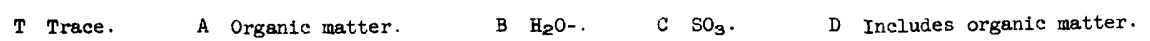


TABLE 10. - Analyses of samples from California 50 percent or more sodium chloride (Group Na), special-rock category- Continued

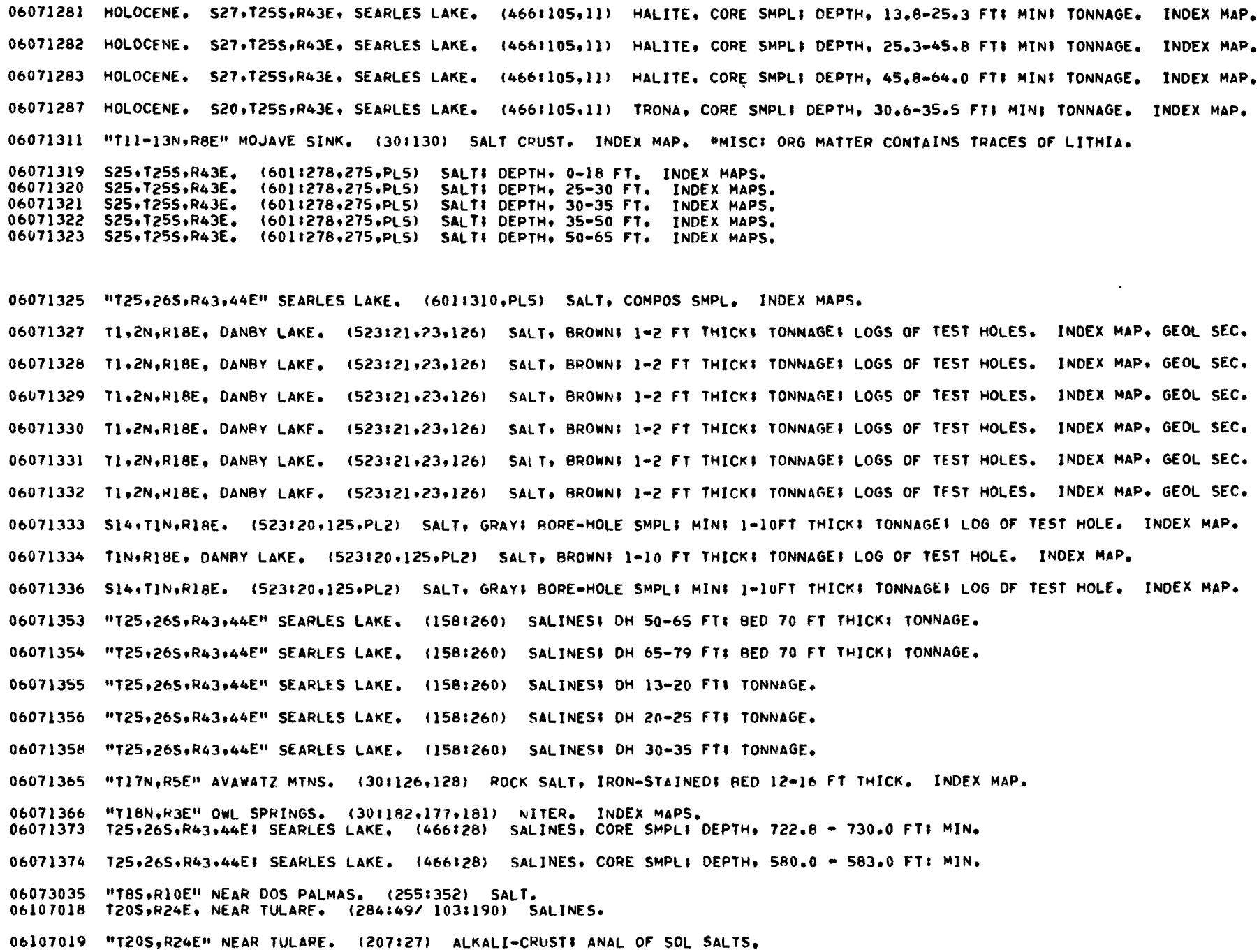


TABLE 11. - Analyses of samples from California containing 50 percent or more miscellaneous sulfate- and carbonate-bearing material (Group S), special-rock category

[Amounts in parenthesis are not included in total]

\begin{tabular}{|c|c|c|c|c|c|c|c|c|c|c|}
\hline Sample & 06025035 & 06025036 & 06027013 & 06027079 & 06027080 & 06027082 & 06027090 & .06027096 & 06029106 & 06029109 \\
\hline $\begin{array}{l}\text { Insoluble } \\
\mathrm{Al}_{2} \mathrm{O}_{3}+\mathrm{Fe}_{2} \mathrm{O}_{3}\end{array}$ & $\begin{array}{c}0.39 \\
.0\end{array}$ & $\begin{array}{rl}0.0 & \mathrm{~T} \\
.0 & \mathrm{~T}\end{array}$ & 17.90 & 0.61 & 0.32 & $\begin{array}{r}0.28 \mathrm{~A} \\
.13 \mathrm{~B}\end{array}$ & $0 . \infty$ & 0.02 & $0.73 \mathrm{~A}$ & 0.2 \\
\hline & $\therefore$ & $\therefore$ & $\cdots$ & $37 . .50$ & 37.00 & $\cdots$ & $\cdots$ & 38.13 & 5.06 & $\cdots$ \\
\hline & $\cdots$ & $\cdots$ & $\cdots$ & $-\frac{0}{2}$ & $\cdots$ & 4.07 & $\cdots$ & $\cdots$ & $\cdots$ & $\cdots$ \\
\hline & .16 & $.0 \mathrm{~T}$ & $\therefore$ & $.0 e^{\circ}$ & $\cdots$ & $\cdots$ & $\cdots$ & $\cdots$ & $\begin{array}{r}.40 \\
. \cdots .\end{array}$ & $\cdots$ \\
\hline $\begin{array}{l}\mathrm{PaCl} \\
\mathrm{Pag} O\end{array}$ & 43.40 & 43.64 & 16.40 & 40.08 & 41.26 & $\begin{array}{l}38.16 \\
\cdots\end{array}$ & $\begin{array}{r}.32 \\
\therefore-.\end{array}$ & 41.00 & 39.64 & $\begin{array}{c}.2 \\
\therefore-\end{array}$ \\
\hline 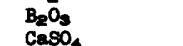 & .0 & .0 & $\cdots$ & $\cdots$ & $\cdots$ & .63 & $\cdots$ & $\cdots$ & -... & $\cdots$ \\
\hline 6504. & $\cdots$ & $\cdots-$ & $\therefore$ & $\cdots$ & $\cdots$ & $\cdots$ & $\cdots$ & $\cdots$ & $\cdots$ & .3 \\
\hline $\mathrm{Ban}_{2} \mathrm{Bu}_{3}$ & ;.:- & $\cdots$ & 1.20 & -:- & -...- & 34.95 & 45.86 & $\cdots$ & -..- & 1.5 \\
\hline 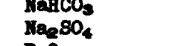 & -..- & -:- & 3.20 & -... & $\cdots$ & $\begin{array}{r}7.40 \\
14.38\end{array}$ & 36.46 & -..- & $\cdots$ & 96.9 \\
\hline 5 & 56.40 & 56.22 & $\cdots$ & .63 & .79 & $\ldots$ & -..; & .70 & $\begin{array}{l}19.52 \\
19.82\end{array}$ & $\cdots$ \\
\hline 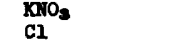 & $\cdots$ & .10 & $\begin{array}{l}61.20 \\
-\cdots . .\end{array}$ &. .21 & 1.57 & $\cdots$ & $\cdots$ & $\because .19$ & 19.20 & $\cdots$ \\
\hline 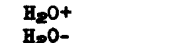 & .35 & .12 & $\ldots$ & \}$_{19.94}$ & 19.62 & -..- & 16.16 & 20.07 & 19.01 & .3 \\
\hline $\mathrm{LeBs} O=\mathrm{Cl}$ & $\therefore$ & $\therefore$ & $\cdots$ & $(.05)$ & $(.35)$ & -.- & -.-- & $(.04)$ & $(4.38)$ & $\cdots$ \\
\hline Total & 100.72 & 100.15 & 100.00 & 99.00 & 100.21 & 100.00 & 98.82 & 100.07 & 100.00 & 100.0 \\
\hline sumple & 06029110 & 06047007 & 06047009 & 06051020 & 06059013 & 06071248 & 06071249 & 06071250 & 06071251 & 06071257 \\
\hline Insoluble & 0.82 & $\cdots$ & $\cdots$ & 3.70 & $\cdots$ & 8.1 & 6.1 & 0.50 & 0.26 & 6.7 \\
\hline CaO & .20 & $\cdots$ & - & a.: & $\cdots$ & 6.0 & 4.7 & .51 & .37 & 6.3 \\
\hline $\begin{array}{l}\mathrm{C}_{2} \\
\mathrm{C}_{2} \mathrm{O}\end{array}$ & 51.15 & 26.19 & $\cdots$ & -... & $\begin{array}{l}28.29 \\
15.67\end{array}$ & $\begin{array}{r}26.6 \\
1.2\end{array}$ & $\begin{array}{l}28.9 \\
.87\end{array}$ & $\begin{array}{l}35.6 \\
.51\end{array}$ & $\begin{array}{l}18.9 \\
.17\end{array}$ & $\begin{array}{l}25.6 \\
.49\end{array}$ \\
\hline & $\cdots$ & 6.81 & $\cdots$ & $\cdots$ & $\cdots$ & 1.0 & 1.6 & .46 & .17 & 2.0 \\
\hline 0 & $\cdots$ & 2.63 & $\cdots$ & $\cdots$ & $\cdots$ & $\cdots$ & $\cdots$ & $\cdots$ & -..- & $\cdots$ \\
\hline $\mathrm{Xacl}_{2}$ & 36.74 & 44.44 & 1.46 & 13.76 & 41.57 & 29.4 & 31.8 & 39.5 & 30.5 & 29.6 \\
\hline & & & 12.98 & $\cdots$ & $\cdots$ & $\cdots$ & $\cdots$ & $\cdots$ & $\cdots$ & $\cdots$ \\
\hline $\mathrm{SO}_{4}$ & $\cdots$ & $\cdots$ & $\begin{array}{l}4.94 \\
4.67\end{array}$ & 11.25 & $\cdots$ & -... & -... & … & $\cdots$ & $\cdots$ \\
\hline & $\cdots$ & $\cdots$ & 75.95 & 70.31 & $\cdots$ & $\cdots$ & $\cdots$ & $\cdots$ & $\cdots$ & $\cdots$ \\
\hline & $\cdots$ & 2.19 & ... & $\cdots$ & 9.47 & $\begin{array}{l}1.9 \\
1.9\end{array}$ & $\begin{array}{l}. .32 \\
3.2\end{array}$ & $\begin{array}{l}.20 \\
3.7\end{array}$ & $\begin{aligned} 22.8 \\
.33\end{aligned}$ & $\begin{array}{l}.49 \\
5.2\end{array}$ \\
\hline & 10.76 & 17.15 & $\because-$ & $\cdots$ & 63.39 & 20.0 & 17.9 & 17.8 & 24.5 & 18.9 \\
\hline Less $0=\mathrm{CI}$ & .... & (.15) & ---. & -..- & $(1.39)$ & (.9) & $(.81)$ & (.61) & (.21) & $(.74)$ \\
\hline rotal & 99.83 & 200.00 & 100.00 & 99.02 & 100.00 & 98.6 . & 98.18 & 100.87 & 98.72 & 97.14 \\
\hline
\end{tabular}

\begin{tabular}{|c|c|c|c|c|c|c|c|c|c|c|}
\hline Sample & 06071258 & 06071259 & 06071262 & 06071267 & 06071268 & 06071269 & 06071270 & 06071275 & 06071279 & 06071284 \\
\hline $\begin{array}{l}\text { Insoluble } \\
\mathrm{CsO}^{2} \mathrm{O} \\
\mathrm{CO}_{2} \\
\mathrm{~K}_{2} \mathrm{O} \\
\mathrm{MgO} \\
\mathrm{Ha}_{2} \mathrm{O} \\
\mathrm{BgO}_{2} \mathrm{O}_{3} \\
\mathrm{SO}_{3} \\
\mathrm{H}_{2} \mathrm{O} \\
\mathrm{Cl} \\
\mathrm{LesB} \quad \mathrm{O}=\mathrm{Cl}\end{array}$ & $\begin{array}{c}4.2 \\
4.0 \\
31.2 \\
.35 \\
1.5 \\
33.4 \\
.28 \\
2.6 \\
19.4 \\
2.2 \\
(.50)\end{array}$ & $\begin{array}{c}6.0 \\
4.8 \\
23.0 \\
.90 \\
1.8 \\
28.3 \\
10.2 \\
1.3 \\
18.8 \\
3.5 \\
(.79)\end{array}$ & $\begin{array}{c}5.0 \\
3.5 \\
32.7 \\
1.2 \\
.78 \\
35.2 \\
.38 \\
2.0 \\
15.7 \\
4.9 \\
(1.11)\end{array}$ & $\begin{array}{c}4.1 \\
4.0 \\
16.7 \\
.55 \\
2.0 \\
35.8 \\
1.8 \\
19.2 \\
9.7 \\
4.3 \\
(.97)\end{array}$ & $\begin{array}{c}6.0 \\
4.3 \\
27.2 \\
.57 \\
1.5 \\
29.9 \\
5.3 \\
1.5 \\
18.6 \\
2.5 \\
(.56)\end{array}$ & $\begin{array}{c}13.7 \\
9.4 \\
24.6 \\
1.06 \\
4.0 \\
20.4 \\
1.4 \\
2.8 \\
15.1 \\
5.0 \\
(1.13)\end{array}$ & $\begin{array}{c}9.2 \\
7.3 \\
25.9 \\
.84 \\
2.0 \\
26.7 \\
.84 \\
2.0 \\
18.8 \\
4.5 \\
(1.01)\end{array}$ & $\begin{array}{c}0.55 \\
.30 \\
23.5 \\
.65 \\
.30 \\
42.7 \\
2.2 \\
13.6 \\
11.6 \\
6.9 \\
(1.56)\end{array}$ & $\begin{array}{c}0.35 \\
.73 \\
24.7 \\
1.1 \\
.64 \\
37.8 \\
7.3 \\
5.0 \\
16.4 \\
7.4 \\
(1.67)\end{array}$ & $\begin{array}{c}0.14 \\
.19 \\
31.4 \\
.78 \\
.22 \\
40.3 \\
1.6 \\
2.7 \\
16.8 \\
7.9 \\
(1.78)\end{array}$ \\
\hline Total & 98.63 & 97.81 & 100.25 & 97.18 & 96.81 & 96.33 & 97.07 & 100.74 & 99.75 & 100.25 \\
\hline Sample & 06071285 & 06071286 & 06071288 & 06071289 & 06071290 & 06071291 & 06071292 & 06071315 & 06071317 & 06071318 \\
\hline $\begin{array}{l}\text { Insoluble } \\
\mathrm{CaO}_{20} \\
\mathrm{CO}_{2} \\
\mathrm{~K}_{2} \mathrm{O} \\
\mathrm{MgO} \\
\mathrm{Ka}_{2} \mathrm{O} \\
\mathrm{B}_{2} \mathrm{O}_{3} \\
\mathrm{SO}_{3} \\
\mathrm{H}_{2} \mathrm{O} \\
\mathrm{Cl}^{2} \\
\mathrm{LeBs} \quad \mathrm{O}=\mathrm{Cl}\end{array}$ & $\begin{array}{c}3.5 \\
1.4 \\
13.8 \\
1.2 \\
.59 \\
37.6 \\
2.4 \\
25.8 \\
8.0 \\
2.2 \\
(.50)\end{array}$ & $\begin{array}{c}2.0 \\
.11 \\
14.8 \\
1.7 \\
.44 \\
40.7 \\
.85 \\
24.0 \\
6.8 \\
9.4 \\
(2.12)\end{array}$ & $\begin{array}{c}2.6 \\
2.5 \\
30.2 \\
.50 \\
1.1 \\
36.2 \\
.28 \\
4.6 \\
17.3 \\
2.7 \\
(.61)\end{array}$ & $\begin{array}{c}0.97 \\
.32 \\
35.2 \\
.53 \\
.08 \\
39.2 \\
.23 \\
1.5 \\
18.3 \\
3.4 \\
(.76)\end{array}$ & $\begin{array}{c}8.8 \\
6.5 \\
25.8 \\
.92 \\
2.3 \\
28.6 \\
.43 \\
2.1 \\
17.5 \\
5.4 \\
(1.22)\end{array}$ & $\begin{array}{c}0.31 \\
.40 \\
35.4 \\
.55 \\
.22 \\
40.5 \\
.24 \\
2.6 \\
17.7 \\
3.6 \\
(.81)\end{array}$ & $\begin{array}{c}6.3 \\
4.7 \\
27.8 \\
.83 \\
1.6 \\
31.3 \\
.40 \\
2.2 \\
17.5 \\
5.3 \\
(1.20)\end{array}$ & 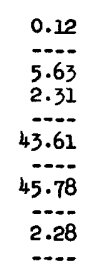 & \begin{tabular}{c}
0.19 \\
\hdashline-.65 \\
2.48 \\
$-\cdots$ \\
43.35
\end{tabular} & 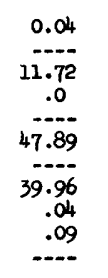 \\
\hline Total & 95.99 & 98.68 & 97.37 & 98.97 & 97.13 & 100.71 & 96.73 & 99.73 & 99.81 & 99.74 \\
\hline
\end{tabular}

T Trace. A $\mathrm{SiO}_{2}$. B Includes $\mathrm{CaO}$ and $\mathrm{MgO}$. 
TABLE 11. -Analyses of samples from California containing 50 percent or more miscellaneous sulfate-and carbonate-bearing material (Group S), special rock category-Continued

06025035 S19,T9S,RI2E. (493891,87,90) SODIUM SULPHATE: MIN3 8 IN - 3 FT THICK. INDEX \& GEOL MAP. USE: 6, 10.

06025036 S19.T9S,R12E. $(493891.90)$ SOOIUM SULFATE: MIN\& 8 IN - 3 FT THICK. INDEX \& GEOL MAP. USE: 6 , 10. SMISC: SIO2 REPT AS INSOL CL $=0,10$, B203 $=0.00$.

06027013 EOCENE. "T2ZN,R2E" AMARGOSA RIVER, $(301164,156)$ NITERI MIN. INDEX MAPS.

06027079 "TI7S,R37E" OWENS LAKE, (84:76) URAO, PINK.

06027080 "T17S,R37E" OWENS LAKE, (101124) URAO.

06027082 "T17S,R37E" OWENS LAKE. (603:762) SALINES.

06027090 "TITS,R37E" OWENS LAKE. (103:193,239) TRONA.

06027096 "T17S.R37E" OWENS LAKE, (101:24) TRONA. B.0. $=2.1473$.

06029106 "TIIN.R12W" MOHAVE. (284:49) SODIUM SALTS. "MISCt HZO INCL ORG MATTER.

06029109 "T9N,R9W" SOUTH OF MUROC. (563:125) THENARDITE.

06029110 "T26S,R43E" SEARLES LAKE: (605:169) SODIUM BICARBONATE: WELL. AT DEPTH OF $120-285$ FT.

06047007 "TTS,RI4E" MERCED BOTTOM, 12841491 SODIUM CARBONATE. "MISC: HZO INCL ORG MATTER.

06047009 "TTS.RIGE" MERCED BDTTOMS. (338:495) INCRUSTATION.

06051020 "T25,R3LE" NEAR BENTON. (6131176) ALKALINE DEPOSIT.

06059013 "TSS.RIIW" WESTMINSTER. (284149) SODIUM CARBONATE.

06071248 PLEISTOCENE, S30,T25S,R44E, SEARLES LAKE, $(466173,11)$ TRONA, CORE SMPL, DEPTH, 109.7-113.4 FT, MIN TONNAGE.

06071249

06071250

06071251

06071257

06071258 INDEX MAP PLE I ST OCENE

PLEISTOCENE. PLEISTOCEN
INDEX MAP. PLEISTOCENE. PLEISTOCENE

INDEX MAP.

PLEISTOCENE

INDEX MAP.

PLEISTOCENE

(466:73.11) TRONA, CORE SMPL, DEPIH, 113.4-116.8 FT. MINE TONNAGE.

(466:73.11) TRONA, CORE SMPL, DEPTH, $216.8 \mathrm{~m} 120.8 \mathrm{FT}$, MINI TONNAGE.

S3,T265,R43E, SEARLES LAKE, (466:73,11) TINCALCONITE, CORE SMPL: DEPTH, 86,9-88.1 FT, MINE TDNNAGE.

53,T26S,R43E, SEARLES LAKE. 1466173,11$)$ TRONA, CORE SMPL, DEPTH, 114.3-119.3 FT: MINI TONNAGE.

06071259

PLEISTOCENE.

S27, T25S,R43E, SEARLES LAKE。

$(466: 73,11)$

TRONA, CORE SMPL DEPTH, 81.1-83.7 FT, MIN: TONNAGE. INDEX MAP.

06071262

PLEISTOCENE.

S27. T25S,R43E, SE.ARLES LAKE.

$(466: 73,11)$

TRONA, CORE SMPL OEPTH, 91.4-92.6 FTI MINE TONNAGE. INDEX MAP.

06071267 PLEISTOCENE.

INDEX MAP.

S27, T25S, R43E, SEARLES LAKE.

$(466173,11)$

BURKEITE, CORE SMPL DEPTH, $111.3-113.8$ FT, MINI TONNAGE.

06071268 PLEISTOCENE.

06071269 PLEISTOCENE.

S27, T25S, R43E, SEARLES LAKE.

$(466173,11)$

TRONA, CORE SMPL, DEPTH $113.8-117.8 \mathrm{FT}$, MINI TONNAGE.

$S 20, T 25 S, R 43 E$, SEARLES LAKE.

(466:73,11) GAYLUSSITE, SMPL; DEPTH 69.9-74.2 FT; MINI TONNAGE.

06071270 INDEX MAP.

(466173,11) TRONA, CORE SMPL, DEPTH, 74.2-77.8 FT, MIN! TONNAGE. INDEX MAP.

HOLOCENE, S30,T255,R44E, SEARLES LAKE

TRNA, CORE SMPLI DEPTH, 68.6-72.7 FTI MINI TONNAGE, INDEX MAP.

0607128

HOLOCENE, S3,T26S,R43E, SEARLES LAKE。

(466:105,

RONA, CORE SMPL: DEPTH, 70.8-76.3 FT: MIN: TONNAGE. INDEX MAP.

HOLOCENE, S27,T2SS,R43E, SEARLES LAKE,

(466:105,11) TRONA, CORE SMPL, DEPTH, 64.0-70.6 FT: MIN: TONNAGE, INDEX MAP.

HOLOCENE. SZO,T25S,R43E, SEARLES LAKE.

(466:105,11) HANKSITE, CORE SMPL, DEPTH, 5.4-10.9 FT: MINI TONNAGE. INDEX MAP.

0607128

HOLOCENE. S20,T25S,R43E, SEARLES LAKE。

(466:105,11) HANKSITE, CORE SMPL, OEPTH, 10.9-30.6 FT: MINI TONNAGE. INDEX MAP.

PLEISTOCENE. S20,T25S,R43t, SEARLES LAKE.

06071290 PLEISTOCENE. S20,T25S,R43E, SEARLES LAKE.

06071291 PLEISTOCENE, S20,T25S,R43E, SEARLES LAKE.

(466:73.11) TRONA, CORE SMPLI OEPTH, 117.5-119.8 FT: MINI TONNAGE.

(466:73.11) TRONA, CORE SMPL, DEPTH, 53.0-56.8 FT: MINI TONNAGE. INDEX MAP.

06071292

(466:73.11) TRONA, CORE SMPL DEPTH, 56.8-61.2 FT: MINI TONNAGE. INDEX MAP.

06071315

TRONA, CORE SMPL: DEPTH, 61.2-65,9 FT: MINI TONNAGE. INDEX MAP.

06071317

"T25S,R43E" SEARLES LAKE. $(1631182)$ HANKSITE, WHITE, VITREOUS: B.D., 2.562 .

06071318

"T25S,R43E" SEARLES LAKE. (1638182) HANKSITE, WHITE, VITREOUS: B.D.. 2.5A2.

"T25S, R43E" SEARLES LAKE. (600:52) BURKEITE. 
TABLE 11. - Analyses of samples from California containing 50 percent or more miscellaneous sulfate- and carbonate-bearing material (Groun S), special-rock categorv- Continued

\begin{tabular}{|c|c|c|c|c|c|c|c|c|c|c|}
\hline \multicolumn{11}{|c|}{ Chemical Analyses } \\
\hline sample & 06071324 & 06071357 & 06071359 & 06071360 & 06071361 & 06071378 & 06079005 & 06079016 & 06079017 & 06079018 \\
\hline Insoluble & $0.0 \mathrm{~T}$ & 1.4 & 3.2 & 6.8 & 7.6 & --- & 0.40 & 0.16 & $-\cdots$ & 0.91 \\
\hline $\mathrm{Al}_{2} \mathrm{O}_{3}$ & $\ldots$ & $\ldots$ & - & $-\ldots$ & -... & $-\cdots$ & .04 & $-\cdots$ & --- & $\cdots$ \\
\hline $\mathrm{CaO}^{\circ}$ & $\ldots$ & $\ldots$ & $-\cdots$ & --- & $--\cdot$ &.$\infty$ & .45 & $\cdots$ & $\cdots$ & .34 \\
\hline $\mathrm{CO}_{2}$ & --- & --- & --- & -... & --- & 5.42 & .0 & $.29 \mathrm{G}$ & --- & $-\cdot-$ \\
\hline $\mathrm{K}_{2} \mathrm{O}$ & --- & $-\ldots$ & $\cdots$ & $\cdots$ & $\cdots$ & $\cdots$ & .28 & --- & $\cdots$ & $\cdots$ \\
\hline $\mathrm{NgO}$ & $-\cdots$ & -- & $-\cdots$ & $=--$ & $\cdots$ &.$\infty 0$ & 1.66 & $\cdots$ & $\cdots$ & 1.00 \\
\hline Hach & 19.0 & 15.3 & 4.5 & 5.1 & 4.0 & $-\cdots$ & --- & .47 & .32 & 5.95 \\
\hline $\mathrm{Na}_{2} \mathrm{~B}_{4} \mathrm{O}_{7}$ & .5 & $.0 \mathrm{~T}$ & $.0 \mathrm{~T}$ & $.0 \mathrm{~T}$ & .0 & --- & $\cdots$ & $-\bar{x}$ & $\cdots$ & --- \\
\hline $\mathrm{NgSO}_{4}$ & $40-$ & $20-$ & $2 \ddot{8.6}$ & 43.5 & 53.0 & -1 & $\cdots$ &. .43 & $\cdots$ & $\cdots$ \\
\hline $\begin{array}{l}\mathrm{Ne}_{2} \mathrm{CO}_{3} \\
\mathrm{NaHCO}\end{array}$ & $\begin{array}{l}40.3 \\
18.5\end{array}$ & $\begin{array}{l}38.7 \\
24.4\end{array}$ & $\begin{array}{l}28.6 \\
10.1\end{array}$ & $\begin{array}{l}43.5 \\
21.0\end{array}$ & $\begin{array}{l}53.0 \\
16.0\end{array}$ & 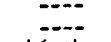 & $\ldots$ & $-\cdots$ & $\cdots$ & $\cdots$ \\
\hline $\mathrm{Fa}_{\mathrm{e}} \mathrm{O}$ & - & --- & $\cdots$ & $-\ldots$ & --- & 46.34 & 40.50 & $\cdots$ & $\cdots$ & $\cdots$ \\
\hline $\mathrm{Ha}_{2} \mathrm{SO}_{4}$ & $7 \cdot 3$ & 4.7 & 38.0 & 6.8 & 2.8 & $\cdots$ & $-\cdots$ & 98.65 & 42.78 & 38.72 \\
\hline $\mathrm{SO}_{3}$ & $\cdots$ & $\cdots$ & $\ldots$ & -.- & $\cdots$ & 45.89 & 46.12 & $\cdots$ & --- & 50.83 \\
\hline C1 & 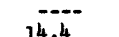 & 5.5 & $-\infty$ & 16.8 & $1 \overline{6.6}$ & 2.36 & $\begin{array}{l}9.27 \\
3.65\end{array}$ & $\cdots$ & 56.90 & $2.65 \mathrm{~B}$ \\
\hline $\begin{array}{l}\mathrm{H}_{2} \mathrm{O} \\
\text { Less }\end{array}$ & $\begin{array}{c}14.4 \\
-\ldots . .\end{array}$ & 15.5 & 10.6 & 10.8 & 10.0 & - & $(2.09)$ & $\ldots$ & -... & $\ldots$ \\
\hline Total & 100.0 & 100.0 & 95.0 & 100.0 & 100.0 & 100.01 & 100.28 & 100.00 & 100.00 & 100.40 \\
\hline Semple & 06097018 & 06107016 & 06107017 & & & & & & & \\
\hline $\mathrm{S}_{2} O_{2}$ & 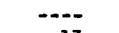 & 2.52 & $-\cdots$ & & & & & & & \\
\hline $\mathrm{CaO}^{-}$ & .13 & $\cdots$ & --- & & & & & & & \\
\hline $\mathrm{CO}_{2}$ & $\cdots$ & 13.20 & 16.69 & & & & & & & \\
\hline Feo & .23 & $\cdots-$ & $\overline{6}=$ & & & & & & & \\
\hline $\mathrm{K}_{2} \mathrm{O}$ & $\cdots$ & $\cdots$ & 6.76 & & & & & & & \\
\hline $\mathbf{1 8 0}$ & 17.91 & $\cdots$ & .35 & & & & & & & \\
\hline MnO & .14 & $=-$ & $\cdots$ & & & & & & & \\
\hline $\mathrm{Ha}_{2} \mathrm{O}$ & $\cdots$ & 48.97 & 27.60 & & & & & & & \\
\hline 110 & .11 & $\ddot{-10}$ & $\overline{5}---$ & & & & & & & \\
\hline $\begin{array}{l}\mathrm{P}_{2} \mathrm{O}_{3} \\
\mathrm{SO}_{3}\end{array}$ & 38.13 & $\begin{array}{r}1.12 \\
24.78\end{array}$ & $\begin{array}{r}2.91 \\
6.43\end{array}$ & & & & & & & \\
\hline $\begin{array}{l}\mathrm{H}_{2} \mathrm{O} \\
\mathrm{Cl}\end{array}$ & 42.97 & $\begin{array}{r}1.54 \\
10.04\end{array}$ & $\begin{array}{r}38.09 \\
1.51\end{array}$ & & & & & & & \\
\hline Less $\mathrm{O}=\mathrm{Cl}$ & -.. & (2.17) & $(.34)$ & & & & & & & \\
\hline Total & 99.62 & 100.00 & 100.00 & & & & & & & \\
\hline Ignition & & H $\mathrm{H}_{2} \mathrm{O}-$. & Trace. & & & & & & & \\
\hline
\end{tabular}

DESCRIPTIVE NOTES

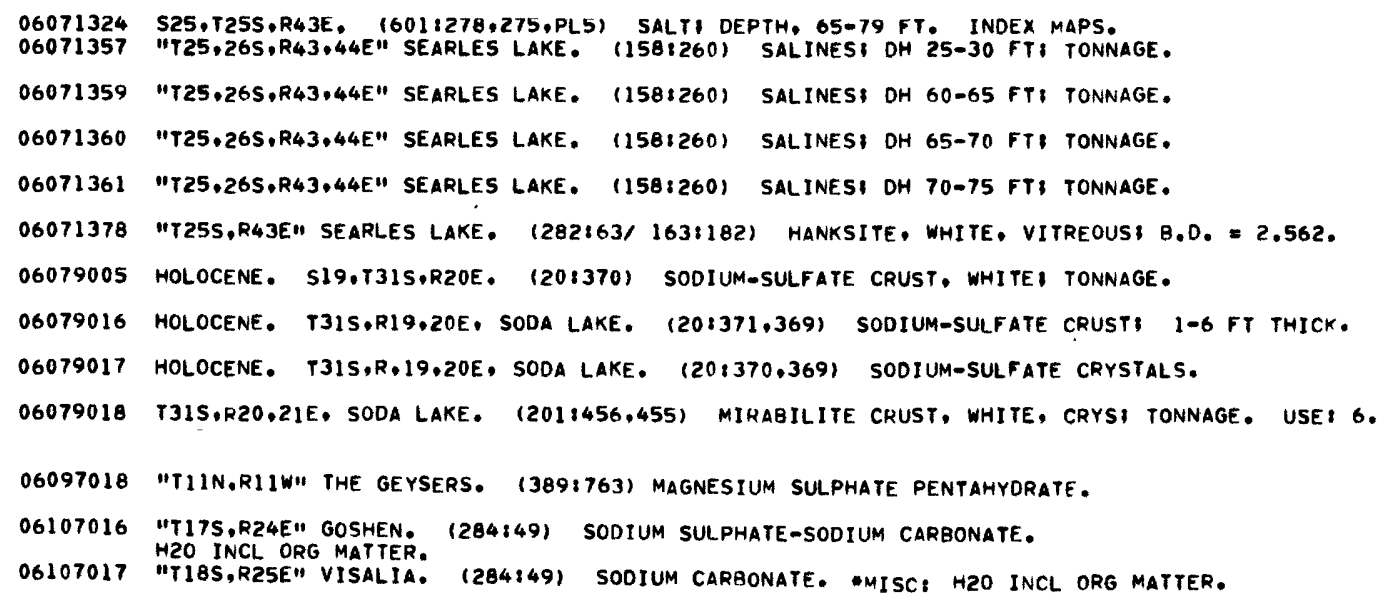


TABLE 12. - Analyses of samples from California containing 50 percent or more gypsum, anhydrite, or gypsite (Group G), special-rock category

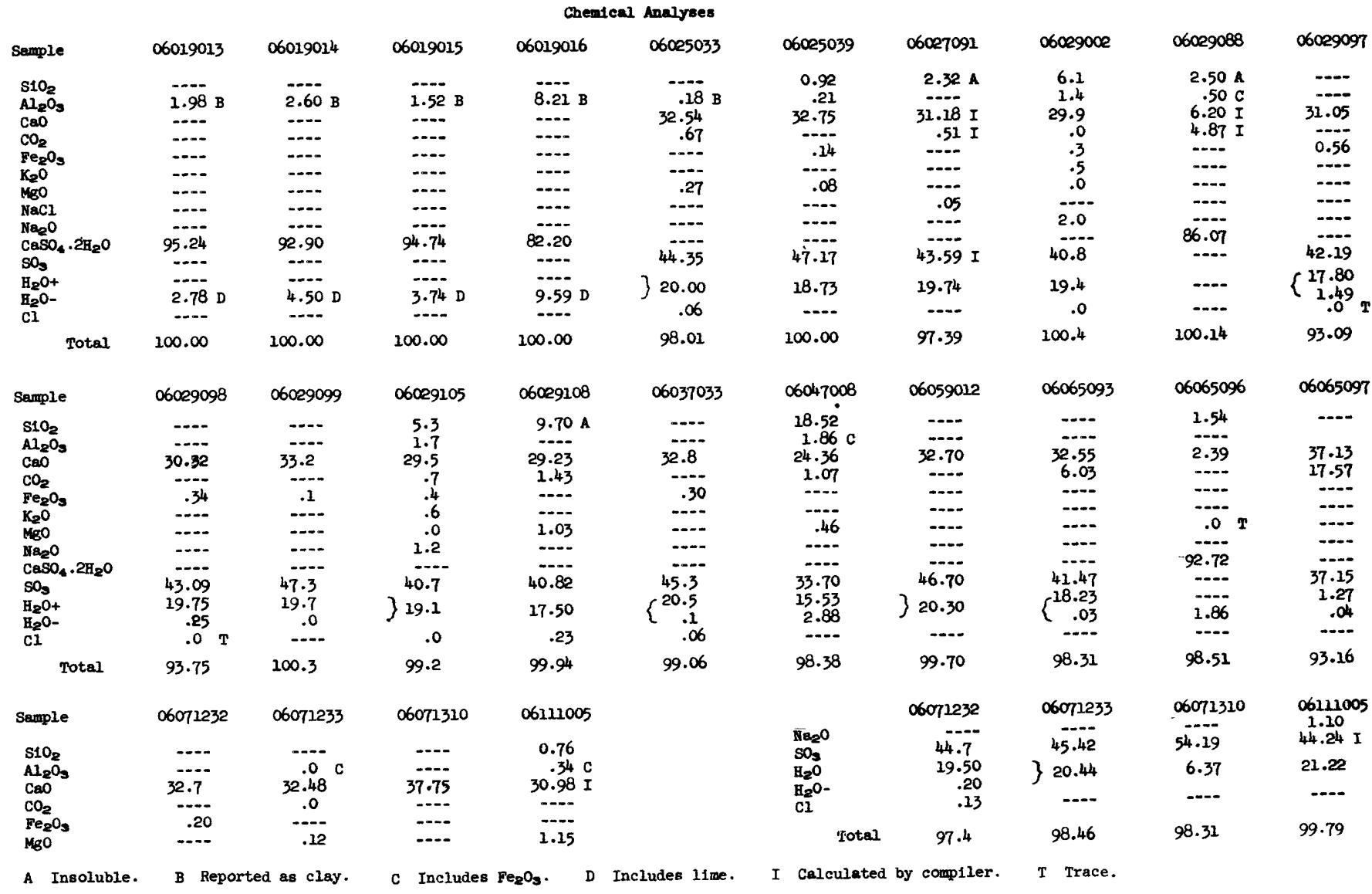

\section{DESCRIPTIVE NOTES}

06019013 TERTIARY. "TI5S,RI3E" PAOLI MINE. (188:122.121.PL21) GYPSUM:30 FT THICK. INDEX MAP. USE:15.

06019014 TERTIARY. "TI5S, RI3E" PAOLI MINE. 1188:122,121,PL21\} GYPSUMI RED 18 FT THICK. INDEX MAP. USE: 15.

06019015 TERTIARY. "T15S,RI3E" PAOLI MINE. (1883122,121,PL21) GYPSUM. INDEX MAP. USE: 15.

06019016 TERTIARY. "TI5S.RI3E" PAOLI MINE. (188:122,121,PL21) GYPSUM. INOEX MAP. USE: 15.

06025033 S29.T135.R9E. (563:122) GYPSIM.

06025039 S19,28,T13S,R9E. (5591739,738) GYPSUM 100 FT THICK.

06027091 "TITN,RGE" AVAWITZ MTNS. (491:115,117) GYPSUMI 250 FT THICK: TONNAGE. USE: 15.

06029002 S30,T26S,R21E. (602:16.PL1) GYPSITE, CREAMY-BUFF, POWDERY 4 FT THICK. INDEX MAP. USE: 15.

06029088 "TIIN,R23W" SUNSET OIL OISTRICT. (129:324) GYPSUM, CHALKYI SEVERAL FT THICK.

06029097 S21.T30S,R22E, 1602117.16, PL1) GYPSITE, BUFF: 2 FT THICK. INDEX MAP. USE: 2 .

06029098 S30,T30S,R22E. (602317,PL1) GYPSITE: 2-4 FT THICK INDEX MAP.

06029099 S26.27,T32S,R27E, $1602: 24.23$,PL1) GYPSITE, CREAMY COLOR, FINE-GRI 4 FT THICKI MIN. INDEX MAP. USE: 15.

06029105 S30.T265,R2IE. $(602: 16$, PL1) GYPSITE, CREAMY-BUFF, POWDERY 4 FT THICK. INDEX MAP. USEI 15.

06029108 "T30S,R38W" SALTDALE. (500:70,69) GYPSUM, WHITE: 9-12 FT THICK. USE: 2.

06037033 MIOCENE. "TSS,RI2W" PALMDALE, 1602:30,29,PL1/4631412.373,395) GYPSUM, YELLOWISH, FINELY CRYS, POROUS, MASSIVEI

BEDS $2-4$ FT THICK. INDEX MAP, GEOL SEC. USE: 15.

06047008 S2,TI2S,RIOE. (437:106,108,109) GYPSITE, 5-10 FT THICK, USE: 2, $M I S C$ SIOZ INCL INSOL.

06059012 TERTIAKY. "T3S,RSW" GYPSUM STATION, (174:60) GYPSUM. USE: 15.

06065093 "T3S,RI9E" PALEN MTNS. (260:415,407-409,414) GYPSUM, WHITE: FINELY CRYSI MIN. INDEX MAP. USE: 15.

06065096 "TGS.RZ3E" NORTH OF BLYTHE. (501:513.511) GYPSUM. WHITE, COMPACT, GRYSI TO FT THICK.

06065097 "T3S,RI9E" PALEN MTNS. (260:415,408,409,414) ANHYORITE S CALCIUM CARBONATE, WHITE, GRANULAR. MIN. INDEX MAP.

06071232 "TSN,R11E" $1602: 27$,PL1) GYPSUMI AFT THICKI MIN. INDEX MAP, USE: 15.

$06071233519, T 135, R 9 E$. 15631122$)$ GYPSUM.

06071310 "TIN,RIBE" DANBY LAKE. (2:1267,1266) BASSANITE, WHITE, FIBROUS: MIN: CORE SMPL DEPTH OF 510 FT. SPECTRO ANAL ALSO

06111005 "T4N.RZ3W" OJAI VALLEY. (165:272) GYPSITE. WHITE. 
TABLE 13. - Analyses of samples of celestite and strontianite (Group Sr) from California, special-rock category

Chemical Analyses

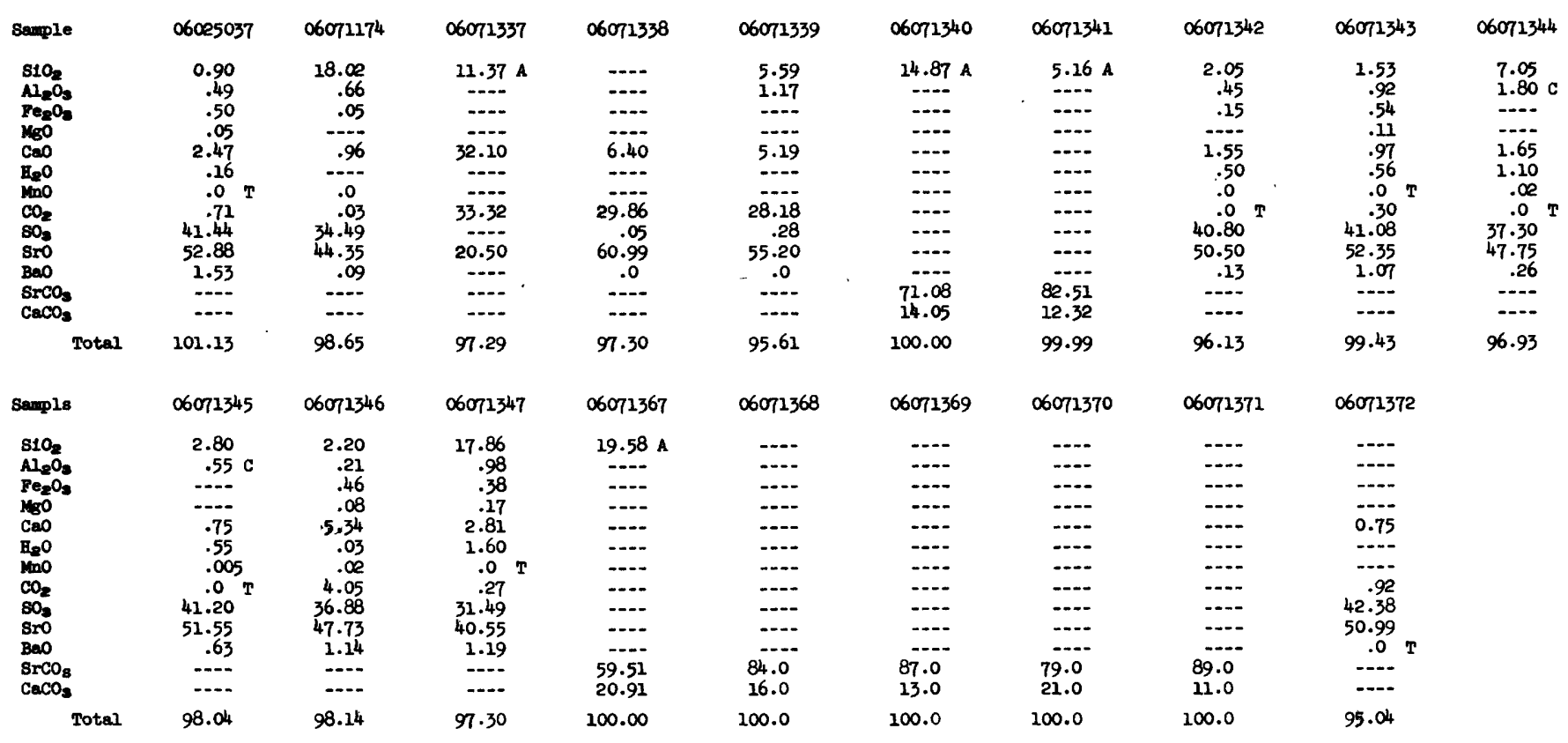

A Insoluble.

C $\mathrm{Al}_{2} \mathrm{O}_{3}+\mathrm{Fe}_{2} \mathrm{O}_{\mathbf{s}}$. T Trace.

DESCRIPTIVE NOTES

06025037 TERTIARY, S18,T13S,R9E. (391:161,154) CELESTITE, WHITE, MASSIVE: 10 FT THICKI REPR SMPL, TONNAGE. INDEX MAP, MEAS SEC.

06071174 MIOCENE, S19,T8N,R7E. (390:371,358-360/ 160:45,37-44,PL8-9/ 390:161,155) CELESTITE, 5.5 FT THICK TONNAGE. INDEX \&

06071337 S30,TIIN,RIW. (160:32,34,23,36,PL2-4) STRONTIANITE, BROWN, DENSE, FIBROUS. TRENCH SAMPLE. TONNAGE. INDEX \& GEOL MAPS, MEAS SEC, GEOL SEC.

06071338 S29.30,TIIN,RIW. (6061262.PL11/ 260:32.23-31,PL3-7) STRONTIANITE. SELECTED SMPL, MIN, TONNAGE. INDEX \& GEOL MAPS,

06071339 MIOCENE, BARSTOW FM. S29,30,T11N,RIW. $1606: 260,261$,PL11/ 390:377,375/ 160132,27-31,PL3-7) STRONTIANITE, MIN3 TONNAGE.

06071340 S20,TIIN,RIW. (160:32,23,27, PL3-7) STRONTIANITE, WHITE, DENSE 2 FTTHICK MIN, TONNAGE. INDEX \& GEOL MAPS. MEAS SEC,

06071341 S20,TIIN,RIW. $(160132,23, P L 3-7)$ STRONTIANITE, BROWN, CRYSI MIN, INDEX \& GEOL MAPS, MEAS SEC, GEOL SEC.

06071342 MIOCENE, S19,T8N,R7E, $1390: 371,358-360 / 160: 45,37-44$, PL8,9) CELESTITE, MASSIVE: 4.5 FT THICK TONNAGE. INDEX \& GEOI

M607 MES, MEAS SEC, GEOL SEC. MIOCENE. SI9,T8N,R7E. (3901371.358-360/ 160145,37-44, PL8.9) CELESTITE, MASSIVE, WEATHERED 3.5 FT THICK TONNAGE.

06071344 MIDCENE. SI9,TQN,R7E. (390:371,356-358, 160:45,37-44, PL8,9) CELESTITE, THINLY LAMINATED, 1.5 FT THICK, TONNAGE.

06071345 MIOCENE. S19,TBN,R7E. $1390,371,358-360 / 160845,37-44$, PL8,9) CELESTITE. THIN-REDDED 1.7 FT THICK IONNAGE. INDEX \&

06071346 MIOCENE. S19,TQN,R7E. 1390:371,358-360/ 160845,37-44, PL8,91 CELESTITE, PINK, MASSIVE, SUGARY: 0.5 FT THICK MIOCENE. S19,TRN,R7E. $1390: 371,358-39$
TONNAGE. INDEX \& GEOL MAP, MEAS SEC.

06071347 MIOCENE: S19,T8N,R7E. (390:371,358-360/ 160145,37-44,PL8,9) CELESTITE, PINK \& BUFF, FINE-GR, MASSIVE 2.25 FT THICK:

06071367 S20,TIIN,RIW. $(160: 32,23-31$, PL 3-7) STRONTIANITE, OROWNI MIN, TONNAGE. INDEX \& GEOL MAPS, MEAS SEC, GEOL SEC.

06071368 S20,TIIN,RIW. $(160: 32,23-31, P L 3-7)$ STRONTIANITE, BROWN. SELECTED SAMPLE. MIN, TONNAGE. INDEX \& GEOL MAPS, MEAS SEC,

06071369 S20,TIIN,RIW. (160:32,23-31, PL3-7) STRONTIANITE, 8ROWNI MIN, TONNAGE. INDEX \& GEOL MAPS, MEAS SEC, GEOL SEC.

06071370 S30,TIIN,RIW. $(160: 32,34,24,36, P L 2)$ STRONTIANITE, BROWNI TONNAGE. INDEX G GEOL MAPS, MEAS SEC, GEOL SEC.

06071371 S30,TIIN,RIW. $(160: 34,23,36, P L 2,3)$ STRONTIANITE, BROWN, TONNAGE. INDEX \& GEOL MAPS, MEAS SEC, GEOL SEC.

06071372 TERTIARY. "TI8N,R5E" DEATH VALLEY. $(160: 21,19$, PLl) CELESTITE, TONNAGE. INDEX \& GEOL MAPS, MEAS SEC. 
TABLE 14. - Analyses of samples of boron-bearing rocks (Group B), from California special-rock category

\begin{tabular}{|c|c|c|c|c|c|c|c|c|c|c|}
\hline Sample & 06027009 & 06027078 & 06027084 & 06027087 & 06027088 & 06029107 & 06037041 & 06037043 & 06037044 & 06037046 \\
\hline $\mathrm{S1O}_{2}$ & 0.65 & $1.40 \mathrm{~A}$ & 0.23 & $\cdots$ & $\cdots$ & $0.25 \mathrm{~A}$ & 13.97 & 14.81 & --- & -..- \\
\hline $\mathrm{Al}_{2} \mathrm{O}_{3}$ & $.60 \mathrm{c}$ & $\ldots$ & $\cdots$ & $\ldots$ & $\ldots$ & $\ldots$ & 1.27 & $\ldots$ & $\cdots$ & $\cdots$ \\
\hline $\mathrm{CaO}$ & 28.43 & --- & 14.06 & 13.8 & 27.2 & $\ldots$ & 23.74 & 28.26 & 27.76 & 14.14 \\
\hline $\mathrm{CO}_{2}$ & $\ldots$ & -.-- & $.0 \mathrm{~T}$ & -..- & -... & -..- & 5.14 &.-- & $-\cdots$ & $\cdots$ \\
\hline $\mathrm{Fe}_{2} \mathrm{O}_{3}$ & 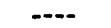 & $-\cdots$ & .12 & $\cdots$ & --- & $\ldots$ & 1.32 & $-\ldots$ & $-\ldots$ & $\cdots$ \\
\hline $\mathrm{NgO}$ & $\ldots$ & -..- & 20.14 & $\ldots$ & -... & $\ldots$ & 2.36 & -..- & $\cdots$ & $7.05 \mathrm{D}$ \\
\hline $\mathrm{NaCl}$ &.-- & 16.98 & -... & $\ldots$ &.-- & $\ldots$ & $\ldots$ & -..- & -.-- & $\cdots$ \\
\hline $\mathrm{Ne}_{2} \mathrm{O}$ & $\cdots$ & $-\cdots$ & $-\cdots$ & 7.7 &.-- & 21.40 & -... & $\ldots$ & $\cdots$ & $\cdots$ \\
\hline $\mathrm{BO}_{3}$ & 48.12 & $\cdots$ & $\cdots$ & $=-$ & $\ldots$ & $=-$ & -- & $\cdots$ & $\cdots$ & $-\cdots$ \\
\hline $\mathrm{B}_{2} \mathrm{O}_{3}$ & $\ldots$ & -.. & 47.71 & 43.0 & 50.9 & 47.26 & 36.10 & 45.56 & 49.45 & 43.13 \\
\hline $\mathrm{H}_{\mathbf{S}} \mathrm{BO}_{3}$ & --- & 36.82 & --- & --- & --. & --- & -... & -..- & $\cdots$ & --.- \\
\hline $\mathrm{Ne}_{2} \mathrm{O}_{3}$ & $\cdots$ & $\cdots$ & --- & 7.7 & $\cdots$ & $\cdots$ & $\cdots$ & $-\infty$ & $-\infty$ & --- \\
\hline $\begin{array}{l}\mathrm{H}_{2} \mathrm{O}^{+} \\
\mathrm{B}_{2} \mathrm{O}-\end{array}$ & 22.20 & 44.80 & $\begin{array}{c}27.37 \\
.0\end{array}$ & 35.5 & 21.9 & 31.01 & 16.10 & 11.37 & 22.48 & 35.68 \\
\hline Total & 100.00 & 100.00 & 96.63 & 100.0 & 100.0 & 99.92 & 100.00 & 100.00 & 99.69 & 100.00 \\
\hline Sample & 06037047 & 06071085 & 06071312 & 06071313 & 06071314 & 06071316 & & & & \\
\hline $\mathrm{SIO}_{2}$ & --- & 1.28 & 15.50 & 15.33 & 15.31 & $\cdots$ & & & & \\
\hline $\begin{array}{l}\mathrm{Al}_{2} \mathrm{O}_{2} \\
\mathrm{CaO}\end{array}$ & 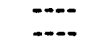 & $\begin{array}{r}.19 \mathrm{C} \\
27.97\end{array}$ & 28.45 & 28.44 & 29.22 & 27.42 & & & & \\
\hline MgO & $\cdots$ & .13 & -..- &.-- & $\cdots$ & $-\cdots$ & & & & \\
\hline $\mathrm{MgO} \cdot \mathrm{Na}_{2} \mathrm{O}$ & -.- & $-\cdots$ & .09 & 1.06 & $\cdots$ & --- & & & & \\
\hline Sro & 32.45 & 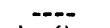 & $\cdots$ & $\cdots$ & $\cdots$ & $\cdots$ & & & & \\
\hline $\begin{array}{l}\mathrm{B}_{2} \mathrm{O}_{3} \\
\mathrm{H}_{2} \mathrm{O}\end{array}$ & $\begin{array}{r}58.16 \\
9.39\end{array}$ & $\begin{array}{l}47.64 \\
22.79\end{array}$ & $\begin{array}{l}44.38 \\
11.58\end{array}$ & $\begin{array}{l}43.78 \\
11.39\end{array}$ & $\begin{array}{l}44.32 \\
11.44\end{array}$ & $\begin{array}{l}49.70 \\
22.26\end{array}$ & & & & \\
\hline Total & 100.00 & 100.00 & 100.00 & 100.00 & 100.29 & 99.38 & & & & \\
\hline
\end{tabular}

A Insoluble. C Includes $\mathrm{Fe}_{2} \mathrm{O}_{3}$. D By difference.

DESCRIPTIVE NOTES

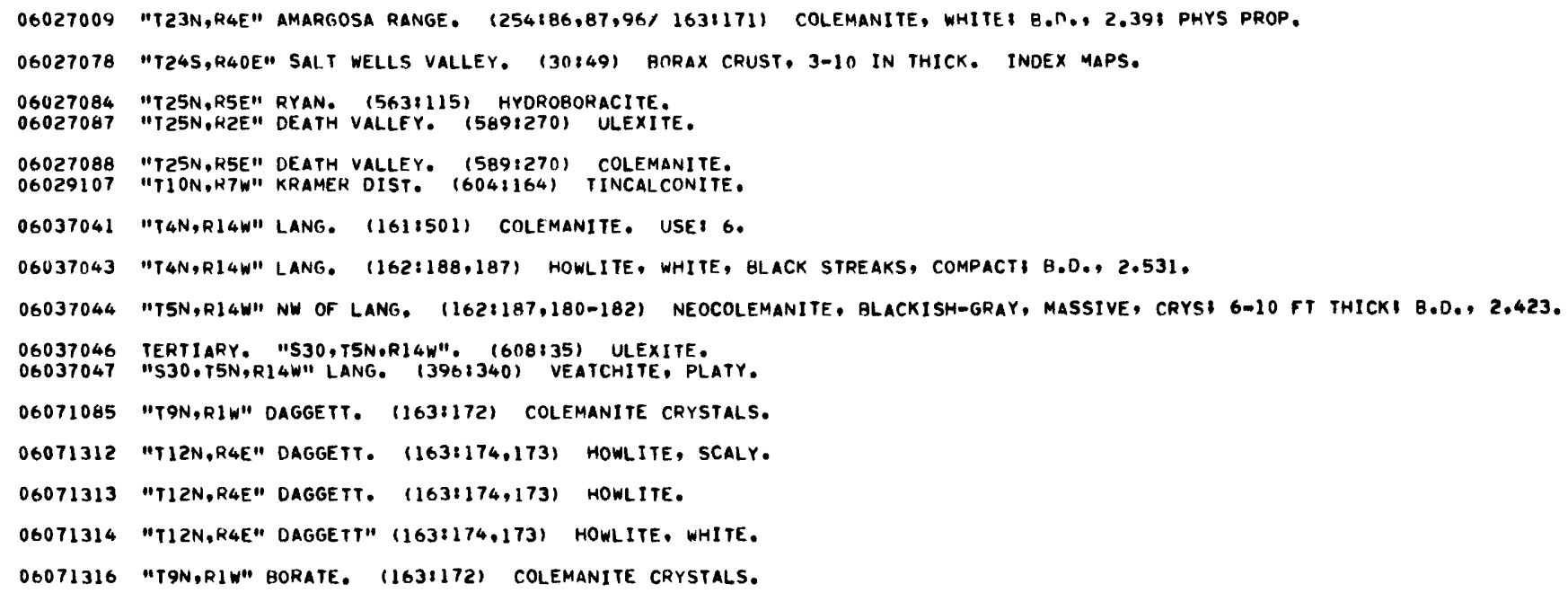


TABLE 15. - Analyses of samples of various types of rocks (Group M), from California and Hawaii, special-rock category

\begin{tabular}{|c|c|c|c|c|c|c|c|c|c|c|}
\hline sample & 06015001 & 06015002 & 06015003 & 06015004 & 06015005 & 06015006 & 06015007 & 06015008 & 06015009 & 06015010 \\
\hline $\begin{array}{l}\mathrm{SHO}_{2} \\
\mathrm{Al}_{2} \mathrm{O}_{2} \\
\mathrm{Cr}_{2} \mathrm{O}_{2} \\
\mathrm{Ag}_{8} \\
\mathrm{Au} \\
\mathrm{Co} \\
\mathrm{Fe} \\
\mathrm{PI} \\
\mathrm{Pt}\end{array}$ & $\begin{array}{c}35.20 \\
5.68 \\
1.0 \\
.0 \mathrm{~T} \\
.0 \mathrm{~T} \\
.06 \\
13.9 \\
.07 \\
<.005\end{array}$ & $\begin{array}{c}28.52 \\
9.08 \\
1.5 \\
.09 \\
.0 \mathrm{~T} \\
.08 \\
20.9 \\
.82 \\
<.005\end{array}$ & $\begin{array}{c}30.36 \\
8.34 \\
1.4 \\
.0 T \\
.0 T \\
.06 \\
16.3 \\
.45 \\
. .005\end{array}$ & $\begin{array}{c}29.94 \\
7.64 \\
1.4 \\
.09 \mathrm{~T} \\
.0 \mathrm{~T} \\
.06 \\
17.6 \\
.68 \\
<.005\end{array}$ & $\begin{array}{c}27.52 \\
7.40 \\
1.5 \\
.0 \\
.00 \\
.00 \\
.11 \\
20.4 \\
.96 \\
<.005\end{array}$ & $\begin{array}{c}10.92 \\
17.32 \\
3.2 \\
.09 \\
.09 \\
.09 \\
37.8 \\
.56 \\
.56 \\
<.005\end{array}$ & $\begin{array}{c}28.24 \\
11.14 \\
1.5 \\
.0 \mathrm{~T} \\
.0 \mathrm{~T} \\
.06 \\
19.5 \\
.55 \\
<.005\end{array}$ & $\begin{array}{c}28.72 \\
11.24 \\
1.7 \\
.09 \\
.09 \\
.08 \\
20.2 \\
.63 \\
<.005\end{array}$ & $\begin{array}{c}37.54 \\
4.84 \\
.86 \\
.0 \mathrm{~T} \\
.0 \mathrm{~T} \\
.05 \\
12.1 \\
.40 \\
<.005\end{array}$ & $\begin{array}{c}38.94 \\
3.40 \\
.59 \\
.0 \mathrm{~T} \\
.0 \mathrm{~T} \\
.044 \\
7.8 \\
.44 \\
<.005\end{array}$ \\
\hline Total & 56.51 & 60.90 & 56.91 & 57.32 & 57.89 & 69.89 & 60.99 & 62.57 & 55.79 & 51.21 \\
\hline Sample & 06015012 & 06015012 & 06015013 & 06015014 & 06015015 & 06015016 & 06015017 & 06015018 & 06015019 & 06015020 \\
\hline $\begin{array}{l}\mathrm{SlO}_{2} \\
\mathrm{Al}_{2} \mathrm{O}_{0} \\
\mathrm{Cr}_{2} \mathrm{O}_{2} \\
\mathrm{Ag}_{2} \\
\mathrm{Au} \\
\mathrm{CO} \\
\mathrm{Fe} \\
\mathrm{MI} \\
\mathrm{Pt}\end{array}$ & $\begin{array}{c}36.52 \\
4.84 \\
.68 \\
.0 \\
.09 \\
.04 \\
9.3 \\
.3 \\
.37 \\
<.005\end{array}$ & $\begin{array}{c}36.04 \\
5.58 \\
.92 \\
.09 \mathrm{~T} \\
.0 \mathrm{~T} \\
.05 \\
11.8 \\
.47 \\
. .005\end{array}$ & $\begin{array}{c}40.44 \\
5.32 \\
.98 \\
.0 \mathrm{~T} \\
.0 \mathrm{~T} \\
.05 \\
14.5 \\
.60 \\
<.005\end{array}$ & $\begin{array}{c}38.20 \\
5.56 \\
.61 \\
.00 \mathrm{~T} \\
.0 \mathrm{~T} \\
.05 \\
8.7 \\
.38 \\
<.005\end{array}$ & $\begin{array}{c}34.26 \\
5.38 \\
1.05 \\
.00 \mathrm{~T} \\
.0 \\
.08 \\
14.8 \\
.59 \\
<.005\end{array}$ & $\begin{array}{c}57.16 \\
4.36 \\
.79 \\
.0 \\
.0 \mathrm{~T} \\
.04 \\
21.04 \\
.39 \\
.005\end{array}$ & $\begin{array}{c}23.16 \\
6.20 \\
2.11 \\
.0 \mathrm{~T} \\
.0 \mathrm{~T} \\
. .11 \\
25.7 \\
1.03 \\
<.005\end{array}$ & $\begin{array}{c}30.04 \\
5.62 \\
1.25 \\
.0 \mathrm{~T} \\
.0 \mathrm{~T} \\
.07 \\
16.3 \\
.61 \\
. .005\end{array}$ & $\begin{array}{c}37.66 \\
3.90 \\
.79 \\
.00 \mathrm{~T} \\
.00 \mathrm{~T} \\
.04 \\
10.1 \\
.52 \\
. .005\end{array}$ & $\begin{array}{c}34.34 \\
6.10 \\
1.06 \\
.0 \mathrm{~T} \\
.0 \mathrm{~T} \\
.07 \\
15.1 \\
.66 \\
<.005\end{array}$ \\
\hline Total & 51.75 & 54.86 & 61.89 & 53.50 & 56.16 & 53.84 & 58.31 & 53.89 & 53.01 & 57.33 \\
\hline Sample & 06015021 & 06015022 & 06015023 & 06015024 & 06015025 & 06015026 & 06015027 & 06015028 & 06015029 & 06015030 \\
\hline 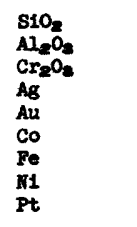 & $\begin{array}{c}35.28 \\
9.06 \\
.81 \\
.0 \\
.0 \mathrm{~T} \\
.04 \mathrm{~T} \\
13.1 \\
1.42 \\
.42 \\
<.005\end{array}$ & $\begin{array}{c}36.94 \\
7.20 \\
.52 \\
.0 \\
.0 \\
.04 \\
.04 \\
7.8 \\
.33 \\
<.005\end{array}$ & $\begin{array}{c}26.52 \\
7.02 \\
1.47 \\
.09 \\
.0 \mathrm{~T} \\
.10 \\
19.2 \\
.67 \\
<.005\end{array}$ & $\begin{array}{c}26.02 \\
7.38 \\
1.49 \\
.00 \mathrm{~T} \\
.0 \mathrm{~T} \\
.09 \\
12.3 \\
.80 \\
<.005\end{array}$ & $\begin{array}{c}26.30 \\
7.20 \\
1.17 \\
.0 \\
.0 \mathrm{~T} \\
.11 \\
22.2 \\
.8 \\
.80 \\
<.005\end{array}$ & $\begin{array}{c}31.72 \\
6.30 \\
.95 \\
.0 \\
.0 \mathrm{~T} \\
.05 \\
15.7 \\
.55 \\
<.005\end{array}$ & $\begin{array}{c}36.02 \\
4.66 \\
.94 \\
.0 \mathrm{~T} \\
.0 \mathrm{~T} \\
.05 \\
14.0 \\
.74 \\
<.005\end{array}$ & $\begin{array}{c}28.24 \\
5.60 \\
1.54 \\
.00 \mathrm{~T} \\
.0 \mathrm{~T} \\
. .11 \\
20.8 \\
.63 \\
<.005\end{array}$ & $\begin{array}{c}23.32 \\
7.24 \\
1.87 \\
.00 T \\
.00 \mathrm{~T} \\
.08 \\
22.5 \\
.69 \\
<.005\end{array}$ & $\begin{array}{c}48.12 \\
3.84 \\
.50 \\
.0 \mathrm{~T} \\
.0 \mathrm{~T} \\
.04 \\
8.4 \\
.33 \\
<.005\end{array}$ \\
\hline Total & 58.71 & 52.83 & 54.98 & 48.08 & .57 .78 & 55.27 & 56.41 & 56.92 & 55.70 & 61.23 \\
\hline Sample & 06015031 & 06015032 & & & & & & & & \\
\hline $\begin{array}{l}\mathrm{SHO}_{2} \\
\mathrm{Al}_{2} \mathrm{O}_{3} \\
\mathrm{Cr}_{2} \mathrm{O}_{3} \\
\mathrm{AB}_{3} \\
\mathrm{Au} \\
\mathrm{Co} \\
\mathrm{Pe} \\
\mathrm{HI} \\
\mathrm{Pt}\end{array}$ & $\begin{array}{c}37.30 \\
3.52 \\
.73 \\
.0 \\
.09 \\
.04 \\
.04 \\
9.6 \\
.42 \\
<.005\end{array}$ & $\begin{array}{c}38.94 \\
3.92 \\
.60 \\
.0 \\
.00 \\
.04 \\
.04 \\
8.1 \\
.33 \\
<.005\end{array}$ & & & & & & & & \\
\hline Total & 51.61 & 51.93 & & & & & & & & \\
\hline
\end{tabular}

\begin{tabular}{|c|c|c|c|c|c|c|c|c|c|c|}
\hline Semple & 06019010 & 06019011 & 06027077 & 06033005 & 06069088 & 06071362 & 06071363 & 06097020 & 06097019 & 20009001 \\
\hline $\begin{array}{l}\mathrm{SHO}_{2} \\
\mathrm{Al}_{2} \mathrm{O}_{3}\end{array}$ & $\begin{array}{l}59.16 \\
14.03\end{array}$ & $\begin{array}{r}72.48 \\
7.69\end{array}$ & $\begin{array}{c}21.40 \mathrm{~A} \\
\ldots \ldots-\end{array}$ & $\begin{array}{l}0.75 \mathrm{~A} \\
.37\end{array}$ & $\begin{array}{l}7.51 \\
2.92\end{array}$ & $\begin{array}{r}7.46 \\
39.02\end{array}$ & $\begin{array}{l}10.27 \\
38.46\end{array}$ & 7.66 & 8.36 & $\begin{array}{r}0.04 \\
34.64\end{array}$ \\
\hline $\mathrm{CoO}^{\circ}$ & 2.68 & 1.46 & $\cdots$ & .25 & .46 & -... & -... & 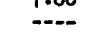 & $\cdots$ & .28 \\
\hline $\mathrm{CO}_{2}$ & .33 & .25 & $\cdots$ & $\cdots$ & $-\cdots$ & $\cdots$ & $\cdots$ & $\cdots$ & $\cdots$ & $\cdots$ \\
\hline Feo & 2.68 & 1.88 & $\cdots$ & 3.28 & $\cdots$ & $\cdots$ & $\cdots$ & 2.01 & 1.56 & $\cdots$ \\
\hline $\mathrm{Fe}_{2} \mathrm{O}_{3}$ & 2.26 & 1.36 & $\cdots$ & 26.79 & .41 & 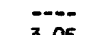 & $\cdots$ & $\cdots$ & $\cdots$ & 2.16 \\
\hline $\mathrm{K}_{2} \mathrm{O}$ & 2.14 & 1.72 &.$\infty$ & $\cdots$ & $\cdots$ & 3.05 & 1.04 & $--\cdot$ & $-\cdots$ & 6.08 \\
\hline Kgo & 5.20 & 6.76 & $\cdots$ & .16 & .35 & $\cdots$ & $\cdots$ & 7.14 & 7.51 & $.0 \mathrm{~T}$ \\
\hline $\operatorname{mon}$ & .03 & .03 & $\cdots$ & $.0 \mathrm{~T}$ & $-\cdots$ & $\cdots$ & --- & -- & -- & $\cdots$ \\
\hline $\mathrm{MaCl}$ & $\cdots$ & $\cdots$ & 25.30 & $\cdots$ & ...- & $\cdots$ & 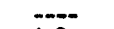 & ---- & -..- & $\cdots$ \\
\hline $\mathrm{Ma}_{2} \mathrm{O}$ & 2.36 & 1.76 & $\cdots$ & $\cdots$ & $\cdots$ & 5.27 & 6.83 & $\cdots$ & $\cdots$ & 4.62 \\
\hline $\mathrm{P}_{2} \mathrm{O}_{3}$ & .49 & .22 & $\cdots$ & $\cdots$ & $.0 \mathrm{~T}$ & $\cdots$ & 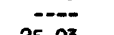 & $-\cdots$ & $\cdots$ & $\cdots$ \\
\hline $\mathrm{THO}_{2}$ & .61 & .23 & $\cdots$ & $-\cdots$ & $\cdots$ & $\cdots$ & 25.03 & $\cdots$ & $\cdots$ & .60 \\
\hline $\mathrm{CaSO}_{4}$ & $\cdots$ & $--\cdot$ & .30 & $\cdots$ & $\cdots$ & $\cdots$ & $\cdots$ & $\cdots$ & $\cdots$ & $\cdots$ \\
\hline 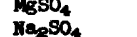 & $\cdots$ & $\cdots$ & 1.20 & $\cdots$ & $\cdots$ & $-\overline{-}$ & $-\cdots$ & $\cdots$ & $\cdots$ & $\cdots$ \\
\hline $\begin{array}{l}\mathrm{Ha}_{2} \mathrm{SO}_{4} \\
\mathrm{SO}_{3}\end{array}$ & .80 & .31 & 5.30 & 38.82 & 29.41 & 30.52 & $-\cdots$ & 38.78 & 38.30 & 37.02 \\
\hline $\mathrm{BaO}$ & -.-- & $\cdots$ & $\cdots$ & $\cdots$ & 57.19 & $\ldots$ & -..-- & - & -..- & .... \\
\hline Hiter & $\cdots$ & $\cdots$ & 46.50 & --- & $\ldots$ & --.. & -... & ---- & -..- & -... \\
\hline & 1.25 & .06 & $\cdots$ & $\cdots$ & $\cdots$ & $\cdots$ & $\cdots$ & $\cdots$ & $-\cdots$ & $\cdots$ \\
\hline 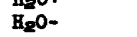 & $\begin{array}{l}2.00 \\
2.50\end{array}$ & $\begin{array}{l}1.44 \\
1.34\end{array}$ & $\cdots$ & ) 29.58 &.-- & 14.87 & 17.60 & 44.41 & 44.27 & 15.38 \\
\hline Total & 99.80 & 99.99 & 100.00 & 100.00 & 98.25 & 100.19 & 99.23 & 100.00 & 100.00 & 100.82 \\
\hline
\end{tabular}

Trace. 
TABLE 15. - Analyses of samples of various types of rocks (Group $M$ ), from California and Hawail, special-rock category-Continued

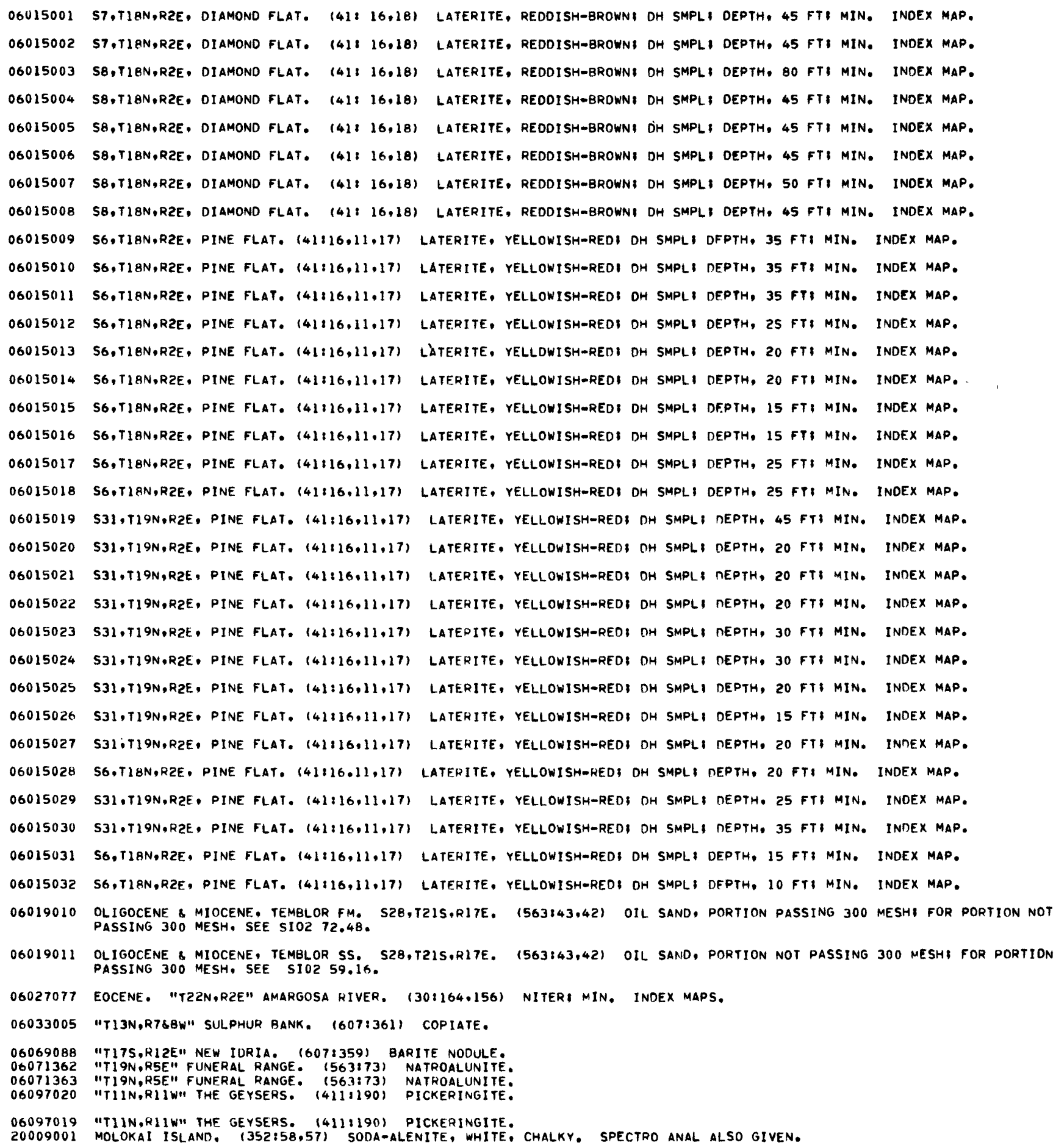


TABLE 16. - Analyses of samples of phosphate rock (Group P) from California, special rock category

[Amounts in parentheses are not included in total]

Chemical Analyses

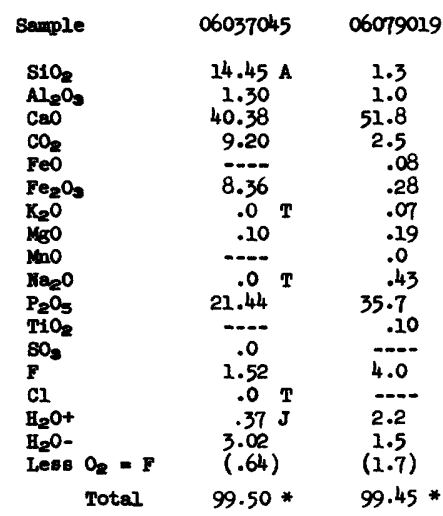

A Insoluble. T Trace. J Organic matter.

06037045 MIOCENE, MODELO FM. RIDGE WEST OF STONE CANYON, (293:106.PL16.17) PHOSPHATE. BROWN. OOLITICI RED 6 IN THICK. INDEX \& GEOL MAP. GEOL SEC. \#MISC: SIOZ = 1.21 .

06079019 MIOCENE, MONTEREY FM. S20.T285,R15E. (224:084,080) PHOSPHATE, PELLETAL, MIN BEDS TO 10 IN THICK. INDEX MAP.

TABLE 17. - Analyses of samples of manganiferous opal (Group Mn) from California, special-rock category

Chemical Anelyses

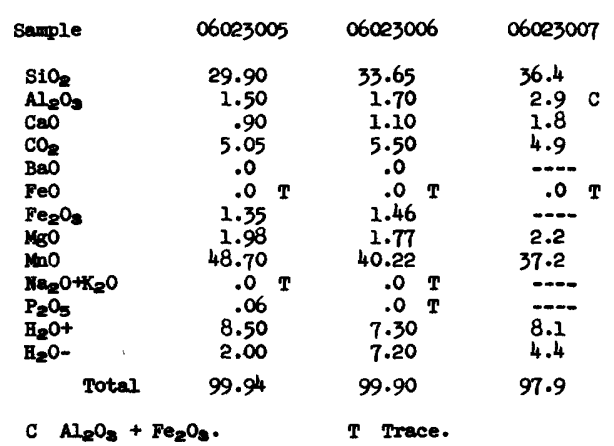

\footnotetext{
06023005 JURASSIC, KNOXVILLE FM. S3.T1S,R4E. $(482: 257.217 .240)$ MANGANIFEROUS OPAL, AROWNI 4 FT THICK. INOEX \& GEOL MAPS. 06023006 JURASSIC, KNOXVILLE FM. S3,TIS,R4E, (482:257,217,240) MANGANIFEROUS OPAL, BROWN 4 FT THICK. INDEX \& GEOL MAP. 06023007 JURASSIC, KNOXVILLE FM. S3.TIS.R4E. (482:257.217.240) MANGANIFEROUS OPAL. RROWN 4 FT THICK. INDEX \& GEOL MAP.
} 
TABLE 23. - Code numbers for state and county

\begin{tabular}{|c|c|}
\hline \multicolumn{2}{|c|}{ State codes } \\
\hline 6. California & 20. Hawaii \\
\hline \multicolumn{2}{|c|}{ California county codes } \\
\hline $\begin{array}{l}\text { 1. Alameda } \\
\text { 3. Alpine } \\
\text { 5. Amador } \\
\text { 7. Butte } \\
\text { 9. Calaveras } \\
\text { 11. Colusa } \\
\text { 13. Contra Costa } \\
\text { 15. Del Norte } \\
\text { 17. El Dorado } \\
\text { 19. Fresno } \\
\text { 21. Glenn } \\
\text { 23. Humboldt } \\
\text { 25. Imperial } \\
\text { 27. Inyo } \\
\text { 29. Kern } \\
\text { 31. Kings } \\
\text { 33. Lake } \\
\text { 35. Lassen } \\
\text { 37. Los Angeles } \\
\text { 39. Madera } \\
\text { 41. Marin } \\
\text { 43. Mariposa } \\
\text { 45. Mendocino } \\
\text { 47. Merced } \\
\text { 49. Modoc } \\
\text { 51. Mono } \\
\text { 53. Monterey } \\
\text { 55. Napa } \\
\text { 57. Nevada }\end{array}$ & $\begin{array}{l}\text { 59. Orange } \\
\text { 61. Placer } \\
\text { 63. Plumas } \\
\text { 65. Riverside } \\
\text { 67. Sacramento } \\
\text { 69. San Benito } \\
\text { 71. San Bernardino } \\
\text { 73. San Diego } \\
\text { 75. San Francisco } \\
\text { 77. San Joaquin } \\
\text { 79. San Luis Obispo } \\
\text { 81. San Mateo } \\
\text { 83. Santa Barbara } \\
\text { 85. Santa Clara } \\
\text { 87. Santa Cruz } \\
\text { 89. Shasta } \\
\text { 91. Sierra } \\
\text { 93. Siskiyou } \\
\text { 95. Solono } \\
\text { 97. Sonoma } \\
\text { 99. Stanislaus } \\
\text { 101. Sutter } \\
\text { 103. Tehama } \\
\text { 105. Trinity } \\
\text { 107. Tulare } \\
\text { 109. Tuolumne } \\
\text { 111. Ventura } \\
\text { 113. Yolo } \\
\text { 115. Yuba }\end{array}$ \\
\hline \multicolumn{2}{|c|}{ Hawaii county codes } \\
\hline $\begin{array}{l}\text { 1. Hawaii } \\
\text { 3. Honolulu }\end{array}$ & $\begin{array}{l}\text { 7. Kauai } \\
\text { 9. Maui }\end{array}$ \\
\hline
\end{tabular}

TABLE 24. - Code numbers for use of rock

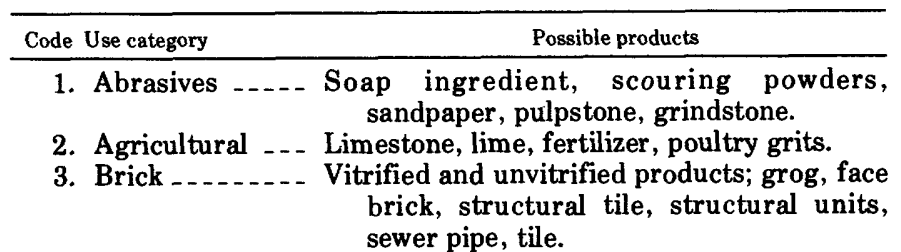

4. Cement material ..... Portland cement, concrete additives, and mixtures.

5. Ceramics -... Porcelain, china, pottery, slag, aggregate, glaze, earthenware, stoneware, terra cotta, whiteware.

6. Chemical and industrial .... Bleaching, clarifying, purification, filtering, suspending agent, carrier, water softener, carbide (acetylene gas), insecticide, manufacture of alkalies, pulp and paper, and sugar.

7. Construction -. - Railroad ballast, rip-rap, crushed stone, heavy concrete aggregate, road metal, dam cores, jetties.

8. Drilling mud -.-

9. Filler ...... Paper, paints, putty, bonding agent, whiting, soap, cosmetics.

10. Glass . . . . . .

11. Insulating material ..... Acoustical, insulating tiles and blocks, lightweight aggregate.

12. Metallurgical -- Flux, foundry sand, molding sands, bonding clays.

13. Pigments -... -

14. Refractories .. - Electrical insulators, refractory brick.

15. Structural ..... Building stone, dimension stone, flagging, roofing, terrazzo, monumental and ornamental stone stucco, plaster.

16. Source of aluminum or some other metal ....... Magnesium, vanadium, fluorine, steel, pig iron.

17. Miscellaneous _. 
TABLE 25. - Code numbers of authors

1. Allen, J. E. (1946)

2. Allen, R. D., and Kramer (1953)

3. Allen, V. T. (1929)

4. Allen, V. T. (1941)

5. Murata and Richter (1966)

6. Ames and others (1958)

7. Anderson, C. A. (1933a)

8. Anderson, C. A. (1933b)

9. Anderson, C. A. (1936)

10. Schneider (1927)

11. Becker (1888)

12. Oesterling and Spurck (1964a)

13. Anderson, C. A., and Russell (1939)

14. Anderson, F. M. (1899)

15. Anderson, F. M. (1911)

16. Anderson, Robert, and Pack (1915)

17. Angel (1908)

18. Arnold and Anderson, Robert (1907)

20. Arnold and Johnson, H. R. (1909)

21. Aubury (1906)

22. Aune (1965)

23. Averill (1929)

25. Averill (1937)

26. Averill (1939)

27. Averill (1941)

28. Averill (1947)

29. Bailey, E. H., and others (1964)

30. Bailey, G. E. ( 1902)

31. Hodge (1938a)

34. Bateman (1965)

35. Sherman and others (1953)

36. Bates, P. H., and others (1923)

37. Bates, T. F. (1945)

40. Bailey, E. H., and Everhart (1964)

41. Benson (1963)

43. Boalich and others (1920)

45. Burgess (1917)

47. Booth (1880)

48. Walker, J. L., and others (1969)

51. Bowen, N. L., and Aurousseau (1923)

52. Bowen (1954)

53. Bowen and Gray (1957)

54. Bowen and Gray (1959)

55. Bowen and Gray (1962)

56. Bowen and Ver Planck (1965)

57. Bradley (1916)

58. Bradley (1925)

60. Bradley and Logan (1919)

61. Bramlette (1946)

65. Burnham (1959)

66. Burnett (1965)

67. Burchard (1911)

68. Burchard (1912)

69. Ross and Hendricks (1941)

\section{California Division of} Mines (1948)

71. California Division of Mines (1957)

72. Irelan (1888)

73. Crawford (1896)

75. Calhoun and Hill (1962)

79. Campbell (1902)

80. Carlson and Clark, W. B. (1954)

82. Chandra (1961)

83. Chapman (1946)

84. Chatard (1890)

86. Chesterman (1943)

87. Chesterman (1956)

93. Clark, L. D. ( 1954)

94. Clark, W. B. (1954)

95. Clark, W. B., and Carlson (1956)

96. Clark, W. B., and Lydon (1962)

98. Clarke $(1890)$

99. Clarke (1891)

101. Clarke (1903)

103. Clarke (1908)

111. Clarke and Hillebrand (1897)

113. Cleveland (1957)

114. Cleveland (1958)

115. Cleveland (1960)

116. Cleveland (1961)

117. Cleveland (1962)

118. Weber (1963b)

120. Cloudman and others (1919)

127. Cross (1915)

129. Crawford (1894)

130. Creely (1965)

131. Cressman (1962)

132. Dana (1852)

137. Davis, E. F. (1918a)

138. Davis, E. F. (1918b)

139. Davis, F. F. (1955)

140. Davis, C. W., and Vacher (1928)

141. Day, A. L., and Allen, E. T. (1925)

142. Day, W. C. (1892)

144. Day, W. C. (1898)

145. Day, W. C. $(1899)$

147. Dietrich (1928)

148. Dietrich and Meyer (1930)

151. Diller (1890)

152. Diller (1891)

154. Diller (1903)

155. Diller (1904)

156. Diller (1906)

157. Diller (1911)

158. Dolbear (1913)

160. Durrell (1953)

161. Dupont (1910)

162. Eakle (1911)

163. Eakle (1914)

164. Eakle (1917)

165. Eakle (1923)

169. Eardley-Wilmot (1928)

170. Eckel and Myers (1946)

172. Eckel (1912)

173. Eckel (1913)

174. Eckel (1928)

TABLE 25. - Code numbers of authors - Continued

175. Eckel (1934)

176. Sherman (1950)

185. Engel and others (1959)

186. Ernst and others (1970)

187. Fairbanks (1896)

188. Fairbanks (1904)

193. Fiske and Koyanagi (1968)

199. Franke (1930)

200. Franke (1931)

201. Franke (1936)

202. Fraser and others (1943)

207. Gale (1912)

209. Gale (1914a)

210. Gale $(1914 b)$

211. Gale (1914c)

213. Gale (1946)

221. Goldman (1959)

222. Goodyear (1890)

224. Gower and Madsen (1964)

228. Gray (1961)

229. Gray (1962c)

230. Gray (1962a)

231. Gray (1962b)

236. Hagner (1939)

250. Hall, C. W., and Sardeson (1895)

251. Hall, W. E., and Stephens (1963)

253. Hanks (1882)

254. Hanks (1883)

255. Hanks (1884)

256. Hanks (1885)

260. Harder (1910)

261. Harder (1912)

264. Hart (1959)

265. Hart (1966)

268. Hay and Iijima (1968)

269. Hazzard (1938)

270. Hay and Jones, B. F. (1962)

273. Heiken (1972)

276. Hewett (1956)

279. Heyl and Walker (1949)

280. Heyl and Wiese (1949)

282. Hidden ( 1885 )

283. Hietanen (1951)

284. Hilgard (1892)

285. Hill (1923)

289. Hinds (1929)

290. Hodge (1938c)

291. Hodge (1935)

292. Hoots (1930)

293. Hoots (1931)

297. Huguenin (1919)

298. Huguenin and Castello (1921a)

299. Huguenin and Castello (1921b)

303. Irelan (1890)

305. Jones, W. F. ( 1911)

306. Johnson and Ricker (1949)

307. Johnston ( 1890)

309. Jones, C. C. (1916)

312. Keenan (1932)

315. Kelley and others (1959)

318. Kerrick and Cotton (1971)

321. Kerr and others (1950)

322. Kessler (1919)

327. King (1948)
329. Kinkel and others (1956)

331. Kivari (1942)

337. Kümmel (1901)

338. Ladoo and Myers (1951)

339. Laizure (1925a)

340. Laizure (1925b)

341. Laizure (1925c)

342. Laizure (1926)

345. Laizure (1929)

346. Lamar (1953)

347. Lamey (1946)

352. Laudermilk (1935)

353. Laudermilk and Woodford (1932)

354. Laudermilk and Woodford ( 1934)

356. Lawson (1893)

361. Lindgren (1896)

362. Lindgren (1898)

364. Linton (1928)

366. Logan (1926)

371. Loney (1968)

372. Lockwood (1971)

374. Lowell (1916)

375. Lyons (1896)

376. Lydon (1964)

377. MacBoyle (1920)

379. Macdonald and Katsura (1965)

386. Murata and others (1972)

388. Merrill (1919)

389. Milton and Johnston (1938)

390. Moore (1935)

391. Moore (1936)

393. Muffler and Doe (1968)

394. Muffler and White (1969)

395. Murdoch and Webb (1948)

396. Murdoch and Webb (1956)

400. Norton (1939)

401. Norman, James, and Ralston (1942)

402. Norman, L. A., Jr., and Stewart (1951)

403. Nutting (1943)

404. Oakeshott (1937)

406. O'Brien (1953)

408. Oesterling and Spurck (1964b)

409. Ogle (1953)

410. Osthaus (1955)

411. Pabst (1938)

412. Pack (1914)

417. Pask and Turner (1952)

420. Pearson and others (1967)

421. Patterson (1971)

423. Powers (1932)

424. Prescott (1908)

428. Ralston and others (1925)

433. Richter and others (1964)

436. Rubey and Callaghan (1936)

437. Rollins (1951)

439. Ross and Hendricks (1945)

440. Ross and Shannon (1926)

443. Russell (1889)

445. Sampson (1937)

446. Sampson and Tucker (1931) 
TABLE 25. - Code numbers of authors - Continued

\begin{tabular}{|c|c|c|c|}
\hline 447. & $\begin{array}{l}\text { Sampson and Tucker } \\
(1942)\end{array}$ & $\begin{array}{l}530 . \\
531 .\end{array}$ & $\begin{array}{l}\text { Watts }(1897) \\
\text { Weaver }(1949)\end{array}$ \\
\hline 453. & $\begin{array}{l}\text { Schroter and Campbell } \\
\text { (1942) }\end{array}$ & $\begin{array}{l}532 . \\
534 .\end{array}$ & $\begin{array}{l}\text { Weber (1963a) } \\
\text { Weigel (1927) }\end{array}$ \\
\hline 457. & Slaughter and Earley & 535. & Weitz $(1942)$ \\
\hline 459. & $\begin{array}{l}(1965) \\
\text { Shelton }(1955)\end{array}$ & 541. & $\begin{array}{l}\text { Whitehouse and others } \\
\quad(1960)\end{array}$ \\
\hline 460. & $\begin{array}{l}\text { Sheppard and Gude } \\
(1965 \mathrm{~b})\end{array}$ & $\begin{array}{l}543 . \\
546 .\end{array}$ & $\begin{array}{l}\text { Williams ( } 1932) \\
\text { Wright, T. L. (1971) }\end{array}$ \\
\hline 461. & $\begin{array}{l}\text { Sheppard and Gude } \\
\text { (1969) }\end{array}$ & $\begin{array}{l}548 . \\
550 .\end{array}$ & $\begin{array}{l}\text { Winston ( } 1947 \text { ) } \\
\text { Woodford and Harris }\end{array}$ \\
\hline 463. & Simpson (1935) & & $(1928)$ \\
\hline $\begin{array}{l}464 . \\
465 .\end{array}$ & $\begin{array}{l}\text { Smith, C. A. (1924) } \\
\text { Sheppard and Gude }\end{array}$ & 552. & $\begin{array}{l}\text { Wright, L. A. and } \\
\text { others (1953) }\end{array}$ \\
\hline 466. & $\begin{array}{l}\text { (1965a) } \\
\text { Smith, G. I. (1973) }\end{array}$ & 553. & $\begin{array}{l}\text { Yale and Stone } \\
(1921)\end{array}$ \\
\hline 467. & Speil and others (1945) & 554. & Youngman (1930) \\
\hline 470. & Spurck (1964) & 555. & Eckel (1905) \\
\hline 478. & Sutherland (1935) & 556. & Logan $(1947)$ \\
\hline 480. & Takahashi (1929) & 557. & Hodge (1944) \\
\hline 481. & Taliaferro (1943) & 558. & Hodge (1938b) \\
\hline 482. & $\begin{array}{l}\text { Taliaferro and Hudson } \\
\text { (1943) }\end{array}$ & $\begin{array}{l}559 . \\
563 .\end{array}$ & $\begin{array}{l}\text { Merrill (1916) } \\
\text { Wells (1937) }\end{array}$ \\
\hline 485. & Townsend (1933) & 564. & Ladoo (1948) \\
\hline 489. & Tucker (1919) & 565. & Clarke (1889) \\
\hline 490. & Tucker (1921) & 566. & Burohfiel (1923) \\
\hline 491. & Tucker (1923a) & 567. & Ladoo (1921) \\
\hline 492. & Tucker (1923b) & 568. & Dickinson (1962) \\
\hline 493. & Tucker (1924) & 569. & Ladoo (1920) \\
\hline 494. & Tucker (1925a) & 570. & Norton (1952) \\
\hline 496. & Tucker (1925b) & 571. & Tischler (1964) \\
\hline 497. & Tucker (1926) & 572. & Goldsmith and others \\
\hline 498. & Tucker (1927a) & & $(1962)$ \\
\hline 499. & Tucker (1927b) & 573. & Foshag and Woodford \\
\hline 500. & Tucker (1929) & & $(1936)$ \\
\hline 501. & $\begin{array}{l}\text { Tucker and Sampson } \\
\text { (1929) }\end{array}$ & $\begin{array}{l}574 . \\
575 .\end{array}$ & $\begin{array}{l}\text { Kerr and Cameron (1936) } \\
\text { Lawson (1914) }\end{array}$ \\
\hline 502. & Tucker and Sampson & 576. & Bloxam (1960) \\
\hline 503. & $\begin{array}{l}(1930) \\
\text { Tucker and Sampson }\end{array}$ & 577. & $\begin{array}{l}\text { Wentworth and others } \\
(1940)\end{array}$ \\
\hline & (1931) & 578. & Bloxam ( 1956) \\
\hline 504. & $\begin{array}{l}\text { Tucker and Sampson } \\
\text { (1933) }\end{array}$ & $\begin{array}{l}580 . \\
583 .\end{array}$ & $\begin{array}{l}\text { Bloxam (1959) } \\
\text { Glaser (1971) }\end{array}$ \\
\hline 505. & $\begin{array}{l}\text { Tucker and Sampson } \\
\text { (1938) }\end{array}$ & $\begin{array}{l}585 . \\
589 .\end{array}$ & $\begin{array}{l}\text { Stewart }(1970) \\
\text { Everhart }(1957)\end{array}$ \\
\hline 506. & $\begin{array}{l}\text { Tucker and Sampson } \\
\text { (1940) }\end{array}$ & $\begin{array}{l}600 . \\
601 .\end{array}$ & $\begin{array}{l}\text { Foshag (1935) } \\
\text { Gale (1915) }\end{array}$ \\
\hline 507. & $\begin{array}{l}\text { Tucker and Sampson } \\
\text { (1944) }\end{array}$ & $\begin{array}{l}602 . \\
603 .\end{array}$ & $\begin{array}{l}\text { Hess }(1910) \\
\text { Phalen }(1911)\end{array}$ \\
\hline 508. & Turner (1891) & 604. & Schaller (1930) \\
\hline 510. & Turner (1898) & 605. & Foshag (1938) \\
\hline 512. & Tyson (1851) & 606. & Knopf ( 1918$)$ \\
\hline 521. & Ver Planck (1955) & 607. & Clarke (1915) \\
\hline 523. & Ver Planck (1958) & 608. & Foshag (1918) \\
\hline 524. & Vitaliano $(1950)$ & 609. & Patterson (1964) \\
\hline 525. & Walker, G. W. (1950a) & 611. & Cathcart (1958) \\
\hline 526. & Walker, G. W. (1950b) & 612. & Osthaus (1956) \\
\hline 529. & Washington (1917) & 613. & Loew (1876) \\
\hline
\end{tabular}



GPO 587-041/19 